In cooperation with the West Virginia Department of Environmental Protection, Division of Water and Waste Management

\title{
Low-Flow Analysis and Selected Flow Statistics Representative of 1930-2002 for Streamflow-Gaging Stations In or Near West Virginia
}

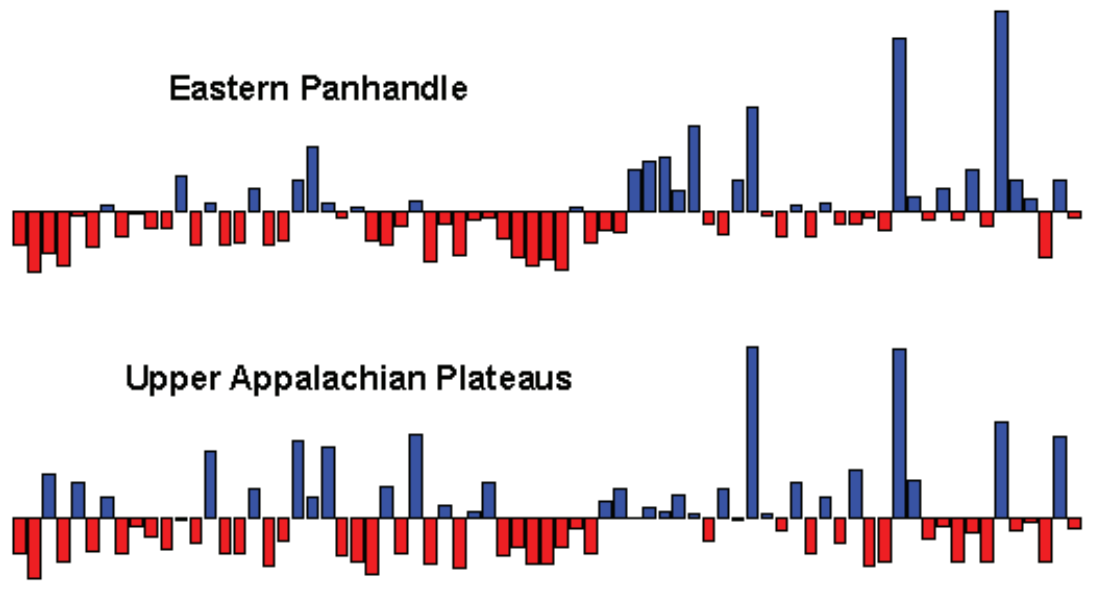

Lower Appalachian Plateaus

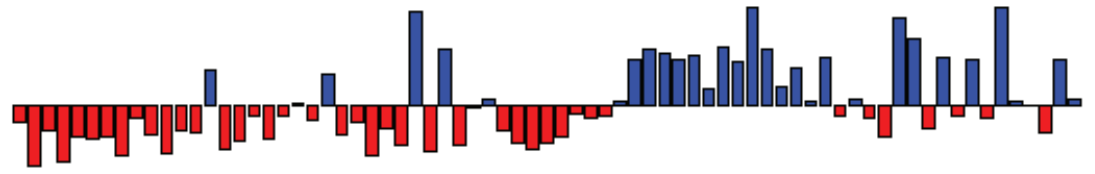

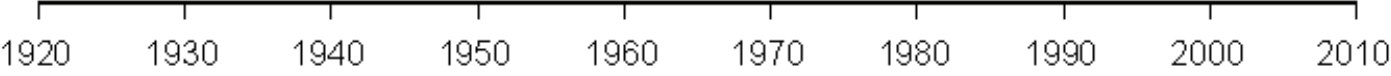

Scientific Investigations Report 2006-5002 


\title{
Low-Flow Analysis and Selected Flow Statistics Representative of 1930-2002 for Streamflow-Gaging Stations In or Near West Virginia
}

\author{
By Jeffrey B. Wiley
}

In cooperation with the West Virginia Department of Environmental Protection, Division Of Water and Waste Management

Scientific Investigations Report 2006-5002 


\title{
U.S. Department of the Interior \\ Gale A. Norton, Secretary \\ U.S. Geological Survey \\ P. Patrick Leahy, Acting Director
}

\section{U.S. Geological Survey, Reston, Virginia: 2006}

\author{
For sale by U.S. Geological Survey, Information Services \\ Box 25286, Denver Federal Center \\ Denver, CO 80225 \\ For more information about the USGS and its products: \\ Telephone: 1-888-ASK-USGS \\ World Wide Web: http://www.usgs.gov/
}

\footnotetext{
Any use of trade, product, or firm names in this publication is for descriptive purposes only and does not imply endorsement by the U.S. Government.

Although this report is in the public domain, permission must be secured from the individual copyright owners to reproduce any copyrighted materials contained within this report.

Suggested citation:

Wiley, J.B., 2006, Low-flow analysis and selected flow statistics representative of 1930-2002 for streamflow-gaging stations in or near West Virginia: U.S. Geological Survey Scientific Investigations Report 2006-5002, 190 p.
} 


\section{Contents}

Abstract

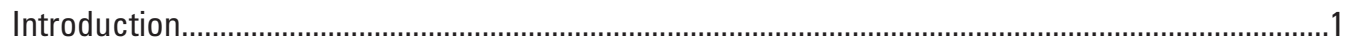

Previous Studies of National Trends in Streamflow Statistics.................................................2

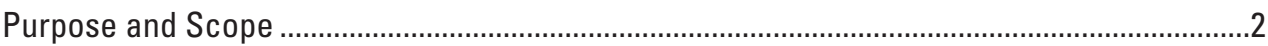

Selected Flow Statistics and Methods of Statistical Analysis...................................................

Patterns in Minimum Flows.........................................................................................................

Increase in Minimum Flows Around 1970 ...........................................................................

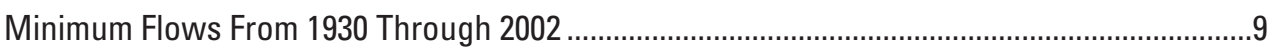

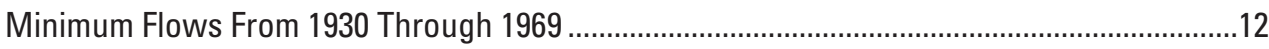

Minimum Flows From 1970 Through 2002 ......................................................................12

Minimum Flows Before 1930 ………............................................................................

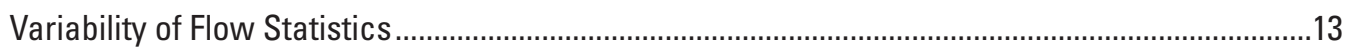

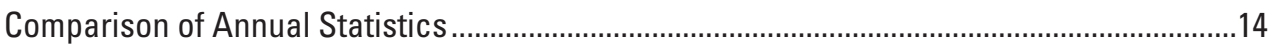

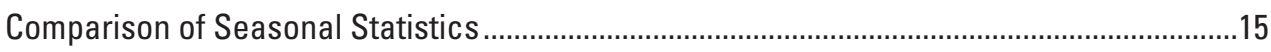

Comparison Between Hydrologically Based and Biologically Based Low Flows ..................20

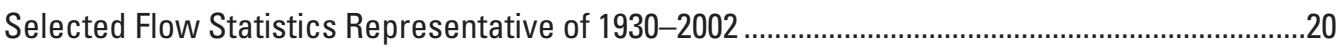

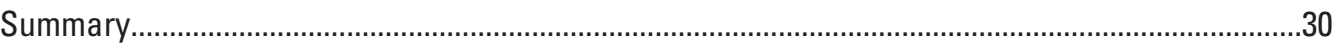

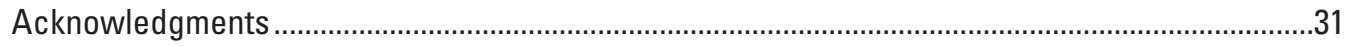

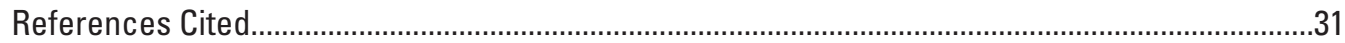

\section{Figures}

1. Map showing U.S. Geological Survey streamflow-gaging stations in or near West Virginia considered in this study .........................................................................

2-7. Graphs showing -

2. Departure of the minimum flows from the record-period average for Greenbrier River at Alderson, West Virginia

3. Departure of the unit mimium flows from the record-period average for the combined stations Bluestone River near Pipestem and at Lilly, West Virginia ........10

4. Average standardized departures of the minimum flows from the record-period average for 1930-2002 for 15 stations in West Virginia

5. Average standardized departures of the minimum flows from the record-period verage for 1930-1969 for 29 stations in West Virginia.

6. Average standardized departures of the minimum flows from the record-period average for 1970-2002 for 32 stations in West Virginia

7. Departure of the minimum flows from the record-period average for Shenandoah River at Millville, West Virginia

8. Map showing U.S. Geological Survey streamflow-gaging stations used to determine three regions of similar minimum flow patterns: Lower Appalachian Plateaus, Upper Appalachian Plateaus, and Eastern Panhandle. 
9-16. Graphs showing -

9. Average standardized departures of the minimum flows from the recordperiod average for 1930-2002 for six stations in the Lower Appalachian Plateaus region

10. Average standardized departures of the minimum flows from the recordperiod average for 1930-2002 for six stations in the Upper Appalachian Plateaus region.

11. Average standardized departures of the minimum flows from the recordperiod average for 1930-2002 for five stations in the Eastern Panhandle region.

12. Record periods for the Lower Appalachian Plateaus region for which the average departure was within 5 percent of the standard deviation of the standardized departures of the minimum flows for 1930-2002. .26

13. Record periods for the Upper Appalachian Plateaus region for which the average departure was within 5 percent of the standard deviation of the standardized departures of the minimum flows for 1930-2002

14. Record periods for the Eastern Panhandle region for which the average departure was within 5 percent of the standard deviation of the standardized departures of the minimum flows for 1930-2002

15. Unit average minimum flows for the entire record period at 110 stations in West Virginia...

16. Unit average minimum flows for the subset record period at 77 stations in West Virginia

\section{Tables}

1. U.S. Geological Survey streamflow-gaging stations in or near West Virginia considered in this study

2. Average differences between selected annual statistics computed for 1930-2002 and the statistics computed for the indicated record periods for 15 stations in West Virginia

3. Differences between selected annual statistics computed for 1930-2002 and the statistics computed for the indicated record periods for 15 stations in West Virginia.

4. Average differences between selected seasonal statistics computed for 1930-2002 and the statistics computed for the indicated record periods for 15 stations in West Virginia

5. Differences between selected seasonal statistics computed for 1930-2002 and the statistics computed for the indicated record periods for 15 stations in West Virginia

6. Average annual and seasonal differences between the 1-day 10-year hydrologically based and 1-day 3-year biologically based low-flow frequencies and between the 7-day 10-year hydrologically based and 4-day 3-year biologically based low-flow frequencies computed for 1930-2002 for 15 stations in West Virginia 
7. Annual and seasonal differences between the 1-day 10-year hydrologically based and 1-day 3-year biologically based low-flow frequencies and between the 7-day 10-year hydrologically based and 4-day 3-year biologically based low-flow frequencies computed for 1930-2002 for 15 stations in West Virginia.

8. Record periods for the Lower Appalachian Plateaus region for which the indicated average departure was within 5 percent of the standard deviation of the standardized departures of the minimum flows for 1930-2002

9. Record periods for the Upper Appalachian Plateaus region for which the indicated average departure was within 5 percent of the standard deviation of the standardized departures of the minimum flows for 1930-2002 .......50

10. Record periods for the Eastern Panhandle region for which the indicated average departure was within 5 percent of the standard deviation of the standardized departures of the minimum flows for 1930-2002

11. Selected annual and seasonal statistics for 77 stations in West Virginia representative of 1930-2002

12. Selected annual and seasonal statistics for 40 stations in surrounding states near West Virginia representative of 1930-2002.

13. Comparison between the average minimum flows computed for the entire record period and the subset record period for 110 stations in West Virginia

\section{Conversion Factors and Datums}

\begin{tabular}{|c|c|c|}
\hline Multiply & By & To obtain \\
\hline \multicolumn{3}{|c|}{ Length } \\
\hline inch (in.) & 2.54 & centimeter $(\mathrm{cm})$ \\
\hline inch (in.) & 25.4 & millimeter (mm) \\
\hline foot $(\mathrm{ft})$ & 0.3048 & meter $(\mathrm{m})$ \\
\hline mile (mi) & 1.609 & kilometer (km) \\
\hline \multicolumn{3}{|c|}{ Area } \\
\hline square foot $\left(\mathrm{ft}^{2}\right)$ & 0.0929 & square meter $\left(\mathrm{m}^{2}\right)$ \\
\hline square mile $\left(\mathrm{mi}^{2}\right)$ & 2.590 & square kilometer $\left(\mathrm{km}^{2}\right)$ \\
\hline \multicolumn{3}{|c|}{ Volume } \\
\hline cubic foot $\left(\mathrm{ft}^{3}\right)$ & 0.02832 & cubic meter $\left(\mathrm{m}^{3}\right)$ \\
\hline \multicolumn{3}{|c|}{ Flow rate } \\
\hline cubic foot per second $\left(\mathrm{ft}^{3} / \mathrm{s}\right)$ & 0.02832 & cubic meter per second $\left(\mathrm{m}^{3} / \mathrm{s}\right)$ \\
\hline
\end{tabular}




\title{
Low-Flow Analysis and Selected Flow Statistics Representative of 1930-2002 for Streamflow-Gaging Stations In or Near West Virginia
}

\author{
By Jeffrey B. Wiley
}

\section{Abstract}

Five time periods between 1930 and 2002 are identified as having distinct patterns of annual minimum daily mean flows (minimum flows). Average minimum flows increased around 1970 at many streamflow-gaging stations in West Virginia. Before 1930, however, there might have been a period of minimum flows greater than any period identified between 1930 and 2002. The effects of climate variability are probably the principal causes of the differences among the five time periods.

Comparisons of selected streamflow statistics are made between values computed for the five identified time periods and values computed for the 1930-2002 interval for 15 streamflow-gaging stations. The average difference between statistics computed for the five time periods and the 1930-2002 interval decreases with increasing magnitude of the low-flow statistic. The greatest individual-station absolute difference was 582.5 percent greater for the 7-day 10-year low flow computed for 1970-1979 compared to the value computed for 1930-2002. The hydrologically based low flows indicate approximately equal or smaller absolute differences than biologically based low flows. The average 1-day 3-year biologically based low flow (1B3) and 4-day 3-year biologically based low flow (4B3) are less than the average 1-day 10-year hydrologically based low flow (1Q10) and 7-day 10-year hydrologic-based low flow (7Q10) respectively, and range between 28.5 percent less and 13.6 percent greater. Seasonally, the average difference between low-flow statistics computed for the five time periods and 1930-2002 is not consistent between magnitudes of low-flow statistics, and the greatest difference is for the summer (July 1-September 30) and fall (October 1-December $31)$ for the same time period as the greatest difference determined in the annual analysis. The greatest average difference between $1 \mathrm{~B} 3$ and 4B3 compared to $1 \mathrm{Q} 10$ and 7Q10, respectively, is in the spring (April 1-June 30), ranging between 11.6 and 102.3 percent greater.

Statistics computed for the individual station's record period may not represent the statistics computed for the period 1930 to 2002 because (1) station records are available predom- inantly after about 1970 when minimum flows were greater than the average between 1930 and 2002 and (2) some shortterm station records are mostly during dry periods, whereas others are mostly during wet periods. A criterion-based sampling of the individual station's record periods at stations was taken to reduce the effects of statistics computed for the entire record periods not representing the statistics computed for 1930-2002. The criterion used to sample the entire record periods is based on a comparison between the regional minimum flows and the minimum flows at the stations. Criterionbased sampling of the available record periods was superior to record-extension techniques for this study because more stations were selected and areal distribution of stations was more widespread. Principal component and correlation analyses of the minimum flows at 20 stations in or near West Virginia identify three regions of the State encompassing stations with similar patterns of minimum flows: the Lower Appalachian Plateaus, the Upper Appalachian Plateaus, and the Eastern Panhandle. All record periods of 10 years or greater between 1930 and 2002 where the average of the regional minimum flows are nearly equal to the average for 1930-2002 are determined as representative of 1930-2002. Selected statistics are presented for the longest representative record period that matches the record period for 77 stations in West Virginia and 40 stations near West Virginia. These statistics can be used to develop equations for estimating flow in ungaged stream locations.

\section{Introduction}

Information on the low-flow statistics of streams is essential for development and management of West Virginia's surface-water resources. Such information is useful for assessing the availability of water for municipal or industrial supplies, irrigation, recreation, aquatic life and wildlife conservation, and disposal of liquid wastes. Low-flow statistics also are useful as a basis for forecasting seasonal low flows, as indicators of the amount of ground-water inflow to streams, and as legal indexes for maintaining water-quality standards. 
Low flows can be affected by mining (Hobba, 1981; Puente and Atkins, 1989; Borchers and others, 1991; Wiley and others, 2001); urbanization; land-use practices such as logging and farming; and construction of small detention reservoirs for flood control, recreation, and low-flow augmentation. Low flows that may be associated with climate change also have been observed (Karl and Knight, 1998; Lins and Slack, 1999; McCabe and Wolock, 2002).

The reliability of low-flow frequency curves is related to the record period, and confidence is increased if the record period includes a substantial drought; a record period representing long-term flow characteristics is desired (Riggs, 1972, p. 6). Arbitrarily using entire record periods at streamflowgaging stations will result in statistics that, for some stations, represent wet or dry periods because all or most of the entire record period is significantly different from average conditions over a longer term. Low-flow statistics representative of average conditions expected over a defined time period that includes a substantial drought are better than arbitrary use of entire record periods from stations that may not represent long-term average conditions.

\section{Previous Studies of National Trends in Streamflow Statistics}

An increase in low flows in the eastern United States around 1970 was identified by McCabe and Wolock (2002). The annual maximum, median, and minimum daily mean flows for 400 streamflow-gaging stations across the entire United States were analyzed for 1941-99. A standardized departure at each station was computed by subtracting the average annual maximum, median, and minimum daily mean flows for 1941-99 from the respective daily flows at each station and then dividing by the respective standard deviation. The standardized departures were averaged for all 400 stations for each year. An abrupt change in the average standardized departures from a negative value to a positive value was apparent at about 1970, indicating an increase in flows. The increase was most evident in the minimum and median flows. The increase in flows around 1970 was coincident with increases in precipitation, particularly in the fall (September 1-November 30), in the eastern United States (Karl and Knight, 1998).

Lins and Slack (1999) studied 395 streamflow-gaging stations across the United States for changes in flow for 1944-93. By the use of the Mann-Kendall test, flows were evaluated at several flow percentiles between and including the annual minimum and annual maximum daily mean flows for six time periods between 1944 and 1993. The study indicated an increase in flow for the annual minimum to about the 50percent flow percentile but no significant increase or decrease in annual maximum flow. Causes for the trends observed were associated with precipitation patterns, variations in ocean characteristics, and increasing atmospheric carbon dioxide in climate model simulations.

\section{Purpose and Scope}

This report, prepared in cooperation with the West Virginia Department of Environmental Protection, Division of Water and Waste Management, evaluates patterns in the annual minimum daily mean flows, referred to as "minimum flows" in this report, and low-flow statistics at U.S. Geological Survey streamflow-gaging stations in or near West Virginia (fig. 1). Five periods consisting of similar patterns in minimum flows are identified, and some flow statistics are computed for those periods. The flow statistics for the periods are compared to the statistics for the long-term period (1930-2002) to quantify the magnitude of statistical variability of low flows. A procedure is developed to select record periods at stations that are representative of streamflows for a long time period (relative to the length of available records) that can be used to develop estimating equations for flow at ungaged stream locations. Selected flow statistics are computed for stations in or near West Virginia. Statistics for stations in West Virginia supersede those published by Friel and others (1989). Statistics for stations in surrounding states can be used to assist in estimating flows for West Virginia but do not supersede the values determined for use in that state.

\section{Selected Flow Statistics and Methods of Statistical Analysis}

The following statistics were computed for this study: the annual-minimum daily-mean flow (minimum flow); the 1-day 2-year (1Q2), 1-day 5-year (1Q5), 1-day 10-year (1Q10), 3-day 2-year (3Q2), 3-day 5-year (3Q5), 3-day 10year (3Q10), 7-day 2-year (7Q2), 7-day 5-year (7Q5), 7-day 10-year (7Q10), 14-day 2-year (14Q2), 14-day 5-year (14Q5), 14-day 10-year (14Q10), 30-day 2-year (30Q2), 30-day 5-year (30Q5), and 30-day 10-year (30Q10) hydrologically based low-flow frequencies; the 1-day 3-year (1B3) and 4-day 3-year (4B3) biologically based low-flow frequencies; the U.S. Environmental Protection Agency (EPA) harmonic-mean flows; the 5-, 10-, 15-, 20-, 25-, 30-, 35-, 40-, 45-, 50-, 55-, 60-, 65-, 70-, 75-, 80-, 85-, 90-, 95-, and 99-percent flow durations; and the variability index. The following statistical methods were used: the Mann-Kendall test for trend (Mann, 1945; Kendall 1975; Hirsch and others, 1982; Helsel and Hirsch, 2002), principal component analysis, and correlation (Helsel and Hirsch, 2002).

Hydrologically based low-flow frequencies were determined by use of methods described by Riggs (1972). The general approach is as follows. An annual series of the minimum n-day (number of consecutive days) daily mean low flows are fitted to a log-Pearson Type III probability curve. A plot of the probability curve and data are reviewed for fitness. Other probability distributions are considered, or a smooth curve is constructed through the data if the data do not fit the log-Pearson Type III probability curve. The frequency of the $\mathrm{n}$ day is computed from the fitted probability curve or read 


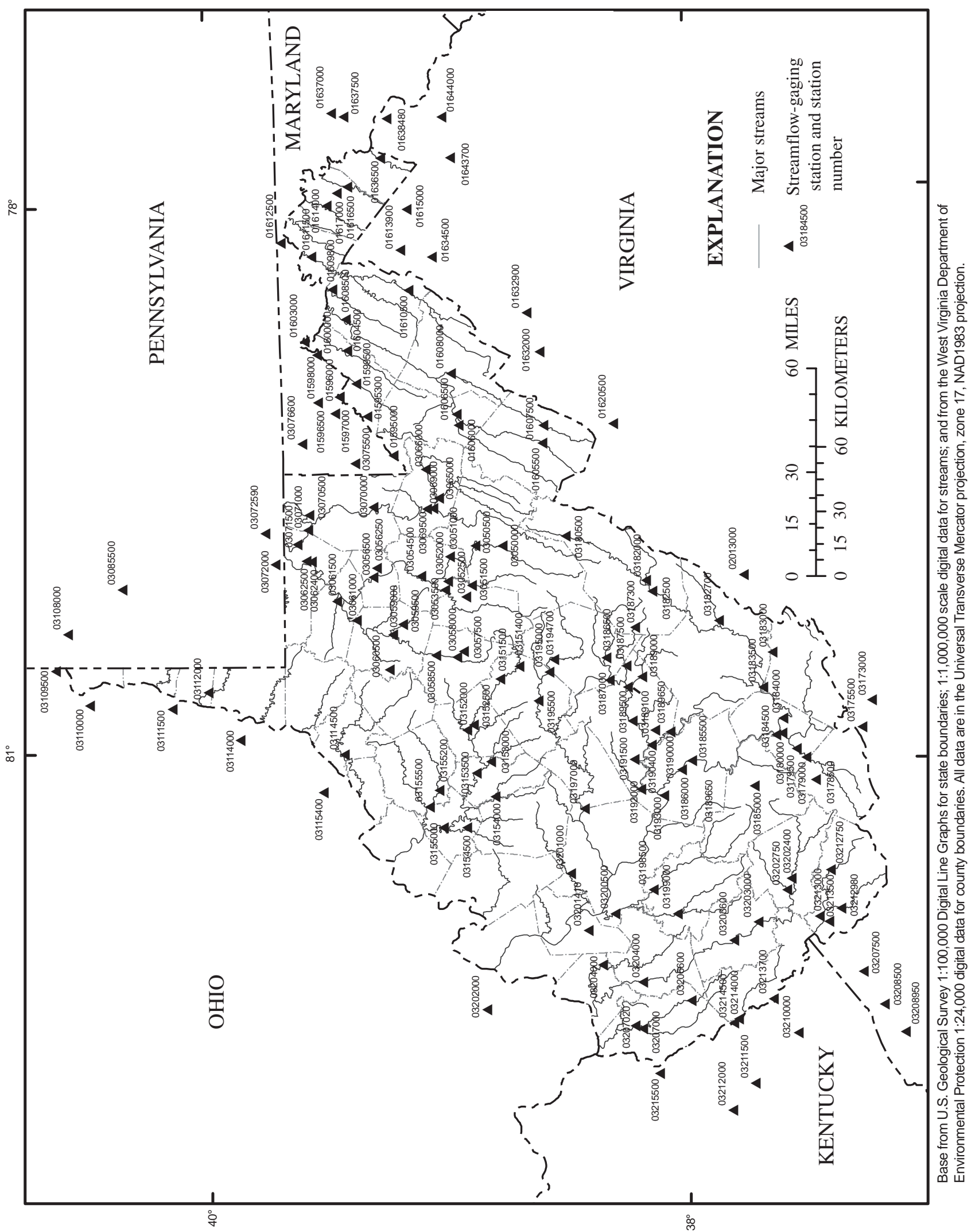

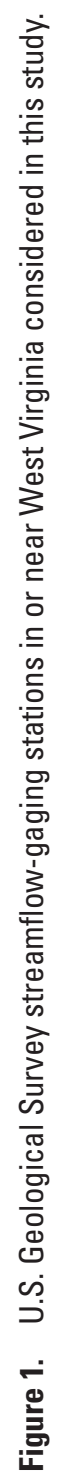


from the smooth curve constructed through the data. (All data fit the log-Pearson Type III probability curve in this study.) For example, The 7Q2 would be the streamflow at the 2-year recurrence interval taken from a frequency curve of annual values of the lowest mean streamflow for 7 consecutive days; the 7-day flow will be less than the 7Q2 at intervals averaging 2 years in length; or the probability is $1 / 2$ that the 7 -day low flow in any one year will be less than 7Q2.

Biologically based low-flow frequencies were determined by use of methods described by the U.S. Environmental Protection Agency (1986). For these statistics, a daily series of average n-day daily mean flows is computed for a streamflow record. Unlike the hydrologically based statistics, the biologically based statistics are computed by identification of "excursions" in the record. An excursion is a low-flow period that is determined to be hydrologically separate from other low-flow periods, typically by a measure of a minimum of 120 days between excursions. (The excursion concept relates to the biological concept of aquatic-life recovery after a period of stress.) The n-day series is evaluated for the desired frequency of occurrence of the excursions on the basis of number of years of record. For example, the 4B3 would be the streamflow at the 3-year recurrence interval of excursions of the lowest mean streamflow for 4 consecutive days; the 4-day flow will be less than the 4B3 at intervals averaging 3 years in length; or the probability is $1 / 3$ that the 4 -day low flow in any one year will be less than 4B3.

The EPA harmonic-mean flows were determined by use of methods described by Rossman (1990). The average of the reciprocals of the daily mean flows is computed for a station record. The harmonic-mean flow is the reciprocal of that average. The EPA harmonic-mean flow is the weighted average of the harmonic mean of the nonzero flows and the arithmetic mean of the zero flows; the harmonic mean of the nonzero flows is multiplied by the number of nonzero days and divided by the total number of days.

Flow durations were determined by use of methods described by Searcy (1959). A yearly record of daily mean flows is divided into 20-30 classes of average flows. Every complete year of record at a station is divided into the same classes. The number of days in each class is computed for the entire record period, and the percentage of the time a flow is in each class is determined. A particular flow duration is extrapolated from the class percentiles, and a log-probability plot of the class percentiles is a flow-duration curve. For example, the 5-percent-duration streamflow is equaled or exceeded 5 percent of the time.

The variability index was determined by use of methods described by Lane and Lei (1950). The variability index is a measure of the slope of the flow-duration curve. The variability index is the standard deviation of the logarithms of flow durations at 10-percent intervals between 5 and 95 percent. A variant of the method used to compute the variability index was necessary when the 95-percent flow duration was 0.00 $\mathrm{ft}^{3} / \mathrm{s}$; the 95 - and 5-percent flow durations were excluded from the computation, and the standard deviation was computed for the logarithms of flow durations at 10-percent intervals between 15 and 85 percent. The variability index increases as the slope of the flow-duration curve increases, indicating that as the variability Index increases, the permeability and storage capacity of the base-flow aquifer for a stream decrease. Flows in streams with higher variability indexes will reduce more rapidly during base-flow periods than flows in streams with lower variability indexes.

The statistics and statistical analyses were computed by use of the computer programs (1) SWSTAT, version 4.1 (Lumb and others, 1990; written commun., from USGS colleagues A.M. Lumb, W.O. Thomas, Jr., and K.M. Flynn, titled "Users manual for SWSTAT, a Computer Program for Interactive Computation of Surface-Water Statistics," June 15, 1995), (2) DFLOW, version 3.0 (Rossman, 1990), and (3) S-PLUS 2000 (MathSoft, Inc., 1999). SWSTAT was used to compute hydrologically based statistics and statistics for the MannKendall test for trend; DFLOW was used to compute biologically based low-flow frequencies and the EPA harmonic-mean flow; and S-PLUS 2000 was used to compute statistics for the principal component and correlation analyses.

DFLOW has the capability to compute both hydrologically and biologically based frequencies. Hydrological frequencies determined with DFLOW were not equal to those determined using SWSTAT because SWSTAT applies an adjustment for zero values along with other computational differences. Hydrological frequencies determined with DFLOW were greater than those determined with SWSTAT if zero values were in the analysis. Hydrological frequencies determined with DFLOW were often greater than twice those determined with SWSTAT. Only the biological frequencies determined with DFLOW are presented in this report, and the hydrological frequencies presented herein were computed with SWSTAT.

\section{Patterns in Minimum Flows}

Patterns of minimum flows at streamflow-gaging stations in West Virginia were investigated by use of methods similar to those used by McCabe and Wolock (2002). The minimum flows for the climatic year were determined for 110 stations in West Virginia having a minimum of 10 years of unregulated low-flow record (table 1). Table 1 contains all stations in West Virginia with at least 10 years of record, although some stations do not have record periods meeting the criteria for analyses or publication of statistics (there are no dots in table 1 for some stations). The climatic year, as used in this study, is April 1 through March 31of the indicated ending year; for example, the 1970 climatic year is April 1, 1969, through March 31, 1970. Use of climatic years maintains a continuous minimum low-flow period within each year because low flows in March and April are higher than average.

The minimum flows for some stations located on the same stream and with drainage areas within 25 percent of one another were combined to increase record lengths. The 25- 
percent criterion was determined as the maximum difference between combined stations-at the drainage areas considered-where the unit minimum flows were approximately equal. The values for the station having the shorter period of record were transferred to the station having the longer period of record. The minimum flows for the station having the shorter period of record were divided by the respective drainage area and multiplied by the drainage area of the station having the longer period of record. Values for the station having the longer period of record were used when the combination of records resulted in more than one value for a particular year. Minimum flows for the following stations in West Virginia were combined: Cheat River near Pisgah (station number, 03071000; drainage area, 1,354 $\mathrm{mi}^{2}$ ) was lengthened using near Morgantown $\left(03071500,1,380 \mathrm{mi}^{2}\right)$; Bluestone River near Pipestem $\left(03179000,395 \mathrm{mi}^{2}\right)$ was lengthened using at Lilly (03179500, $438 \mathrm{mi}^{2}$ ); New River at Caperton (03185500, $\left.6,826 \mathrm{mi}^{2}\right)$ was lengthened using at Fayette $(03186000,6,850$ mi2); Gauley River near Summersville (03189500, $680 \mathrm{mi}^{2}$ ) was lengthened using near Craigsville (03189100, $529 \mathrm{mi}$ ) and; Meadow River at Nallen $\left(03190000,287 \mathrm{mi}^{2}\right)$ was lengthened using near Mount Lookout (03190400, $\left.365 \mathrm{mi}^{2}\right)$; Elk River below Webster Springs $\left(03194700,266 \mathrm{mi}^{2}\right)$ was lengthened using at Centralia $\left(03195000,281 \mathrm{mi}^{2}\right)$; Guyandotte River at Man $\left(03203000,758 \mathrm{mi}^{2}\right)$ was lengthened using at Logan $\left(03203600,833 \mathrm{mi}^{2}\right)$; and Tug Fork near Kermit $\left(03214000,1,188 \mathrm{mi}^{2}\right)$ was lengthened using at Kermit $\left(03214500,1,280 \mathrm{mi}^{2}\right)$. Combined records were used for analyzing the minimum flows and were not used to compute other statistics.

The minimum flows were analyzed for the increase in minimum flows around 1970 identified by McCabe and Wolock (2002) and for having patterns of similar flows for different time periods.

Table 1. U.S. Geological Survey streamflow-gaging stations in or near West Virginia considered in this study.

[MD, Maryland; WV, West Virginia; VA, Virginia; PA, Pennsylvania; OH, Ohio; KY, Kentucky; $\bullet$, indicates that station is included in the indicated criterion]

\begin{tabular}{|c|c|c|c|c|c|c|c|c|}
\hline \multirow[b]{2}{*}{$\begin{array}{l}\text { Station } \\
\text { number }\end{array}$} & \multirow[b]{2}{*}{ Station name } & \multirow[b]{2}{*}{$\begin{array}{l} \pm \\
\text { के }\end{array}$} & \multicolumn{5}{|c|}{ Analysis } & \multirow[b]{2}{*}{$\begin{array}{l}\text { Selected- } \\
\text { stream- } \\
\text { flow } \\
\text { statistics } \\
\text { published } \\
\text { in this } \\
\text { report }\end{array}$} \\
\hline & & & 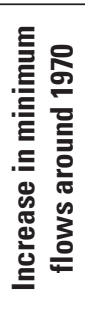 & 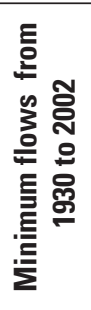 & 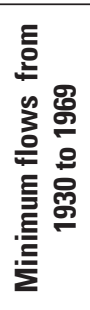 & 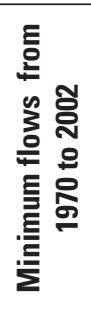 & 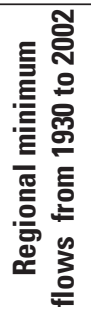 & \\
\hline 01595000 & North Branch Potomac River at Steyer & MD & & & & & & $\bullet$ \\
\hline 01595300 & Abram Creek at Oakmont & WV & $\bullet$ & & & & & $\bullet$ \\
\hline 01596000 & North Branch Potomac River at Bloomington & MD & & & & & & $\bullet$ \\
\hline 01596500 & Savage River near Barton & MD & & & & & & • \\
\hline 01597000 & Crabtree Creek near Swanton & MD & & & & & & $\bullet$ \\
\hline 01598000 & Savage River at Bloomington & MD & & & & & & - \\
\hline 01599500 & New Creek near Keyser & WV & & & & & & • \\
\hline 01600000 & North Branch Potomac River at Pinto & MD & & & & & & - \\
\hline 01603000 & North Branch Potomac River near Cumberland & MD & & & & & & $\bullet$ \\
\hline 01604500 & Patterson Creek near Headsville & WV & $\bullet$ & & & $\bullet$ & & $\cdot$ \\
\hline 01605500 & South Branch Potomac River at Franklin & WV & $\bullet$ & & & & & $\bullet$ \\
\hline 01606000 & North Fork South Branch Potomac River at Cabins & WV & & & & & & $\bullet$ \\
\hline 01606500 & South Branch Potomac River near Petersburg & WV & $\bullet$ & - & - & $\bullet$ & - & $\bullet$ \\
\hline 01607500 & South Fork South Branch Potomac River at Brandywine & WV & - & & & - & & - \\
\hline 01608000 & South Fork South Branch Potomac River near Moorefield & WV & • & & & • & & - \\
\hline 01608500 & South Branch Potomac River near Springfield & WV & • & $\bullet$ & - & $\bullet$ & $\bullet$ & • \\
\hline 01609800 & Little Cacapon River near Levels & WV & & & & & & \\
\hline 01610500 & Cacapon River at Yellow Spring & WV & & & & & & $\bullet$ \\
\hline 01611500 & Cacapon River near Great Cacapon & WV & $\bullet$ & $\bullet$ & $\bullet$ & $\bullet$ & $\cdot$ & $\bullet$ \\
\hline 01612500 & Little Tonoloway Creek near Hancock & MD & & & & & & $\bullet$ \\
\hline 01613900 & Hogue Creek near Hayfield & VA & & & & & & - \\
\hline
\end{tabular}


Table 1. U.S. Geological Survey streamflow-gaging stations in or near West Virginia considered in this study.-Continued

[MD, Maryland; WV, West Virginia; VA, Virginia; PA, Pennsylvania; OH, Ohio; KY, Kentucky;•, indicates that station is included in the indicated criterion]

\begin{tabular}{|c|c|c|c|c|c|c|c|c|}
\hline $\begin{array}{l}\text { Station } \\
\text { number }\end{array}$ & Station name & 志 & \multicolumn{5}{|c|}{ Analysis } & $\begin{array}{l}\text { Selected- } \\
\text { stream-flow } \\
\text { statistics } \\
\text { published in } \\
\text { this report }\end{array}$ \\
\hline 01614000 & Back Creek near Jones Springs & WV & $\bullet$ & & & & & $\bullet$ \\
\hline 01615000 & Opequon Creek near Berryville & VA & & & & & & $\bullet$ \\
\hline 01616500 & Opequon Creek near Martinsburg & WV & $\bullet$ & & & $\bullet$ & & $\bullet$ \\
\hline 01617000 & Tuscarora Creek above Martinsburg & WV & $\bullet$ & & & & & \\
\hline 01620500 & North River near Stokesville & VA & & & & & & $\bullet$ \\
\hline 01636500 & Shenandoah River at Millville & WV & $\bullet$ & $\bullet$ & $\bullet$ & $\bullet$ & $\bullet$ & $\bullet$ \\
\hline 01637000 & Little Catoctin Creek at Harmony & MD & & & & & & $\bullet$ \\
\hline 01637500 & Catoctin Creek near Middletown & MD & & & & & & $\bullet$ \\
\hline 01638480 & Catoctin Creek at Taylorstown & VA & & & & & & $\bullet$ \\
\hline 01643700 & Goose Creek near Middleburg & VA & & & & & & $\bullet$ \\
\hline 01644000 & Goose Creek near Leesburg & VA & & & & & $\bullet$ & $\bullet$ \\
\hline 02013000 & Dunlap Creek near Covington & VA & & & & & $\bullet$ & \\
\hline 03050000 & Tygart Valley River near Dailey & WV & $\bullet$ & & $\bullet$ & & & $\bullet$ \\
\hline 03050500 & Tygart Valley River near Elkins & WV & $\bullet$ & & & $\bullet$ & & • \\
\hline 03051000 & Tygart Valley River at Belington & WV & • & • & • & - & • & • \\
\hline 03056500 & Tygart Valley River at Fetterman & WV & & & & & & \\
\hline 03057500 & Skin Creek near Brownsville & WV & & & & & & • \\
\hline 03058000 & West Fork River below Stonewall Jackson Dam near Weston & WV & $\bullet$ & & & & & $\bullet$ \\
\hline 03058500 & West Fork River at Butcherville & WV & $\bullet$ & & $\bullet$ & & & $\bullet$ \\
\hline 03059000 & West Fork River at Clarksburg & WV & $\bullet$ & & $\bullet$ & & & $\bullet$ \\
\hline 03059500 & Elk Creek at Quiet Dell & WV & & & & & & \\
\hline 03060500 & Salem Creek at Salem & WV & & & & & & \\
\hline 03061000 & West Fork River at Enterprise & WV & $\bullet$ & & & & & $\bullet$ \\
\hline 03061500 & Buffalo Creek at Barrackville & WV & $\bullet$ & $\bullet$ & & $\bullet$ & $\bullet$ & $\bullet$ \\
\hline 03062400 & Cobun Creek at Morgantown & WV & & & & & & \\
\hline 03062500 & Deckers Creek at Morgantown & WV & & & & & & \\
\hline 03065000 & Dry Fork at Hendricks & WV & $\bullet$ & & & & & $\bullet$ \\
\hline 03066000 & Blackwater River at Davis & WV & $\bullet$ & $\bullet$ & $\bullet$ & $\bullet$ & $\bullet$ & $\bullet$ \\
\hline 03069000 & Shavers Fork at Parsons & WV & $\bullet$ & & & & & $\bullet$ \\
\hline 03069500 & Cheat River near Parsons & WV & $\bullet$ & $\bullet$ & $\bullet$ & $\bullet$ & $\bullet$ & $\bullet$ \\
\hline 03070000 & Cheat River at Rowlesburg & WV & $\bullet$ & & $\bullet$ & & & - \\
\hline 03070500 & Big Sandy Creek at Rockville & WV & $\bullet$ & • & • & - & • & • \\
\hline
\end{tabular}


Table 1. U.S. Geological Survey streamflow-gaging stations in or near West Virginia considered in this study.—Continued

[MD, Maryland; WV, West Virginia; VA, Virginia; PA, Pennsylvania; OH, Ohio; KY, Kentucky; ; indicates that station is included in the indicated criterion]

\begin{tabular}{|c|c|c|c|c|c|c|c|c|}
\hline \multirow[b]{2}{*}{$\begin{array}{l}\text { Station } \\
\text { number }\end{array}$} & \multirow[b]{2}{*}{ Station name } & \multirow[b]{2}{*}{ 壱 } & \multicolumn{5}{|c|}{ Analysis } & \multirow[b]{2}{*}{$\begin{array}{l}\text { Selected- } \\
\text { stream-flow } \\
\text { statistics } \\
\text { published in } \\
\text { this report }\end{array}$} \\
\hline & & & 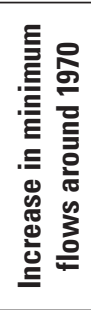 & 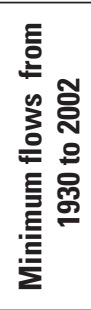 & 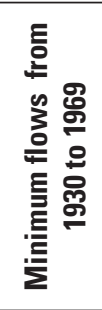 & 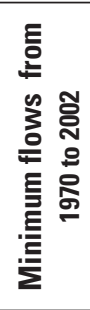 & 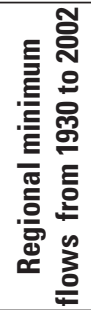 & \\
\hline 03071000 & Cheat River near Pisgah & WV & & & & & & $\bullet$ \\
\hline 03071500 & Cheat River near Morgantown & WV & & & & & & \\
\hline 03072000 & Dunkard Creek at Shannopin & PA & & & & & & $\bullet$ \\
\hline 03072590 & Georges Creek at Smithfield & PA & & & & & & $\bullet$ \\
\hline 03075500 & Youghiogheny River near Oakland & MD & & & & & & - \\
\hline 03109500 & Little Beaver Creek near East Liverpool & $\mathrm{OH}$ & & & & & $\bullet$ & $\bullet$ \\
\hline 03110000 & Yellow Creek near Hammondsville & $\mathrm{OH}$ & & & & & & $\bullet$ \\
\hline 03111500 & Short Creak near Dillonvale & $\mathrm{OH}$ & & & & & & $\bullet$ \\
\hline 03112000 & Wheeling Creek at Elm Grove & WV & $\cdot$ & & & $\bullet$ & & $\bullet$ \\
\hline 03114000 & Captina Creek at Armstrongs Mills & $\mathrm{OH}$ & & & & & & $\bullet$ \\
\hline 03114500 & Middle Island Creek at Little & WV & $\cdot$ & & $\bullet$ & & & $\bullet$ \\
\hline 03115400 & Little Muskingum River at Bloomfield & $\mathrm{OH}$ & & & & & & $\bullet$ \\
\hline 03151400 & Little Kanawha River near Wildcat & WV & & & & & & \\
\hline 03151500 & Little Kanawha River near Burnsville & WV & & & & & & \\
\hline 03152000 & Little Kanawha River at Glenville & WV & $\cdot$ & & $\cdot$ & & & $\bullet$ \\
\hline 03155500 & Hughes River at Cisco & WV & $\cdot$ & & & & & $\bullet$ \\
\hline 03173000 & Walker Creek at Bane & VA & & & & & & $\bullet$ \\
\hline 03175500 & Wolf Creek near Narrows & VA & & & & & & $\bullet$ \\
\hline 03178500 & Camp Creek near Camp Creek & WV & & & & & & \\
\hline 03179000 & Bluestone River near Pipestem & WV & $\mathrm{a}_{\bullet}$ & & & $\bullet$ & & $\bullet$ \\
\hline 03179500 & Bluestone River at Lilly & WV & & & & & & \\
\hline 03180000 & New River at Bluestone Dam & WV & & & & & & \\
\hline 03180500 & Greenbrier River at Durbin & WV & $\bullet$ & & & $\bullet$ & & $\bullet$ \\
\hline 03182000 & Knapp Creek at Marlinton & WV & & & & & & $\bullet$ \\
\hline 03182500 & Greenbrier River at Buckeye & WV & • & $\bullet$ & $\bullet$ & $\bullet$ & $\bullet$ & - \\
\hline 03182700 & Anthony Creek near Anthony & WV & & & & & & \\
\hline 03183000 & Second Creek near Second Creek & WV & & & & & & $\bullet$ \\
\hline 03183500 & Greenbrier River at Alderson & WV & $\cdot$ & $\bullet$ & $\bullet$ & $\bullet$ & $\bullet$ & $\bullet$ \\
\hline 03184000 & Greenbrier River at Hilldale & WV & $\cdot$ & & & $\bullet$ & & $\bullet$ \\
\hline 03184500 & New River at Hinton & WV & & & & & & \\
\hline 03185000 & Piney Creek at Raleigh & WV & $\bullet$ & & & & & $\bullet$ \\
\hline 03185500 & New River at Caperton & WV & & & & & & \\
\hline
\end{tabular}


Table 1. U.S. Geological Survey streamflow-gaging stations in or near West Virginia considered in this study.-Continued

[MD, Maryland; WV, West Virginia; VA, Virginia; PA, Pennsylvania; OH, Ohio; KY, Kentucky; $\bullet$, indicates that station is included in the indicated criterion]

\begin{tabular}{|c|c|c|c|c|c|c|c|c|}
\hline $\begin{array}{l}\text { Station } \\
\text { number }\end{array}$ & Station name & 志 & \multicolumn{5}{|c|}{ Analysis } & $\begin{array}{l}\text { Selected- } \\
\text { stream-flow } \\
\text { statistics } \\
\text { published in } \\
\text { this report }\end{array}$ \\
\hline 03186000 & New River at Fayette & WV & & & & & & \\
\hline 03186500 & Williams River at Dyer & WV & $\bullet$ & $\bullet$ & $\bullet$ & $\bullet$ & $\bullet$ & $\bullet$ \\
\hline 03187000 & Gauley River at Camden On Gauley & WV & $\bullet$ & & $\bullet$ & & & $\bullet$ \\
\hline 03187300 & North Fork Cranberry River near Hillsboro & WV & & & & & & \\
\hline 03187500 & Cranberry River near Richwood & WV & $\bullet$ & & & $\bullet$ & & $\bullet$ \\
\hline 03189650 & Collison Creek near Nallen & WV & & & & & & $\bullet$ \\
\hline 03190000 & Meadow River at Nallen & WV & $a_{\bullet}$ & & $\bullet$ & & & $\bullet$ \\
\hline 03190400 & Meadow River near Mount Lookout & WV & & & & & & $\bullet$ \\
\hline 03191500 & Peters Creek near Lockwood & WV & $\bullet$ & & & & & $\cdot$ \\
\hline 03192000 & Gauley River above Belva & WV & & & & & & $\bullet$ \\
\hline 03193000 & Kanawha River at Kanawha Falls & WV & & & & & & \\
\hline 03194700 & Elk River below Webster Springs & WV & ${ }^{\mathrm{a}} \bullet$ & & & $\bullet$ & & $\bullet$ \\
\hline 03195000 & Elk River at Centralia & WV & & & & & & $\bullet$ \\
\hline 03195500 & Elk River at Sutton & WV & & & & & & $\bullet$ \\
\hline 03197000 & Elk River at Queen Shoals & WV & & & & & & $\bullet$ \\
\hline 03202750 & Clear Fork at Clear Fork & WV & & & & & & \\
\hline 03203000 & Guyandotte River at Man & WV & ${ }^{\mathrm{a}} \bullet$ & & ${ }^{\mathrm{a}} \bullet$ & & & \\
\hline 03203600 & Guyandotte River at Logan & WV & & & & & & $\bullet$ \\
\hline 03204000 & Guyandotte River at Branchland & WV & $\bullet$ & & $\bullet$ & & & $\bullet$ \\
\hline 03204500 & Mud River near Milton & WV & $\bullet$ & & & & & $\bullet$ \\
\hline 03206600 & East Fork Twelvepole Creek near Dunlow & WV & & & & $\bullet$ & & \\
\hline 03207000 & Twelvepole Creek at Wayne & WV & & & & & & \\
\hline 03207020 & Twelvepole Creek below Wayne & WV & & & & & & \\
\hline 03207500 & Levisa Fork near Grundy & VA & & & & & & $\bullet$ \\
\hline 03208500 & Russell Fork at Haysi & VA & & & & & $\bullet$ & $\bullet$ \\
\hline 03208950 & Cranes Nest River near Clintwood & VA & & & & & & $\bullet$ \\
\hline 03210000 & Johns Creek near Meta & KY & & & & & & $\bullet$ \\
\hline 03211500 & Johns Creek near Van Lear & KY & & & & & & $\cdot$ \\
\hline 03212000 & Paint Creek at Staffordsville & KY & & & & & & $\bullet$ \\
\hline 03212750 & Tug Fork at Welch & WV & & & & & & \\
\hline 03212980 & Dry Fork at Beartown & WV & & & & & & \\
\hline 03213000 & Tug Fork at Litwar & WV & $\bullet$ & & $\bullet$ & & & • \\
\hline
\end{tabular}


Table 1. U.S. Geological Survey streamflow-gaging stations in or near West Virginia considered in this study.-Continued

[MD, Maryland; WV, West Virginia; VA, Virginia; PA, Pennsylvania; OH, Ohio; KY, Kentucky; $\bullet$, indicates that station is included in the indicated criterion]

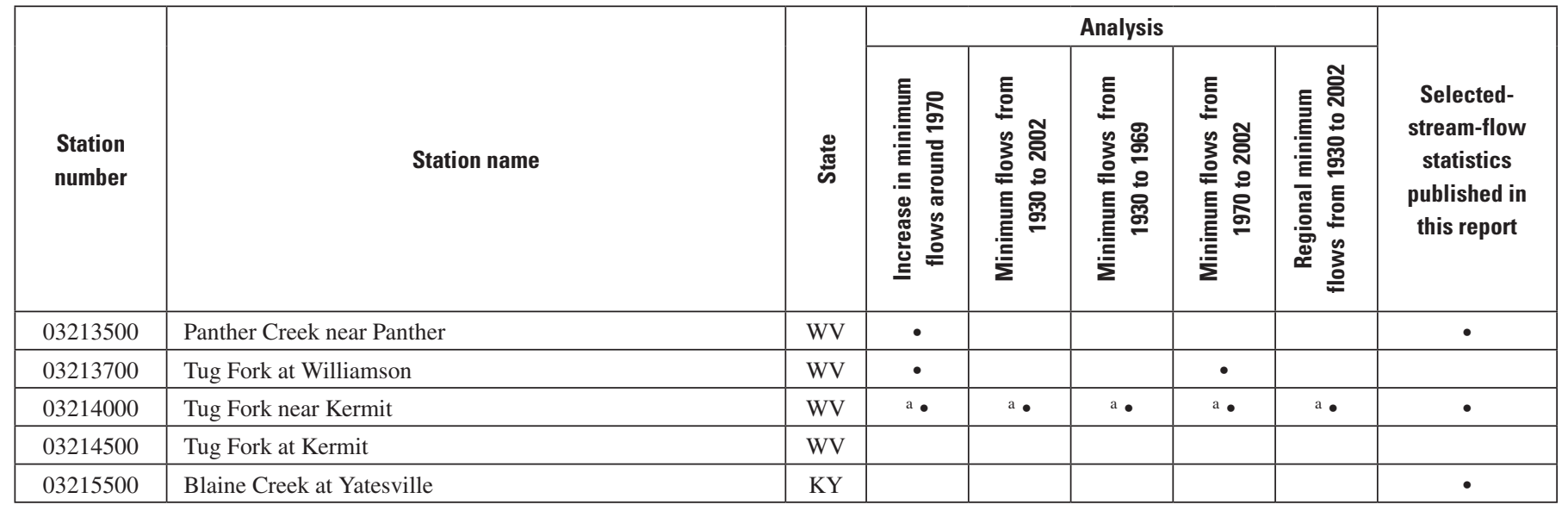

a Station record was lengthened using a nearby station for this analysis.

\section{Increase in Minimum Flows Around 1970}

Stations in West Virginia having a minimum of 5 years of record before 1970 and 5 years after 1969 were investigated to determine whether there was an increase in minimum flows around 1970 as identified by McCabe and Wolock (2002) (table 1). Departures of the minimum flows from the average minimum flows for the period of record were plotted against time for each station and were visually inspected for the increase in minimum flows around 1970.

The visual patterns of the increase in minimum flows around 1970 in the station plots of departures are not uniform throughout the State. Well-defined increases in minimum flows around 1970 are apparent for many station plots statewide, but some station plots show no noticeable increase. Some station plots in the eastern panhandle of the State indicate a well-defined increase in minimum flows around 1972. The plot for Greenbrier River at Alderson (03183500, 1,364 $\mathrm{mi}^{2}$ ) does not show an increase in minimum flows (fig. 2) such as the one apparent in the plot for the nearby combined stations Bluestone River near Pipestem (03179000, record years 1952-2002, $\left.395 \mathrm{mi}^{2}\right)$ and at Lilly $(03179500,1910-48$, $438 \mathrm{mi}^{2}$ )(fig. 3). The average minimum flows before the 1970 increase were less than the average minimum flows after the 1970 increase at both Greenbrier River at Alderson and the combined Bluestone River near Pipestem and Bluestone River at Lilly, although the difference at Alderson of about $1 \mathrm{ft}^{3} / \mathrm{s}$ (about 1 percent of the average minimum flow) is insignificant.

The principal cause of the minimum-flow patterns associated with this analysis is probably climate variability. Mining is probably the greatest land-use change affecting low flows in West Virginia. In small drainage basins (drainage areas less than $10 \mathrm{mi}^{2}$ ) mining has transferred water between basins sometimes decreasing low flows and sometimes increasing low flows. The net result of underground mining was an increase in ground-water storage that would return to the surface at a lower elevation due to gravity or would return to the surface from mine pumpage. The water may return to the surface in a different drainage basin, thus in small drainage basins sometimes decreasing low flows and sometimes increasing low flows (Puente and Adkins, 1989; Borchers and others, 1991). In small drainage basins, the net result of valley fills from mountaintop mining was an increase in low flows (Wiley and others, 2001). At large drainage basins (drainage areas greater than $100 \mathrm{mi}^{2}$ ) the net result from mining should show an increase in low flows because of ground-water storage returning to the surface at a lower elevation from underground mines and the increase in low flows from valley fills. A visual inspection of the pattern in plots of the minimum flows for large drainage basins affected by mining did not show a general increase through time corresponding to an increase in mining through time. The pattern in plots of the minimum flows probably did not show the changes associated with mining because these changes were indistinguishable from more significant affects, probably those associated with climate variability.

\section{Minimum Flows From 1930 Through 2002}

The stations used for the 1930-2002 analysis were limited to those in West Virginia with mostly complete records for the longest possible continuous time period. There were 14 stations with no more than a total of 4 years of missing record for the period from 1930 through 2002. The 14 stations were equally distributed across the State except for a lack of coverage along the western border with Ohio and the southwest border with Kentucky. The combined record for station 


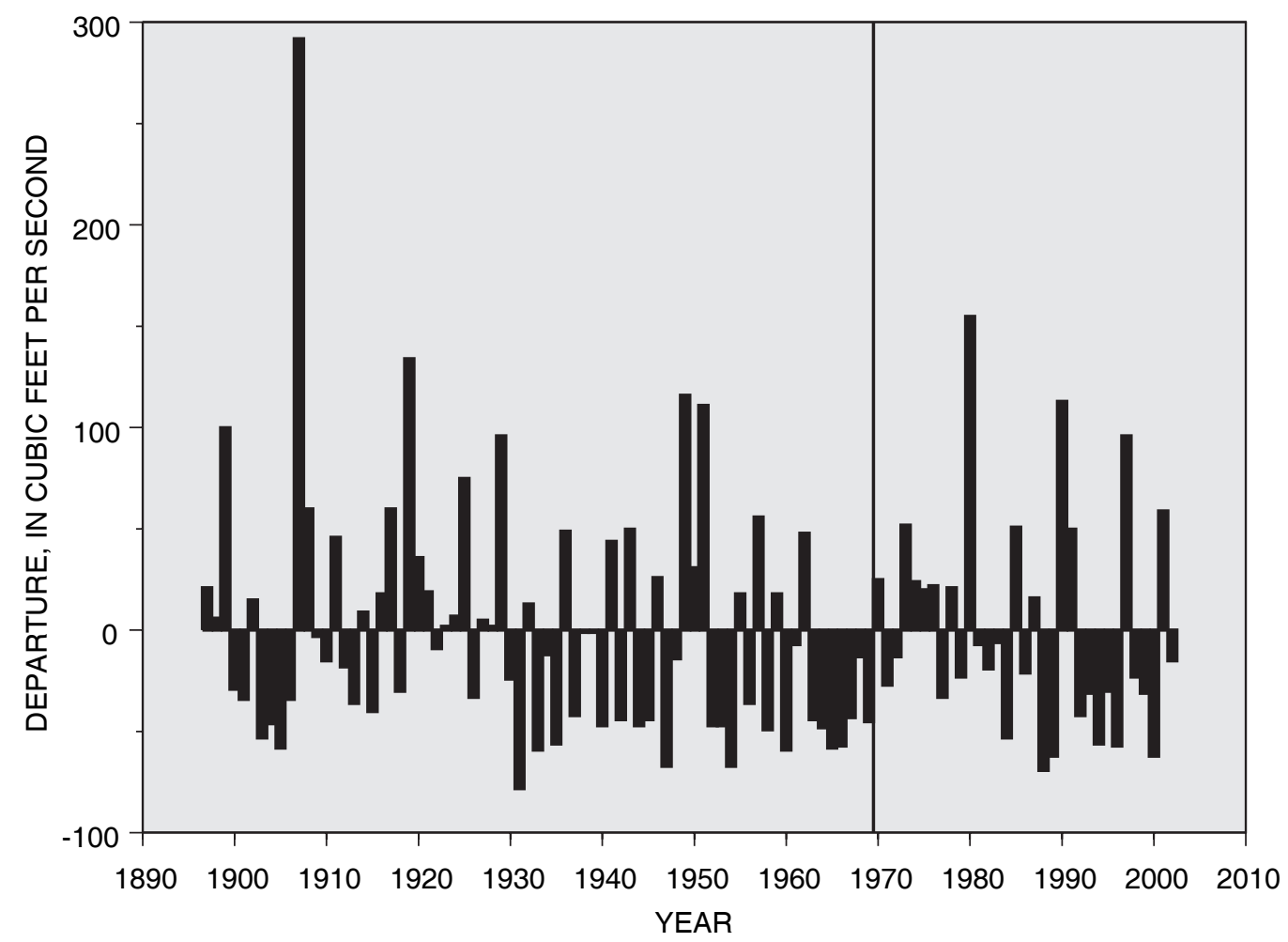

Figure 2. Departure of the minimum flows from the record-period average for Greenbrier River at Alderson, West Virginia (Vertical line between 1969 and 1970 separates periods described in text).

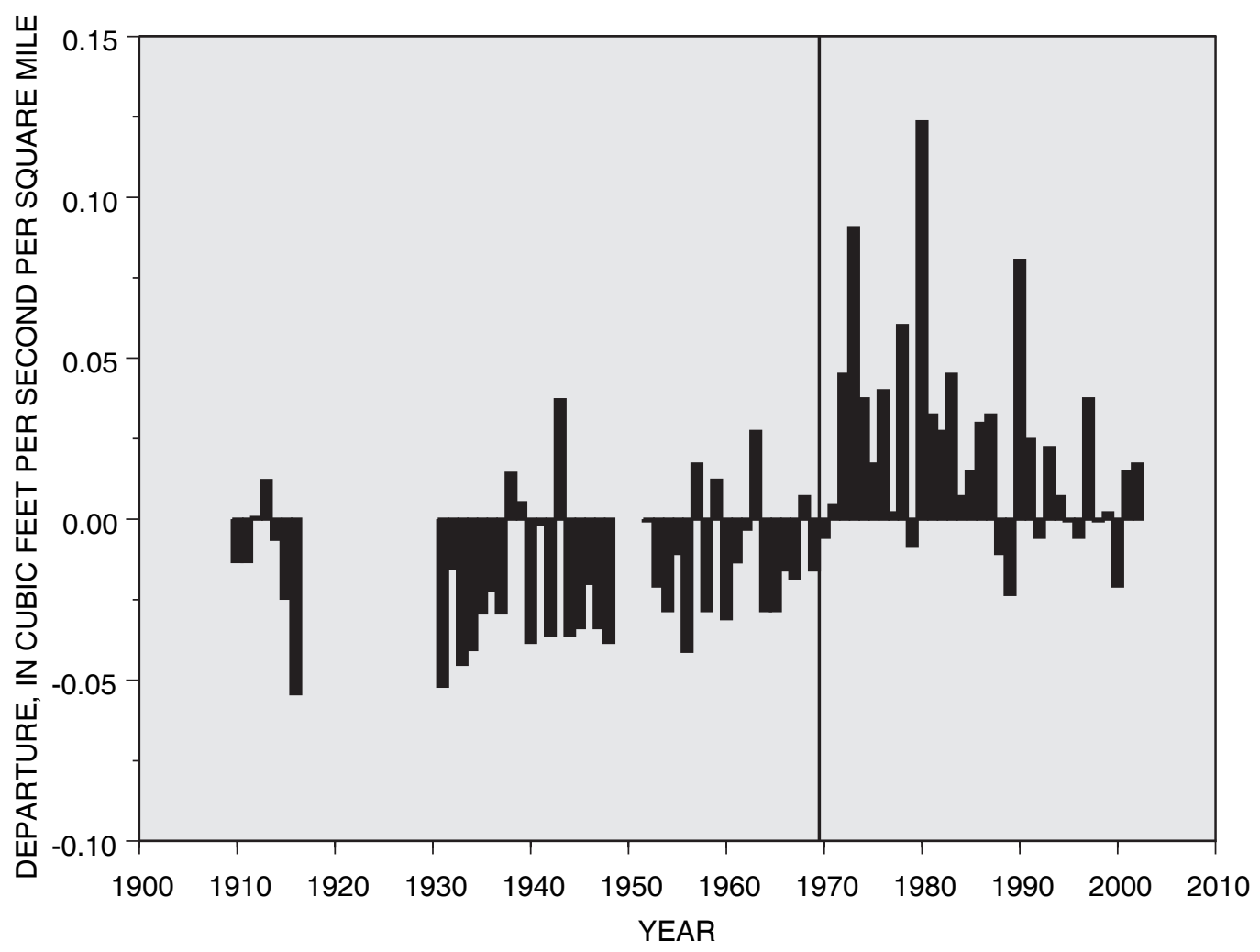

Figure 3. Departure of the unit minimum flows from the record-period average for the combined stations Bluestone River near Pipestem and at Lilly, West Virginia (Vertical line between 1969 and 1970 separates periods described in text). 
Tug Fork near Kermit (03214000) lengthened using at Kermit (03214500) was included to improve the areal distribution along the south-west border, but there are no data available to improve the distribution along the western border. Equally distributed station locations and minimizing use of combined stations are necessary for studying the variability of flow statistics and calculating a regional average of low flows discussed later in this report. The station set consisted of 15 stations with the combined records for Tug Fork near and at Kermit counted as 1 station (table 1). The standardized departure at each station was computed by subtracting the respective average minimum flow for 1930-2002 from the minimum flow at each station and then dividing by the respective standard deviation of the minimum flow. The standardized departures were averaged for all 15 stations for each year (fig. 4). of the time periods investigated. Finally, the period 1980-2002 indicates variation between positive and negative departures, with the positive departures that are the most positive of any of the periods investigated and an average positive departure slightly less than the average for 1970-79. The average departure for the years before 1970 is negative, and the average departure for the years after 1969 is positive.

A statistical trend analysis of the minimum flows for the 15 stations was done by use of the Mann-Kendall test for the record periods 1930-2002, 1930-63, 1930-69, 1930-42, 1943-62, 1943-69, 1963-69, 1963-79, 1970-2002, 1970-79, and 1980-2002. An alpha value of 0.0667 was selected so that 1 station out of the 15 would be expected to indicate a trend by chance. No stations indicated a trend for 1943-62, 1970-79, or 1980-2002. Only one station indicated a trend for 1930-69

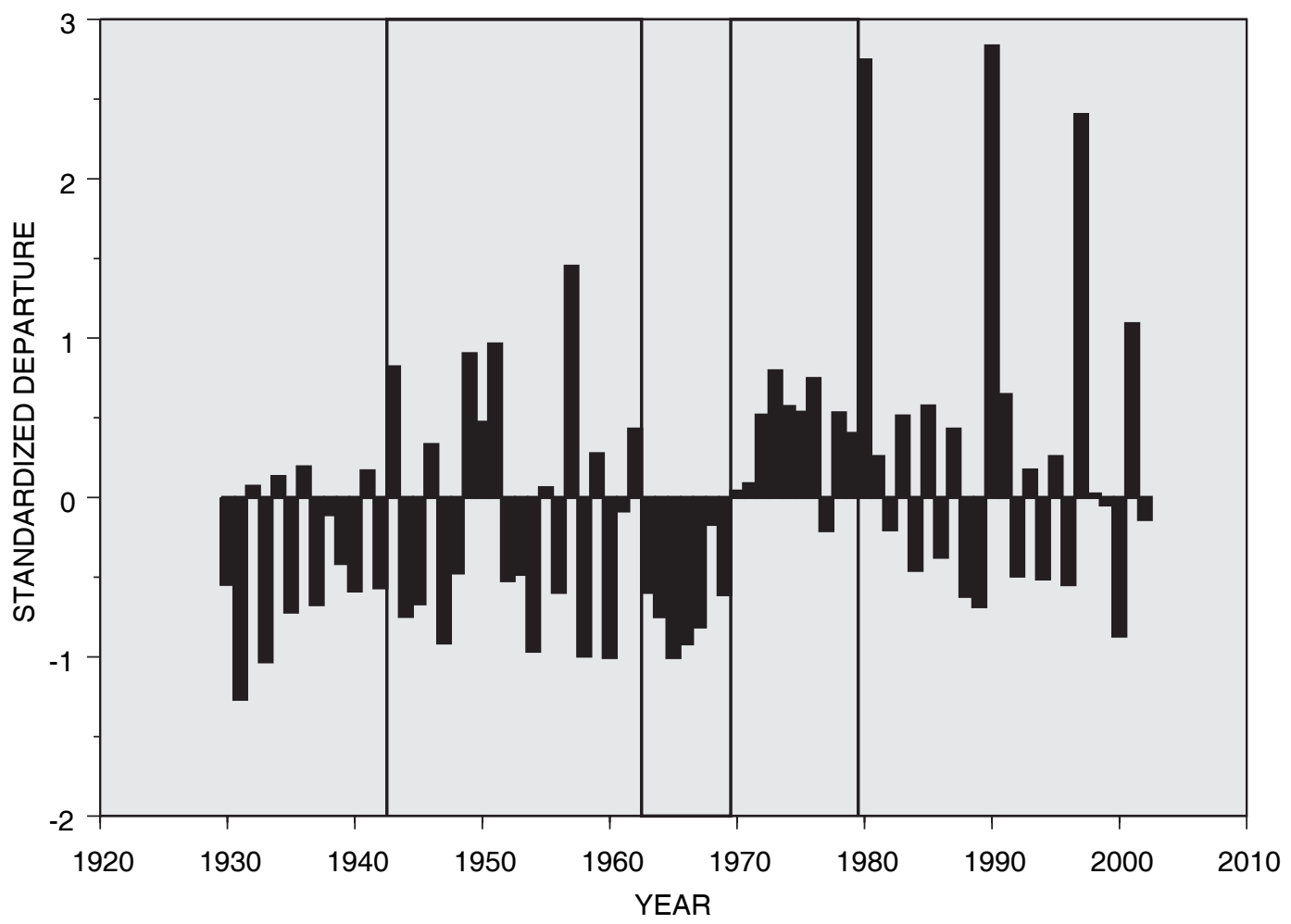

Figure 4. Average standardized departures of the minimum flows from the record-period average for 1930-2002 for 15 stations in West Virginia. (Vertical lines separate subperiods described in text.)

The change from the general negative departures to positive departures between the 1969 and 1970 climatic years is apparent in fig. 4. The period 1930-42 indicates mostly negative departure. The period 1943-62 indicates variation between positive and negative departures that average slightly less than the mean. The period 1963-69 indicates consistently negative departure and the most negative average of any of the time periods investigated. The period 1970-79 indicates largely positive departure and the most positive average of any and 1963-69 (a different station for each period). Only two stations indicated a trend for 1930-42, 1930-63, 1943-69, and 1970-2002 (only one station indicated a trend for two periods). One or two stations indicating a trend is close enough to the one station expected by chance that the conclusion of no trends was supported.

In contrast, statistically significant positive trends were found for all 15 stations for 1963-79 (14 stations at an alpha value of 0.05 , and 6 stations at an alpha value of 0.01 ) and 6 
stations for 1930-2002 (5 stations at an alpha value of 0.05, and 4 stations at an alpha value of 0.01). All 15 stations indicating a trend for 1963-79 is expected because the change from negative departures to positive departures around 1970 was visible in plots of departures for many stations across the State. The six stations indicating a positive trend for 19302002 were Cacapon River near Great Cacapon (station number 01611500), Shenandoah River at Milliville (01636500), Buffalo Creek at Barrackville (03061500), Big Sandy Creek at Rockville (03070500), Big Coal River at Ashford (03198500), and the combined stations Tug Fork near Kermit (03214000) and at Kermit (03214500). Two stations are located in the eastern panhandle (01611500 and 01636500), two are in the north-central area (03061500 and 03070500), and two are in the southwest area of the State $(03198500$ and combined stations 03214000 and 03214500). These six stations are distributed across the State, but none is near the east-central area of the State, which has the highest annual precipitation.

The Mann-Kendall test did not indicate a trend for 1970-2002, although the average departures of minimum flows for 1970-79 are greater than the average departures for 1980-2002. The annual variation of selected flow statistics discussed later in this report also indicates decreases in low flows between 1970-79 and 1980-2002. The Mann-Kendall test did not indicate a negative trend for the decrease in the minimum flows between the periods, probably because the trend was sudden and not gradual. The Mann-Kendall test is better suited for detecting monotonic trends.

\section{Minimum Flows From 1930 Through 1969}

The stations used in this analysis were limited to those in West Virginia with mostly complete records for the period 1930-69. There are 29 stations (the combined records for Tug Fork near and at Kermit counted as 1 station) with no more than a total of 2 years of missing record for the 1930-69 period (table 1). The standardized departures were computed for the 29 stations and averaged (fig. 5).

The same time periods (1930-42, 1943-62, and 1963-69) show similar patterns of departures as those determined by analyzing the characteristic from 1930 through 2002 (fig. 5). The average departure for 1963-69 is the most negative.

\section{Minimum Flows From 1970 Through 2002}

The stations used in this analysis were limited to those in West Virginia with mostly complete records for the period 1970-2002. There are 32 stations (or combined stations counted as 1 station) with no more than a total of 2 years of missing record for the 1970-2002 period (table 1). The standardized departures were computed for the 32 stations and averaged (fig. 6).

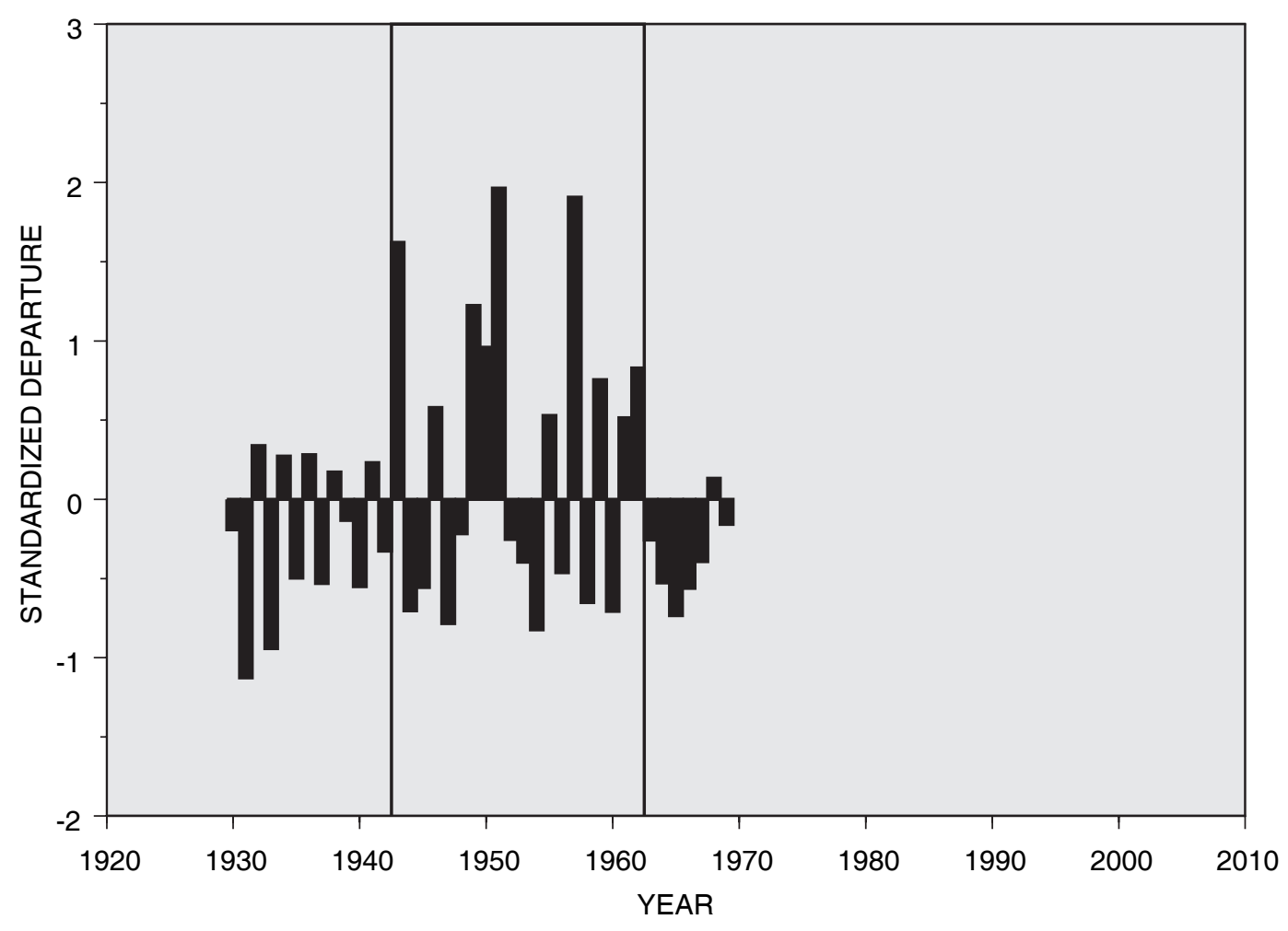

Figure 5. Average standardized departures of the minimum flows from the record-period average for 1930-1969 for 29 stations in West Virginia. (Vertical lines separate subperiods described in text.) 
The same time periods (1970-79 and 1980-2002) show similar patterns of departures as those determined by analyzing the characteristic from 1930 through 2002 (fig. 6). The average departure for 1970-79 is the most positive.

\section{Minimum Flows Before 1930}

Few West Virginia stations with long periods of records include the period before 1930. Shenandoah River at Millville (01636500) has record starting in 1896 and extending through 2002 (fig. 7) and indicates an increase in minimum flow between the 1971 and 1972 climatic years; however, record is missing from 1910 through 1929 . The average positive departures for 1896-1909 are greater than the average departures for 1972-79 and 1972-2002. Greenbrier River at Alderson (03183500) has record from 1897 through 2002 and does not show an increase in minimum flow around 1970 (fig. 2). The average positive departures for 1896-1909 and 1896-1929 are greater than the average departures for the periods 1970-79 and 1970-2002. The analysis for these two stations indicates that there were probably periods in West Virginia between 1896 and 1930 when minimum flows were greater than during any periods between 1930 and 2002.

The Shenandoah River at Millville and Greenbrier River at Alderson are most likely representative of the regional pattern of minimum flows because the $\log 10$-transformed flow values are fairly well correlated with concurrent $\log 10$ transformed flow values at other stations. The correlation coefficient of minimum flows at Shenandoah River at Millville with minimum flows at Kanawha River at Kanawha Falls (03193000) is 0.75 for the record period 1878-1948, sharing 35 years of concurrent record. Correlation coefficients of 0.84 and 0.82 , respectively, were found for minimum flows at Greenbrier River at Alderson and minimum flows at Cheat River near Parsons (03069500), record period 1914-2002 (89 years of concurrent record), and with minimum flows at Tygart Valley River at Belington (03051000), record period 1909-2002 (94 years of concurrent record).

\section{Variability of Flow Statistics}

Several streamflow statistics were computed for 15 stations (combined records for Tug Fork near and at Kermit counted as 1 station) to study the variability of determined values for 1930-2002. The 15 stations are the same as those used for the minimum-flow analysis for 1930-2002 (table 1). The annual and seasonal 1Q10, 7Q10, 30Q5, 1B3, 4B3, and the EPA harmonic-mean flows were computed for 1930-2002, 1930-42, 1943-62, 1963-69, 1970-79, and 1980-2002.

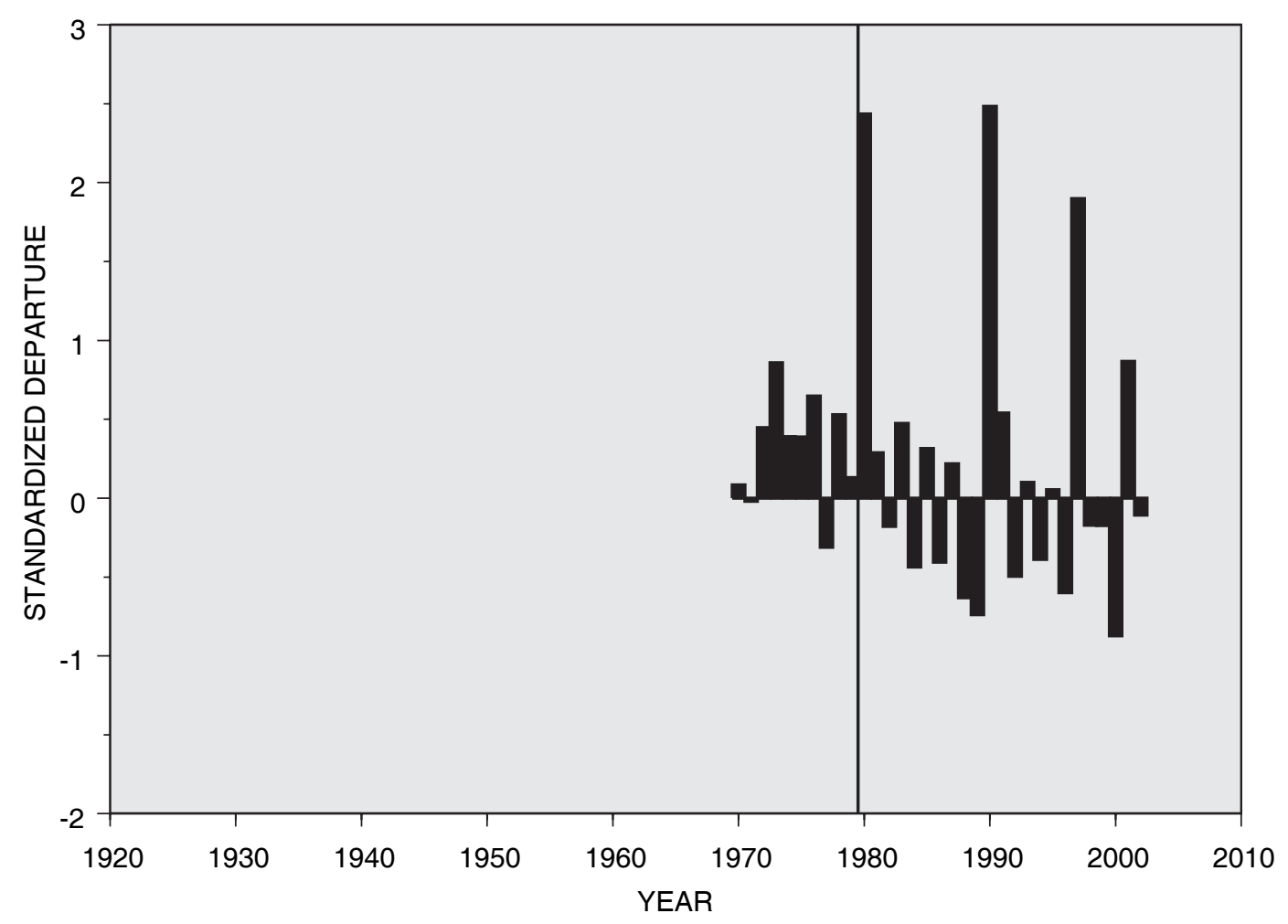

Figure 6. Average standardized departures of the minimum flows from the record-period average for 1970-2002 for 32 stations in West Virginia (Vertical line separate subperiods described in text). 


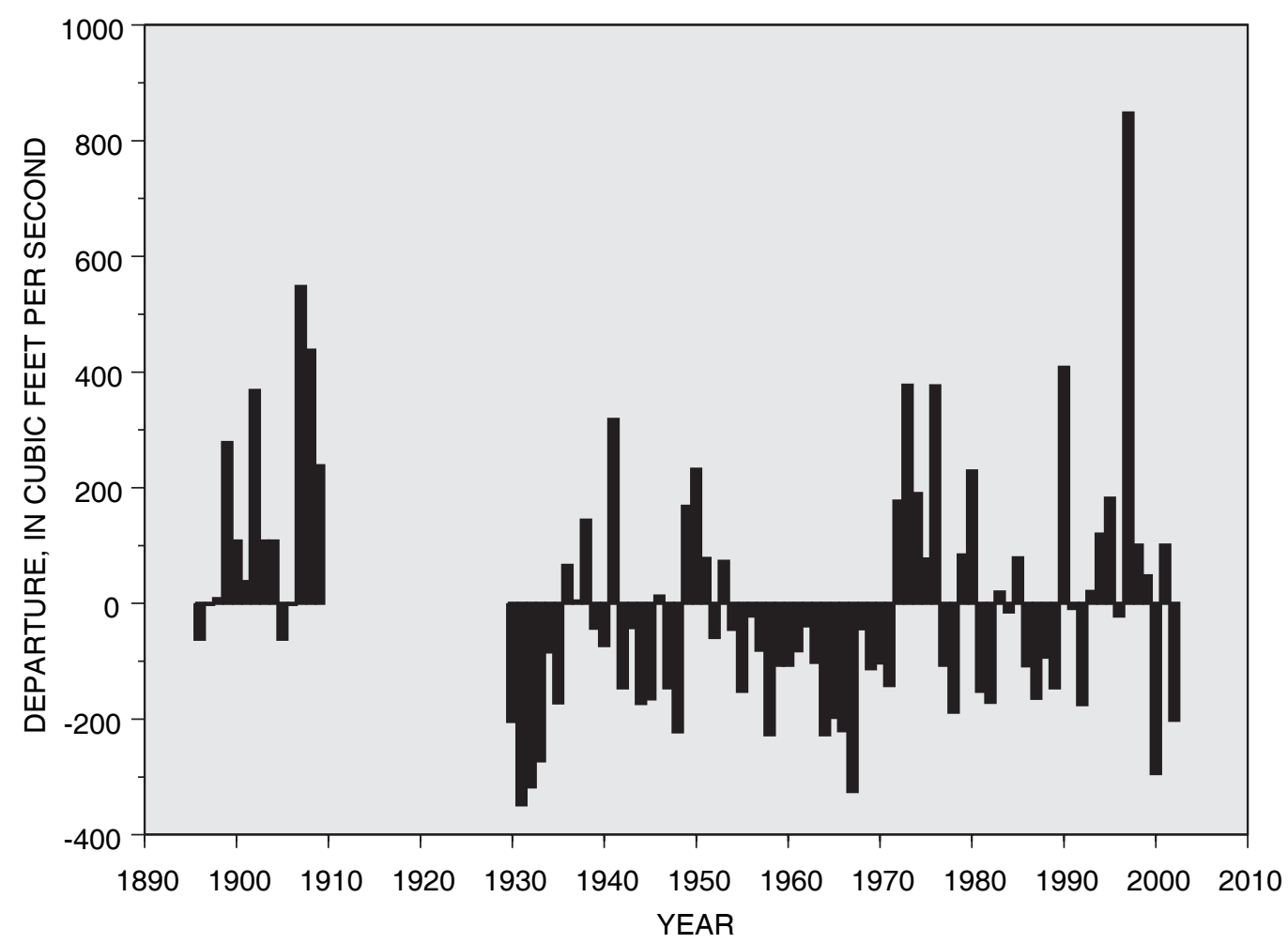

Figure 7. Departure of the minimum flows from the record-period average for Shenandoah River at Millville, West Virginia.

\section{Comparison of Annual Statistics}

The values of the selected annual statistics (climatic year) for the 15 stations were averaged and compared to values computed for 1930-2002 to assess variability (table 2). Differences of the average values for the period 1930-42 compared to the average values for the period 1943-62 mostly increase; differences include both increases and decreases between 1943-62 and 1963-69; differences all increase between 1963-69 and 1970-79, and all decrease between 1970-79 and 1980-2002. Generally, the relative differences in values between successive periods follow the trend of the departures of the minimum flows (fig. 4).

The increases and decreases between values for 1943-62 and 1963-69 are inconsistent with the trend of the departures of the minimum flows (fig. 4), where a marked decrease between the periods is apparent in the plot. The increases are for the values of lower flows (1Q10, 7Q10, 1B3, and 4B3), whereas the decreases are for higher flows (30Q5 and the EPA harmonic-mean flow).

No consistent trend was found between statistics for lower flows and those for higher flows. For 1930-42, the absolute differences of values for the lower flows are equal or greater than the absolute differences for higher flows. For 1943-62, the absolute differences are approximately equal. In contrast, for 1970-79 and 1980-2002, the differences of values for the lower flows are about twice the differences for the higher flows.

There is a consistent trend in the statistics for the hydrologically based flows $1 \mathrm{Q} 10$ and 7Q10 and the biologically based flows 1B3 and 4B3: absolute differences for hydrologically based flows are approximately equal to or smaller than those for biologically based flows for all periods.

The variability of the differences between annual statistics computed for each subperiod compared to the values computed for 1930-2002 decreases with increasing flows; the values in table 2 get closer to zero when comparing the values for 1Q10, 7Q10, 1B3, and 4B3 to the higher flow values for $30 \mathrm{Q} 5$ and the EPA harmonic-mean flow. The values for 1Q10, 7Q10, 1B3, and 4B3 range from -36.9 to 200.7 percent for the average of stations for each period from the values computed for 1930-2002, and the values for 30Q5 and the EPA harmonic-mean flow range from -21.0 to 115.1 percent (table 2). The values for 1Q10, 7Q10, 1B3, and 4B3 range from -93.9 to 582.5 percent for the individual stations for each period from the values computed for 1930-2002, and the values for $30 \mathrm{Q} 5$ and the EPA harmonic-mean flow range from -62.3 to 281.2 percent (table 3 ). Table 3 contains no values for the period 1930-42 for the combined station Tug Fork near Kermit (03214000) and at Kermit (03214500) because the combined station was only used for analyzing the minimum flows and was not used to compute other statistics, and because there 
Table 2. Average differences between selected annual statistics computed for 1930-2002 and the statistics computed for the indicated record periods for 15 stations in West Virginia.

[Station numbers for the 15 stations are $01606500,01608500,01611500,01636500,03051000,03053500,03061500,03066000$, 03069500, 03070500, 03182500, 03183500, 03186500, 03198500, and combined stations 03214000 and 03214500; a negative value means the average for the indicated record period is less than the average for 1930-2002; a positive value means the average for the indicated record period is greater than the average for 1930-2002; EPA, U.S. Environmental Protection Agency]

\begin{tabular}{|c|c|c|c|c|c|}
\hline \multirow{2}{*}{$\begin{array}{l}\text { Streamflow } \\
\text { statistic }\end{array}$} & \multicolumn{5}{|c|}{ Difference for the indicated period, in percent } \\
\hline & $1930-42$ & 1943-62 & 1963-69 & $1970-79$ & 1980-2002 \\
\hline $\begin{array}{l}\text { 1-day } 10 \text {-year } \\
\text { hydrologically } \\
\text { based low } \\
\text { flow (1Q10) }\end{array}$ & -23.7 & -11.2 & 5.4 & 187.0 & 52.3 \\
\hline $\begin{array}{l}\text { 7-day } 10 \text {-year } \\
\text { hydrologically } \\
\text { based low } \\
\text { flow (7Q10) }\end{array}$ & -20.6 & -13.6 & 2.4 & 182.8 & 47.7 \\
\hline $\begin{array}{l}\text { 30-day 5-year } \\
\text { hydrologically } \\
\text { based low } \\
\text { flow (30Q5) }\end{array}$ & -9.8 & -12.7 & -19.2 & 115.1 & 24.1 \\
\hline $\begin{array}{l}\text { 1-day 3-year } \\
\text { biologically } \\
\text { based low } \\
\text { flow (1B3) }\end{array}$ & -36.9 & -10.7 & 9.2 & 200.7 & 67.4 \\
\hline $\begin{array}{l}\text { 4-day 3-year } \\
\text { biologically } \\
\text { based low } \\
\text { flow (4B3) }\end{array}$ & -32.1 & -13.5 & 8.9 & 190.3 & 58.9 \\
\hline $\begin{array}{l}\text { EPA harmonic } \\
\text { mean flow }\end{array}$ & -21.0 & -7.1 & -15.2 & 90.7 & 31.6 \\
\hline
\end{tabular}

were not sufficient years of record at either station to make an estimate representative of the period.

An increase in departures around 1970 is not obvious in the plot of departures for Greenbrier River at Alderson (03183500) (fig. 2), but values for the selected annual statistics indicate an increase between 1963-69 and 1970-79. The greatest difference was found for 1970-79, where the selected statistics are between 40.6 and 70.5 percent greater than those for 1930-2002 (table 3).

\section{Comparison of Seasonal Statistics}

Selected statistics were computed for January 1 through March 31 (winter), April 1 through June 30 (spring), July 1 through September 30 (summer), and October 1 through December 31 (fall). The average differences (table 4) and individual-station differences (table 5 at back of report) of the selected statistics were compared to values computed for 1930-2002 for the 15 stations to measure variability.
Generally, the relative average difference in values between successive fall periods (table 4 ) follows the trend of the departures of the minimum flows (fig. 4) because the minimum flows commonly take place in the fall. The average differences of values for all seasons for all statistics are negative for 1930-42 (table 4); differences for the individual stations range from -95.7 to 43.3 percent (table 5 at back of report). The average differences of values are mixed for 1943-62 and 1963-69; differences for the individual stations range from -86.9 to 231.2 percent. The average differences for values of 30Q5 are negative for all seasons, and those for 1B3 are positive for all seasons for 1963-69. The average differences of values for all seasons for all statistics are positive for 1970-79; differences for the individual stations range from -44.6 to 683.6 percent. The average differences of values for the summer and fall for 1970-79 are the greatest for all periods, ranging from 85.4 to 207.7 percent for all statistics. The average differences of values for the spring for 1970-79 vary between the biologically based flows (range, 0.6 to 4.7 percent) and hydrologically based flows (range, 5.1 to 32.4 
Table 3. Differences between selected annual statistics computed for 1930-2002 and the statistics computed for the indicated record periods for 15 stations in West Virginia.

[Station numbers for the 15 stations are $01606500,01608500,01611500,01636500,03051000,03053500,03061500,03066000$, 03069500, 03070500, 03182500, 03183500, 03186500, 03198500, and combined stations 03214000 and 03214500; a negative value means the average for the indicated record period is less than the average for 1930-2002; a positive value means the average for the indicated record period is greater than the average for 1930-2002]

\begin{tabular}{|c|c|c|c|c|c|}
\hline \multirow{2}{*}{ Station number } & \multicolumn{5}{|c|}{ Difference for the indicated period, in percent } \\
\hline & $1930-42$ & 1943-62 & $1963-69$ & 1970-79 & 1980-2002 \\
\hline \multicolumn{6}{|c|}{ 1-day 10-year hydrologically based low flow (1010) } \\
\hline 01606500 & -2.8 & 4.2 & -17.7 & 26.5 & 4.4 \\
\hline 01608500 & -3.5 & 6.1 & -28.2 & 62.0 & 10.8 \\
\hline 01611500 & -1.8 & 7.5 & -31.4 & 19.9 & 18.3 \\
\hline 01636500 & -21.5 & 15.5 & -19.0 & 23.6 & 10.8 \\
\hline 03051000 & -56.5 & 16.0 & 74.6 & 524.4 & 7.0 \\
\hline 03053500 & -56.8 & -17.8 & 33.5 & 366.3 & 45.7 \\
\hline 03061500 & -66.7 & -20.7 & 50.2 & 351.5 & 149.1 \\
\hline 03066000 & 4.6 & -32.0 & 7.8 & 98.1 & 14.4 \\
\hline 03069500 & -24.9 & -18.9 & -11.6 & 170.1 & 25.1 \\
\hline 03070500 & -7.2 & -56.3 & 31.8 & 217.4 & 93.8 \\
\hline 03182500 & -35.4 & 7.3 & -28.9 & 115.6 & 28.6 \\
\hline 03183500 & -13.3 & -2.1 & 4.3 & 70.5 & -1.9 \\
\hline 03186500 & -1.1 & -30.4 & 23.3 & 312.8 & -6.3 \\
\hline 03198500 & -45.3 & -37.7 & -28.2 & 339.7 & 250.0 \\
\hline $\begin{array}{l}{ }^{\mathrm{a}} 03214000 \text { and } \\
03214500\end{array}$ & No value & b -8.6 & b 20.5 & b 107.0 & c 134.3 \\
\hline \multicolumn{6}{|c|}{ 7-day 10-year hydrologically based low flow (7010) } \\
\hline 01606500 & -2.1 & 2.6 & -19.2 & 27.2 & 5.7 \\
\hline 01608500 & -5.5 & 5.7 & -26.1 & 59.6 & 11.6 \\
\hline 01611500 & -2.2 & 5.1 & -29.4 & 21.0 & 18.1 \\
\hline 01636500 & -7.1 & 14.0 & -24.6 & 14.0 & 4.4 \\
\hline 03051000 & -53.9 & -4.9 & 55.7 & 582.5 & 24.3 \\
\hline 03053500 & -56.3 & -20.2 & 33.3 & 387.1 & 45.6 \\
\hline 03061500 & -59.0 & -20.1 & 41.5 & 269.0 & 98.7 \\
\hline 03066000 & 2.2 & -26.6 & -7.7 & 101.7 & 13.6 \\
\hline 03069500 & -25.2 & -23.0 & -4.4 & 182.3 & 30.6 \\
\hline 03070500 & -7.5 & -56.2 & 30.5 & 213.9 & 98.5 \\
\hline 03182500 & -26.9 & 0.9 & -26.9 & 117.2 & 19.0 \\
\hline 03183500 & -14.3 & -4.5 & 3.4 & 63.1 & 3.1 \\
\hline 03186500 & 12.0 & -34.0 & 19.5 & 302.9 & -3.2 \\
\hline 03198500 & -42.3 & -36.2 & -27.1 & 293.1 & 217.8 \\
\hline $\begin{array}{l}{ }^{\mathrm{b}} 03214000 \text { and } \\
03214500\end{array}$ & No value & b -5.9 & b 17.6 & b 107.5 & c 127.7 \\
\hline
\end{tabular}


Table 3. Differences between selected annual statistics computed for 1930-2002 and the statistics computed for the indicated record periods for 15 stations in West Virginia.-Continued

[Station numbers for the 15 stations are 01606500, 01608500, 01611500, 01636500, 03051000, 03053500, 03061500, 03066000, 03069500, 03070500, 03182500, 03183500, 03186500, 03198500, and combined stations 03214000 and 03214500; a negative value means the average for the indicated record period is less than the average for 1930-2002; a positive value means the average for the indicated record period is greater than the average for 1930-2002]

\begin{tabular}{|c|c|c|c|c|c|}
\hline \multirow{2}{*}{ Station number } & \multicolumn{5}{|c|}{ Difference for the indicated period, in percent } \\
\hline & $1930-42$ & $1943-62$ & $1963-69$ & 1970-79 & 1980-2002 \\
\hline \multicolumn{6}{|c|}{ 30-day 5-year hydrologically based low flow (3005) } \\
\hline 01606500 & -0.2 & 4.8 & -19.0 & 25.7 & 2.8 \\
\hline 01608500 & -1.6 & 4.4 & -25.2 & 39.3 & 4.7 \\
\hline 01611500 & -3.0 & -0.4 & -28.5 & 30.0 & 12.1 \\
\hline 01636500 & -1.5 & 6.9 & -23.2 & 13.9 & 3.0 \\
\hline 03051000 & -21.6 & -21.9 & -18.0 & 281.2 & 9.2 \\
\hline 03053500 & -35.3 & -23.8 & -5.3 & 215.2 & 20.2 \\
\hline 03061500 & -50.6 & -19.6 & -8.0 & 220.6 & 58.1 \\
\hline 03066000 & 7.6 & -25.2 & -16.6 & 93.6 & 11.2 \\
\hline 03069500 & 2.3 & -22.7 & -14.4 & 115.3 & 11.6 \\
\hline 03070500 & -14.9 & -35.4 & -37.2 & 134.8 & 54.2 \\
\hline 03182500 & 0.7 & 5.9 & -37.1 & 65.5 & 2.1 \\
\hline 03183500 & 2.2 & 2.3 & -10.0 & 53.6 & -2.1 \\
\hline 03186500 & 11.1 & -26.1 & -20.8 & 188.7 & 0.9 \\
\hline 03198500 & -32.2 & -34.4 & -27.6 & 149.2 & 97.3 \\
\hline $\begin{array}{c}{ }^{\mathrm{a}} 03214000 \text { and } \\
03214500\end{array}$ & No value & b -5.6 & b 3.2 & b 100.4 & с 76.1 \\
\hline \multicolumn{6}{|c|}{ 1-day 3-year biologically based low flow (1B3) } \\
\hline 01606500 & -6.3 & 5.7 & -10.2 & 34.5 & 1.8 \\
\hline 01608500 & -8.2 & 4.9 & -11.2 & 85.9 & 33.1 \\
\hline 01611500 & -3.0 & 2.4 & -27.0 & 18.6 & 24.1 \\
\hline 01636500 & -21.1 & 21.4 & -28.6 & 21.7 & 18.1 \\
\hline 03051000 & -93.9 & -7.1 & 75.0 & 541.9 & -20.6 \\
\hline 03053500 & -92.7 & -11.0 & 32.2 & 265.7 & 12.7 \\
\hline 03061500 & -60.0 & -10.9 & 60.0 & 480.0 & 223.6 \\
\hline 03066000 & -15.8 & -26.3 & 12.5 & 101.8 & 21.8 \\
\hline 03069500 & -59.5 & -29.4 & -14.5 & 159.9 & 33.5 \\
\hline 03070500 & -43.8 & -42.6 & 24.9 & 145.4 & 71.1 \\
\hline 03182500 & -42.3 & 0.0 & -16.5 & 153.6 & 36.4 \\
\hline 03183500 & -38.0 & -13.3 & 4.0 & 61.8 & 2.0 \\
\hline 03186500 & 21.2 & -25.6 & 34.6 & 384.0 & 39.1 \\
\hline 03198500 & -52.9 & -26.5 & -30.0 & 458.8 & 367.6 \\
\hline $\begin{array}{c}{ }^{\mathrm{a}} 03214000 \text { and } \\
03214500\end{array}$ & No value & b -2.7 & b 32.4 & b 97.3 & c 147.5 \\
\hline
\end{tabular}


Table 3. Differences between selected annual statistics computed for 1930-2002 and the statistics computed for the indicated record periods for 15 stations in West Virginia.-Continued

[Station numbers for the 15 stations are $01606500,01608500,01611500,01636500,03051000,03053500,03061500,03066000$, 03069500, 03070500, 03182500, 03183500, 03186500, 03198500, and combined stations 03214000 and 03214500; a negative value means the average for the indicated record period is less than the average for 1930-2002; a positive value means the average for the indicated record period is greater than the average for 1930-2002]

\begin{tabular}{|c|c|c|c|c|c|}
\hline \multirow{2}{*}{ Station number } & \multicolumn{5}{|c|}{ Difference for the indicated period, in percent } \\
\hline & $1930-42$ & 1943-62 & $1963-69$ & 1970-79 & 1980-2002 \\
\hline \multicolumn{6}{|c|}{ 4-day 3-year biologically based low flow (4B3) } \\
\hline 01606500 & -8.5 & 6.3 & -12.1 & 34.1 & 8.5 \\
\hline 01608500 & -4.3 & 6.8 & -9.7 & 79.3 & 36.8 \\
\hline 01611500 & -0.5 & 3.4 & -25.3 & 25.5 & 24.0 \\
\hline 01636500 & -13.4 & 17.2 & -30.9 & 13.4 & 12.2 \\
\hline 03051000 & -83.4 & -10.0 & 66.6 & 580.0 & -20.0 \\
\hline 03053500 & -91.3 & -17.7 & 15.3 & 360.0 & 20.7 \\
\hline 03061500 & -40.5 & -12.7 & 51.9 & 350.6 & 155.7 \\
\hline 03066000 & -7.1 & -23.5 & 7.9 & 114.0 & 22.2 \\
\hline 03069500 & -62.2 & -38.7 & 8.9 & 164.6 & 23.8 \\
\hline 03070500 & -30.0 & -52.3 & 33.6 & 105.5 & 52.6 \\
\hline 03182500 & -37.4 & -6.7 & -6.7 & 145.0 & 55.8 \\
\hline 03183500 & -39.5 & -14.4 & 2.1 & 68.9 & 2.1 \\
\hline 03186500 & 16.8 & -30.2 & 34.2 & 326.7 & 23.3 \\
\hline 03198500 & -48.6 & -30.4 & -35.1 & 383.5 & 305.7 \\
\hline $\begin{array}{c}{ }^{\mathrm{a}} 03214000 \text { and } \\
03214500\end{array}$ & No value & b -0.3 & b 32.3 & b 103.3 & c 159.9 \\
\hline \multicolumn{6}{|c|}{ U.S. Environmental Protection Agency harmonic-mean flow } \\
\hline 01606500 & -5.6 & 0.9 & -26.2 & 22.3 & 5.6 \\
\hline 01608500 & -7.4 & 0.0 & -30.1 & 29.6 & 8.2 \\
\hline 01611500 & -10.7 & -1.3 & -24.5 & 35.8 & 6.3 \\
\hline 01636500 & -7.3 & 3.3 & -27.1 & 23.6 & 4.1 \\
\hline 03051000 & -57.7 & 16.4 & 11.0 & 222.2 & 46.1 \\
\hline 03053500 & -62.3 & 7.8 & 30.4 & 244.3 & 97.2 \\
\hline 03061500 & -49.9 & -11.9 & -28.9 & 156.3 & 56.3 \\
\hline 03066000 & -7.2 & -15.9 & -17.2 & 49.1 & 15.1 \\
\hline 03069500 & -23.1 & -9.0 & -16.2 & 54.4 & 18.8 \\
\hline 03070500 & 17.4 & -45.4 & -19.2 & 159.0 & 78.5 \\
\hline 03182500 & -18.8 & 2.8 & -29.9 & 45.1 & 10.4 \\
\hline 03183500 & -14.7 & 1.6 & -19.9 & 40.6 & 3.5 \\
\hline 03186500 & -7.0 & -23.5 & -8.5 & 121.4 & 10.4 \\
\hline 03198500 & -39.9 & -21.5 & -19.2 & 93.5 & 72.0 \\
\hline $\begin{array}{c}{ }^{a} 03214000 \text { and } \\
03214500\end{array}$ & No value & b -10.1 & $\mathrm{~b}-2.7$ & b 63.4 & c 41.3 \\
\hline
\end{tabular}

a 1930-2002 period estimated from record for 1936-85 at Tug Fork near Kermit (03214000).

${ }^{\mathrm{b}}$ Computed from record for Tug Fork near Kermit (03214000).

${ }^{\mathrm{c}}$ Estimated from record for 1986-2002 at Tug Fork at Kermit (03214500). 
Table 4. Average differences between selected seasonal statistics computed for 1930-2002 and the statistics computed for the indicated record periods for 15 stations in West Virginia.

[Winter, January 1-March 31; spring, April 1-June 30; summer, July 1-September 30; fall, October 1-December 31; station numbers for the 15 stations are $01606500,01608500,01611500,01636500,03051000,03053500,03061500,03066000,03069500,03070500$, $03182500,03183500,03186500,03198500$, and combined stations 03214000 and 03214500 ; a negative value means the average for the indicated record period is less than the average for 1930-2002; a positive value means the average for the indicated record period is greater than the average for 1930-2002]

\begin{tabular}{|c|c|c|c|c|c|}
\hline \multirow{2}{*}{ Season } & \multicolumn{5}{|c|}{ Difference for the indicated period, in percent } \\
\hline & 1930-42 & $1943-62$ & 1963-69 & 1970-79 & 1980-2002 \\
\hline \multicolumn{6}{|c|}{ 1-day 10-year hydrologically based low flow (1010) } \\
\hline Winter & -9.7 & 6.0 & -11.5 & 69.4 & -2.6 \\
\hline Spring & -12.5 & 35.7 & -29.5 & 32.4 & 9.2 \\
\hline Summer & -24.8 & -6.5 & -3.6 & 175.4 & 44.8 \\
\hline Fall & -26.2 & -18.8 & 6.3 & 167.2 & 62.2 \\
\hline \multicolumn{6}{|c|}{ 7-day 10-year hydrologically based low flow (7010) } \\
\hline Winter & -12.2 & -4.4 & -17.3 & 58.5 & 0.1 \\
\hline Spring & -17.6 & 19.5 & -29.2 & 27.1 & 3.4 \\
\hline Summer & -21.8 & -8.3 & -6.0 & 176.2 & 39.2 \\
\hline Fall & -25.6 & -20.4 & 10.6 & 140.4 & 55.3 \\
\hline \multicolumn{6}{|c|}{ 30-day 5-year hydrologically based low flow (3005) } \\
\hline Winter & -19.2 & 2.3 & -31.4 & 31.7 & 6.5 \\
\hline Spring & -18.7 & 13.1 & -34.3 & 5.1 & 6.8 \\
\hline Summer & -12.5 & -6.3 & -29.2 & 85.4 & 20.0 \\
\hline Fall & -25.6 & -17.5 & -16.5 & 104.6 & 22.9 \\
\hline \multicolumn{6}{|c|}{ 1-day 3-year biologically based low flow (1B3) } \\
\hline Winter & -11.4 & -4.2 & 1.7 & 73.8 & 5.8 \\
\hline Spring & -30.2 & 15.7 & 4.3 & 4.7 & 57.9 \\
\hline Summer & -35.0 & -5.4 & 0.8 & 170.2 & 50.8 \\
\hline Fall & -35.7 & -15.1 & 7.8 & 207.7 & 72.2 \\
\hline \multicolumn{6}{|c|}{ 4-day 3-year biologically based low flow (4B3) } \\
\hline Winter & -8.1 & -4.4 & -13.2 & 59.1 & 5.1 \\
\hline Spring & -24.3 & 18.6 & 3.0 & 0.6 & 53.7 \\
\hline Summer & -33.2 & -5.8 & -2.8 & 159.3 & 39.1 \\
\hline Fall & -40.0 & -20.3 & 7.3 & 170.2 & 54.1 \\
\hline \multicolumn{6}{|c|}{ U.S. Environmental Protection Agency harmonic-mean flow } \\
\hline Winter & -5.7 & 5.5 & -19.5 & 37.9 & -1.9 \\
\hline Spring & -25.3 & 4.0 & -14.9 & 3.8 & 28.6 \\
\hline Summer & -21.9 & 1.0 & -27.9 & 86.3 & 31.8 \\
\hline Fall & -18.3 & -14.3 & 2.9 & 142.5 & 41.0 \\
\hline
\end{tabular}


percent). The average differences of values for all statistics are less for 1980-2002 than for 1970-79 except for spring values of 30Q5, biologically based flows, and the EPA harmonicmean flow. The differences of values for all seasons for all statistics for the individual stations range from -45.5 to 603.4 percent for 1980-2002.

Karl and Knight (1998) found that the increases in low flows around 1970 coincide with increased rainfall, primarily in the fall (September 1-November 30) when the lowest streamflows typically occur. As table 4 indicates, the average differences for the period 1970-79 in the summer (July 1-September 30) and fall (October 1-December 31) are the greatest differences for any of the periods investigated. The results of this study are similar to the findings by Karl and Knight (1998).

\section{Comparison Between Hydrologically Based and Biologically Based Low Flows}

The 1Q10 and 1B3 were found to be nearly equal, on average, in an analysis of 60 streams across the United States by the U.S. Environmental Protection Agency (1986). The 7Q10 and 4B3 were also found to be nearly equal.

In this study, averaged-station comparisons (table 6) and individual-station comparisons (table 7) between 1Q10/1B3 and 7Q10/4B3 were made for 1930-2002 for the 15 stations. The $1 \mathrm{~B} 3$ and 4B3 average 6.2- and 7.9-percent less than the 1Q10 and 7Q10 (table 6), respectively; differences for the individual stations range from 28.5 percent less to 13.6 percent greater (table 7). Generally, the biologically based flow was less than the hydrologically based flow in the winter and fall, greater in the spring, and varied among the individual stations during the summer.
The results of this study are consistent with the findings by the U.S. Environmental Protection Agency (1986) for the analysis of hydrologically and biologically based flows. There were no statistics computed seasonally in the study by the U.S. Environmental Protection Agency that could be compared to the results from this study.

\section{Selected Flow Statistics Representative of 1930-2002}

The sample of available streamflow records is not representative of the entire population of streamflow records over the time period encompassing the operation of all the stations. Therefore, estimating equations developed by using the entire sample of station records would not be representative of the overall period when stations were operating. Estimating equations should be developed by use of station records that are representative of streamflows during a particular period of interest. These representative station records could not be acquired by means of record-extension techniques; rather, they were acquired for this analysis by way of a purposeful sampling (criterion based for this application) of the available station records.

Record periods for unregulated stations throughout a state or other large area differ because of differing times that stations were operating at particular locations. The number of active stations in a given area changes through time because of budget constraints, interests in particular hydrologic investigations, and stream regulation. The number of stations in West Virginia was about 5 between 1900 and 1910, increased to about 20 between 1910 and 1920, increased to about 40 in the

Table 6.-Average annual and seasonal differences between the 1-day 10-year hydrologically based and 1-day 3year biologically based low-flow frequencies and between the 7-day 10-year hydrologically based and 4-day 3-year biologically based low-flow frequencies computed for 1930-2002 for 15 stations in West Virginia.

[Winter, January 1-March 31; spring, April 1-June 30; summer, July 1-September 30; fall, October 1-December 31; 1Q10, 1-day 10-year hydrologically based low-flow frequency; 1B3, 1-day 3-year biologically based low-flow frequency; 7Q10, 7-day 10-year hydrologically based low-flow frequency; 4B3, 4-day 3-year biologically based low-flow frequency; negative numbers indicate the biologically based flow is less than the hydrologically based flow, and positive numbers indicated the biologically based flow is greater than the hydrologically based flow; station numbers for the 15 stations are $01606500,01608500,01611500,01636500,03051000,03053500,03061500$, 03066000, 03069500, 03070500, 03182500, 03183500, 03186500, 03198500, and combined stations 03214000 and 03214500]

\begin{tabular}{cccccc}
\hline \multirow{2}{*}{$\begin{array}{c}\text { Compared } \\
\text { streamflow } \\
\text { statistics }\end{array}$} & Annual & \multicolumn{5}{c}{ Average difference, in percent } \\
\cline { 2 - 6 } & & Winter & Spring & Summer & Fall \\
\cline { 2 - 6 } & -6.2 & -23.1 & 59.9 & 2.8 & -23.9 \\
1Q10 and 1B3 & -7.9 & -20.9 & 49.6 & 4.1 & -21.9 \\
\hline
\end{tabular}




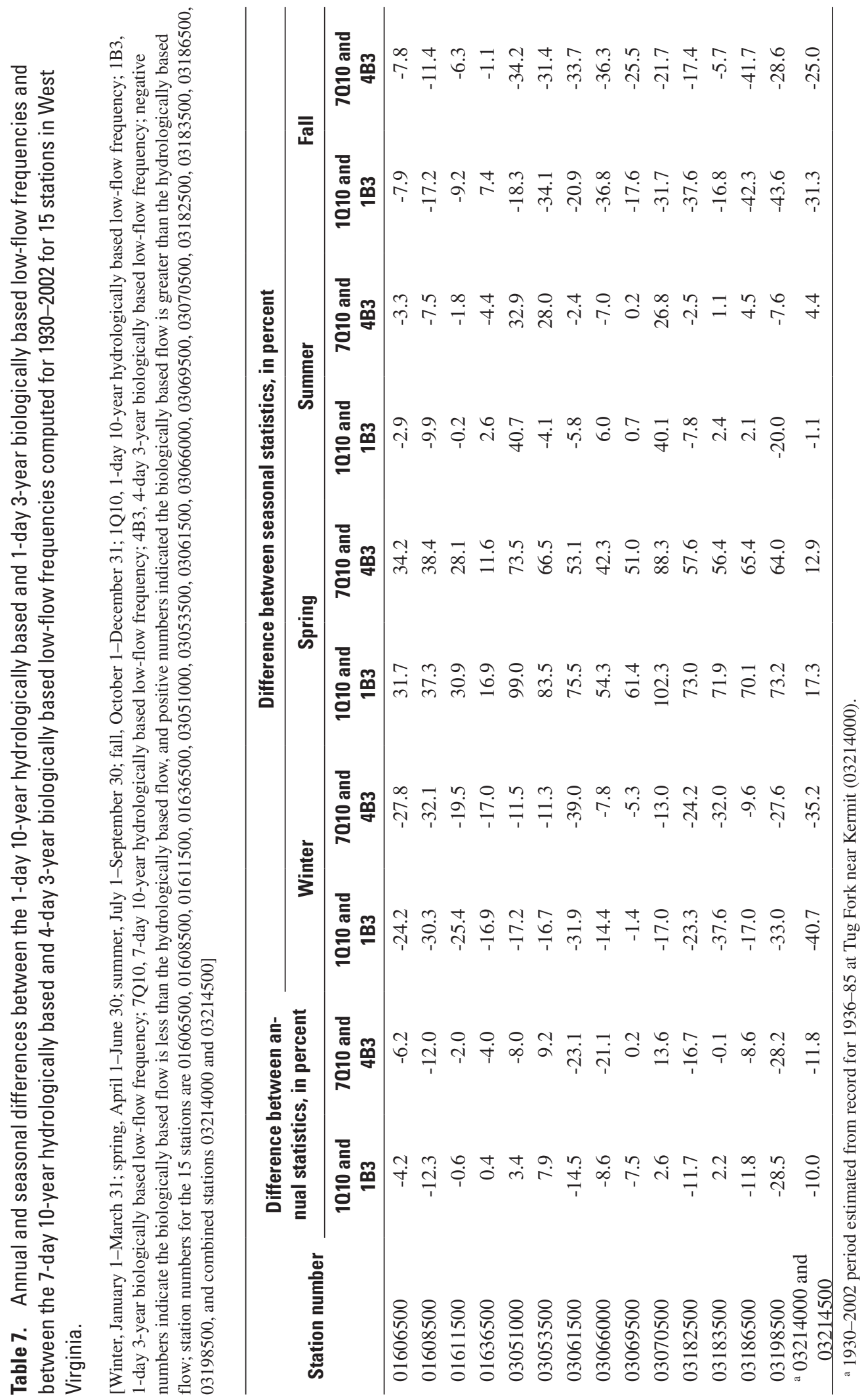


early 1930s, increased to about 95 in the late 1940s, was about 95 between 1950 and 1960, increased to about 110 around 1970 , remained at 110 through the early 1980 s, decreased to about 70 in the middle 1980s (Runner and others, 1989, fig.1), and decreased to about 60 in 2002. The greatest number of stations was operating after a sudden increase in minimum flows was observed around 1970, and many fewer stations were operating during the 1930s, during a significant drought. Therefore, the entire sample of station records is not random and does not represent the entire population; generally, the entire sample of station records has a positive bias over the period encompassing the operation of the stations.

Record periods in West Virginia can be divided into three general groups based on length: those near the length of the overall time period encompassing the operation of the stations, those near the median length of record periods for all stations, and those near the length of the 10-year minimum record period. Record periods near the length of the overall time period will be representative of the entire population. Record periods near the median length of record periods for all stations will be biased toward the period during the 1970s when minimum flows were much greater than average and biased away from the 1930s when there was a significant drought, if the record periods are equally distributed across the entire sample of station records. Bias in record periods near the 10year minimum will be similar to those near the median length of record periods for all stations if they are equally distributed across the entire sample of station records. Some record periods near the 10-year minimum will be during consistently wet or dry periods and will be significantly different than the average of the entire population.

Log10-transformed minimum flows for the entire record period at the 15 stations with record during the period 19302002 (fig. 4) and five stations from outside the State were correlated with $\log 10$-transformed minimum flows for the entire record period at the remaining stations to study recordextension capabilities. Four stations were in Virginia: North Fork Shenandoah River at Cootes Store (01632000), Goose Creek near Leesburg (01644000), Dunlap Creek near Covington (02013000), and Russell Fork at Haysi (03208500). One station was in Ohio; Little Beaver Creek near East Liverpool (03109500). Correlation coefficients of 0.80 or greater were considered reliable for extending the station record at the remaining stations to the period 1930-2002. There were 47 stations in West Virginia with correlation coefficients of 0.80 or greater, but none of these stations were in the northern panhandle and only 4 stations were within $50 \mathrm{mi}$ of the Ohio River along the western border of the State. Although records could be extended at the 47 stations, the areal distribution of stations was insufficient for developing estimating equations for ungaged-stream locations.

A criterion-based sampling (Goetz and LeCompte, 1984, p. 77) of the entire sample of station records can provide record periods representative of a defined time period. The criterion would reduce the influence caused particularly by the positive bias inherent in the entire sample of station records but also by the bias resulting when individual station records were collected only during a consistently wet or dry period. The longest time period with a sufficient number of active stations well distributed across the State was needed to develop a criterion for sampling. This period also should include a significant drought. The period 1930-2002 has record periods at 15 stations distributed across the State and includes the drought of the 1930s.

Correlation between the log 10-transformed average minimum flow and the log 10-transformed 1Q10, 7Q10, 30Q5, 1B3, and 4B3, and the EPA harmonic-mean flows for 110 stations (listed in table 1) having a minimum of 10 years of low-flow record in West Virginia resulted in correlation coefficients ranging from 0.987 to 0.998 . The high correlation between the average minimum flow and the selected statistics indicates that the minimum flow can be used as a surrogate for statistical analysis. A sampling criterion based on record periods determined from analyzing the minimum flow will be applicable to low-flow statistics. A sampling criterion that the average minimum flow of the record period is nearly equal to the average minimum flow for 1930-2002 will provide representative record periods of the entire population for 1930-2002.

The standardized departures of the minimum flows for the 15 stations during 1930-2002 (fig. 4) were combined with the standardized departures of five stations outside the State (identified above) to determine regional subsets of record periods that could represent 1930-2002.

Principal component and correlation analyses were done on the standardized departures of the 20 stations. Three groups of stations representing three regions of the State were identified, and three stations were removed from consideration in the regional analysis. The three regions are identified in this report as the Lower Appalachian Plateaus, the Upper Appalachian Plateaus, and the Eastern Panhandle (fig. 8). The main stem of the Cheat, Monongahela, and Elk Rivers downstream from the boundary between the Lower Appalachian Plateaus and Upper Appalachian Plateaus are part of the Upper Appalachian Plateaus, but the tributaries to these Rivers downstream from the boundary are part of the Lower Appalachian Plateaus. Greenbrier River at Alderson (03183500) was removed from consideration in the Upper Appalachian Plateaus because it was on the same stream and highly correlated with Greenbrier River at Buckeye (03182500) (correlation coefficient of 0.92). South Branch Potomac River near Springfield (01608500) was removed from consideration in the Eastern Panhandle because it was on the same stream and highly correlated with South Branch Potomac River near Petersburg (01606500) (correlation coefficient of 0.95). Dunlap Creek near Covington, Va. (02013000) was removed from the analysis because it was grouped with the Eastern Panhandle, although it is closer to the Upper Appalachian Plateaus; moreover, its unusually high minimum flow for 1930, which is apparently accurate (D.C. Hayes, U.S. Geological Survey, oral commun., September 10, 2004), is inconsistent with concurrent minimum flows at other stations in the study area. 


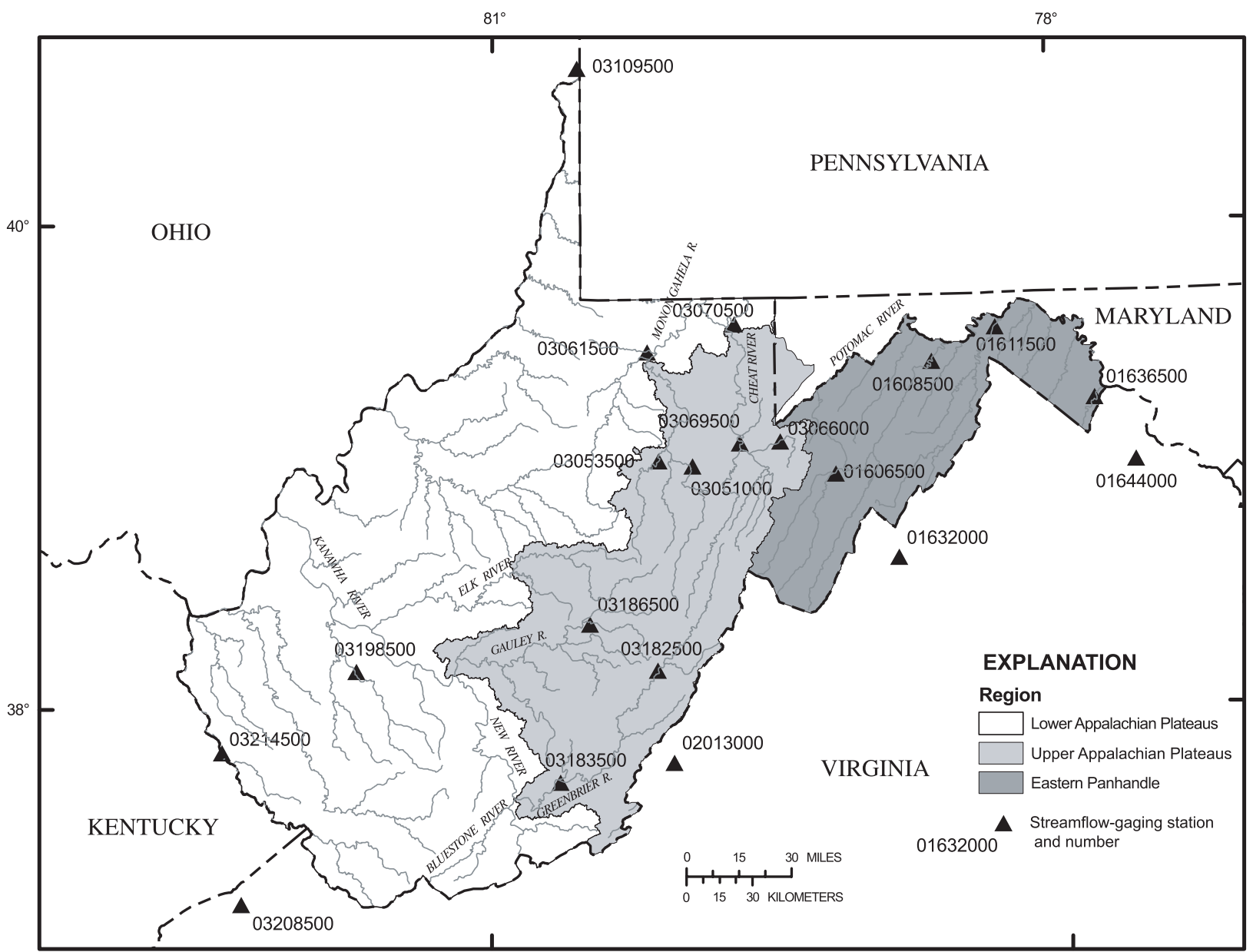

Base from U.S. Geological Survey 1:100,000 Digital Line Graphs for state boundaries and 1:1,000,000 scale digital data for streams; All data are in the Universal Transverse Mercator projection, zone 17, NAD1983 projection.

Figure 8. U.S. Geological Survey streamflow-gaging stations used to determine three regions of similar mimimum flow patterns: Lower Appalachian Plateaus, Upper Appalachian Plateaus, and Eastern Panhandle.

Generally, the Lower Appalachian Plateaus has less annual precipitation-between about 40 and 50 in.- than the Upper Appalachian Plateaus, where the annual precipitation is between about 50 and 60 in. (U.S. Department of Agriculture, Natural Resources Conservation Service, 2004). The exception is the Greenbrier River in the Upper Appalachian Plateaus where annual total precipitation is about 42-48 in.

The standardized departures were averaged for the three regions, and plots of the departures (figs. 9-11) show the increase around 1970 is most apparent in the Lower Appalachian Plateaus. The increase in minimum flows around 1970 and the greater-than-average minimum flows for 1970-79 probably dissipated from west to east, as evidenced by the absence of strong correlation between stations in the Lower Appalachian Plateaus and the difference between patterns of the departures for the Lower and Upper Appalachian Plateaus (figs. 9 and 10). There was also probably a north-to-south difference in minimum flows within the Lower Appalachian Plateaus, as evidenced by the absence of strong correlation between stations in that region. The east-to-west and northto-south variability in minimum flows in the Lower Appalachian Plateaus reduced the effectiveness of record-extension techniques. The seasonal increase in atmospheric moisture after about 1970 (Karl and Knight, 1998) could have resulted in storms having clouds with greater densities. Precipitation that previously did not fall until reaching the area of the State where mean annual precipitation is the greatest could have began falling sooner, resulting in greater relative increases in minimum flows in the Lower Appalachian Plateaus (fig. 9) and less or no relative increases in minimum flows in the Upper Appalachian Plateaus (fig. 10). This meteorological hypothesis could help explain why the plot of departures 
for the Greenbrier River at Alderson (03183500) did not show the increase in minimum flows around 1970 (fig. 2) evident in the plot of departures for the nearby combined stations Bluestone River near Pipestem (03179000) and Bluestone River at Lilly (03179500)(fig. 3). The Greenbrier River Basin receives annual precipitation of about $42-48$ in. and is within a significant rain shadow caused by mountainous terrain (annual precipitation reaches about $60 \mathrm{in}$. on the windward side), whereas the Bluestone River Basin receives annual precipitation of about 38-40 in. and is not within as significant of a rain shadow (annual precipitation reaches only about 46 in. on the windward side). There is little or no increase in minimum flows for the Greenbrier River because the increased moisture was probably depleted from crossing the mountainous terrain before reaching the basin, unlike the situation for the Bluestone River. The Upper Appalachian Plateaus is significantly different from the other regions in that standardized departures for the period 1970-79 are much lower, close to the average for 1930-2002. Also, none of the stations indicating a trend for 1930-2002 by use of the Mann-Kendall test (discussed earlier in this report) are in the Upper Appalachian Plateaus Region.

The sampling criterion was based on determining where the average minimum flow of a record period is nearly equal to that for 1930-2002 in each region. The standardized depar- tures for every 10-year or greater record period between 1930 and 2002 were averaged and compared to the standard deviation of the standardized departures for 1930-2002 for the three regions. The subset of record periods with average departures within 5 percent of the standard deviation of the departures were determined to be representative of 1930-2002 for the three regions (figs. 12-14). The subset of record periods may account for changes in low flow associated with climate variability, but changes in land use - particularly associated with mining_-probably remain.

The Lower Appalachian Plateaus, with six stations, has the most restrictive subset of record periods (fig. 12) compared to the other regions (figs. 13-14). There are no 10-year or greater record periods beginning after 1965 or ending before 1974. The record periods for the Lower Appalachian Plateaus are roughly balanced around about 1970 when there was an increase from negative to positive departures (fig. 9). The Upper Appalachian Plateaus has the least restrictive subset of record periods (fig. 13), with the subset of record periods that include the maximum possible beginning year, 1993. The Eastern Panhandle does not have any subset of record periods beginning after 1980 .

The subsets of record periods for the three regions were used to determine subset record periods for all stations in West Virginia. Subset record periods for all stations were selected

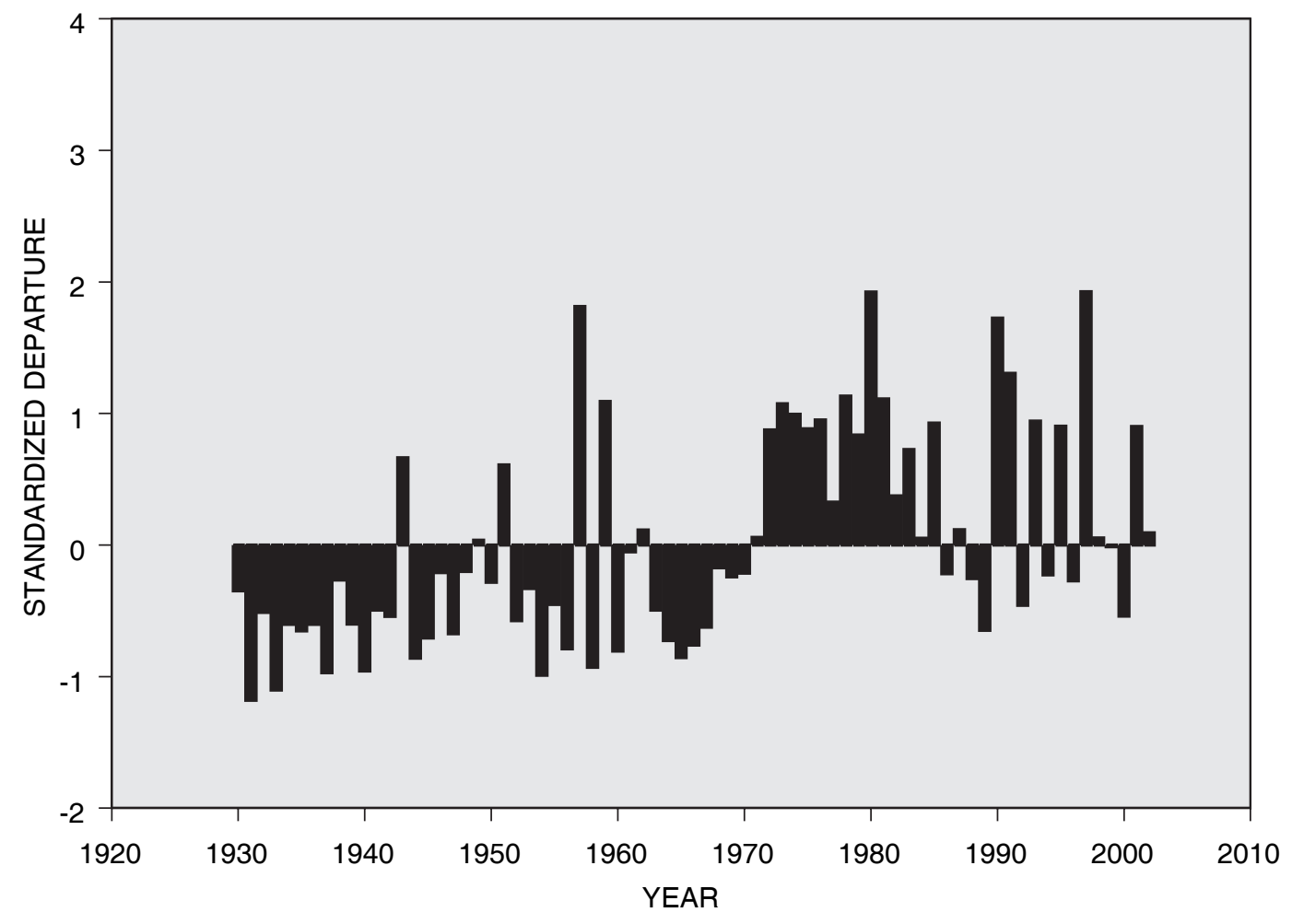

Figure 9. Average standardized departures of the minimum flows from the record-period average for 1930-2002 for six stations in the Lower Appalachian Plateaus region. 


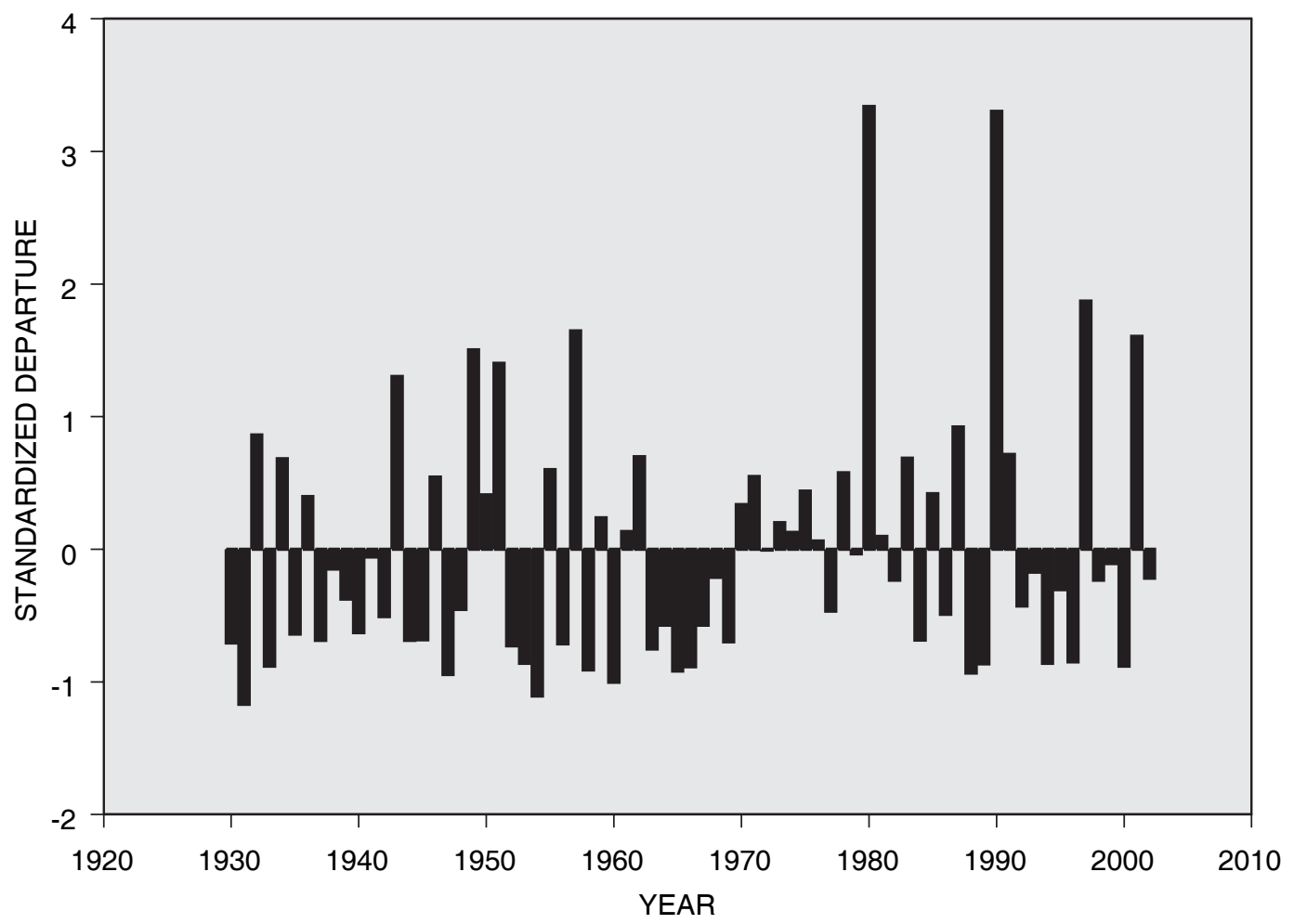

Figure 10. Average standardized departures of the minimum flows from the record-period average for 1930-2002 for six stations in the Upper Appalachian Plateaus region.

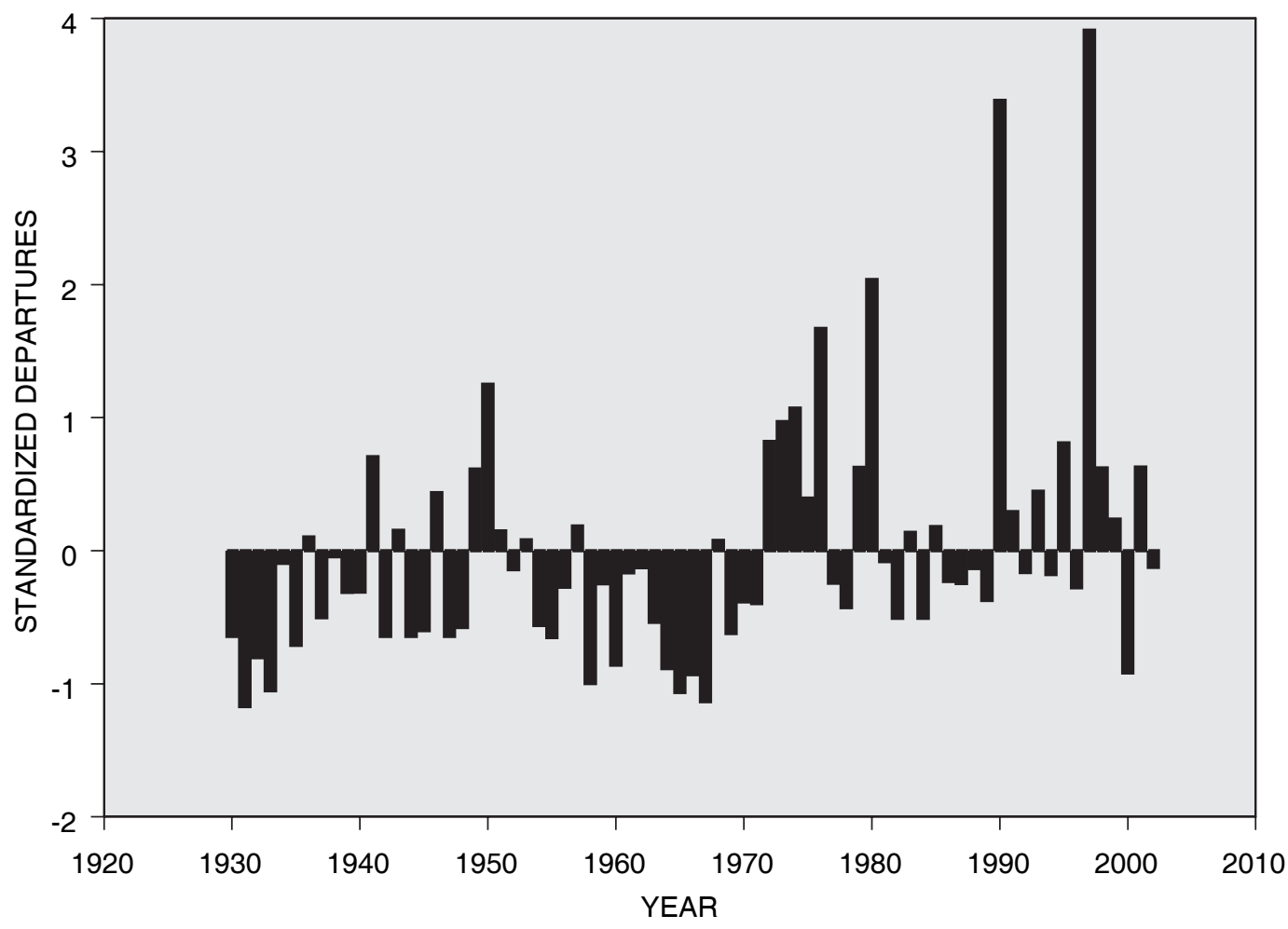

Figure 11. Average standardized departures of the minimum flows from the record-period average for 1930-2002 for five stations in the Eastern Panhandle region. 


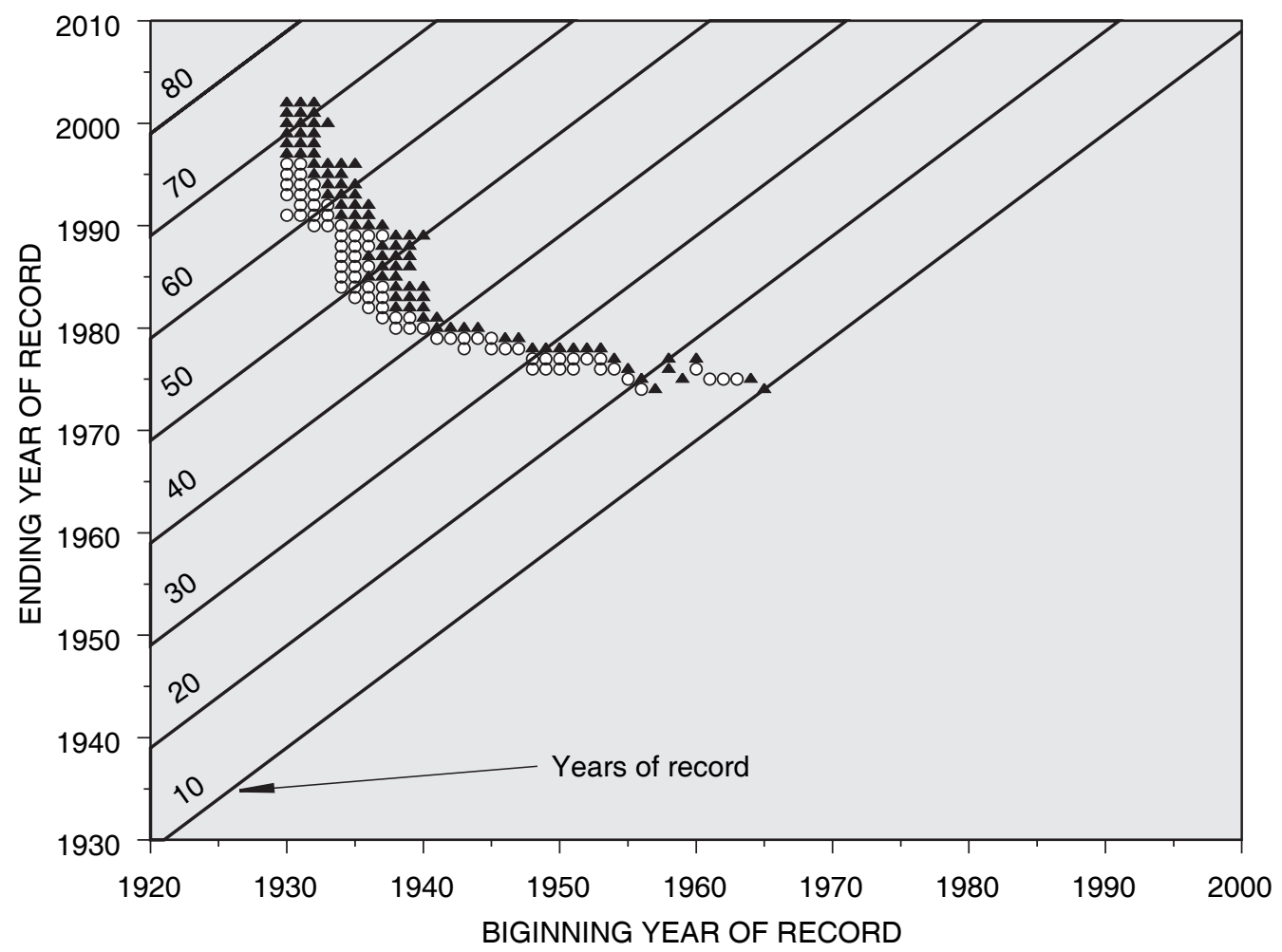

Figure 12. Record periods for the Lower Appalachian Plateaus region for which the average departure was within 5 percent of the standard deviation of the standardized departures of the minimum flows for 1930-2002 (Six stations in West Virginia, Ohio, and Virginia; open circle indicates negative departure; solid triangle indicates positive departure).

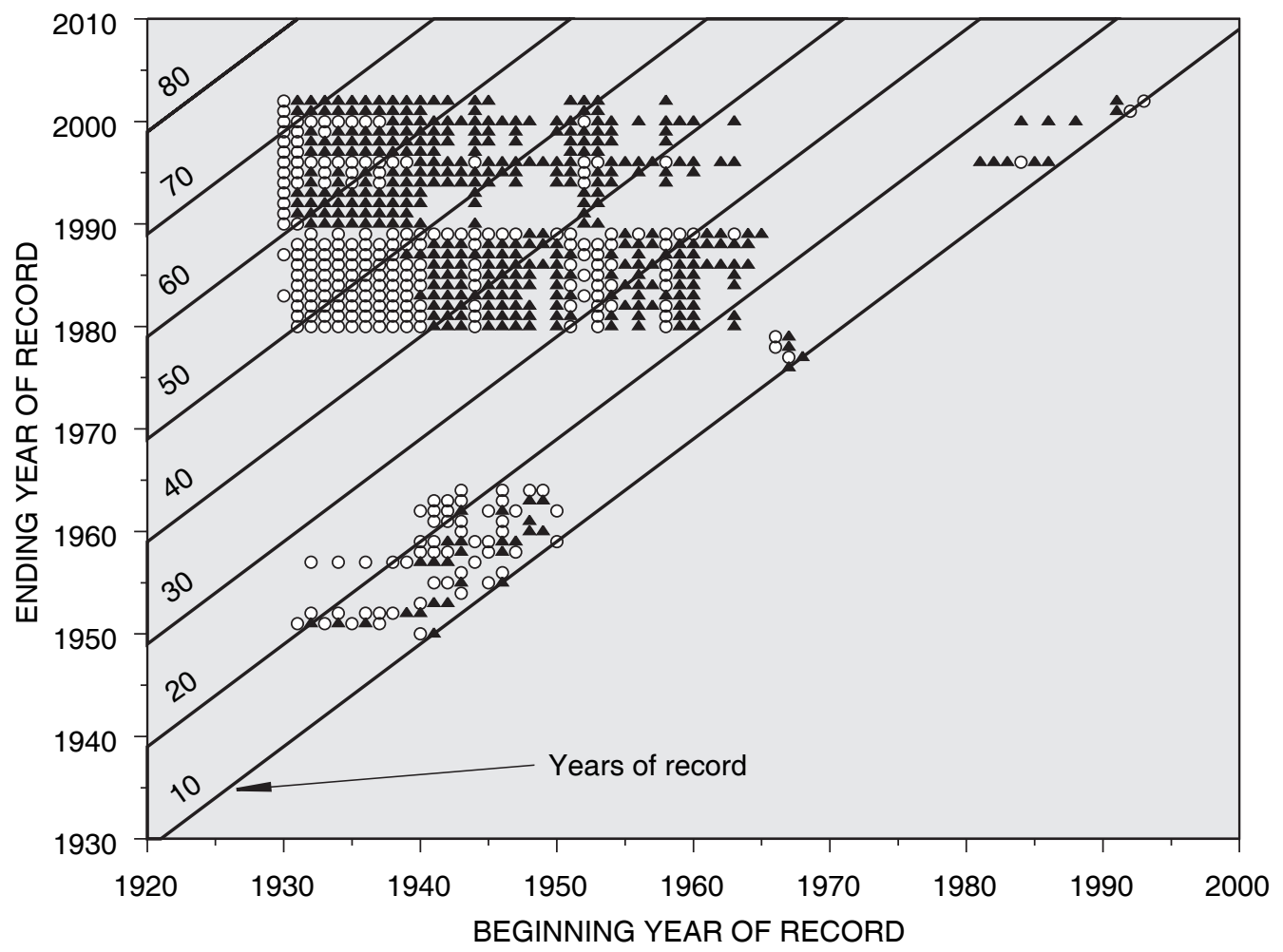

Figure 13. Record periods for the Upper Appalachian Plateaus region for which the average departure was within 5 percent of the standard deviation of the standardized departures of the mimimum flows for 1930-2002 (Six stations in West Virginia; open circle indicates negative departure; solid triangle indicates positive departure. 


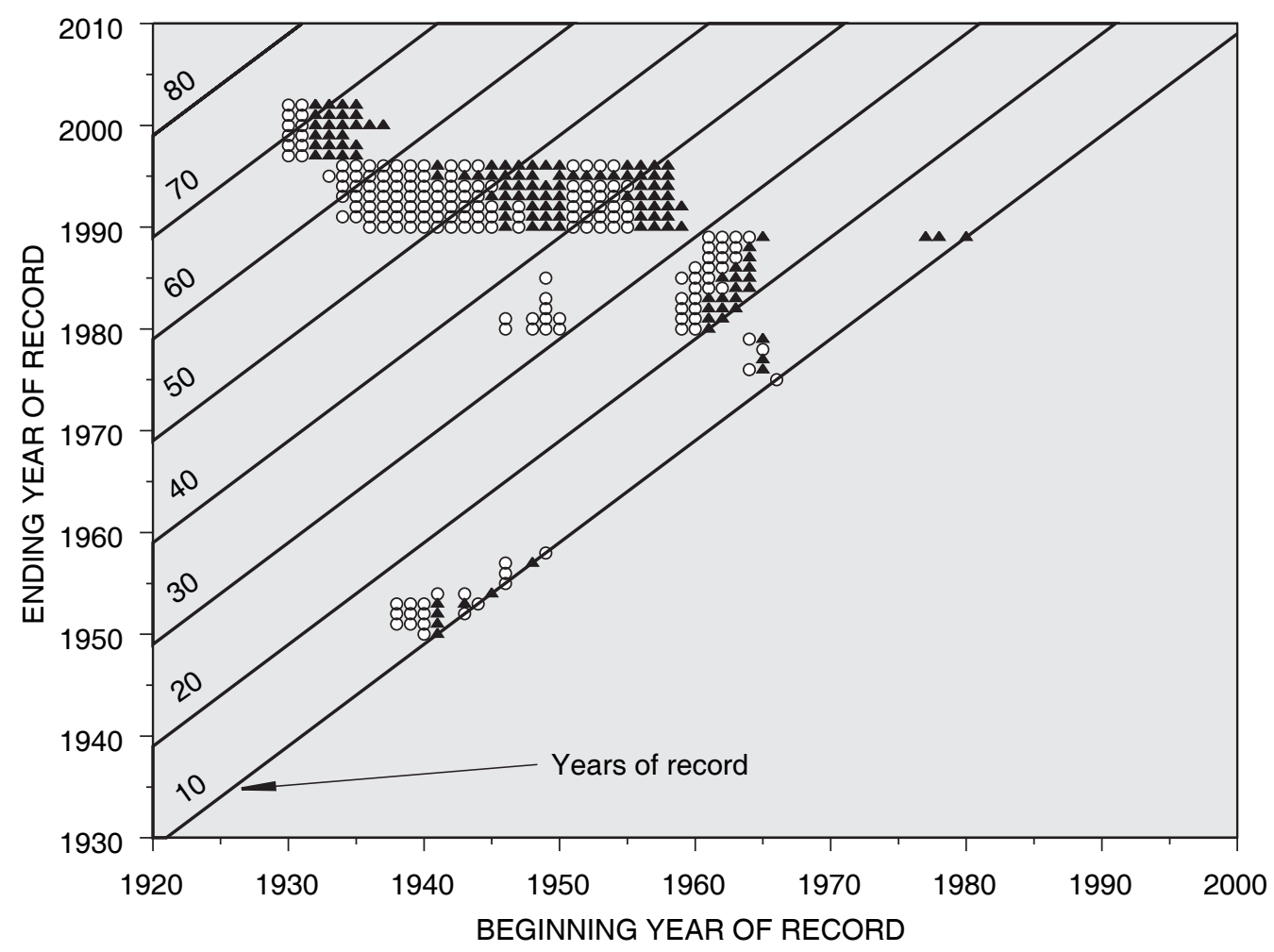

Figure 14. Record periods for the Eastern Panhandle region for which the average departure was within 5 percent of the standard deviation of the standardized departures of the minimum flows for 1930-2002 (Five stations in West Virginia; open circle indicates negative departure; solid triangle indicates positive departure).

from the subset of record periods determined by the maximum record length with the minimum departure for the appropriate region (figs. 12-14; tables 8-10, near the end of this report). The dark shaded areas in tables 8-10 indicate 10-year record periods not feasible through 2002, and the light shaded area in table 8 is for the first example presented below.

The subset record period for a particular station can be determined by means of the appropriate regional table. For example, if a station is in the Lower Appalachian Plateaus and the station has a record length of 26 years beginning in climatic year 1949, all record periods of 10 years or more between 1949 and 1974 are feasible. The feasible record periods can be found in table 8 by locating the table entry where the first year is 1949 and the years of record are 26. The field defined by every table entry to the left and increasing by one entry down with each entry to the left indicates the feasible record periods, and these entries are lightly shaded in table 8 for this example. The record period representing 1930-2002 is either the period with the maximum number of record years or the period with the tabled average departure nearest to zero when more than one period is available for the maximum number of years. This example indicates that the maximum number of years is 19 beginning in 1956, or a subset record period of $1956-74$, because the near-zero value of -0.034 is tabulated as the feasible record period having the maximum number of record years.

There is no subset record for Leading Creek near Glenville (03152500). There are 13 years of record 1939-51, and the station is located in the Lower Appalachian Plateaus (figs. 1 and 8). The feasible record periods can be found in table 8 by locating the table entry where the first year is 1939 and the years of record are 13; there are no values in the field defined by every table entry to the left and increasing by one entry down with each entry to the left. There is no subset record period because there are no values in the feasiblerecord field.

Selected statistics were computed for all stations where all or some part of the record period was included in the subset of record periods. Only continuous record periods were considered; therefore, a break in the record period could result in more than one selected record period for a station. The longest selected record period was used if more than one selected record period was considered for a station, or the selected record period with the closest departure to zero was used if the record periods were equal. For example, South Branch Potomac River at Franklin (01605500) is located in the East- 
ern Panhandle with record periods 1942-69 and 1978-2002. The feasible record periods for 1942-69 can be found in table 10 by locating the table entry where the first year is 1942 and the years of record are 28; the feasible records are in the field defined by every table entry to the left and increasing by one entry down with each entry to the left. There are 2 record periods with the maximum number of record years, 12 years beginning in 1943 with the table value of -0.040 and 12 years beginning in 1946 with the table value of -0.011 . The feasible record period for 1942-69 is 1946-57 with the tabled value of -0.011 because the tabled average departure is nearest to zero. The feasible record periods for 1978-2002 can be found in table 10 by locating the table entry where the first year is 1978 and the years of record are 25; the feasible records are in the field defined by every table entry to the left and increasing by one entry down with each entry to the left. The feasible record period for 1978-2002 is the 12 years 1978-89 with the tabled value of 0.039 because this is the period with the maximum number of record years. Finally, the subset record period is 1946-57 with the tabled value of -0.011 because the feasible records determined for the station record periods 1942-69 and 1978-2002 both have record lengths of 12 years and the feasible record period is the tabled value nearest zero.

Table 1 lists stations where streamflow statistics are published in this report; 77 of the 110 stations with a minimum of 10 years of record in West Virginia have a period of record that was included in the subset of record periods. The stations are areally distributed across the State, including the northern panhandle and the area along the Ohio River. Table 11, near the end of this report, lists the selected statistics that are representative of 1930-2002, and these values supersede those published by Friel and others (1989).

Record periods for 40 stations near but outside West Virginia were selected from the subset of record periods for the appropriate region, and the streamflow statistics computed from these record periods (table 12, at the end of this report) are representative of 1930-2002. These statistics can be used to assist in determining flows for West Virginia, but they do not supersede the values determined for use by the particular state.

Criterion-based sampling of the available record periods was superior to record-extension techniques for this study because more stations were selected and areal distribution of stations was more widespread. Climate variability is likely the primary cause of the regional patterns in the standardized departures of minimum flows. Comparisons of minimum flows between stations considered for record extension could have different slopes for different time periods (Riggs, 1972). For example, one station could be in the Lower Appalachian Plateaus and the other station in the Upper Appalachian Plateaus with record periods 1963-69 and 1963-79, respectively. Stations in both regions have much less than average minimum flows for 1963-69, but the minimum flows for the Lower Appalachian Plateaus are much greater than those for the Upper Appalachian Plateaus for 1970-79 (figs. 9 and 10). These stations could be in the same basin, and the correlation between the stations could be 0.80 or greater, but the extension of the station in the Lower Appalachian Plateaus for the period 1970-79 would reflect the lower flow of the station in the Upper Appalachian Plateaus. Station statistics computed with this type of error from record-extension techniques can make it more difficult to determine significant independent variables when developing estimating equations for ungaged stations. Criterion-based sampling of the available record is not prone to this error. Also, the criterion-based sampling allows for computation of medium-flow statistics, such as 50percent flow duration and EPA harmonic-mean flow, whereas record-extension techniques are limited to computation of only low-flow statistics; medium flows are also affected by climate variability (Lins and Slack, 1999; McCabe and Wolock, 2002).

The average minimum flows were computed for the entire record period and the subset record period for stations in West Virginia (table 13 at back of report) to quantify some of the effects resulting from criterion-based sampling. The entire record periods for 13 stations were included in the feasible record periods (figs. 12-14 and tables 8-10) making the entire record period equal to the subset record period. There were no subset record periods for 33 stations. The average minimum flows were divided by the respective drainage areas for the stations and plotted for the entire record period (fig. 15) and the subset record period (fig. 16) for comparison.

Two stations have much greater minimum flows than all other stations with respect to drainage area (identified as No. 1 in fig. 15): Tuscarora Creek above Martinsburg (01617000) and North Fork Cranberry River near Hillsboro (03187300). Tuscarora Creek is in a limestone area of karst topography, and its contributing drainage area probably is greater than the drainage area determined from topography. Tuscarora Creek would not be considered for developing estimating equations even if a subset record period had been determined because of insufficient information for streams in karst areas of the State. The record period for North Fork Cranberry River, 1970-82 (table 13), was a consistently wet period of high standardized departures without a subset record period.

Figures 15 and 16 identify stations within the Potomac River Basin as dark-shaded circles. Two stations are identified as outliers from this plot (No. 2 in fig. 15): Tuscarora Creek above Martinsburg (01617000) and Little Cacapon River near Levels (016098000). Tuscarora Creek is a high outlier as discussed above, and Little Cacapon River has lower minimum flows than the rest of the Potomic River Basin. The record period for Little Cacapon River, 1968-77 (table 13), incorporates the period when minimum flows increased suddenly around 1970. Neither of these stations has subset record periods.

East Fork Twelvepole Creek near Dunlow (03206600) is a minor stream flowing directly into the Ohio River with a record period of 1966-2002 (No. 3 in fig. 15). The record period was a consistently wet period of high standardized departures without a subset record period. East Fork Twelvepole Creek has a minimum flow higher than other streams 


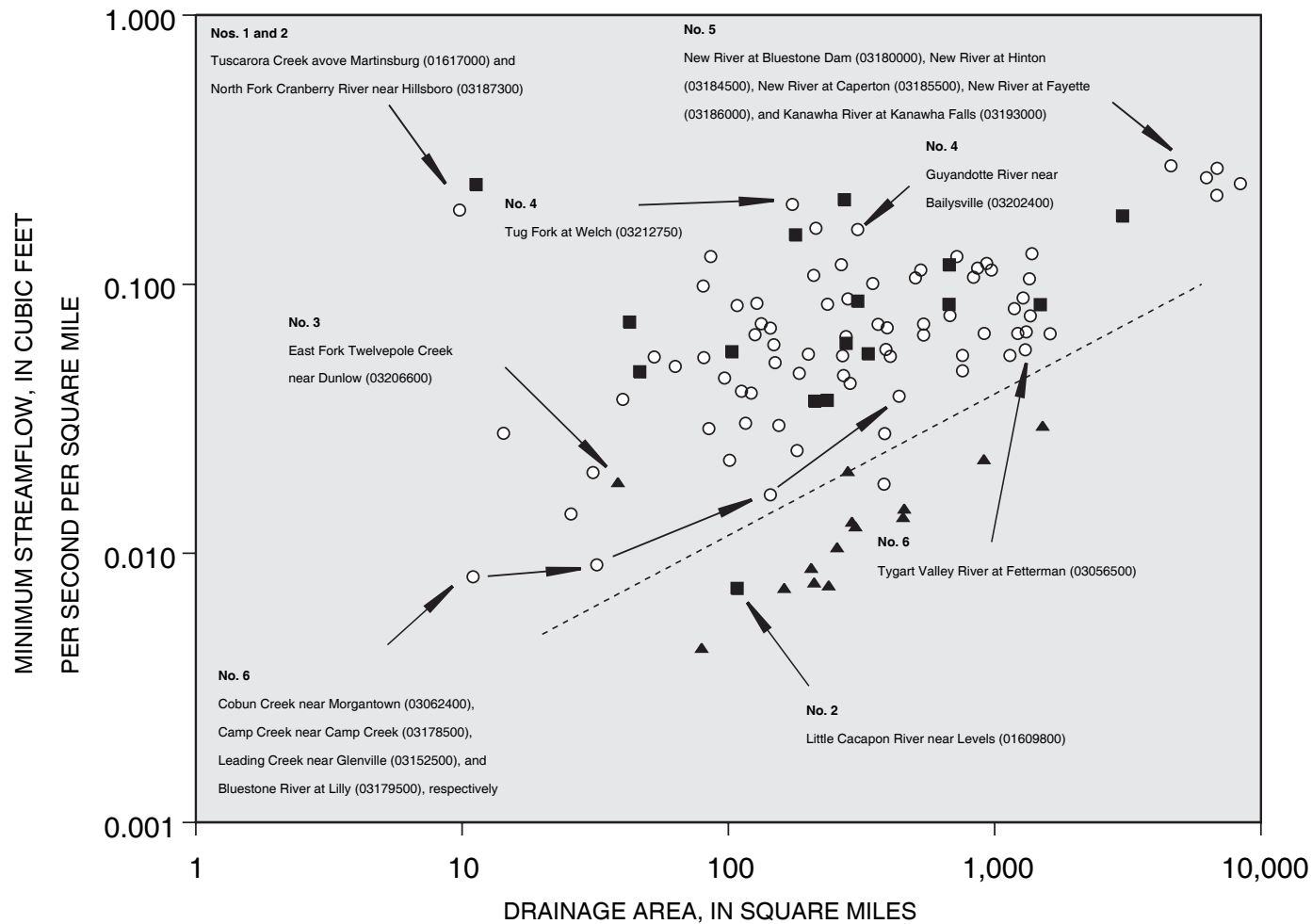

Figure 15. Unit average minimum flows for the entire record period at 110 stations in West Virginia (Open circle indicates station outside the Potomac River Basin and 50 miles or greater from the Ohio River; solid square indicates station within the Potomac River basin; solid triangle indicates station within 50 miles of the Ohio River; generally, points below the dashed line represent stations within 50 miles of the Ohio River; the numbered stations correspond to numbered discussions in text).,

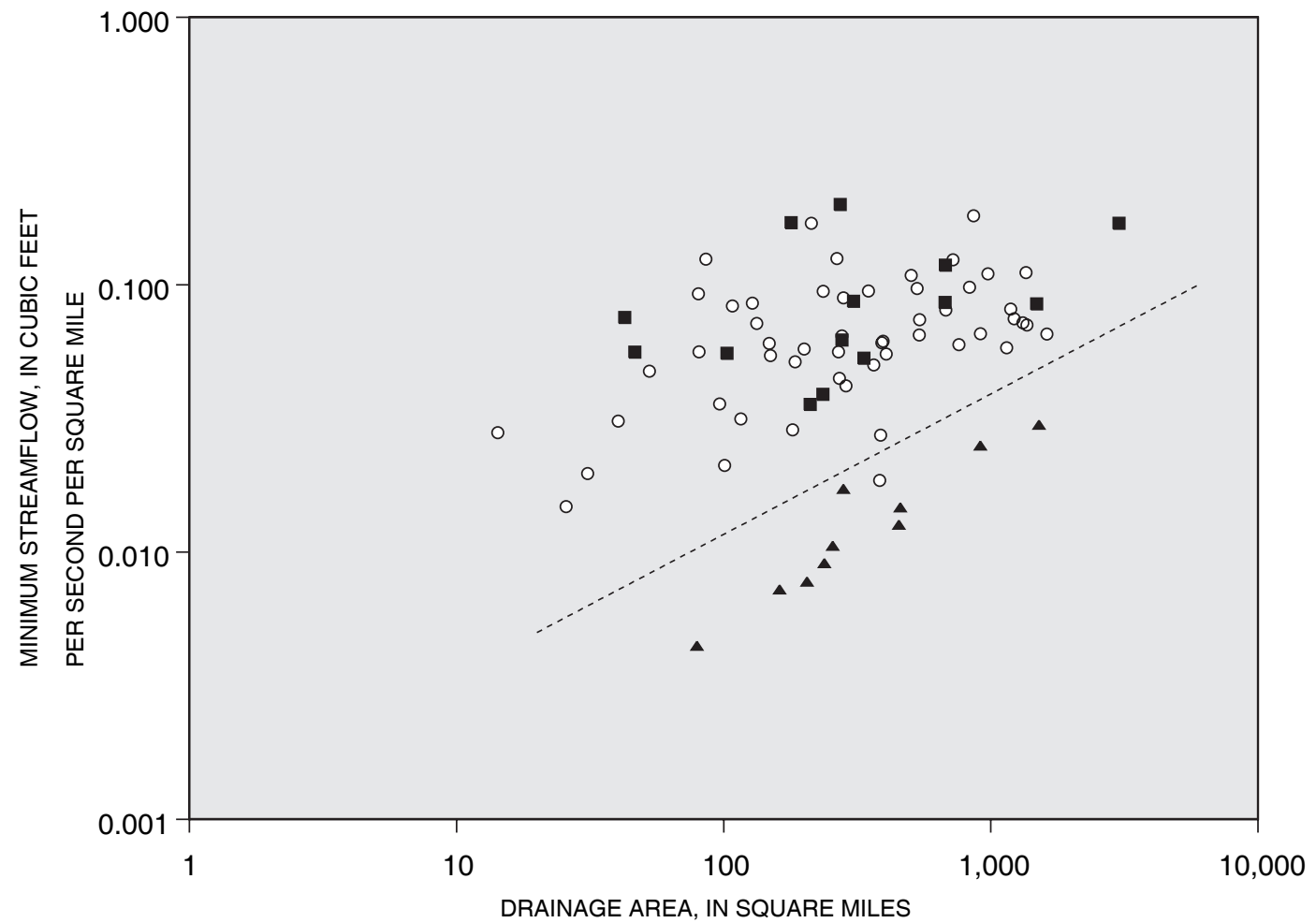

Figure 16. Unit average minimum flows for the subset record period at 77 stations in West Virginia (Open circle indicates station outside the Potomac River Basin and 50 miles or greater from the Ohio River; solid square indicates station within the Potomac River Basin solid triangle indicates station within 50 miles of the Ohio River; generally, points below dashed line represent stations within 50 miles of the Ohio River). 
within $50 \mathrm{mi}$ of the Ohio River with respect to drainage area (note dashed line in figs. 15 and 16).

Guyandotte River near Bailysville (03202400) and Tug Fork at Welch (03212750) had different record periods between 1970 and 2002 during a consistently wet period of high standardized departures (No. 4 in fig. 15). The minimum flows were in the high range compared with other stations and there are no subset record periods for either station.

New River at Bluestone Dam (0318000), New River at Hinton (031845000), New River at Caperton (03185500), and Kanawha River at Kanawha Falls (03193000) are the stations with the greatest drainage areas, and their record periods ended in 1948 because of regulation (No. 5 in fig. 15). These stations had record periods during a consistently dry period of low standardized departures without subset record periods.

Cobun Creek at Morgantown (03062400), Camp Creek near Camp Creek (03178500), Leading Creek near Glenville (03152500), Bluestone River at Lilly (03179500), and Tygart Valley River at Fetterman (03056500) are all stations near the line below which minor streams flowing directly to the Ohio River plotted (No. 6 in fig. 15). Record periods for these stations spanned either the sudden increase in minimum flows around 1970 or a consistently dry period of low standardized departures, and there are no subset record periods.

The plot of subset record periods (fig. 16) identifies two subpopulations - stations within $50 \mathrm{mi}$ of the Ohio River and stations in the remainder of the State-that are not easily discerned from the plot of the entire record periods (fig. 15). The purpose sampling of the entire record periods eliminates outliers that are not included in the subpopulation of records that represent 1930-2002.

\section{Summary}

The U.S. Geological Survey, in cooperation with the West Virginia Department of Environmental Protection, Division of Water and Waste Management, evaluated the low-flow patterns and statistics at U.S. Geological Survey streamflowgaging stations in or near West Virginia. The effects of climate variability were probably the principal causes of the low-flow patterns observed at stations across the State.

Five time periods were identified between 1930 and 2002 with similar low-flow patterns, and average minimum flows for 15 stations were computed for the 5 time periods and the period 1930-2002. Average minimum flows for 1930-42, 1943-62, and 1963-69 were less than the average minimum flows for 1930-2002, and average minimum flows for 1970-79 and 1980-2002 were greater than the average minimum flows for 1930-2002. An abrupt change from a period of less-than-average to greater-than-average minimum flows was noted around 1970 . The period of lowest minimum flows was 1963-69, and the period of greatest minimum flows was 1970-79; however, there was probably a period before 1930 with minimum flows greater than during 1970-79.
Variability of selected low-flow statistics for the 5 time periods identified between 1930 and 2002 at 15 stations, was analyzed both annually and seasonally. The selected low-flow statistics were the 1Q10, 7Q10, 7Q30, 1B3, 4B3, and the EPA harmonic-mean flow. Annually, the magnitude of the variability decreases with increasing magnitudes of the flow statistic. The values for 1Q10, 7Q10, 1B3, and 4B3 range from -93.9 to 582.5 percent for the individual stations for each selected time period from the values computed for 1930-2002, and the values for 30Q5 and the EPA harmonic-mean flow range from -62.3 to 281.2 percent. The maximum variation for an individual station is 582.5 percent greater for 7Q10 for the period 1970-79. A consistent trend was noted in the variability between the hydrologically based flows 1Q10 and 7Q10 and the biologically based flows 1B3 and 4B3. Hydrologically based flows indicate approximately equal or smaller absolute differences than biologically based flows for all selected time periods. Seasonally, there is no consistent trend between lower and higher low flows, and the average differences for 15 stations for 1970-79 in the summer (July 1-September 30) and fall (October 1-December 31) are the greatest average differences for any of the periods investigated. The selected statistics range from -95.7 to 683.6 percent for the individual stations for the selected time periods.

Comparisons between the $1 \mathrm{Q} 10$ and $1 \mathrm{~B} 3$ and the $7 \mathrm{Q} 10$ and 4B3 were made for 15 stations. Annually, the $1 \mathrm{~B} 3$ and $4 \mathrm{~B} 3$ average less than the 1Q10 and 7Q10, respectively, and range from 28.5 percent less to 13.6 percent greater. Seasonally, the biologically based flow was less than the hydrologically based flow in the winter (January 1-March 31) and fall, greater in the spring (April 1-June 30), and varied among the individual stations during the summer.

Station records are available predominantly after about 1970, when minimum flows are greater than the 1930-2002 average. Some short-term station records are mostly during dry periods, whereas others are mostly during wet periods.

Record extension of stations as a means of deriving data representative of 1930-2002 was not feasible because no stations in the northern panhandle and only 4 stations within 50 mi of the Ohio River could be extended to 1930-2002. The areal distribution of stations was insufficient for developing estimating equations for ungaged stream locations. Instead, a criterion-based sampling of the entire record periods at stations was taken to reduce the effects of the entire record periods not representing those between 1930 and 2002. The sampling criterion reduced the inherent bias of entire record periods towards the higher-than-average minimum flows after about 1970 and was based on a comparison between regional minimum flows and station minimum flows. The regional minimum flows were determined from an analysis of 15 stations in West Virginia and 5 stations from surrounding states.

Principal component and correlation analyses on the 20 stations reduced the number of stations to 17 and identified three regions of the State: the Lower Appalachian Plateaus, the Upper Appalachian Plateaus, and the Eastern Panhandle. Generally, the Lower Appalachian Plateaus had less annual 
precipitation (about 40 to 50 in.) compared to the Upper Appalachian Plateaus (about 50 to 60 in.). The Greenbrier River Basin was included in the Upper Appalachian Plateaus with an annual precipitation of about 42-48 in. because of rainshadow effects.

The difference in standardized departures of the minimum flows between the Lower and Upper Appalachian Plateaus regions may be a result of increased storm-cloud densities in the summer and fall after about 1970. Precipitation that typically did not fall until reaching the central mountainous area of the State (where mean annual precipitation is the greatest) could have started falling sooner, resulting in greater relative increases in low flows in the Lower Appalachian Plateaus and lesser or no relative increases in low flows in the Upper Appalachian Plateaus.

Record periods of 10 years or greater between 1930 and 2002 were determined for stations in or near West Virginia on the basis of sampling criterion. The samples of station records are representative of the population for 1930-2002. The record periods for 77 stations in West Virginia and 40 stations near West Virginia, and selected statistics for these record periods are presented. These statistics can be used to develop equations to estimate flow at ungaged stream locations.

\section{Acknowledgments}

The author thanks William H. Kirby for assistance in developing technical discussions, particularly those for criterion-based sampling. Thanks also to William P. Bartlett for help adding clarity to the report with additional discussions and revisions to presentations.

\section{References Cited}

Borchers, J.W., Ehlke, T.A., Mathes, M.V. Jr., and Downs, S.C., 1991, The effects of coal mining on the hydrologic environment of selected stream basins in southern West Virginia: U.S. Geological Survey Water-Resources Investigations Report 84-4300, 119 p.

Friel, E.A., Embree, W.N., Jack, A.R., and Atkins, J.T. Jr., 1989, Low-flow characteristics of streams in West Virginia: U.S. Geological Survey Water-Resources Investigations Report 88-4072, 34 p.

Goetz, J. P., and LeCompte, M. D., 1984, Ethnography and qualitative design in educational research: Academic Press, Orlando, Fla., 292 p.

Helsel, D.R., and Hirsch, R.M., 2002, Statistical methods in water resources: U.S. Geological Survey Techniques of Water-Resources Investigations, book 4, chap. A3, 510 p.
Hirsch, R. M., Slack, J.R., and Smith, R.A., 1982, Techniques of trend analysis for monthly water quality data: Water Resources Research, v. 18, no. 1, p. 107-121.

Hobba, W.A., Jr., 1981, Effects of underground mining and mine collapse on the hydrology of selected basins in West Virginia: West Virginia Geologic and Economic Survey Report of Investigations RI-33, 77 p.

Karl, T.R., and Knight, W.R., 1998, Secular trends of precipitation amount, frequency, and intensity in the USA: Bulletin of the American Meteorological Society, v. 79, p. 231-241.

Kendall, M.G., 1975, Rank correlation methods (4th ed.): London, Charles Griffin, 202 p.

Lane, E.W., and Lei, Kai, 1950, Stream flow variability: American Society of Civil Engineers, Transactions, v. 115, p. 1084-1134.

Lins, H.F., and Slack, J.R., 1999, Streamflow trends in the United States: Geophysical Research Letters, v. 26, p. 227-230.

Mann, H.B., 1945, Non-parametric test against trend: Econometrica, v. 13, p. 245-259.

MathSoft, 1999, S-PLUS 2000 guide to statistics: Seattle, Washington, volumes I, II, and III [variously paged].

McCabe, G.J., and Wolock, D.M., 2002, A step increase in streamflow in the conterminous United States: Geophysical Research Letters, v. 29, no. 24, 2185, doi:1029/ 200 GL015999.

Puente, C., and Atkins, J.T., 1989, Simulation of rainfall-runoff response in mined and unmined watersheds in coal areas of West Virginia: U.S. Geological Survey Water-Supply Paper 2298, 48 p.

Riggs, H.C., 1972, Low-flow investigations: U.S. Geological Survey Techniques of Water-Resources Investigations, book 4, chap. B1, 18 p.

Rossman, L.A., 1990, DFLOW user's manual: U.S. Environmental Protection Agency, 26 p.

Runner, G.S., Bragg, R.L., and Atkins, J.T., Jr., 1989, Cost effectiveness of the stream-gaging program in West Virginia: U.S. Geological Survey Water-Resources Investigations Report 87-4089, 57 p.

Searcy, J.K., 1959, Flow-duration curves; manual of hydrology, part 2, Low-flow techniques: U.S. Geological Survey Water-Supply Paper 1542-A, 33 p.

U.S. Department of Agriculture, Natural Resources Conservation Service, 2004, West Virginia precipitation data/maps, accessed September 13, 2004, at URL http://www.ncgc. nrcs.usda.gov/branch/gdb/products/climate/data/precipitation-state/wv.html 
U.S. Environmental Protection Agency, 1986, Technical guidance manual for performing wasteload allocationsBook VI, design conditions, chapter 1, stream design flow for steady-state modeling: Washington, D.C., U.S. Environmental Protection Agency, Office of Water, EPA 440/4-86014 [variously paged].
Wiley, J.B., Evaldi, R.D., Eychaner, J.H., and Chambers, D.B., 2001, Reconnaissance of stream geomorphology, low streamflow, and stream temperature in the mountaintop coal-mining region, southern West Virginia, 1999-2000: U.S. Geological Survey Water-Resources Investigations Report 01-4092, 34 p.

Table 5. Differences between selected seasonal statistics computed for 1930-2002 and the statistics computed for the indicated record periods for 15 stations in West Virginia.

[Winter, January 1-March 31; spring, April 1-June 30; summer, July 1-September 30; fall, October 1-December 31; station numbers for the 15 stations are 01606500, 01608500, 01611500, 01636500, 03051000, 03053500, 03061500, 03066000, 03069500, 03070500, 03182500, 03183500, 03186500, 03198500, and combined stations 03214000 and 03214500; a negative value means the average for the indicated record period is less than the average for 1930-2002; a positive value means the average for the indicated record period is greater than the average for 1930-2002]

\begin{tabular}{|c|c|c|c|c|c|}
\hline \multirow{2}{*}{ Station number } & \multicolumn{5}{|c|}{ Difference for the indicated period, in percent } \\
\hline & $1930-42$ & 1943-62 & $1963-69$ & $1970-79$ & 1980-2002 \\
\hline \multicolumn{6}{|c|}{ 1-day 10-year hydrologically based low flow (1010) for winter } \\
\hline 01606500 & -8.5 & -2.2 & -16.8 & 60.2 & -3.7 \\
\hline 01608500 & 1.5 & -6.7 & -25.3 & 58.5 & -2.3 \\
\hline 01611500 & -8.6 & -8.6 & -16.7 & 95.5 & 1.5 \\
\hline 01636500 & -22.4 & 3.6 & -23.6 & 78.0 & 3.4 \\
\hline 03051000 & 12.3 & 15.1 & -17.5 & 50.6 & -20.3 \\
\hline 03053500 & -6.7 & 24.0 & -17.7 & 36.7 & -14.1 \\
\hline 03061500 & -20.2 & -0.4 & -39.8 & 83.6 & 27.6 \\
\hline 03066000 & -7.5 & 14.0 & 1.2 & 20.9 & -13.0 \\
\hline 03069500 & -13.4 & 18.9 & 3.9 & 29.6 & -13.0 \\
\hline 03070500 & -13.2 & -6.3 & 14.1 & 40.4 & -0.2 \\
\hline 03182500 & 5.8 & 2.4 & 18.2 & 55.3 & -21.1 \\
\hline 03183500 & -6.0 & 12.0 & -9.5 & 50.0 & -16.9 \\
\hline 03186500 & -10.5 & 25.5 & 30.0 & 45.1 & -20.9 \\
\hline 03198500 & -38.7 & -1.9 & -37.7 & 156.1 & 20.2 \\
\hline $\begin{array}{c}{ }^{\mathrm{a}} 03214000 \text { and } \\
03214500\end{array}$ & No value & b 0.1 & b -35.5 & b 181.1 & c 33.5 \\
\hline \multicolumn{6}{|c|}{ 1-day 10 -year hydrologically based low flow (1010) for spring } \\
\hline 01606500 & 4.0 & 28.6 & -26.8 & -6.5 & -1.2 \\
\hline 01608500 & -0.8 & 32.6 & -28.7 & -0.1 & -2.8 \\
\hline 01611500 & 1.8 & 15.3 & -22.8 & -7.9 & 6.8 \\
\hline 01636500 & -13.7 & 19.0 & -15.5 & 8.9 & 1.6 \\
\hline 03051000 & -1.2 & 52.4 & -44.6 & 9.6 & -6.3 \\
\hline 03053500 & -14.5 & 74.2 & -43.0 & -10.8 & -0.1 \\
\hline 03061500 & -67.3 & 9.8 & -1.6 & 135.3 & 36.7 \\
\hline 03066000 & -3.3 & 18.7 & -32.1 & 7.2 & -4.1 \\
\hline 03069500 & -3.5 & 46.9 & -34.8 & 9.7 & -9.3 \\
\hline 03070500 & 8.5 & 1.8 & -51.4 & 101.0 & 4.3 \\
\hline 03182500 & -0.2 & 55.5 & -35.5 & 16.3 & -9.5 \\
\hline 03183500 & -0.9 & 59.4 & -37.6 & 22.5 & -12.3 \\
\hline 03186500 & -21.2 & 80.1 & -45.4 & 70.4 & -10.5 \\
\hline 03198500 & -63.3 & 39.1 & -31.6 & 66.0 & 86.9 \\
\hline $\begin{array}{c}{ }^{\mathrm{a}} 03214000 \text { and } \\
03214500\end{array}$ & No value & b 1.9 & b 8.7 & ${ }^{\mathrm{b}} 63.8$ & c 57.7 \\
\hline
\end{tabular}


Table 5. Differences between selected seasonal statistics computed for 1930-2002 and the statistics computed for the indicated record periods for 15 stations in West Virginia.-Continued

[Winter, January 1-March 31; spring, April 1-June 30; summer, July 1-September 30; fall, October 1-December 31; station numbers for the 15 stations are 01606500, 01608500, 01611500, 01636500, 03051000, 03053500, 03061500, 03066000, 03069500, 03070500, 03182500, 03183500, 03186500, 03198500, and combined stations 03214000 and 03214500; a negative value means the average for the indicated record period is less than the average for 1930-2002; a positive value means the average for the indicated record period is greater than the average for 1930-2002]

\begin{tabular}{|c|c|c|c|c|c|}
\hline \multirow{2}{*}{ Station number } & \multicolumn{5}{|c|}{ Difference for the indicated period, in percent } \\
\hline & $1930-42$ & $1943-62$ & $1963-69$ & 1970-79 & 1980-2002 \\
\hline \multicolumn{6}{|c|}{ 1-day 10 -year hydrologically based low flow (1010) for summer } \\
\hline 01606500 & -3.6 & 6.1 & -18.3 & 28.0 & 5.0 \\
\hline 01608500 & -2.5 & 6.6 & -29.7 & 61.5 & 11.7 \\
\hline 01611500 & -1.6 & 8.3 & -33.0 & 22.2 & 17.8 \\
\hline 01636500 & -17.8 & 14.5 & -23.4 & 22.4 & 10.6 \\
\hline 03051000 & -62.2 & 19.7 & 58.5 & 557.4 & 13.4 \\
\hline 03053500 & -52.6 & 3.7 & 15.9 & 288.4 & 15.7 \\
\hline 03061500 & -66.5 & -23.6 & 23.2 & 301.0 & 141.6 \\
\hline 03066000 & 3.2 & -27.3 & -1.4 & 84.9 & 10.8 \\
\hline 03069500 & -28.9 & -12.0 & -16.3 & 159.3 & 21.0 \\
\hline 03070500 & 1.5 & -43.6 & 6.3 & 146.2 & 60.9 \\
\hline 03182500 & -38.8 & 8.4 & -31.7 & 109.7 & 31.8 \\
\hline 03183500 & -17.1 & 2.3 & 0.2 & 64.5 & -0.1 \\
\hline 03186500 & -11.3 & -15.7 & 9.8 & 267.5 & -13.2 \\
\hline 03198500 & -49.1 & -33.9 & -30.6 & 396.9 & 218.9 \\
\hline $\begin{array}{c}03214000 \text { and } \\
03214500\end{array}$ & No value & b -10.8 & b 16.2 & b 121.5 & c 126.6 \\
\hline \multicolumn{6}{|c|}{ 1-day 10-year hydrologically based low flow (1010) for fall } \\
\hline 01606500 & -7.9 & 0.0 & -17.9 & 25.8 & 6.0 \\
\hline 01608500 & -14.6 & 1.2 & -25.7 & 47.6 & 12.3 \\
\hline 01611500 & -13.4 & 0.4 & -16.4 & 12.8 & 9.3 \\
\hline 01636500 & -26.0 & 6.6 & -5.7 & 31.6 & 12.4 \\
\hline 03051000 & -48.2 & -12.8 & -2.5 & 385.1 & 23.9 \\
\hline 03053500 & -68.2 & -34.4 & -2.8 & 493.3 & 124.4 \\
\hline 03061500 & -52.0 & -28.9 & 21.8 & 437.3 & 146.1 \\
\hline 03066000 & -9.4 & -40.0 & 39.7 & 164.6 & 41.8 \\
\hline 03069500 & -22.7 & -33.0 & 5.6 & 153.6 & 40.6 \\
\hline 03070500 & -16.5 & -54.8 & 33.6 & 241.6 & 99.6 \\
\hline 03182500 & -21.5 & -2.0 & -33.7 & 56.5 & 23.5 \\
\hline 03183500 & -14.0 & -9.3 & -6.0 & 43.7 & 10.0 \\
\hline 03186500 & -9.6 & -45.3 & 43.2 & 158.0 & 36.9 \\
\hline 03198500 & -43.3 & -31.6 & 35.6 & 168.3 & 210.6 \\
\hline $\begin{array}{c}03214000 \text { and } \\
03214500\end{array}$ & No value & b 1.1 & b 25.3 & b 88.9 & c 135.2 \\
\hline
\end{tabular}


Table 5. Differences between selected seasonal statistics computed for 1930-2002 and the statistics computed for the indicated record periods for 15 stations in West Virginia.-Continued

[Winter, January 1-March 31; spring, April 1-June 30; summer, July 1-September 30; fall, October 1-December 31; station numbers for the 15 stations are $01606500,01608500,01611500,01636500,03051000,03053500,03061500,03066000,03069500,03070500$, $03182500,03183500,03186500,03198500$, and combined stations 03214000 and 03214500 ; a negative value means the average for the indicated record period is less than the average for 1930-2002; a positive value means the average for the indicated record period is greater than the average for 1930-2002]

\begin{tabular}{|c|c|c|c|c|c|}
\hline \multirow{2}{*}{ Station number } & \multicolumn{5}{|c|}{ Difference for the indicated period, in percent } \\
\hline & 1930-42 & 1943-62 & $1963-69$ & 1970-79 & 1980-2002 \\
\hline \multicolumn{6}{|c|}{ 7-day 10 -year hydrologically based low flow (7010) for winter } \\
\hline 01606500 & 2.0 & -8.1 & -16.1 & 63.7 & -4.9 \\
\hline 01608500 & 9.1 & -11.2 & -26.0 & 69.5 & -1.1 \\
\hline 01611500 & -10.0 & -9.0 & -22.8 & 113.8 & 4.0 \\
\hline 01636500 & -17.8 & 2.6 & -19.8 & 73.7 & -0.8 \\
\hline 03051000 & 11.3 & 13.9 & -25.2 & 41.1 & -17.1 \\
\hline 03053500 & -1.4 & 17.9 & -25.8 & 26.8 & -11.4 \\
\hline 03061500 & -25.3 & -3.2 & -38.8 & 58.1 & 30.3 \\
\hline 03066000 & 16.5 & 3.0 & -17.2 & 4.1 & -6.7 \\
\hline 03069500 & -1.5 & 12.4 & -11.1 & 12.8 & -10.3 \\
\hline 03070500 & 5.9 & -10.1 & -3.6 & 18.0 & -0.2 \\
\hline 03182500 & 11.1 & -1.4 & 5.9 & 39.4 & -17.6 \\
\hline 03183500 & -9.2 & 7.4 & -6.8 & 23.6 & -12.6 \\
\hline 03186500 & -79.9 & -52.5 & 11.5 & 26.9 & -10.0 \\
\hline 03198500 & -81.1 & -28.8 & -34.7 & 143.4 & 26.3 \\
\hline $\begin{array}{c}{ }^{\mathrm{a}} 03214000 \text { and } \\
03214500\end{array}$ & No value & b 1.4 & b -29.8 & b 162.3 & c 33.0 \\
\hline \multicolumn{6}{|c|}{ 7-day 10 -year hydrologically based low flow (7010) for spring } \\
\hline 01606500 & 3.3 & 42.5 & -25.1 & -8.4 & -6.5 \\
\hline 01608500 & -0.4 & 39.8 & -26.9 & -5.7 & -6.0 \\
\hline 01611500 & 3.2 & 17.9 & -24.9 & -9.0 & 3.9 \\
\hline 01636500 & 3.6 & 23.2 & -21.0 & -3.9 & -4.0 \\
\hline 03051000 & -5.7 & 58.8 & -40.9 & 16.3 & -15.2 \\
\hline 03053500 & -17.7 & 86.7 & -47.3 & -10.4 & -3.2 \\
\hline 03061500 & -61.0 & 2.5 & -2.1 & 128.2 & 29.8 \\
\hline 03066000 & -6.1 & 22.5 & -34.1 & 15.1 & -5.6 \\
\hline 03069500 & -3.7 & 53.9 & -34.6 & 6.8 & -13.1 \\
\hline 03070500 & 6.5 & -2.4 & -54.4 & 108.4 & 5.3 \\
\hline 03182500 & 6.2 & 60.4 & -27.3 & 10.2 & -16.4 \\
\hline 03183500 & -1.0 & 53.1 & -28.8 & 11.2 & -14.4 \\
\hline 03186500 & -84.5 & -86.9 & -44.6 & 52.0 & -14.8 \\
\hline 03198500 & -89.3 & -86.9 & -29.2 & 45.1 & 68.8 \\
\hline $\begin{array}{c}\text { a } 03214000 \text { and } \\
03214500\end{array}$ & No value & b 8.1 & b 3.6 & b 49.9 & c 42.4 \\
\hline
\end{tabular}


Table 5. Differences between selected seasonal statistics computed for 1930-2002 and the statistics computed for the indicated record periods for 15 stations in West Virginia.-Continued

[Winter, January 1-March 31; spring, April 1-June 30; summer, July 1-September 30; fall, October 1-December 31; station numbers for the 15 stations are $01606500,01608500,01611500,01636500,03051000,03053500,03061500,03066000,03069500,03070500$, $03182500,03183500,03186500,03198500$, and combined stations 03214000 and 03214500 ; a negative value means the average for the indicated record period is less than the average for 1930-2002; a positive value means the average for the indicated record period is greater than the average for 1930-2002]

\begin{tabular}{|c|c|c|c|c|c|}
\hline \multirow{2}{*}{ Station number } & \multicolumn{5}{|c|}{ Difference for the indicated period, in percent } \\
\hline & $1930-42$ & 1943-62 & $1963-69$ & 1970-79 & 1980-2002 \\
\hline \multicolumn{6}{|c|}{ 7-day 10-year hydrologically based low flow (7010) for summer } \\
\hline 01606500 & -3.0 & 5.3 & -19.6 & 30.7 & 6.6 \\
\hline 01608500 & -5.4 & 6.9 & -27.7 & 59.5 & 12.5 \\
\hline 01611500 & -1.2 & 5.8 & -31.3 & 22.7 & 17.2 \\
\hline 01636500 & 1.3 & 12.1 & -28.4 & 12.1 & 3.9 \\
\hline 03051000 & -61.9 & -0.9 & 40.3 & 649.3 & 30.7 \\
\hline 03053500 & -53.7 & 0.1 & 30.4 & 298.1 & 16.9 \\
\hline 03061500 & -60.1 & -16.7 & 22.1 & 230.8 & 98.7 \\
\hline 03066000 & 5.4 & -22.0 & -16.9 & 84.0 & 8.8 \\
\hline 03069500 & -24.8 & -17.1 & -14.5 & 175.3 & 23.8 \\
\hline 03070500 & -4.9 & -39.4 & 2.0 & 129.2 & 59.9 \\
\hline 03182500 & -30.4 & 2.1 & -30.0 & 116.6 & 20.7 \\
\hline 03183500 & -17.7 & 1.9 & -2.5 & 60.5 & 2.0 \\
\hline 03186500 & -3.5 & -18.1 & 2.3 & 279.2 & -12.7 \\
\hline 03198500 & -46.0 & -34.1 & -29.8 & 380.0 & 182.7 \\
\hline $\begin{array}{c}03214000 \text { and } \\
03214500\end{array}$ & No value & b -9.8 & b 12.9 & b 115.8 & c 115.9 \\
\hline \multicolumn{6}{|c|}{ 7-day 10 -year hydrologically based low flow (7010) for fall } \\
\hline 01606500 & -6.1 & 0.9 & -17.1 & 18.3 & 6.5 \\
\hline 01608500 & -13.0 & 3.5 & -25.9 & 37.2 & 11.0 \\
\hline 01611500 & -11.2 & 3.2 & -17.1 & 14.9 & 12.4 \\
\hline 01636500 & -12.1 & 8.2 & -14.4 & 16.2 & 4.0 \\
\hline 03051000 & -51.4 & -22.9 & 34.0 & 270.4 & 24.6 \\
\hline 03053500 & -70.5 & -33.4 & 2.4 & 428.6 & 114.1 \\
\hline 03061500 & -48.0 & -30.7 & 39.7 & 369.1 & 94.9 \\
\hline 03066000 & -5.1 & -39.8 & 35.5 & 138.5 & 32.7 \\
\hline 03069500 & -25.1 & -34.6 & 23.7 & 121.4 & 37.4 \\
\hline 03070500 & -20.8 & -58.7 & 15.2 & 260.6 & 112.1 \\
\hline 03182500 & -25.7 & -6.2 & -23.0 & 37.6 & 24.0 \\
\hline 03183500 & -18.0 & -11.3 & 0.4 & 33.4 & 14.5 \\
\hline 03186500 & -13.6 & -50.0 & 66.7 & 146.3 & 45.5 \\
\hline 03198500 & -38.0 & -33.1 & 15.1 & 128.0 & 172.7 \\
\hline $\begin{array}{c}{ }^{\mathrm{a}} 03214000 \text { and } \\
03214500\end{array}$ & No value & $\mathrm{b}-0.8$ & b 23.8 & b 85.8 & c 123.5 \\
\hline
\end{tabular}


Table 5. Differences between selected seasonal statistics computed for 1930-2002 and the statistics computed for the indicated record periods for 15 stations in West Virginia.-Continued

[Winter, January 1-March 31; spring, April 1-June 30; summer, July 1-September 30; fall, October 1-December 31; station numbers for the 15 stations are $01606500,01608500,01611500,01636500,03051000,03053500,03061500,03066000,03069500,03070500$, $03182500,03183500,03186500,03198500$, and combined stations 03214000 and 03214500 ; a negative value means the average for the indicated record period is less than the average for 1930-2002; a positive value means the average for the indicated record period is greater than the average for 1930-2002]

\begin{tabular}{|c|c|c|c|c|c|}
\hline \multirow{2}{*}{ Station number } & \multicolumn{5}{|c|}{ Difference for the indicated period, in percent } \\
\hline & $1930-42$ & 1943-62 & $1963-69$ & 1970-79 & 1980-2002 \\
\hline \multicolumn{6}{|c|}{ 30-day 5-year hydrologically based low flow (3005) for winter } \\
\hline 01606500 & -9.6 & 3.9 & -30.2 & 57.8 & -2.1 \\
\hline 01608500 & -10.8 & 1.2 & -29.6 & 61.4 & -0.4 \\
\hline 01611500 & -18.2 & 3.5 & -23.3 & 111.6 & -8.4 \\
\hline 01636500 & -18.0 & -3.0 & -4.4 & 57.2 & -2.0 \\
\hline 03051000 & -9.1 & 16.1 & -35.6 & 5.1 & 8.0 \\
\hline 03053500 & -12.3 & 25.9 & -39.8 & -0.7 & 8.7 \\
\hline 03061500 & -14.9 & 30.1 & -65.4 & 13.1 & 25.4 \\
\hline 03066000 & -5.7 & 6.2 & -28.2 & 4.2 & 13.9 \\
\hline 03069500 & -8.0 & 10.9 & -35.4 & 7.1 & 12.2 \\
\hline 03070500 & 8.8 & 5.3 & -24.4 & 0.2 & 5.4 \\
\hline 03182500 & -1.2 & 5.9 & -25.2 & 18.8 & 0.5 \\
\hline 03183500 & -17.3 & 13.3 & -24.8 & 13.4 & 7.7 \\
\hline 03186500 & -33.0 & 49.3 & 63.4 & 175.7 & 152.9 \\
\hline 03198500 & -46.1 & 10.8 & 52.9 & 294.6 & 208.6 \\
\hline $\begin{array}{c}{ }^{\mathrm{a}} 03214000 \text { and } \\
03214500\end{array}$ & No value & b 13.1 & b -28.2 & ${ }^{\mathrm{b}} 66.6$ & c 10.7 \\
\hline \multicolumn{6}{|c|}{ 30-day 5-year hydrologically based low flow (3005) for spring } \\
\hline 01606500 & -7.9 & 38.8 & -28.9 & -17.5 & -1.1 \\
\hline 01608500 & -7.5 & 35.8 & -32.0 & -17.0 & 2.3 \\
\hline 01611500 & -7.7 & 26.2 & -17.7 & -4.2 & -0.8 \\
\hline 01636500 & -2.7 & 21.0 & -26.7 & -8.0 & 4.4 \\
\hline 03051000 & 3.0 & 23.2 & -36.0 & 12.9 & -10.5 \\
\hline 03053500 & -16.0 & 45.7 & -46.5 & 9.8 & -3.6 \\
\hline 03061500 & -20.9 & 5.0 & -44.1 & 51.5 & 10.8 \\
\hline 03066000 & -14.1 & 26.2 & -38.2 & 6.8 & 1.2 \\
\hline 03069500 & -5.8 & 47.9 & -40.3 & -4.0 & -8.8 \\
\hline 03070500 & -4.3 & 5.9 & -48.1 & 38.9 & 9.4 \\
\hline 03182500 & -6.3 & 57.2 & -39.3 & -11.9 & -9.5 \\
\hline 03183500 & -11.7 & 37.6 & -30.0 & -8.6 & -2.5 \\
\hline 03186500 & 12.5 & -14.6 & 231.2 & 486.7 & 520.4 \\
\hline 03198500 & -10.3 & -39.2 & 188.5 & 355.0 & 603.4 \\
\hline $\begin{array}{c}\text { a } 03214000 \text { and } \\
03214500\end{array}$ & No value & b -2.6 & b -10.1 & b 22.9 & c 43.0 \\
\hline
\end{tabular}


Table 5. Differences between selected seasonal statistics computed for 1930-2002 and the statistics computed for the indicated record periods for 15 stations in West Virginia.-Continued

[Winter, January 1-March 31; spring, April 1-June 30; summer, July 1-September 30; fall, October 1-December 31; station numbers for the 15 stations are $01606500,01608500,01611500,01636500,03051000,03053500,03061500,03066000,03069500,03070500$, 03182500, 03183500, 03186500, 03198500, and combined stations 03214000 and 03214500; a negative value means the average for the indicated record period is less than the average for 1930-2002; a positive value means the average for the indicated record period is greater than the average for 1930-2002]

\begin{tabular}{|c|c|c|c|c|c|}
\hline \multirow{2}{*}{ Station number } & \multicolumn{5}{|c|}{ Difference for the indicated period, in percent } \\
\hline & 1930-42 & 1943-62 & $1963-69$ & $1970-79$ & 1980-2002 \\
\hline \multicolumn{6}{|c|}{ 30-day 5-year hydrologically based low flow (3005) for summer } \\
\hline 01606500 & -1.2 & 8.7 & -24.3 & 29.8 & 3.3 \\
\hline 01608500 & -2.6 & 6.6 & -30.4 & 43.3 & 5.3 \\
\hline 01611500 & -1.9 & -0.3 & -33.4 & 31.0 & 10.2 \\
\hline 01636500 & 3.9 & 4.4 & -28.3 & 17.1 & 3.7 \\
\hline 03051000 & -39.8 & -9.0 & -30.9 & 193.9 & 15.7 \\
\hline 03053500 & -42.6 & -3.7 & -25.7 & 128.7 & 25.4 \\
\hline 03061500 & -54.8 & -10.0 & -29.9 & 148.0 & 46.0 \\
\hline 03066000 & -1.8 & -13.8 & -29.5 & 69.5 & 7.2 \\
\hline 03069500 & -19.5 & -8.1 & -29.1 & 86.2 & 12.6 \\
\hline 03070500 & -3.4 & -31.9 & -38.2 & 81.5 & 56.9 \\
\hline 03182500 & -4.8 & 16.6 & -45.9 & 60.2 & 1.6 \\
\hline 03183500 & -9.4 & 17.8 & -23.7 & 46.0 & -2.7 \\
\hline 03186500 & 12.5 & -14.6 & -32.6 & 129.3 & -4.6 \\
\hline 03198500 & -10.3 & -39.2 & -32.0 & 137.8 & 62.4 \\
\hline $\begin{array}{c}03214000 \text { and } \\
03214500\end{array}$ & No value & b -17.6 & b -4.2 & b 78.2 & c 56.8 \\
\hline \multicolumn{6}{|c|}{ 30-day 5-year hydrologically based low flow (3005) for fall } \\
\hline 01606500 & -12.4 & -3.6 & -20.0 & 38.4 & 3.9 \\
\hline 01608500 & -12.7 & -2.7 & -22.7 & 44.7 & 2.8 \\
\hline 01611500 & -12.4 & -4.4 & -16.7 & 49.9 & -8.5 \\
\hline 01636500 & -9.6 & 9.5 & -22.6 & 23.8 & 0.3 \\
\hline 03051000 & -37.2 & -25.9 & -17.9 & 152.0 & 21.9 \\
\hline 03053500 & -43.3 & -39.3 & -32.3 & 192.6 & 34.4 \\
\hline 03061500 & -45.0 & -10.5 & -40.0 & 210.9 & 37.8 \\
\hline 03066000 & 0.8 & -40.7 & 7.0 & 101.1 & 19.3 \\
\hline 03069500 & -16.5 & -33.4 & -2.7 & 96.6 & 21.7 \\
\hline 03070500 & -25.7 & -30.7 & -52.8 & 132.4 & 40.5 \\
\hline 03182500 & -36.8 & -5.7 & -15.6 & 73.6 & 14.2 \\
\hline 03183500 & -27.7 & -9.8 & -0.8 & 68.7 & 5.7 \\
\hline 03186500 & -31.0 & -35.5 & 7.3 & 170.9 & 21.9 \\
\hline 03198500 & -49.3 & -25.0 & -12.7 & 104.4 & 67.9 \\
\hline $\begin{array}{c}\text { a } 03214000 \text { and } \\
03214500\end{array}$ & No value & b -5.1 & $\mathrm{~b}-5.6$ & b 109.0 & c 60.4 \\
\hline
\end{tabular}


Table 5. Differences between selected seasonal statistics computed for 1930-2002 and the statistics computed for the indicated record periods for 15 stations in West Virginia._- Continued

[Winter, January 1-March 31; spring, April 1-June 30; summer, July 1-September 30; fall, October 1-December 31; station numbers for the 15 stations are $01606500,01608500,01611500,01636500,03051000,03053500,03061500,03066000,03069500,03070500$, 03182500, 03183500, 03186500, 03198500, and combined stations 03214000 and 03214500; a negative value means the average for the indicated record period is less than the average for 1930-2002; a positive value means the average for the indicated record period is greater than the average for 1930-2002]

\begin{tabular}{|c|c|c|c|c|c|}
\hline \multirow{2}{*}{ Station number } & \multicolumn{5}{|c|}{ Difference for the indicated period, in percent } \\
\hline & $1930-42$ & 1943-62 & $1963-69$ & $1970-79$ & 1980-2002 \\
\hline \multicolumn{6}{|c|}{ 1-day 3-year biologically based low flow (1B3) for winter } \\
\hline 01606500 & 7.6 & -4.8 & -2.5 & 68.7 & 3.4 \\
\hline 01608500 & 20.0 & -6.4 & -8.6 & 60.7 & -0.7 \\
\hline 01611500 & 16.4 & -8.3 & -9.8 & 100.3 & 3.2 \\
\hline 01636500 & -16.8 & 13.6 & -17.1 & 75.1 & -2.0 \\
\hline 03051000 & -22.3 & 14.4 & -22.2 & 94.4 & -7.0 \\
\hline 03053500 & 0.0 & 21.0 & -12.6 & 62.7 & -0.1 \\
\hline 03061500 & -15.6 & -33.6 & -8.6 & 22.4 & 42.9 \\
\hline 03066000 & 4.4 & 0.4 & 20.1 & 40.2 & -15.7 \\
\hline 03069500 & -21.7 & -0.4 & -0.4 & 30.0 & -19.6 \\
\hline 03070500 & -13.8 & -29.9 & 30.1 & 31.9 & 24.0 \\
\hline 03182500 & 3.5 & -15.0 & 34.1 & 58.5 & -24.6 \\
\hline 03183500 & -14.3 & 8.6 & 42.1 & 56.4 & -12.9 \\
\hline 03186500 & -41.8 & 16.8 & 51.4 & 77.1 & -13.8 \\
\hline 03198500 & -65.6 & -19.8 & -48.7 & 243.8 & 40.1 \\
\hline $\begin{array}{c}{ }^{\mathrm{a}} 03214000 \text { and } \\
03214500\end{array}$ & No value & b -20.2 & b -21.6 & b 84.0 & c 69.3 \\
\hline \multicolumn{6}{|c|}{ 1-day 3-year biologically based low flow (1B3) for spring } \\
\hline 01606500 & -22.2 & 37.5 & -2.1 & -14.6 & 35.4 \\
\hline 01608500 & -32.1 & 27.8 & -3.6 & -27.0 & 40.9 \\
\hline 01611500 & -19.7 & 14.6 & 12.5 & -36.2 & 15.6 \\
\hline 01636500 & -14.6 & 13.6 & 15.8 & -2.2 & -3.7 \\
\hline 03051000 & -34.7 & 13.3 & -24.9 & 0.0 & 51.6 \\
\hline 03053500 & -6.6 & 21.1 & 0.0 & -44.6 & 79.6 \\
\hline 03061500 & -56.2 & -5.3 & 48.2 & 127.1 & 127.1 \\
\hline 03066000 & -14.8 & 4.3 & -4.8 & 4.8 & 51.9 \\
\hline 03069500 & -35.3 & 9.6 & 0.0 & -20.9 & 26.2 \\
\hline 03070500 & -21.1 & -3.6 & -14.0 & 14.7 & 90.0 \\
\hline 03182500 & -43.5 & 26.2 & -14.0 & -10.8 & 29.5 \\
\hline 03183500 & -18.9 & 17.2 & 1.6 & -16.8 & 26.2 \\
\hline 03186500 & -34.2 & 36.8 & -5.3 & 21.1 & 57.4 \\
\hline 03198500 & -68.8 & 8.7 & 0.0 & 26.7 & 104.2 \\
\hline $\begin{array}{c}{ }^{\mathrm{a}} 03214000 \text { and } \\
03214500\end{array}$ & No value & b 14.4 & b 55.5 & b 49.3 & c 137.0 \\
\hline
\end{tabular}


Table 5. Differences between selected seasonal statistics computed for 1930-2002 and the statistics computed for the indicated record periods for 15 stations in West Virginia.—Continued

[Winter, January 1-March 31; spring, April 1-June 30; summer, July 1-September 30; fall, October 1-December 31; station numbers for the 15 stations are $01606500,01608500,01611500,01636500,03051000,03053500,03061500,03066000,03069500,03070500$, 03182500, 03183500, 03186500, 03198500, and combined stations 03214000 and 03214500 ; a negative value means the average for the indicated record period is less than the average for 1930-2002; a positive value means the average for the indicated record period is greater than the average for 1930-2002]

\begin{tabular}{|c|c|c|c|c|c|}
\hline \multirow{2}{*}{ Station number } & \multicolumn{5}{|c|}{ Difference for the indicated period, in percent } \\
\hline & $1930-42$ & 1943-62 & $1963-69$ & 1970-79 & 1980-2002 \\
\hline \multicolumn{6}{|c|}{ 1-day 3-year biologically based low flow (1B3) for summer } \\
\hline 01606500 & -6.0 & 4.0 & -12.2 & 31.8 & -0.2 \\
\hline 01608500 & -12.2 & 6.4 & -15.4 & 77.1 & 27.0 \\
\hline 01611500 & -2.9 & 15.5 & -26.3 & 15.8 & 21.1 \\
\hline 01636500 & -18.4 & 18.7 & -34.4 & 11.8 & 19.9 \\
\hline 03051000 & -95.7 & 10.5 & 17.8 & 330.5 & -9.1 \\
\hline 03053500 & -87.9 & -3.5 & 54.0 & 211.1 & 10.4 \\
\hline 03061500 & -62.7 & -13.4 & 47.8 & 407.5 & 179.1 \\
\hline 03066000 & -9.3 & -14.4 & 0.0 & 64.3 & 0.0 \\
\hline 03069500 & -51.8 & -19.1 & -25.6 & 126.2 & 19.7 \\
\hline 03070500 & -3.0 & -63.0 & -26.5 & 85.8 & 17.7 \\
\hline 03182500 & -38.9 & 17.6 & 0.8 & 134.5 & 42.9 \\
\hline 03183500 & -40.3 & -6.2 & 2.3 & 55.4 & -1.9 \\
\hline 03186500 & 0.5 & -14.6 & 39.2 & 331.2 & 8.5 \\
\hline 03198500 & -61.7 & -28.9 & -29.2 & 543.5 & 306.7 \\
\hline $\begin{array}{c}{ }^{\mathrm{a}} 03214000 \text { and } \\
03214500\end{array}$ & No value & b 9.8 & b 19.7 & b 127.1 & c 120.6 \\
\hline \multicolumn{6}{|c|}{ 1-day 3-year biologically based low flow (1B3) for fall } \\
\hline 01606500 & -17.9 & 3.8 & -21.5 & 21.6 & 9.1 \\
\hline 01608500 & -13.3 & 9.5 & -15.8 & 68.7 & 25.4 \\
\hline 01611500 & -12.2 & 2.0 & -14.6 & 19.0 & 19.0 \\
\hline 01636500 & -32.0 & 7.9 & -10.6 & 11.1 & 6.0 \\
\hline 03051000 & -84.0 & -8.5 & 27.7 & 469.8 & -45.5 \\
\hline 03053500 & -93.3 & -3.4 & 20.9 & 683.6 & 30.2 \\
\hline 03061500 & -35.1 & -23.4 & 55.8 & 416.9 & 193.5 \\
\hline 03066000 & -14.3 & -31.7 & -1.1 & 217.5 & 105.0 \\
\hline 03069500 & -69.4 & -44.4 & 13.6 & 158.1 & 21.9 \\
\hline 03070500 & -49.3 & -46.4 & 61.2 & 123.9 & 54.3 \\
\hline 03182500 & -41.4 & -7.6 & -22.8 & 135.3 & 101.7 \\
\hline 03183500 & -41.5 & -16.7 & 2.3 & 67.0 & 24.6 \\
\hline 03186500 & 16.8 & -47.3 & -0.9 & 244.5 & 40.0 \\
\hline 03198500 & -13.4 & -34.6 & -32.9 & 347.1 & 276.5 \\
\hline $\begin{array}{c}{ }^{\mathrm{a}} 03214000 \text { and } \\
03214500\end{array}$ & No value & b 14.2 & b 55.4 & b 131.1 & c 221.5 \\
\hline
\end{tabular}


Table 5. Differences between selected seasonal statistics computed for 1930-2002 and the statistics computed for the indicated record periods for 15 stations in West Virginia._Continued

[Winter, January 1-March 31; spring, April 1-June 30; summer, July 1-September 30; fall, October 1-December 31; station numbers for the 15 stations are $01606500,01608500,01611500,01636500,03051000,03053500,03061500,03066000,03069500,03070500$, 03182500, 03183500, 03186500, 03198500, and combined stations 03214000 and 03214500; a negative value means the average for the indicated record period is less than the average for 1930-2002; a positive value means the average for the indicated record period is greater than the average for 1930-2002]

\begin{tabular}{|c|c|c|c|c|c|}
\hline \multirow{2}{*}{ Station number } & \multicolumn{5}{|c|}{ Difference for the indicated period, in percent } \\
\hline & $1930-42$ & $1943-62$ & $1963-69$ & 1970-79 & 1980-2002 \\
\hline \multicolumn{6}{|c|}{ 4-day 3-year biologically based low flow (4B3) for winter } \\
\hline 01606500 & 19.1 & -4.6 & -3.9 & 57.2 & -0.2 \\
\hline 01608500 & 43.3 & -5.7 & -17.8 & 54.1 & 0.0 \\
\hline 01611500 & 5.9 & -14.3 & -21.5 & 120.3 & 3.3 \\
\hline 01636500 & -14.0 & 11.3 & -26.4 & 74.3 & -3.6 \\
\hline 03051000 & -20.8 & 2.7 & -36.2 & 66.7 & -1.8 \\
\hline 03053500 & -1.4 & 12.7 & -24.0 & 48.2 & -4.3 \\
\hline 03061500 & 17.3 & -8.2 & -10.4 & 74.5 & 83.6 \\
\hline 03066000 & 10.8 & 11.7 & -8.4 & 10.8 & -13.2 \\
\hline 03069500 & -6.8 & 0.0 & -12.5 & 16.6 & -0.8 \\
\hline 03070500 & -9.1 & -24.3 & 10.1 & 8.8 & 4.0 \\
\hline 03182500 & -5.7 & -16.3 & 20.4 & 32.4 & -27.2 \\
\hline 03183500 & -33.0 & -0.5 & 13.0 & 46.5 & -10.3 \\
\hline 03186500 & -46.4 & 19.5 & 24.8 & 44.8 & -13.8 \\
\hline 03198500 & -72.5 & -26.3 & -62.7 & 154.2 & 21.3 \\
\hline $\begin{array}{c}\text { a } 03214000 \text { and } \\
03214500\end{array}$ & No value & b -22.9 & b -43.1 & b 76.3 & c 39.5 \\
\hline \multicolumn{6}{|c|}{ 4-day 3-year biologically based low flow (4B3) for spring } \\
\hline 01606500 & -17.6 & 40.0 & -9.4 & -23.5 & 31.8 \\
\hline 01608500 & -22.9 & 26.3 & -9.6 & -29.0 & 31.7 \\
\hline 01611500 & -18.3 & 22.2 & 16.7 & -32.5 & 27.8 \\
\hline 01636500 & -8.5 & 13.3 & 7.2 & -7.1 & -3.4 \\
\hline 03051000 & -23.6 & 10.9 & -10.1 & -1.5 & 33.1 \\
\hline 03053500 & -15.1 & 23.8 & -14.1 & -43.0 & 73.5 \\
\hline 03061500 & -61.2 & -18.6 & 37.0 & 99.4 & 102.2 \\
\hline 03066000 & -14.6 & 15.8 & -10.7 & -1.2 & 49.0 \\
\hline 03069500 & -15.9 & 19.9 & -4.0 & -19.9 & 23.9 \\
\hline 03070500 & -26.6 & -5.3 & -11.9 & 13.3 & 69.5 \\
\hline 03182500 & -18.9 & 26.6 & -12.8 & -15.5 & 37.6 \\
\hline 03183500 & -13.4 & 26.5 & -1.1 & -8.6 & 46.3 \\
\hline 03186500 & -26.8 & 49.8 & 18.8 & -4.2 & 66.3 \\
\hline 03198500 & -56.7 & 9.9 & 3.3 & 17.4 & 95.6 \\
\hline $\begin{array}{c}03214000 \text { and } \\
03214500\end{array}$ & No value & b 17.8 & b 45.6 & ${ }^{\mathrm{b}} 64.5$ & c 120.7 \\
\hline
\end{tabular}


Table 5. Differences between selected seasonal statistics computed for 1930-2002 and the statistics computed for the indicated record periods for 15 stations in West Virginia._-Continued

[Winter, January 1-March 31; spring, April 1-June 30; summer, July 1-September 30; fall, October 1-December 31; station numbers for the 15 stations are $01606500,01608500,01611500,01636500,03051000,03053500,03061500,03066000,03069500,03070500$, 03182500, 03183500, 03186500, 03198500, and combined stations 03214000 and 03214500; a negative value means the average for the indicated record period is less than the average for 1930-2002; a positive value means the average for the indicated record period is greater than the average for 1930-2002]

\begin{tabular}{|c|c|c|c|c|c|}
\hline \multirow{2}{*}{ Station number } & \multicolumn{5}{|c|}{ Difference for the indicated period, in percent } \\
\hline & 1930-42 & $1943-62$ & $1963-69$ & 1970-79 & 1980-2002 \\
\hline \multicolumn{6}{|c|}{ 4-day 3-year biologically based low flow (4B3) for summer } \\
\hline 01606500 & -5.7 & 7.2 & -12.7 & 33.7 & 4.2 \\
\hline 01608500 & -9.1 & 17.0 & -16.1 & 66.7 & 27.3 \\
\hline 01611500 & -2.8 & 16.5 & -24.8 & 34.7 & 22.8 \\
\hline 01636500 & -12.4 & 19.6 & -34.3 & 7.7 & 16.9 \\
\hline 03051000 & -88.3 & -4.3 & 6.0 & 347.3 & -1.6 \\
\hline 03053500 & -87.1 & -7.4 & 10.8 & 289.8 & -0.4 \\
\hline 03061500 & -57.4 & -36.1 & 11.1 & 300.0 & 87.0 \\
\hline 03066000 & -7.8 & -9.4 & 11.9 & 69.8 & -3.4 \\
\hline 03069500 & -51.3 & -14.8 & -1.6 & 139.0 & 24.5 \\
\hline 03070500 & -1.9 & -64.2 & -10.1 & 100.8 & 26.3 \\
\hline 03182500 & -41.0 & 3.4 & -15.4 & 118.8 & 28.9 \\
\hline 03183500 & -39.2 & 11.5 & 0.8 & 66.6 & -1.6 \\
\hline 03186500 & -2.3 & -22.3 & 16.2 & 300.0 & 8.3 \\
\hline 03198500 & -59.2 & -5.1 & -27.0 & 405.7 & 245.3 \\
\hline $\begin{array}{c}03214000 \text { and } \\
03214500\end{array}$ & No value & b 1.2 & b 43.3 & b 109.6 & c 101.6 \\
\hline \multicolumn{6}{|c|}{ 4-day 3-year biologically based low flow (4B3) for fall } \\
\hline 01606500 & -22.0 & 1.4 & -20.3 & 16.9 & 6.9 \\
\hline 01608500 & -15.1 & 5.1 & -21.5 & 52.0 & 15.2 \\
\hline 01611500 & -5.5 & -1.1 & -16.3 & 16.6 & 16.6 \\
\hline 01636500 & -20.9 & 5.0 & -13.3 & 9.5 & 4.5 \\
\hline 03051000 & -84.8 & -15.2 & 41.9 & 455.3 & -43.1 \\
\hline 03053500 & -92.6 & -22.3 & -4.9 & 509.9 & 35.7 \\
\hline 03061500 & -40.2 & -26.5 & 46.1 & 395.1 & 154.9 \\
\hline 03066000 & -28.4 & -33.8 & 29.0 & 166.0 & 72.8 \\
\hline 03069500 & -65.0 & -28.8 & 16.0 & 142.9 & 26.4 \\
\hline 03070500 & -42.1 & -50.8 & 15.2 & 135.8 & 39.8 \\
\hline 03182500 & -49.9 & -34.3 & -37.6 & 68.5 & 41.6 \\
\hline 03183500 & -49.2 & -21.8 & 0.9 & 41.5 & 11.2 \\
\hline 03186500 & -4.6 & -43.9 & 5.4 & 223.2 & 42.1 \\
\hline 03198500 & -39.7 & -46.0 & 13.4 & 206.0 & 200.0 \\
\hline $\begin{array}{c}03214000 \text { and } \\
03214500\end{array}$ & No value & b 8.2 & b 55.1 & b 114.3 & c 186.6 \\
\hline
\end{tabular}


Table 5. Differences between selected seasonal statistics computed for 1930-2002 and the statistics computed for the indicated record periods for 15 stations in West Virginia._Continued

[Winter, January 1-March 31; spring, April 1-June 30; summer, July 1-September 30; fall, October 1-December 31; station numbers for the 15 stations are $01606500,01608500,01611500,01636500,03051000,03053500,03061500,03066000,03069500,03070500$, 03182500, 03183500, 03186500, 03198500, and combined stations 03214000 and 03214500; a negative value means the average for the indicated record period is less than the average for 1930-2002; a positive value means the average for the indicated record period is greater than the average for 1930-2002]

\begin{tabular}{|c|c|c|c|c|c|}
\hline \multirow{2}{*}{ Station number } & \multicolumn{5}{|c|}{ Difference for the indicated period, in percent } \\
\hline & $1930-42$ & 1943-62 & $1963-69$ & $1970-79$ & 1980-2002 \\
\hline \multicolumn{6}{|c|}{ U.S. Environmental Protection Agency harmonic-mean flow for winter } \\
\hline 01606500 & -0.2 & -0.2 & -18.2 & 40.4 & -5.1 \\
\hline 01608500 & 0.5 & -1.9 & -19.8 & 42.3 & -3.9 \\
\hline 01611500 & -8.5 & -1.1 & -22.1 & 78.3 & -3.6 \\
\hline 01636500 & -11.7 & 3.5 & -16.4 & 48.5 & -4.7 \\
\hline 03051000 & -1.5 & 9.6 & -19.6 & 24.3 & -7.3 \\
\hline 03053500 & -7.3 & 13.7 & -20.1 & 15.6 & -4.1 \\
\hline 03061500 & -2.6 & 5.9 & -39.9 & 45.6 & 3.0 \\
\hline 03066000 & -6.7 & 6.0 & -12.7 & 7.5 & -0.7 \\
\hline 03069500 & -6.5 & 10.2 & -17.3 & 11.1 & -1.9 \\
\hline 03070500 & -0.7 & -1.4 & -12.9 & 16.4 & 0.4 \\
\hline 03182500 & 7.9 & 1.0 & -9.6 & 27.3 & -9.6 \\
\hline 03183500 & -4.6 & 6.4 & -12.5 & 28.4 & -6.4 \\
\hline 03186500 & -4.1 & 8.1 & -13.1 & 15.3 & -5.9 \\
\hline 03198500 & -33.5 & 11.4 & -33.5 & 82.4 & 12.9 \\
\hline $\begin{array}{c}{ }^{a} 03214000 \text { and } \\
03214500 \\
\end{array}$ & No value & b 11.7 & b -25.3 & b 85.6 & c 8.9 \\
\hline \multicolumn{6}{|c|}{ U.S. Environmental Protection Agency harmonic-mean flow for spring } \\
\hline 01606500 & -16.6 & 8.1 & -15.1 & -7.4 & 16.0 \\
\hline 01608500 & -17.2 & 7.6 & -15.4 & -10.3 & 19.0 \\
\hline 01611500 & -17.9 & 7.5 & -12.1 & -3.1 & 14.0 \\
\hline 01636500 & -11.8 & 6.1 & -18.4 & 1.3 & 9.6 \\
\hline 03051000 & -22.5 & 4.0 & -19.4 & 1.2 & 25.1 \\
\hline 03053500 & -25.8 & 11.1 & -24.7 & -8.5 & 34.7 \\
\hline 03061500 & -46.6 & -7.1 & -13.9 & 42.6 & 48.7 \\
\hline 03066000 & -21.8 & -1.6 & -12.2 & -2.4 & 27.6 \\
\hline 03069500 & -19.3 & 6.3 & -14.9 & -9.0 & 22.5 \\
\hline 03070500 & -25.4 & -4.0 & -17.9 & 16.1 & 30.8 \\
\hline 03182500 & -24.8 & 8.0 & -13.1 & -8.0 & 24.1 \\
\hline 03183500 & -25.0 & 6.4 & -13.6 & -6.4 & 28.0 \\
\hline 03186500 & -36.6 & 9.7 & -11.4 & 4.6 & 31.4 \\
\hline 03198500 & -43.0 & 0.7 & -15.0 & 14.3 & 53.8 \\
\hline $\begin{array}{c}{ }^{\mathrm{a}} 03214000 \text { and } \\
03214500\end{array}$ & No value & $\mathrm{b}-2.6$ & $\mathrm{~b}-5.9$ & b 32.4 & c 43.7 \\
\hline
\end{tabular}


Table 5. Differences between selected seasonal statistics computed for 1930-2002 and the statistics computed for the indicated record periods for 15 stations in West Virginia._- Continued

[Winter, January 1-March 31; spring, April 1-June 30; summer, July 1-September 30; fall, October 1-December 31; station numbers for the 15 stations are $01606500,01608500,01611500,01636500,03051000,03053500,03061500,03066000,03069500,03070500$, 03182500, 03183500, 03186500, 03198500, and combined stations 03214000 and 03214500 ; a negative value means the average for the indicated record period is less than the average for 1930-2002; a positive value means the average for the indicated record period is greater than the average for 1930-2002]

\begin{tabular}{|c|c|c|c|c|c|}
\hline \multirow{2}{*}{ Station number } & \multicolumn{5}{|c|}{ Difference for the indicated period, in percent } \\
\hline & 1930-42 & 1943-62 & 1963-69 & 1970-79 & 1980-2002 \\
\hline \multicolumn{6}{|c|}{ U.S. Environmental Protection Agency harmonic-mean flow for summer } \\
\hline 01606500 & -4.0 & 3.3 & -36.5 & 16.7 & 12.0 \\
\hline 01608500 & -7.1 & 2.5 & -41.1 & 27.4 & 17.0 \\
\hline 01611500 & -10.6 & 0.0 & -33.1 & 32.0 & 11.7 \\
\hline 01636500 & 0.4 & 2.7 & -37.0 & 16.2 & 9.6 \\
\hline 03051000 & -64.6 & 49.2 & 5.0 & 263.6 & 71.8 \\
\hline 03053500 & -56.0 & 29.7 & -8.9 & 139.2 & 42.4 \\
\hline 03061500 & -54.8 & -3.4 & -33.0 & 169.3 & 61.3 \\
\hline 03066000 & -8.2 & -9.4 & -27.7 & 49.6 & 13.3 \\
\hline 03069500 & -25.3 & 1.8 & -30.2 & 59.6 & 17.3 \\
\hline 03070500 & 5.4 & -25.8 & -45.4 & 120.8 & 41.2 \\
\hline 03182500 & -18.8 & 9.4 & -42.6 & 55.9 & 13.4 \\
\hline 03183500 & -13.4 & 6.7 & -35.7 & 50.4 & 5.8 \\
\hline 03186500 & -13.6 & -12.3 & -24.3 & 141.3 & 6.0 \\
\hline 03198500 & -35.9 & -26.5 & -28.2 & 98.1 & 93.4 \\
\hline $\begin{array}{c}{ }^{\mathrm{a}} 03214000 \text { and } \\
03214500\end{array}$ & No value & b -12.5 & ${ }^{\mathrm{b}} 0.0$ & b 53.7 & ${ }^{\mathrm{c}} 61.1$ \\
\hline \multicolumn{6}{|c|}{ U.S. Environmental Protection Agency harmonic-mean flow for fall } \\
\hline 01606500 & -6.6 & -2.0 & -18.5 & 33.8 & 1.3 \\
\hline 01608500 & -7.1 & -2.9 & -21.6 & 42.3 & 2.9 \\
\hline 01611500 & -9.4 & -3.8 & -17.0 & 42.5 & 3.8 \\
\hline 01636500 & -8.4 & 2.5 & -22.7 & 31.0 & 1.8 \\
\hline 03051000 & -50.3 & -5.6 & 26.7 & 284.8 & 31.2 \\
\hline 03053500 & -66.9 & -2.3 & 90.4 & 589.3 & 177.4 \\
\hline 03061500 & -46.4 & -21.0 & -24.0 & 196.6 & 61.5 \\
\hline 03066000 & -1.6 & -28.8 & -2.9 & 89.0 & 18.8 \\
\hline 03069500 & -23.8 & -22.5 & 6.5 & 92.6 & 25.5 \\
\hline 03070500 & 36.5 & -56.0 & 20.8 & 273.6 & 143.3 \\
\hline 03182500 & -20.9 & -4.0 & -16.1 & 52.3 & 9.1 \\
\hline 03183500 & -15.0 & -4.2 & 0.5 & 46.0 & 0.5 \\
\hline 03186500 & 7.1 & -35.4 & 16.8 & 163.7 & 14.6 \\
\hline 03198500 & -43.3 & -22.9 & -7.3 & 110.8 & 72.0 \\
\hline $\begin{array}{c}03214000 \text { and } \\
03214500\end{array}$ & No value & b -5.4 & b 12.2 & b 89.9 & c 50.7 \\
\hline
\end{tabular}

a 1930-2002 period estimated from record for 1936-85 at Tug Fork near Kermit (03214000).

${ }^{\mathrm{b}}$ Computed from record for Tug Fork near Kermit (03214000).

${ }^{\mathrm{c}}$ Estimated from record for 1986-2002 at Tug Fork at Kermit (03214500). 
Table 8. Record periods for the Lower Appalachian Plateaus region for which the indicated average departure was within 5 percent of the standard deviation of the standardized departures of the minimum flows for 1930-2002 (six stations in West Virginia, Ohio, and Virginia).

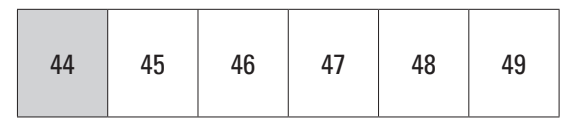

Table layout (page no.)

[No value indicates the average departure was greater than 5 percent; light shading is for example presented in text; station numbers for the six stations are 03061500, 03070500, 03109500, 03198500, 03208500, and combined stations 03214000 and 03214500]

\begin{tabular}{|c|c|c|c|c|c|c|c|c|c|c|c|}
\hline \multirow{2}{*}{ First year } & \multicolumn{11}{|c|}{ Years of record } \\
\hline & 10 & 11 & 12 & 13 & 14 & 15 & 16 & 17 & 18 & 19 & 20 \\
\hline \multicolumn{12}{|l|}{1930} \\
\hline \multicolumn{12}{|l|}{1931} \\
\hline \multicolumn{12}{|l|}{1932} \\
\hline \multicolumn{12}{|l|}{1933} \\
\hline \multicolumn{12}{|l|}{1934} \\
\hline \multicolumn{12}{|l|}{1935} \\
\hline \multicolumn{12}{|l|}{1936} \\
\hline \multicolumn{12}{|l|}{1937} \\
\hline \multicolumn{12}{|l|}{1938} \\
\hline \multicolumn{12}{|l|}{1939} \\
\hline \multicolumn{12}{|l|}{1940} \\
\hline \multicolumn{12}{|l|}{1941} \\
\hline \multicolumn{12}{|l|}{1942} \\
\hline \multicolumn{12}{|l|}{1943} \\
\hline \multicolumn{12}{|l|}{1944} \\
\hline \multicolumn{12}{|l|}{1945} \\
\hline \multicolumn{12}{|l|}{1946} \\
\hline \multicolumn{12}{|l|}{1947} \\
\hline \multicolumn{12}{|l|}{1948} \\
\hline \multicolumn{12}{|l|}{1949} \\
\hline \multicolumn{12}{|l|}{1950} \\
\hline \multicolumn{12}{|l|}{1951} \\
\hline 1952 & & & & & & & & & & & \\
\hline 1953 & & & & & & & & & & & \\
\hline 1954 & & & & & & & & & & & \\
\hline 1955 & & & & & & & & & & & \\
\hline 1956 & & & & & & & & & & -0.034 & 0.012 \\
\hline 1957 & & & & & & & & & 0.008 & & \\
\hline 1958 & & & & & & & & & & 0.008 & 0.024 \\
\hline 1959 & & & & & & & & 0.008 & & & \\
\hline 1960 & & & & & & & & 0.000 & 0.018 & & \\
\hline 1961 & & & & & & -0.010 & & & & & \\
\hline 1962 & & & & & -0.007 & & & & & & \\
\hline 1963 & & & & -0.016 & & & & & & & \\
\hline 1964 & & & 0.024 & & & & & & & & \\
\hline 1965 & 0.013 & & & & & & & & & & \\
\hline
\end{tabular}


Table 8. Record periods for the Lower Appalachian Plateaus region for which the indicated average departure was within 5 percent of the standard deviation of the standardized departures of the minimum flows for 1930-2002 (six stations in West Virginia, Ohio, and Virginia).—Continued

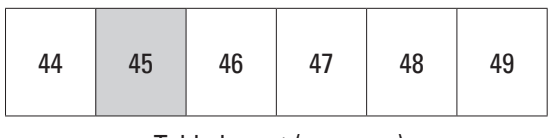

Table layout (page no.)

[No value indicates the average departure was greater than 5 percent; light shading is for example presented in text; station numbers for the six stations are 03061500, 03070500, 03109500, 03198500, 03208500, and combined stations 03214000 and 03214500]

\begin{tabular}{|c|c|c|c|c|c|c|c|c|c|c|c|}
\hline \multirow{2}{*}{ First year } & \multicolumn{11}{|c|}{ Years of record } \\
\hline & 21 & 22 & 23 & 24 & 25 & 26 & 27 & 28 & 29 & 30 & 31 \\
\hline \multicolumn{12}{|l|}{1930} \\
\hline \multicolumn{12}{|l|}{1931} \\
\hline \multicolumn{12}{|l|}{1932} \\
\hline \multicolumn{12}{|l|}{1933} \\
\hline \multicolumn{12}{|l|}{1934} \\
\hline \multicolumn{12}{|l|}{1935} \\
\hline \multicolumn{12}{|l|}{1936} \\
\hline \multicolumn{12}{|l|}{1937} \\
\hline \multicolumn{12}{|l|}{1938} \\
\hline \multicolumn{12}{|l|}{1939} \\
\hline \multicolumn{12}{|l|}{1940} \\
\hline \multicolumn{12}{|l|}{1941} \\
\hline \multicolumn{12}{|l|}{1942} \\
\hline \multicolumn{12}{|l|}{1943} \\
\hline \multicolumn{12}{|l|}{1944} \\
\hline \multicolumn{12}{|l|}{1945} \\
\hline \multicolumn{12}{|l|}{1946} \\
\hline \multicolumn{12}{|l|}{1947} \\
\hline 1948 & & & & & & & & & -0.035 & -0.023 & 0.015 \\
\hline 1949 & & & & & & & & -0.029 & -0.016 & 0.022 & \\
\hline 1950 & & & & & & & -0.031 & -0.018 & 0.021 & & \\
\hline 1951 & & & & & & -0.021 & -0.008 & 0.032 & & & \\
\hline 1952 & & & & & & -0.032 & 0.011 & & & & \\
\hline 1953 & & & & -0.024 & -0.010 & 0.033 & & & & & \\
\hline 1954 & & & -0.011 & 0.003 & & & & & & & \\
\hline 1955 & -0.010 & 0.033 & & & & & & & & & \\
\hline \multicolumn{12}{|l|}{1956} \\
\hline \multicolumn{12}{|l|}{1957} \\
\hline \multicolumn{12}{|l|}{1958} \\
\hline \multicolumn{12}{|l|}{1959} \\
\hline 1960 & & & & & & & & & & & \\
\hline 1961 & & & & & & & & & & & \\
\hline 1962 & & & & & & & & & & & \\
\hline 1963 & & & & & & & & & & & \\
\hline 1964 & & & & & & & & & & & \\
\hline 1965 & & & & & & & & & & & \\
\hline
\end{tabular}


Table 8. Record periods for the Lower Appalachian Plateaus region for which the indicated average departure was within 5 percent of the standard deviation of the standardized departures of the minimum flows for 1930-2002 (six stations in West Virginia, Ohio, and Virginia).-Continued

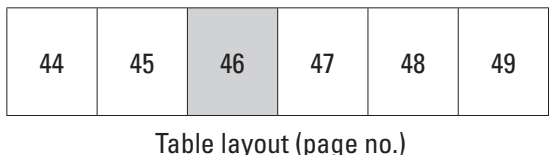

Table layout (page no.)

[No value indicates the average departure was greater than 5 percent; dark shading indicates period outside the limit of this study; station numbers for the six stations are $03061500,03070500,03109500,03198500,03208500$, and combined stations 03214000 and 03214500 ]

\begin{tabular}{|c|c|c|c|c|c|c|c|c|c|c|c|}
\hline \multirow{2}{*}{ First year } & \multicolumn{11}{|c|}{ Years of record } \\
\hline & 32 & 33 & 34 & 35 & 36 & 37 & 38 & 39 & 40 & 41 & 42 \\
\hline \multicolumn{12}{|l|}{1930} \\
\hline \multicolumn{12}{|l|}{1931} \\
\hline \multicolumn{12}{|l|}{1932} \\
\hline \multicolumn{12}{|l|}{1933} \\
\hline \multicolumn{12}{|l|}{1934} \\
\hline \multicolumn{12}{|l|}{1935} \\
\hline \multicolumn{12}{|l|}{1936} \\
\hline \multicolumn{12}{|l|}{1937} \\
\hline \multicolumn{12}{|l|}{1938} \\
\hline 1939 & & & & & & & & & & & -0.028 \\
\hline 1940 & & & & & & & & & & -0.014 & 0.013 \\
\hline 1941 & & & & & & & & -0.039 & 0.010 & 0.037 & \\
\hline 1942 & & & & & & & -0.027 & 0.023 & & & \\
\hline 1943 & & & & & -0.037 & -0.013 & 0.038 & & & & \\
\hline 1944 & & & & & -0.032 & 0.021 & & & & & \\
\hline 1945 & & & -0.033 & -0.009 & & & & & & & \\
\hline 1946 & & -0.013 & 0.012 & & & & & & & & \\
\hline 1947 & -0.007 & 0.019 & & & & & & & & & \\
\hline \multicolumn{12}{|l|}{1948} \\
\hline \multicolumn{12}{|l|}{1949} \\
\hline \multicolumn{12}{|l|}{1950} \\
\hline \multicolumn{12}{|l|}{1951} \\
\hline \multicolumn{12}{|l|}{1952} \\
\hline \multicolumn{12}{|l|}{1953} \\
\hline \multicolumn{12}{|l|}{1954} \\
\hline \multicolumn{12}{|l|}{1955} \\
\hline \multicolumn{12}{|l|}{1956} \\
\hline \multicolumn{12}{|l|}{1957} \\
\hline \multicolumn{12}{|l|}{1958} \\
\hline \multicolumn{12}{|l|}{1959} \\
\hline \multicolumn{12}{|l|}{1960} \\
\hline 1961 & & & & & & & & & & & \\
\hline 1962 & & & & & & & & & & & \\
\hline 1963 & & & & & & & & & & & \\
\hline 1964 & & & & & & & & & & & \\
\hline 1965 & & & & & & & & & & & \\
\hline
\end{tabular}


Table 8. Record periods for the Lower Appalachian Plateaus region for which the indicated average departure was within 5 percent of the standard deviation of the standardized departures of the minimum flows for 1930-2002 (six stations in West Virginia, Ohio, and Virginia).-Continued

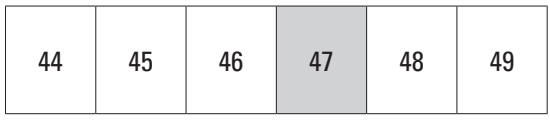

Table layout (page no.)

[No value indicates the average departure was greater than 5 percent; dark shading indicates period outside the limit of this study; station numbers for the six stations are $03061500,03070500,03109500,03198500,03208500$, and combined stations 03214000 and 03214500$]$

\begin{tabular}{|c|c|c|c|c|c|c|c|c|c|c|c|}
\hline \multirow{2}{*}{ First year } & \multicolumn{11}{|c|}{ Years of record } \\
\hline & 43 & 44 & 45 & 46 & 47 & 48 & 49 & 50 & 51 & 52 & 53 \\
\hline \multicolumn{12}{|l|}{1930} \\
\hline \multicolumn{12}{|l|}{1931} \\
\hline \multicolumn{12}{|l|}{1932} \\
\hline \multicolumn{12}{|l|}{1933} \\
\hline 1934 & & & & & & & & & -0.039 & -0.021 & -0.024 \\
\hline 1935 & & & & & & & -0.030 & -0.028 & -0.009 & -0.013 & -0.011 \\
\hline 1936 & & & & & -0.032 & -0.017 & -0.015 & 0.004 & -0.001 & 0.002 & -0.003 \\
\hline 1937 & & & -0.029 & -0.020 & -0.004 & -0.003 & 0.016 & 0.011 & 0.013 & 0.008 & -0.004 \\
\hline 1938 & -0.033 & -0.007 & 0.001 & 0.017 & 0.018 & 0.037 & 0.031 & 0.033 & 0.027 & 0.014 & \\
\hline 1939 & -0.001 & 0.007 & 0.023 & 0.024 & & 0.038 & 0.039 & 0.033 & 0.020 & & \\
\hline 1940 & 0.021 & 0.037 & 0.038 & & & & & 0.032 & & & \\
\hline \multicolumn{12}{|l|}{1941} \\
\hline \multicolumn{12}{|l|}{1942} \\
\hline \multicolumn{12}{|l|}{1943} \\
\hline \multicolumn{12}{|l|}{1944} \\
\hline \multicolumn{12}{|l|}{1945} \\
\hline \multicolumn{12}{|l|}{1946} \\
\hline \multicolumn{12}{|l|}{1947} \\
\hline \multicolumn{12}{|l|}{1948} \\
\hline \multicolumn{12}{|l|}{1949} \\
\hline \multicolumn{12}{|l|}{1950} \\
\hline \multicolumn{12}{|l|}{1951} \\
\hline \multicolumn{12}{|l|}{1952} \\
\hline \multicolumn{12}{|l|}{1953} \\
\hline \multicolumn{12}{|l|}{1954} \\
\hline \multicolumn{12}{|l|}{1955} \\
\hline \multicolumn{12}{|l|}{1956} \\
\hline \multicolumn{12}{|l|}{1957} \\
\hline \multicolumn{12}{|l|}{1958} \\
\hline \multicolumn{12}{|l|}{1959} \\
\hline 1960 & & & & & & & & & & & \\
\hline
\end{tabular}


Table 8. Record periods for the Lower Appalachian Plateaus region for which the indicated average departure was within 5 percent of the standard deviation of the standardized departures of the minimum flows for 1930-2002 (six stations in West Virginia, Ohio, and Virginia)._-Continued

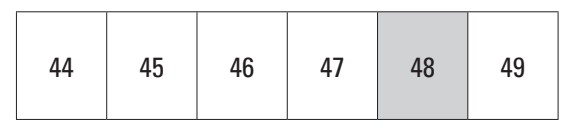

Table layout (page no.)

[No value indicates the average departure was greater than 5 percent; dark shading indicates period outside the limit of this study; station numbers for the six stations are 03061500, 03070500, 03109500, 03198500, 03208500, and combined stations 03214000 and 03214500]

\begin{tabular}{|c|c|c|c|c|c|c|c|c|c|c|c|}
\hline \multirow{2}{*}{ First year } & \multicolumn{11}{|c|}{ Years of record } \\
\hline & 54 & 55 & 56 & 57 & 58 & 59 & 60 & 61 & 62 & 63 & 64 \\
\hline 1930 & & & & & & & & & -0.035 & & -0.027 \\
\hline 1931 & & & & & & & & -0.030 & -0.037 & -0.022 & -0.025 \\
\hline 1932 & & & & & & -0.033 & -0.011 & -0.018 & -0.003 & -0.006 & 0.008 \\
\hline 1933 & & & & & -0.025 & -0.003 & -0.010 & 0.005 & 0.002 & 0.016 & 0.012 \\
\hline 1934 & -0.022 & -0.026 & -0.037 & -0.006 & 0.016 & 0.008 & 0.024 & 0.020 & 0.034 & 0.029 & \\
\hline 1935 & -0.015 & -0.027 & 0.004 & 0.027 & 0.019 & 0.035 & 0.030 & & 0.039 & & \\
\hline 1936 & -0.015 & 0.016 & 0.039 & 0.031 & & & & & & & \\
\hline 1937 & 0.028 & & & & & & & & & & \\
\hline 1938 & & & & & & & & & & & \\
\hline 1939 & & & & & & & & & & & \\
\hline 1940 & & & & & & & & & & & \\
\hline 1941 & & & & & & & & & & & \\
\hline 1942 & & & & & & & & & & & \\
\hline 1943 & & & & & & & & & & & \\
\hline 1944 & & & & & & & & & & & \\
\hline 1945 & & & & & & & & & & & \\
\hline 1946 & & & & & & & & & & & \\
\hline 1947 & & & & & & & & & & & \\
\hline 1948 & & & & & & & & & & & \\
\hline 1949 & & & & & & & & & & & \\
\hline
\end{tabular}


Table 8. Record periods for the Lower Appalachian Plateaus region for which the indicated average departure was within 5 percent of the standard deviation of the standardized departures of the minimum flows for 1930-2002 (six stations in West Virginia, Ohio, and Virginia)._-Continued

\begin{tabular}{|l|l|l|l|l|l|}
\hline 44 & 45 & 46 & 47 & 48 & 49 \\
\hline
\end{tabular}

Table layout (page no.)

[No value indicates the average departure was greater than 5 percent; dark shading indicates period outside the limit of this study; station numbers for the six stations are 03061500, 03070500, 03109500, 03198500, 03208500, and combined stations 03214000 and 03214500]

\begin{tabular}{|c|c|c|c|c|c|c|c|c|c|}
\hline \multirow{2}{*}{ First year } & \multicolumn{9}{|c|}{ Years of record } \\
\hline & 65 & 66 & 67 & 68 & 69 & 70 & 71 & 72 & 73 \\
\hline 1930 & -0.030 & -0.016 & -0.019 & 0.009 & 0.010 & 0.009 & 0.002 & 0.014 & 0.015 \\
\hline 1931 & -0.011 & -0.014 & 0.014 & 0.015 & 0.015 & 0.007 & 0.019 & 0.020 & \\
\hline 1932 & 0.003 & 0.033 & 0.033 & 0.032 & 0.024 & 0.036 & 0.037 & & \\
\hline 1933 & & & & 0.032 & & & & & \\
\hline 1934 & & & & & & & & & \\
\hline 1935 & & & & & & & & & \\
\hline 1936 & & & & & & & & & \\
\hline 1937 & & & & & & & & & \\
\hline 1938 & & & & & & & & & \\
\hline
\end{tabular}


Table 9. Record periods for the Upper Appalachian Plateaus region for which the indicated average departure was within 5 percent of the standard deviation of the standardized departures of the minimum flows for 1930-2002 (six stations in West Virginia).

[No value indicates the average departure was greater than 5 percent; station numbers for the six stations are 03051000, 03053500, 03066000, 03069500, 03182500, and 03186500]

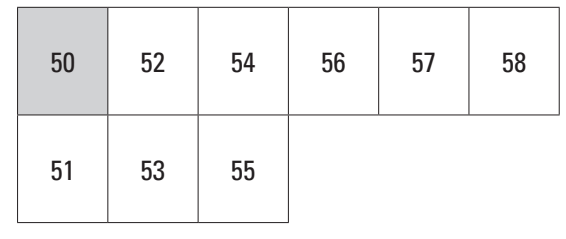

Table layout (page no.)

\begin{tabular}{|c|c|c|c|c|c|c|c|c|c|c|c|}
\hline \multirow{2}{*}{ First year } & \multicolumn{11}{|c|}{ Years of record } \\
\hline & 10 & 11 & 12 & 13 & 14 & 15 & 16 & 17 & 18 & 19 & 20 \\
\hline \multicolumn{12}{|l|}{1930} \\
\hline \multicolumn{12}{|l|}{1931} \\
\hline 1932 & & & & & & & & & & & 0.021 \\
\hline 1933 & & & & & & & & & & -0.024 & \\
\hline 1934 & & & & & & & & & 0.024 & -0.016 & \\
\hline 1935 & & & & & & & & -0.015 & & & \\
\hline 1936 & & & & & & & 0.024 & -0.020 & & & \\
\hline 1937 & & & & & & -0.001 & -0.046 & & & & \\
\hline 1938 & & & & & & -0.003 & & & & & -0.024 \\
\hline 1939 & & & & & 0.007 & & & & & -0.018 & \\
\hline 1940 & & -0.018 & & 0.036 & -0.028 & & & & 0.002 & -0.046 & -0.032 \\
\hline 1941 & 0.043 & & & 0.019 & & -0.017 & & 0.040 & -0.013 & 0.000 & \\
\hline 1942 & & & 0.025 & & -0.015 & & 0.046 & -0.011 & 0.003 & & -0.041 \\
\hline 1943 & & & -0.025 & 0.023 & -0.029 & & 0.020 & 0.033 & -0.024 & -0.016 & 0.020 \\
\hline 1944 & & & & & -0.005 & & -0.046 & & & & \\
\hline 1945 & & -0.028 & & & -0.021 & -0.003 & & & -0.012 & & \\
\hline 1946 & 0.037 & -0.031 & & 0.030 & 0.045 & -0.025 & -0.015 & 0.027 & -0.016 & -0.045 & \\
\hline 1947 & & & -0.013 & 0.007 & & & -0.005 & & & & \\
\hline 1948 & & & & 0.002 & 0.012 & & 0.007 & -0.027 & & & \\
\hline 1949 & & & 0.040 & & & 0.037 & -0.001 & & & & \\
\hline 1950 & -0.002 & & & -0.015 & & & & & & & \\
\hline \multicolumn{12}{|l|}{1951} \\
\hline \multicolumn{12}{|l|}{1952} \\
\hline \multicolumn{12}{|l|}{1953} \\
\hline \multicolumn{12}{|l|}{1954} \\
\hline \multicolumn{12}{|l|}{1955} \\
\hline \multicolumn{12}{|l|}{1956} \\
\hline \multicolumn{12}{|l|}{1957} \\
\hline \multicolumn{12}{|l|}{1958} \\
\hline \multicolumn{12}{|l|}{1959} \\
\hline \multicolumn{12}{|l|}{1960} \\
\hline \multicolumn{12}{|l|}{1961} \\
\hline \multicolumn{12}{|l|}{1962} \\
\hline 1963 & & & & & & & & & 0.029 & 0.032 & 0.019 \\
\hline \multicolumn{12}{|l|}{1964} \\
\hline 1965 & & & & & & & & & & & \\
\hline
\end{tabular}


Table 9. Record periods for the Upper Appalachian Plateaus region for which the indicated average departure was within 5 percent of the standard deviation of the standardized departures of the minimum flows for 1930-2002 (six stations in West Virginia).-Continued

[No value indicates the average departure was greater than 5 percent; dark shading indicates period outside the limit of this study; station numbers for the six stations are 03051000, 03053500, 03066000, 03069500, 03182500, and 03186500]

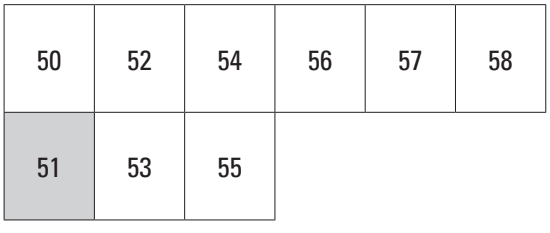

Table layout (page no.)

\begin{tabular}{|c|c|c|c|c|c|c|c|c|c|c|c|}
\hline \multirow{2}{*}{ First year } & \multicolumn{11}{|c|}{ Years of record } \\
\hline & 10 & 11 & 12 & 13 & 14 & 15 & 16 & 17 & 18 & 19 & 20 \\
\hline 1966 & & & & -0.042 & -0.042 & & & & & & \\
\hline 1967 & 0.022 & -0.022 & 0.028 & 0.023 & & & & & & & \\
\hline 1968 & 0.033 & & & & & & & & & & \\
\hline \multicolumn{12}{|l|}{1969} \\
\hline \multicolumn{12}{|l|}{1970} \\
\hline \multicolumn{12}{|l|}{1971} \\
\hline \multicolumn{12}{|l|}{1972} \\
\hline \multicolumn{12}{|l|}{1973} \\
\hline \multicolumn{12}{|l|}{1974} \\
\hline \multicolumn{12}{|l|}{1975} \\
\hline \multicolumn{12}{|l|}{1976} \\
\hline \multicolumn{12}{|l|}{1977} \\
\hline \multicolumn{12}{|l|}{1978} \\
\hline \multicolumn{12}{|l|}{1979} \\
\hline \multicolumn{12}{|l|}{1980} \\
\hline 1981 & & & & & & & 0.021 & & & & \\
\hline 1982 & & & & & & 0.015 & & & & & \\
\hline 1983 & & & & & 0.033 & & & & & & \\
\hline 1984 & & & & -0.017 & & & & 0.025 & & & \\
\hline 1985 & & & 0.038 & & & & & & & & \\
\hline 1986 & & 0.004 & & & & 0.046 & & & & & \\
\hline \multicolumn{12}{|l|}{1987} \\
\hline 1988 & & & & 0.020 & & & & & & & \\
\hline \multicolumn{12}{|l|}{1989} \\
\hline \multicolumn{12}{|l|}{1990} \\
\hline 1991 & & 0.032 & 0.012 & & & & & & & & \\
\hline 1992 & -0.036 & & & & & & & & & & \\
\hline 1993 & -0.015 & & & & & & & & & & \\
\hline
\end{tabular}


Table 9. Record periods for the Upper Appalachian Plateaus region for which the indicated average departure was within 5 percent of the standard deviation of the standardized departures of the minimum flows for 1930-2002 (six stations in West Virginia)._-Continued

[No value indicates the average departure was greater than 5 percent; station numbers for the six stations are $03051000,03053500,03066000,03069500,03182500$, and 03186500]

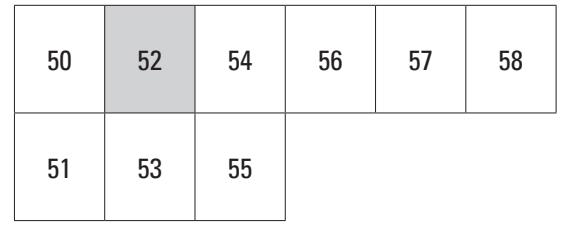

Table layout (page no.)

\begin{tabular}{|c|c|c|c|c|c|c|c|c|c|c|c|}
\hline \multirow{2}{*}{ First year } & \multicolumn{11}{|c|}{ Years of record } \\
\hline & 21 & 22 & 23 & 24 & 25 & 26 & 27 & 28 & 29 & 30 & 31 \\
\hline \multicolumn{12}{|l|}{1930} \\
\hline 1931 & -0.036 & & & & & & & & & & \\
\hline 1932 & -0.015 & & & & & -0.029 & & & & & \\
\hline \multicolumn{12}{|l|}{1933} \\
\hline 1934 & & & & -0.030 & & & & & & & \\
\hline \multicolumn{12}{|l|}{1935} \\
\hline 1936 & & -0.035 & & & & & & & & & \\
\hline \multicolumn{12}{|l|}{1937} \\
\hline \multicolumn{12}{|l|}{1938} \\
\hline \multicolumn{12}{|l|}{1939} \\
\hline 1940 & & & -0.035 & & & & & & & & \\
\hline 1941 & -0.041 & -0.008 & -0.040 & & & & & & & & \\
\hline 1942 & -0.006 & -0.039 & & & & & & & & & \\
\hline 1943 & -0.017 & -0.042 & & & & & & & & & \\
\hline \multicolumn{12}{|l|}{1944} \\
\hline \multicolumn{12}{|l|}{1945} \\
\hline \multicolumn{12}{|l|}{1946} \\
\hline \multicolumn{12}{|l|}{1947} \\
\hline \multicolumn{12}{|l|}{1948} \\
\hline \multicolumn{12}{|l|}{1949} \\
\hline 1950 & & & & & & & & & & & 0.010 \\
\hline 1951 & & & & & & & & & & -0.003 & 0.000 \\
\hline \multicolumn{12}{|l|}{1952} \\
\hline 1953 & & & & & & & & -0.027 & -0.023 & -0.030 & -0.007 \\
\hline 1954 & & & & & & & 0.004 & 0.007 & -0.001 & 0.022 & -0.001 \\
\hline 1955 & & & & & & & & 0.038 & & 0.036 & \\
\hline 1956 & & & & & 0.024 & 0.027 & 0.017 & 0.041 & 0.016 & 0.030 & 0.013 \\
\hline 1957 & & & & & & 0.045 & & 0.042 & & 0.037 & \\
\hline 1958 & & & -0.014 & -0.010 & -0.019 & 0.008 & -0.017 & -0.002 & -0.018 & 0.013 & -0.018 \\
\hline 1959 & & 0.026 & 0.029 & 0.018 & 0.045 & 0.017 & 0.032 & 0.013 & 0.045 & 0.012 & -0.016 \\
\hline 1960 & 0.016 & 0.020 & 0.009 & 0.037 & 0.008 & 0.024 & 0.005 & 0.038 & 0.004 & -0.024 & \\
\hline 1961 & & & & & & 0.044 & & 0.040 & 0.009 & & \\
\hline 1962 & & & & & 0.040 & & 0.037 & 0.005 & & & \\
\hline 1963 & & 0.017 & 0.035 & 0.013 & & 0.012 & -0.021 & & & & \\
\hline 1964 & & & 0.046 & & 0.042 & 0.007 & & & & & \\
\hline 1965 & & & & & 0.030 & & & & & & \\
\hline
\end{tabular}


Table 9. Record periods for the Upper Appalachian Plateaus region for which the indicated average departure was within 5 percent of the standard deviation of the standardized departures of the minimum flows for 1930-2002 (six stations in West Virginia).-Continued

[No value indicates the average departure was greater than 5 percent; dark shading indicates period outside the limit of this study; station numbers for the six stations are 03051000, 03053500, 03066000, 03069500, 03182500, and 03186500]

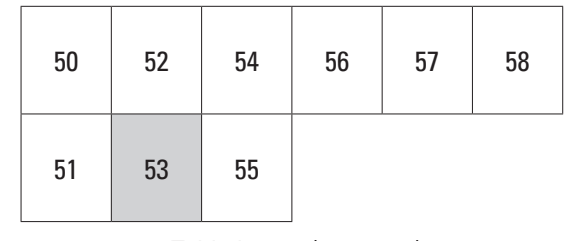

Table layout (page no.)

\begin{tabular}{|c|c|c|c|c|c|c|c|c|c|c|c|}
\hline \multirow{2}{*}{ First year } & \multicolumn{11}{|c|}{ Years of record } \\
\hline & 21 & 22 & 23 & 24 & 25 & 26 & 27 & 28 & 29 & 30 & 31 \\
\hline 1966 & & & & & & & & & & & \\
\hline 1967 & & & & & & & & & & & \\
\hline 1968 & & & & & & & & & & & \\
\hline 1969 & & & & & & & & & & & \\
\hline 1970 & & & & & & & & & & & \\
\hline 1971 & & & & & & & & & & & \\
\hline 1972 & & & & & & & & & & & \\
\hline 1973 & & & & & & & & & & & \\
\hline 1974 & & & & & & & & & & & \\
\hline 1975 & & & & & & & & & & & \\
\hline 1976 & & & & & & & & & & & \\
\hline 1977 & & & & & & & & & & & \\
\hline 1978 & & & & & & & & & & & \\
\hline 1979 & & & & & & & & & & & \\
\hline 1980 & & & & & & & & & & & \\
\hline 1981 & & & & & & & & & & & \\
\hline 1982 & & & & & & & & & & & \\
\hline
\end{tabular}


Table 9. Record periods for the Upper Appalachian Plateaus region for which the indicated average departure was within 5 percent of the standard deviation of the standardized departures of the minimum flows for 1930-2002 (six stations in West Virginia)._-Continued

[No value indicates the average departure was greater than 5 percent; dark shading indicates period outside the limit of this study; station numbers for the six stations are 03051000, 03053500, 03066000, 03069500, 03182500, and 03186500]

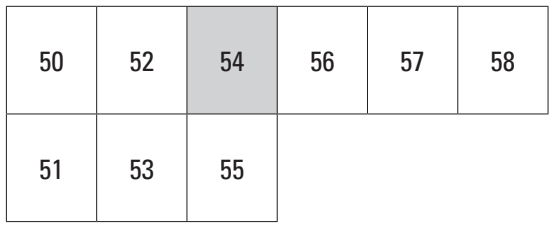

Table layout (page no.)

\begin{tabular}{|c|c|c|c|c|c|c|c|c|c|c|c|}
\hline \multirow{2}{*}{ First year } & \multicolumn{11}{|c|}{ Years of record } \\
\hline & 32 & 33 & 34 & 35 & 36 & 37 & 38 & 39 & 40 & 41 & 42 \\
\hline \multicolumn{12}{|l|}{1930} \\
\hline \multicolumn{12}{|l|}{1931} \\
\hline \multicolumn{12}{|l|}{1932} \\
\hline \multicolumn{12}{|l|}{1933} \\
\hline \multicolumn{12}{|l|}{1934} \\
\hline \multicolumn{12}{|l|}{1935} \\
\hline \multicolumn{12}{|l|}{1936} \\
\hline \multicolumn{12}{|l|}{1937} \\
\hline \multicolumn{12}{|l|}{1938} \\
\hline 1939 & & & & & & & & & & & -0.016 \\
\hline 1940 & & & & & & & & & & -0.007 & -0.005 \\
\hline 1941 & & & & & & & & & 0.008 & 0.011 & 0.005 \\
\hline 1942 & & & & & & & & 0.010 & 0.012 & 0.006 & 0.023 \\
\hline 1943 & & & & & & & 0.024 & 0.026 & 0.019 & 0.036 & 0.018 \\
\hline 1944 & & & & & & -0.011 & -0.008 & -0.014 & 0.004 & -0.013 & -0.003 \\
\hline 1945 & & & & & 0.008 & 0.010 & 0.004 & 0.022 & 0.004 & 0.014 & 0.002 \\
\hline 1946 & & & & 0.028 & 0.030 & 0.023 & 0.040 & 0.022 & 0.031 & 0.019 & 0.040 \\
\hline 1947 & & & 0.013 & 0.015 & 0.008 & 0.026 & 0.008 & 0.018 & 0.006 & 0.028 & 0.005 \\
\hline 1948 & & 0.042 & 0.043 & 0.035 & & 0.034 & 0.044 & 0.030 & & 0.028 & 0.007 \\
\hline 1949 & & & & & & & 0.043 & & 0.040 & 0.018 & \\
\hline 1950 & 0.013 & 0.006 & 0.026 & 0.005 & 0.017 & 0.003 & 0.027 & 0.003 & -0.019 & & \\
\hline 1951 & -0.007 & 0.014 & -0.007 & 0.006 & -0.008 & 0.017 & -0.008 & -0.030 & & & \\
\hline 1952 & -0.029 & & -0.035 & & -0.021 & -0.046 & & 0.019 & 0.036 & 0.025 & 0.020 \\
\hline 1953 & -0.028 & -0.014 & -0.028 & -0.001 & -0.027 & & 0.039 & & 0.044 & 0.038 & 0.017 \\
\hline 1954 & 0.012 & -0.003 & 0.024 & -0.003 & -0.027 & & & & & 0.038 & 0.030 \\
\hline 1955 & 0.031 & & 0.029 & 0.004 & & & & & & & 0.036 \\
\hline 1956 & 0.041 & 0.012 & -0.014 & & & & & & 0.044 & 0.023 & \\
\hline 1957 & 0.034 & 0.007 & & & & & & & 0.041 & & \\
\hline 1958 & -0.044 & & & & & 0.031 & 0.022 & 0.000 & & 0.040 & 0.036 \\
\hline 1959 & & & & & & & 0.024 & & & & 0.037 \\
\hline 1960 & & & & & 0.042 & 0.018 & & & & 0.032 & \\
\hline \multicolumn{12}{|l|}{1961} \\
\hline 1962 & & & & 0.044 & & & & & & & \\
\hline 1963 & & & 0.025 & & & & 0.039 & & & & \\
\hline \multicolumn{12}{|l|}{1964} \\
\hline 1965 & & & & & & & & & & & \\
\hline
\end{tabular}


Table 9. Record periods for the Upper Appalachian Plateaus region for which the indicated average departure was within 5 percent of the standard deviation of the standardized departures of the minimum flows for 1930-2002 (six stations in West Virginia).-Continued

[No value indicates the average departure was greater than 5 percent; dark shading indicates period outside the limit of this study; station numbers for the six stations are 03051000, 03053500, 03066000, 03069500, 03182500, and 03186500]

\begin{tabular}{|l|l|l|l|l|l|}
\hline 50 & 52 & 54 & 56 & 57 & 58 \\
\hline 51 & 53 & 55 & \multicolumn{3}{|l}{} \\
\hline
\end{tabular}

Table layout (page no.)

\begin{tabular}{|c|c|c|c|c|c|c|c|c|c|c|c|}
\hline \multirow{2}{*}{ First year } & \multicolumn{11}{|c|}{ Years of record } \\
\hline & 32 & 33 & 34 & 35 & 36 & 37 & 38 & 39 & 40 & 41 & 42 \\
\hline \multicolumn{12}{|l|}{1966} \\
\hline \multicolumn{12}{|l|}{1967} \\
\hline \multicolumn{12}{|l|}{1968} \\
\hline \multicolumn{12}{|l|}{1969} \\
\hline \multicolumn{12}{|l|}{1970} \\
\hline 1971 & & & & & & & & & & & \\
\hline
\end{tabular}


Table 9. Record periods for the Upper Appalachian Plateaus region for which the indicated average departure was within 5 percent of the standard deviation of the standardized departures of the minimum flows for 1930-2002 (six stations in West Virginia)._-Continued

[No value indicates the average departure was greater than 5 percent; dark shading indicates period outside the limit of this study; station numbers for the six stations are 03051000, 03053500, 03066000, 03069500, 03182500, and 03186500]

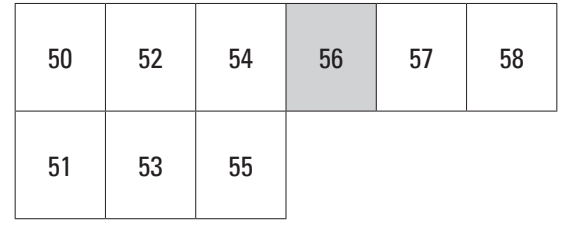

Table layout (page no.)

\begin{tabular}{|c|c|c|c|c|c|c|c|c|c|c|c|}
\hline \multirow{2}{*}{ First year } & \multicolumn{11}{|c|}{ Years of record } \\
\hline & 43 & 44 & 45 & 46 & 47 & 48 & 49 & 50 & 51 & 52 & 53 \\
\hline \multicolumn{12}{|l|}{1930} \\
\hline 1931 & & & & & & & & -0.045 & -0.042 & -0.046 & -0.032 \\
\hline 1932 & & & & & & & -0.022 & -0.020 & -0.024 & -0.010 & -0.023 \\
\hline 1933 & & & & & & -0.040 & -0.038 & -0.042 & -0.027 & -0.040 & -0.031 \\
\hline 1934 & & & & & -0.023 & -0.020 & -0.024 & -0.010 & -0.023 & -0.015 & -0.024 \\
\hline 1935 & & & & -0.038 & -0.035 & -0.039 & -0.024 & -0.038 & -0.029 & -0.037 & -0.019 \\
\hline 1936 & & & -0.025 & -0.022 & -0.026 & -0.012 & -0.025 & -0.016 & -0.026 & -0.007 & -0.025 \\
\hline 1937 & & -0.034 & -0.031 & -0.036 & -0.020 & -0.034 & -0.025 & -0.034 & -0.015 & -0.033 & \\
\hline 1938 & -0.019 & -0.016 & -0.021 & -0.006 & -0.020 & -0.011 & -0.021 & -0.002 & -0.020 & -0.036 & 0.027 \\
\hline 1939 & -0.013 & -0.018 & -0.003 & -0.017 & -0.008 & -0.018 & 0.001 & -0.018 & -0.034 & 0.030 & 0.043 \\
\hline 1940 & -0.010 & 0.006 & -0.009 & 0.000 & -0.010 & 0.009 & -0.010 & -0.027 & 0.038 & & 0.042 \\
\hline 1941 & 0.021 & 0.005 & 0.014 & 0.003 & 0.023 & 0.003 & -0.015 & & & & \\
\hline 1942 & 0.006 & 0.016 & 0.004 & 0.024 & 0.004 & -0.014 & & & & & 0.035 \\
\hline 1943 & 0.028 & 0.016 & 0.036 & 0.015 & -0.004 & & & & & 0.046 & 0.039 \\
\hline 1944 & -0.014 & 0.007 & -0.014 & -0.032 & 0.039 & & 0.043 & 0.039 & 0.021 & 0.015 & -0.001 \\
\hline 1945 & 0.023 & 0.002 & -0.017 & & & & & 0.035 & 0.029 & 0.012 & \\
\hline 1946 & 0.018 & -0.002 & & & & & & 0.043 & 0.025 & & \\
\hline 1947 & -0.015 & & & & & 0.040 & 0.033 & 0.015 & & 0.046 & 0.043 \\
\hline 1948 & & & & & & & 0.035 & & & & 0.044 \\
\hline 1949 & & & & & & 0.045 & & & & & \\
\hline 1950 & & & 0.040 & 0.033 & 0.014 & & & 0.044 & 0.025 & & \\
\hline 1951 & & 0.032 & 0.024 & 0.005 & 0.045 & 0.039 & 0.036 & 0.018 & & 0.044 & \\
\hline 1952 & 0.000 & -0.007 & -0.026 & 0.015 & 0.010 & 0.008 & -0.011 & 0.022 & 0.017 & & \\
\hline 1953 & 0.010 & -0.010 & 0.032 & 0.026 & 0.023 & 0.004 & 0.037 & 0.032 & & & \\
\hline 1954 & 0.010 & & 0.046 & 0.042 & 0.023 & & & & & & \\
\hline \multicolumn{12}{|l|}{1955} \\
\hline 1956 & & & 0.035 & & & & & & & & \\
\hline \multicolumn{12}{|l|}{1957} \\
\hline 1958 & 0.015 & & 0.045 & & & & & & & & \\
\hline \multicolumn{12}{|l|}{1959} \\
\hline 1960 & & & & & & & & & & & \\
\hline
\end{tabular}


Table 9. Record periods for the Upper Appalachian Plateaus region for which the indicated average departure was within 5 percent of the standard deviation of the standardized departures of the minimum flows for 1930-2002 (six stations in West Virginia).-Continued

[No value indicates the average departure was greater than 5 percent; dark shading indicates period outside the limit of this study; station numbers for the six stations are 03051000, 03053500, 03066000, 03069500, 03182500, and 03186500]

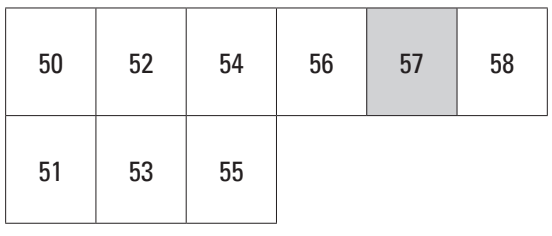

Table layout (page no.)

\begin{tabular}{|l|l|l|l|l|l|l|l|l|l|l|c|}
\hline \multirow{2}{*}{ First year } & \multicolumn{9}{|c|}{ Years of record } \\
\cline { 2 - 14 } & $\mathbf{5 4}$ & $\mathbf{5 5}$ & $\mathbf{5 6}$ & $\mathbf{5 7}$ & $\mathbf{5 8}$ & $\mathbf{5 9}$ & $\mathbf{6 0}$ & $\mathbf{6 1}$ & $\mathbf{6 2}$ & $\mathbf{6 3}$ & $\mathbf{6 4}$ \\
\hline 1930 & -0.045 & & & & -0.039 & & & -0.012 & 0.000 & -0.007 & -0.010 \\
\hline 1931 & -0.044 & -0.036 & -0.044 & -0.027 & -0.042 & & 0.000 & 0.011 & 0.004 & 0.001 & -0.012 \\
\hline 1932 & -0.015 & -0.023 & -0.006 & -0.023 & -0.037 & 0.019 & 0.031 & 0.024 & 0.020 & 0.006 & 0.002 \\
\hline 1933 & -0.040 & -0.022 & -0.038 & & 0.005 & 0.017 & 0.010 & 0.007 & -0.007 & -0.012 & -0.025 \\
\hline 1934 & -0.006 & -0.023 & -0.038 & 0.020 & 0.032 & 0.025 & 0.021 & 0.007 & 0.002 & -0.012 & 0.018 \\
\hline 1935 & -0.036 & & 0.009 & 0.021 & 0.013 & 0.010 & -0.004 & -0.009 & -0.023 & 0.007 & 0.003 \\
\hline 1936 & -0.040 & 0.020 & 0.033 & 0.025 & 0.021 & 0.006 & 0.001 & -0.013 & 0.018 & 0.014 & 0.012 \\
\hline 1937 & 0.013 & 0.026 & 0.018 & 0.015 & 0.000 & -0.006 & -0.020 & 0.011 & 0.007 & 0.006 & -0.008 \\
\hline 1938 & 0.039 & 0.031 & 0.027 & 0.012 & 0.006 & -0.008 & 0.023 & 0.019 & 0.017 & 0.003 & 0.028 \\
\hline 1939 & 0.034 & 0.030 & 0.015 & 0.009 & -0.006 & 0.026 & 0.022 & 0.020 & 0.005 & 0.030 & 0.027 \\
\hline 1940 & 0.038 & 0.022 & 0.016 & 0.001 & 0.033 & 0.028 & 0.026 & 0.011 & 0.037 & 0.033 \\
\hline 1941 & 0.034 & 0.028 & 0.012 & 0.045 & 0.040 & 0.037 & 0.022 & & 0.044 & \\
\hline 1942 & 0.029 & 0.013 & & 0.041 & 0.039 & 0.023 & & 0.045 & & \\
\hline 1943 & 0.023 & & & & 0.032 & & & & & \\
\hline 1944 & 0.033 & 0.028 & 0.026 & 0.010 & 0.038 & 0.033 & & & & \\
\hline 1945 & 0.042 & 0.039 & 0.023 & & 0.046 & & & & & &
\end{tabular}


Table 9. Record periods for the Upper Appalachian Plateaus region for which the indicated average departure was within 5 percent of the standard deviation of the standardized departures of the minimum flows for 1930-2002 (six stations in West Virginia)._-Continued

[No value indicates the average departure was greater than 5 percent; dark shading indicates period outside the limit of this study; station numbers for the six stations are 03051000, 03053500, 03066000, 03069500, 03182500, and 03186500]

\begin{tabular}{|l|l|l|l|l|l|}
\hline 50 & 52 & 54 & 56 & 57 & 58 \\
\hline 51 & 53 & 55 & \multicolumn{2}{|l}{} \\
\cline { 1 - 3 } & &
\end{tabular}

Table layout (page no.)

\begin{tabular}{|c|c|c|c|c|c|c|c|c|c|}
\hline \multirow{2}{*}{ First year } & \multicolumn{9}{|c|}{ Years of record } \\
\hline & 65 & 66 & 67 & 68 & 69 & 70 & 71 & 72 & 73 \\
\hline 1930 & -0.023 & -0.027 & -0.039 & -0.011 & -0.014 & -0.016 & -0.028 & -0.005 & -0.008 \\
\hline 1931 & -0.016 & -0.029 & -0.001 & -0.004 & -0.006 & -0.018 & 0.005 & 0.002 & \\
\hline 1932 & -0.012 & 0.017 & 0.013 & 0.011 & -0.001 & 0.021 & 0.018 & & \\
\hline 1933 & 0.004 & 0.000 & -0.001 & -0.014 & 0.009 & 0.006 & & & \\
\hline 1934 & 0.014 & 0.012 & -0.001 & 0.022 & 0.019 & & & & \\
\hline 1935 & 0.002 & -0.012 & 0.013 & 0.009 & & & & & \\
\hline 1936 & -0.002 & 0.022 & 0.019 & & & & & & \\
\hline 1937 & 0.017 & 0.013 & & & & & & & \\
\hline 1938 & 0.024 & & & & & & & & \\
\hline
\end{tabular}


Table 10. Record periods for the Eastern Panhandle region for which the indicated average departure was within 5 percent of the standard deviation of the standardized departures of the minimum flows for 1930-2002 (five stations in West Virginia and Virginia).

[No value indicates the average departure was greater than 5 percent; station numbers for the five stations are 01606500, 01611500, 01632000, 01636500, and 01644000]

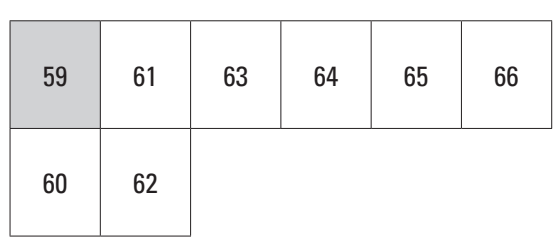

Table layout (page no.)

\begin{tabular}{|c|c|c|c|c|c|c|c|c|c|c|c|}
\hline \multirow{2}{*}{ First year } & \multicolumn{11}{|c|}{ Years of record } \\
\hline & 10 & 11 & 12 & 13 & 14 & 15 & 16 & 17 & 18 & 19 & 20 \\
\hline \multicolumn{12}{|l|}{1930} \\
\hline \multicolumn{12}{|l|}{1931} \\
\hline \multicolumn{12}{|l|}{1932} \\
\hline \multicolumn{12}{|l|}{1933} \\
\hline \multicolumn{12}{|l|}{$\begin{array}{l}1934 \\
\end{array}$} \\
\hline \multicolumn{12}{|l|}{1935} \\
\hline \multicolumn{12}{|l|}{1936} \\
\hline \multicolumn{12}{|l|}{1937} \\
\hline 1938 & & & & & -0.034 & -0.041 & -0.033 & & & & \\
\hline 1939 & & & & -0.033 & -0.041 & -0.032 & & & & & \\
\hline 1940 & & -0.024 & -0.009 & -0.019 & -0.012 & & & & & & \\
\hline 1941 & 0.005 & 0.018 & 0.005 & 0.011 & -0.030 & & & & & & \\
\hline \multicolumn{12}{|l|}{1942} \\
\hline 1943 & 0.000 & 0.007 & -0.040 & & & & & & & & \\
\hline 1944 & -0.007 & & & & & & & & & & \\
\hline 1945 & 0.001 & & & & & & & & & & \\
\hline 1946 & -0.004 & -0.029 & -0.011 & & & & & & & & \\
\hline \multicolumn{12}{|l|}{1947} \\
\hline 1948 & 0.007 & & & & & & & & & & \\
\hline 1949 & -0.035 & & & & & & & & & & \\
\hline \multicolumn{12}{|l|}{1950} \\
\hline \multicolumn{12}{|l|}{1951} \\
\hline \multicolumn{12}{|l|}{1952} \\
\hline \multicolumn{12}{|l|}{1953} \\
\hline \multicolumn{12}{|l|}{1954} \\
\hline \multicolumn{12}{|l|}{1955} \\
\hline \multicolumn{12}{|l|}{1956} \\
\hline \multicolumn{12}{|l|}{1957} \\
\hline \multicolumn{12}{|l|}{1958} \\
\hline \multicolumn{12}{|l|}{1959} \\
\hline \multicolumn{12}{|l|}{1960} \\
\hline 1961 & & & & & & & & & & & 0.037 \\
\hline 1962 & & & & & & & & & & & 0.042 \\
\hline 1963 & & & & & & & & & & & 0.023 \\
\hline 1964 & & & & -0.032 & & & -0.029 & & & & \\
\hline 1965 & & & 0.040 & 0.018 & -0.014 & 0.029 & & & & & \\
\hline
\end{tabular}


Table 10. Record periods for the Eastern Panhandle region for which the indicated average departure was within 5 percent of the standard deviation of the standardized departures of the minimum flows for 1930-2002 (five stations in West Virginia and Virginia)._-Continued

[No value indicates the average departure was greater than 5 percent; station numbers for the five stations are $01606500,01611500,01632000,01636500$, and 01644000]

\begin{tabular}{|l|l|l|l|l|l|}
\hline 59 & 61 & 63 & 64 & 65 & 66 \\
\hline 60 & 62 & \multicolumn{4}{|c|}{ Table layout (page no.) }
\end{tabular}

\begin{tabular}{|c|c|c|c|c|c|c|c|c|c|c|c|}
\hline \multirow{2}{*}{ First year } & \multicolumn{11}{|c|}{ Years of record } \\
\hline & 10 & 11 & 12 & 13 & 14 & 15 & 16 & 17 & 18 & 19 & 20 \\
\hline 1966 & -0.013 & & & & & & & & & & \\
\hline 1967 & & & & & & & & & & & \\
\hline 1968 & & & & & & & & & & & \\
\hline 1969 & & & & & & & & & & & \\
\hline 1970 & & & & & & & & & & & \\
\hline 1971 & & & & & & & & & & & \\
\hline 1972 & & & & & & & & & & & \\
\hline 1973 & & & & & & & & & & & \\
\hline 1974 & & & & & & & & & & & \\
\hline 1975 & & & & & & & & & & & \\
\hline 1976 & & & & & & & & & & & \\
\hline 1977 & & & & 0.017 & & & & & & & \\
\hline 1978 & & & 0.039 & & & & & & & & \\
\hline 1979 & & & & & & & & & & & \\
\hline 1980 & 0.027 & & & & & & & & & & \\
\hline
\end{tabular}


Table 10. Record periods for the Eastern Panhandle region for which the indicated average departure was within 5 percent of the standard deviation of the standardized departures of the minimum flows for 1930-2002 (five stations in West Virginia and Virginia)._-Continued

[No value indicates the average departure was greater than 5 percent; station numbers for the five stations are $01606500,01611500,01632000,01636500$, and 01644000]

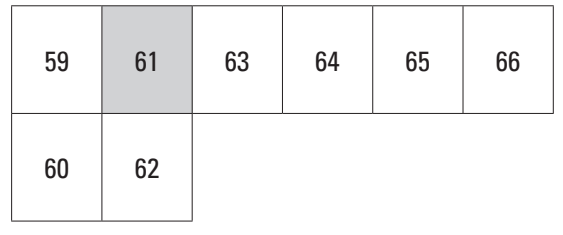

Table layout (page no.)

\begin{tabular}{|c|c|c|c|c|c|c|c|c|c|c|c|}
\hline \multirow{2}{*}{ First year } & \multicolumn{11}{|c|}{ Years of record } \\
\hline & 21 & 22 & 23 & 24 & 25 & 26 & 27 & 28 & 29 & 30 & 31 \\
\hline \multicolumn{12}{|l|}{1930} \\
\hline \multicolumn{12}{|l|}{1931} \\
\hline \multicolumn{12}{|l|}{1932} \\
\hline \multicolumn{12}{|l|}{1933} \\
\hline \multicolumn{12}{|l|}{1934} \\
\hline \multicolumn{12}{|l|}{1935} \\
\hline \multicolumn{12}{|l|}{1936} \\
\hline \multicolumn{12}{|l|}{1937} \\
\hline \multicolumn{12}{|l|}{1938} \\
\hline \multicolumn{12}{|l|}{1939} \\
\hline \multicolumn{12}{|l|}{1940} \\
\hline \multicolumn{12}{|l|}{1941} \\
\hline \multicolumn{12}{|l|}{1942} \\
\hline \multicolumn{12}{|l|}{1943} \\
\hline \multicolumn{12}{|l|}{1944} \\
\hline \multicolumn{12}{|l|}{1945} \\
\hline \multicolumn{12}{|l|}{1946} \\
\hline \multicolumn{12}{|l|}{1947} \\
\hline \multicolumn{12}{|l|}{1948} \\
\hline \multicolumn{12}{|l|}{1949} \\
\hline 1950 & & & & & & & & & & & -0.043 \\
\hline \multicolumn{12}{|l|}{1951} \\
\hline \multicolumn{12}{|l|}{1952} \\
\hline 1953 & & & & & & & & & & & \\
\hline 1954 & & & & & & & & & & & \\
\hline 1955 & & & & & & & & & & & \\
\hline 1956 & & & & & & & & & & & \\
\hline 1957 & & & & & & & & & & & \\
\hline 1958 & & & & & & & & & & & \\
\hline 1959 & & -0.016 & -0.019 & -0.040 & -0.033 & & -0.042 & & & & \\
\hline 1960 & -0.005 & -0.009 & -0.031 & -0.024 & -0.043 & -0.034 & -0.042 & & & & \\
\hline 1961 & 0.032 & 0.007 & 0.013 & -0.009 & -0.001 & -0.010 & -0.019 & -0.023 & -0.035 & & \\
\hline 1962 & 0.015 & 0.021 & -0.002 & 0.006 & -0.004 & -0.013 & -0.018 & -0.030 & & & \\
\hline 1963 & 0.028 & 0.004 & 0.011 & 0.001 & -0.009 & -0.013 & -0.027 & & & & \\
\hline 1964 & 0.029 & 0.036 & 0.025 & 0.014 & 0.008 & -0.007 & & & & & \\
\hline 1965 & & & & & 0.028 & & & & & & \\
\hline
\end{tabular}


Table 10. Record periods for the Eastern Panhandle region for which the indicated average departure was within 5 percent of the standard deviation of the standardized departures of the minimum flows for 1930-2002 (five stations in West Virginia and Virginia)._-Continued

[No value indicates the average departure was greater than 5 percent; dark shading indicates period outside the limit of this study; station numbers for the five stations are 01606500, 01611500, 01632000, 01636500, and 01644000]

\begin{tabular}{|c|c|c|c|c|c|}
\hline 59 & 61 & 63 & 64 & 65 & 66 \\
\hline 60 & 62 & \multicolumn{2}{|l}{} \\
\cline { 1 - 2 }
\end{tabular}

Table layout (page no.)

\begin{tabular}{|c|c|c|c|c|c|c|c|c|c|c|c|}
\hline \multirow{2}{*}{ First year } & \multicolumn{11}{|c|}{ Years of record } \\
\hline & 21 & 22 & 23 & 24 & 25 & 26 & 27 & 28 & 29 & 30 & 31 \\
\hline \multicolumn{12}{|l|}{1966} \\
\hline \multicolumn{12}{|l|}{1967} \\
\hline \multicolumn{12}{|l|}{1968} \\
\hline \multicolumn{12}{|l|}{1969} \\
\hline \multicolumn{12}{|l|}{1970} \\
\hline \multicolumn{12}{|l|}{1971} \\
\hline \multicolumn{12}{|l|}{1972} \\
\hline \multicolumn{12}{|l|}{1973} \\
\hline \multicolumn{12}{|l|}{1974} \\
\hline \multicolumn{12}{|l|}{1975} \\
\hline \multicolumn{12}{|l|}{1976} \\
\hline \multicolumn{12}{|l|}{1977} \\
\hline \multicolumn{12}{|l|}{1978} \\
\hline \multicolumn{12}{|l|}{1979} \\
\hline 1980 & & & & & & & & & & & \\
\hline
\end{tabular}


Table 10. Record periods for the Eastern Panhandle region for which the indicated average departure was within 5 percent of the standard deviation of the standardized departures of the minimum flows for 1930-2002 (five stations in West Virginia and Virginia).-Continued

[No value indicates the average departure was greater than 5 percent; dark shading indicates period outside the limit of this study; station numbers for the five stations are 01606500, 01611500, 01632000,01636500, and 01644000]

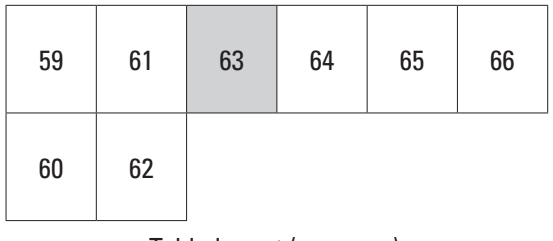

Table layout (page no.)

\begin{tabular}{|c|c|c|c|c|c|c|c|c|c|c|c|}
\hline \multirow{2}{*}{ First year } & \multicolumn{11}{|c|}{ Years of record } \\
\hline & 32 & 33 & 34 & 35 & 36 & 37 & 38 & 39 & 40 & 41 & 42 \\
\hline \multicolumn{12}{|l|}{1930} \\
\hline \multicolumn{12}{|l|}{1931} \\
\hline \multicolumn{12}{|l|}{1932} \\
\hline \multicolumn{12}{|l|}{1933} \\
\hline \multicolumn{12}{|l|}{1934} \\
\hline \multicolumn{12}{|l|}{1935} \\
\hline \multicolumn{12}{|l|}{1936} \\
\hline \multicolumn{12}{|l|}{1937} \\
\hline \multicolumn{12}{|l|}{1938} \\
\hline \multicolumn{12}{|l|}{1939} \\
\hline \multicolumn{12}{|l|}{1940} \\
\hline \multicolumn{12}{|l|}{1941} \\
\hline \multicolumn{12}{|l|}{1942} \\
\hline \multicolumn{12}{|l|}{1943} \\
\hline \multicolumn{12}{|l|}{1944} \\
\hline \multicolumn{12}{|l|}{1945} \\
\hline 1946 & & & & -0.043 & -0.044 & & & & & & \\
\hline \multicolumn{12}{|l|}{1947} \\
\hline 1948 & & -0.039 & -0.040 & & & & & & & & \\
\hline 1949 & -0.022 & -0.024 & -0.038 & -0.033 & & -0.040 & & & & & 0.022 \\
\hline 1950 & -0.044 & & & & & & & & & 0.007 & 0.014 \\
\hline 1951 & & & & & & & & & -0.024 & -0.016 & -0.020 \\
\hline 1952 & & & & & & & & -0.028 & -0.020 & -0.024 & -0.013 \\
\hline 1953 & & & & & & & -0.025 & -0.017 & -0.021 & -0.009 & -0.013 \\
\hline 1954 & & & & & & -0.028 & -0.020 & -0.023 & -0.012 & -0.016 & 0.004 \\
\hline 1955 & & & & & -0.013 & -0.005 & -0.009 & 0.002 & -0.002 & 0.018 & 0.011 \\
\hline 1956 & & & & 0.005 & 0.013 & 0.008 & 0.020 & 0.015 & 0.035 & 0.027 & \\
\hline 1957 & & & 0.013 & 0.021 & 0.016 & 0.028 & 0.022 & 0.042 & 0.034 & & \\
\hline 1958 & & 0.008 & 0.016 & 0.011 & 0.023 & 0.018 & 0.039 & 0.031 & & & \\
\hline 1959 & 0.039 & & 0.041 & & & & & & & & \\
\hline \multicolumn{12}{|l|}{1960} \\
\hline \multicolumn{12}{|l|}{1961} \\
\hline \multicolumn{12}{|l|}{1962} \\
\hline \multicolumn{12}{|l|}{1963} \\
\hline \multicolumn{12}{|l|}{1964} \\
\hline 1965 & & & & & & & & & & & \\
\hline
\end{tabular}


Table 10. Record periods for the Eastern Panhandle region for which the indicated average departure was within 5 percent of the standard deviation of the standardized departures of the minimum flows for 1930-2002 (five stations in West Virginia and Virginia).-Continued

[No value indicates the average departure was greater than 5 percent; dark shading indicates period outside the limit of this study; station numbers for the five stations are 01606500, 01611500, 01632000, 01636500, and 01644000]

\begin{tabular}{|l|l|l|l|l|l|}
\hline 59 & 61 & 63 & 64 & 65 & 66 \\
\hline 60 & 62 & \multicolumn{2}{|l}{} \\
\hline
\end{tabular}

\begin{tabular}{|c|c|c|c|c|c|c|c|c|c|c|c|}
\hline \multirow{2}{*}{ First year } & \multicolumn{11}{|c|}{ Years of record } \\
\hline & 32 & 33 & 34 & 35 & 36 & 37 & 38 & 39 & 40 & 41 & 42 \\
\hline \multicolumn{12}{|l|}{1966} \\
\hline \multicolumn{12}{|l|}{1967} \\
\hline \multicolumn{12}{|l|}{1968} \\
\hline \multicolumn{12}{|l|}{1969} \\
\hline \multicolumn{12}{|l|}{1970} \\
\hline 1971 & & & & & & & & & & & \\
\hline
\end{tabular}


Table 10. Record periods for the Eastern Panhandle region for which the indicated average departure was within 5 percent of the standard deviation of the standardized departures of the minimum flows for 1930-2002 (five stations in West Virginia and Virginia).-Continued

[No value indicates the average departure was greater than 5 percent; dark shading indicates period outside the limit of this study; station numbers for the five stations are 01606500, 01611500, 01632000, 01636500, and 01644000]

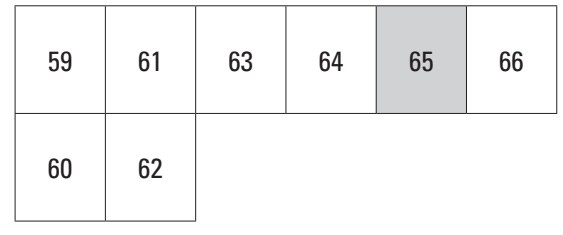

Table layout (page no.)

\begin{tabular}{|c|c|c|c|c|c|c|c|c|c|c|c|}
\hline \multirow{2}{*}{ First year } & \multicolumn{11}{|c|}{ Years of record } \\
\hline & 43 & 44 & 45 & 46 & 47 & 48 & 49 & 50 & 51 & 52 & 53 \\
\hline \multicolumn{12}{|l|}{1930} \\
\hline \multicolumn{12}{|l|}{1931} \\
\hline \multicolumn{12}{|l|}{1932} \\
\hline \multicolumn{12}{|l|}{1933} \\
\hline \multicolumn{12}{|l|}{1934} \\
\hline \multicolumn{12}{|l|}{1935} \\
\hline \multicolumn{12}{|l|}{1936} \\
\hline \multicolumn{12}{|l|}{1937} \\
\hline 1938 & & & & & & & & & & & -0.030 \\
\hline 1939 & & & & & & & & & & -0.029 & -0.023 \\
\hline 1940 & & & & & & & & & -0.024 & -0.018 & -0.020 \\
\hline 1941 & & & & & & & & -0.018 & -0.012 & -0.015 & -0.006 \\
\hline 1942 & & & & & & & -0.033 & -0.026 & -0.029 & -0.020 & -0.023 \\
\hline 1943 & & & & & & -0.020 & -0.014 & -0.017 & -0.008 & -0.011 & 0.005 \\
\hline 1944 & & & & & -0.024 & -0.017 & -0.020 & -0.011 & -0.014 & 0.002 & -0.004 \\
\hline 1945 & & & & -0.010 & -0.004 & -0.007 & 0.002 & -0.002 & 0.014 & 0.009 & \\
\hline 1946 & & & 0.003 & 0.009 & 0.005 & 0.015 & 0.011 & 0.027 & 0.021 & & \\
\hline 1947 & & -0.007 & 0.000 & -0.004 & 0.006 & 0.002 & 0.018 & 0.012 & & & \\
\hline 1948 & 0.008 & 0.014 & 0.010 & 0.020 & 0.016 & 0.032 & 0.026 & & & & \\
\hline 1949 & 0.028 & 0.024 & 0.033 & 0.028 & & 0.038 & & & & & \\
\hline 1950 & 0.010 & 0.020 & 0.015 & 0.033 & 0.026 & & & & & & \\
\hline 1951 & -0.009 & -0.013 & 0.006 & 0.000 & & & & & & & \\
\hline 1952 & -0.016 & 0.002 & -0.004 & & & & & & & & \\
\hline 1953 & 0.006 & -0.001 & & & & & & & & & \\
\hline 1954 & -0.003 & & & & & & & & & & \\
\hline \multicolumn{12}{|l|}{1955} \\
\hline \multicolumn{12}{|l|}{1956} \\
\hline \multicolumn{12}{|l|}{1957} \\
\hline \multicolumn{12}{|l|}{1958} \\
\hline \multicolumn{12}{|l|}{1959} \\
\hline 1960 & & & & & & & & & & & \\
\hline
\end{tabular}


Table 10. Record periods for the Eastern Panhandle region for which the indicated average departure was within 5 percent of the standard deviation of the standardized departures of the minimum flows for 1930-2002 (five stations in West Virginia and Virginia)._-Continued

[No value indicates the average departure was greater than 5 percent; dark shading indicates period outside the limit of this study; station numbers for the five stations are 01606500, 01611500, 01632000, 01636500, and 01644000]

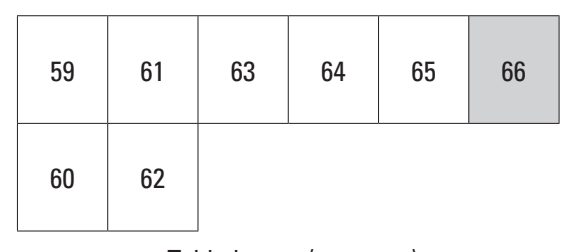

Table layout (page no.)

\begin{tabular}{|c|c|c|c|c|c|c|c|c|c|c|c|}
\hline \multirow{2}{*}{ First year } & \multicolumn{11}{|c|}{ Years of record } \\
\hline & 54 & 55 & 56 & 57 & 58 & 59 & 60 & 61 & 62 & 63 & 64 \\
\hline \multicolumn{12}{|l|}{1930} \\
\hline \multicolumn{12}{|l|}{1931} \\
\hline \multicolumn{12}{|l|}{1932} \\
\hline 1933 & & & & & & & & & & -0.042 & \\
\hline 1934 & & & & & -0.043 & & -0.037 & -0.039 & -0.025 & -0.029 & 0.032 \\
\hline 1935 & & & & -0.042 & -0.044 & -0.036 & -0.038 & -0.024 & -0.028 & 0.034 & 0.043 \\
\hline 1936 & & -0.036 & -0.030 & -0.032 & -0.024 & -0.027 & -0.013 & -0.017 & & & \\
\hline 1937 & -0.038 & -0.032 & -0.035 & -0.026 & -0.029 & -0.015 & -0.019 & & & & 0.042 \\
\hline 1938 & -0.024 & -0.026 & -0.018 & -0.021 & -0.006 & -0.011 & & & & & \\
\hline 1939 & -0.026 & -0.017 & -0.020 & -0.006 & -0.010 & & & & & & \\
\hline 1940 & -0.012 & -0.015 & 0.000 & -0.005 & & & & & & & \\
\hline 1941 & -0.009 & 0.006 & 0.001 & & & & & & & & \\
\hline 1942 & -0.007 & -0.012 & & & & & & & & & \\
\hline 1943 & -0.001 & & & & & & & & & & \\
\hline \multicolumn{12}{|l|}{1944} \\
\hline \multicolumn{12}{|l|}{1945} \\
\hline \multicolumn{12}{|l|}{1946} \\
\hline \multicolumn{12}{|l|}{1947} \\
\hline \multicolumn{12}{|l|}{1948} \\
\hline 1949 & & & & & & & & & & & \\
\hline
\end{tabular}

Table 10. Record periods for the Eastern Panhandle region for which the indicated average departure was within 5 percent of the standard deviation of the standardized departures of the minimum flows for 1930-2002 (five stations in West Virginia and Virginia).-Continued

[No value indicates the average departure was greater than 5 percent; dark shading indicates period outside the limit of this study; station numbers for the five stations are 01606500, 01611500, 01632000, 01636500, and 01644000]

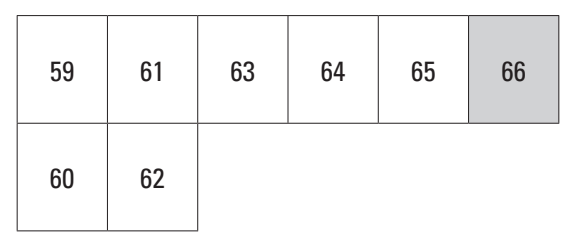

Table layout (page no.)

\begin{tabular}{|c|c|c|c|c|c|c|c|c|c|}
\hline \multirow{2}{*}{ First year } & \multicolumn{9}{|c|}{ Years of record } \\
\cline { 2 - 10 } & $\mathbf{6 5}$ & $\mathbf{6 6}$ & $\mathbf{6 7}$ & $\mathbf{6 8}$ & $\mathbf{6 9}$ & $\mathbf{7 0}$ & $\mathbf{7 1}$ & $\mathbf{7 2}$ & $\mathbf{7 3}$ \\
\hline 1930 & & & & -0.024 & -0.014 & -0.011 & -0.023 & -0.014 & -0.016 \\
\hline 1931 & & & -0.014 & -0.005 & -0.002 & -0.015 & -0.006 & -0.007 & \\
\hline 1932 & & 0.003 & 0.012 & 0.016 & 0.002 & 0.011 & 0.009 & & \\
\hline 1933 & 0.016 & 0.025 & 0.028 & 0.014 & 0.023 & 0.021 & & & \\
\hline 1934 & 0.041 & 0.044 & 0.030 & 0.039 & 0.036 & & & \\
\hline 1935 & & 0.032 & 0.041 & 0.038 & & & & \\
& & & & & & & \\
\end{tabular}


Table 11. Selected annual and seasonal statistics for 77 stations in West Virginia representative of 1930-2002.

[Winter, January 1-March 31; spring, April 1-June 30; summer, July 1-September 30; fall, October 1-December 31; streamflow statistics are in cubic feet per second, except for variability index, which is dimensionless; the record period is in climatic years, April 1 through March 31of the indicated ending year; EPA, U.S. Environmental Protection Agency]

\begin{tabular}{|c|c|c|c|c|c|}
\hline \multirow{2}{*}{ Streamflow statistic } & \multirow{2}{*}{ Annual } & \multicolumn{4}{|c|}{ Season } \\
\hline & & Winter & Spring & Summer & Fall \\
\hline \multicolumn{6}{|c|}{01595300 Abram Creek at Oakmont, WV, $1959-1982$} \\
\hline 1-day 2-year hydrologically based flow & 2.20 & 26.0 & 9.92 & 2.30 & 4.82 \\
\hline 1-day 5-year hydrologically based flow & 0.77 & 12.6 & 4.86 & 0.78 & 2.06 \\
\hline 1-day 10-year hydrologically based flow & 0.41 & 7.61 & 3.24 & 0.41 & 1.35 \\
\hline 3-day 2-year hydrologically based flow & 2.37 & 25.0 & 11.1 & 2.52 & 5.22 \\
\hline 3-day 5-year hydrologically based flow & 0.83 & 14.7 & 5.48 & 0.85 & 2.29 \\
\hline 3-day 10 -year hydrologically based flow & 0.44 & 11.0 & 3.66 & 0.45 & 1.51 \\
\hline 7-day 2-year hydrologically based flow & 2.72 & 28.2 & 13.6 & 2.96 & 6.04 \\
\hline 7-day 5-year hydrologically based flow & 0.95 & 16.1 & 6.73 & 0.98 & 2.64 \\
\hline 7-day 10-year hydrologically based flow & 0.50 & 11.8 & 4.51 & 0.51 & 1.75 \\
\hline 14-day 2-year hydrologically based flow & 3.25 & 32.3 & 18.1 & 3.70 & 7.14 \\
\hline 14-day 5-year hydrologically based flow & 1.18 & 18.2 & 9.32 & 1.26 & 3.14 \\
\hline 14-day 10 -year hydrologically based flow & 0.64 & 13.3 & 6.48 & 0.66 & 2.08 \\
\hline 30-day 2-year hydrologically based flow & 4.56 & 46.1 & 29.2 & 5.73 & 12.2 \\
\hline 30-day 5-year hydrologically based flow & 1.97 & 27.0 & 14.9 & 2.29 & 5.12 \\
\hline 30-day 10 -year hydrologically based flow & 1.23 & 20.3 & 10.3 & 1.34 & 3.22 \\
\hline 1-day 3-year biologically based flow & 0.17 & 4.99 & 7.50 & 0.29 & 0.19 \\
\hline 4-day 3-year biologically based flow & 0.37 & 5.89 & 10.4 & 0.44 & 0.94 \\
\hline EPA harmonic-mean flow & 9.11 & 37.1 & 52.4 & 4.55 & 5.80 \\
\hline 5-percent-duration flow & 251 & 374 & 260 & 74.7 & 166 \\
\hline 10-percent-duration flow & 168 & 264 & 195 & 45.6 & 111 \\
\hline 15-percent-duration flow & 127 & 210 & 158 & 32.7 & 83.9 \\
\hline 20-percent-duration flow & 104 & 173 & 131 & 25.4 & 68.2 \\
\hline 25-percent-duration flow & 85.8 & 146 & 112 & 21.0 & 57.3 \\
\hline 30-percent-duration flow & 71.3 & 126 & 95.4 & 17.8 & 48.7 \\
\hline 35-percent-duration flow & 60.7 & 112 & 84.5 & 15.0 & 42.2 \\
\hline 40-percent-duration flow & 51.4 & 99.9 & 74.7 & 13.1 & 36.6 \\
\hline 45-percent-duration flow & 43.6 & 89.0 & 65.6 & 11.3 & 31.6 \\
\hline 50-percent-duration flow & 36.9 & 78.8 & 58.0 & 9.5 & 26.5 \\
\hline 55-percent-duration flow & 30.7 & 71.0 & 51.4 & 8.1 & 21.9 \\
\hline 60-percent-duration flow & 25.2 & 63.3 & 45.4 & 6.8 & 17.5 \\
\hline 65-percent-duration flow & 20.1 & 55.7 & 40.4 & 5.8 & 13.4 \\
\hline 70-percent-duration flow & 15.7 & 49.3 & 34.8 & 4.8 & 10.2 \\
\hline 75-percent-duration flow & 12.1 & 43.5 & 29.3 & 3.9 & 7.8 \\
\hline 80-percent-duration flow & 8.7 & 36.5 & 24.0 & 3.1 & 6.3 \\
\hline 85-percent-duration flow & 6.3 & 31.0 & 18.8 & 2.2 & 5.1 \\
\hline 90-percent-duration flow & 4.3 & 25.6 & 14.0 & 1.6 & 3.9 \\
\hline 95-percent-duration flow & 2.3 & 18.3 & 9.3 & 1.0 & 2.5 \\
\hline 99-percent-duration flow & 0.9 & 11.1 & 3.9 & 0.3 & 1.5 \\
\hline Variability index & 0.62 & 0.39 & 0.44 & 0.56 & 0.57 \\
\hline
\end{tabular}


Table 11. Selected annual and seasonal statistics for 77 stations in West Virginia representative of 1930-2002.—Continued

[Winter, January 1-March 31; spring, April 1-June 30; summer, July 1-September 30; fall, October 1-December 31; streamflow statistics are in cubic feet per second, except for variability index, which is dimensionless; the record period is in climatic years, April 1 through March 31 of the indicated ending year; EPA, U.S. Environmental Protection Agency]

\begin{tabular}{|c|c|c|c|c|c|}
\hline \multirow{2}{*}{ Streamflow statistic } & \multirow[b]{2}{*}{ Annual } & \multicolumn{4}{|c|}{$\begin{array}{c}\text { Season } \\
\end{array}$} \\
\hline & & Winter & Spring & Summer & Fall \\
\hline \multicolumn{6}{|c|}{01599500 New Creek near Keyser, WV, 1949 -1958 } \\
\hline 1-day 2-year hydrologically based flow & 2.17 & 14.5 & 8.72 & 2.23 & 2.78 \\
\hline 1-day 5-year hydrologically based flow & 1.49 & 6.67 & 6.71 & 1.47 & 1.73 \\
\hline 1-day 10-year hydrologically based flow & 1.24 & 4.18 & 5.82 & 1.21 & 1.37 \\
\hline 3-day 2-year hydrologically based flow & 2.24 & 15.1 & 9.55 & 2.33 & 2.91 \\
\hline 3-day 5-year hydrologically based flow & 1.50 & 6.91 & 7.18 & 1.51 & 1.80 \\
\hline 3-day 10-year hydrologically based flow & 1.23 & 4.33 & 6.14 & 1.23 & 1.41 \\
\hline 7-day 2-year hydrologically based flow & 2.43 & 16.8 & 11.5 & 2.53 & 3.58 \\
\hline 7-day 5-year hydrologically based flow & 1.61 & 7.45 & 8.67 & 1.62 & 2.25 \\
\hline 7-day 10-year hydrologically based flow & 1.31 & 4.72 & 7.31 & 1.31 & 1.75 \\
\hline 14-day 2-year hydrologically based flow & 2.68 & 24.8 & 13.5 & 2.92 & 4.04 \\
\hline 14-day 5-year hydrologically based flow & 1.74 & 10.1 & 10.2 & 1.82 & 2.43 \\
\hline 14-day 10 -year hydrologically based flow & 1.40 & 5.82 & 8.71 & 1.44 & 1.90 \\
\hline 30-day 2-year hydrologically based flow & 3.15 & 34.9 & 21.5 & 3.46 & 6.10 \\
\hline 30-day 5-year hydrologically based flow & 2.05 & 15.9 & 16.6 & 2.16 & 3.35 \\
\hline 30-day 10 -year hydrologically based flow & 1.67 & 9.98 & 14.7 & 1.73 & 2.40 \\
\hline 1-day 3-year biologically based flow & 1.09 & 2.97 & 6.70 & 1.05 & 1.39 \\
\hline 4-day 3-year biologically based flow & 1.30 & 3.40 & 7.80 & 1.35 & 1.66 \\
\hline EPA harmonic-mean flow & 8.40 & 22.1 & 34.3 & 5.03 & 4.95 \\
\hline 5-percent-duration flow & 168 & 245 & 192 & 37.9 & 90.2 \\
\hline 10-percent-duration flow & 115 & 170 & 122 & 18.1 & 51.5 \\
\hline 15-percent-duration flow & 87.0 & 146 & 98.0 & 13.7 & 37.7 \\
\hline 20-percent-duration flow & 69.8 & 127 & 82.2 & 10.9 & 28.0 \\
\hline 25-percent-duration flow & 57.6 & 111 & 69.9 & 9.2 & 21.8 \\
\hline 30-percent-duration flow & 47.4 & 98.6 & 60.6 & 8.2 & 16.4 \\
\hline 35-percent-duration flow & 37.8 & 88.2 & 53.8 & 7.3 & 13.4 \\
\hline 40-percent-duration flow & 30.3 & 80.4 & 47.8 & 6.7 & 11.4 \\
\hline 45-percent-duration flow & 24.4 & 72.6 & 42.3 & 6.1 & 9.5 \\
\hline 50-percent-duration flow & 19.3 & 64.9 & 37.5 & 5.5 & 8.2 \\
\hline 55-percent-duration flow & 15.0 & 58.4 & 33.7 & 5.0 & 7.3 \\
\hline 60-percent-duration flow & 11.8 & 52.4 & 30.0 & 4.4 & 6.4 \\
\hline 65-percent-duration flow & 9.2 & 47.0 & 26.3 & 3.9 & 5.5 \\
\hline 70-percent-duration flow & 7.3 & 39.9 & 23.1 & 3.4 & 4.9 \\
\hline 75-percent-duration flow & 6.0 & 32.9 & 20.5 & 3.0 & 4.3 \\
\hline 80 -percent-duration flow & 4.9 & 27.6 & 17.8 & 2.7 & 3.6 \\
\hline 85-percent-duration flow & 3.9 & 21.6 & 15.4 & 2.4 & 2.9 \\
\hline 90-percent-duration flow & 2.9 & 12.5 & 13.7 & 2.2 & 2.6 \\
\hline 95-percent-duration flow & 2.3 & 5.1 & 10.8 & 1.8 & 2.2 \\
\hline 99-percent-duration flow & 1.6 & 3.5 & 6.5 & 1.4 & 1.9 \\
\hline Variability index & 0.61 & 0.48 & 0.38 & 0.39 & 0.51 \\
\hline
\end{tabular}


Table 11. Selected annual and seasonal statistics for 77 stations in West Virginia representative of 1930-2002. - Continued

[Winter, January 1-March 31; spring, April 1-June 30; summer, July 1-September 30; fall, October 1-December 31; streamflow statistics are in cubic feet per second, except for variability index, which is dimensionless; the record period is in climatic years, April 1 through March 31of the indicated ending year; EPA, U.S. Environmental Protection Agency]

\begin{tabular}{|c|c|c|c|c|c|}
\hline \multirow{2}{*}{ Streamflow statistic } & \multirow{2}{*}{ Annual } & \multicolumn{4}{|c|}{ Season } \\
\hline & & Winter & Spring & Summer & Fall \\
\hline \multicolumn{6}{|c|}{01604500 Patterson Creek near Headsville, WV, 1940-1996 } \\
\hline 1-day 2-year hydrologically based flow & 6.03 & 39.0 & 22.3 & 6.29 & 9.65 \\
\hline 1-day 5-year hydrologically based flow & 3.31 & 20.8 & 12.7 & 3.43 & 5.45 \\
\hline 1-day 10-year hydrologically based flow & 2.40 & 14.7 & 8.97 & 2.48 & 4.12 \\
\hline 3-day 2-year hydrologically based flow & 6.29 & 41.8 & 24.1 & 6.56 & 10.3 \\
\hline 3-day 5-year hydrologically based flow & 3.53 & 22.1 & 13.8 & 3.64 & 5.89 \\
\hline 3-day 10 -year hydrologically based flow & 2.60 & 15.5 & 9.80 & 2.67 & 4.51 \\
\hline 7-day 2-year hydrologically based flow & 6.76 & 48.1 & 28.2 & 7.12 & 11.5 \\
\hline 7-day 5-year hydrologically based flow & 3.88 & 24.6 & 16.4 & 4.04 & 6.69 \\
\hline 7-day 10-year hydrologically based flow & 2.91 & 16.9 & 11.6 & 3.01 & 5.15 \\
\hline 14-day 2-year hydrologically based flow & 7.56 & 59.6 & 34.0 & 8.17 & 13.0 \\
\hline 14-day 5-year hydrologically based flow & 4.39 & 29.1 & 19.6 & 4.64 & 7.52 \\
\hline 14-day 10 -year hydrologically based flow & 3.35 & 19.5 & 14.0 & 3.51 & 5.82 \\
\hline 30-day 2-year hydrologically based flow & 9.76 & 95.1 & 53.8 & 11.1 & 20.1 \\
\hline 30-day 5-year hydrologically based flow & 5.88 & 46.9 & 31.2 & 6.47 & 10.1 \\
\hline 30-day 10-year hydrologically based flow & 4.62 & 31.8 & 23.0 & 5.02 & 7.10 \\
\hline 1-day 3-year biologically based flow & 1.96 & 9.98 & 15.9 & 2.13 & 3.10 \\
\hline 4-day 3-year biologically based flow & 2.40 & 11.4 & 19.1 & 2.87 & 4.51 \\
\hline EPA harmonic-mean flow & 25.4 & 72.0 & 90.0 & 14.6 & 15.8 \\
\hline 5-percent-duration flow & 694 & 1,100 & 789 & 191 & 434 \\
\hline 10-percent-duration flow & 436 & 737 & 520 & 91.1 & 248 \\
\hline 15-percent-duration flow & 308 & 566 & 380 & 58.3 & 166 \\
\hline 20-percent-duration flow & 226 & 456 & 294 & 44.1 & 119 \\
\hline 25-percent-duration flow & 175 & 379 & 234 & 36.6 & 93.3 \\
\hline 30-percent-duration flow & 139 & 314 & 189 & 30.7 & 74.3 \\
\hline 35-percent-duration flow & 111 & 261 & 156 & 26.1 & 59.8 \\
\hline 40-percent-duration flow & 90.5 & 221 & 132 & 22.9 & 47.7 \\
\hline 45-percent-duration flow & 73.8 & 191 & 114 & 20.1 & 38.5 \\
\hline 50-percent-duration flow & 60.0 & 167 & 97.6 & 17.8 & 31.6 \\
\hline 55-percent-duration flow & 48.7 & 147 & 85.9 & 15.7 & 26.3 \\
\hline 60-percent-duration flow & 38.8 & 129 & 75.0 & 14.0 & 22.7 \\
\hline 65-percent-duration flow & 31.5 & 113 & 65.8 & 12.3 & 19.5 \\
\hline 70-percent-duration flow & 25.4 & 97.0 & 57.7 & 10.7 & 17.1 \\
\hline 75-percent-duration flow & 20.4 & 80.7 & 50.3 & 9.2 & 15.1 \\
\hline 80-percent-duration flow & 16.3 & 65.9 & 42.3 & 8.1 & 13.4 \\
\hline 85-percent-duration flow & 13.1 & 52.8 & 34.9 & 7.0 & 11.6 \\
\hline 90-percent-duration flow & 9.9 & 40.8 & 28.2 & 5.9 & 9.5 \\
\hline 95-percent-duration flow & 7.1 & 26.7 & 21.1 & 4.6 & 7.3 \\
\hline 99-percent-duration flow & 3.5 & 12.5 & 10.2 & 2.6 & 3.3 \\
\hline Variability index & 0.63 & 0.49 & 0.48 & 0.48 & 0.55 \\
\hline
\end{tabular}


Table 11. Selected annual and seasonal statistics for 77 stations in West Virginia representative of 1930-2002. - Continued

[Winter, January 1-March 31; spring, April 1-June 30; summer, July 1-September 30; fall, October 1-December 31; streamflow statistics are in cubic feet per second, except for variability index, which is dimensionless; the record period is in climatic years, April 1 through March 31of the indicated ending year; EPA, U.S. Environmental Protection Agency]

\begin{tabular}{|c|c|c|c|c|c|}
\hline \multirow{2}{*}{ Streamflow statistic } & \multirow{2}{*}{ Annual } & \multicolumn{4}{|c|}{ Season } \\
\hline & & Winter & Spring & Summer & Fall \\
\hline \multicolumn{6}{|c|}{01605500 South Branch Potomac River at Franklin, WV, 1946-1957 } \\
\hline 1-day 2-year hydrologically based flow & 29.3 & 60.0 & 51.0 & 30.9 & 32.7 \\
\hline 1-day 5-year hydrologically based flow & 25.2 & 36.4 & 41.6 & 26.7 & 26.4 \\
\hline 1-day 10-year hydrologically based flow & 23.5 & 27.7 & 37.8 & 25.4 & 24.1 \\
\hline 3-day 2-year hydrologically based flow & 29.9 & 64.9 & 52.9 & 31.6 & 33.4 \\
\hline 3-day 5-year hydrologically based flow & 26.2 & 40.2 & 43.3 & 27.1 & 27.0 \\
\hline 3-day 10-year hydrologically based flow & 24.9 & 31.0 & 39.7 & 25.6 & 24.8 \\
\hline 7-day 2-year hydrologically based flow & 30.7 & 73.0 & 59.7 & 32.9 & 34.5 \\
\hline 7-day 5-year hydrologically based flow & 26.9 & 43.5 & 48.4 & 27.9 & 27.9 \\
\hline 7-day 10-year hydrologically based flow & 25.6 & 32.7 & 43.9 & 26.2 & 25.6 \\
\hline 14-day 2-year hydrologically based flow & 31.9 & 87.6 & 68.9 & 34.3 & 35.9 \\
\hline 14-day 5-year hydrologically based flow & 27.7 & 48.8 & 56.9 & 28.7 & 29.1 \\
\hline 14-day 10-year hydrologically based flow & 26.2 & 34.8 & 52.4 & 26.8 & 26.8 \\
\hline 30-day 2-year hydrologically based flow & 33.7 & 132 & 92.2 & 39.0 & 42.2 \\
\hline 30-day 5-year hydrologically based flow & 28.9 & 73.2 & 72.0 & 31.2 & 31.6 \\
\hline 30-day 10-year hydrologically based flow & 27.1 & 51.4 & 64.8 & 28.6 & 28.4 \\
\hline 1-day 3-year biologically based flow & 24.3 & 25.0 & 52.9 & 26.0 & 24.9 \\
\hline 4-day 3-year biologically based flow & 25.8 & 27.8 & 61.0 & 26.0 & 26.2 \\
\hline EPA harmonic-mean flow & 73.7 & 119 & 148 & 52.2 & 50.4 \\
\hline 5-percent-duration flow & 527 & 740 & 524 & 221 & 385 \\
\hline 10-percent-duration flow & 366 & 539 & 357 & 138 & 222 \\
\hline 15-percent-duration flow & 289 & 448 & 282 & 108 & 163 \\
\hline 20-percent-duration flow & 237 & 390 & 241 & 87.4 & 128 \\
\hline 25 -percent-duration flow & 200 & 349 & 211 & 74.6 & 108 \\
\hline 30-percent-duration flow & 173 & 316 & 188 & 65.8 & 93.1 \\
\hline 35-percent-duration flow & 149 & 292 & 174 & 59.9 & 81.7 \\
\hline 40-percent-duration flow & 128 & 268 & 161 & 55.2 & 73.2 \\
\hline 45-percent-duration flow & 112 & 245 & 149 & 51.6 & 66.2 \\
\hline 50-percent-duration flow & 96.6 & 224 & 138 & 48.1 & 59.5 \\
\hline 55-percent-duration flow & 83.2 & 205 & 128 & 45.8 & 53.6 \\
\hline 60-percent-duration flow & 72.2 & 190 & 118 & 43.5 & 47.4 \\
\hline 65-percent-duration flow & 63.3 & 173 & 109 & 41.4 & 42.2 \\
\hline 70-percent-duration flow & 55.2 & 152 & 101 & 39.4 & 38.9 \\
\hline 75-percent-duration flow & 48.0 & 128 & 92.7 & 37.5 & 36.4 \\
\hline 80-percent-duration flow & 42.1 & 107 & 84.1 & 35.5 & 34.1 \\
\hline 85-percent-duration flow & 38.0 & 79.7 & 75.5 & 33.5 & 32.2 \\
\hline 90-percent-duration flow & 34.3 & 58.4 & 66.8 & 31.4 & 30.6 \\
\hline 95-percent-duration flow & 30.7 & 42.6 & 56.9 & 28.7 & 28.9 \\
\hline 99-percent-duration flow & 26.7 & 29.2 & 43.7 & 25.1 & 25.8 \\
\hline Variability index & 0.40 & 0.36 & 0.28 & 0.27 & 0.35 \\
\hline
\end{tabular}


Table 11. Selected annual and seasonal statistics for 77 stations in West Virginia representative of 1930-2002.—Continued

[Winter, January 1-March 31; spring, April 1-June 30; summer, July 1-September 30; fall, October 1-December 31; streamflow statistics are in cubic feet per second, except for variability index, which is dimensionless; the record period is in climatic years, April 1 through March 31 of the indicated ending year; EPA, U.S. Environmental Protection Agency]

\begin{tabular}{|c|c|c|c|c|c|}
\hline \multirow{2}{*}{ Streamflow statistic } & \multirow{2}{*}{ Annual } & \multicolumn{4}{|c|}{ Season } \\
\hline & & Winter & Spring & Summer & Fall \\
\hline \multicolumn{6}{|c|}{01606000 North Fork South Branch Potomac River at Cabins, WV, 1941-1954 } \\
\hline 1-day 2-year hydrologically based flow & 15.2 & 114 & 74.7 & 16.0 & 24.5 \\
\hline 1-day 5-year hydrologically based flow & 8.75 & 65.9 & 58.1 & 9.31 & 10.8 \\
\hline 1-day 10-year hydrologically based flow & 6.49 & 47.8 & 51.9 & 6.89 & 7.06 \\
\hline 3-day 2-year hydrologically based flow & 15.7 & 121 & 83.3 & 16.7 & 25.2 \\
\hline 3-day 5-year hydrologically based flow & 9.04 & 68.3 & 64.0 & 9.74 & 11.1 \\
\hline 3-day 10-year hydrologically based flow & 6.67 & 48.8 & 56.3 & 7.20 & 7.25 \\
\hline 7-day 2-year hydrologically based flow & 17.4 & 140 & 96.6 & 19.0 & 26.4 \\
\hline 7-day 5-year hydrologically based flow & 9.61 & 74.6 & 72.1 & 10.7 & 11.5 \\
\hline 7-day 10-year hydrologically based flow & 6.97 & 51.5 & 62.2 & 7.78 & 7.41 \\
\hline 14-day 2-year hydrologically based flow & 19.7 & 175 & 127 & 23.9 & 29.5 \\
\hline 14-day 5-year hydrologically based flow & 10.5 & 87.0 & 92.3 & 12.9 & 12.3 \\
\hline 14-day 10-year hydrologically based flow & 7.39 & 58.6 & 77.5 & 9.00 & 7.88 \\
\hline 30-day 2-year hydrologically based flow & 23.4 & 270 & 200 & 36.4 & 40.4 \\
\hline 30-day 5-year hydrologically based flow & 11.8 & 166 & 151 & 17.7 & 15.7 \\
\hline 30-day 10 -year hydrologically based flow & 8.22 & 130 & 131 & 11.5 & 9.86 \\
\hline 1-day 3-year biologically based flow & 4.98 & 25.9 & 65.0 & 6.16 & 4.97 \\
\hline 4-day 3-year biologically based flow & 5.49 & 33.5 & 76.7 & 6.78 & 5.50 \\
\hline EPA harmonic-mean flow & 65.7 & 230 & 300 & 42.9 & 33.6 \\
\hline 5-percent-duration flow & 1,370 & 1,970 & 1,500 & 460 & 872 \\
\hline 10-percent-duration flow & 942 & 1,430 & 1,050 & 258 & 495 \\
\hline 15-percent-duration flow & 703 & 1,150 & 844 & 181 & 366 \\
\hline 20-percent-duration flow & 560 & 969 & 681 & 145 & 296 \\
\hline 25 -percent-duration flow & 459 & 840 & 562 & 116 & 244 \\
\hline 30-percent-duration flow & 385 & 733 & 505 & 96.3 & 206 \\
\hline 35-percent-duration flow & 325 & 649 & 447 & 82.1 & 179 \\
\hline 40-percent-duration flow & 274 & 580 & 397 & 72.4 & 155 \\
\hline 45-percent-duration flow & 228 & 520 & 357 & 64.0 & 129 \\
\hline 50-percent-duration flow & 193 & 462 & 322 & 57.9 & 104 \\
\hline 55-percent-duration flow & 162 & 410 & 289 & 51.8 & 83.7 \\
\hline 60-percent-duration flow & 132 & 367 & 258 & 45.4 & 61.8 \\
\hline 65-percent-duration flow & 105 & 330 & 228 & 39.4 & 49.2 \\
\hline 70-percent-duration flow & 84.0 & 293 & 203 & 33.9 & 40.9 \\
\hline 75-percent-duration flow & 65.4 & 257 & 180 & 29.2 & 31.8 \\
\hline 80-percent-duration flow & 49.0 & 210 & 160 & 25.2 & 23.7 \\
\hline 85-percent-duration flow & 36.1 & 164 & 138 & 20.9 & 19.2 \\
\hline 90-percent-duration flow & 24.6 & 123 & 112 & 15.9 & 14.8 \\
\hline 95-percent-duration flow & 15.4 & 90.9 & 85.6 & 10.3 & 11.8 \\
\hline 99-percent-duration flow & 7.1 & 46.9 & 64.3 & 6.3 & 5.8 \\
\hline Variability index & 0.60 & 0.40 & 0.37 & 0.48 & 0.59 \\
\hline
\end{tabular}


Table 11. Selected annual and seasonal statistics for 77 stations in West Virginia representative of 1930-2002. - Continued

[Winter, January 1-March 31; spring, April 1-June 30; summer, July 1-September 30; fall, October 1-December 31; streamflow statistics are in cubic feet per second, except for variability index, which is dimensionless; the record period is in climatic years, April 1 through March 31 of the indicated ending year; EPA, U.S. Environmental Protection Agency]

\begin{tabular}{|c|c|c|c|c|c|}
\hline \multirow{2}{*}{ Streamflow statistic } & \multirow{2}{*}{ Annual } & \multicolumn{4}{|c|}{ Season } \\
\hline & & Winter & Spring & Summer & Fall \\
\hline \multicolumn{6}{|c|}{01606500 South Branch Potomac River near Petersburg, WV, 1930-2002 } \\
\hline 1-day 2-year hydrologically based flow & 72.6 & 239 & 175 & 76.7 & 90.3 \\
\hline 1-day 5-year hydrologically based flow & 56.9 & 152 & 129 & 58.4 & 67.9 \\
\hline 1-day 10-year hydrologically based flow & 51.2 & 117 & 109 & 51.5 & 60.7 \\
\hline 3-day 2-year hydrologically based flow & 74.1 & 253 & 187 & 78.4 & 93.9 \\
\hline 3-day 5-year hydrologically based flow & 57.9 & 161 & 137 & 59.5 & 69.9 \\
\hline 3-day 10 -year hydrologically based flow & 52.0 & 125 & 115 & 52.4 & 62.1 \\
\hline 7-day 2-year hydrologically based flow & 76.2 & 284 & 211 & 82.2 & 101 \\
\hline 7-day 5-year hydrologically based flow & 59.6 & 178 & 152 & 61.9 & 72.9 \\
\hline 7-day 10-year hydrologically based flow & 53.8 & 138 & 127 & 54.4 & 64.1 \\
\hline 14-day 2-year hydrologically based flow & 79.8 & 336 & 259 & 88.7 & 110 \\
\hline 14-day 5-year hydrologically based flow & 62.3 & 201 & 182 & 65.7 & 76.7 \\
\hline 14-day 10 -year hydrologically based flow & 56.7 & 154 & 149 & 57.6 & 66.0 \\
\hline 30-day 2-year hydrologically based flow & 88.1 & 498 & 374 & 102 & 152 \\
\hline 30-day 5-year hydrologically based flow & 68.3 & 295 & 246 & 73.8 & 92.3 \\
\hline 30-day 10-year hydrologically based flow & 62.3 & 223 & 194 & 65.5 & 72.9 \\
\hline 1-day 3-year biologically based flow & 49.0 & 88.9 & 144 & 50.0 & 55.9 \\
\hline 4-day 3-year biologically based flow & 50.5 & 99.9 & 170 & 52.6 & 59.1 \\
\hline EPA harmonic-mean flow & 233 & 455 & 608 & 150 & 151 \\
\hline 5-percent-duration flow & 2,510 & 3,820 & 2,840 & 840 & 1,610 \\
\hline 10-percent-duration flow & 1,660 & 2,590 & 1,990 & 489 & 1,030 \\
\hline 15-percent-duration flow & 1,280 & 2,050 & 1,540 & 355 & 782 \\
\hline 20-percent-duration flow & 1,030 & 1,710 & 1,250 & 288 & 623 \\
\hline 25-percent-duration flow & 847 & 1,470 & 1,070 & 248 & 522 \\
\hline 30-percent-duration flow & 709 & 1,300 & 930 & 217 & 443 \\
\hline 35-percent-duration flow & 593 & 1,150 & 820 & 192 & 380 \\
\hline 40-percent-duration flow & 509 & 1,020 & 726 & 174 & 328 \\
\hline 45-percent-duration flow & 437 & 911 & 644 & 158 & 283 \\
\hline 50-percent-duration flow & 375 & 813 & 579 & 146 & 242 \\
\hline 55-percent-duration flow & 320 & 724 & 521 & 134 & 205 \\
\hline 60-percent-duration flow & 271 & 640 & 472 & 124 & 173 \\
\hline 65-percent-duration flow & 228 & 564 & 429 & 114 & 150 \\
\hline 70-percent-duration flow & 190 & 497 & 388 & 106 & 132 \\
\hline 75-percent-duration flow & 160 & 433 & 345 & 98.3 & 116 \\
\hline 80-percent-duration flow & 134 & 371 & 303 & 90.4 & 104 \\
\hline 85-percent-duration flow & 113 & 311 & 262 & 81.4 & 93.8 \\
\hline 90-percent-duration flow & 95.1 & 255 & 220 & 71.7 & 84.9 \\
\hline 95-percent-duration flow & 78.3 & 190 & 173 & 60.7 & 72.8 \\
\hline 99-percent-duration flow & 56.1 & 115 & 113 & 49.2 & 56.8 \\
\hline Variability index & 0.48 & 0.39 & 0.37 & 0.34 & 0.43 \\
\hline
\end{tabular}


Table 11. Selected annual and seasonal statistics for 77 stations in West Virginia representative of 1930-2002._Continued

[Winter, January 1-March 31; spring, April 1-June 30; summer, July 1-September 30; fall, October 1-December 31; streamflow statistics are in cubic feet per second, except for variability index, which is dimensionless; the record period is in climatic years, April 1 through March 31of the indicated ending year; EPA, U.S. Environmental Protection Agency]

\begin{tabular}{|c|c|c|c|c|c|}
\hline \multirow{2}{*}{ Streamflow statistic } & \multirow{2}{*}{ Annual } & \multicolumn{4}{|c|}{ Season } \\
\hline & & Winter & Spring & Summer & Fall \\
\hline \multicolumn{6}{|c|}{01607500 South Fork South Branch Potomac River at Brandywine, WV, 1945-1996 } \\
\hline 1-day 2-year hydrologically based flow & 5.02 & 23.5 & 13.4 & 5.22 & 8.15 \\
\hline 1-day 5-year hydrologically based flow & 3.08 & 12.9 & 8.64 & 3.16 & 5.14 \\
\hline 1-day 10-year hydrologically based flow & 2.36 & 8.91 & 6.80 & 2.41 & 4.11 \\
\hline 3-day 2-year hydrologically based flow & 5.20 & 25.7 & 14.4 & 5.44 & 8.41 \\
\hline 3-day 5-year hydrologically based flow & 3.21 & 14.1 & 9.20 & 3.29 & 5.25 \\
\hline 3-day 10 -year hydrologically based flow & 2.49 & 9.90 & 7.20 & 2.53 & 4.19 \\
\hline 7-day 2-year hydrologically based flow & 5.47 & 30.3 & 16.5 & 5.86 & 9.01 \\
\hline 7-day 5-year hydrologically based flow & 3.40 & 16.2 & 10.6 & 3.52 & 5.59 \\
\hline 7-day 10 -year hydrologically based flow & 2.64 & 11.1 & 8.25 & 2.69 & 4.47 \\
\hline 14-day 2-year hydrologically based flow & 6.02 & 35.2 & 20.3 & 6.48 & 10.0 \\
\hline 14-day 5-year hydrologically based flow & 3.77 & 17.9 & 12.8 & 3.87 & 6.11 \\
\hline 14-day 10 -year hydrologically based flow & 2.97 & 12.1 & 9.97 & 3.01 & 4.86 \\
\hline 30-day 2-year hydrologically based flow & 7.03 & 54.1 & 34.2 & 8.15 & 15.5 \\
\hline 30-day 5-year hydrologically based flow & 4.53 & 27.0 & 19.5 & 4.88 & 8.31 \\
\hline 30-day 10-year hydrologically based flow & 3.69 & 17.9 & 14.6 & 3.85 & 6.17 \\
\hline 1-day 3-year biologically based flow & 1.98 & 5.74 & 10.9 & 1.91 & 3.39 \\
\hline 4-day 3-year biologically based flow & 2.08 & 6.52 & 13.7 & 2.07 & 4.00 \\
\hline EPA harmonic-mean flow & 19.1 & 41.5 & 58.2 & 10.6 & 13.6 \\
\hline 5-percent-duration flow & 383 & 588 & 417 & 118 & 315 \\
\hline 10-percent-duration flow & 221 & 345 & 250 & 63.9 & 167 \\
\hline 15-percent-duration flow & 159 & 255 & 184 & 44.2 & 114 \\
\hline 20-percent-duration flow & 123 & 207 & 144 & 33.0 & 86.4 \\
\hline 25-percent-duration flow & 99.4 & 174 & 119 & 25.3 & 68.1 \\
\hline 30-percent-duration flow & 81.3 & 148 & 100 & 21.2 & 55.6 \\
\hline 35-percent-duration flow & 68.0 & 131 & 85.6 & 17.9 & 46.5 \\
\hline 40-percent-duration flow & 57.3 & 115 & 75.4 & 15.6 & 38.9 \\
\hline 45-percent-duration flow & 48.1 & 103 & 65.9 & 14.0 & 32.4 \\
\hline 50-percent-duration flow & 40.7 & 91.3 & 58.7 & 12.7 & 27.9 \\
\hline 55-percent-duration flow & 33.7 & 80.9 & 52.0 & 11.4 & 23.5 \\
\hline 60-percent-duration flow & 27.4 & 71.4 & 46.4 & 10.0 & 19.3 \\
\hline 65-percent-duration flow & 22.2 & 62.5 & 41.2 & 8.8 & 15.8 \\
\hline 70-percent-duration flow & 17.8 & 55.1 & 36.6 & 7.9 & 13.6 \\
\hline 75-percent-duration flow & 14.5 & 47.9 & 31.9 & 7.1 & 11.4 \\
\hline 80-percent-duration flow & 11.7 & 40.2 & 26.9 & 6.3 & 9.5 \\
\hline 85-percent-duration flow & 9.3 & 31.7 & 21.8 & 5.6 & 8.4 \\
\hline 90-percent-duration flow & 7.4 & 24.4 & 17.4 & 4.7 & 7.2 \\
\hline 95-percent-duration flow & 5.6 & 16.1 & 13.2 & 3.6 & 6.0 \\
\hline 99-percent-duration flow & 3.1 & 7.3 & 7.8 & 2.0 & 4.4 \\
\hline Variability index & 0.57 & 0.45 & 0.45 & 0.45 & 0.54 \\
\hline
\end{tabular}


Table 11. Selected annual and seasonal statistics for 77 stations in West Virginia representative of 1930-2002.—Continued

[Winter, January 1-March 31; spring, April 1-June 30; summer, July 1-September 30; fall, October 1-December 31; streamflow statistics are in cubic feet per second, except for variability index, which is dimensionless; the record period is in climatic years, April 1 through March 31 of the indicated ending year; EPA, U.S. Environmental Protection Agency]

\begin{tabular}{|c|c|c|c|c|c|}
\hline \multirow{2}{*}{ Streamflow statistic } & \multirow{2}{*}{ Annual } & \multicolumn{4}{|c|}{ Season } \\
\hline & & Winter & Spring & Summer & Fall \\
\hline \multicolumn{6}{|c|}{01608000 South Fork South Branch Potomac River near Moorefield, WV, 1940-1996 } \\
\hline 1-day 2-year hydrologically based flow & 15.7 & 58.7 & 41.5 & 17.2 & 21.8 \\
\hline 1-day 5-year hydrologically based flow & 10.4 & 33.9 & 28.6 & 11.2 & 14.1 \\
\hline 1-day 10 -year hydrologically based flow & 8.29 & 24.7 & 23.3 & 8.73 & 11.5 \\
\hline 3-day 2-year hydrologically based flow & 16.1 & 62.1 & 44.0 & 17.7 & 22.7 \\
\hline 3-day 5 -year hydrologically based flow & 10.7 & 35.8 & 30.3 & 11.5 & 14.7 \\
\hline 3-day 10 -year hydrologically based flow & 8.55 & 26.0 & 24.6 & 8.99 & 12.0 \\
\hline 7-day 2-year hydrologically based flow & 16.8 & 71.7 & 49.3 & 18.4 & 24.1 \\
\hline 7-day 5-year hydrologically based flow & 11.2 & 40.2 & 33.8 & 12.0 & 15.5 \\
\hline 7-day 10 -year hydrologically based flow & 9.09 & 28.6 & 27.5 & 9.46 & 12.7 \\
\hline 14-day 2-year hydrologically based flow & 17.8 & 81.8 & 59.2 & 19.8 & 26.7 \\
\hline 14-day 5-year hydrologically based flow & 12.2 & 43.6 & 40.0 & 12.9 & 17.0 \\
\hline 14-day 10 -year hydrologically based flow & 10.1 & 30.6 & 32.2 & 10.4 & 13.9 \\
\hline 30-day 2-year hydrologically based flow & 20.4 & 120 & 95.2 & 23.8 & 38.0 \\
\hline 30-day 5-year hydrologically based flow & 14.1 & 62.4 & 58.5 & 15.4 & 21.4 \\
\hline 30-day 10-year hydrologically based flow & 11.9 & 43.2 & 44.8 & 12.7 & 16.4 \\
\hline 1-day 3-year biologically based flow & 7.80 & 14.9 & 33.0 & 7.86 & 11.0 \\
\hline 4-day 3-year biologically based flow & 8.44 & 18.4 & 40.7 & 8.82 & 11.4 \\
\hline EPA harmonic-mean flow & 54.2 & 101 & 153 & 33.8 & 36.1 \\
\hline 5-percent-duration flow & 840 & 1,270 & 998 & 291 & 623 \\
\hline 10-percent-duration flow & 513 & 777 & 640 & 163 & 351 \\
\hline 15-percent-duration flow & 374 & 584 & 478 & 112 & 239 \\
\hline 20-percent-duration flow & 290 & 472 & 378 & 84.2 & 181 \\
\hline 25-percent-duration flow & 233 & 394 & 309 & 69.0 & 145 \\
\hline 30-percent-duration flow & 191 & 338 & 258 & 58.6 & 119 \\
\hline 35-percent-duration flow & 161 & 295 & 223 & 50.6 & 99.4 \\
\hline 40-percent-duration flow & 136 & 261 & 195 & 45.5 & 85.0 \\
\hline 45-percent-duration flow & 115 & 232 & 170 & 40.6 & 72.3 \\
\hline 50-percent-duration flow & 97.9 & 204 & 152 & 36.6 & 61.1 \\
\hline 55-percent-duration flow & 82.3 & 179 & 134 & 32.7 & 51.0 \\
\hline 60-percent-duration flow & 68.0 & 159 & 121 & 29.6 & 43.9 \\
\hline 65-percent-duration flow & 55.6 & 141 & 108 & 26.7 & 38.0 \\
\hline 70-percent-duration flow & 47.0 & 126 & 96.6 & 24.3 & 33.4 \\
\hline 75-percent-duration flow & 39.2 & 109 & 85.0 & 22.0 & 29.5 \\
\hline 80-percent-duration flow & 32.5 & 90.7 & 73.4 & 19.9 & 25.9 \\
\hline 85-percent-duration flow & 26.6 & 72.5 & 61.8 & 17.8 & 22.9 \\
\hline 90-percent-duration flow & 22.0 & 52.5 & 51.8 & 15.4 & 20.1 \\
\hline 95-percent-duration flow & 17.2 & 36.9 & 39.7 & 12.5 & 17.0 \\
\hline 99-percent-duration flow & 11.2 & 22.1 & 25.1 & 8.1 & 13.2 \\
\hline Variability index & 0.53 & 0.45 & 0.42 & 0.41 & 0.49 \\
\hline
\end{tabular}


Table 11. Selected annual and seasonal statistics for 77 stations in West Virginia representative of 1930-2002.—Continued

[Winter, January 1-March 31; spring, April 1-June 30; summer, July 1-September 30; fall, October 1-December 31; streamflow statistics are in cubic feet per second, except for variability index, which is dimensionless; the record period is in climatic years, April 1 through March 31of the indicated ending year; EPA, U.S. Environmental Protection Agency]

\begin{tabular}{|c|c|c|c|c|c|}
\hline \multirow{2}{*}{ Streamflow statistic } & \multirow{2}{*}{ Annual } & \multicolumn{4}{|c|}{ Season } \\
\hline & & Winter & Spring & Summer & Fall \\
\hline \multicolumn{6}{|c|}{01608500 South Branch Potomac River near Springfield, WV, 1930-2002 } \\
\hline 1-day 2-year hydrologically based flow & 115 & 431 & 310 & 122 & 146 \\
\hline 1-day 5-year hydrologically based flow & 82.2 & 267 & 221 & 85.4 & 104 \\
\hline 1-day 10-year hydrologically based flow & 69.4 & 201 & 184 & 70.8 & 90.2 \\
\hline 3-day 2-year hydrologically based flow & 118 & 458 & 329 & 125 & 151 \\
\hline 3-day 5-year hydrologically based flow & 83.9 & 282 & 233 & 87.4 & 106 \\
\hline 3-day 10 -year hydrologically based flow & 70.7 & 212 & 193 & 72.3 & 91.8 \\
\hline 7-day 2-year hydrologically based flow & 121 & 510 & 372 & 131 & 161 \\
\hline 7-day 5-year hydrologically based flow & 86.7 & 307 & 259 & 90.9 & 111 \\
\hline 7-day 10-year hydrologically based flow & 73.5 & 231 & 212 & 75.3 & 95.1 \\
\hline 14-day 2-year hydrologically based flow & 127 & 602 & 454 & 141 & 177 \\
\hline 14-day 5-year hydrologically based flow & 91.1 & 352 & 310 & 96.6 & 118 \\
\hline 14-day 10 -year hydrologically based flow & 78.2 & 263 & 249 & 80.2 & 100 \\
\hline 30-day 2-year hydrologically based flow & 141 & 885 & 655 & 169 & 246 \\
\hline 30-day 5-year hydrologically based flow & 102 & 507 & 426 & 113 & 143 \\
\hline 30-day 10 -year hydrologically based flow & 90.5 & 373 & 332 & 94.8 & 111 \\
\hline 1-day 3-year biologically based flow & 61.0 & 140 & 253 & 64.0 & 74.8 \\
\hline 4-day 3-year biologically based flow & 64.7 & 157 & 292 & 69.7 & 84.2 \\
\hline EPA harmonic-mean flow & 378 & 767 & 1,040 & 241 & 241 \\
\hline 5-percent-duration flow & 4,750 & 7,030 & 5,310 & 1,640 & 3,010 \\
\hline 10-percent-duration flow & 3,090 & 4,770 & 3,620 & 920 & 1,860 \\
\hline 15-percent-duration flow & 2,300 & 3,700 & 2,820 & 663 & 1,350 \\
\hline 20-percent-duration flow & 1,820 & 3,070 & 2,290 & 528 & 1,070 \\
\hline 25-percent-duration flow & 1,490 & 2,640 & 1,920 & 443 & 888 \\
\hline 30-percent-duration flow & 1,240 & 2,300 & 1,650 & 381 & 750 \\
\hline 35-percent-duration flow & 1,040 & 2,010 & 1,430 & 335 & 637 \\
\hline 40-percent-duration flow & 893 & 1,780 & 1,270 & 302 & 542 \\
\hline 45-percent-duration flow & 767 & 1,590 & 1,130 & 271 & 465 \\
\hline 50-percent-duration flow & 654 & 1,410 & 1,010 & 247 & 393 \\
\hline 55-percent-duration flow & 554 & 1,250 & 914 & 223 & 331 \\
\hline 60-percent-duration flow & 467 & 1,110 & 826 & 204 & 280 \\
\hline 65-percent-duration flow & 389 & 987 & 752 & 186 & 244 \\
\hline 70-percent-duration flow & 321 & 871 & 679 & 170 & 214 \\
\hline 75-percent-duration flow & 265 & 758 & 607 & 156 & 188 \\
\hline 80-percent-duration flow & 221 & 649 & 530 & 141 & 169 \\
\hline 85-percent-duration flow & 183 & 541 & 452 & 127 & 152 \\
\hline 90-percent-duration flow & 152 & 428 & 378 & 110 & 134 \\
\hline 95-percent-duration flow & 121 & 310 & 295 & 88.6 & 114 \\
\hline 99-percent-duration flow & 80.0 & 193 & 188 & 64.6 & 85.8 \\
\hline Variability index & 0.50 & 0.40 & 0.38 & 0.37 & 0.45 \\
\hline
\end{tabular}


Table 11. Selected annual and seasonal statistics for 77 stations in West Virginia representative of 1930-2002.—Continued

[Winter, January 1-March 31; spring, April 1-June 30; summer, July 1-September 30; fall, October 1-December 31; streamflow statistics are in cubic feet per second, except for variability index, which is dimensionless; the record period is in climatic years, April 1 through March 31 of the indicated ending year; EPA, U.S. Environmental Protection Agency]

\begin{tabular}{|c|c|c|c|c|c|}
\hline \multirow{2}{*}{ Streamflow statistic } & \multirow{2}{*}{ Annual } & \multicolumn{4}{|c|}{ Season } \\
\hline & & Winter & Spring & Summer & Fall \\
\hline \multicolumn{6}{|c|}{01610500 Cacapon River at Yellow Spring, WV, 1941-1951 } \\
\hline 1-day 2-year hydrologically based flow & 25.2 & 77.7 & 51.7 & 25.5 & 34.0 \\
\hline 1-day 5-year hydrologically based flow & 21.3 & 45.5 & 43.5 & 21.8 & 25.9 \\
\hline 1-day 10-year hydrologically based flow & 19.8 & 33.8 & 39.8 & 20.5 & 22.2 \\
\hline 3-day 2-year hydrologically based flow & 26.2 & 81.4 & 55.6 & 26.4 & 35.0 \\
\hline 3-day 5-year hydrologically based flow & 22.2 & 48.5 & 46.5 & 22.4 & 26.8 \\
\hline 3-day 10-year hydrologically based flow & 20.5 & 36.4 & 42.5 & 20.9 & 23.0 \\
\hline 7-day 2-year hydrologically based flow & 27.3 & 89.5 & 65.3 & 27.4 & 37.0 \\
\hline 7-day 5-year hydrologically based flow & 23.0 & 51.5 & 53.0 & 23.0 & 26.9 \\
\hline 7-day 10-year hydrologically based flow & 21.2 & 38.6 & 47.8 & 21.4 & 23.3 \\
\hline 14-day 2-year hydrologically based flow & 29.3 & 102 & 77.3 & 30.1 & 42.7 \\
\hline 14-day 5-year hydrologically based flow & 23.9 & 59.1 & 61.7 & 24.4 & 27.8 \\
\hline 14-day 10 -year hydrologically based flow & 21.6 & 44.1 & 56.0 & 22.2 & 23.3 \\
\hline 30-day 2-year hydrologically based flow & 32.9 & 151 & 128 & 36.7 & 49.7 \\
\hline 30-day 5-year hydrologically based flow & 25.6 & 83.1 & 92.1 & 27.6 & 30.2 \\
\hline 30-day 10 -year hydrologically based flow & 22.6 & 59.3 & 77.6 & 24.2 & 24.7 \\
\hline 1-day 3-year biologically based flow & 20.9 & 30.0 & 45.9 & 21.0 & 23.0 \\
\hline 4-day 3-year biologically based flow & 21.0 & 35.5 & 51.3 & 21.0 & 23.0 \\
\hline EPA harmonic-mean flow & 80.4 & 144 & 183 & 52.1 & 55.6 \\
\hline 5-percent-duration flow & 867 & 1,110 & 1,020 & 389 & 706 \\
\hline 10-percent-duration flow & 572 & 793 & 641 & 238 & 438 \\
\hline 15-percent-duration flow & 439 & 645 & 472 & 154 & 306 \\
\hline 20-percent-duration flow & 351 & 544 & 398 & 113 & 225 \\
\hline 25-percent-duration flow & 286 & 484 & 341 & 91.7 & 182 \\
\hline 30-percent-duration flow & 237 & 424 & 299 & 79.0 & 150 \\
\hline 35-percent-duration flow & 200 & 370 & 266 & 69.4 & 122 \\
\hline 40-percent-duration flow & 174 & 323 & 237 & 62.2 & 97.8 \\
\hline 45-percent-duration flow & 148 & 278 & 212 & 56.8 & 80.2 \\
\hline 50-percent-duration flow & 123 & 247 & 190 & 52.0 & 70.3 \\
\hline 55-percent-duration flow & 101 & 218 & 174 & 47.7 & 61.4 \\
\hline 60-percent-duration flow & 84.3 & 195 & 157 & 44.1 & 54.3 \\
\hline 65-percent-duration flow & 71.8 & 176 & 139 & 40.5 & 49.9 \\
\hline 70-percent-duration flow & 61.6 & 160 & 123 & 36.9 & 45.5 \\
\hline 75-percent-duration flow & 53.7 & 135 & 111 & 33.6 & 41.9 \\
\hline 80-percent-duration flow & 46.6 & 110 & 96.9 & 30.9 & 38.2 \\
\hline 85-percent-duration flow & 39.9 & 83.4 & 84.1 & 28.8 & 34.3 \\
\hline 90-percent-duration flow & 33.3 & 68.5 & 71.9 & 27.0 & 30.4 \\
\hline 95-percent-duration flow & 27.8 & 52.1 & 58.8 & 24.4 & 26.6 \\
\hline 99-percent-duration flow & 23.1 & 40.1 & 46.8 & 20.8 & 22.2 \\
\hline Variability index & 0.48 & 0.41 & 0.37 & 0.37 & 0.45 \\
\hline
\end{tabular}


Table 11. Selected annual and seasonal statistics for 77 stations in West Virginia representative of 1930-2002.-Continued

[Winter, January 1-March 31; spring, April 1-June 30; summer, July 1-September 30; fall, October 1-December 31; streamflow statistics are in cubic feet per second, except for variability index, which is dimensionless; the record period is in climatic years, April 1 through March 31of the indicated ending year; EPA, U.S. Environmental Protection Agency]

\begin{tabular}{|c|c|c|c|c|c|}
\hline \multirow{2}{*}{ Streamflow statistic } & \multirow{2}{*}{ Annual } & \multicolumn{4}{|c|}{ Season } \\
\hline & & Winter & Spring & Summer & Fall \\
\hline \multicolumn{6}{|c|}{01611500 Cacapon River near Great Cacapon, WV, 1933-1995 } \\
\hline 1-day 2-year hydrologically based flow & 54.1 & 178 & 116 & 56.6 & 62.0 \\
\hline 1-day 5-year hydrologically based flow & 42.1 & 112 & 87.0 & 43.3 & 49.1 \\
\hline 1-day 10-year hydrologically based flow & 37.2 & 86.2 & 74.8 & 37.8 & 45.6 \\
\hline 3-day 2-year hydrologically based flow & 55.1 & 187 & 122 & 57.4 & 63.4 \\
\hline 3-day 5-year hydrologically based flow & 42.9 & 117 & 91.4 & 44.0 & 49.9 \\
\hline 3-day 10-year hydrologically based flow & 38.0 & 88.8 & 78.4 & 38.5 & 46.1 \\
\hline 7-day 2-year hydrologically based flow & 56.8 & 207 & 137 & 59.8 & 68.8 \\
\hline 7-day 5-year hydrologically based flow & 44.2 & 125 & 101 & 45.7 & 52.0 \\
\hline 7-day 10-year hydrologically based flow & 39.2 & 93.3 & 86.0 & 39.9 & 47.2 \\
\hline 14-day 2-year hydrologically based flow & 59.5 & 236 & 164 & 64.4 & 75.6 \\
\hline 14-day 5-year hydrologically based flow & 46.1 & 137 & 116 & 48.3 & 54.5 \\
\hline 14-day 10 -year hydrologically based flow & 40.9 & 101 & 98.1 & 41.9 & 48.0 \\
\hline 30-day 2-year hydrologically based flow & 66.1 & 338 & 253 & 76.5 & 99.6 \\
\hline 30-day 5-year hydrologically based flow & 50.3 & 185 & 164 & 55.8 & 62.9 \\
\hline 30-day 10-year hydrologically based flow & 44.6 & 131 & 131 & 47.7 & 51.9 \\
\hline 1-day 3-year biologically based flow & 36.8 & 59.9 & 101 & 37.8 & 42.0 \\
\hline 4-day 3-year biologically based flow & 38.5 & 70.0 & 112 & 39.9 & 43.4 \\
\hline EPA harmonic-mean flow & 159 & 301 & 424 & 103 & 108 \\
\hline 5-percent-duration flow & 2,170 & 3,190 & 2,620 & 609 & 1,480 \\
\hline 10-percent-duration flow & 1,380 & 2,100 & 1,760 & 334 & 851 \\
\hline 15-percent-duration flow & 992 & 1,600 & 1,330 & 238 & 580 \\
\hline 20-percent-duration flow & 765 & 1,280 & 1,080 & 191 & 443 \\
\hline 25-percent-duration flow & 609 & 1,070 & 888 & 163 & 350 \\
\hline 30-percent-duration flow & 502 & 914 & 743 & 142 & 287 \\
\hline 35-percent-duration flow & 419 & 790 & 632 & 128 & 242 \\
\hline 40-percent-duration flow & 352 & 688 & 547 & 116 & 202 \\
\hline 45-percent-duration flow & 296 & 612 & 477 & 107 & 170 \\
\hline 50-percent-duration flow & 249 & 542 & 417 & 97.9 & 146 \\
\hline 55-percent-duration flow & 207 & 479 & 372 & 91.1 & 126 \\
\hline 60-percent-duration flow & 173 & 427 & 331 & 84.3 & 112 \\
\hline 65-percent-duration flow & 146 & 382 & 297 & 78.6 & 99.9 \\
\hline 70-percent-duration flow & 123 & 341 & 265 & 73.5 & 90.9 \\
\hline 75-percent-duration flow & 106 & 295 & 234 & 68.5 & 82.5 \\
\hline 80-percent-duration flow & 91.2 & 248 & 203 & 63.6 & 75.8 \\
\hline 85-percent-duration flow & 78.5 & 199 & 173 & 58.8 & 69.2 \\
\hline 90-percent-duration flow & 67.8 & 159 & 144 & 53.4 & 62.5 \\
\hline 95-percent-duration flow & 56.6 & 122 & 115 & 47.2 & 54.7 \\
\hline 99-percent-duration flow & 42.8 & 68.2 & 81.7 & 35.6 & 43.5 \\
\hline Variability index & 0.51 & 0.43 & 0.42 & 0.33 & 0.45 \\
\hline
\end{tabular}


Table 11. Selected annual and seasonal statistics for 77 stations in West Virginia representative of 1930-2002.—Continued

[Winter, January 1-March 31; spring, April 1-June 30; summer, July 1-September 30; fall, October 1-December 31; streamflow statistics are in cubic feet per second, except for variability index, which is dimensionless; the record period is in climatic years, April 1 through March 31 of the indicated ending year; EPA, U.S. Environmental Protection Agency]

\begin{tabular}{|c|c|c|c|c|c|}
\hline \multirow{2}{*}{ Streamflow statistic } & \multirow{2}{*}{ Annual } & \multicolumn{4}{|c|}{ Season } \\
\hline & & Winter & Spring & Summer & Fall \\
\hline \multicolumn{6}{|c|}{01614000 Back Creek near Jones Springs, WV, 1940-1953 } \\
\hline 1-day 2-year hydrologically based flow & 7.90 & 55.1 & 25.2 & 8.04 & 13.9 \\
\hline 1-day 5-year hydrologically based flow & 5.08 & 32.5 & 19.3 & 5.21 & 7.89 \\
\hline 1-day 10-year hydrologically based flow & 4.07 & 24.4 & 16.8 & 4.22 & 5.78 \\
\hline 3-day 2-year hydrologically based flow & 8.35 & 59.5 & 27.5 & 8.49 & 14.5 \\
\hline 3-day 5-year hydrologically based flow & 5.36 & 35.2 & 21.0 & 5.45 & 8.34 \\
\hline 3-day 10 -year hydrologically based flow & 4.28 & 26.5 & 18.2 & 4.39 & 6.17 \\
\hline 7-day 2-year hydrologically based flow & 9.02 & 66.4 & 33.3 & 9.21 & 16.8 \\
\hline 7-day 5-year hydrologically based flow & 5.85 & 38.4 & 25.0 & 5.95 & 9.16 \\
\hline 7-day 10-year hydrologically based flow & 4.73 & 28.8 & 21.3 & 4.83 & 6.65 \\
\hline 14-day 2-year hydrologically based flow & 10.4 & 78.9 & 42.2 & 11.0 & 20.3 \\
\hline 14-day 5-year hydrologically based flow & 6.44 & 44.9 & 32.0 & 6.75 & 9.92 \\
\hline 14-day 10 -year hydrologically based flow & 5.07 & 33.3 & 27.4 & 5.30 & 6.98 \\
\hline 30-day 2-year hydrologically based flow & 14.1 & 118 & 75.4 & 17.0 & 29.8 \\
\hline 30-day 5-year hydrologically based flow & 8.25 & 66.7 & 54.1 & 10.6 & 13.1 \\
\hline 30-day 10-year hydrologically based flow & 6.22 & 49.0 & 45.2 & 8.24 & 8.88 \\
\hline 1-day 3-year biologically based flow & 3.88 & 14.0 & 19.9 & 3.88 & 4.76 \\
\hline 4-day 3-year biologically based flow & 4.55 & 22.5 & 23.7 & 4.91 & 5.32 \\
\hline EPA harmonic-mean flow & 37.6 & 116 & 106 & 21.5 & 24.2 \\
\hline 5-percent-duration flow & 801 & 1,090 & 828 & 276 & 654 \\
\hline 10-percent-duration flow & 494 & 760 & 519 & 133 & 358 \\
\hline 15-percent-duration flow & 356 & 614 & 385 & 85.6 & 249 \\
\hline 20-percent-duration flow & 275 & 494 & 304 & 65.6 & 188 \\
\hline 25-percent-duration flow & 222 & 411 & 252 & 51.7 & 152 \\
\hline 30-percent-duration flow & 184 & 355 & 214 & 42.2 & 122 \\
\hline 35-percent-duration flow & 151 & 310 & 185 & 35.6 & 98.2 \\
\hline 40-percent-duration flow & 124 & 267 & 159 & 31.3 & 81.1 \\
\hline 45-percent-duration flow & 102 & 242 & 136 & 27.6 & 65.0 \\
\hline 50-percent-duration flow & 83.8 & 218 & 117 & 24.6 & 52.1 \\
\hline 55-percent-duration flow & 69.2 & 195 & 103 & 22.0 & 42.5 \\
\hline 60-percent-duration flow & 56.3 & 172 & 90.6 & 19.5 & 35.2 \\
\hline 65-percent-duration flow & 45.2 & 148 & 80.7 & 17.6 & 30.0 \\
\hline 70-percent-duration flow & 36.4 & 130 & 72.3 & 15.7 & 25.9 \\
\hline 75-percent-duration flow & 29.9 & 114 & 63.9 & 14.0 & 22.3 \\
\hline 80-percent-duration flow & 24.2 & 98.9 & 55.2 & 12.5 & 19.5 \\
\hline 85-percent-duration flow & 19.3 & 78.1 & 47.0 & 10.2 & 16.5 \\
\hline 90-percent-duration flow & 14.8 & 54.7 & 39.1 & 8.5 & 13.2 \\
\hline 95-percent-duration flow & 9.9 & 37.7 & 30.2 & 6.9 & 9.1 \\
\hline 99-percent-duration flow & 5.8 & 27.5 & 20.9 & 4.8 & 5.9 \\
\hline Variability index & 0.59 & 0.43 & 0.44 & 0.47 & 0.57 \\
\hline
\end{tabular}


Table 11. Selected annual and seasonal statistics for 77 stations in West Virginia representative of 1930-2002.—Continued

[Winter, January 1-March 31; spring, April 1-June 30; summer, July 1-September 30; fall, October 1-December 31; streamflow statistics are in cubic feet per second, except for variability index, which is dimensionless; the record period is in climatic years, April 1 through March 31of the indicated ending year; EPA, U.S. Environmental Protection Agency]

\begin{tabular}{|c|c|c|c|c|c|}
\hline \multirow{2}{*}{ Streamflow statistic } & \multirow{2}{*}{ Annual } & \multicolumn{4}{|c|}{ Season } \\
\hline & & Winter & Spring & Summer & Fall \\
\hline \multicolumn{6}{|c|}{01616500 Opequon Creek near Martinsburg, WV, 1949-1996 } \\
\hline 1-day 2-year hydrologically based flow & 50.6 & 98.6 & 104 & 59.8 & 52.8 \\
\hline 1-day 5-year hydrologically based flow & 38.4 & 62.7 & 76.5 & 44.7 & 39.9 \\
\hline 1-day 10-year hydrologically based flow & 33.5 & 48.9 & 64.4 & 38.2 & 35.6 \\
\hline 3-day 2-year hydrologically based flow & 51.7 & 103 & 106 & 61.1 & 54.0 \\
\hline 3-day 5-year hydrologically based flow & 39.4 & 65.0 & 78.4 & 45.8 & 40.8 \\
\hline 3-day 10-year hydrologically based flow & 34.5 & 50.6 & 66.3 & 39.3 & 36.3 \\
\hline 7-day 2-year hydrologically based flow & 53.6 & 113 & 111 & 63.0 & 57.1 \\
\hline 7-day 5-year hydrologically based flow & 40.8 & 70.5 & 82.7 & 47.4 & 42.5 \\
\hline 7-day 10-year hydrologically based flow & 35.8 & 54.0 & 70.5 & 40.9 & 37.5 \\
\hline 14-day 2-year hydrologically based flow & 55.9 & 133 & 121 & 66.1 & 61.2 \\
\hline 14-day 5-year hydrologically based flow & 42.4 & 81.1 & 90.1 & 49.9 & 44.0 \\
\hline 14-day 10 -year hydrologically based flow & 37.1 & 60.7 & 76.8 & 43.3 & 38.3 \\
\hline 30-day 2-year hydrologically based flow & 61.1 & 169 & 144 & 73.4 & 74.6 \\
\hline 30-day 5-year hydrologically based flow & 46.0 & 101 & 106 & 54.9 & 50.1 \\
\hline 30-day 10-year hydrologically based flow & 40.0 & 74.4 & 89.8 & 47.3 & 41.9 \\
\hline 1-day 3-year biologically based flow & 32.0 & 44.0 & 75.8 & 42.0 & 35.0 \\
\hline 4-day 3-year biologically based flow & 33.9 & 45.6 & 79.4 & 44.9 & 35.4 \\
\hline EPA harmonic-mean flow & 115 & 152 & 201 & 95.1 & 79.0 \\
\hline 5-percent-duration flow & 700 & 1,030 & 773 & 273 & 547 \\
\hline 10-percent-duration flow & 468 & 696 & 514 & 195 & 360 \\
\hline 15-percent-duration flow & 362 & 548 & 410 & 161 & 281 \\
\hline 20-percent-duration flow & 300 & 461 & 345 & 142 & 228 \\
\hline 25-percent-duration flow & 256 & 399 & 302 & 129 & 190 \\
\hline 30-percent-duration flow & 224 & 351 & 269 & 119 & 161 \\
\hline 35-percent-duration flow & 198 & 314 & 245 & 111 & 135 \\
\hline 40-percent-duration flow & 176 & 282 & 224 & 104 & 112 \\
\hline 45-percent-duration flow & 157 & 258 & 206 & 96.7 & 98.1 \\
\hline 50-percent-duration flow & 140 & 236 & 190 & 91.5 & 89.1 \\
\hline 55-percent-duration flow & 125 & 216 & 176 & 86.4 & 81.0 \\
\hline 60-percent-duration flow & 113 & 196 & 163 & 81.3 & 74.5 \\
\hline 65-percent-duration flow & 101 & 180 & 151 & 76.9 & 69.1 \\
\hline 70-percent-duration flow & 91.2 & 164 & 140 & 72.4 & 65.0 \\
\hline 75-percent-duration flow & 82.4 & 146 & 129 & 67.9 & 60.8 \\
\hline 80-percent-duration flow & 73.9 & 127 & 118 & 63.2 & 56.7 \\
\hline 85-percent-duration flow & 65.5 & 108 & 109 & 58.5 & 52.6 \\
\hline 90-percent-duration flow & 57.5 & 87.7 & 97.8 & 52.9 & 48.1 \\
\hline 95-percent-duration flow & 49.0 & 65.7 & 84.2 & 46.4 & 43.0 \\
\hline 99-percent-duration flow & 37.5 & 44.2 & 62.5 & 36.5 & 33.8 \\
\hline Variability index & 0.35 & 0.35 & 0.29 & 0.23 & 0.35 \\
\hline
\end{tabular}


Table 11. Selected annual and seasonal statistics for 77 stations in West Virginia representative of 1930-2002. Continued

[Winter, January 1-March 31; spring, April 1-June 30; summer, July 1-September 30; fall, October 1-December 31; streamflow statistics are in cubic feet per second, except for variability index, which is dimensionless; the record period is in climatic years, April 1 through March 31of the indicated ending year; EPA, U.S. Environmental Protection Agency]

\begin{tabular}{|c|c|c|c|c|c|}
\hline \multirow{2}{*}{ Streamflow statistic } & \multirow{2}{*}{ Annual } & \multicolumn{4}{|c|}{ Season } \\
\hline & & Winter & Spring & Summer & Fall \\
\hline \multicolumn{6}{|c|}{01636500 Shenandoah River at Millville, WV, 1930-2002 } \\
\hline 1-day 2-year hydrologically based flow & 478 & 1,160 & 980 & 522 & 573 \\
\hline 1-day 5-year hydrologically based flow & 354 & 721 & 700 & 381 & 405 \\
\hline 1-day 10-year hydrologically based flow & 303 & 550 & 578 & 323 & 344 \\
\hline 3-day 2-year hydrologically based flow & 513 & 1,210 & 1,030 & 557 & 605 \\
\hline 3-day 5-year hydrologically based flow & 387 & 770 & 754 & 412 & 439 \\
\hline 3-day 10-year hydrologically based flow & 335 & 596 & 633 & 352 & 380 \\
\hline 7-day 2-year hydrologically based flow & 541 & 1,290 & 1,110 & 590 & 638 \\
\hline 7-day 5-year hydrologically based flow & 410 & 824 & 822 & 440 & 462 \\
\hline 7-day 10-year hydrologically based flow & 357 & 639 & 693 & 379 & 402 \\
\hline 14-day 2-year hydrologically based flow & 568 & 1,430 & 1,250 & 628 & 681 \\
\hline 14-day 5-year hydrologically based flow & 432 & 889 & 918 & 465 & 484 \\
\hline 14-day 10-year hydrologically based flow & 378 & 692 & 771 & 398 & 419 \\
\hline 30-day 2-year hydrologically based flow & 615 & 1,770 & 1,580 & 712 & 822 \\
\hline 30-day 5-year hydrologically based flow & 466 & 1,060 & 1,130 & 520 & 545 \\
\hline 30-day 10-year hydrologically based flow & 409 & 804 & 934 & 446 & 455 \\
\hline 1-day 3-year biologically based flow & 304 & 457 & 676 & 331 & 369 \\
\hline 4-day 3-year biologically based flow & 343 & 530 & 773 & 362 & 398 \\
\hline EPA harmonic-mean flow & 1,230 & 1,710 & 2,280 & 912 & 878 \\
\hline 5-percent-duration flow & 8,040 & 11,400 & 8,970 & 3,730 & 6,510 \\
\hline 10-percent-duration flow & 5,480 & 7,670 & 6,310 & 2,440 & 4,180 \\
\hline 15-percent-duration flow & 4,300 & 6,120 & 4,990 & 1,870 & 3,140 \\
\hline 20-percent-duration flow & 3,530 & 5,200 & 4,170 & 1,600 & 2,510 \\
\hline 25-percent-duration flow & 2,980 & 4,520 & 3,610 & 1,410 & 2,140 \\
\hline 30-percent-duration flow & 2,570 & 4,030 & 3,190 & 1,260 & 1,840 \\
\hline 35-percent-duration flow & 2,270 & 3,610 & 2,850 & 1,150 & 1,590 \\
\hline 40-percent-duration flow & 2,000 & 3,220 & 2,600 & 1,050 & 1,380 \\
\hline 45-percent-duration flow & 1,770 & 2,920 & 2,370 & 977 & 1,190 \\
\hline 50-percent-duration flow & 1,590 & 2,640 & 2,190 & 900 & 1,070 \\
\hline 55-percent-duration flow & 1,410 & 2,410 & 2,020 & 842 & 955 \\
\hline 60-percent-duration flow & 1,250 & 2,190 & 1,870 & 792 & 872 \\
\hline 65-percent-duration flow & 1,100 & 1,980 & 1,720 & 741 & 790 \\
\hline 70-percent-duration flow & 970 & 1,770 & 1,600 & 691 & 732 \\
\hline 75-percent-duration flow & 863 & 1,570 & 1,470 & 641 & 677 \\
\hline 80-percent-duration flow & 764 & 1,390 & 1,340 & 590 & 620 \\
\hline 85-percent-duration flow & 678 & 1,160 & 1,210 & 538 & 563 \\
\hline 90-percent-duration flow & 585 & 908 & 1,060 & 482 & 502 \\
\hline 95-percent-duration flow & 482 & 707 & 872 & 416 & 436 \\
\hline 99-percent-duration flow & 367 & 488 & 629 & 324 & 361 \\
\hline Variability index & 0.38 & 0.36 & 0.30 & 0.28 & 0.36 \\
\hline
\end{tabular}


Table 11. elected annual and seasonal statistics for 77 stations in West Virginia representative of 1930-2002. Continued

[Winter, January 1-March 31; spring, April 1-June 30; summer, July 1-September 30; fall, October 1-December 31; streamflow statistics are in cubic feet per second, except for variability index, which is dimensionless; the record period is in climatic years, April 1 through March 31 of the indicated ending year; EPA, U.S. Environmental Protection Agency]

\begin{tabular}{|c|c|c|c|c|c|}
\hline \multirow{2}{*}{ Streamflow statistic } & \multirow{2}{*}{ Annual } & \multicolumn{4}{|c|}{ Season } \\
\hline & & Winter & Spring & Summer & Fall \\
\hline \multicolumn{6}{|c|}{03050000 Tygart Valley River near Dailey, WV, 1932-1957 } \\
\hline 1-day 2-year hydrologically based flow & 6.27 & 97.9 & 37.8 & 7.68 & 8.94 \\
\hline 1-day 5-year hydrologically based flow & 1.89 & 62.6 & 22.7 & 2.26 & 3.02 \\
\hline 1-day 10 -year hydrologically based flow & 0.83 & 47.6 & 17.2 & 0.95 & 1.48 \\
\hline 3-day 2-year hydrologically based flow & 6.79 & 105 & 43.1 & 9.38 & 10.1 \\
\hline 3-day 5-year hydrologically based flow & 2.02 & 65.4 & 25.7 & 2.17 & 3.52 \\
\hline 3-day 10 -year hydrologically based flow & 0.89 & 49.1 & 19.4 & 0.76 & 1.77 \\
\hline 7-day 2-year hydrologically based flow & 7.87 & 122 & 55.5 & 10.9 & 13.2 \\
\hline 7-day 5-year hydrologically based flow & 2.38 & 73.7 & 33.2 & 2.64 & 4.45 \\
\hline 7-day 10-year hydrologically based flow & 1.07 & 54.1 & 24.8 & 1.01 & 2.22 \\
\hline 14-day 2-year hydrologically based flow & 10.1 & 164 & 78.4 & 15.2 & 16.9 \\
\hline 14-day 5-year hydrologically based flow & 3.04 & 93.7 & 45.7 & 3.66 & 5.52 \\
\hline 14-day 10 -year hydrologically based flow & 1.40 & 67.8 & 34.0 & 1.45 & 2.74 \\
\hline 30-day 2-year hydrologically based flow & 14.8 & 282 & 148 & 28.2 & 37.2 \\
\hline 30-day 5-year hydrologically based flow & 5.08 & 178 & 83.3 & 7.80 & 12.0 \\
\hline 30-day 10-year hydrologically based flow & 2.61 & 135 & 61.0 & 3.36 & 5.87 \\
\hline 1-day 3-year biologically based flow & 0.51 & 35.0 & 23.0 & 1.19 & 0.58 \\
\hline 4-day 3-year biologically based flow & 0.81 & 41.1 & 30.2 & 1.87 & 0.86 \\
\hline EPA harmonic-mean flow & 21.5 & 230 & 171 & 15.9 & 8.78 \\
\hline 5-percent-duration flow & 1.260 & 1.920 & 1.200 & 560 & 812 \\
\hline 10-percent-duration flow & 818 & 1,300 & 812 & 285 & 515 \\
\hline 15-percent-duration flow & 606 & 999 & 646 & 191 & 374 \\
\hline 20-percent-duration flow & 471 & 822 & 533 & 147 & 286 \\
\hline 25-percent-duration flow & 392 & 694 & 437 & 119 & 227 \\
\hline 30-percent-duration flow & 323 & 604 & 378 & 96.0 & 189 \\
\hline 35-percent-duration flow & 268 & 539 & 327 & 80.7 & 159 \\
\hline 40-percent-duration flow & 225 & 483 & 277 & 68.1 & 132 \\
\hline 45-percent-duration flow & 189 & 431 & 243 & 58.3 & 111 \\
\hline 50-percent-duration flow & 159 & 383 & 214 & 49.3 & 90.9 \\
\hline 55-percent-duration flow & 132 & 345 & 189 & 42.1 & 74.5 \\
\hline 60 -percent-duration flow & 109 & 310 & 167 & 35.5 & 61.1 \\
\hline 65-percent-duration flow & 87.7 & 278 & 147 & 29.8 & 49.4 \\
\hline 70-percent-duration flow & 69.0 & 248 & 129 & 24.4 & 39.1 \\
\hline 75-percent-duration flow & 53.5 & 219 & 113 & 19.3 & 30.3 \\
\hline 80 -percent-duration flow & 40.0 & 189 & 96.4 & 15.0 & 21.9 \\
\hline 85-percent-duration flow & 27.8 & 155 & 76.7 & 10.7 & 15.5 \\
\hline 90-percent-duration flow & 17.3 & 125 & 59.5 & 6.8 & 8.8 \\
\hline 95-percent-duration flow & 7.4 & 91.2 & 40.8 & 3.6 & 3.5 \\
\hline 99-percent-duration flow & 0.9 & 44.7 & 22.4 & 0.5 & 0.2 \\
\hline Variability index & 0.66 & 0.39 & 0.44 & 0.63 & 0.70 \\
\hline
\end{tabular}


Table 11. Selected annual and seasonal statistics for 77 stations in West Virginia representative of 1930-2002.Continued

[Winter, January 1-March 31; spring, April 1-June 30; summer, July 1-September 30; fall, October 1-December 31; streamflow statistics are in cubic feet per second, except for variability index, which is dimensionless; the record period is in climatic years, April 1 through March 31 of the indicated ending year; EPA, U.S. Environmental Protection Agency]

\begin{tabular}{|c|c|c|c|c|c|}
\hline \multirow{2}{*}{ Streamflow statistic } & \multirow{2}{*}{ Annual } & \multicolumn{4}{|c|}{ Season } \\
\hline & & Winter & Spring & Summer & Fall \\
\hline \multicolumn{6}{|c|}{03050500 Tygart Valley River near Elkins, WV, 1946-2000 } \\
\hline 1-day 2-year hydrologically based flow & 6.45 & 142 & 39.8 & 8.15 & 16.4 \\
\hline 1-day 5-year hydrologically based flow & 1.79 & 90.5 & 17.0 & 2.26 & 4.94 \\
\hline 1-day 10-year hydrologically based flow & 0.85 & 67.0 & 10.2 & 1.04 & 2.46 \\
\hline 3-day 2-year hydrologically based flow & 8.11 & 153 & 49.0 & 11.1 & 19.9 \\
\hline 3-day 5-year hydrologically based flow & 2.34 & 98.7 & 23.1 & 3.14 & 6.38 \\
\hline 3-day 10-year hydrologically based flow & 1.13 & 75.2 & 14.8 & 1.44 & 3.31 \\
\hline 7-day 2-year hydrologically based flow & 12.2 & 173 & 64.3 & 16.5 & 27.3 \\
\hline 7-day 5-year hydrologically based flow & 3.66 & 107 & 31.7 & 4.84 & 9.45 \\
\hline 7-day 10-year hydrologically based flow & 1.75 & 82.1 & 21.2 & 2.20 & 5.14 \\
\hline 14-day 2-year hydrologically based flow & 14.9 & 239 & 92.3 & 21.6 & 37.8 \\
\hline 14-day 5-year hydrologically based flow & 5.22 & 137 & 46.0 & 7.30 & 12.5 \\
\hline 14-day 10 -year hydrologically based flow & 2.97 & 102 & 30.9 & 3.90 & 6.51 \\
\hline 30-day 2-year hydrologically based flow & 23.7 & 439 & 179 & 37.4 & 86.9 \\
\hline 30-day 5-year hydrologically based flow & 8.31 & 272 & 91.9 & 12.2 & 26.9 \\
\hline 30-day 10 -year hydrologically based flow & 4.67 & 204 & 62.6 & 6.39 & 13.1 \\
\hline 1-day 3-year biologically based flow & 1.08 & 62.0 & 28.0 & 1.78 & 1.13 \\
\hline 4-day 3-year biologically based flow & 1.38 & 70.9 & 42.2 & 2.54 & 2.11 \\
\hline EPA harmonic-mean flow & 36.5 & 333 & 270 & 25.8 & 15.6 \\
\hline 5-percent-duration flow & 1,960 & 2,930 & 1,990 & 773 & 1,600 \\
\hline 10-percent-duration flow & 1,270 & 1,980 & 1,280 & 425 & 1,000 \\
\hline 15-percent-duration flow & 939 & 1,500 & 977 & 286 & 739 \\
\hline 20-percent-duration flow & 753 & 1,230 & 810 & 220 & 575 \\
\hline 25-percent-duration flow & 617 & 1,060 & 685 & 174 & 458 \\
\hline 30-percent-duration flow & 506 & 921 & 581 & 143 & 372 \\
\hline 35-percent-duration flow & 426 & 808 & 499 & 119 & 315 \\
\hline 40-percent-duration flow & 352 & 711 & 429 & 98.8 & 262 \\
\hline 45-percent-duration flow & 298 & 638 & 371 & 82.8 & 223 \\
\hline 50-percent-duration flow & 247 & 569 & 324 & 68.3 & 186 \\
\hline 55-percent-duration flow & 209 & 507 & 283 & 57.4 & 159 \\
\hline 60-percent-duration flow & 172 & 452 & 246 & 47.2 & 132 \\
\hline 65-percent-duration flow & 141 & 400 & 210 & 40.1 & 106 \\
\hline 70-percent-duration flow & 111 & 352 & 180 & 32.9 & 83.5 \\
\hline 75-percent-duration flow & 83.4 & 310 & 151 & 26.3 & 65.1 \\
\hline 80-percent-duration flow & 61.6 & 267 & 124 & 20.0 & 49.4 \\
\hline 85-percent-duration flow & 42.4 & 222 & 98.2 & 14.4 & 33.6 \\
\hline 90-percent-duration flow & 26.1 & 183 & 70.9 & 8.4 & 19.2 \\
\hline 95-percent-duration flow & 11.7 & 135 & 42.8 & 4.2 & 8.5 \\
\hline 99-percent-duration flow & 2.8 & 81.8 & 18.0 & 1.6 & 2.3 \\
\hline Variability index & 0.66 & 0.40 & 0.49 & 0.66 & 0.67 \\
\hline
\end{tabular}


Table 11. Selected annual and seasonal statistics for 77 stations in West Virginia representative of 1930-2002. Continued

[Winter, January 1-March 31; spring, April 1-June 30; summer, July 1-September 30; fall, October 1-December 31; streamflow statistics are in cubic feet per second, except for variability index, which is dimensionless; the record period is in climatic years, April 1 through March 31 of the indicated ending year; EPA, U.S. Environmental Protection Agency]

\begin{tabular}{|c|c|c|c|c|c|}
\hline \multirow{2}{*}{ Streamflow statistic } & \multirow{2}{*}{ Annual } & \multicolumn{4}{|c|}{ Season } \\
\hline & & Winter & Spring & Summer & Fall \\
\hline \multicolumn{6}{|c|}{03051000 Tygart Valley River at Belington, WV, 1930-2002 } \\
\hline 1-day 2-year hydrologically based flow & 16.6 & 227 & 71.9 & 19.8 & 28.3 \\
\hline 1-day 5-year hydrologically based flow & 5.69 & 145 & 37.5 & 6.47 & 9.86 \\
\hline 1-day 10 -year hydrologically based flow & 2.86 & 109 & 26.1 & 3.12 & 5.35 \\
\hline 3-day 2-year hydrologically based flow & 17.8 & 244 & 83.0 & 21.7 & 32.1 \\
\hline 3-day 5-year hydrologically based flow & 6.28 & 153 & 43.4 & 7.28 & 11.1 \\
\hline 3-day 10 -year hydrologically based flow & 3.31 & 114 & 30.1 & 3.65 & 6.06 \\
\hline 7-day 2-year hydrologically based flow & 21.6 & 277 & 109 & 27.7 & 42.2 \\
\hline 7-day 5-year hydrologically based flow & 7.41 & 167 & 57.0 & 8.97 & 14.4 \\
\hline 7-day 10-year hydrologically based flow & 3.81 & 125 & 39.5 & 4.36 & 7.69 \\
\hline 14-day 2-year hydrologically based flow & 26.8 & 375 & 158 & 37.2 & 57.5 \\
\hline 14-day 5-year hydrologically based flow & 9.28 & 223 & 81.0 & 12.1 & 18.8 \\
\hline 14-day 10 -year hydrologically based flow & 4.95 & 169 & 55.4 & 6.00 & 9.66 \\
\hline 30-day 2-year hydrologically based flow & 41.7 & 677 & 290 & 67.6 & 133 \\
\hline 30-day 5-year hydrologically based flow & 14.2 & 434 & 155 & 21.1 & 41.6 \\
\hline 30-day 10-year hydrologically based flow & 7.51 & 336 & 109 & 10.3 & 20.1 \\
\hline 1-day 3-year biologically based flow & 2.96 & 90.0 & 51.9 & 4.39 & 4.37 \\
\hline 4-day 3-year biologically based flow & 3.50 & 111 & 68.5 & 5.79 & 5.06 \\
\hline EPA harmonic-mean flow & 62.7 & 531 & 423 & 31.9 & 35.6 \\
\hline 5-percent-duration flow & 3,010 & 4,390 & 3,080 & 1,300 & 2,420 \\
\hline 10-percent-duration flow & 2,000 & 2,970 & 2,090 & 713 & 1,590 \\
\hline 15-percent-duration flow & 1,500 & 2,330 & 1,610 & 471 & 1,150 \\
\hline 20-percent-duration flow & 1,210 & 1,940 & 1,310 & 350 & 885 \\
\hline 25-percent-duration flow & 971 & 1,650 & 1,110 & 275 & 701 \\
\hline 30-percent-duration flow & 810 & 1,440 & 946 & 220 & 581 \\
\hline 35-percent-duration flow & 667 & 1,270 & 806 & 186 & 481 \\
\hline 40-percent-duration flow & 564 & 1,110 & 687 & 153 & 408 \\
\hline 45-percent-duration flow & 468 & 992 & 600 & 131 & 346 \\
\hline 50-percent-duration flow & 397 & 884 & 522 & 110 & 292 \\
\hline 55-percent-duration flow & 327 & 788 & 453 & 93.5 & 246 \\
\hline 60-percent-duration flow & 273 & 703 & 395 & 77.6 & 202 \\
\hline 65-percent-duration flow & 221 & 622 & 341 & 65.7 & 161 \\
\hline 70-percent-duration flow & 175 & 547 & 293 & 54.2 & 126 \\
\hline 75-percent-duration flow & 135 & 482 & 247 & 43.9 & 96.2 \\
\hline 80-percent-duration flow & 98.1 & 420 & 205 & 33.4 & 69.9 \\
\hline 85-percent-duration flow & 67.6 & 354 & 164 & 23.6 & 48.1 \\
\hline 90-percent-duration flow & 42.5 & 289 & 120 & 15.0 & 28.7 \\
\hline 95-percent-duration flow & 19.6 & 218 & 77.0 & 8.2 & 14.6 \\
\hline 99-percent-duration flow & 5.2 & 128 & 34.0 & 2.5 & 3.7 \\
\hline Variability index & 0.65 & 0.39 & 0.48 & 0.64 & 0.66 \\
\hline
\end{tabular}


Table 11. Selected annual and seasonal statistics for 77 stations in West Virginia representative of 1930-2002.—Continued

[Winter, January 1-March 31; spring, April 1-June 30; summer, July 1-September 30; fall, October 1-December 31; streamflow statistics are in cubic feet per second, except for variability index, which is dimensionless; the record period is in climatic years, April 1 through March 31 of the indicated ending year; EPA, U.S. Environmental Protection Agency]

\begin{tabular}{|c|c|c|c|c|c|}
\hline \multirow{2}{*}{ Streamflow statistic } & \multirow{2}{*}{ Annual } & \multicolumn{4}{|c|}{ Season } \\
\hline & & Winter & Spring & Summer & Fall \\
\hline \multicolumn{6}{|c|}{03052000 Middle Fork River at Audra, WV, 1943-1964 } \\
\hline 1-day 2-year hydrologically based flow & 4.05 & 112 & 33.3 & 5.15 & 9.56 \\
\hline 1-day 5-year hydrologically based flow & 1.04 & 79.0 & 19.1 & 1.43 & 2.36 \\
\hline 1-day 10 -year hydrologically based flow & 0.49 & 65.7 & 14.3 & 0.69 & 1.05 \\
\hline 3-day 2-year hydrologically based flow & 4.46 & 121 & 39.2 & 5.76 & 10.8 \\
\hline 3-day 5 -year hydrologically based flow & 1.14 & 83.8 & 22.0 & 1.60 & 2.54 \\
\hline 3-day 10 -year hydrologically based flow & 0.53 & 69.1 & 16.3 & 0.77 & 1.09 \\
\hline 7-day 2-year hydrologically based flow & 5.65 & 140 & 52.1 & 7.50 & 13.9 \\
\hline 7-day 5-year hydrologically based flow & 1.37 & 92.9 & 29.8 & 2.02 & 3.02 \\
\hline 7-day 10 -year hydrologically based flow & 0.62 & 74.9 & 22.3 & 0.96 & 1.24 \\
\hline 14-day 2-year hydrologically based flow & 7.61 & 193 & 70.3 & 11.0 & 19.4 \\
\hline 14-day 5-year hydrologically based flow & 1.75 & 120 & 40.0 & 2.82 & 3.98 \\
\hline 14-day 10 -year hydrologically based flow & 0.77 & 92.3 & 29.9 & 1.30 & 1.53 \\
\hline 30-day 2-year hydrologically based flow & 12.4 & 315 & 136 & 20.8 & 43.9 \\
\hline 30-day 5-year hydrologically based flow & 3.09 & 240 & 73.5 & 5.77 & 9.24 \\
\hline 30-day 10-year hydrologically based flow & 1.41 & 208 & 53.1 & 2.72 & 3.49 \\
\hline 1-day 3-year biologically based flow & 0.42 & 49.9 & 22.0 & 0.55 & 0.55 \\
\hline 4-day 3-year biologically based flow & 0.54 & 64.7 & 33.7 & 0.72 & 1.22 \\
\hline EPA harmonic-mean flow & 18.5 & 281 & 188 & 13.2 & 7.61 \\
\hline 5-percent-duration flow & 1,250 & 1,800 & 1,140 & 490 & 1,010 \\
\hline 10-percent-duration flow & 821 & 1,300 & 813 & 296 & 665 \\
\hline 15-percent-duration flow & 647 & 1,040 & 667 & 193 & 491 \\
\hline 20-percent-duration flow & 529 & 858 & 567 & 142 & 386 \\
\hline 25-percent-duration flow & 435 & 740 & 483 & 112 & 315 \\
\hline 30-percent-duration flow & 369 & 650 & 416 & 89.8 & 253 \\
\hline 35-percent-duration flow & 311 & 582 & 358 & 73.9 & 207 \\
\hline 40-percent-duration flow & 261 & 522 & 310 & 60.1 & 172 \\
\hline 45-percent-duration flow & 218 & 469 & 269 & 48.2 & 148 \\
\hline 50-percent-duration flow & 180 & 426 & 234 & 38.8 & 127 \\
\hline 55-percent-duration flow & 152 & 388 & 203 & 32.6 & 109 \\
\hline 60-percent-duration flow & 126 & 353 & 180 & 27.1 & 90.9 \\
\hline 65-percent-duration flow & 102 & 319 & 161 & 22.6 & 73.9 \\
\hline 70-percent-duration flow & 78.9 & 287 & 142 & 18.0 & 57.6 \\
\hline 75-percent-duration flow & 57.3 & 252 & 123 & 14.4 & 41.3 \\
\hline 80-percent-duration flow & 38.4 & 219 & 103 & 10.9 & 25.0 \\
\hline 85-percent-duration flow & 24.9 & 188 & 79.5 & 7.8 & 14.2 \\
\hline 90-percent-duration flow & 14.1 & 154 & 57.8 & 4.8 & 6.6 \\
\hline 95-percent-duration flow & 5.3 & 120 & 36.8 & 2.1 & 2.5 \\
\hline 99-percent-duration flow & 1.1 & 68.9 & 19.3 & 0.7 & 0.5 \\
\hline Variability index & 0.71 & 0.35 & 0.45 & 0.69 & 0.77 \\
\hline
\end{tabular}


Table 11. Selected annual and seasonal statistics for 77 stations in West Virginia representative of 1930-2002. - Continued

[Winter, January 1-March 31; spring, April 1-June 30; summer, July 1-September 30; fall, October 1-December 31; streamflow statistics are in cubic feet per second, except for variability index, which is dimensionless; the record period is in climatic years, April 1 through March 31 of the indicated ending year; EPA, U.S. Environmental Protection Agency]

\begin{tabular}{|c|c|c|c|c|c|}
\hline \multirow{2}{*}{ Streamflow statistic } & \multirow{2}{*}{ Annual } & \multicolumn{4}{|c|}{ Season } \\
\hline & & Winter & Spring & Summer & Fall \\
\hline \multicolumn{6}{|c|}{03052500 Sand Run near Buckhannon, WV, 1948-2000 } \\
\hline 1-day 2-year hydrologically based flow & 0.22 & 7.62 & 1.12 & 0.25 & 1.05 \\
\hline 1-day 5-year hydrologically based flow & 0.01 & 5.07 & 0.44 & 0.02 & 0.27 \\
\hline 1-day 10-year hydrologically based flow & 0.00 & 3.96 & 0.25 & 0.00 & 0.10 \\
\hline 3-day 2-year hydrologically based flow & 0.24 & 8.28 & 1.65 & 0.30 & 1.18 \\
\hline 3-day 5-year hydrologically based flow & 0.03 & 5.45 & 0.51 & 0.03 & 0.31 \\
\hline 3-day 10-year hydrologically based flow & 0.00 & 4.27 & 0.21 & 0.00 & 0.11 \\
\hline 7-day 2-year hydrologically based flow & 0.33 & 9.95 & 2.03 & 0.43 & 1.54 \\
\hline 7-day 5-year hydrologically based flow & 0.06 & 6.28 & 0.70 & 0.07 & 0.49 \\
\hline 7-day 10-year hydrologically based flow & 0.02 & 4.89 & 0.35 & 0.02 & 0.23 \\
\hline 14-day 2-year hydrologically based flow & 0.51 & 13.6 & 2.93 & 0.81 & 2.04 \\
\hline 14-day 5-year hydrologically based flow & 0.11 & 8.25 & 1.15 & 0.12 & 0.69 \\
\hline 14-day 10 -year hydrologically based flow & 0.04 & 6.34 & 0.65 & 0.03 & 0.34 \\
\hline 30-day 2-year hydrologically based flow & 0.96 & 24.4 & 5.91 & 1.59 & 5.55 \\
\hline 30-day 5-year hydrologically based flow & 0.28 & 16.1 & 2.60 & 0.42 & 1.52 \\
\hline 30-day 10-year hydrologically based flow & 0.13 & 12.5 & 1.67 & 0.19 & 0.59 \\
\hline 1-day 3-year biologically based flow & 0.00 & 3.48 & 0.79 & 0.00 & 0.00 \\
\hline 4-day 3-year biologically based flow & 0.00 & 4.23 & 1.23 & 0.00 & 0.00 \\
\hline EPA harmonic-mean flow & 1.15 & 19.4 & 9.81 & 0.50 & 0.74 \\
\hline 5-percent-duration flow & 101 & 156 & 103 & 39.6 & 91.6 \\
\hline 10-percent-duration flow & 65.8 & 99.4 & 65.4 & 21.9 & 56.7 \\
\hline 15-percent-duration flow & 48.9 & 74.9 & 49.8 & 14.5 & 42.4 \\
\hline 20-percent-duration flow & 39.8 & 61.3 & 40.9 & 10.4 & 34.3 \\
\hline 25-percent-duration flow & 32.5 & 53.2 & 33.3 & 7.7 & 27.7 \\
\hline 30-percent-duration flow & 27.4 & 46.5 & 28.5 & 6.2 & 23.3 \\
\hline 35-percent-duration flow & 23.0 & 41.0 & 23.8 & 5.0 & 19.2 \\
\hline 40-percent-duration flow & 19.3 & 36.9 & 20.6 & 4.0 & 16.0 \\
\hline 45-percent-duration flow & 16.0 & 33.4 & 17.3 & 3.3 & 13.1 \\
\hline 50-percent-duration flow & 13.2 & 30.3 & 15.1 & 2.8 & 10.7 \\
\hline 55-percent-duration flow & 10.7 & 27.5 & 12.9 & 2.3 & 8.9 \\
\hline 60-percent-duration flow & 8.3 & 25.1 & 10.8 & 2.0 & 7.3 \\
\hline 65 -percent-duration flow & 6.6 & 22.7 & 8.8 & 1.6 & 5.8 \\
\hline 70-percent-duration flow & 4.9 & 20.4 & 7.3 & 1.3 & 4.5 \\
\hline 75-percent-duration flow & 3.6 & 18.0 & 5.8 & 0.9 & 3.5 \\
\hline 80-percent-duration flow & 2.6 & 15.6 & 4.3 & 0.6 & 2.6 \\
\hline 85-percent-duration flow & 1.8 & 13.2 & 3.1 & 0.4 & 1.8 \\
\hline 90-percent-duration flow & 1.0 & 10.7 & 2.1 & 0.2 & 1.2 \\
\hline 95-percent-duration flow & 0.4 & 8.0 & 1.2 & 0.1 & 0.5 \\
\hline 99-percent-duration flow & 0.0 & 4.7 & 0.4 & 0.0 & 0.0 \\
\hline Variability index & 0.72 & 0.38 & 0.58 & 0.76 & 0.68 \\
\hline
\end{tabular}


Table 11. Selected annual and seasonal statistics for 77 stations in West Virginia representative of 1930-2002.—Continued

[Winter, January 1-March 31; spring, April 1-June 30; summer, July 1-September 30; fall, October 1-December 31; streamflow statistics are in cubic feet per second, except for variability index, which is dimensionless; the record period is in climatic years, April 1 through March 31 of the indicated ending year; EPA, U.S. Environmental Protection Agency]

\begin{tabular}{|c|c|c|c|c|c|}
\hline \multirow{2}{*}{ Streamflow statistic } & \multirow{2}{*}{ Annual } & \multicolumn{4}{|c|}{ Season } \\
\hline & & Winter & Spring & Summer & Fall \\
\hline \multicolumn{6}{|c|}{03053500 Buckhannon River at Hall, WV, 1930-2002 } \\
\hline 1-day 2-year hydrologically based flow & 12.3 & 171 & 49.5 & 14.3 & 30.2 \\
\hline 1-day 5-year hydrologically based flow & 4.28 & 118 & 23.8 & 5.33 & 8.94 \\
\hline 1-day 10-year hydrologically based flow & 2.27 & 95.9 & 15.7 & 3.01 & 4.07 \\
\hline 3-day 2-year hydrologically based flow & 13.3 & 186 & 56.2 & 15.9 & 33.1 \\
\hline 3-day 5-year hydrologically based flow & 4.64 & 126 & 27.0 & 5.82 & 9.66 \\
\hline 3-day 10 -year hydrologically based flow & 2.44 & 101 & 17.7 & 3.24 & 4.37 \\
\hline 7-day 2-year hydrologically based flow & 15.5 & 216 & 70.8 & 19.3 & 41.2 \\
\hline 7-day 5-year hydrologically based flow & 5.29 & 140 & 33.8 & 6.80 & 11.9 \\
\hline 7-day 10-year hydrologically based flow & 2.75 & 111 & 22.2 & 3.69 & 5.31 \\
\hline 14-day 2-year hydrologically based flow & 20.3 & 293 & 96.1 & 26.9 & 54.1 \\
\hline 14-day 5-year hydrologically based flow & 6.77 & 182 & 47.5 & 9.09 & 15.5 \\
\hline 14-day 10-year hydrologically based flow & 3.47 & 143 & 32.0 & 4.80 & 6.85 \\
\hline 30-day 2-year hydrologically based flow & 34.0 & 510 & 180 & 53.2 & 129 \\
\hline 30-day 5-year hydrologically based flow & 11.2 & 333 & 90.6 & 17.6 & 36.5 \\
\hline 30-day 10 -year hydrologically based flow & 5.52 & 260 & 62.3 & 8.91 & 14.8 \\
\hline 1-day 3-year biologically based flow & 2.45 & 79.9 & 28.9 & 2.89 & 2.68 \\
\hline 4-day 3-year biologically based flow & 3.00 & 98.5 & 37.0 & 4.72 & 3.64 \\
\hline EPA harmonic-mean flow & 42.4 & 437 & 271 & 31.6 & 17.7 \\
\hline 5-percent-duration flow & 2,150 & 3,030 & 2,090 & 940 & 1,880 \\
\hline 10-percent-duration flow & 1,450 & 2,120 & 1,430 & 551 & 1,190 \\
\hline 15-percent-duration flow & 1,100 & 1,640 & 1,120 & 387 & 901 \\
\hline 20-percent-duration flow & 866 & 1,370 & 918 & 288 & 722 \\
\hline 25-percent-duration flow & 736 & 1,190 & 768 & 220 & 595 \\
\hline 30-percent-duration flow & 610 & 1,050 & 649 & 177 & 499 \\
\hline 35-percent-duration flow & 522 & 934 & 556 & 147 & 410 \\
\hline 40-percent-duration flow & 435 & 832 & 480 & 121 & 352 \\
\hline 45-percent-duration flow & 370 & 753 & 412 & 103 & 294 \\
\hline 50-percent-duration flow & 307 & 682 & 355 & 86.9 & 251 \\
\hline 55-percent-duration flow & 257 & 623 & 310 & 73.9 & 209 \\
\hline 60-percent-duration flow & 211 & 563 & 268 & 61.9 & 175 \\
\hline 65-percent-duration flow & 172 & 504 & 232 & 51.2 & 143 \\
\hline 70-percent-duration flow & 137 & 449 & 198 & 42.3 & 113 \\
\hline 75-percent-duration flow & 105 & 395 & 166 & 33.9 & 88.9 \\
\hline 80-percent-duration flow & 77.2 & 340 & 135 & 26.1 & 66.6 \\
\hline 85-percent-duration flow & 53.9 & 285 & 106 & 19.4 & 45.5 \\
\hline 90-percent-duration flow & 34.0 & 229 & 77.6 & 13.1 & 27.8 \\
\hline 95-percent-duration flow & 16.4 & 178 & 47.4 & 7.3 & 11.7 \\
\hline 99-percent-duration flow & 3.9 & 107 & 19.5 & 2.7 & 0.8 \\
\hline Variability index & 0.64 & 0.37 & 0.49 & 0.62 & 0.65 \\
\hline
\end{tabular}


Table 11. Selected annual and seasonal statistics for 77 stations in West Virginia representative of 1930-2002. - Continued

[Winter, January 1-March 31; spring, April 1-June 30; summer, July 1-September 30; fall, October 1-December 31; streamflow statistics are in cubic feet per second, except for variability index, which is dimensionless; the record period is in climatic years, April 1 through March 31 of the indicated ending year; EPA, U.S. Environmental Protection Agency]

\begin{tabular}{|c|c|c|c|c|c|}
\hline \multirow{2}{*}{ Streamflow statistic } & \multirow{2}{*}{ Annual } & \multicolumn{4}{|c|}{ Season } \\
\hline & & Winter & Spring & Summer & Fall \\
\hline \multicolumn{6}{|c|}{03054500 Tygart Valley River at Philippi, WV, 1941-2000 } \\
\hline 1-day 2-year hydrologically based flow & 39.3 & 544 & 178 & 47.8 & 91.3 \\
\hline 1-day 5-year hydrologically based flow & 15.6 & 377 & 91.8 & 18.3 & 33.3 \\
\hline 1-day 10-year hydrologically based flow & 9.37 & 310 & 63.0 & 10.6 & 18.5 \\
\hline 3-day 2-year hydrologically based flow & 42.5 & 587 & 201 & 52.3 & 101 \\
\hline 3-day 5-year hydrologically based flow & 16.8 & 399 & 103 & 19.9 & 36.6 \\
\hline 3-day 10-year hydrologically based flow & 10.0 & 324 & 70.7 & 11.4 & 20.3 \\
\hline 7-day 2-year hydrologically based flow & 49.7 & 684 & 256 & 62.9 & 119 \\
\hline 7-day 5-year hydrologically based flow & 19.3 & 442 & 128 & 23.6 & 42.5 \\
\hline 7-day 10-year hydrologically based flow & 11.5 & 354 & 86.2 & 13.5 & 23.7 \\
\hline 14-day 2-year hydrologically based flow & 63.4 & 944 & 354 & 86.6 & 160 \\
\hline 14-day 5-year hydrologically based flow & 24.3 & 563 & 173 & 31.7 & 55.9 \\
\hline 14-day 10 -year hydrologically based flow & 14.4 & 429 & 116 & 18.0 & 30.1 \\
\hline 30-day 2-year hydrologically based flow & 103 & 1,670 & 634 & 158 & 360 \\
\hline 30-day 5-year hydrologically based flow & 38.4 & 1,070 & 329 & 57.5 & 120 \\
\hline 30-day 10-year hydrologically based flow & 22.4 & 822 & 230 & 32.5 & 60.8 \\
\hline 1-day 3-year biologically based flow & 8.20 & 260 & 121 & 11.0 & 10.0 \\
\hline 4-day 3-year biologically based flow & 10.5 & 302 & 161 & 14.7 & 12.3 \\
\hline EPA harmonic-mean flow & 224 & 1,400 & 1,030 & 131 & 118 \\
\hline 5-percent-duration flow & 6,800 & 9,770 & 6,720 & 2,930 & 5,940 \\
\hline 10-percent-duration flow & 4,580 & 6,720 & 4,700 & 1,740 & 3,930 \\
\hline 15-percent-duration flow & 3,490 & 5,270 & 3,610 & 1,230 & 2,850 \\
\hline 20-percent-duration flow & 2,810 & 4,450 & 2,980 & 902 & 2,290 \\
\hline 25-percent-duration flow & 2,370 & 3,850 & 2,530 & 692 & 1,940 \\
\hline 30-percent-duration flow & 2,000 & 3,400 & 2,180 & 550 & 1,630 \\
\hline 35-percent-duration flow & 1,720 & 3,020 & 1,910 & 449 & 1,370 \\
\hline 40-percent-duration flow & 1,470 & 2,690 & 1,670 & 371 & 1,170 \\
\hline 45-percent-duration flow & 1,250 & 2,430 & 1,460 & 310 & 981 \\
\hline 50-percent-duration flow & 1,040 & 2,210 & 1,280 & 265 & 819 \\
\hline 55-percent-duration flow & 851 & 2,020 & 1,110 & 225 & 688 \\
\hline 60-percent-duration flow & 699 & 1,840 & 958 & 189 & 570 \\
\hline 65 -percent-duration flow & 557 & 1,670 & 828 & 159 & 455 \\
\hline 70-percent-duration flow & 438 & 1,470 & 706 & 132 & 362 \\
\hline 75-percent-duration flow & 332 & 1,300 & 581 & 109 & 281 \\
\hline 80-percent-duration flow & 246 & 1,120 & 463 & 85.4 & 208 \\
\hline 85-percent-duration flow & 173 & 917 & 365 & 62.8 & 152 \\
\hline 90-percent-duration flow & 111 & 726 & 265 & 40.3 & 89.6 \\
\hline 95-percent-duration flow & 54.8 & 548 & 176 & 24.5 & 46.6 \\
\hline 99-percent-duration flow & 16.1 & 339 & 75.6 & 11.2 & 12.6 \\
\hline Variability index & 0.63 & 0.37 & 0.48 & 0.62 & 0.63 \\
\hline
\end{tabular}


Table 11. Selected annual and seasonal statistics for 77 stations in West Virginia representative of 1930-2002. - Continued

[Winter, January 1-March 31; spring, April 1-June 30; summer, July 1-September 30; fall, October 1-December 31; streamflow statistics are in cubic feet per second, except for variability index, which is dimensionless; the record period is in climatic years, April 1 through March 31 of the indicated ending year; EPA, U.S. Environmental Protection Agency]

\begin{tabular}{|c|c|c|c|c|c|}
\hline \multirow{2}{*}{ Streamflow statistic } & \multirow{2}{*}{ Annual } & \multicolumn{4}{|c|}{ Season } \\
\hline & & Winter & Spring & Summer & Fall \\
\hline \multicolumn{6}{|c|}{03056250 Three Fork Creek near Grafton, WV, 1986-2000 } \\
\hline 1-day 2-year hydrologically based flow & 2.58 & 47.2 & 10.6 & 2.61 & 7.75 \\
\hline 1-day 5-year hydrologically based flow & 1.11 & 31.1 & 5.26 & 1.11 & 3.49 \\
\hline 1-day 10-year hydrologically based flow & 0.68 & 24.2 & 3.51 & 0.68 & 2.24 \\
\hline 3-day 2-year hydrologically based flow & 2.83 & 55.3 & 12.2 & 2.86 & 8.30 \\
\hline 3-day 5-year hydrologically based flow & 1.19 & 36.2 & 5.92 & 1.19 & 3.84 \\
\hline 3-day 10 -year hydrologically based flow & 0.73 & 28.1 & 3.85 & 0.73 & 2.51 \\
\hline 7-day 2-year hydrologically based flow & 3.45 & 63.9 & 16.2 & 3.53 & 9.84 \\
\hline 7-day 5-year hydrologically based flow & 1.39 & 40.1 & 7.68 & 1.39 & 4.81 \\
\hline 7-day 10-year hydrologically based flow & 0.83 & 30.9 & 4.94 & 0.82 & 3.29 \\
\hline 14-day 2-year hydrologically based flow & 4.58 & 90.6 & 21.1 & 4.83 & 11.8 \\
\hline 14-day 5-year hydrologically based flow & 1.86 & 51.0 & 9.72 & 1.88 & 5.76 \\
\hline 14-day 10 -year hydrologically based flow & 1.11 & 37.7 & 6.33 & 1.10 & 4.02 \\
\hline 30-day 2-year hydrologically based flow & 7.37 & 170 & 40.7 & 8.83 & 20.6 \\
\hline 30-day 5-year hydrologically based flow & 3.11 & 104 & 18.1 & 3.27 & 9.33 \\
\hline 30-day 10-year hydrologically based flow & 1.87 & 79.8 & 11.4 & 1.93 & 6.56 \\
\hline 1-day 3-year biologically based flow & 0.56 & 17.0 & 13.3 & 0.52 & 1.16 \\
\hline 4-day 3-year biologically based flow & 0.69 & 21.6 & 17.3 & 0.81 & 1.14 \\
\hline EPA harmonic-mean flow & 15.0 & 116 & 93.6 & 6.86 & 9.85 \\
\hline 5-percent-duration flow & 692 & 1,030 & 640 & 211 & 608 \\
\hline 10-percent-duration flow & 432 & 688 & 402 & 114 & 371 \\
\hline 15-percent-duration flow & 325 & 540 & 310 & 74.6 & 272 \\
\hline 20-percent-duration flow & 254 & 440 & 242 & 55.0 & 215 \\
\hline 25-percent-duration flow & 202 & 378 & 201 & 42.0 & 175 \\
\hline 30-percent-duration flow & 169 & 331 & 173 & 31.9 & 146 \\
\hline 35-percent-duration flow & 142 & 293 & 150 & 25.6 & 121 \\
\hline 40-percent-duration flow & 121 & 254 & 131 & 21.1 & 97.9 \\
\hline 45-percent-duration flow & 101 & 225 & 116 & 17.8 & 80.1 \\
\hline 50-percent-duration flow & 82.7 & 201 & 101 & 15.2 & 66.7 \\
\hline 55-percent-duration flow & 67.1 & 180 & 87.3 & 12.7 & 54.3 \\
\hline 60-percent-duration flow & 52.2 & 159 & 74.2 & 10.1 & 41.2 \\
\hline 65-percent-duration flow & 38.8 & 141 & 61.7 & 8.1 & 30.8 \\
\hline 70-percent-duration flow & 28.1 & 127 & 50.8 & 6.5 & 24.6 \\
\hline 75-percent-duration flow & 20.8 & 113 & 41.0 & 5.1 & 18.1 \\
\hline 80-percent-duration flow & 14.9 & 99.4 & 30.8 & 3.9 & 13.5 \\
\hline 85-percent-duration flow & 10.1 & 85.0 & 23.0 & 2.9 & 10.5 \\
\hline 90-percent-duration flow & 6.6 & 70.3 & 17.0 & 2.0 & 8.1 \\
\hline 95-percent-duration flow & 3.2 & 53.4 & 10.0 & 1.3 & 5.8 \\
\hline 99-percent-duration flow & 1.1 & 30.2 & 3.6 & 0.7 & 2.4 \\
\hline Variability index & 0.71 & 0.39 & 0.54 & 0.67 & 0.64 \\
\hline
\end{tabular}


Table 11. Selected annual and seasonal statistics for 77 stations in West Virginia representative of 1930-2002._Continued

[Winter, January 1-March 31; spring, April 1-June 30; summer, July 1-September 30; fall, October 1-December 31; streamflow statistics are in cubic feet per second, except for variability index, which is dimensionless; the record period is in climatic years, April 1 through March 31 of the indicated ending year; EPA, U.S. Environmental Protection Agency]

\begin{tabular}{|c|c|c|c|c|c|}
\hline \multirow{2}{*}{ Streamflow statistic } & \multirow{2}{*}{ Annual } & \multicolumn{4}{|c|}{ Season } \\
\hline & & Winter & Spring & Summer & Fall \\
\hline \multicolumn{6}{|c|}{03057500 Skin Creek near Brownsville, WV, 1948-1960 } \\
\hline 1-day 2-year hydrologically based flow & 0.18 & 9.04 & 0.96 & 0.20 & 0.45 \\
\hline 1-day 5-year hydrologically based flow & 0.00 & 5.20 & 0.44 & 0.00 & 0.00 \\
\hline 1-day 10-year hydrologically based flow & 0.00 & 3.75 & 0.30 & 0.00 & 0.00 \\
\hline 3-day 2-year hydrologically based flow & 0.25 & 9.84 & 1.13 & 0.27 & 0.44 \\
\hline 3-day 5-year hydrologically based flow & 0.00 & 5.48 & 0.53 & 0.00 & 0.00 \\
\hline 3-day 10-year hydrologically based flow & 0.00 & 3.91 & 0.37 & 0.00 & 0.00 \\
\hline 7-day 2-year hydrologically based flow & 0.34 & 12.4 & 1.79 & 0.15 & 1.29 \\
\hline 7-day 5-year hydrologically based flow & 0.00 & 6.82 & 0.81 & 0.00 & 0.00 \\
\hline 7-day 10-year hydrologically based flow & 0.00 & 4.72 & 0.54 & 0.00 & 0.00 \\
\hline 14-day 2-year hydrologically based flow & 0.21 & 22.5 & 2.41 & 0.42 & 3.04 \\
\hline 14-day 5-year hydrologically based flow & 0.00 & 11.6 & 1.21 & 0.00 & 0.05 \\
\hline 14-day 10 -year hydrologically based flow & 0.00 & 7.63 & 0.88 & 0.00 & 0.00 \\
\hline 30-day 2-year hydrologically based flow & 0.49 & 40.9 & 6.76 & 1.11 & 4.79 \\
\hline 30-day 5-year hydrologically based flow & 0.01 & 30.3 & 2.86 & 0.07 & 0.19 \\
\hline 30-day 10-year hydrologically based flow & 0.00 & 25.1 & 1.87 & 0.00 & 0.00 \\
\hline 1-day 3-year biologically based flow & 0.00 & 2.53 & 0.39 & 0.00 & 0.00 \\
\hline 4-day 3-year biologically based flow & 0.00 & 3.09 & 0.50 & 0.00 & 0.00 \\
\hline EPA harmonic-mean flow & 0.96 & 21.2 & 6.84 & 0.52 & 0.44 \\
\hline 5-percent-duration flow & 185 & 330 & 159 & 67.3 & 142 \\
\hline 10-percent-duration flow & 105 & 196 & 95.7 & 30.3 & 74.2 \\
\hline 15-percent-duration flow & 67.8 & 140 & 62.0 & 16.5 & 49.7 \\
\hline 20-percent-duration flow & 51.8 & 107 & 46.9 & 10.9 & 36.5 \\
\hline 25-percent-duration flow & 40.4 & 85.4 & 36.4 & 7.9 & 28.0 \\
\hline 30-percent-duration flow & 31.7 & 68.9 & 28.9 & 5.8 & 20.9 \\
\hline 35-percent-duration flow & 25.8 & 59.3 & 23.8 & 4.5 & 16.8 \\
\hline 40-percent-duration flow & 20.9 & 51.9 & 19.8 & 3.6 & 13.5 \\
\hline 45-percent-duration flow & 16.7 & 45.4 & 16.5 & 2.8 & 11.2 \\
\hline 50-percent-duration flow & 13.4 & 40.2 & 14.1 & 2.2 & 9.4 \\
\hline 55-percent-duration flow & 10.5 & 36.1 & 12.3 & 1.6 & 8.0 \\
\hline 60-percent-duration flow & 7.9 & 32.2 & 10.4 & 1.2 & 6.7 \\
\hline 65 -percent-duration flow & 6.1 & 29.1 & 8.5 & 0.9 & 5.4 \\
\hline 70-percent-duration flow & 4.5 & 26.0 & 6.8 & 0.6 & 4.3 \\
\hline 75-percent-duration flow & 3.2 & 23.1 & 5.1 & 0.4 & 3.2 \\
\hline 80-percent-duration flow & 2.0 & 20.2 & 3.9 & 0.2 & 2.2 \\
\hline 85-percent-duration flow & 1.1 & 17.0 & 2.8 & 0.1 & 0.6 \\
\hline 90-percent-duration flow & 0.3 & 13.7 & 1.9 & 0.0 & 0.0 \\
\hline 95-percent-duration flow & 0.0 & 9.4 & 1.0 & 0.0 & 0.0 \\
\hline 99-percent-duration flow & 0.0 & 4.0 & 0.4 & 0.0 & 0.0 \\
\hline Variability index & 0.59 & 0.45 & 0.65 & 0.72 & 0.60 \\
\hline
\end{tabular}


Table 11. Selected annual and seasonal statistics for 77 stations in West Virginia representative of 1930-2002.—Continued

[Winter, January 1-March 31; spring, April 1-June 30; summer, July 1-September 30; fall, October 1-December 31; streamflow statistics are in cubic feet per second, except for variability index, which is dimensionless; the record period is in climatic years, April 1 through March 31 of the indicated ending year; EPA, U.S. Environmental Protection Agency]

\begin{tabular}{|c|c|c|c|c|c|}
\hline \multirow{2}{*}{ Streamflow statistic } & \multirow{2}{*}{ Annual } & \multicolumn{4}{|c|}{ Season } \\
\hline & & Winter & Spring & Summer & Fall \\
\hline \multicolumn{6}{|c|}{03058000 WEST Fork River below Stonewall Jackson Dam near Weston, WV, 1948-1978 } \\
\hline 1-day 2-year hydrologically based flow & 1.03 & 33.3 & 4.61 & 1.51 & 4.38 \\
\hline 1-day 5-year hydrologically based flow & 0.10 & 19.6 & 1.72 & 0.25 & 0.78 \\
\hline 1-day 10-year hydrologically based flow & 0.00 & 14.3 & 0.92 & 0.01 & 0.03 \\
\hline 3-day 2-year hydrologically based flow & 1.26 & 35.6 & 5.31 & 1.77 & 4.76 \\
\hline 3-day 5-year hydrologically based flow & 0.22 & 21.2 & 2.03 & 0.42 & 0.86 \\
\hline 3-day 10-year hydrologically based flow & 0.00 & 15.8 & 1.12 & 0.14 & 0.04 \\
\hline 7-day 2-year hydrologically based flow & 1.64 & 46.6 & 7.19 & 2.15 & 5.50 \\
\hline 7-day 5-year hydrologically based flow & 0.40 & 25.7 & 3.10 & 0.54 & 1.08 \\
\hline 7-day 10-year hydrologically based flow & 0.13 & 18.1 & 1.98 & 0.22 & 0.35 \\
\hline 14-day 2-year hydrologically based flow & 2.34 & 73.8 & 10.6 & 3.05 & 8.83 \\
\hline 14-day 5-year hydrologically based flow & 0.57 & 36.6 & 4.94 & 0.80 & 1.79 \\
\hline 14-day 10-year hydrologically based flow & 0.23 & 24.0 & 3.33 & 0.38 & 0.56 \\
\hline 30-day 2-year hydrologically based flow & 4.39 & 139 & 24.3 & 6.32 & 19.7 \\
\hline 30-day 5-year hydrologically based flow & 1.21 & 74.8 & 10.1 & 1.91 & 3.87 \\
\hline 30-day 10 -year hydrologically based flow & 0.58 & 48.4 & 6.42 & 0.96 & 1.35 \\
\hline 1-day 3-year biologically based flow & 0.00 & 13.9 & 1.86 & 0.09 & 0.00 \\
\hline 4-day 3-year biologically based flow & 0.00 & 14.9 & 3.89 & 0.19 & 0.00 \\
\hline EPA harmonic-mean flow & 5.51 & 71.6 & 35.6 & 2.94 & 2.90 \\
\hline 5-percent-duration flow & 710 & 1,110 & 697 & 225 & 575 \\
\hline 10-percent-duration flow & 419 & 712 & 403 & 106 & 332 \\
\hline 15-percent-duration flow & 286 & 535 & 268 & 62.4 & 229 \\
\hline 20-percent-duration flow & 211 & 409 & 199 & 44.0 & 163 \\
\hline 25-percent-duration flow & 163 & 330 & 155 & 33.2 & 126 \\
\hline 30-percent-duration flow & 128 & 281 & 125 & 25.9 & 100 \\
\hline 35-percent-duration flow & 104 & 241 & 102 & 20.5 & 80.7 \\
\hline 40-percent-duration flow & 84.2 & 208 & 83.4 & 15.9 & 67.2 \\
\hline 45-percent-duration flow & 67.2 & 180 & 71.9 & 13.3 & 54.7 \\
\hline 50-percent-duration flow & 55.0 & 158 & 61.3 & 10.9 & 44.0 \\
\hline 55-percent-duration flow & 43.8 & 140 & 52.0 & 8.9 & 36.5 \\
\hline 60-percent-duration flow & 34.1 & 124 & 43.2 & 7.4 & 29.8 \\
\hline 65-percent-duration flow & 26.7 & 110 & 35.3 & 6.0 & 24.7 \\
\hline 70-percent-duration flow & 19.8 & 95.6 & 28.8 & 4.7 & 19.3 \\
\hline 75-percent-duration flow & 14.4 & 81.9 & 22.6 & 3.5 & 14.6 \\
\hline 80-percent-duration flow & 9.5 & 68.3 & 16.2 & 2.6 & 10.1 \\
\hline 85-percent-duration flow & 6.1 & 54.7 & 11.7 & 1.7 & 4.7 \\
\hline 90-percent-duration flow & 3.4 & 43.3 & 7.8 & 0.8 & 2.3 \\
\hline 95-percent-duration flow & 1.1 & 31.6 & 4.7 & 0.4 & 0.6 \\
\hline 99-percent-duration flow & 0.2 & 14.1 & 1.7 & 0.1 & 0.1 \\
\hline Variability index & 0.83 & 0.46 & 0.65 & 0.79 & 0.86 \\
\hline
\end{tabular}


Table 11. Selected annual and seasonal statistics for 77 stations in West Virginia representative of 1930-2002.—Continued

[Winter, January 1-March 31; spring, April 1-June 30; summer, July 1-September 30; fall, October 1-December 31; streamflow statistics are in cubic feet per second, except for variability index, which is dimensionless; the record period is in climatic years, April 1 through March 31of the indicated ending year; EPA, U.S. Environmental Protection Agency]

\begin{tabular}{|c|c|c|c|c|c|}
\hline \multirow{2}{*}{ Streamflow statistic } & \multirow{2}{*}{ Annual } & \multicolumn{4}{|c|}{ Season } \\
\hline & & Winter & Spring & Summer & Fall \\
\hline \multicolumn{6}{|c|}{03058500 West Fork River at Butcherville, WV, 1934-1989 } \\
\hline 1-day 2-year hydrologically based flow & 3.13 & 61.9 & 11.1 & 3.62 & 7.80 \\
\hline 1-day 5-year hydrologically based flow & 1.14 & 37.1 & 4.73 & 1.25 & 2.42 \\
\hline 1-day 10-year hydrologically based flow & 0.66 & 26.9 & 2.86 & 0.70 & 1.23 \\
\hline 3-day 2-year hydrologically based flow & 3.56 & 66.4 & 12.9 & 4.16 & 8.96 \\
\hline 3-day 5-year hydrologically based flow & 1.28 & 40.0 & 5.47 & 1.42 & 2.72 \\
\hline 3-day 10-year hydrologically based flow & 0.73 & 29.5 & 3.29 & 0.79 & 1.37 \\
\hline 7-day 2-year hydrologically based flow & 4.43 & 82.2 & 17.6 & 5.49 & 12.0 \\
\hline 7-day 5-year hydrologically based flow & 1.57 & 47.8 & 7.28 & 1.82 & 3.65 \\
\hline 7-day 10-year hydrologically based flow & 0.88 & 34.9 & 4.33 & 1.00 & 1.82 \\
\hline 14-day 2-year hydrologically based flow & 6.47 & 123 & 25.5 & 8.60 & 16.9 \\
\hline 14-day 5-year hydrologically based flow & 2.13 & 67.7 & 10.9 & 2.75 & 4.94 \\
\hline 14-day 10-year hydrologically based flow & 1.14 & 47.4 & 6.67 & 1.44 & 2.36 \\
\hline 30-day 2-year hydrologically based flow & 12.4 & 244 & 60.3 & 20.5 & 44.8 \\
\hline 30-day 5-year hydrologically based flow & 3.88 & 140 & 25.0 & 6.30 & 11.8 \\
\hline 30-day 10-year hydrologically based flow & 1.94 & 96.3 & 15.1 & 3.14 & 4.91 \\
\hline 1-day 3-year biologically based flow & 0.59 & 22.0 & 3.36 & 0.69 & 0.88 \\
\hline 4-day 3-year biologically based flow & 0.75 & 25.1 & 5.89 & 0.92 & 1.08 \\
\hline EPA harmonic-mean flow & 16.8 & 151 & 64.1 & 9.24 & 9.27 \\
\hline 5-percent-duration flow & 1,270 & 1,830 & 1,240 & 544 & 1,040 \\
\hline 10-percent-duration flow & 738 & 1,210 & 728 & 248 & 598 \\
\hline 15-percent-duration flow & 511 & 911 & 505 & 143 & 412 \\
\hline 20-percent-duration flow & 383 & 717 & 380 & 99.9 & 311 \\
\hline 25-percent-duration flow & 307 & 582 & 302 & 76.0 & 246 \\
\hline 30-percent-duration flow & 248 & 489 & 243 & 60.8 & 198 \\
\hline 35-percent-duration flow & 201 & 420 & 195 & 51.5 & 162 \\
\hline 40-percent-duration flow & 163 & 366 & 158 & 43.6 & 132 \\
\hline 45-percent-duration flow & 131 & 321 & 133 & 37.0 & 108 \\
\hline 50-percent-duration flow & 106 & 280 & 112 & 31.2 & 89.0 \\
\hline 55-percent-duration flow & 85.8 & 250 & 93.9 & 26.0 & 72.3 \\
\hline 60-percent-duration flow & 69.0 & 222 & 80.9 & 21.3 & 58.6 \\
\hline 65-percent-duration flow & 55.2 & 199 & 68.3 & 17.4 & 47.9 \\
\hline 70-percent-duration flow & 44.1 & 174 & 56.7 & 13.9 & 38.4 \\
\hline 75-percent-duration flow & 33.9 & 148 & 46.3 & 10.3 & 30.0 \\
\hline 80-percent-duration flow & 24.7 & 124 & 36.5 & 7.1 & 22.0 \\
\hline 85-percent-duration flow & 16.6 & 103 & 26.9 & 4.7 & 13.9 \\
\hline 90-percent-duration flow & 9.4 & 81.0 & 18.6 & 2.9 & 7.2 \\
\hline 95-percent-duration flow & 3.7 & 58.4 & 10.7 & 1.6 & 2.7 \\
\hline 99-percent-duration flow & 1.1 & 29.1 & 3.3 & 0.8 & 0.6 \\
\hline Variability index & 0.75 & 0.45 & 0.61 & 0.73 & 0.75 \\
\hline
\end{tabular}


Table 11. Selected annual and seasonal statistics for 77 stations in West Virginia representative of 1930-2002._Continued

[Winter, January 1-March 31; spring, April 1-June 30; summer, July 1-September 30; fall, October 1-December 31; streamflow statistics are in cubic feet per second, except for variability index, which is dimensionless; the record period is in climatic years, April 1 through March 31 of the indicated ending year; EPA, U.S. Environmental Protection Agency]

\begin{tabular}{|c|c|c|c|c|c|}
\hline \multirow{2}{*}{ Streamflow statistic } & \multirow{2}{*}{ Annual } & \multicolumn{4}{|c|}{ Season } \\
\hline & & Winter & Spring & Summer & Fall \\
\hline \multicolumn{6}{|c|}{03059000 West Fork River at Clarksburg, WV, 1935-1983 } \\
\hline 1-day 2-year hydrologically based flow & 2.22 & 138 & 16.4 & 3.13 & 10.7 \\
\hline 1-day 5-year hydrologically based flow & 0.00 & 77.4 & 3.85 & 0.00 & 0.00 \\
\hline 1-day 10-year hydrologically based flow & 0.00 & 52.2 & 1.49 & 0.00 & 0.00 \\
\hline 3-day 2-year hydrologically based flow & 2.87 & 147 & 20.3 & 3.85 & 12.8 \\
\hline 3-day 5-year hydrologically based flow & 0.00 & 82.4 & 4.93 & 0.00 & 0.26 \\
\hline 3-day 10 -year hydrologically based flow & 0.00 & 57.7 & 1.95 & 0.00 & 0.00 \\
\hline 7-day 2-year hydrologically based flow & 4.21 & 182 & 31.6 & 5.79 & 20.8 \\
\hline 7-day 5-year hydrologically based flow & 0.00 & 96.0 & 8.47 & 0.23 & 1.19 \\
\hline 7-day 10-year hydrologically based flow & 0.00 & 65.1 & 3.56 & 0.00 & 0.00 \\
\hline 14-day 2-year hydrologically based flow & 7.56 & 255 & 51.4 & 10.5 & 29.5 \\
\hline 14-day 5-year hydrologically based flow & 0.57 & 128 & 16.1 & 1.14 & 2.34 \\
\hline 14-day 10 -year hydrologically based flow & 0.00 & 84.6 & 7.74 & 0.00 & 0.00 \\
\hline 30-day 2-year hydrologically based flow & 20.3 & 495 & 122 & 31.2 & 75.5 \\
\hline 30-day 5-year hydrologically based flow & 2.22 & 271 & 43.5 & 6.38 & 17.3 \\
\hline 30-day 10 -year hydrologically based flow & 0.00 & 180 & 22.9 & 1.96 & 3.39 \\
\hline 1-day 3-year biologically based flow & 0.00 & 40.0 & 1.78 & 0.00 & 0.00 \\
\hline 4-day 3-year biologically based flow & 0.00 & 49.6 & 4.31 & 0.00 & 0.00 \\
\hline EPA harmonic-mean flow & 17.3 & 291 & 51.0 & 7.35 & 13.3 \\
\hline 5-percent-duration flow & 2,410 & 3,720 & 2,300 & 1,020 & 1,990 \\
\hline 10-percent-duration flow & 1,500 & 2,470 & 1,490 & 506 & 1,120 \\
\hline 15-percent-duration flow & 1,050 & 1,830 & 1,060 & 320 & 750 \\
\hline 20-percent-duration flow & 786 & 1,470 & 789 & 222 & 582 \\
\hline 25-percent-duration flow & 618 & 1,220 & 605 & 164 & 457 \\
\hline 30-percent-duration flow & 502 & 1,030 & 495 & 126 & 359 \\
\hline 35-percent-duration flow & 401 & 875 & 402 & 100 & 297 \\
\hline 40-percent-duration flow & 331 & 765 & 331 & 82.2 & 239 \\
\hline 45-percent-duration flow & 267 & 674 & 279 & 67.2 & 196 \\
\hline 50-percent-duration flow & 217 & 592 & 235 & 55.4 & 161 \\
\hline 55-percent-duration flow & 174 & 531 & 201 & 44.8 & 134 \\
\hline 60-percent-duration flow & 137 & 471 & 171 & 36.5 & 109 \\
\hline 65-percent-duration flow & 109 & 417 & 144 & 28.5 & 85.8 \\
\hline 70-percent-duration flow & 83.4 & 364 & 120 & 21.1 & 65.8 \\
\hline 75-percent-duration flow & 61.8 & 314 & 96.1 & 14.5 & 50.2 \\
\hline 80-percent-duration flow & 43.2 & 264 & 73.4 & 8.2 & 34.2 \\
\hline 85-percent-duration flow & 26.7 & 218 & 53.4 & 3.1 & 19.2 \\
\hline 90-percent-duration flow & 12.1 & 173 & 33.6 & 1.5 & 6.8 \\
\hline 95-percent-duration flow & 1.9 & 118 & 13.3 & 0.1 & 0.1 \\
\hline 99-percent-duration flow & 0.0 & 49.7 & 1.5 & 0.0 & 0.0 \\
\hline Variability index & 0.89 & 0.45 & 0.65 & 1.13 & 1.19 \\
\hline
\end{tabular}


Table 11. Selected annual and seasonal statistics for 77 stations in West Virginia representative of 1930-2002.—Continued

[Winter, January 1-March 31; spring, April 1-June 30; summer, July 1-September 30; fall, October 1-December 31; streamflow statistics are in cubic feet per second, except for variability index, which is dimensionless; the record period is in climatic years, April 1 through March 31 of the indicated ending year; EPA, U.S. Environmental Protection Agency]

\begin{tabular}{|c|c|c|c|c|c|}
\hline \multirow{2}{*}{ Streamflow statistic } & \multirow{2}{*}{ Annual } & \multicolumn{4}{|c|}{ Season } \\
\hline & & Winter & Spring & Summer & Fall \\
\hline \multicolumn{6}{|c|}{03061000 West Fork River at Enterprise, WV, 1935-1983 } \\
\hline 1-day 2-year hydrologically based flow & 39.7 & 316 & 115 & 41.7 & 58.4 \\
\hline 1-day 5-year hydrologically based flow & 18.9 & 186 & 59.2 & 20.5 & 27.8 \\
\hline 1-day 10-year hydrologically based flow & 12.0 & 134 & 38.7 & 13.4 & 18.0 \\
\hline 3-day 2-year hydrologically based flow & 42.1 & 336 & 124 & 44.2 & 63.0 \\
\hline 3-day 5-year hydrologically based flow & 20.5 & 199 & 64.3 & 22.3 & 29.9 \\
\hline 3-day 10-year hydrologically based flow & 13.2 & 146 & 42.5 & 14.9 & 19.5 \\
\hline 7-day 2-year hydrologically based flow & 47.0 & 400 & 152 & 51.1 & 76.5 \\
\hline 7-day 5-year hydrologically based flow & 23.6 & 222 & 77.2 & 26.5 & 35.7 \\
\hline 7-day 10-year hydrologically based flow & 15.6 & 159 & 50.9 & 18.2 & 22.9 \\
\hline 14-day 2-year hydrologically based flow & 57.5 & 535 & 193 & 67.6 & 92.6 \\
\hline 14-day 5-year hydrologically based flow & 30.6 & 290 & 98.6 & 35.7 & 43.3 \\
\hline 14-day 10 -year hydrologically based flow & 21.5 & 206 & 66.3 & 24.8 & 28.2 \\
\hline 30-day 2-year hydrologically based flow & 82.5 & 982 & 330 & 111 & 170 \\
\hline 30-day 5-year hydrologically based flow & 44.3 & 564 & 165 & 57.7 & 73.1 \\
\hline 30-day 10-year hydrologically based flow & 31.3 & 397 & 111 & 39.7 & 45.3 \\
\hline 1-day 3-year biologically based flow & 9.51 & 89.7 & 69.9 & 9.98 & 15.0 \\
\hline 4-day 3-year biologically based flow & 15.9 & 142 & 65.7 & 19.0 & 19.9 \\
\hline EPA harmonic-mean flow & 176 & 697 & 407 & 104 & 109 \\
\hline 5-percent-duration flow & 4,420 & 6,880 & 4,490 & 1,850 & 3,500 \\
\hline 10-percent-duration flow & 2,840 & 4,560 & 2,900 & 1,000 & 2,090 \\
\hline 15-percent-duration flow & 2,090 & 3,500 & 2,170 & 665 & 1,490 \\
\hline 20-percent-duration flow & 1,610 & 2,840 & 1,660 & 480 & 1,110 \\
\hline 25-percent-duration flow & 1,300 & 2,410 & 1,340 & 367 & 881 \\
\hline 30-percent-duration flow & 1,060 & 2,090 & 1,110 & 296 & 723 \\
\hline 35-percent-duration flow & 868 & 1,830 & 930 & 245 & 595 \\
\hline 40-percent-duration flow & 721 & 1,610 & 782 & 205 & 487 \\
\hline 45-percent-duration flow & 591 & 1,430 & 668 & 180 & 401 \\
\hline 50-percent-duration flow & 480 & 1,290 & 572 & 157 & 329 \\
\hline 55-percent-duration flow & 391 & 1,150 & 494 & 138 & 273 \\
\hline 60-percent-duration flow & 317 & 1,030 & 426 & 120 & 229 \\
\hline 65 -percent-duration flow & 260 & 916 & 363 & 104 & 192 \\
\hline 70-percent-duration flow & 211 & 807 & 308 & 90.3 & 159 \\
\hline 75-percent-duration flow & 168 & 699 & 265 & 77.7 & 130 \\
\hline 80-percent-duration flow & 132 & 587 & 224 & 66.0 & 105 \\
\hline 85-percent-duration flow & 101 & 474 & 179 & 53.5 & 76.3 \\
\hline 90-percent-duration flow & 72.6 & 375 & 138 & 40.9 & 51.3 \\
\hline 95-percent-duration flow & 43.0 & 264 & 96.7 & 28.6 & 33.2 \\
\hline 99-percent-duration flow & 20.9 & 120 & 40.3 & 13.8 & 19.1 \\
\hline Variability index & 0.62 & 0.42 & 0.51 & 0.54 & 0.61 \\
\hline
\end{tabular}


Table 11. Selected annual and seasonal statistics for 77 stations in West Virginia representative of 1930-2002.—Continued

[Winter, January 1-March 31; spring, April 1-June 30; summer, July 1-September 30; fall, October 1-December 31; streamflow statistics are in cubic feet per second, except for variability index, which is dimensionless; the record period is in climatic years, April 1 through March 31 of the indicated ending year; EPA, U.S. Environmental Protection Agency]

\begin{tabular}{|c|c|c|c|c|c|}
\hline \multirow{2}{*}{ Streamflow statistic } & \multirow{2}{*}{ Annual } & \multicolumn{4}{|c|}{ Season } \\
\hline & & Winter & Spring & Summer & Fall \\
\hline \multicolumn{6}{|c|}{03061500 Buffalo Creek at Barrackville, WV, 1934-1996 } \\
\hline 1-day 2-year hydrologically based flow & 2.57 & 36.4 & 10.5 & 2.91 & 4.40 \\
\hline 1-day 5-year hydrologically based flow & 1.02 & 20.6 & 4.88 & 1.14 & 1.61 \\
\hline 1-day 10-year hydrologically based flow & 0.60 & 14.7 & 3.07 & 0.66 & 0.93 \\
\hline 3-day 2-year hydrologically based flow & 2.92 & 39.7 & 11.9 & 3.22 & 4.97 \\
\hline 3-day 5-year hydrologically based flow & 1.21 & 22.2 & 5.57 & 1.31 & 1.91 \\
\hline 3-day 10-year hydrologically based flow & 0.72 & 15.8 & 3.52 & 0.79 & 1.14 \\
\hline 7-day 2-year hydrologically based flow & 3.49 & 46.5 & 15.0 & 3.86 & 6.19 \\
\hline 7-day 5-year hydrologically based flow & 1.55 & 25.4 & 7.09 & 1.66 & 2.46 \\
\hline 7-day 10-year hydrologically based flow & 0.98 & 18.1 & 4.53 & 1.04 & 1.50 \\
\hline 14-day 2-year hydrologically based flow & 4.33 & 60.8 & 20.8 & 4.99 & 7.78 \\
\hline 14-day 5-year hydrologically based flow & 1.94 & 31.1 & 9.66 & 2.14 & 3.08 \\
\hline 14-day 10 -year hydrologically based flow & 1.24 & 21.5 & 6.29 & 1.35 & 1.88 \\
\hline 30-day 2-year hydrologically based flow & 6.26 & 127 & 40.1 & 8.23 & 15.9 \\
\hline 30-day 5-year hydrologically based flow & 2.70 & 65.5 & 18.0 & 3.45 & 5.97 \\
\hline 30-day 10-year hydrologically based flow & 1.72 & 44.0 & 11.6 & 2.20 & 3.51 \\
\hline 1-day 3-year biologically based flow & 0.57 & 10.0 & 5.50 & 0.66 & 0.70 \\
\hline 4-day 3-year biologically based flow & 0.69 & 11.2 & 6.86 & 0.84 & 0.92 \\
\hline EPA harmonic-mean flow & 13.1 & 81.4 & 55.4 & 6.83 & 7.86 \\
\hline 5-percent-duration flow & 711 & 1,160 & 744 & 236 & 485 \\
\hline 10-percent-duration flow & 415 & 715 & 451 & 106 & 271 \\
\hline 15-percent-duration flow & 285 & 533 & 311 & 62.8 & 186 \\
\hline 20-percent-duration flow & 213 & 419 & 232 & 45.4 & 137 \\
\hline 25-percent-duration flow & 168 & 341 & 183 & 35.2 & 109 \\
\hline 30-percent-duration flow & 134 & 286 & 154 & 28.4 & 88.6 \\
\hline 35-percent-duration flow & 110 & 243 & 129 & 23.1 & 72.2 \\
\hline 40-percent-duration flow & 90.5 & 213 & 110 & 19.0 & 60.4 \\
\hline 45-percent-duration flow & 73.2 & 186 & 94.3 & 16.1 & 49.5 \\
\hline 50-percent-duration flow & 60.0 & 164 & 80.5 & 13.5 & 39.7 \\
\hline 55-percent-duration flow & 47.7 & 144 & 69.2 & 11.3 & 32.4 \\
\hline 60-percent-duration flow & 37.0 & 127 & 58.3 & 9.2 & 25.7 \\
\hline 65-percent-duration flow & 28.7 & 112 & 49.1 & 7.8 & 19.8 \\
\hline 70-percent-duration flow & 21.9 & 98.4 & 40.8 & 6.5 & 15.2 \\
\hline 75-percent-duration flow & 16.6 & 85.2 & 33.2 & 5.5 & 11.9 \\
\hline 80-percent-duration flow & 12.0 & 72.6 & 26.1 & 4.5 & 9.1 \\
\hline 85-percent-duration flow & 8.3 & 59.7 & 20.2 & 3.5 & 6.7 \\
\hline 90-percent-duration flow & 5.4 & 46.4 & 15.1 & 2.7 & 4.4 \\
\hline 95-percent-duration flow & 3.1 & 30.0 & 9.6 & 1.8 & 2.7 \\
\hline 99-percent-duration flow & 1.1 & 14.6 & 3.7 & 0.7 & 0.9 \\
\hline Variability index & 0.72 & 0.47 & 0.56 & 0.63 & 0.69 \\
\hline
\end{tabular}


Table 11. Selected annual and seasonal statistics for 77 stations in West Virginia representative of 1930-2002.—Continued

[Winter, January 1-March 31; spring, April 1-June 30; summer, July 1-September 30; fall, October 1-December 31; streamflow statistics are in cubic feet per second, except for variability index, which is dimensionless; the record period is in climatic years, April 1 through March 31 of the indicated ending year; EPA, U.S. Environmental Protection Agency]

\begin{tabular}{|c|c|c|c|c|c|}
\hline \multirow{2}{*}{ Streamflow statistic } & \multirow{2}{*}{ Annual } & \multicolumn{4}{|c|}{ Season } \\
\hline & & Winter & Spring & Summer & Fall \\
\hline \multicolumn{6}{|c|}{03065000 Dry Fork at Hendricks, WV, 1944-1993 } \\
\hline 1-day 2-year hydrologically based flow & 26.3 & 203 & 96.7 & 28.6 & 55.7 \\
\hline 1-day 5-year hydrologically based flow & 13.5 & 149 & 56.9 & 14.4 & 25.5 \\
\hline 1-day 10-year hydrologically based flow & 9.29 & 127 & 42.7 & 9.76 & 16.4 \\
\hline 3-day 2-year hydrologically based flow & 27.9 & 213 & 111 & 31.1 & 62.0 \\
\hline 3-day 5-year hydrologically based flow & 14.3 & 154 & 64.3 & 15.5 & 27.1 \\
\hline 3-day 10-year hydrologically based flow & 9.87 & 131 & 47.6 & 10.5 & 17.0 \\
\hline 7-day 2-year hydrologically based flow & 32.3 & 241 & 146 & 37.3 & 76.2 \\
\hline 7-day 5-year hydrologically based flow & 16.3 & 166 & 81.9 & 18.3 & 32.7 \\
\hline 7-day 10-year hydrologically based flow & 11.2 & 139 & 58.8 & 12.3 & 20.2 \\
\hline 14-day 2-year hydrologically based flow & 39.4 & 314 & 199 & 48.8 & 98.5 \\
\hline 14-day 5-year hydrologically based flow & 19.7 & 195 & 107 & 23.4 & 40.7 \\
\hline 14-day 10 -year hydrologically based flow & 13.6 & 154 & 75.0 & 15.6 & 24.6 \\
\hline 30-day 2-year hydrologically based flow & 59.2 & 533 & 345 & 83.4 & 201 \\
\hline 30-day 5-year hydrologically based flow & 28.1 & 326 & 188 & 39.7 & 79.3 \\
\hline 30-day 10-year hydrologically based flow & 18.7 & 246 & 132 & 25.6 & 44.8 \\
\hline 1-day 3-year biologically based flow & 7.09 & 117 & 68.8 & 8.00 & 12.0 \\
\hline 4-day 3-year biologically based flow & 9.13 & 132 & 87.8 & 10.7 & 13.4 \\
\hline EPA harmonic-mean flow & 148 & 503 & 494 & 82.6 & 92.6 \\
\hline 5-percent-duration flow & 2,740 & 4,000 & 2,680 & 1,100 & 2,280 \\
\hline 10-percent-duration flow & 1,800 & 2,750 & 1,930 & 675 & 1,500 \\
\hline 15-percent-duration flow & 1,380 & 2,170 & 1,520 & 487 & 1,150 \\
\hline 20-percent-duration flow & 1,140 & 1,770 & 1,270 & 379 & 918 \\
\hline 25-percent-duration flow & 945 & 1,490 & 1,100 & 302 & 760 \\
\hline 30-percent-duration flow & 789 & 1,300 & 959 & 250 & 641 \\
\hline 35-percent-duration flow & 673 & 1,150 & 837 & 211 & 552 \\
\hline 40-percent-duration flow & 576 & 1,020 & 744 & 179 & 484 \\
\hline 45-percent-duration flow & 493 & 906 & 655 & 155 & 426 \\
\hline 50-percent-duration flow & 424 & 806 & 574 & 134 & 373 \\
\hline 55-percent-duration flow & 360 & 718 & 511 & 115 & 322 \\
\hline 60-percent-duration flow & 304 & 637 & 448 & 99.6 & 277 \\
\hline 65-percent-duration flow & 254 & 562 & 389 & 85.7 & 236 \\
\hline 70-percent-duration flow & 209 & 494 & 339 & 73.3 & 197 \\
\hline 75-percent-duration flow & 169 & 431 & 290 & 61.9 & 160 \\
\hline 80-percent-duration flow & 130 & 369 & 243 & 50.4 & 122 \\
\hline 85-percent-duration flow & 96.0 & 307 & 198 & 39.2 & 86.3 \\
\hline 90-percent-duration flow & 64.3 & 251 & 149 & 28.5 & 54.1 \\
\hline 95-percent-duration flow & 35.7 & 185 & 99.3 & 19.6 & 32.0 \\
\hline 99-percent-duration flow & 14.2 & 137 & 47.8 & 9.9 & 12.9 \\
\hline Variability index & 0.56 & 0.40 & 0.43 & 0.52 & 0.55 \\
\hline
\end{tabular}


Table 11. Selected annual and seasonal statistics for 77 stations in West Virginia representative of 1930-2002.—Continued

[Winter, January 1-March 31; spring, April 1-June 30; summer, July 1-September 30; fall, October 1-December 31; streamflow statistics are in cubic feet per second, except for variability index, which is dimensionless; the record period is in climatic years, April 1 through March 31 of the indicated ending year; EPA, U.S. Environmental Protection Agency]

\begin{tabular}{|c|c|c|c|c|c|}
\hline \multirow{2}{*}{ Streamflow statistic } & \multirow{2}{*}{ Annual } & \multicolumn{4}{|c|}{ Season } \\
\hline & & Winter & Spring & Summer & Fall \\
\hline \multicolumn{6}{|c|}{03066000 Blackwater River at Davis, WV, 1930-2002 } \\
\hline 1-day 2-year hydrologically based flow & 9.08 & 54.4 & 28.0 & 9.60 & 17.3 \\
\hline 1-day 5-year hydrologically based flow & 5.08 & 36.5 & 17.6 & 5.33 & 8.78 \\
\hline 1-day 10-year hydrologically based flow & 3.66 & 29.1 & 13.6 & 3.86 & 5.98 \\
\hline 3-day 2-year hydrologically based flow & 9.64 & 57.8 & 31.5 & 10.2 & 19.6 \\
\hline 3-day 5-year hydrologically based flow & 5.50 & 39.9 & 19.5 & 5.84 & 9.74 \\
\hline 3-day 10 -year hydrologically based flow & 4.05 & 32.5 & 14.9 & 4.35 & 6.50 \\
\hline 7-day 2-year hydrologically based flow & 11.0 & 65.7 & 38.7 & 12.0 & 23.9 \\
\hline 7-day 5-year hydrologically based flow & 6.39 & 44.5 & 23.5 & 6.88 & 11.8 \\
\hline 7-day 10 -year hydrologically based flow & 4.79 & 36.1 & 17.8 & 5.13 & 7.85 \\
\hline 14-day 2-year hydrologically based flow & 13.2 & 84.6 & 51.0 & 15.2 & 30.6 \\
\hline 14-day 5-year hydrologically based flow & 7.51 & 53.7 & 30.9 & 8.22 & 14.5 \\
\hline 14-day 10 -year hydrologically based flow & 5.55 & 42.5 & 23.5 & 5.95 & 9.27 \\
\hline 30-day 2-year hydrologically based flow & 18.6 & 144 & 82.9 & 23.3 & 55.2 \\
\hline 30-day 5-year hydrologically based flow & 9.87 & 89.8 & 50.0 & 11.9 & 24.3 \\
\hline 30-day 10-year hydrologically based flow & 7.11 & 68.9 & 37.7 & 8.50 & 14.6 \\
\hline 1-day 3-year biologically based flow & 3.35 & 24.9 & 21.0 & 4.09 & 3.78 \\
\hline 4-day 3-year biologically based flow & 3.78 & 33.3 & 25.3 & 4.77 & 5.00 \\
\hline EPA harmonic-mean flow & 45.8 & 134 & 123 & 25.6 & 30.9 \\
\hline 5-percent-duration flow & 718 & 1,040 & 684 & 361 & 577 \\
\hline 10-percent-duration flow & 479 & 740 & 492 & 212 & 376 \\
\hline 15-percent-duration flow & 361 & 574 & 388 & 150 & 284 \\
\hline 20-percent-duration flow & 289 & 465 & 319 & 111 & 229 \\
\hline 25-percent-duration flow & 239 & 393 & 270 & 87.4 & 193 \\
\hline 30-percent-duration flow & 204 & 340 & 233 & 71.2 & 165 \\
\hline 35-percent-duration flow & 174 & 296 & 203 & 59.2 & 145 \\
\hline 40-percent-duration flow & 149 & 260 & 177 & 51.2 & 125 \\
\hline 45-percent-duration flow & 129 & 234 & 158 & 43.8 & 109 \\
\hline 50-percent-duration flow & 110 & 210 & 140 & 37.5 & 94.7 \\
\hline 55-percent-duration flow & 95.1 & 189 & 123 & 32.5 & 82.2 \\
\hline 60-percent-duration flow & 80.8 & 169 & 109 & 27.9 & 71.6 \\
\hline 65-percent-duration flow & 67.5 & 151 & 96.4 & 23.9 & 61.4 \\
\hline 70-percent-duration flow & 56.4 & 132 & 84.3 & 20.4 & 51.6 \\
\hline 75-percent-duration flow & 45.9 & 116 & 72.4 & 17.3 & 42.9 \\
\hline 80-percent-duration flow & 36.1 & 101 & 61.7 & 14.8 & 34.0 \\
\hline 85-percent-duration flow & 26.9 & 85.9 & 51.6 & 12.2 & 24.8 \\
\hline 90-percent-duration flow & 18.3 & 67.0 & 40.5 & 9.3 & 17.5 \\
\hline 95-percent-duration flow & 11.9 & 51.0 & 29.0 & 6.8 & 12.1 \\
\hline 99-percent-duration flow & 5.4 & 34.1 & 15.6 & 4.2 & 4.6 \\
\hline Variability index & 0.54 & 0.39 & 0.41 & 0.52 & 0.50 \\
\hline
\end{tabular}


Table 11. Selected annual and seasonal statistics for 77 stations in West Virginia representative of 1930-2002.—Continued

[Winter, January 1-March 31; spring, April 1-June 30; summer, July 1-September 30; fall, October 1-December 31; streamflow statistics are in cubic feet per second, except for variability index, which is dimensionless; the record period is in climatic years, April 1 through March 31of the indicated ending year; EPA, U.S. Environmental Protection Agency]

\begin{tabular}{|c|c|c|c|c|c|}
\hline \multirow{2}{*}{ Streamflow statistic } & \multirow{2}{*}{ Annual } & \multicolumn{4}{|c|}{ Season } \\
\hline & & Winter & Spring & Summer & Fall \\
\hline \multicolumn{6}{|c|}{03069000 Shavers Fork at Parsons, WV, 1944-1993 } \\
\hline 1-day 2-year hydrologically based flow & 28.9 & 138 & 94.3 & 32.4 & 57.7 \\
\hline 1-day 5-year hydrologically based flow & 13.8 & 98.1 & 55.7 & 15.6 & 25.4 \\
\hline 1-day 10-year hydrologically based flow & 9.02 & 82.2 & 41.5 & 10.2 & 15.4 \\
\hline 3-day 2-year hydrologically based flow & 30.7 & 148 & 104 & 35.2 & 64.4 \\
\hline 3-day 5-year hydrologically based flow & 14.6 & 103 & 60.7 & 16.8 & 28.0 \\
\hline 3-day 10 -year hydrologically based flow & 9.52 & 85.4 & 44.8 & 11.0 & 16.8 \\
\hline 7-day 2-year hydrologically based flow & 35.9 & 169 & 129 & 42.8 & 76.1 \\
\hline 7-day 5-year hydrologically based flow & 16.8 & 113 & 73.8 & 20.1 & 32.7 \\
\hline 7-day 10-year hydrologically based flow & 10.8 & 92.7 & 53.7 & 12.9 & 19.4 \\
\hline 14-day 2-year hydrologically based flow & 44.1 & 219 & 164 & 55.0 & 97.8 \\
\hline 14-day 5-year hydrologically based flow & 20.5 & 133 & 95.5 & 25.9 & 40.9 \\
\hline 14-day 10 -year hydrologically based flow & 13.1 & 103 & 70.0 & 16.7 & 23.7 \\
\hline 30-day 2-year hydrologically based flow & 65.3 & 364 & 268 & 90.1 & 188 \\
\hline 30-day 5-year hydrologically based flow & 30.1 & 221 & 165 & 43.9 & 77.0 \\
\hline 30-day 10-year hydrologically based flow & 19.2 & 166 & 125 & 28.7 & 42.2 \\
\hline 1-day 3-year biologically based flow & 7.00 & 75.0 & 63.0 & 10.9 & 13.0 \\
\hline 4-day 3-year biologically based flow & 10.0 & 91.1 & 83.4 & 13.4 & 14.1 \\
\hline EPA harmonic-mean flow & 142 & 340 & 387 & 90.1 & 86.3 \\
\hline 5-percent-duration flow & 1,850 & 2,620 & 1,910 & 868 & 1,590 \\
\hline 10-percent-duration flow & 1,260 & 1,790 & 1,330 & 551 & 1,060 \\
\hline 15-percent-duration flow & 962 & 1,430 & 1,040 & 415 & 781 \\
\hline 20-percent-duration flow & 779 & 1,170 & 875 & 336 & 630 \\
\hline 25-percent-duration flow & 649 & 1,000 & 763 & 283 & 528 \\
\hline 30-percent-duration flow & 555 & 870 & 672 & 240 & 455 \\
\hline 35-percent-duration flow & 481 & 760 & 593 & 209 & 398 \\
\hline 40-percent-duration flow & 415 & 675 & 524 & 183 & 354 \\
\hline 45-percent-duration flow & 364 & 605 & 470 & 162 & 313 \\
\hline 50-percent-duration flow & 317 & 542 & 419 & 142 & 281 \\
\hline 55-percent-duration flow & 279 & 483 & 374 & 125 & 252 \\
\hline 60-percent-duration flow & 243 & 431 & 335 & 109 & 225 \\
\hline 65-percent-duration flow & 210 & 385 & 298 & 93.1 & 199 \\
\hline 70-percent-duration flow & 181 & 336 & 265 & 79.3 & 172 \\
\hline 75-percent-duration flow & 153 & 292 & 234 & 68.2 & 144 \\
\hline 80-percent-duration flow & 124 & 252 & 204 & 56.6 & 117 \\
\hline 85-percent-duration flow & 97.9 & 207 & 173 & 45.0 & 88.4 \\
\hline 90-percent-duration flow & 69.0 & 169 & 136 & 34.0 & 56.8 \\
\hline 95-percent-duration flow & 39.3 & 127 & 96.0 & 22.2 & 31.7 \\
\hline 99-percent-duration flow & 15.6 & 86.5 & 48.0 & 10.6 & 14.0 \\
\hline Variability index & 0.49 & 0.40 & 0.38 & 0.47 & 0.48 \\
\hline
\end{tabular}


Table 11. Selected annual and seasonal statistics for 77 stations in West Virginia representative of 1930-2002.-Continued

[Winter, January 1-March 31; spring, April 1-June 30; summer, July 1-September 30; fall, October 1-December 31; streamflow statistics are in cubic feet per second, except for variability index, which is dimensionless; the record period is in climatic years, April 1 through March 31of the indicated ending year; EPA, U.S. Environmental Protection Agency]

\begin{tabular}{|c|c|c|c|c|c|}
\hline \multirow{2}{*}{ Streamflow statistic } & \multirow{2}{*}{ Annual } & \multicolumn{4}{|c|}{ Season } \\
\hline & & Winter & Spring & Summer & Fall \\
\hline \multicolumn{6}{|c|}{03069500 Cheat River near Parsons, WV, 1930-2002 } \\
\hline 1-day 2-year hydrologically based flow & 75.5 & 418 & 246 & 82.0 & 138 \\
\hline 1-day 5-year hydrologically based flow & 41.1 & 285 & 151 & 43.9 & 67.2 \\
\hline 1-day 10-year hydrologically based flow & 29.1 & 233 & 116 & 30.7 & 43.7 \\
\hline 3-day 2-year hydrologically based flow & 79.7 & 451 & 278 & 87.8 & 155 \\
\hline 3-day 5-year hydrologically based flow & 43.0 & 307 & 168 & 46.7 & 72.7 \\
\hline 3-day 10-year hydrologically based flow & 30.4 & 251 & 127 & 32.6 & 46.5 \\
\hline 7-day 2-year hydrologically based flow & 90.6 & 519 & 351 & 103 & 190 \\
\hline 7-day 5-year hydrologically based flow & 47.9 & 345 & 203 & 53.4 & 87.0 \\
\hline 7-day 10-year hydrologically based flow & 33.5 & 280 & 150 & 37.1 & 54.5 \\
\hline 14-day 2-year hydrologically based flow & 112 & 685 & 463 & 133 & 243 \\
\hline 14-day 5-year hydrologically based flow & 57.5 & 426 & 265 & 67.4 & 107 \\
\hline 14-day 10 -year hydrologically based flow & 39.5 & 336 & 193 & 46.3 & 64.8 \\
\hline 30-day 2-year hydrologically based flow & 163 & 1,200 & 772 & 220 & 468 \\
\hline 30-day 5-year hydrologically based flow & 79.3 & 752 & 457 & 105 & 191 \\
\hline 30-day 10-year hydrologically based flow & 53.0 & 575 & 339 & 70.2 & 108 \\
\hline 1-day 3-year biologically based flow & 26.9 & 230 & 187 & 30.9 & 36.0 \\
\hline 4-day 3-year biologically based flow & 33.6 & 265 & 226 & 37.2 & 40.6 \\
\hline EPA harmonic-mean flow & 377 & 1,080 & 1,110 & 225 & 231 \\
\hline 5-percent-duration flow & 5,880 & 8,370 & 5,890 & 2,960 & 4,800 \\
\hline 10-percent-duration flow & 3,980 & 5,900 & 4,210 & 1,790 & 3,170 \\
\hline 15-percent-duration flow & 3,060 & 4,600 & 3,340 & 1,310 & 2,390 \\
\hline 20-percent-duration flow & 2,480 & 3,780 & 2,810 & 994 & 1,950 \\
\hline 25-percent-duration flow & 2,090 & 3,230 & 2,450 & 786 & 1,640 \\
\hline 30-percent-duration flow & 1,780 & 2,840 & 2,140 & 650 & 1,380 \\
\hline 35-percent-duration flow & 1,520 & 2,510 & 1,890 & 545 & 1,200 \\
\hline 40-percent-duration flow & 1,310 & 2,210 & 1,660 & 460 & 1,040 \\
\hline 45-percent-duration flow & 1,120 & 1,990 & 1,470 & 400 & 899 \\
\hline 50-percent-duration flow & 961 & 1,780 & 1,300 & 347 & 785 \\
\hline 55-percent-duration flow & 818 & 1,600 & 1,150 & 302 & 680 \\
\hline 60-percent-duration flow & 692 & 1,430 & 1,010 & 260 & 590 \\
\hline 65-percent-duration flow & 585 & 1,260 & 882 & 223 & 504 \\
\hline 70-percent-duration flow & 486 & 1,100 & 769 & 193 & 427 \\
\hline 75-percent-duration flow & 398 & 953 & 664 & 163 & 351 \\
\hline 80-percent-duration flow & 316 & 819 & 564 & 134 & 278 \\
\hline 85-percent-duration flow & 237 & 680 & 464 & 106 & 206 \\
\hline 90-percent-duration flow & 167 & 530 & 358 & 79.0 & 137 \\
\hline 95-percent-duration flow & 96.1 & 398 & 248 & 56.1 & 82.8 \\
\hline 99-percent-duration flow & 39.9 & 273 & 129 & 28.7 & 30.5 \\
\hline Variability index & 0.54 & 0.40 & 0.41 & 0.52 & 0.52 \\
\hline
\end{tabular}


Table 11. elected annual and seasonal statistics for 77 stations in West Virginia representative of 1930-2002.—Continued

[Winter, January 1-March 31; spring, April 1-June 30; summer, July 1-September 30; fall, October 1-December 31; streamflow statistics are in cubic feet per second, except for variability index, which is dimensionless; the record period is in climatic years, April 1 through March 31 of the indicated ending year; EPA, U.S. Environmental Protection Agency]

\begin{tabular}{|c|c|c|c|c|c|}
\hline \multirow{2}{*}{ Streamflow statistic } & \multirow{2}{*}{ Annual } & \multicolumn{4}{|c|}{ Season } \\
\hline & & Winter & Spring & Summer & Fall \\
\hline \multicolumn{6}{|c|}{03070000 Cheat River at Rowlesburg, WV, 1930-1996 } \\
\hline 1-day 2-year hydrologically based flow & 93.1 & 612 & 325 & 99.6 & 169 \\
\hline 1-day 5-year hydrologically based flow & 49.6 & 415 & 202 & 54.3 & 78.6 \\
\hline 1-day 10-year hydrologically based flow & 34.2 & 337 & 156 & 38.4 & 49.5 \\
\hline 3-day 2-year hydrologically based flow & 97.0 & 660 & 361 & 105 & 186 \\
\hline 3-day 5-year hydrologically based flow & 51.5 & 447 & 219 & 57.2 & 83.8 \\
\hline 3-day 10-year hydrologically based flow & 35.5 & 364 & 167 & 40.5 & 52.2 \\
\hline 7-day 2-year hydrologically based flow & 109 & 757 & 451 & 123 & 226 \\
\hline 7-day 5-year hydrologically based flow & 56.3 & 509 & 263 & 64.9 & 98.5 \\
\hline 7-day 10-year hydrologically based flow & 38.4 & 415 & 195 & 45.4 & 59.8 \\
\hline 14-day 2-year hydrologically based flow & 135 & 996 & 596 & 162 & 293 \\
\hline 14-day 5-year hydrologically based flow & 68.0 & 619 & 339 & 83.3 & 121 \\
\hline 14-day 10 -year hydrologically based flow & 45.8 & 486 & 248 & 57.6 & 70.9 \\
\hline 30-day 2-year hydrologically based flow & 201 & 1,700 & 1,010 & 276 & 605 \\
\hline 30-day 5-year hydrologically based flow & 96.7 & 1,060 & 591 & 133 & 235 \\
\hline 30-day 10-year hydrologically based flow & 63.6 & 809 & 437 & 87.3 & 127 \\
\hline 1-day 3-year biologically based flow & 35.0 & 309 & 229 & 40.9 & 41.9 \\
\hline 4-day 3-year biologically based flow & 39.6 & 380 & 280 & 50.6 & 47.8 \\
\hline EPA harmonic-mean flow & 471 & 1,550 & 1,470 & 283 & 276 \\
\hline 5-percent-duration flow & 8,030 & 11,400 & 7,860 & 3,690 & 6,590 \\
\hline 10-percent-duration flow & 5,430 & 7,950 & 5,690 & 2,260 & 4,450 \\
\hline 15-percent-duration flow & 4,160 & 6,290 & 4,540 & 1,660 & 3,370 \\
\hline 20-percent-duration flow & 3,380 & 5,210 & 3,770 & 1,270 & 2,710 \\
\hline 25-percent-duration flow & 2,810 & 4,450 & 3,240 & 1,020 & 2,230 \\
\hline 30-percent-duration flow & 2,390 & 3,880 & 2,830 & 834 & 1,920 \\
\hline 35-percent-duration flow & 2,060 & 3,410 & 2,490 & 699 & 1,660 \\
\hline 40-percent-duration flow & 1,760 & 3,050 & 2,210 & 592 & 1,440 \\
\hline 45-percent-duration flow & 1,530 & 2,730 & 1,960 & 504 & 1,250 \\
\hline 50-percent-duration flow & 1,300 & 2,440 & 1,740 & 433 & 1,070 \\
\hline 55-percent-duration flow & 1,120 & 2,200 & 1,530 & 379 & 932 \\
\hline 60-percent-duration flow & 942 & 1,970 & 1,340 & 329 & 798 \\
\hline 65 -percent-duration flow & 783 & 1,750 & 1,190 & 283 & 677 \\
\hline 70-percent-duration flow & 648 & 1,560 & 1,030 & 242 & 571 \\
\hline 75-percent-duration flow & 522 & 1,370 & 888 & 203 & 467 \\
\hline 80-percent-duration flow & 408 & 1,180 & 752 & 166 & 357 \\
\hline 85-percent-duration flow & 305 & 981 & 616 & 130 & 258 \\
\hline 90-percent-duration flow & 208 & 768 & 472 & 97.4 & 168 \\
\hline 95-percent-duration flow & 116 & 573 & 313 & 68.3 & 97.1 \\
\hline 99-percent-duration flow & 49.0 & 395 & 180 & 36.2 & 31.0 \\
\hline Variability index & 0.55 & 0.39 & 0.42 & 0.52 & 0.54 \\
\hline
\end{tabular}


Table 11. Selected annual and seasonal statistics for 77 stations in West Virginia representative of 1930-2002.—Continued

[Winter, January 1-March 31; spring, April 1-June 30; summer, July 1-September 30; fall, October 1-December 31; streamflow statistics are in cubic feet per second, except for variability index, which is dimensionless; the record period is in climatic years, April 1 through March 31of the indicated ending year; EPA, U.S. Environmental Protection Agency]

\begin{tabular}{|c|c|c|c|c|c|}
\hline \multirow{2}{*}{ Streamflow statistic } & \multirow{2}{*}{ Annual } & \multicolumn{4}{|c|}{ Season } \\
\hline & & Winter & Spring & Summer & Fall \\
\hline \multicolumn{6}{|c|}{03070500 Big Sandy Creek at Rockville, WV, 1930-2002 } \\
\hline 1-day 2-year hydrologically based flow & 10.6 & 112 & 38.1 & 9.89 & 23.5 \\
\hline 1-day 5-year hydrologically based flow & 4.48 & 75.3 & 19.8 & 4.74 & 8.27 \\
\hline 1-day 10-year hydrologically based flow & 2.43 & 60.1 & 13.8 & 3.07 & 4.04 \\
\hline 3-day 2-year hydrologically based flow & 11.4 & 121 & 43.8 & 10.7 & 25.9 \\
\hline 3-day 5-year hydrologically based flow & 4.79 & 81.6 & 22.4 & 5.14 & 8.79 \\
\hline 3-day 10 -year hydrologically based flow & 2.58 & 65.6 & 15.4 & 3.37 & 4.23 \\
\hline 7-day 2-year hydrologically based flow & 13.5 & 138 & 55.9 & 12.8 & 31.6 \\
\hline 7-day 5-year hydrologically based flow & 5.48 & 91.7 & 28.3 & 5.94 & 10.7 \\
\hline 7-day 10-year hydrologically based flow & 2.88 & 74.1 & 19.2 & 3.83 & 5.04 \\
\hline 14-day 2-year hydrologically based flow & 16.8 & 178 & 75.3 & 16.8 & 39.6 \\
\hline 14-day 5-year hydrologically based flow & 6.74 & 111 & 38.2 & 7.49 & 13.2 \\
\hline 14-day 10 -year hydrologically based flow & 3.59 & 87.7 & 26.0 & 4.73 & 6.24 \\
\hline 30-day 2-year hydrologically based flow & 23.5 & 306 & 136 & 27.5 & 75.8 \\
\hline 30-day 5-year hydrologically based flow & 9.90 & 195 & 71.7 & 11.9 & 24.2 \\
\hline 30-day 10-year hydrologically based flow & 5.54 & 151 & 50.3 & 7.39 & 11.4 \\
\hline 1-day 3-year biologically based flow & 2.49 & 49.9 & 27.9 & 4.30 & 2.76 \\
\hline 4-day 3-year biologically based flow & 3.27 & 64.5 & 36.1 & 4.86 & 3.94 \\
\hline EPA harmonic-mean flow & 39.0 & 280 & 224 & 26.0 & 17.8 \\
\hline 5-percent-duration flow & 1,500 & 2,170 & 1,480 & 555 & 1,230 \\
\hline 10-percent-duration flow & 957 & 1,500 & 1,030 & 315 & 805 \\
\hline 15-percent-duration flow & 755 & 1,170 & 819 & 212 & 609 \\
\hline 20-percent-duration flow & 610 & 965 & 679 & 156 & 483 \\
\hline 25-percent-duration flow & 506 & 820 & 571 & 120 & 407 \\
\hline 30-percent-duration flow & 427 & 713 & 491 & 95.3 & 336 \\
\hline 35-percent-duration flow & 360 & 636 & 431 & 78.9 & 286 \\
\hline 40-percent-duration flow & 303 & 571 & 376 & 65.3 & 237 \\
\hline 45-percent-duration flow & 253 & 514 & 331 & 53.9 & 202 \\
\hline 50-percent-duration flow & 210 & 462 & 289 & 45.8 & 168 \\
\hline 55-percent-duration flow & 172 & 414 & 251 & 38.7 & 142 \\
\hline 60-percent-duration flow & 140 & 369 & 215 & 33.0 & 116 \\
\hline 65-percent-duration flow & 111 & 326 & 183 & 27.8 & 93.8 \\
\hline 70-percent-duration flow & 86.4 & 286 & 155 & 22.9 & 72.6 \\
\hline 75-percent-duration flow & 64.6 & 248 & 130 & 18.9 & 54.3 \\
\hline 80-percent-duration flow & 46.5 & 214 & 106 & 15.2 & 40.2 \\
\hline 85-percent-duration flow & 32.4 & 177 & 82.3 & 12.0 & 29.5 \\
\hline 90-percent-duration flow & 20.7 & 144 & 60.9 & 9.2 & 19.7 \\
\hline 95-percent-duration flow & 11.6 & 109 & 38.4 & 6.4 & 11.4 \\
\hline 99-percent-duration flow & 4.7 & 68.2 & 15.6 & 2.8 & 2.4 \\
\hline Variability index & 0.65 & 0.39 & 0.48 & 0.59 & 0.62 \\
\hline
\end{tabular}


Table 11. Selected annual and seasonal statistics for 77 stations in West Virginia representative of 1930-2002.—Continued

[Winter, January 1-March 31; spring, April 1-June 30; summer, July 1-September 30; fall, October 1-December 31; streamflow statistics are in cubic feet per second, except for variability index, which is dimensionless; the record period is in climatic years, April 1 through March 31 of the indicated ending year; EPA, U.S. Environmental Protection Agency]

\begin{tabular}{|c|c|c|c|c|c|}
\hline \multirow{2}{*}{ Streamflow statistic } & \multirow{2}{*}{ Annual } & \multicolumn{4}{|c|}{ Season } \\
\hline & & Winter & Spring & Summer & Fall \\
\hline \multicolumn{6}{|c|}{03071000 Cheat River near Pisgah, WV, 1932-1957 } \\
\hline 1-day 2-year hydrologically based flow & 130 & 886 & 551 & 132 & 192 \\
\hline 1-day 5-year hydrologically based flow & 64.9 & 605 & 320 & 72.0 & 85.7 \\
\hline 1-day 10-year hydrologically based flow & 42.6 & 497 & 235 & 51.9 & 52.3 \\
\hline 3-day 2-year hydrologically based flow & 137 & 948 & 609 & 141 & 205 \\
\hline 3-day 5-year hydrologically based flow & 68.0 & 644 & 350 & 75.8 & 89.9 \\
\hline 3-day 10-year hydrologically based flow & 44.4 & 529 & 254 & 54.4 & 54.7 \\
\hline 7-day 2-year hydrologically based flow & 154 & 1,080 & 726 & 164 & 252 \\
\hline 7-day 5-year hydrologically based flow & 73.3 & 720 & 419 & 84.9 & 101 \\
\hline 7-day 10-year hydrologically based flow & 46.9 & 585 & 304 & 60.0 & 59.2 \\
\hline 14-day 2-year hydrologically based flow & 189 & 1,450 & 911 & 213 & 317 \\
\hline 14-day 5-year hydrologically based flow & 85.7 & 895 & 547 & 107 & 120 \\
\hline 14-day 10-year hydrologically based flow & 53.6 & 697 & 410 & 74.7 & 67.6 \\
\hline 30-day 2-year hydrologically based flow & 273 & 2,330 & 1,510 & 378 & 609 \\
\hline 30-day 5-year hydrologically based flow & 120 & 1,620 & 919 & 177 & 220 \\
\hline 30-day 10 -year hydrologically based flow & 72.4 & 1,330 & 693 & 115 & 114 \\
\hline 1-day 3-year biologically based flow & 41.0 & 399 & 299 & 46.8 & 40.9 \\
\hline 4-day 3-year biologically based flow & 49.6 & 484 & 357 & 65.4 & 68.7 \\
\hline EPA harmonic-mean flow & 597 & 2,280 & 1,910 & 415 & 301 \\
\hline 5-percent-duration flow & 10,000 & 15,800 & 9,440 & 5,100 & 7,140 \\
\hline 10-percent-duration flow & 6,900 & 11,200 & 7,020 & 3,140 & 4,560 \\
\hline 15-percent-duration flow & 5,380 & 8,310 & 5,660 & 2,340 & 3,720 \\
\hline 20-percent-duration flow & 4,440 & 7,100 & 4,820 & 1,830 & 3,030 \\
\hline 25-percent-duration flow & 3,770 & 6,040 & 4,220 & 1,440 & 2,510 \\
\hline 30-percent-duration flow & 3,260 & 5,360 & 3,740 & 1,160 & 2,120 \\
\hline 35-percent-duration flow & 2,780 & 4,870 & 3,330 & 988 & 1,860 \\
\hline 40-percent-duration flow & 2,400 & 4,420 & 3,000 & 845 & 1,610 \\
\hline 45-percent-duration flow & 2,070 & 4,010 & 2,690 & 723 & 1,410 \\
\hline 50-percent-duration flow & 1,790 & 3,610 & 2,420 & 634 & 1,210 \\
\hline 55-percent-duration flow & 1,510 & 3,250 & 2,170 & 549 & 1,060 \\
\hline 60-percent-duration flow & 1,260 & 2,960 & 1,920 & 470 & 904 \\
\hline 65-percent-duration flow & 1,060 & 2,700 & 1,690 & 406 & 759 \\
\hline 70-percent-duration flow & 882 & 2,430 & 1,470 & 341 & 635 \\
\hline 75-percent-duration flow & 712 & 2,080 & 1,290 & 278 & 512 \\
\hline 80-percent-duration flow & 553 & 1,810 & 1,120 & 226 & 365 \\
\hline 85-percent-duration flow & 401 & 1,500 & 949 & 183 & 252 \\
\hline 90-percent-duration flow & 260 & 1,160 & 766 & 138 & 170 \\
\hline 95-percent-duration flow & 148 & 842 & 499 & 97.1 & 101 \\
\hline 99-percent-duration flow & 63.3 & 504 & 286 & 50.3 & 23.1 \\
\hline Variability index & 0.55 & 0.37 & 0.38 & 0.52 & 0.55 \\
\hline
\end{tabular}


Table 11. Selected annual and seasonal statistics for 77 stations in West Virginia representative of 1930-2002. - Continued

[Winter, January 1-March 31; spring, April 1-June 30; summer, July 1-September 30; fall, October 1-December 31; streamflow statistics are in cubic feet per second, except for variability index, which is dimensionless; the record period is in climatic years, April 1 through March 31of the indicated ending year; EPA, U.S. Environmental Protection Agency]

\begin{tabular}{|c|c|c|c|c|c|}
\hline \multirow{2}{*}{ Streamflow statistic } & \multirow{2}{*}{ Annual } & \multicolumn{4}{|c|}{ Season } \\
\hline & & Winter & Spring & Summer & Fall \\
\hline \multicolumn{6}{|c|}{03112000 Wheeling Creek at Elm Grove, WV, 1942-1980 } \\
\hline 1-day 2-year hydrologically based flow & 2.25 & 80.9 & 29.4 & 2.53 & 6.52 \\
\hline 1-day 5-year hydrologically based flow & 0.68 & 35.9 & 13.5 & 0.74 & 1.64 \\
\hline 1-day 10-year hydrologically based flow & 0.36 & 21.1 & 8.01 & 0.37 & 0.78 \\
\hline 3-day 2-year hydrologically based flow & 2.64 & 86.1 & 34.0 & 2.90 & 7.64 \\
\hline 3-day 5-year hydrologically based flow & 0.82 & 38.0 & 15.5 & 0.89 & 1.95 \\
\hline 3-day 10-year hydrologically based flow & 0.43 & 22.8 & 9.21 & 0.46 & 0.91 \\
\hline 7-day 2-year hydrologically based flow & 3.29 & 96.7 & 43.1 & 3.66 & 9.43 \\
\hline 7-day 5-year hydrologically based flow & 1.09 & 46.2 & 20.2 & 1.23 & 2.42 \\
\hline 7-day 10-year hydrologically based flow & 0.60 & 30.2 & 12.2 & 0.68 & 1.12 \\
\hline 14-day 2-year hydrologically based flow & 5.10 & 129 & 59.1 & 5.85 & 13.7 \\
\hline 14-day 5-year hydrologically based flow & 1.69 & 59.8 & 28.8 & 1.99 & 3.47 \\
\hline 14-day 10-year hydrologically based flow & 0.91 & 38.8 & 18.2 & 1.11 & 1.53 \\
\hline 30-day 2-year hydrologically based flow & 8.77 & 234 & 104 & 11.5 & 28.0 \\
\hline 30-day 5-year hydrologically based flow & 2.81 & 110 & 51.0 & 3.82 & 6.83 \\
\hline 30-day 10-year hydrologically based flow & 1.47 & 70.2 & 34.0 & 2.05 & 2.88 \\
\hline 1-day 3-year biologically based flow & 0.30 & 11.0 & 21.0 & 0.55 & 0.35 \\
\hline 4-day 3-year biologically based flow & 0.37 & 16.7 & 28.0 & 0.84 & 0.46 \\
\hline EPA harmonic-mean flow & 13.9 & 131 & 165 & 8.96 & 6.13 \\
\hline 5-percent-duration flow & 1,320 & 2,030 & 1,420 & 423 & 820 \\
\hline 10-percent-duration flow & 858 & 1,400 & 922 & 213 & 507 \\
\hline 15-percent-duration flow & 621 & 1,080 & 701 & 134 & 350 \\
\hline 20-percent-duration flow & 472 & 883 & 544 & 95.2 & 261 \\
\hline 25-percent-duration flow & 374 & 744 & 441 & 73.2 & 200 \\
\hline 30-percent-duration flow & 302 & 637 & 371 & 57.9 & 155 \\
\hline 35-percent-duration flow & 241 & 556 & 318 & 45.8 & 126 \\
\hline 40-percent-duration flow & 196 & 488 & 274 & 36.8 & 102 \\
\hline 45-percent-duration flow & 157 & 435 & 235 & 30.0 & 82.1 \\
\hline 50-percent-duration flow & 126 & 387 & 202 & 24.3 & 68.4 \\
\hline 55-percent-duration flow & 99.4 & 339 & 175 & 19.6 & 57.2 \\
\hline 60-percent-duration flow & 77.4 & 292 & 152 & 15.7 & 48.1 \\
\hline 65-percent-duration flow & 60.5 & 255 & 130 & 12.7 & 39.1 \\
\hline 70-percent-duration flow & 46.8 & 219 & 111 & 10.0 & 30.2 \\
\hline 75-percent-duration flow & 34.3 & 186 & 94.3 & 7.8 & 22.2 \\
\hline 80-percent-duration flow & 23.4 & 154 & 78.0 & 5.8 & 16.2 \\
\hline 85-percent-duration flow & 14.7 & 112 & 60.6 & 3.9 & 8.7 \\
\hline 90-percent-duration flow & 7.8 & 76.5 & 44.3 & 2.6 & 4.3 \\
\hline 95-percent-duration flow & 3.1 & 53.6 & 27.8 & 1.5 & 1.9 \\
\hline 99-percent-duration flow & 0.9 & 21.4 & 10.9 & 0.5 & 0.6 \\
\hline Variability index & 0.79 & 0.47 & 0.51 & 0.73 & 0.78 \\
\hline
\end{tabular}


Table 11. Selected annual and seasonal statistics for 77 stations in West Virginia representative of 1930-2002.—Continued

[Winter, January 1-March 31; spring, April 1-June 30; summer, July 1-September 30; fall, October 1-December 31; streamflow statistics are in cubic feet per second, except for variability index, which is dimensionless; the record period is in climatic years, April 1 through March 31 of the indicated ending year; EPA, U.S. Environmental Protection Agency]

\begin{tabular}{|c|c|c|c|c|c|}
\hline \multirow{2}{*}{ Streamflow statistic } & \multirow{2}{*}{ Annual } & \multicolumn{4}{|c|}{ Season } \\
\hline & & Winter & Spring & Summer & Fall \\
\hline \multicolumn{6}{|c|}{03114500 Middle Island Creek at Little, WV, 1930-1995 } \\
\hline 1-day 2-year hydrologically based flow & 3.08 & 138 & 28.4 & 4.48 & 7.14 \\
\hline 1-day 5-year hydrologically based flow & 0.68 & 65.3 & 9.65 & 1.01 & 1.69 \\
\hline 1-day 10-year hydrologically based flow & 0.27 & 34.0 & 4.57 & 0.39 & 0.70 \\
\hline 3-day 2-year hydrologically based flow & 3.46 & 149 & 30.1 & 4.97 & 7.78 \\
\hline 3-day 5-year hydrologically based flow & 0.79 & 67.8 & 10.7 & 1.10 & 1.93 \\
\hline 3-day 10-year hydrologically based flow & 0.32 & 35.2 & 5.49 & 0.42 & 0.84 \\
\hline 7-day 2-year hydrologically based flow & 4.14 & 174 & 39.7 & 6.05 & 9.58 \\
\hline 7-day 5-year hydrologically based flow & 0.97 & 74.2 & 13.6 & 1.38 & 2.41 \\
\hline 7-day 10-year hydrologically based flow & 0.40 & 39.0 & 6.88 & 0.55 & 1.05 \\
\hline 14-day 2-year hydrologically based flow & 5.79 & 214 & 55.8 & 8.96 & 14.5 \\
\hline 14-day 5-year hydrologically based flow & 1.45 & 91.0 & 20.4 & 2.09 & 3.99 \\
\hline 14-day 10-year hydrologically based flow & 0.63 & 53.5 & 11.3 & 0.85 & 1.83 \\
\hline 30-day 2-year hydrologically based flow & 11.6 & 521 & 125 & 22.6 & 43.8 \\
\hline 30-day 5-year hydrologically based flow & 3.14 & 230 & 46.9 & 6.08 & 11.0 \\
\hline 30-day 10 -year hydrologically based flow & 1.43 & 129 & 26.6 & 2.67 & 4.64 \\
\hline 1-day 3-year biologically based flow & 0.17 & 27.9 & 11.9 & 0.19 & 0.27 \\
\hline 4-day 3-year biologically based flow & 0.23 & 40.0 & 15.9 & 0.56 & 0.46 \\
\hline EPA harmonic-mean flow & 12.8 & 127 & 139 & 7.11 & 6.40 \\
\hline 5-percent-duration flow & 2,940 & 4,820 & 2,880 & 912 & 2,070 \\
\hline 10-percent-duration flow & 1,560 & 2,950 & 1,750 & 424 & 1,120 \\
\hline 15-percent-duration flow & 1,070 & 2,120 & 1,190 & 260 & 757 \\
\hline 20-percent-duration flow & 807 & 1,570 & 873 & 178 & 543 \\
\hline 25-percent-duration flow & 625 & 1,250 & 676 & 128 & 405 \\
\hline 30-percent-duration flow & 485 & 1,060 & 550 & 98.2 & 311 \\
\hline 35-percent-duration flow & 388 & 887 & 443 & 78.0 & 252 \\
\hline 40-percent-duration flow & 313 & 776 & 375 & 61.5 & 202 \\
\hline 45-percent-duration flow & 249 & 664 & 312 & 49.7 & 160 \\
\hline 50-percent-duration flow & 201 & 589 & 266 & 39.8 & 128 \\
\hline 55-percent-duration flow & 156 & 515 & 221 & 31.3 & 99.6 \\
\hline 60-percent-duration flow & 119 & 450 & 186 & 26.0 & 76.7 \\
\hline 65-percent-duration flow & 89.5 & 390 & 152 & 21.0 & 58.2 \\
\hline 70-percent-duration flow & 65.5 & 335 & 127 & 16.9 & 43.2 \\
\hline 75-percent-duration flow & 46.6 & 285 & 101 & 13.2 & 30.9 \\
\hline 80-percent-duration flow & 31.6 & 237 & 75.9 & 9.9 & 21.2 \\
\hline 85-percent-duration flow & 20.5 & 189 & 54.9 & 6.8 & 13.8 \\
\hline 90-percent-duration flow & 11.8 & 134 & 36.5 & 4.0 & 6.1 \\
\hline 95-percent-duration flow & 4.4 & 85.2 & 20.6 & 1.5 & 2.3 \\
\hline 99-percent-duration flow & 0.4 & 32.9 & 6.9 & 0.2 & 0.2 \\
\hline Variability index & 0.84 & 0.51 & 0.64 & 0.80 & 0.87 \\
\hline
\end{tabular}


Table 11. Selected annual and seasonal statistics for 77 stations in West Virginia representative of 1930-2002.-Continued

[Winter, January 1-March 31; spring, April 1-June 30; summer, July 1-September 30; fall, October 1-December 31; streamflow statistics are in cubic feet per second, except for variability index, which is dimensionless; the record period is in climatic years, April 1 through March 31of the indicated ending year; EPA, U.S. Environmental Protection Agency]

\begin{tabular}{|c|c|c|c|c|c|}
\hline \multirow{2}{*}{ Streamflow statistic } & \multirow{2}{*}{ Annual } & \multicolumn{4}{|c|}{ Season } \\
\hline & & Winter & Spring & Summer & Fall \\
\hline \multicolumn{6}{|c|}{03152000 Little Kanawha River at Glenville, WV, 1943-1978 } \\
\hline 1-day 2-year hydrologically based flow & 5.89 & 153 & 30.8 & 7.40 & 15.7 \\
\hline 1-day 5-year hydrologically based flow & 1.36 & 88.7 & 15.6 & 1.81 & 4.15 \\
\hline 1-day 10-year hydrologically based flow & 0.55 & 63.2 & 10.8 & 0.74 & 1.80 \\
\hline 3-day 2-year hydrologically based flow & 6.51 & 170 & 35.0 & 8.15 & 18.1 \\
\hline 3-day 5-year hydrologically based flow & 1.59 & 97.9 & 17.3 & 2.11 & 4.89 \\
\hline 3-day 10-year hydrologically based flow & 0.68 & 69.5 & 11.7 & 0.91 & 2.16 \\
\hline 7-day 2-year hydrologically based flow & 7.68 & 212 & 44.3 & 9.56 & 23.3 \\
\hline 7-day 5-year hydrologically based flow & 2.00 & 116 & 21.2 & 2.67 & 6.29 \\
\hline 7-day 10-year hydrologically based flow & 0.90 & 79.8 & 14.0 & 1.25 & 2.76 \\
\hline 14-day 2-year hydrologically based flow & 10.4 & 300 & 65.6 & 13.6 & 35.9 \\
\hline 14-day 5-year hydrologically based flow & 2.57 & 156 & 30.0 & 3.62 & 9.24 \\
\hline 14-day 10-year hydrologically based flow & 1.13 & 104 & 19.2 & 1.65 & 3.80 \\
\hline 30-day 2-year hydrologically based flow & 19.0 & 531 & 149 & 28.9 & 83.0 \\
\hline 30-day 5-year hydrologically based flow & 5.00 & 304 & 64.7 & 8.29 & 18.4 \\
\hline 30-day 10-year hydrologically based flow & 2.24 & 207 & 39.9 & 3.92 & 6.68 \\
\hline 1-day 3-year biologically based flow & 0.27 & 43.9 & 17.9 & 0.70 & 0.57 \\
\hline 4-day 3-year biologically based flow & 0.37 & 52.2 & 20.9 & 0.88 & 1.25 \\
\hline EPA harmonic-mean flow & 22.0 & 334 & 192 & 12.3 & 10.8 \\
\hline 5-percent-duration flow & 2,600 & 3,690 & 2,480 & 927 & 2,100 \\
\hline 10-percent-duration flow & 1,520 & 2,570 & 1,530 & 449 & 1,200 \\
\hline 15-percent-duration flow & 1,100 & 1,940 & 1,090 & 286 & 840 \\
\hline 20-percent-duration flow & 848 & 1,540 & 817 & 194 & 646 \\
\hline 25-percent-duration flow & 670 & 1,300 & 643 & 141 & 508 \\
\hline 30-percent-duration flow & 540 & 1,100 & 522 & 108 & 402 \\
\hline 35-percent-duration flow & 446 & 940 & 432 & 89.6 & 334 \\
\hline 40-percent-duration flow & 361 & 823 & 367 & 73.6 & 272 \\
\hline 45-percent-duration flow & 296 & 733 & 314 & 61.4 & 219 \\
\hline 50-percent-duration flow & 237 & 651 & 268 & 50.6 & 180 \\
\hline 55-percent-duration flow & 191 & 580 & 231 & 41.9 & 146 \\
\hline 60-percent-duration flow & 152 & 523 & 200 & 34.7 & 117 \\
\hline 65-percent-duration flow & 118 & 470 & 172 & 28.6 & 92.3 \\
\hline 70-percent-duration flow & 88.8 & 416 & 146 & 23.6 & 71.1 \\
\hline 75-percent-duration flow & 64.8 & 361 & 122 & 18.9 & 53.2 \\
\hline 80-percent-duration flow & 45.7 & 307 & 96.4 & 14.8 & 38.3 \\
\hline 85-percent-duration flow & 29.9 & 253 & 72.8 & 10.2 & 24.7 \\
\hline 90-percent-duration flow & 18.7 & 195 & 49.4 & 6.6 & 13.3 \\
\hline 95-percent-duration flow & 8.1 & 141 & 26.7 & 3.1 & 4.1 \\
\hline 99-percent-duration flow & 1.2 & 69.0 & 12.5 & 0.6 & 0.6 \\
\hline Variability index & 0.75 & 0.42 & 0.58 & 0.72 & 0.79 \\
\hline
\end{tabular}


Table 11. Selected annual and seasonal statistics for 77 stations in West Virginia representative of 1930-2002.—Continued

[Winter, January 1-March 31; spring, April 1-June 30; summer, July 1-September 30; fall, October 1-December 31; streamflow statistics are in cubic feet per second, except for variability index, which is dimensionless; the record period is in climatic years, April 1 through March 31 of the indicated ending year; EPA, U.S. Environmental Protection Agency]

\begin{tabular}{|c|c|c|c|c|c|}
\hline \multirow{2}{*}{ Streamflow statistic } & \multirow{2}{*}{ Annual } & \multicolumn{4}{|c|}{ Season } \\
\hline & & Winter & Spring & Summer & Fall \\
\hline \multicolumn{6}{|c|}{03153000 Steer Creek near Grantsville, WV, 1956-1974 } \\
\hline 1-day 2-year hydrologically based flow & 0.55 & 37.3 & 4.55 & 0.67 & 1.72 \\
\hline 1-day 5-year hydrologically based flow & 0.00 & 15.4 & 1.36 & 0.00 & 0.46 \\
\hline 1-day 10-year hydrologically based flow & 0.00 & 8.53 & 0.61 & 0.00 & 0.21 \\
\hline 3-day 2-year hydrologically based flow & 0.63 & 39.8 & 5.24 & 0.77 & 2.11 \\
\hline 3-day 5-year hydrologically based flow & 0.00 & 18.2 & 1.53 & 0.00 & 0.57 \\
\hline 3-day 10-year hydrologically based flow & 0.00 & 11.4 & 0.66 & 0.00 & 0.26 \\
\hline 7-day 2-year hydrologically based flow & 0.63 & 53.7 & 7.19 & 0.87 & 3.19 \\
\hline 7-day 5-year hydrologically based flow & 0.03 & 23.1 & 2.23 & 0.03 & 0.88 \\
\hline 7-day 10-year hydrologically based flow & 0.00 & 13.7 & 1.03 & 0.00 & 0.39 \\
\hline 14-day 2-year hydrologically based flow & 0.79 & 82.8 & 11.5 & 1.11 & 5.81 \\
\hline 14-day 5-year hydrologically based flow & 0.06 & 34.7 & 3.70 & 0.08 & 1.50 \\
\hline 14-day 10 -year hydrologically based flow & 0.01 & 19.5 & 1.78 & 0.01 & 0.62 \\
\hline 30-day 2-year hydrologically based flow & 1.95 & 183 & 20.0 & 3.84 & 18.3 \\
\hline 30-day 5-year hydrologically based flow & 0.22 & 80.7 & 9.23 & 0.39 & 3.88 \\
\hline 30-day 10-year hydrologically based flow & 0.06 & 44.1 & 6.34 & 0.09 & 1.38 \\
\hline 1-day 3-year biologically based flow & 0.00 & 8.20 & 3.30 & 0.00 & 0.00 \\
\hline 4-day 3-year biologically based flow & 0.00 & 8.84 & 4.53 & 0.00 & 0.06 \\
\hline EPA harmonic-mean flow & 1.32 & 57.3 & 42.1 & 0.41 & 1.76 \\
\hline 5-percent-duration flow & 957 & 1,580 & 949 & 291 & 665 \\
\hline 10-percent-duration flow & 529 & 953 & 571 & 136 & 353 \\
\hline 15-percent-duration flow & 360 & 706 & 367 & 70.7 & 224 \\
\hline 20-percent-duration flow & 275 & 557 & 273 & 44.7 & 158 \\
\hline 25-percent-duration flow & 208 & 448 & 209 & 31.1 & 118 \\
\hline 30-percent-duration flow & 158 & 378 & 163 & 22.5 & 91.6 \\
\hline 35-percent-duration flow & 125 & 326 & 128 & 16.6 & 71.9 \\
\hline 40-percent-duration flow & 95.8 & 283 & 104 & 12.3 & 57.8 \\
\hline 45-percent-duration flow & 72.9 & 248 & 83.4 & 9.1 & 44.9 \\
\hline 50-percent-duration flow & 55.5 & 216 & 68.2 & 7.0 & 34.4 \\
\hline 55-percent-duration flow & 41.3 & 190 & 55.8 & 5.7 & 27.2 \\
\hline 60-percent-duration flow & 30.9 & 165 & 45.0 & 4.5 & 22.2 \\
\hline 65-percent-duration flow & 22.7 & 145 & 36.8 & 3.4 & 18.0 \\
\hline 70-percent-duration flow & 16.0 & 126 & 29.2 & 2.6 & 13.6 \\
\hline 75-percent-duration flow & 10.6 & 107 & 22.5 & 1.8 & 9.8 \\
\hline 80-percent-duration flow & 6.9 & 85.9 & 16.4 & 1.1 & 6.7 \\
\hline 85-percent-duration flow & 4.3 & 64.5 & 11.2 & 0.5 & 3.8 \\
\hline 90-percent-duration flow & 2.1 & 45.5 & 7.5 & 0.2 & 2.1 \\
\hline 95-percent-duration flow & 0.6 & 29.7 & 4.6 & 0.0 & 0.9 \\
\hline 99-percent-duration flow & 0.0 & 6.9 & 0.6 & 0.0 & 0.2 \\
\hline Variability index & 0.96 & 0.50 & 0.70 & 0.69 & 0.85 \\
\hline
\end{tabular}


Table 11. Selected annual and seasonal statistics for 77 stations in West Virginia representative of 1930-2002.-Continued

[Winter, January 1-March 31; spring, April 1-June 30; summer, July 1-September 30; fall, October 1-December 31; streamflow statistics are in cubic feet per second, except for variability index, which is dimensionless; the record period is in climatic years, April 1 through March 31of the indicated ending year; EPA, U.S. Environmental Protection Agency]

\begin{tabular}{|c|c|c|c|c|c|}
\hline \multirow{2}{*}{ Streamflow statistic } & \multirow{2}{*}{ Annual } & \multicolumn{4}{|c|}{ Season } \\
\hline & & Winter & Spring & Summer & Fall \\
\hline \multicolumn{6}{|c|}{03153500 Little Kanawha River at Grantsville, WV, 1943-1978 } \\
\hline 1-day 2-year hydrologically based flow & 14.7 & 313 & 67.7 & 20.6 & 37.1 \\
\hline 1-day 5-year hydrologically based flow & 2.31 & 174 & 32.7 & 3.66 & 8.48 \\
\hline 1-day 10-year hydrologically based flow & 0.63 & 121 & 21.5 & 1.04 & 2.93 \\
\hline 3-day 2-year hydrologically based flow & 15.0 & 347 & 75.4 & 20.6 & 40.5 \\
\hline 3-day 5-year hydrologically based flow & 2.60 & 187 & 36.0 & 4.06 & 8.99 \\
\hline 3-day 10-year hydrologically based flow & 0.80 & 127 & 23.6 & 1.32 & 3.09 \\
\hline 7-day 2-year hydrologically based flow & 17.3 & 433 & 92.2 & 23.9 & 49.3 \\
\hline 7-day 5-year hydrologically based flow & 3.12 & 221 & 43.5 & 4.99 & 10.6 \\
\hline 7-day 10-year hydrologically based flow & 1.00 & 145 & 28.2 & 1.75 & 3.55 \\
\hline 14-day 2-year hydrologically based flow & 22.8 & 605 & 136 & 31.9 & 73.7 \\
\hline 14-day 5-year hydrologically based flow & 4.42 & 306 & 60.0 & 7.48 & 15.1 \\
\hline 14-day 10 -year hydrologically based flow & 1.52 & 203 & 37.5 & 3.02 & 4.77 \\
\hline 30-day 2-year hydrologically based flow & 42.0 & 1,130 & 304 & 65.2 & 181 \\
\hline 30-day 5-year hydrologically based flow & 9.09 & 607 & 134 & 17.5 & 34.0 \\
\hline 30-day 10-year hydrologically based flow & 3.26 & 402 & 82.6 & 7.67 & 9.75 \\
\hline 1-day 3-year biologically based flow & 0.78 & 79.9 & 36.9 & 2.20 & 1.00 \\
\hline 4-day 3-year biologically based flow & 0.90 & 91.1 & 45.3 & 2.55 & 1.66 \\
\hline EPA harmonic-mean flow & 16.6 & 645 & 393 & 10.4 & 7.10 \\
\hline 5-percent-duration flow & 6,130 & 9,000 & 5,610 & 2,040 & 4,630 \\
\hline 10-percent-duration flow & 3,590 & 6,040 & 3,440 & 1,010 & 2,590 \\
\hline 15-percent-duration flow & 2,500 & 4,510 & 2,430 & 590 & 1,760 \\
\hline 20-percent-duration flow & 1,830 & 3,550 & 1,810 & 406 & 1,300 \\
\hline 25-percent-duration flow & 1,450 & 2,930 & 1,420 & 321 & 999 \\
\hline 30-percent-duration flow & 1,140 & 2,480 & 1,150 & 247 & 803 \\
\hline 35-percent-duration flow & 940 & 2,100 & 940 & 202 & 650 \\
\hline 40-percent-duration flow & 750 & 1,840 & 777 & 159 & 521 \\
\hline 45-percent-duration flow & 612 & 1,610 & 652 & 134 & 425 \\
\hline 50-percent-duration flow & 482 & 1,430 & 558 & 109 & 337 \\
\hline 55-percent-duration flow & 391 & 1,270 & 477 & 91.8 & 277 \\
\hline 60-percent-duration flow & 305 & 1,130 & 412 & 76.4 & 220 \\
\hline 65-percent-duration flow & 239 & 992 & 350 & 62.9 & 175 \\
\hline 70-percent-duration flow & 181 & 872 & 292 & 52.4 & 136 \\
\hline 75-percent-duration flow & 132 & 757 & 238 & 42.1 & 101 \\
\hline 80-percent-duration flow & 94.7 & 638 & 193 & 33.1 & 72.1 \\
\hline 85-percent-duration flow & 64.5 & 514 & 145 & 23.7 & 46.3 \\
\hline 90-percent-duration flow & 40.3 & 390 & 96.4 & 14.0 & 22.4 \\
\hline 95-percent-duration flow & 16.2 & 278 & 57.0 & 5.6 & 8.5 \\
\hline 99-percent-duration flow & 1.9 & 114 & 25.9 & 1.2 & 0.2 \\
\hline Variability index & 0.77 & 0.45 & 0.59 & 0.73 & 0.80 \\
\hline
\end{tabular}


Table 11. Selected annual and seasonal statistics for 77 stations in West Virginia representative of 1930-2002.—Continued

[Winter, January 1-March 31; spring, April 1-June 30; summer, July 1-September 30; fall, October 1-December 31; streamflow statistics are in cubic feet per second, except for variability index, which is dimensionless; the record period is in climatic years, April 1 through March 31of the indicated ending year; EPA, U.S. Environmental Protection Agency]

\begin{tabular}{|c|c|c|c|c|c|}
\hline \multirow{2}{*}{ Streamflow statistic } & \multirow{2}{*}{ Annual } & \multicolumn{4}{|c|}{ Season } \\
\hline & & Winter & Spring & Summer & Fall \\
\hline \multicolumn{6}{|c|}{03154000 West Fork Little Kanawha River at Rocksdale, WV, 1955-1975 } \\
\hline 1-day 2-year hydrologically based flow & 0.90 & 45.9 & 5.38 & 1.15 & 2.68 \\
\hline 1-day 5-year hydrologically based flow & 0.10 & 20.8 & 1.86 & 0.12 & 0.64 \\
\hline 1-day 10-year hydrologically based flow & 0.00 & 12.7 & 1.00 & 0.00 & 0.23 \\
\hline 3-day 2-year hydrologically based flow & 1.00 & 51.3 & 6.20 & 1.29 & 3.30 \\
\hline 3-day 5-year hydrologically based flow & 0.11 & 22.4 & 2.09 & 0.12 & 0.76 \\
\hline 3-day 10 -year hydrologically based flow & 0.00 & 13.4 & 1.10 & 0.00 & 0.25 \\
\hline 7-day 2-year hydrologically based flow & 1.20 & 68.3 & 8.50 & 1.66 & 4.89 \\
\hline 7-day 5-year hydrologically based flow & 0.16 & 28.4 & 2.88 & 0.19 & 1.44 \\
\hline 7-day 10-year hydrologically based flow & 0.01 & 16.3 & 1.50 & 0.01 & 0.60 \\
\hline 14-day 2-year hydrologically based flow & 1.67 & 106 & 13.2 & 2.44 & 7.23 \\
\hline 14-day 5-year hydrologically based flow & 0.24 & 46.0 & 4.50 & 0.28 & 1.90 \\
\hline 14-day 10 -year hydrologically based flow & 0.01 & 26.2 & 2.34 & 0.01 & 0.73 \\
\hline 30-day 2-year hydrologically based flow & 3.52 & 229 & 26.0 & 5.11 & 21.6 \\
\hline 30-day 5-year hydrologically based flow & 0.44 & 103 & 10.9 & 0.82 & 5.78 \\
\hline 30-day 10 -year hydrologically based flow & 0.11 & 57.7 & 6.90 & 0.28 & 2.58 \\
\hline 1-day 3-year biologically based flow & 0.00 & 6.58 & 4.57 & 0.00 & 0.00 \\
\hline 4-day 3-year biologically based flow & 0.00 & 11.4 & 6.00 & 0.00 & 0.36 \\
\hline EPA harmonic-mean flow & 3.90 & 72.7 & 50.2 & 1.98 & 2.03 \\
\hline 5-percent-duration flow & 1,170 & 1,970 & 1,150 & 319 & 863 \\
\hline 10-percent-duration flow & 669 & 1,190 & 634 & 148 & 452 \\
\hline 15-percent-duration flow & 445 & 886 & 434 & 84.1 & 293 \\
\hline 20-percent-duration flow & 328 & 696 & 319 & 53.7 & 209 \\
\hline 25-percent-duration flow & 254 & 567 & 242 & 40.4 & 157 \\
\hline 30-percent-duration flow & 194 & 478 & 188 & 31.1 & 121 \\
\hline 35-percent-duration flow & 154 & 409 & 151 & 24.2 & 96.1 \\
\hline 40-percent-duration flow & 120 & 357 & 120 & 19.7 & 74.5 \\
\hline 45-percent-duration flow & 91.2 & 311 & 96.6 & 15.7 & 57.3 \\
\hline 50-percent-duration flow & 69.6 & 272 & 78.4 & 12.5 & 44.2 \\
\hline 55-percent-duration flow & 52.0 & 237 & 64.4 & 9.7 & 34.5 \\
\hline 60-percent-duration flow & 38.3 & 206 & 53.4 & 7.3 & 26.6 \\
\hline 65-percent-duration flow & 28.7 & 180 & 43.6 & 5.5 & 20.6 \\
\hline 70-percent-duration flow & 21.0 & 156 & 34.4 & 3.8 & 15.8 \\
\hline 75-percent-duration flow & 14.7 & 133 & 26.2 & 2.7 & 11.8 \\
\hline 80-percent-duration flow & 9.7 & 111 & 19.8 & 2.0 & 8.1 \\
\hline 85-percent-duration flow & 6.1 & 87.2 & 13.5 & 1.2 & 5.6 \\
\hline 90-percent-duration flow & 3.1 & 62.5 & 8.5 & 0.6 & 3.2 \\
\hline 95-percent-duration flow & 1.1 & 36.7 & 4.7 & 0.2 & 1.5 \\
\hline 99-percent-duration flow & 0.1 & 8.2 & 1.0 & 0.0 & 0.1 \\
\hline Variability index & 0.91 & 0.50 & 0.72 & 0.93 & 0.83 \\
\hline
\end{tabular}


Table 11. Selected annual and seasonal statistics for 77 stations in West Virginia representative of 1930-2002.-Continued

[Winter, January 1-March 31; spring, April 1-June 30; summer, July 1-September 30; fall, October 1-December 31; streamflow statistics are in cubic feet per second, except for variability index, which is dimensionless; the record period is in climatic years, April 1 through March 31 of the indicated ending year; EPA, U.S. Environmental Protection Agency]

\begin{tabular}{|c|c|c|c|c|c|}
\hline \multirow{2}{*}{ Streamflow statistic } & \multirow{2}{*}{ Annual } & \multicolumn{4}{|c|}{ Season } \\
\hline & & Winter & Spring & Summer & Fall \\
\hline \multicolumn{6}{|c|}{03154500 Reedy Creek near Reedy, WV, 1953-1978 } \\
\hline 1-day 2-year hydrologically based flow & 0.22 & 11.3 & 1.24 & 0.34 & 0.64 \\
\hline 1-day 5-year hydrologically based flow & 0.05 & 5.17 & 0.41 & 0.08 & 0.13 \\
\hline 1-day 10-year hydrologically based flow & 0.00 & 3.23 & 0.21 & 0.00 & 0.05 \\
\hline 3-day 2-year hydrologically based flow & 0.26 & 12.0 & 1.41 & 0.36 & 0.73 \\
\hline 3-day 5-year hydrologically based flow & 0.06 & 5.48 & 0.50 & 0.10 & 0.17 \\
\hline 3-day 10-year hydrologically based flow & 0.00 & 3.46 & 0.28 & 0.00 & 0.07 \\
\hline 7-day 2-year hydrologically based flow & 0.32 & 15.6 & 1.92 & 0.45 & 1.09 \\
\hline 7-day 5-year hydrologically based flow & 0.08 & 7.19 & 0.72 & 0.12 & 0.26 \\
\hline 7-day 10-year hydrologically based flow & 0.00 & 4.64 & 0.41 & 0.00 & 0.10 \\
\hline 14-day 2-year hydrologically based flow & 0.44 & 26.7 & 3.19 & 0.76 & 1.61 \\
\hline 14-day 5-year hydrologically based flow & 0.11 & 11.5 & 1.18 & 0.18 & 0.37 \\
\hline 14-day 10 -year hydrologically based flow & 0.00 & 7.03 & 0.66 & 0.00 & 0.15 \\
\hline 30-day 2-year hydrologically based flow & 0.95 & 62.6 & 9.08 & 2.34 & 5.32 \\
\hline 30-day 5-year hydrologically based flow & 0.13 & 26.3 & 3.21 & 0.37 & 1.12 \\
\hline 30-day 10-year hydrologically based flow & 0.03 & 15.1 & 1.75 & 0.10 & 0.44 \\
\hline 1-day 3-year biologically based flow & 0.00 & 1.15 & 0.80 & 0.00 & 0.00 \\
\hline 4-day 3-year biologically based flow & 0.00 & 1.59 & 1.07 & 0.00 & 0.00 \\
\hline EPA harmonic-mean flow & 1.65 & 22.1 & 11.4 & 0.90 & 0.83 \\
\hline 5-percent-duration flow & 434 & 762 & 428 & 116 & 297 \\
\hline 10-percent-duration flow & 220 & 433 & 211 & 50.3 & 143 \\
\hline 15-percent-duration flow & 141 & 298 & 137 & 25.8 & 92.1 \\
\hline 20-percent-duration flow & 103 & 228 & 98.1 & 16.8 & 64.9 \\
\hline 25-percent-duration flow & 76.6 & 181 & 71.7 & 11.8 & 48.2 \\
\hline 30-percent-duration flow & 57.7 & 148 & 54.2 & 8.3 & 37.1 \\
\hline 35-percent-duration flow & 43.7 & 121 & 42.7 & 6.2 & 28.9 \\
\hline 40-percent-duration flow & 34.0 & 104 & 34.7 & 4.9 & 22.5 \\
\hline 45-percent-duration flow & 25.8 & 90.6 & 28.3 & 3.9 & 17.5 \\
\hline 50-percent-duration flow & 19.5 & 79.1 & 22.9 & 3.2 & 13.6 \\
\hline 55-percent-duration flow & 14.8 & 68.7 & 18.2 & 2.5 & 9.8 \\
\hline 60-percent-duration flow & 10.5 & 58.9 & 14.9 & 2.1 & 7.1 \\
\hline 65-percent-duration flow & 7.3 & 50.0 & 11.6 & 1.6 & 5.1 \\
\hline 70-percent-duration flow & 5.0 & 42.2 & 8.8 & 1.2 & 3.6 \\
\hline 75-percent-duration flow & 3.5 & 34.7 & 6.5 & 0.9 & 2.4 \\
\hline 80-percent-duration flow & 2.3 & 26.7 & 4.6 & 0.6 & 1.6 \\
\hline 85-percent-duration flow & 1.4 & 20.5 & 3.3 & 0.4 & 1.0 \\
\hline 90-percent-duration flow & 0.8 & 15.5 & 2.0 & 0.2 & 0.5 \\
\hline 95-percent-duration flow & 0.3 & 8.7 & 1.0 & 0.1 & 0.2 \\
\hline 99-percent-duration flow & 0.0 & 2.8 & 0.3 & 0.0 & 0.1 \\
\hline Variability index & 0.96 & 0.57 & 0.78 & 0.89 & 0.95 \\
\hline
\end{tabular}


Table 11. Selected annual and seasonal statistics for 77 stations in West Virginia representative of 1930-2002. - Continued

[Winter, January 1-March 31; spring, April 1-June 30; summer, July 1-September 30; fall, October 1-December 31; streamflow statistics are in cubic feet per second, except for variability index, which is dimensionless; the record period is in climatic years, April 1 through March 31of the indicated ending year; EPA, U.S. Environmental Protection Agency]

\begin{tabular}{|c|c|c|c|c|c|}
\hline \multirow{2}{*}{ Streamflow statistic } & \multirow{2}{*}{ Annual } & \multicolumn{4}{|c|}{ Season } \\
\hline & & Winter & Spring & Summer & Fall \\
\hline \multicolumn{6}{|c|}{03155000 Little Kanawha River at Palestine, WV, 1943-1978 } \\
\hline 1-day 2-year hydrologically based flow & 29.2 & 524 & 152 & 38.4 & 62.8 \\
\hline 1-day 5-year hydrologically based flow & 7.63 & 289 & 65.9 & 10.7 & 17.6 \\
\hline 1-day 10-year hydrologically based flow & 3.30 & 198 & 36.7 & 4.70 & 8.03 \\
\hline 3-day 2-year hydrologically based flow & 30.8 & 563 & 169 & 40.8 & 67.8 \\
\hline 3-day 5-year hydrologically based flow & 8.05 & 306 & 72.3 & 11.5 & 18.6 \\
\hline 3-day 10 -year hydrologically based flow & 3.49 & 211 & 39.6 & 5.06 & 8.37 \\
\hline 7-day 2-year hydrologically based flow & 34.3 & 674 & 203 & 46.8 & 76.8 \\
\hline 7-day 5-year hydrologically based flow & 8.86 & 351 & 86.0 & 12.9 & 20.4 \\
\hline 7-day 10-year hydrologically based flow & 3.86 & 237 & 47.6 & 5.71 & 9.02 \\
\hline 14-day 2-year hydrologically based flow & 40.8 & 947 & 274 & 62.5 & 105 \\
\hline 14-day 5-year hydrologically based flow & 10.7 & 471 & 118 & 17.2 & 26.8 \\
\hline 14-day 10 -year hydrologically based flow & 4.83 & 307 & 68.1 & 7.79 & 11.4 \\
\hline 30-day 2-year hydrologically based flow & 64.5 & 1,740 & 524 & 116 & 225 \\
\hline 30-day 5-year hydrologically based flow & 17.0 & 912 & 245 & 36.5 & 54.4 \\
\hline 30-day 10 -year hydrologically based flow & 7.74 & 603 & 154 & 17.6 & 21.4 \\
\hline 1-day 3-year biologically based flow & 1.65 & 120 & 74.9 & 2.40 & 3.29 \\
\hline 4-day 3-year biologically based flow & 2.05 & 151 & 92.7 & 3.42 & 4.76 \\
\hline EPA harmonic-mean flow & 93.0 & 1,030 & 714 & 54.8 & 44.8 \\
\hline 5-percent-duration flow & 9,000 & 14,100 & 8,670 & 2,790 & 7,050 \\
\hline 10-percent-duration flow & 5,740 & 9,660 & 5,600 & 1,480 & 3,960 \\
\hline 15-percent-duration flow & 4,010 & 7,440 & 3,980 & 962 & 2,620 \\
\hline 20-percent-duration flow & 2,940 & 6,020 & 2,960 & 712 & 1,930 \\
\hline 25-percent-duration flow & 2,260 & 5,020 & 2,280 & 545 & 1,490 \\
\hline 30-percent-duration flow & 1,790 & 4,230 & 1,840 & 440 & 1,190 \\
\hline 35-percent-duration flow & 1,460 & 3,590 & 1,520 & 358 & 957 \\
\hline 40-percent-duration flow & 1,170 & 3,110 & 1,270 & 293 & 805 \\
\hline 45-percent-duration flow & 955 & 2,680 & 1,080 & 248 & 666 \\
\hline 50-percent-duration flow & 786 & 2,330 & 919 & 208 & 548 \\
\hline 55-percent-duration flow & 636 & 2,050 & 802 & 175 & 448 \\
\hline 60-percent-duration flow & 513 & 1,810 & 695 & 147 & 355 \\
\hline 65-percent-duration flow & 406 & 1,590 & 606 & 121 & 275 \\
\hline 70-percent-duration flow & 313 & 1,390 & 517 & 100 & 215 \\
\hline 75-percent-duration flow & 235 & 1,200 & 429 & 80.5 & 162 \\
\hline 80-percent-duration flow & 169 & 1,010 & 347 & 62.8 & 122 \\
\hline 85-percent-duration flow & 118 & 800 & 273 & 47.3 & 77.7 \\
\hline 90-percent-duration flow & 74.3 & 621 & 191 & 28.2 & 33.3 \\
\hline 95-percent-duration flow & 29.3 & 430 & 116 & 11.9 & 12.6 \\
\hline 99-percent-duration flow & 4.40 & 177 & 53.2 & 2.7 & 2.6 \\
\hline Variability index & 0.74 & 0.46 & 0.56 & 0.68 & 0.79 \\
\hline
\end{tabular}


Table 11. Selected annual and seasonal statistics for 77 stations in West Virginia representative of 1930-2002.—Continued

[Winter, January 1-March 31; spring, April 1-June 30; summer, July 1-September 30; fall, October 1-December 31; streamflow statistics are in cubic feet per second, except for variability index, which is dimensionless; the record period is in climatic years, April 1 through March 31 of the indicated ending year; EPA, U.S. Environmental Protection Agency]

\begin{tabular}{|c|c|c|c|c|c|}
\hline \multirow{2}{*}{ Streamflow statistic } & \multirow{2}{*}{ Annual } & \multicolumn{4}{|c|}{ Season } \\
\hline & & Winter & Spring & Summer & Fall \\
\hline \multicolumn{6}{|c|}{03155500 Hughes River at Cisco, WV, 1940-1989 } \\
\hline 1-day 2-year hydrologically based flow & 3.75 & 92.0 & 20.9 & 4.94 & 6.67 \\
\hline 1-day 5-year hydrologically based flow & 1.24 & 47.0 & 9.41 & 1.74 & 2.06 \\
\hline 1-day 10 -year hydrologically based flow & 0.64 & 31.7 & 5.85 & 0.91 & 1.08 \\
\hline 3-day 2-year hydrologically based flow & 4.06 & 99.9 & 23.6 & 5.41 & 7.29 \\
\hline 3-day 5-year hydrologically based flow & 1.31 & 49.8 & 10.3 & 1.87 & 2.20 \\
\hline 3-day 10 -year hydrologically based flow & 0.66 & 33.3 & 6.31 & 0.97 & 1.13 \\
\hline 7-day 2-year hydrologically based flow & 4.66 & 122 & 30.0 & 6.51 & 8.72 \\
\hline 7-day 5-year hydrologically based flow & 1.45 & 59.0 & 12.7 & 2.19 & 2.57 \\
\hline 7-day 10 -year hydrologically based flow & 0.73 & 39.0 & 7.73 & 1.12 & 1.29 \\
\hline 14-day 2-year hydrologically based flow & 5.83 & 176 & 45.1 & 8.57 & 11.8 \\
\hline 14-day 5-year hydrologically based flow & 1.83 & 80.7 & 18.6 & 2.84 & 3.44 \\
\hline 14-day 10 -year hydrologically based flow & 0.94 & 51.7 & 11.4 & 1.48 & 1.69 \\
\hline 30-day 2-year hydrologically based flow & 9.45 & 419 & 105 & 18.9 & 39.6 \\
\hline 30-day 5-year hydrologically based flow & 2.97 & 208 & 44.5 & 6.41 & 10.5 \\
\hline 30-day 10-year hydrologically based flow & 1.55 & 133 & 27.5 & 3.41 & 4.75 \\
\hline 1-day 3-year biologically based flow & 0.37 & 19.0 & 15.0 & 0.89 & 0.43 \\
\hline 4-day 3-year biologically based flow & 0.48 & 23.0 & 19.4 & 1.33 & 0.77 \\
\hline EPA harmonic-mean flow & 18.5 & 208 & 151 & 10.9 & 8.87 \\
\hline 5-percent-duration flow & 2,620 & 3,940 & 2,670 & 777 & 1,980 \\
\hline 10-percent-duration flow & 1,480 & 2,570 & 1,570 & 354 & 1,010 \\
\hline 15-percent-duration flow & 976 & 1,920 & 1,030 & 223 & 674 \\
\hline 20-percent-duration flow & 707 & 1,440 & 758 & 156 & 487 \\
\hline 25-percent-duration flow & 546 & 1,130 & 597 & 114 & 365 \\
\hline 30-percent-duration flow & 434 & 930 & 483 & 87.1 & 281 \\
\hline 35-percent-duration flow & 341 & 787 & 392 & 69.1 & 224 \\
\hline 40-percent-duration flow & 275 & 677 & 323 & 54.9 & 178 \\
\hline 45-percent-duration flow & 220 & 588 & 274 & 43.8 & 139 \\
\hline 50-percent-duration flow & 174 & 517 & 232 & 35.9 & 107 \\
\hline 55-percent-duration flow & 137 & 451 & 197 & 29.1 & 80.8 \\
\hline 60-percent-duration flow & 104 & 395 & 163 & 24.2 & 59.1 \\
\hline 65-percent-duration flow & 77.4 & 345 & 135 & 19.8 & 43.3 \\
\hline 70-percent-duration flow & 55.6 & 298 & 111 & 16.2 & 31.7 \\
\hline 75-percent-duration flow & 38.6 & 252 & 88.7 & 12.8 & 24.3 \\
\hline 80-percent-duration flow & 27.3 & 208 & 69.4 & 9.6 & 17.8 \\
\hline 85-percent-duration flow & 19.0 & 164 & 48.1 & 7.0 & 11.7 \\
\hline 90-percent-duration flow & 11.6 & 119 & 32.3 & 4.4 & 6.4 \\
\hline 95-percent-duration flow & 4.9 & 75.2 & 20.2 & 2.4 & 2.4 \\
\hline 99-percent-duration flow & 1.1 & 29.1 & 6.9 & 0.8 & 0.6 \\
\hline Variability index & 0.82 & 0.51 & 0.63 & 0.74 & 0.87 \\
\hline
\end{tabular}


Table 11. Selected annual and seasonal statistics for 77 stations in West Virginia representative of 1930-2002.—Continued

[Winter, January 1-March 31; spring, April 1-June 30; summer, July 1-September 30; fall, October 1-December 31; streamflow statistics are in cubic feet per second, except for variability index, which is dimensionless; the record period is in climatic years, April 1 through March 31of the indicated ending year; EPA, U.S. Environmental Protection Agency]

\begin{tabular}{|c|c|c|c|c|c|}
\hline \multirow{2}{*}{ Streamflow statistic } & \multirow{2}{*}{ Annual } & \multicolumn{4}{|c|}{ Season } \\
\hline & & Winter & Spring & Summer & Fall \\
\hline \multicolumn{6}{|c|}{03179000 Bluestone River near Pipestem, WV, 1952-1978 } \\
\hline 1-day 2-year hydrologically based flow & 21.6 & 131 & 72.4 & 23.1 & 31.7 \\
\hline 1-day 5-year hydrologically based flow & 14.0 & 63.8 & 47.6 & 14.7 & 17.7 \\
\hline 1-day 10-year hydrologically based flow & 11.1 & 40.5 & 37.5 & 11.5 & 13.5 \\
\hline 3-day 2-year hydrologically based flow & 22.4 & 144 & 80.3 & 24.5 & 32.9 \\
\hline 3-day 5-year hydrologically based flow & 14.6 & 69.3 & 51.9 & 15.5 & 18.3 \\
\hline 3-day 10 -year hydrologically based flow & 11.6 & 44.0 & 40.4 & 12.1 & 14.0 \\
\hline 7-day 2-year hydrologically based flow & 24.1 & 183 & 95.0 & 26.8 & 36.5 \\
\hline 7-day 5-year hydrologically based flow & 15.7 & 84.5 & 61.4 & 16.9 & 19.5 \\
\hline 7-day 10-year hydrologically based flow & 12.6 & 51.2 & 48.0 & 13.2 & 14.7 \\
\hline 14-day 2-year hydrologically based flow & 26.9 & 243 & 118 & 32.5 & 42.8 \\
\hline 14-day 5-year hydrologically based flow & 17.4 & 108 & 74.3 & 20.4 & 22.1 \\
\hline 14-day 10 -year hydrologically based flow & 14.0 & 63.8 & 58.9 & 15.8 & 16.4 \\
\hline 30-day 2-year hydrologically based flow & 35.6 & 389 & 179 & 43.9 & 68.8 \\
\hline 30-day 5-year hydrologically based flow & 22.7 & 188 & 108 & 29.1 & 30.2 \\
\hline 30-day 10 -year hydrologically based flow & 17.9 & 116 & 84.0 & 23.3 & 19.9 \\
\hline 1-day 3-year biologically based flow & 12.0 & 24.9 & 63.9 & 13.0 & 12.0 \\
\hline 4-day 3-year biologically based flow & 12.5 & 28.0 & 75.2 & 17.1 & 13.1 \\
\hline EPA harmonic-mean flow & 90.3 & 222 & 304 & 59.1 & 51.3 \\
\hline 5-percent-duration flow & 1,790 & 3,080 & 1,870 & 327 & 1,300 \\
\hline 10-percent-duration flow & 1,110 & 1,960 & 1,210 & 211 & 784 \\
\hline 15-percent-duration flow & 824 & 1,490 & 889 & 163 & 556 \\
\hline 20-percent-duration flow & 652 & 1,210 & 738 & 133 & 420 \\
\hline 25-percent-duration flow & 531 & 1,010 & 625 & 114 & 326 \\
\hline 30-percent-duration flow & 438 & 880 & 535 & 99.9 & 253 \\
\hline 35-percent-duration flow & 365 & 779 & 470 & 88.3 & 203 \\
\hline 40-percent-duration flow & 299 & 684 & 412 & 79.2 & 169 \\
\hline 45-percent-duration flow & 244 & 608 & 361 & 71.4 & 136 \\
\hline 50-percent-duration flow & 198 & 555 & 318 & 64.3 & 112 \\
\hline 55-percent-duration flow & 160 & 501 & 281 & 58.0 & 95.0 \\
\hline 60-percent-duration flow & 130 & 448 & 248 & 51.9 & 80.7 \\
\hline 65-percent-duration flow & 106 & 400 & 217 & 47.1 & 67.2 \\
\hline 70-percent-duration flow & 87.6 & 356 & 185 & 42.2 & 55.4 \\
\hline 75-percent-duration flow & 70.8 & 304 & 160 & 37.2 & 45.1 \\
\hline 80-percent-duration flow & 56.5 & 253 & 137 & 32.6 & 37.3 \\
\hline 85-percent-duration flow & 43.9 & 205 & 116 & 28.4 & 31.1 \\
\hline 90-percent-duration flow & 33.4 & 152 & 96.7 & 24.3 & 26.4 \\
\hline 95-percent-duration flow & 24.7 & 92.2 & 73.0 & 19.4 & 18.9 \\
\hline 99-percent-duration flow & 15.2 & 26.5 & 43.9 & 13.2 & 14.3 \\
\hline Variability index & 0.59 & 0.44 & 0.42 & 0.37 & 0.58 \\
\hline
\end{tabular}


Table 11. Selected annual and seasonal statistics for 77 stations in West Virginia representative of 1930-2002.—Continued

[Winter, January 1-March 31; spring, April 1-June 30; summer, July 1-September 30; fall, October 1-December 31; streamflow statistics are in cubic feet per second, except for variability index, which is dimensionless; the record period is in climatic years, April 1 through March 31 of the indicated ending year; EPA, U.S. Environmental Protection Agency]

\begin{tabular}{|c|c|c|c|c|c|}
\hline \multirow{2}{*}{ Streamflow statistic } & \multirow{2}{*}{ Annual } & \multicolumn{4}{|c|}{ Season } \\
\hline & & Winter & Spring & Summer & Fall \\
\hline \multicolumn{6}{|c|}{03180500 Greenbrier River at Durbin, WV, 1944-2002 } \\
\hline 1-day 2-year hydrologically based flow & 7.76 & 69.5 & 31.4 & 8.40 & 16.5 \\
\hline 1-day 5-year hydrologically based flow & 3.07 & 45.8 & 18.8 & 3.37 & 6.38 \\
\hline 1-day 10 -year hydrologically based flow & 1.71 & 36.4 & 14.0 & 1.90 & 3.59 \\
\hline 3-day 2-year hydrologically based flow & 8.33 & 75.0 & 34.8 & 9.13 & 17.7 \\
\hline 3-day 5 -year hydrologically based flow & 3.37 & 48.7 & 20.7 & 3.72 & 6.90 \\
\hline 3-day 10 -year hydrologically based flow & 1.91 & 38.5 & 15.4 & 2.11 & 3.92 \\
\hline 7-day 2-year hydrologically based flow & 9.57 & 86.0 & 42.1 & 10.7 & 20.1 \\
\hline 7-day 5-year hydrologically based flow & 3.94 & 54.9 & 25.3 & 4.36 & 8.30 \\
\hline 7-day 10 -year hydrologically based flow & 2.27 & 42.9 & 19.2 & 2.49 & 5.08 \\
\hline 14-day 2-year hydrologically based flow & 11.7 & 113 & 56.8 & 13.9 & 24.4 \\
\hline 14-day 5-year hydrologically based flow & 4.80 & 68.2 & 34.0 & 5.57 & 9.91 \\
\hline 14-day 10 -year hydrologically based flow & 2.82 & 52.1 & 25.6 & 3.18 & 6.05 \\
\hline 30-day 2-year hydrologically based flow & 16.2 & 202 & 108 & 21.0 & 45.4 \\
\hline 30-day 5-year hydrologically based flow & 7.36 & 121 & 63.4 & 9.29 & 17.2 \\
\hline 30-day 10-year hydrologically based flow & 4.67 & 88.7 & 45.9 & 5.93 & 9.91 \\
\hline 1-day 3-year biologically based flow & 1.58 & 30.0 & 28.0 & 1.64 & 1.96 \\
\hline 4-day 3-year biologically based flow & 1.79 & 36.2 & 35.7 & 2.09 & 3.78 \\
\hline EPA harmonic-mean flow & 36.4 & 166 & 180 & 20.5 & 20.3 \\
\hline 5-percent-duration flow & 955 & 1,450 & 953 & 310 & 757 \\
\hline 10-percent-duration flow & 615 & 966 & 653 & 183 & 480 \\
\hline 15-percent-duration flow & 472 & 756 & 509 & 131 & 362 \\
\hline 20-percent-duration flow & 381 & 622 & 419 & 101 & 287 \\
\hline 25-percent-duration flow & 311 & 531 & 357 & 80.3 & 240 \\
\hline 30-percent-duration flow & 264 & 464 & 310 & 65.5 & 200 \\
\hline 35-percent-duration flow & 222 & 415 & 276 & 54.8 & 173 \\
\hline 40-percent-duration flow & 191 & 370 & 244 & 46.7 & 148 \\
\hline 45-percent-duration flow & 162 & 329 & 215 & 40.0 & 127 \\
\hline 50-percent-duration flow & 138 & 295 & 192 & 34.7 & 106 \\
\hline 55-percent-duration flow & 114 & 264 & 170 & 29.7 & 86.6 \\
\hline 60-percent-duration flow & 92.6 & 235 & 150 & 25.7 & 70.9 \\
\hline 65-percent-duration flow & 74.8 & 210 & 131 & 22.0 & 57.3 \\
\hline 70-percent-duration flow & 59.2 & 185 & 113 & 18.9 & 45.5 \\
\hline 75-percent-duration flow & 45.8 & 161 & 97.5 & 16.0 & 36.2 \\
\hline 80-percent-duration flow & 34.3 & 140 & 80.4 & 13.5 & 27.7 \\
\hline 85-percent-duration flow & 24.8 & 118 & 64.3 & 10.4 & 18.9 \\
\hline 90-percent-duration flow & 16.5 & 92.6 & 49.0 & 7.5 & 13.3 \\
\hline 95-percent-duration flow & 9.9 & 67.8 & 33.6 & 4.9 & 8.9 \\
\hline 99-percent-duration flow & 3.5 & 38.3 & 17.5 & 1.6 & 4.3 \\
\hline Variability index & 0.60 & 0.39 & 0.43 & 0.53 & 0.59 \\
\hline
\end{tabular}


Table 11. Selected annual and seasonal statistics for 77 stations in West Virginia representative of 1930-2002.-Continued

[Winter, January 1-March 31; spring, April 1-June 30; summer, July 1-September 30; fall, October 1-December 31; streamflow statistics are in cubic feet per second, except for variability index, which is dimensionless; the record period is in climatic years, April 1 through March 31of the indicated ending year; EPA, U.S. Environmental Protection Agency]

\begin{tabular}{|c|c|c|c|c|c|}
\hline \multirow{2}{*}{ Streamflow statistic } & \multirow{2}{*}{ Annual } & \multicolumn{4}{|c|}{ Season } \\
\hline & & Winter & Spring & Summer & Fall \\
\hline \multicolumn{6}{|c|}{03182000 Knapp Creek at Marlinton, WV, 1947-1958 } \\
\hline 1-day 2-year hydrologically based flow & 8.94 & 40.3 & 20.4 & 9.28 & 11.2 \\
\hline 1-day 5-year hydrologically based flow & 5.32 & 22.7 & 16.2 & 5.35 & 7.78 \\
\hline 1-day 10-year hydrologically based flow & 3.77 & 15.4 & 14.3 & 3.75 & 6.59 \\
\hline 3-day 2-year hydrologically based flow & 9.36 & 44.7 & 22.0 & 9.81 & 11.5 \\
\hline 3-day 5-year hydrologically based flow & 5.48 & 25.3 & 17.5 & 5.55 & 7.92 \\
\hline 3-day 10-year hydrologically based flow & 3.85 & 17.0 & 15.4 & 3.84 & 6.71 \\
\hline 7-day 2-year hydrologically based flow & 10.0 & 53.3 & 25.5 & 10.5 & 12.5 \\
\hline 7-day 5-year hydrologically based flow & 5.81 & 28.1 & 19.7 & 5.91 & 8.39 \\
\hline 7-day 10 -year hydrologically based flow & 4.10 & 18.5 & 17.3 & 4.11 & 6.90 \\
\hline 14-day 2-year hydrologically based flow & 11.5 & 74.6 & 33.1 & 12.0 & 15.8 \\
\hline 14-day 5-year hydrologically based flow & 6.51 & 36.1 & 25.0 & 6.63 & 10.1 \\
\hline 14-day 10 -year hydrologically based flow & 4.59 & 22.2 & 21.8 & 4.61 & 7.88 \\
\hline 30-day 2-year hydrologically based flow & 13.2 & 114 & 55.9 & 14.3 & 24.0 \\
\hline 30-day 5-year hydrologically based flow & 8.11 & 58.4 & 41.3 & 8.49 & 12.2 \\
\hline 30-day 10-year hydrologically based flow & 6.40 & 37.2 & 35.9 & 6.52 & 8.45 \\
\hline 1-day 3-year biologically based flow & 2.14 & 8.40 & 20.0 & 2.08 & 3.38 \\
\hline 4-day 3-year biologically based flow & 3.62 & 9.05 & 22.7 & 5.56 & 6.99 \\
\hline EPA harmonic-mean flow & 30.6 & 82.0 & 85.1 & 17.7 & 20.1 \\
\hline 5-percent-duration flow & 579 & 895 & 507 & 126 & 455 \\
\hline 10-percent-duration flow & 344 & 594 & 336 & 72.1 & 239 \\
\hline 15-percent-duration flow & 251 & 469 & 247 & 52.1 & 175 \\
\hline 20-percent-duration flow & 195 & 374 & 198 & 42.2 & 134 \\
\hline 25-percent-duration flow & 160 & 314 & 166 & 35.7 & 102 \\
\hline 30-percent-duration flow & 133 & 275 & 143 & 30.2 & 82.8 \\
\hline 35-percent-duration flow & 109 & 242 & 124 & 26.3 & 69.3 \\
\hline 40-percent-duration flow & 90.4 & 209 & 110 & 23.3 & 57.7 \\
\hline 45-percent-duration flow & 74.8 & 189 & 97.3 & 21.3 & 46.6 \\
\hline 50-percent-duration flow & 62.9 & 170 & 87.3 & 19.4 & 39.5 \\
\hline 55-percent-duration flow & 52.9 & 154 & 79.5 & 17.5 & 31.7 \\
\hline 60-percent-duration flow & 44.0 & 139 & 71.2 & 15.9 & 26.7 \\
\hline 65-percent-duration flow & 34.4 & 126 & 63.8 & 14.5 & 22.2 \\
\hline 70-percent-duration flow & 27.5 & 110 & 57.4 & 13.3 & 18.8 \\
\hline 75-percent-duration flow & 22.2 & 93.1 & 51.4 & 12.2 & 16.0 \\
\hline 80-percent-duration flow & 17.9 & 78.8 & 45.2 & 11.0 & 13.7 \\
\hline 85-percent-duration flow & 14.5 & 65.4 & 37.6 & 9.8 & 12.3 \\
\hline 90-percent-duration flow & 11.9 & 50.2 & 30.0 & 8.5 & 10.7 \\
\hline 95-percent-duration flow & 9.0 & 35.1 & 24.1 & 7.1 & 8.5 \\
\hline 99-percent-duration flow & 6.4 & 9.5 & 17.4 & 3.0 & 7.1 \\
\hline Variability index & 0.57 & 0.41 & 0.39 & 0.37 & 0.55 \\
\hline
\end{tabular}


Table 11. Selected annual and seasonal statistics for 77 stations in West Virginia representative of 1930-2002.Continued

[Winter, January 1-March 31; spring, April 1-June 30; summer, July 1-September 30; fall, October 1-December 31; streamflow statistics are in cubic feet per second, except for variability index, which is dimensionless; the record period is in climatic years, April 1 through March 31 of the indicated ending year; EPA, U.S. Environmental Protection Agency]

\begin{tabular}{|c|c|c|c|c|c|}
\hline \multirow{2}{*}{ Streamflow statistic } & \multirow{2}{*}{ Annual } & \multicolumn{4}{|c|}{ Season } \\
\hline & & Winter & Spring & Summer & Fall \\
\hline \multicolumn{6}{|c|}{03182500 Greenbrier River at Buckeye, WV, 1931-2002 } \\
\hline 1-day 2-year hydrologically based flow & 29.7 & 234 & 108 & 32.4 & 49.2 \\
\hline 1-day 5-year hydrologically based flow & 17.0 & 143 & 68.5 & 18.0 & 26.4 \\
\hline 1-day 10-year hydrologically based flow & 12.6 & 107 & 53.1 & 12.9 & 19.2 \\
\hline 3-day 2-year hydrologically based flow & 30.7 & 254 & 120 & 33.8 & 51.7 \\
\hline 3-day 5-year hydrologically based flow & 17.9 & 155 & 75.5 & 18.9 & 27.5 \\
\hline 3-day 10-year hydrologically based flow & 13.3 & 116 & 58.2 & 13.8 & 20.0 \\
\hline 7-day 2-year hydrologically based flow & 33.4 & 299 & 143 & 37.7 & 58.6 \\
\hline 7-day 5-year hydrologically based flow & 19.3 & 178 & 89.9 & 21.0 & 30.2 \\
\hline 7-day 10 -year hydrologically based flow & 14.5 & 132 & 69.2 & 15.3 & 21.7 \\
\hline 14-day 2-year hydrologically based flow & 38.4 & 392 & 192 & 45.7 & 69.9 \\
\hline 14-day 5-year hydrologically based flow & 21.7 & 220 & 116 & 24.5 & 34.4 \\
\hline 14-day 10 -year hydrologically based flow & 16.3 & 159 & 88.0 & 17.7 & 24.1 \\
\hline 30-day 2-year hydrologically based flow & 50.8 & 696 & 341 & 68.3 & 129 \\
\hline 30-day 5-year hydrologically based flow & 28.4 & 389 & 196 & 34.7 & 53.8 \\
\hline 30-day 10 -year hydrologically based flow & 21.4 & 272 & 141 & 24.9 & 33.9 \\
\hline 1-day 3-year biologically based flow & 11.0 & 82.0 & 91.9 & 11.9 & 11.9 \\
\hline 4-day 3-year biologically based flow & 15.4 & 115 & 112 & 17.1 & 17.4 \\
\hline EPA harmonic-mean flow & 144 & 521 & 540 & 82.1 & 84.7 \\
\hline 5-percent-duration flow & 3,310 & 5,020 & 3,250 & 1,020 & 2,580 \\
\hline 10-percent-duration flow & 2,100 & 3,440 & 2,160 & 588 & 1,520 \\
\hline 15-percent-duration flow & 1,550 & 2,640 & 1,660 & 406 & 1,100 \\
\hline 20-percent-duration flow & 1,240 & 2,170 & 1,350 & 310 & 855 \\
\hline 25-percent-duration flow & 1,010 & 1,840 & 1,150 & 252 & 684 \\
\hline 30-percent-duration flow & 836 & 1,610 & 993 & 209 & 571 \\
\hline 35-percent-duration flow & 689 & 1,420 & 860 & 176 & 481 \\
\hline 40-percent-duration flow & 586 & 1,250 & 748 & 149 & 406 \\
\hline 45-percent-duration flow & 492 & 1,110 & 653 & 129 & 336 \\
\hline 50-percent-duration flow & 414 & 990 & 576 & 112 & 277 \\
\hline 55-percent-duration flow & 341 & 877 & 515 & 96.8 & 227 \\
\hline 60-percent-duration flow & 277 & 781 & 458 & 84.9 & 183 \\
\hline 65-percent-duration flow & 223 & 691 & 401 & 73.5 & 149 \\
\hline 70-percent-duration flow & 175 & 605 & 348 & 63.0 & 119 \\
\hline 75-percent-duration flow & 136 & 524 & 299 & 53.3 & 95.1 \\
\hline 80-percent-duration flow & 103 & 453 & 251 & 44.6 & 75.0 \\
\hline 85-percent-duration flow & 76.7 & 371 & 201 & 37.3 & 58.5 \\
\hline 90-percent-duration flow & 53.8 & 296 & 155 & 30.2 & 43.6 \\
\hline 95-percent-duration flow & 34.7 & 207 & 112 & 21.8 & 31.7 \\
\hline 99-percent-duration flow & 16.9 & 101 & 60.1 & 12.1 & 16.8 \\
\hline Variability index & 0.61 & 0.41 & 0.44 & 0.50 & 0.59 \\
\hline
\end{tabular}


Table 11. Selected annual and seasonal statistics for 77 stations in West Virginia representative of 1930-2002.Continued

[Winter, January 1-March 31; spring, April 1-June 30; summer, July 1-September 30; fall, October 1-December 31; streamflow statistics are in cubic feet per second, except for variability index, which is dimensionless; the record period is in climatic years, April 1 through March 31 of the indicated ending year; EPA, U.S. Environmental Protection Agency]

\begin{tabular}{|c|c|c|c|c|c|}
\hline \multirow{2}{*}{ Streamflow statistic } & \multirow{2}{*}{ Annual } & \multicolumn{4}{|c|}{ Season } \\
\hline & & Winter & Spring & Summer & Fall \\
\hline \multicolumn{6}{|c|}{03183000 Second Creek near Second Creek, WV, 1948-1964 } \\
\hline 1-day 2-year hydrologically based flow & 4.18 & 20.7 & 12.1 & 4.51 & 5.36 \\
\hline 1-day 5-year hydrologically based flow & 3.04 & 9.25 & 8.26 & 3.40 & 3.46 \\
\hline 1-day 10-year hydrologically based flow & 2.58 & 5.65 & 6.77 & 2.98 & 2.73 \\
\hline 3-day 2-year hydrologically based flow & 4.71 & 23.9 & 13.2 & 5.01 & 6.06 \\
\hline 3-day 5-year hydrologically based flow & 3.58 & 11.1 & 9.03 & 3.83 & 4.07 \\
\hline 3-day 10-year hydrologically based flow & 3.15 & 6.95 & 7.37 & 3.37 & 3.31 \\
\hline 7-day 2-year hydrologically based flow & 5.22 & 29.6 & 15.4 & 5.51 & 6.76 \\
\hline 7-day 5-year hydrologically based flow & 3.99 & 14.0 & 10.7 & 4.19 & 4.65 \\
\hline 7-day 10-year hydrologically based flow & 3.51 & 8.92 & 8.86 & 3.67 & 3.82 \\
\hline 14-day 2-year hydrologically based flow & 5.72 & 43.1 & 19.6 & 6.07 & 7.61 \\
\hline 14-day 5-year hydrologically based flow & 4.29 & 19.6 & 13.6 & 4.51 & 5.01 \\
\hline 14-day 10-year hydrologically based flow & 3.74 & 12.0 & 11.2 & 3.97 & 4.08 \\
\hline 30-day 2-year hydrologically based flow & 6.58 & 64.5 & 28.5 & 7.28 & 11.1 \\
\hline 30-day 5-year hydrologically based flow & 4.87 & 33.7 & 19.5 & 5.69 & 5.94 \\
\hline 30-day 10-year hydrologically based flow & 4.19 & 22.5 & 15.7 & 5.14 & 4.33 \\
\hline 1-day 3-year biologically based flow & 2.68 & 4.09 & 10.9 & 3.39 & 2.66 \\
\hline 4-day 3-year biologically based flow & 3.22 & 4.95 & 12.2 & 4.26 & 3.23 \\
\hline EPA harmonic-mean flow & 16.4 & 37.8 & 48.9 & 10.4 & 10.0 \\
\hline 5-percent-duration flow & 297 & 548 & 292 & 55.4 & 200 \\
\hline 10-percent-duration flow & 183 & 332 & 193 & 32.7 & 106 \\
\hline 15-percent-duration flow & 135 & 246 & 143 & 24.6 & 72.6 \\
\hline 20-percent-duration flow & 106 & 201 & 114 & 20.5 & 54.6 \\
\hline 25-percent-duration flow & 86.7 & 175 & 97.1 & 17.6 & 44.6 \\
\hline 30-percent-duration flow & 70.4 & 154 & 84.4 & 15.4 & 36.3 \\
\hline 35-percent-duration flow & 57.6 & 136 & 73.1 & 13.8 & 29.0 \\
\hline 40-percent-duration flow & 47.4 & 123 & 63.9 & 12.4 & 23.5 \\
\hline 45-percent-duration flow & 39.0 & 110 & 56.6 & 11.2 & 19.6 \\
\hline 50-percent-duration flow & 31.7 & 99.2 & 50.4 & 9.9 & 16.6 \\
\hline 55-percent-duration flow & 25.5 & 88.3 & 45.3 & 9.1 & 14.1 \\
\hline 60-percent-duration flow & 20.3 & 79.8 & 40.5 & 8.4 & 12.2 \\
\hline 65-percent-duration flow & 16.7 & 71.3 & 35.8 & 7.7 & 10.2 \\
\hline 70-percent-duration flow & 13.7 & 63.2 & 31.3 & 7.1 & 8.6 \\
\hline 75-percent-duration flow & 11.4 & 54.9 & 27.4 & 6.7 & 7.7 \\
\hline 80-percent-duration flow & 8.9 & 46.4 & 23.2 & 6.2 & 6.8 \\
\hline 85-percent-duration flow & 7.5 & 37.6 & 19.8 & 5.8 & 6.0 \\
\hline 90-percent-duration flow & 6.3 & 27.9 & 16.3 & 5.2 & 5.3 \\
\hline 95-percent-duration flow & 5.1 & 16.4 & 12.5 & 4.5 & 4.4 \\
\hline 99-percent-duration flow & 3.6 & 5.5 & 8.6 & 3.4 & 3.2 \\
\hline Variability index & 0.57 & 0.43 & 0.41 & 0.33 & 0.52 \\
\hline
\end{tabular}


Table 11. Selected annual and seasonal statistics for 77 stations in West Virginia representative of 1930-2002.Continued

[Winter, January 1-March 31; spring, April 1-June 30; summer, July 1-September 30; fall, October 1-December 31; streamflow statistics are in cubic feet per second, except for variability index, which is dimensionless; the record period is in climatic years, April 1 through March 31 of the indicated ending year; EPA, U.S. Environmental Protection Agency]

\begin{tabular}{|c|c|c|c|c|c|}
\hline \multirow{2}{*}{ Streamflow statistic } & \multirow{2}{*}{ Annual } & \multicolumn{4}{|c|}{ Season } \\
\hline & & Winter & Spring & Summer & Fall \\
\hline \multicolumn{6}{|c|}{03183500 Greenbrier River at Alderson, WV, 1930-2002 } \\
\hline 1-day 2-year hydrologically based flow & 84.1 & 540 & 281 & 92.8 & 126 \\
\hline 1-day 5-year hydrologically based flow & 54.9 & 313 & 180 & 58.5 & 74.8 \\
\hline 1-day 10-year hydrologically based flow & 44.0 & 224 & 142 & 45.8 & 57.6 \\
\hline 3-day 2-year hydrologically based flow & 86.7 & 577 & 302 & 96.2 & 130 \\
\hline 3-day 5-year hydrologically based flow & 57.0 & 336 & 193 & 61.1 & 76.5 \\
\hline 3-day 10-year hydrologically based flow & 46.0 & 241 & 152 & 48.2 & 59.2 \\
\hline 7-day 2-year hydrologically based flow & 91.1 & 683 & 346 & 103 & 140 \\
\hline 7-day 5-year hydrologically based flow & 59.3 & 384 & 218 & 64.8 & 80.1 \\
\hline 7-day 10 -year hydrologically based flow & 48.0 & 272 & 171 & 50.9 & 61.4 \\
\hline 14-day 2-year hydrologically based flow & 101 & 879 & 446 & 120 & 163 \\
\hline 14-day 5-year hydrologically based flow & 64.4 & 465 & 277 & 73.2 & 88.2 \\
\hline 14-day 10 -year hydrologically based flow & 52.1 & 321 & 216 & 56.9 & 65.5 \\
\hline 30-day 2-year hydrologically based flow & 125 & 1,560 & 756 & 167 & 272 \\
\hline 30-day 5-year hydrologically based flow & 77.5 & 836 & 447 & 94.1 & 124 \\
\hline 30-day 10 -year hydrologically based flow & 62.0 & 568 & 334 & 72.3 & 82.6 \\
\hline 1-day 3-year biologically based flow & 45.0 & 140 & 244 & 46.9 & 47.9 \\
\hline 4-day 3-year biologically based flow & 47.9 & 185 & 268 & 51.5 & 57.9 \\
\hline EPA harmonic-mean flow & 367 & 1,090 & 1,250 & 224 & 213 \\
\hline 5-percent-duration flow & 7,110 & 11,400 & 7,090 & 2,190 & 5,340 \\
\hline 10-percent-duration flow & 4,600 & 7,620 & 4,770 & 1,250 & 3,140 \\
\hline 15-percent-duration flow & 3,420 & 6,020 & 3,660 & 872 & 2,290 \\
\hline 20-percent-duration flow & 2,700 & 4,950 & 2,980 & 680 & 1,800 \\
\hline 25-percent-duration flow & 2,190 & 4,170 & 2,530 & 555 & 1,460 \\
\hline 30-percent-duration flow & 1,840 & 3,600 & 2,190 & 466 & 1,200 \\
\hline 35-percent-duration flow & 1,540 & 3,170 & 1,910 & 401 & 978 \\
\hline 40-percent-duration flow & 1,290 & 2,810 & 1,680 & 347 & 795 \\
\hline 45-percent-duration flow & 1,080 & 2,490 & 1,490 & 301 & 668 \\
\hline 50-percent-duration flow & 895 & 2,230 & 1,320 & 264 & 549 \\
\hline 55-percent-duration flow & 739 & 1,980 & 1,170 & 233 & 444 \\
\hline 60-percent-duration flow & 603 & 1,770 & 1,040 & 206 & 359 \\
\hline 65-percent-duration flow & 483 & 1,560 & 918 & 183 & 293 \\
\hline 70-percent-duration flow & 382 & 1,370 & 804 & 161 & 241 \\
\hline 75-percent-duration flow & 301 & 1,180 & 692 & 141 & 198 \\
\hline 80-percent-duration flow & 235 & 989 & 588 & 121 & 165 \\
\hline 85-percent-duration flow & 182 & 821 & 479 & 102 & 132 \\
\hline 90-percent-duration flow & 135 & 654 & 376 & 84.1 & 106 \\
\hline 95-percent-duration flow & 92.6 & 444 & 274 & 66.3 & 81.1 \\
\hline 99-percent-duration flow & 54.7 & 193 & 161 & 44.2 & 50.1 \\
\hline Variability index & 0.59 & 0.42 & 0.42 & 0.45 & 0.57 \\
\hline
\end{tabular}


Table 11. Selected annual and seasonal statistics for 77 stations in West Virginia representative of 1930-2002. Continued

[Winter, January 1-March 31; spring, April 1-June 30; summer, July 1-September 30; fall, October 1-December 31; streamflow statistics are in cubic feet per second, except for variability index, which is dimensionless; the record period is in climatic years, April 1 through March 31 of the indicated ending year; EPA, U.S. Environmental Protection Agency]

\begin{tabular}{|c|c|c|c|c|c|}
\hline \multirow{2}{*}{ Streamflow statistic } & \multirow{2}{*}{ Annual } & \multicolumn{4}{|c|}{ Season } \\
\hline & & Winter & Spring & Summer & Fall \\
\hline \multicolumn{6}{|c|}{03184000 Greenbrier River at Hilldale, WV, 1938-2002 } \\
\hline 1-day 2-year hydrologically based flow & 91.1 & 622 & 329 & 101 & 132 \\
\hline 1-day 5-year hydrologically based flow & 59.6 & 349 & 210 & 64.3 & 78.1 \\
\hline 1-day 10-year hydrologically based flow & 48.2 & 245 & 165 & 50.8 & 61.4 \\
\hline 3-day 2-year hydrologically based flow & 93.4 & 667 & 352 & 105 & 137 \\
\hline 3-day 5-year hydrologically based flow & 60.7 & 375 & 223 & 66.1 & 80.1 \\
\hline 3-day 10-year hydrologically based flow & 49.0 & 262 & 175 & 52.0 & 62.8 \\
\hline 7-day 2-year hydrologically based flow & 97.9 & 796 & 400 & 113 & 148 \\
\hline 7-day 5-year hydrologically based flow & 63.4 & 437 & 252 & 70.6 & 83.6 \\
\hline 7-day 10-year hydrologically based flow & 51.5 & 303 & 197 & 55.6 & 64.6 \\
\hline 14-day 2-year hydrologically based flow & 108 & 1,050 & 518 & 132 & 171 \\
\hline 14-day 5-year hydrologically based flow & 68.2 & 528 & 319 & 79.5 & 91.0 \\
\hline 14-day 10-year hydrologically based flow & 55.2 & 354 & 246 & 61.9 & 68.0 \\
\hline 30-day 2-year hydrologically based flow & 136 & 1,870 & 913 & 186 & 288 \\
\hline 30-day 5-year hydrologically based flow & 82.6 & 995 & 530 & 106 & 128 \\
\hline 30-day 10-year hydrologically based flow & 65.9 & 670 & 391 & 80.7 & 85.7 \\
\hline 1-day 3-year biologically based flow & 45.9 & 170 & 293 & 49.9 & 48.0 \\
\hline 4-day 3-year biologically based flow & 49.9 & 203 & 337 & 57.8 & 58.3 \\
\hline EPA harmonic-mean flow & 413 & 1,250 & 1,530 & 261 & 229 \\
\hline 5-percent-duration flow & 8,300 & 13,200 & 8,560 & 2,460 & 6,110 \\
\hline 10-percent-duration flow & 5,510 & 8,720 & 5,670 & 1,420 & 3,750 \\
\hline 15-percent-duration flow & 4,140 & 7,030 & 4,440 & 1,000 & 2,740 \\
\hline 20-percent-duration flow & 3,270 & 5,920 & 3,600 & 774 & 2,100 \\
\hline 25-percent-duration flow & 2,670 & 5,050 & 3,030 & 629 & 1,680 \\
\hline 30-percent-duration flow & 2,220 & 4,400 & 2,610 & 525 & 1,380 \\
\hline 35-percent-duration flow & 1,840 & 3,890 & 2,280 & 451 & 1,110 \\
\hline 40-percent-duration flow & 1,530 & 3,460 & 2,020 & 390 & 908 \\
\hline 45-percent-duration flow & 1,270 & 3,090 & 1,790 & 339 & 753 \\
\hline 50-percent-duration flow & 1,050 & 2,760 & 1,590 & 299 & 625 \\
\hline 55-percent-duration flow & 864 & 2,450 & 1,420 & 265 & 507 \\
\hline 60-percent-duration flow & 696 & 2,170 & 1,250 & 234 & 406 \\
\hline 65-percent-duration flow & 555 & 1,910 & 1,100 & 207 & 329 \\
\hline 70-percent-duration flow & 434 & 1,670 & 962 & 182 & 265 \\
\hline 75-percent-duration flow & 342 & 1,430 & 831 & 159 & 216 \\
\hline 80-percent-duration flow & 265 & 1,180 & 700 & 136 & 178 \\
\hline 85-percent-duration flow & 202 & 981 & 563 & 114 & 142 \\
\hline 90-percent-duration flow & 148 & 764 & 437 & 93.6 & 108 \\
\hline 95-percent-duration flow & 99.1 & 496 & 324 & 73.7 & 84.7 \\
\hline 99-percent-duration flow & 61.7 & 205 & 177 & 51.3 & 56.8 \\
\hline Variability index & 0.60 & 0.42 & 0.43 & 0.46 & 0.59 \\
\hline
\end{tabular}


Table 11. Selected annual and seasonal statistics for 77 stations in West Virginia representative of 1930-2002.-Continued

[Winter, January 1-March 31; spring, April 1-June 30; summer, July 1-September 30; fall, October 1-December 31; streamflow statistics are in cubic feet per second, except for variability index, which is dimensionless; the record period is in climatic years, April 1 through March 31of the indicated ending year; EPA, U.S. Environmental Protection Agency]

\begin{tabular}{|c|c|c|c|c|c|}
\hline \multirow{2}{*}{ Streamflow statistic } & \multirow{2}{*}{ Annual } & \multicolumn{4}{|c|}{ Season } \\
\hline & & Winter & Spring & Summer & Fall \\
\hline \multicolumn{6}{|c|}{03185000 Piney Creek at Raleigh, WV, 1953-1978 } \\
\hline 1-day 2-year hydrologically based flow & 1.85 & 19.3 & 9.95 & 2.40 & 2.69 \\
\hline 1-day 5-year hydrologically based flow & 0.86 & 7.91 & 5.39 & 1.12 & 1.16 \\
\hline 1-day 10-year hydrologically based flow & 0.56 & 4.22 & 3.55 & 0.70 & 0.78 \\
\hline 3-day 2-year hydrologically based flow & 1.99 & 20.9 & 11.3 & 2.58 & 2.96 \\
\hline 3-day 5-year hydrologically based flow & 0.93 & 8.47 & 6.16 & 1.19 & 1.31 \\
\hline 3-day 10-year hydrologically based flow & 0.61 & 4.48 & 4.04 & 0.74 & 0.90 \\
\hline 7-day 2-year hydrologically based flow & 2.19 & 26.3 & 13.7 & 2.99 & 3.30 \\
\hline 7-day 5-year hydrologically based flow & 1.00 & 10.4 & 7.74 & 1.36 & 1.43 \\
\hline 7-day 10-year hydrologically based flow & 0.64 & 5.28 & 5.26 & 0.82 & 0.96 \\
\hline 14-day 2-year hydrologically based flow & 2.60 & 34.7 & 17.0 & 3.85 & 3.77 \\
\hline 14-day 5-year hydrologically based flow & 1.16 & 13.6 & 9.77 & 1.71 & 1.61 \\
\hline 14-day 10 -year hydrologically based flow & 0.73 & 6.68 & 6.91 & 1.00 & 1.09 \\
\hline 30-day 2-year hydrologically based flow & 3.74 & 55.8 & 26.0 & 6.30 & 7.28 \\
\hline 30-day 5-year hydrologically based flow & 1.73 & 26.1 & 14.5 & 2.91 & 2.76 \\
\hline 30-day 10-year hydrologically based flow & 1.10 & 14.7 & 10.3 & 1.71 & 1.64 \\
\hline 1-day 3-year biologically based flow & 0.54 & 3.08 & 6.88 & 0.77 & 0.66 \\
\hline 4-day 3-year biologically based flow & 0.63 & 3.88 & 8.43 & 0.87 & 0.80 \\
\hline EPA harmonic-mean flow & 8.41 & 19.5 & 39.7 & 5.80 & 4.43 \\
\hline 5-percent-duration flow & 217 & 384 & 210 & 66.4 & 157 \\
\hline 10-percent-duration flow & 143 & 244 & 148 & 39.4 & 103 \\
\hline 15-percent-duration flow & 110 & 182 & 116 & 28.0 & 75.9 \\
\hline 20-percent-duration flow & 88.2 & 149 & 95.5 & 22.9 & 57.8 \\
\hline 25-percent-duration flow & 71.7 & 129 & 82.0 & 18.9 & 45.9 \\
\hline 30-percent-duration flow & 60.3 & 114 & 71.0 & 16.0 & 36.7 \\
\hline 35-percent-duration flow & 50.5 & 101 & 62.5 & 13.6 & 30.0 \\
\hline 40-percent-duration flow & 41.9 & 90.8 & 54.9 & 11.7 & 25.0 \\
\hline 45-percent-duration flow & 35.2 & 81.6 & 48.0 & 10.0 & 19.6 \\
\hline 50-percent-duration flow & 29.2 & 73.3 & 42.6 & 8.5 & 14.8 \\
\hline 55-percent-duration flow & 24.2 & 66.2 & 37.5 & 7.7 & 11.4 \\
\hline 60-percent-duration flow & 19.4 & 59.2 & 33.3 & 6.8 & 8.9 \\
\hline 65-percent-duration flow & 15.4 & 53.1 & 29.5 & 6.2 & 7.3 \\
\hline 70-percent-duration flow & 11.9 & 47.0 & 26.3 & 5.5 & 5.9 \\
\hline 75-percent-duration flow & 8.9 & 41.5 & 23.2 & 4.7 & 4.8 \\
\hline 80-percent-duration flow & 6.9 & 35.9 & 20.1 & 4.0 & 3.9 \\
\hline 85-percent-duration flow & 5.3 & 30.4 & 16.4 & 3.2 & 3.0 \\
\hline 90-percent-duration flow & 3.7 & 20.7 & 13.3 & 2.4 & 2.2 \\
\hline 95-percent-duration flow & 2.2 & 13.2 & 9.1 & 1.5 & 1.3 \\
\hline 99-percent-duration flow & 0.8 & 1.7 & 4.9 & 0.4 & 0.8 \\
\hline Variability index & 0.62 & 0.41 & 0.41 & 0.48 & 0.65 \\
\hline
\end{tabular}


Table 11. Selected annual and seasonal statistics for 77 stations in West Virginia representative of 1930-2002.—Continued

[Winter, January 1-March 31; spring, April 1-June 30; summer, July 1-September 30; fall, October 1-December 31; streamflow statistics are in cubic feet per second, except for variability index, which is dimensionless; the record period is in climatic years, April 1 through March 31 of the indicated ending year; EPA, U.S. Environmental Protection Agency]

\begin{tabular}{|c|c|c|c|c|c|}
\hline \multirow{2}{*}{ Streamflow statistic } & \multirow{2}{*}{ Annual } & \multicolumn{4}{|c|}{ Season } \\
\hline & & Winter & Spring & Summer & Fall \\
\hline \multicolumn{6}{|c|}{03186500 Williams River at Dyer, WV, 1931-2002 } \\
\hline 1-day 2-year hydrologically based flow & 7.15 & 94.3 & 30.9 & 7.78 & 18.1 \\
\hline 1-day 5-year hydrologically based flow & 2.91 & 64.8 & 16.2 & 3.18 & 6.72 \\
\hline 1-day 10-year hydrologically based flow & 1.77 & 51.6 & 11.2 & 1.95 & 3.81 \\
\hline 3-day 2-year hydrologically based flow & 7.69 & 102 & 35.3 & 8.52 & 19.5 \\
\hline 3-day 5-year hydrologically based flow & 3.13 & 70.0 & 18.3 & 3.47 & 7.15 \\
\hline 3-day 10-year hydrologically based flow & 1.91 & 55.6 & 12.5 & 2.13 & 4.02 \\
\hline 7-day 2-year hydrologically based flow & 9.04 & 117 & 46.4 & 10.5 & 24.8 \\
\hline 7-day 5-year hydrologically based flow & 3.63 & 78.0 & 23.5 & 4.18 & 8.84 \\
\hline 7-day 10-year hydrologically based flow & 2.21 & 61.9 & 15.8 & 2.54 & 4.81 \\
\hline 14-day 2-year hydrologically based flow & 11.9 & 150 & 67.6 & 14.9 & 33.7 \\
\hline 14-day 5-year hydrologically based flow & 4.71 & 93.6 & 34.5 & 5.78 & 11.6 \\
\hline 14-day 10 -year hydrologically based flow & 2.81 & 73.1 & 23.4 & 3.42 & 6.18 \\
\hline 30-day 2-year hydrologically based flow & 20.1 & 258 & 128 & 31.6 & 77.2 \\
\hline 30-day 5-year hydrologically based flow & 8.12 & 164 & 67.2 & 11.8 & 25.1 \\
\hline 30-day 10-year hydrologically based flow & 5.00 & 126 & 45.4 & 6.81 & 12.5 \\
\hline 1-day 3-year biologically based flow & 1.56 & 42.8 & 19.0 & 1.99 & 2.20 \\
\hline 4-day 3-year biologically based flow & 2.02 & 56.0 & 26.1 & 2.65 & 2.80 \\
\hline EPA harmonic-mean flow & 41.2 & 222 & 175 & 23.5 & 22.6 \\
\hline 5-percent-duration flow & 1,190 & 1,740 & 1,090 & 580 & 1,020 \\
\hline 10-percent-duration flow & 776 & 1,180 & 776 & 340 & 656 \\
\hline 15-percent-duration flow & 588 & 910 & 614 & 244 & 491 \\
\hline 20-percent-duration flow & 478 & 750 & 510 & 184 & 388 \\
\hline 25-percent-duration flow & 401 & 641 & 434 & 142 & 327 \\
\hline 30-percent-duration flow & 335 & 557 & 374 & 114 & 277 \\
\hline 35-percent-duration flow & 290 & 494 & 330 & 93.3 & 235 \\
\hline 40-percent-duration flow & 246 & 440 & 289 & 77.6 & 205 \\
\hline 45-percent-duration flow & 212 & 392 & 254 & 64.1 & 178 \\
\hline 50-percent-duration flow & 180 & 354 & 224 & 53.5 & 154 \\
\hline 55-percent-duration flow & 153 & 317 & 196 & 44.4 & 131 \\
\hline 60-percent-duration flow & 129 & 282 & 173 & 36.5 & 113 \\
\hline 65-percent-duration flow & 107 & 250 & 150 & 30.0 & 96.4 \\
\hline 70-percent-duration flow & 87.1 & 220 & 129 & 24.3 & 79.4 \\
\hline 75-percent-duration flow & 68.2 & 192 & 108 & 19.3 & 61.2 \\
\hline 80-percent-duration flow & 49.7 & 165 & 88.5 & 15.0 & 42.7 \\
\hline 85-percent-duration flow & 32.8 & 139 & 69.9 & 10.7 & 26.7 \\
\hline 90-percent-duration flow & 19.8 & 118 & 51.1 & 7.1 & 15.9 \\
\hline 95-percent-duration flow & 9.5 & 91.9 & 32.1 & 4.3 & 8.8 \\
\hline 99-percent-duration flow & 3.1 & 59.3 & 12.1 & 2.1 & 2.9 \\
\hline Variability index & 0.62 & 0.39 & 0.46 & 0.64 & 0.61 \\
\hline
\end{tabular}


Table 11. Selected annual and seasonal statistics for 77 stations in West Virginia representative of 1930-2002.-Continued

[Winter, January 1-March 31; spring, April 1-June 30; summer, July 1-September 30; fall, October 1-December 31; streamflow statistics are in cubic feet per second, except for variability index, which is dimensionless; the record period is in climatic years, April 1 through March 31of the indicated ending year; EPA, U.S. Environmental Protection Agency]

\begin{tabular}{|c|c|c|c|c|c|}
\hline \multirow{2}{*}{ Streamflow statistic } & \multirow{2}{*}{ Annual } & \multicolumn{4}{|c|}{ Season } \\
\hline & & Winter & Spring & Summer & Fall \\
\hline \multicolumn{6}{|c|}{03187000 Gauley River at Camden On Gauley, WV, 1932-1957 } \\
\hline 1-day 2-year hydrologically based flow & 16.4 & 173 & 63.5 & 16.7 & 25.9 \\
\hline 1-day 5-year hydrologically based flow & 5.30 & 112 & 37.8 & 6.74 & 7.85 \\
\hline 1-day 10-year hydrologically based flow & 2.57 & 87.1 & 28.7 & 4.04 & 3.57 \\
\hline 3-day 2-year hydrologically based flow & 17.6 & 196 & 72.7 & 18.3 & 28.4 \\
\hline 3-day 5-year hydrologically based flow & 5.56 & 127 & 43.1 & 7.25 & 8.53 \\
\hline 3-day 10-year hydrologically based flow & 2.66 & 97.6 & 32.5 & 4.28 & 3.80 \\
\hline 7-day 2-year hydrologically based flow & 20.4 & 226 & 94.2 & 22.6 & 35.9 \\
\hline 7-day 5-year hydrologically based flow & 6.31 & 140 & 56.0 & 8.80 & 10.3 \\
\hline 7-day 10-year hydrologically based flow & 3.01 & 105 & 41.9 & 5.12 & 4.44 \\
\hline 14-day 2-year hydrologically based flow & 26.7 & 302 & 140 & 33.2 & 45.7 \\
\hline 14-day 5-year hydrologically based flow & 8.21 & 173 & 78.1 & 12.6 & 12.8 \\
\hline 14-day 10-year hydrologically based flow & 3.92 & 125 & 56.3 & 7.13 & 5.50 \\
\hline 30-day 2-year hydrologically based flow & 39.1 & 519 & 235 & 67.8 & 98.5 \\
\hline 30-day 5-year hydrologically based flow & 12.6 & 324 & 136 & 24.9 & 27.3 \\
\hline 30-day 10-year hydrologically based flow & 6.30 & 241 & 98.7 & 13.7 & 11.9 \\
\hline 1-day 3-year biologically based flow & 3.45 & 80.0 & 29.9 & 4.18 & 4.35 \\
\hline 4-day 3-year biologically based flow & 4.15 & 95.8 & 50.7 & 5.39 & 4.95 \\
\hline EPA harmonic-mean flow & 57.8 & 430 & 292 & 49.7 & 23.1 \\
\hline 5-percent-duration flow & 2,040 & 3,070 & 1,820 & 1,100 & 1,510 \\
\hline 10-percent-duration flow & 1,370 & 2,140 & 1,290 & 668 & 958 \\
\hline 15-percent-duration flow & 1,050 & 1,650 & 1,020 & 483 & 720 \\
\hline 20-percent-duration flow & 859 & 1,400 & 859 & 367 & 595 \\
\hline 25-percent-duration flow & 724 & 1,210 & 742 & 291 & 488 \\
\hline 30-percent-duration flow & 607 & 1,060 & 642 & 236 & 405 \\
\hline 35-percent-duration flow & 523 & 960 & 560 & 192 & 348 \\
\hline 40-percent-duration flow & 446 & 869 & 497 & 159 & 302 \\
\hline 45-percent-duration flow & 383 & 789 & 443 & 132 & 259 \\
\hline 50-percent-duration flow & 326 & 714 & 394 & 109 & 219 \\
\hline 55-percent-duration flow & 275 & 643 & 352 & 91.0 & 187 \\
\hline 60-percent-duration flow & 229 & 579 & 311 & 75.4 & 160 \\
\hline 65-percent-duration flow & 189 & 521 & 272 & 61.4 & 133 \\
\hline 70-percent-duration flow & 151 & 468 & 238 & 49.2 & 105 \\
\hline 75-percent-duration flow & 117 & 411 & 199 & 39.6 & 73.8 \\
\hline 80-percent-duration flow & 86.5 & 351 & 163 & 30.4 & 47.6 \\
\hline 85-percent-duration flow & 57.1 & 293 & 133 & 22.1 & 31.4 \\
\hline 90-percent-duration flow & 35.2 & 233 & 100 & 15.1 & 21.0 \\
\hline 95-percent-duration flow & 17.9 & 169 & 70.6 & 9.5 & 12.2 \\
\hline 99-percent-duration flow & 5.4 & 68.6 & 35.0 & 3.8 & 1.4 \\
\hline Variability index & 0.62 & 0.37 & 0.42 & 0.63 & 0.63 \\
\hline
\end{tabular}


Table 11. Selected annual and seasonal statistics for 77 stations in West Virginia representative of 1930-2002. - Continued

[Winter, January 1-March 31; spring, April 1-June 30; summer, July 1-September 30; fall, October 1-December 31; streamflow statistics are in cubic feet per second, except for variability index, which is dimensionless; the record period is in climatic years, April 1 through March 31of the indicated ending year; EPA, U.S. Environmental Protection Agency]

\begin{tabular}{|c|c|c|c|c|c|}
\hline \multirow{2}{*}{ Streamflow statistic } & \multirow{2}{*}{ Annual } & \multicolumn{4}{|c|}{ Season } \\
\hline & & Winter & Spring & Summer & Fall \\
\hline \multicolumn{6}{|c|}{03187500 Cranberry River near Richwood, WV, 1966-1979 } \\
\hline 1-day 2-year hydrologically based flow & 6.44 & 68.0 & 22.2 & 6.82 & 27.8 \\
\hline 1-day 5-year hydrologically based flow & 3.82 & 44.6 & 10.1 & 3.89 & 12.7 \\
\hline 1-day 10-year hydrologically based flow & 2.86 & 35.7 & 5.96 & 2.87 & 7.82 \\
\hline 3-day 2-year hydrologically based flow & 7.13 & 70.8 & 25.9 & 7.65 & 31.1 \\
\hline 3-day 5-year hydrologically based flow & 4.13 & 45.7 & 11.4 & 4.21 & 13.9 \\
\hline 3-day 10 -year hydrologically based flow & 3.03 & 36.1 & 6.64 & 3.03 & 8.48 \\
\hline 7-day 2-year hydrologically based flow & 8.64 & 77.4 & 30.8 & 9.58 & 38.3 \\
\hline 7-day 5-year hydrologically based flow & 4.86 & 47.0 & 13.0 & 5.49 & 18.0 \\
\hline 7-day 10-year hydrologically based flow & 3.47 & 36.4 & 7.69 & 4.14 & 11.7 \\
\hline 14-day 2-year hydrologically based flow & 12.1 & 93.2 & 41.5 & 14.3 & 48.9 \\
\hline 14-day 5-year hydrologically based flow & 6.66 & 52.7 & 17.9 & 8.06 & 22.0 \\
\hline 14-day 10 -year hydrologically based flow & 4.72 & 39.1 & 11.1 & 6.02 & 13.8 \\
\hline 30-day 2-year hydrologically based flow & 21.0 & 150 & 75.7 & 26.7 & 113 \\
\hline 30-day 5-year hydrologically based flow & 12.6 & 74.8 & 35.9 & 17.2 & 59.8 \\
\hline 30-day 10-year hydrologically based flow & 9.25 & 49.7 & 23.2 & 14.0 & 37.1 \\
\hline 1-day 3-year biologically based flow & 2.07 & 32.0 & 14.9 & 2.09 & 4.49 \\
\hline 4-day 3-year biologically based flow & 3.31 & 34.9 & 16.6 & 3.31 & 5.26 \\
\hline EPA harmonic-mean flow & 52.4 & 139 & 124 & 26.1 & 44.2 \\
\hline 5-percent-duration flow & 818 & 1,140 & 775 & 409 & 783 \\
\hline 10-percent-duration flow & 564 & 803 & 568 & 258 & 541 \\
\hline 15-percent-duration flow & 433 & 635 & 460 & 191 & 411 \\
\hline 20-percent-duration flow & 359 & 514 & 383 & 150 & 342 \\
\hline 25-percent-duration flow & 303 & 437 & 325 & 122 & 282 \\
\hline 30-percent-duration flow & 257 & 384 & 285 & 98.2 & 246 \\
\hline 35-percent-duration flow & 221 & 343 & 250 & 81.0 & 215 \\
\hline 40-percent-duration flow & 189 & 309 & 218 & 67.8 & 188 \\
\hline 45-percent-duration flow & 161 & 279 & 188 & 57.7 & 167 \\
\hline 50-percent-duration flow & 140 & 249 & 163 & 49.6 & 149 \\
\hline 55-percent-duration flow & 120 & 216 & 140 & 42.6 & 135 \\
\hline 60-percent-duration flow & 101 & 191 & 119 & 36.6 & 120 \\
\hline 65-percent-duration flow & 83.7 & 167 & 100 & 31.2 & 105 \\
\hline 70-percent-duration flow & 68.7 & 142 & 83.5 & 26.0 & 88.8 \\
\hline 75-percent-duration flow & 56.0 & 118 & 69.1 & 21.3 & 76.0 \\
\hline 80-percent-duration flow & 45.0 & 97.0 & 56.9 & 17.4 & 65.0 \\
\hline 85-percent-duration flow & 34.6 & 78.1 & 44.2 & 13.7 & 53.0 \\
\hline 90-percent-duration flow & 23.6 & 56.6 & 31.9 & 9.7 & 37.1 \\
\hline 95-percent-duration flow & 12.9 & 43.5 & 19.5 & 6.8 & 19.1 \\
\hline 99-percent-duration flow & 5.5 & 34.3 & 6.5 & 3.8 & 8.1 \\
\hline Variability index & 0.54 & 0.43 & 0.49 & 0.54 & 0.46 \\
\hline
\end{tabular}


Table 11. Selected annual and seasonal statistics for 77 stations in West Virginia representative of 1930-2002.-Continued

[Winter, January 1-March 31; spring, April 1-June 30; summer, July 1-September 30; fall, October 1-December 31; streamflow statistics are in cubic feet per second, except for variability index, which is dimensionless; the record period is in climatic years, April 1 through March 31of the indicated ending year; EPA, U.S. Environmental Protection Agency]

\begin{tabular}{|c|c|c|c|c|c|}
\hline \multirow{2}{*}{ Streamflow statistic } & \multirow{2}{*}{ Annual } & \multicolumn{4}{|c|}{ Season } \\
\hline & & Winter & Spring & Summer & Fall \\
\hline \multicolumn{6}{|c|}{03189000 Cherry River at Fenwick, WV, 1932-1957 } \\
\hline 1-day 2-year hydrologically based flow & 4.44 & 107 & 29.8 & 5.56 & 8.03 \\
\hline 1-day 5-year hydrologically based flow & 1.49 & 67.8 & 14.1 & 2.13 & 2.22 \\
\hline 1-day 10 -year hydrologically based flow & 0.82 & 52.0 & 9.34 & 1.27 & 1.12 \\
\hline 3-day 2-year hydrologically based flow & 5.32 & 124 & 37.1 & 6.32 & 8.77 \\
\hline 3-day 5 -year hydrologically based flow & 2.03 & 75.9 & 18.0 & 2.48 & 2.86 \\
\hline 3-day 10 -year hydrologically based flow & 1.23 & 56.4 & 12.1 & 1.50 & 1.64 \\
\hline 7-day 2-year hydrologically based flow & 6.55 & 154 & 55.0 & 8.22 & 12.0 \\
\hline 7-day 5-year hydrologically based flow & 2.46 & 87.1 & 26.4 & 3.29 & 3.74 \\
\hline 7-day 10-year hydrologically based flow & 1.48 & 62.0 & 17.0 & 2.03 & 2.01 \\
\hline 14-day 2-year hydrologically based flow & 8.71 & 200 & 95.2 & 11.9 & 19.8 \\
\hline 14-day 5-year hydrologically based flow & 3.20 & 113 & 43.5 & 4.59 & 5.53 \\
\hline 14-day 10 -year hydrologically based flow & 1.87 & 82.1 & 26.1 & 2.71 & 2.72 \\
\hline 30-day 2-year hydrologically based flow & 14.6 & 358 & 161 & 29.9 & 46.1 \\
\hline 30-day 5-year hydrologically based flow & 5.02 & 209 & 76.4 & 10.5 & 12.1 \\
\hline 30-day 10-year hydrologically based flow & 2.82 & 152 & 47.1 & 5.67 & 5.62 \\
\hline 1-day 3-year biologically based flow & 1.00 & 44.9 & 11.0 & 1.48 & 1.18 \\
\hline 4-day 3-year biologically based flow & 1.18 & 54.8 & 19.2 & 2.10 & 1.42 \\
\hline EPA harmonic-mean flow & 29.3 & 286 & 166 & 19.7 & 13.1 \\
\hline 5-percent-duration flow & 1,450 & 2,280 & 1,380 & 757 & 1,130 \\
\hline 10-percent-duration flow & 1,020 & 1,590 & 970 & 448 & 740 \\
\hline 15-percent-duration flow & 779 & 1,230 & 787 & 312 & 556 \\
\hline 20-percent-duration flow & 648 & 1,060 & 664 & 224 & 438 \\
\hline 25-percent-duration flow & 535 & 900 & 566 & 164 & 351 \\
\hline 30-percent-duration flow & 451 & 808 & 488 & 125 & 291 \\
\hline 35-percent-duration flow & 379 & 724 & 425 & 98.3 & 245 \\
\hline 40-percent-duration flow & 317 & 650 & 373 & 78.0 & 209 \\
\hline 45-percent-duration flow & 267 & 591 & 326 & 62.0 & 177 \\
\hline 50-percent-duration flow & 221 & 534 & 288 & 49.4 & 147 \\
\hline 55-percent-duration flow & 181 & 479 & 254 & 40.6 & 119 \\
\hline 60-percent-duration flow & 146 & 428 & 224 & 33.1 & 95.7 \\
\hline 65-percent-duration flow & 114 & 381 & 195 & 26.8 & 74.0 \\
\hline 70-percent-duration flow & 86.1 & 341 & 166 & 21.1 & 53.8 \\
\hline 75-percent-duration flow & 62.1 & 297 & 139 & 16.0 & 35.7 \\
\hline 80-percent-duration flow & 41.2 & 241 & 109 & 12.3 & 19.6 \\
\hline 85-percent-duration flow & 25.5 & 188 & 80.7 & 8.5 & 12.7 \\
\hline 90-percent-duration flow & 14.0 & 144 & 56.6 & 5.6 & 8.4 \\
\hline 95-percent-duration flow & 6.6 & 102 & 34.4 & 3.4 & 4.5 \\
\hline 99-percent-duration flow & 2.0 & 52.9 & 12.6 & 1.6 & 0.7 \\
\hline Variability index & 0.71 & 0.40 & 0.48 & 0.72 & 0.75 \\
\hline
\end{tabular}


Table 11. Selected annual and seasonal statistics for 77 stations in West Virginia representative of 1930-2002.—Continued

[Winter, January 1-March 31; spring, April 1-June 30; summer, July 1-September 30; fall, October 1-December 31; streamflow statistics are in cubic feet per second, except for variability index, which is dimensionless; the record period is in climatic years, April 1 through March 31 of the indicated ending year; EPA, U.S. Environmental Protection Agency]

\begin{tabular}{|c|c|c|c|c|c|}
\hline \multirow{2}{*}{ Streamflow statistic } & \multirow{2}{*}{ Annual } & \multicolumn{4}{|c|}{ Season } \\
\hline & & Winter & Spring & Summer & Fall \\
\hline \multicolumn{6}{|c|}{03189100 Gauley River near Craigsville, WV, 1966-1979 } \\
\hline 1-day 2-year hydrologically based flow & 45.4 & 433 & 141 & 50.8 & 153 \\
\hline 1-day 5-year hydrologically based flow & 26.4 & 334 & 74.4 & 27.9 & 69.6 \\
\hline 1-day 10-year hydrologically based flow & 19.5 & 295 & 49.9 & 19.9 & 43.5 \\
\hline 3-day 2-year hydrologically based flow & 48.9 & 457 & 159 & 55.1 & 168 \\
\hline 3-day 5-year hydrologically based flow & 29.1 & 345 & 83.8 & 30.4 & 73.6 \\
\hline 3-day 10-year hydrologically based flow & 21.7 & 299 & 56.3 & 21.9 & 45.8 \\
\hline 7-day 2-year hydrologically based flow & 57.2 & 510 & 182 & 68.4 & 197 \\
\hline 7-day 5-year hydrologically based flow & 33.3 & 353 & 91.9 & 36.6 & 90.5 \\
\hline 7-day 10-year hydrologically based flow & 24.5 & 298 & 61.9 & 25.8 & 59.2 \\
\hline 14-day 2-year hydrologically based flow & 79.6 & 630 & 250 & 100 & 254 \\
\hline 14-day 5-year hydrologically based flow & 45.5 & 400 & 124 & 53.4 & 113 \\
\hline 14-day 10 -year hydrologically based flow & 32.6 & 318 & 85.1 & 37.2 & 72.0 \\
\hline 30-day 2-year hydrologically based flow & 129 & 1,020 & 462 & 162 & 599 \\
\hline 30-day 5-year hydrologically based flow & 81.6 & 588 & 241 & 105 & 307 \\
\hline 30-day 10-year hydrologically based flow & 63.1 & 429 & 165 & 85.7 & 194 \\
\hline 1-day 3-year biologically based flow & 17.0 & 249 & 97.7 & 22.9 & 17.0 \\
\hline 4-day 3-year biologically based flow & 23.9 & 261 & 122 & 27.7 & 30.8 \\
\hline EPA harmonic-mean flow & 340 & 981 & 788 & 182 & 253 \\
\hline 5-percent-duration flow & 4,870 & 6,730 & 4,590 & 2,470 & 4,620 \\
\hline 10-percent-duration flow & 3,340 & 4,820 & 3,410 & 1,370 & 3,140 \\
\hline 15-percent-duration flow & 2,610 & 3,670 & 2,700 & 998 & 2,470 \\
\hline 20-percent-duration flow & 2,140 & 3,130 & 2,270 & 743 & 2,060 \\
\hline 25-percent-duration flow & 1,820 & 2,650 & 1,950 & 615 & 1,750 \\
\hline 30-percent-duration flow & 1,560 & 2,370 & 1,680 & 520 & 1,520 \\
\hline 35-percent-duration flow & 1,320 & 2,100 & 1,470 & 440 & 1,310 \\
\hline 40-percent-duration flow & 1,130 & 1,890 & 1,270 & 373 & 1,120 \\
\hline 45-percent-duration flow & 966 & 1,700 & 1,100 & 326 & 1,000 \\
\hline 50-percent-duration flow & 820 & 1,520 & 960 & 289 & 893 \\
\hline 55-percent-duration flow & 692 & 1,350 & 825 & 257 & 802 \\
\hline 60-percent-duration flow & 590 & 1,210 & 706 & 222 & 709 \\
\hline 65 -percent-duration flow & 503 & 1,080 & 604 & 190 & 616 \\
\hline 70-percent-duration flow & 426 & 939 & 517 & 161 & 513 \\
\hline 75-percent-duration flow & 356 & 781 & 440 & 137 & 427 \\
\hline 80-percent-duration flow & 278 & 636 & 363 & 112 & 352 \\
\hline 85-percent-duration flow & 211 & 534 & 278 & 89.4 & 279 \\
\hline 90-percent-duration flow & 145 & 469 & 199 & 68.5 & 200 \\
\hline 95-percent-duration flow & 83.4 & 404 & 131 & 48.8 & 96.1 \\
\hline 99-percent-duration flow & 39.4 & 293 & 57.4 & 22.9 & 50.6 \\
\hline Variability index & 0.53 & 0.38 & 0.47 & 0.51 & 0.49 \\
\hline
\end{tabular}


Table 11. Selected annual and seasonal statistics for 77 stations in West Virginia representative of 1930-2002.—Continued

[Winter, January 1-March 31; spring, April 1-June 30; summer, July 1-September 30; fall, October 1-December 31; streamflow statistics are in cubic feet per second, except for variability index, which is dimensionless; the record period is in climatic years, April 1 through March 31of the indicated ending year; EPA, U.S. Environmental Protection Agency]

\begin{tabular}{|c|c|c|c|c|c|}
\hline \multirow{2}{*}{ Streamflow statistic } & \multirow{2}{*}{ Annual } & \multicolumn{4}{|c|}{ Season } \\
\hline & & Winter & Spring & Summer & Fall \\
\hline \multicolumn{6}{|c|}{03189500 Gauley River near Summersville, WV, 1932-1957 } \\
\hline 1-day 2-year hydrologically based flow & 34.0 & 536 & 189 & 37.7 & 53.5 \\
\hline 1-day 5-year hydrologically based flow & 10.5 & 343 & 102 & 13.5 & 15.3 \\
\hline 1-day 10-year hydrologically based flow & 5.19 & 259 & 70.4 & 7.56 & 7.11 \\
\hline 3-day 2-year hydrologically based flow & 37.7 & 585 & 221 & 42.4 & 61.1 \\
\hline 3-day 5-year hydrologically based flow & 11.3 & 368 & 119 & 14.9 & 17.2 \\
\hline 3-day 10-year hydrologically based flow & 5.49 & 273 & 79.8 & 8.32 & 7.84 \\
\hline 7-day 2-year hydrologically based flow & 46.1 & 663 & 295 & 53.1 & 80.4 \\
\hline 7-day 5-year hydrologically based flow & 13.4 & 396 & 161 & 18.6 & 21.4 \\
\hline 7-day 10-year hydrologically based flow & 6.30 & 287 & 105 & 10.3 & 9.11 \\
\hline 14-day 2-year hydrologically based flow & 61.5 & 863 & 417 & 77.4 & 108 \\
\hline 14-day 5-year hydrologically based flow & 17.3 & 485 & 213 & 26.7 & 27.5 \\
\hline 14-day 10 -year hydrologically based flow & 7.85 & 340 & 136 & 14.4 & 11.3 \\
\hline 30-day 2-year hydrologically based flow & 91.2 & 1,410 & 646 & 165 & 235 \\
\hline 30-day 5-year hydrologically based flow & 26.5 & 882 & 348 & 56.1 & 56.4 \\
\hline 30-day 10-year hydrologically based flow & 12.3 & 659 & 237 & 29.1 & 22.1 \\
\hline 1-day 3-year biologically based flow & 6.43 & 229 & 105 & 6.72 & 8.99 \\
\hline 4-day 3-year biologically based flow & 9.21 & 260 & 129 & 12.2 & 9.84 \\
\hline EPA harmonic-mean flow & 114 & 1,180 & 786 & 113 & 41.6 \\
\hline 5-percent-duration flow & 5,500 & 8,410 & 5,020 & 2,810 & 4,070 \\
\hline 10-percent-duration flow & 3,710 & 5,780 & 3,540 & 1,670 & 2,630 \\
\hline 15-percent-duration flow & 2,880 & 4,530 & 2,830 & 1,220 & 2,010 \\
\hline 20-percent-duration flow & 2,360 & 3,830 & 2,390 & 931 & 1,590 \\
\hline 25-percent-duration flow & 1,990 & 3,310 & 2,060 & 752 & 1,280 \\
\hline 30-percent-duration flow & 1,660 & 2,950 & 1,810 & 605 & 1,090 \\
\hline 35-percent-duration flow & 1,420 & 2,670 & 1,580 & 503 & 915 \\
\hline 40-percent-duration flow & 1,190 & 2,420 & 1,370 & 420 & 792 \\
\hline 45-percent-duration flow & 1,030 & 2,190 & 1,210 & 359 & 669 \\
\hline 50-percent-duration flow & 869 & 2,010 & 1,070 & 301 & 572 \\
\hline 55-percent-duration flow & 736 & 1,820 & 958 & 249 & 478 \\
\hline 60-percent-duration flow & 607 & 1,630 & 850 & 209 & 403 \\
\hline 65-percent-duration flow & 500 & 1,460 & 746 & 172 & 329 \\
\hline 70-percent-duration flow & 403 & 1,300 & 644 & 137 & 254 \\
\hline 75-percent-duration flow & 316 & 1,150 & 543 & 104 & 177 \\
\hline 80-percent-duration flow & 231 & 983 & 449 & 78.4 & 113 \\
\hline 85-percent-duration flow & 153 & 809 & 365 & 51.0 & 69.3 \\
\hline 90-percent-duration flow & 85.1 & 653 & 286 & 31.7 & 45.1 \\
\hline 95-percent-duration flow & 36.8 & 485 & 190 & 18.0 & 25.4 \\
\hline 99-percent-duration flow & 10.8 & 165 & 90.2 & 8.6 & 2.0 \\
\hline Variability index & 0.64 & 0.36 & 0.43 & 0.66 & 0.67 \\
\hline
\end{tabular}


Table 11. Selected annual and seasonal statistics for 77 stations in West Virginia representative of 1930-2002.—Continued

[Winter, January 1-March 31; spring, April 1-June 30; summer, July 1-September 30; fall, October 1-December 31; streamflow statistics are in cubic feet per second, except for variability index, which is dimensionless; the record period is in climatic years, April 1 through March 31 of the indicated ending year; EPA, U.S. Environmental Protection Agency]

\begin{tabular}{|c|c|c|c|c|c|}
\hline \multirow{2}{*}{ Streamflow statistic } & \multirow{2}{*}{ Annual } & \multicolumn{4}{|c|}{ Season } \\
\hline & & Winter & Spring & Summer & Fall \\
\hline \multicolumn{6}{|c|}{03189650 Collison Creek near Nallen, WV, 1968-1977 } \\
\hline 1-day 2-year hydrologically based flow & 0.04 & 0.85 & 0.18 & 0.04 & 0.06 \\
\hline 1-day 5-year hydrologically based flow & 0.01 & 0.19 & 0.07 & 0.01 & 0.03 \\
\hline 1-day 10-year hydrologically based flow & 0.00 & 0.07 & 0.04 & 0.00 & 0.02 \\
\hline 3-day 2-year hydrologically based flow & 0.04 & 0.97 & 0.23 & 0.05 & 0.08 \\
\hline 3-day 5-year hydrologically based flow & 0.02 & 0.22 & 0.08 & 0.02 & 0.03 \\
\hline 3-day 10-year hydrologically based flow & 0.00 & 0.08 & 0.04 & 0.00 & 0.02 \\
\hline 7-day 2-year hydrologically based flow & 0.05 & 1.15 & 0.33 & 0.06 & 0.12 \\
\hline 7-day 5-year hydrologically based flow & 0.02 & 0.26 & 0.13 & 0.03 & 0.04 \\
\hline 7-day 10-year hydrologically based flow & 0.00 & 0.09 & 0.07 & 0.00 & 0.02 \\
\hline 14-day 2-year hydrologically based flow & 0.10 & 2.24 & 0.52 & 0.13 & 0.18 \\
\hline 14-day 5-year hydrologically based flow & 0.02 & 0.69 & 0.19 & 0.03 & 0.06 \\
\hline 14-day 10 -year hydrologically based flow & 0.01 & 0.27 & 0.10 & 0.01 & 0.04 \\
\hline 30-day 2-year hydrologically based flow & 0.16 & 3.60 & 1.37 & 0.23 & 0.68 \\
\hline 30-day 5-year hydrologically based flow & 0.08 & 1.71 & 0.61 & 0.10 & 0.25 \\
\hline 30-day 10-year hydrologically based flow & 0.05 & 0.99 & 0.36 & 0.06 & 0.14 \\
\hline 1-day 3-year biologically based flow & 0.04 & 0.00 & 0.00 & 0.03 & 0.05 \\
\hline 4-day 3-year biologically based flow & 0.04 & 0.00 & 0.00 & 0.03 & 0.05 \\
\hline EPA harmonic-mean flow & 0.28 & 0.61 & 0.87 & 0.16 & 0.19 \\
\hline 5-percent-duration flow & 17.8 & 25.1 & 15.6 & 8.8 & 19.3 \\
\hline 10-percent-duration flow & 11.1 & 16.0 & 10.0 & 3.9 & 11.5 \\
\hline 15-percent-duration flow & 8.2 & 12.5 & 8.0 & 2.4 & 7.8 \\
\hline 20-percent-duration flow & 6.5 & 10.1 & 6.6 & 1.8 & 6.3 \\
\hline 25-percent-duration flow & 5.3 & 8.4 & 5.5 & 1.4 & 5.2 \\
\hline 30-percent-duration flow & 4.3 & 7.3 & 4.5 & 1.0 & 4.3 \\
\hline 35-percent-duration flow & 3.7 & 6.4 & 3.8 & 0.8 & 3.7 \\
\hline 40-percent-duration flow & 3.1 & 5.6 & 3.3 & 0.6 & 3.1 \\
\hline 45-percent-duration flow & 2.6 & 4.9 & 2.8 & 0.5 & 2.5 \\
\hline 50-percent-duration flow & 2.1 & 4.3 & 2.3 & 0.4 & 2.0 \\
\hline 55-percent-duration flow & 1.7 & 4.0 & 2.0 & 0.3 & 1.6 \\
\hline 60-percent-duration flow & 1.3 & 3.6 & 1.7 & 0.3 & 1.3 \\
\hline 65-percent-duration flow & 1.0 & 3.3 & 1.4 & 0.2 & 0.9 \\
\hline 70-percent-duration flow & 0.7 & 2.9 & 1.1 & 0.2 & 0.5 \\
\hline 75-percent-duration flow & 0.4 & 2.6 & 0.9 & 0.1 & 0.4 \\
\hline 80-percent-duration flow & 0.3 & 2.2 & 0.7 & 0.1 & 0.3 \\
\hline 85-percent-duration flow & 0.2 & 1.9 & 0.4 & 0.1 & 0.2 \\
\hline 90-percent-duration flow & 0.1 & 1.3 & 0.3 & 0.1 & 0.1 \\
\hline 95-percent-duration flow & 0.1 & 0.3 & 0.1 & 0.0 & 0.0 \\
\hline 99-percent-duration flow & 0.0 & 0.0 & 0.0 & 0.0 & 0.0 \\
\hline Variability index & 0.72 & 0.52 & 0.65 & 0.51 & 0.55 \\
\hline
\end{tabular}


Table 11. Selected annual and seasonal statistics for 77 stations in West Virginia representative of 1930-2002.—Continued

[Winter, January 1-March 31; spring, April 1-June 30; summer, July 1-September 30; fall, October 1-December 31; streamflow statistics are in cubic feet per second, except for variability index, which is dimensionless; the record period is in climatic years, April 1 through March 31of the indicated ending year; EPA, U.S. Environmental Protection Agency]

\begin{tabular}{|c|c|c|c|c|c|}
\hline \multirow{2}{*}{ Streamflow statistic } & \multirow{2}{*}{ Annual } & \multicolumn{4}{|c|}{ Season } \\
\hline & & Winter & Spring & Summer & Fall \\
\hline \multicolumn{6}{|c|}{03190000 Meadow River at Nallen, WV, 1932-1957 } \\
\hline 1-day 2-year hydrologically based flow & 8.54 & 184 & 54.1 & 10.2 & 14.1 \\
\hline 1-day 5-year hydrologically based flow & 2.34 & 96.8 & 27.1 & 3.31 & 4.60 \\
\hline 1-day 10-year hydrologically based flow & 0.91 & 62.5 & 17.7 & 1.63 & 2.19 \\
\hline 3-day 2-year hydrologically based flow & 8.88 & 204 & 61.5 & 11.2 & 14.5 \\
\hline 3-day 5-year hydrologically based flow & 2.88 & 108 & 30.9 & 3.72 & 4.93 \\
\hline 3-day 10-year hydrologically based flow & 1.45 & 68.9 & 20.1 & 1.88 & 2.65 \\
\hline 7-day 2-year hydrologically based flow & 10.2 & 242 & 80.1 & 13.8 & 16.9 \\
\hline 7-day 5-year hydrologically based flow & 3.55 & 120 & 40.5 & 4.85 & 5.75 \\
\hline 7-day 10-year hydrologically based flow & 1.90 & 74.0 & 26.2 & 2.58 & 3.09 \\
\hline 14-day 2-year hydrologically based flow & 12.4 & 331 & 113 & 17.7 & 22.4 \\
\hline 14-day 5-year hydrologically based flow & 4.79 & 156 & 55.4 & 6.64 & 7.84 \\
\hline 14-day 10 -year hydrologically based flow & 2.77 & 92.3 & 35.4 & 3.77 & 4.24 \\
\hline 30-day 2-year hydrologically based flow & 18.3 & 537 & 184 & 34.5 & 43.1 \\
\hline 30-day 5-year hydrologically based flow & 6.86 & 288 & 89.2 & 11.9 & 13.0 \\
\hline 30-day 10 -year hydrologically based flow & 4.00 & 190 & 58.3 & 6.56 & 6.79 \\
\hline 1-day 3-year biologically based flow & 0.96 & 39.9 & 20.9 & 1.72 & 1.28 \\
\hline 4-day 3-year biologically based flow & 1.28 & 48.1 & 33.6 & 2.95 & 1.45 \\
\hline EPA harmonic-mean flow & 41.1 & 341 & 242 & 26.0 & 19.4 \\
\hline 5-percent-duration flow & 1,980 & 3,030 & 1,900 & 914 & 1,340 \\
\hline 10-percent-duration flow & 1,380 & 2,220 & 1,340 & 503 & 879 \\
\hline 15-percent-duration flow & 1,050 & 1,780 & 1,050 & 330 & 645 \\
\hline 20-percent-duration flow & 847 & 1,460 & 859 & 225 & 505 \\
\hline 25-percent-duration flow & 699 & 1,280 & 722 & 171 & 396 \\
\hline 30-percent-duration flow & 573 & 1,130 & 616 & 138 & 312 \\
\hline 35-percent-duration flow & 475 & 1,000 & 520 & 113 & 251 \\
\hline 40-percent-duration flow & 390 & 907 & 454 & 92.1 & 205 \\
\hline 45-percent-duration flow & 314 & 818 & 396 & 76.7 & 167 \\
\hline 50-percent-duration flow & 258 & 742 & 345 & 63.6 & 131 \\
\hline 55-percent-duration flow & 208 & 668 & 306 & 53.4 & 95.7 \\
\hline 60-percent-duration flow & 163 & 598 & 269 & 43.9 & 76.0 \\
\hline 65-percent-duration flow & 126 & 534 & 234 & 35.6 & 58.1 \\
\hline 70-percent-duration flow & 92.6 & 476 & 201 & 29.5 & 44.8 \\
\hline 75-percent-duration flow & 67.6 & 411 & 168 & 24.5 & 31.0 \\
\hline 80-percent-duration flow & 48.5 & 340 & 138 & 19.7 & 22.2 \\
\hline 85-percent-duration flow & 31.7 & 283 & 111 & 14.0 & 16.7 \\
\hline 90-percent-duration flow & 20.0 & 221 & 83.9 & 8.2 & 12.4 \\
\hline 95-percent-duration flow & 10.1 & 147 & 52.7 & 4.8 & 6.9 \\
\hline 99-percent-duration flow & 2.9 & 39.5 & 20.4 & 2.3 & 1.6 \\
\hline Variability index & 0.71 & 0.39 & 0.47 & 0.67 & 0.73 \\
\hline
\end{tabular}


Table 11. Selected annual and seasonal statistics for 77 stations in West Virginia representative of 1930-2002.—Continued

[Winter, January 1-March 31; spring, April 1-June 30; summer, July 1-September 30; fall, October 1-December 31; streamflow statistics are in cubic feet per second, except for variability index, which is dimensionless; the record period is in climatic years, April 1 through March 31of the indicated ending year; EPA, U.S. Environmental Protection Agency]

\begin{tabular}{|c|c|c|c|c|c|}
\hline \multirow{2}{*}{ Streamflow statistic } & \multirow{2}{*}{ Annual } & \multicolumn{4}{|c|}{ Season } \\
\hline & & Winter & Spring & Summer & Fall \\
\hline \multicolumn{6}{|c|}{03190400 Meadow River near Mount Lookout, WV, 1988-2000 } \\
\hline 1-day 2-year hydrologically based flow & 12.2 & 196 & 74.4 & 14.7 & 24.5 \\
\hline 1-day 5-year hydrologically based flow & 6.40 & 141 & 37.7 & 7.32 & 11.0 \\
\hline 1-day 10-year hydrologically based flow & 4.74 & 120 & 25.6 & 5.15 & 7.53 \\
\hline 3-day 2-year hydrologically based flow & 12.7 & 235 & 83.2 & 15.5 & 25.9 \\
\hline 3-day 5-year hydrologically based flow & 6.64 & 168 & 41.1 & 7.66 & 11.6 \\
\hline 3-day 10 -year hydrologically based flow & 4.92 & 143 & 27.4 & 5.39 & 7.82 \\
\hline 7-day 2-year hydrologically based flow & 13.9 & 330 & 106 & 17.3 & 31.0 \\
\hline 7-day 5-year hydrologically based flow & 7.32 & 215 & 51.9 & 8.67 & 13.2 \\
\hline 7-day 10-year hydrologically based flow & 5.52 & 172 & 34.0 & 6.21 & 8.51 \\
\hline 14-day 2-year hydrologically based flow & 16.4 & 469 & 144 & 21.2 & 39.4 \\
\hline 14-day 5-year hydrologically based flow & 8.54 & 273 & 71.3 & 10.5 & 14.6 \\
\hline 14-day 10 -year hydrologically based flow & 6.51 & 204 & 46.8 & 7.62 & 8.84 \\
\hline 30-day 2-year hydrologically based flow & 25.2 & 761 & 275 & 34.5 & 70.2 \\
\hline 30-day 5-year hydrologically based flow & 12.8 & 497 & 128 & 16.3 & 22.8 \\
\hline 30-day 10-year hydrologically based flow & 9.60 & 391 & 80.5 & 11.6 & 12.5 \\
\hline 1-day 3-year biologically based flow & 5.15 & 97.8 & 99.8 & 6.86 & 5.76 \\
\hline 4-day 3-year biologically based flow & 6.48 & 112 & 133 & 8.38 & 6.81 \\
\hline EPA harmonic-mean flow & 77.4 & 515 & 571 & 44.6 & 39.2 \\
\hline 5-percent-duration flow & 2,520 & 3,430 & 2,510 & 734 & 1,880 \\
\hline 10-percent-duration flow & 1,820 & 2,670 & 1,820 & 399 & 1,210 \\
\hline 15-percent-duration flow & 1,380 & 2,260 & 1,410 & 270 & 902 \\
\hline 20-percent-duration flow & 1,100 & 1,940 & 1,180 & 192 & 688 \\
\hline 25-percent-duration flow & 901 & 1,760 & 1,000 & 152 & 541 \\
\hline 30-percent-duration flow & 756 & 1,560 & 865 & 124 & 434 \\
\hline 35-percent-duration flow & 637 & 1,370 & 773 & 101 & 355 \\
\hline 40-percent-duration flow & 534 & 1,210 & 689 & 83.1 & 296 \\
\hline 45-percent-duration flow & 441 & 1,080 & 612 & 70.2 & 249 \\
\hline 50-percent-duration flow & 360 & 954 & 546 & 60.3 & 212 \\
\hline 55-percent-duration flow & 285 & 855 & 482 & 51.0 & 179 \\
\hline 60-percent-duration flow & 222 & 776 & 423 & 44.2 & 144 \\
\hline 65-percent-duration flow & 175 & 707 & 363 & 37.1 & 115 \\
\hline 70-percent-duration flow & 127 & 634 & 306 & 30.3 & 90.9 \\
\hline 75-percent-duration flow & 89.5 & 563 & 261 & 25.0 & 63.7 \\
\hline 80-percent-duration flow & 61.3 & 500 & 215 & 20.5 & 44.1 \\
\hline 85-percent-duration flow & 41.4 & 435 & 173 & 16.9 & 28.8 \\
\hline 90-percent-duration flow & 25.7 & 350 & 119 & 14.1 & 20.3 \\
\hline 95-percent-duration flow & 15.6 & 223 & 64.6 & 11.0 & 13.0 \\
\hline 99-percent-duration flow & 8.1 & 162 & 28.3 & 7.1 & 6.9 \\
\hline Variability index & 0.70 & 0.35 & 0.46 & 0.56 & 0.67 \\
\hline
\end{tabular}


Table 11. Selected annual and seasonal statistics for 77 stations in West Virginia representative of 1930-2002.-Continued

[Winter, January 1-March 31; spring, April 1-June 30; summer, July 1-September 30; fall, October 1-December 31; streamflow statistics are in cubic feet per second, except for variability index, which is dimensionless; the record period is in climatic years, April 1 through March 31of the indicated ending year; EPA, U.S. Environmental Protection Agency]

\begin{tabular}{|c|c|c|c|c|c|}
\hline \multirow{2}{*}{ Streamflow statistic } & \multirow{2}{*}{ Annual } & \multicolumn{4}{|c|}{ Season } \\
\hline & & Winter & Spring & Summer & Fall \\
\hline \multicolumn{6}{|c|}{03191500 Peters Creek near Lockwood, WV, 1948-1964 } \\
\hline 1-day 2-year hydrologically based flow & 1.13 & 21.5 & 3.89 & 1.15 & 1.96 \\
\hline 1-day 5-year hydrologically based flow & 0.20 & 11.5 & 2.32 & 0.39 & 0.47 \\
\hline 1-day 10-year hydrologically based flow & 0.04 & 7.65 & 1.82 & 0.16 & 0.16 \\
\hline 3-day 2-year hydrologically based flow & 1.34 & 23.2 & 4.55 & 1.32 & 2.29 \\
\hline 3-day 5-year hydrologically based flow & 0.24 & 12.3 & 2.81 & 0.43 & 0.54 \\
\hline 3-day 10-year hydrologically based flow & 0.04 & 8.18 & 2.24 & 0.17 & 0.18 \\
\hline 7-day 2-year hydrologically based flow & 1.44 & 25.7 & 6.08 & 1.93 & 2.61 \\
\hline 7-day 5-year hydrologically based flow & 0.25 & 13.5 & 3.76 & 0.41 & 0.66 \\
\hline 7-day 10-year hydrologically based flow & 0.07 & 9.21 & 2.99 & 0.12 & 0.24 \\
\hline 14-day 2-year hydrologically based flow & 1.87 & 32.3 & 9.79 & 2.70 & 4.10 \\
\hline 14-day 5-year hydrologically based flow & 0.38 & 18.2 & 5.94 & 0.68 & 0.95 \\
\hline 14-day 10-year hydrologically based flow & 0.12 & 13.1 & 4.61 & 0.24 & 0.32 \\
\hline 30-day 2-year hydrologically based flow & 3.02 & 66.5 & 21.7 & 4.80 & 7.17 \\
\hline 30-day 5-year hydrologically based flow & 0.84 & 46.3 & 11.0 & 1.58 & 1.76 \\
\hline 30-day 10-year hydrologically based flow & 0.36 & 37.1 & 7.65 & 0.72 & 0.73 \\
\hline 1-day 3-year biologically based flow & 0.00 & 6.97 & 3.57 & 0.07 & 0.14 \\
\hline 4-day 3-year biologically based flow & 0.00 & 7.60 & 4.30 & 0.10 & 0.22 \\
\hline EPA harmonic-mean flow & 2.07 & 40.7 & 25.2 & 1.17 & 0.99 \\
\hline 5-percent-duration flow & 273 & 403 & 257 & 112 & 203 \\
\hline 10-percent-duration flow & 167 & 271 & 166 & 50.8 & 112 \\
\hline 15-percent-duration flow & 120 & 205 & 123 & 32.0 & 75.4 \\
\hline 20-percent-duration flow & 96.6 & 166 & 93.8 & 23.4 & 54.1 \\
\hline 25-percent-duration flow & 77.0 & 140 & 78.7 & 17.6 & 41.1 \\
\hline 30-percent-duration flow & 61.5 & 123 & 64.3 & 14.0 & 32.7 \\
\hline 35-percent-duration flow & 50.2 & 111 & 53.9 & 11.5 & 27.4 \\
\hline 40-percent-duration flow & 40.0 & 99.9 & 45.0 & 9.4 & 22.7 \\
\hline 45-percent-duration flow & 32.9 & 90.4 & 38.2 & 8.0 & 19.8 \\
\hline 50-percent-duration flow & 26.2 & 81.1 & 33.2 & 6.8 & 16.9 \\
\hline 55-percent-duration flow & 21.4 & 72.8 & 28.8 & 5.7 & 14.5 \\
\hline 60-percent-duration flow & 17.3 & 65.1 & 25.0 & 4.8 & 12.3 \\
\hline 65-percent-duration flow & 13.9 & 58.0 & 21.8 & 4.0 & 10.0 \\
\hline 70-percent-duration flow & 10.8 & 51.6 & 18.8 & 3.5 & 7.5 \\
\hline 75-percent-duration flow & 8.0 & 45.5 & 15.5 & 2.9 & 5.4 \\
\hline 80-percent-duration flow & 5.8 & 39.8 & 12.7 & 2.3 & 3.7 \\
\hline 85-percent-duration flow & 4.0 & 33.7 & 9.4 & 1.7 & 2.8 \\
\hline 90-percent-duration flow & 2.8 & 24.9 & 6.9 & 1.1 & 1.6 \\
\hline 95-percent-duration flow & 1.3 & 16.0 & 5.0 & 0.3 & 0.6 \\
\hline 99-percent-duration flow & 0.1 & 8.6 & 3.0 & 0.1 & 0.1 \\
\hline Variability index & 0.70 & 0.40 & 0.52 & 0.71 & 0.73 \\
\hline
\end{tabular}


Table 11. Selected annual and seasonal statistics for 77 stations in West Virginia representative of 1930-2002. - Continued

[Winter, January 1-March 31; spring, April 1-June 30; summer, July 1-September 30; fall, October 1-December 31; streamflow statistics are in cubic feet per second, except for variability index, which is dimensionless; the record period is in climatic years, April 1 through March 31of the indicated ending year; EPA, U.S. Environmental Protection Agency]

\begin{tabular}{|c|c|c|c|c|c|}
\hline \multirow{2}{*}{ Streamflow statistic } & \multirow{2}{*}{ Annual } & \multicolumn{4}{|c|}{ Season } \\
\hline & & Winter & Spring & Summer & Fall \\
\hline \multicolumn{6}{|c|}{03192000 Gauley River above Belva, WV, 1932-1957 } \\
\hline 1-day 2-year hydrologically based flow & 66.1 & 916 & 346 & 78.3 & 89.0 \\
\hline 1-day 5-year hydrologically based flow & 24.8 & 555 & 194 & 32.4 & 30.6 \\
\hline 1-day 10-year hydrologically based flow & 14.0 & 400 & 134 & 19.6 & 16.5 \\
\hline 3-day 2-year hydrologically based flow & 68.9 & 998 & 386 & 84.6 & 92.5 \\
\hline 3-day 5-year hydrologically based flow & 25.8 & 601 & 216 & 34.6 & 31.8 \\
\hline 3-day 10 -year hydrologically based flow & 14.6 & 428 & 149 & 20.7 & 17.2 \\
\hline 7-day 2-year hydrologically based flow & 76.8 & 1,150 & 492 & 100 & 110 \\
\hline 7-day 5-year hydrologically based flow & 28.2 & 648 & 272 & 39.7 & 36.5 \\
\hline 7-day 10-year hydrologically based flow & 15.8 & 447 & 180 & 23.2 & 19.3 \\
\hline 14-day 2-year hydrologically based flow & 94.6 & 1,530 & 682 & 134 & 141 \\
\hline 14-day 5-year hydrologically based flow & 33.9 & 805 & 352 & 51.7 & 44.8 \\
\hline 14-day 10 -year hydrologically based flow & 18.7 & 528 & 230 & 29.7 & 23.0 \\
\hline 30-day 2-year hydrologically based flow & 134 & 2,460 & 1,070 & 267 & 290 \\
\hline 30-day 5-year hydrologically based flow & 45.8 & 1,450 & 574 & 94.3 & 78.8 \\
\hline 30-day 10 -year hydrologically based flow & 24.8 & 1,040 & 395 & 50.5 & 36.8 \\
\hline 1-day 3-year biologically based flow & 15.0 & 325 & 191 & 24.0 & 15.0 \\
\hline 4-day 3-year biologically based flow & 17.0 & 375 & 231 & 29.2 & 17.0 \\
\hline EPA harmonic-mean flow & 264 & 1,900 & 1,370 & 226 & 105 \\
\hline 5-percent-duration flow & 9,560 & 14,800 & 8,910 & 4,530 & 6,770 \\
\hline 10-percent-duration flow & 6,510 & 10,300 & 6,270 & 2,760 & 4,220 \\
\hline 15-percent-duration flow & 4,980 & 8,130 & 4,990 & 2,010 & 3,200 \\
\hline 20-percent-duration flow & 4,070 & 6,820 & 4,180 & 1,530 & 2,540 \\
\hline 25-percent-duration flow & 3,420 & 5,910 & 3,590 & 1,220 & 2,060 \\
\hline 30-percent-duration flow & 2,850 & 5,220 & 3,150 & 982 & 1,680 \\
\hline 35-percent-duration flow & 2,400 & 4,650 & 2,730 & 788 & 1,400 \\
\hline 40-percent-duration flow & 2,020 & 4,240 & 2,350 & 662 & 1,180 \\
\hline 45-percent-duration flow & 1,700 & 3,860 & 2,080 & 557 & 989 \\
\hline 50-percent-duration flow & 1,430 & 3,520 & 1,850 & 469 & 825 \\
\hline 55-percent-duration flow & 1,180 & 3,180 & 1,640 & 398 & 674 \\
\hline 60-percent-duration flow & 977 & 2,850 & 1,460 & 339 & 541 \\
\hline 65-percent-duration flow & 789 & 2,560 & 1,280 & 283 & 430 \\
\hline 70-percent-duration flow & 620 & 2,280 & 1,090 & 227 & 338 \\
\hline 75-percent-duration flow & 474 & 1,960 & 910 & 179 & 250 \\
\hline 80-percent-duration flow & 354 & 1,660 & 760 & 137 & 149 \\
\hline 85-percent-duration flow & 244 & 1,370 & 611 & 96.5 & 98.3 \\
\hline 90-percent-duration flow & 138 & 1,080 & 487 & 63.0 & 66.2 \\
\hline 95-percent-duration flow & 63.4 & 773 & 328 & 36.2 & 38.0 \\
\hline 99-percent-duration flow & 20.1 & 217 & 184 & 18.7 & 6.8 \\
\hline Variability index & 0.65 & 0.38 & 0.43 & 0.63 & 0.69 \\
\hline
\end{tabular}


Table 11. Selected annual and seasonal statistics for 77 stations in West Virginia representative of 1930-2002.-Continued

[Winter, January 1-March 31; spring, April 1-June 30; summer, July 1-September 30; fall, October 1-December 31; streamflow statistics are in cubic feet per second, except for variability index, which is dimensionless; the record period is in climatic years, April 1 through March 31of the indicated ending year; EPA, U.S. Environmental Protection Agency]

\begin{tabular}{|c|c|c|c|c|c|}
\hline \multirow{2}{*}{ Streamflow statistic } & \multirow{2}{*}{ Annual } & \multicolumn{4}{|c|}{ Season } \\
\hline & & Winter & Spring & Summer & Fall \\
\hline \multicolumn{6}{|c|}{03194700 Elk River below Webster Springs, WV, 1962-1984 } \\
\hline 1-day 2-year hydrologically based flow & 27.6 & 189 & 73.0 & 30.5 & 61.3 \\
\hline 1-day 5-year hydrologically based flow & 15.7 & 140 & 41.6 & 16.7 & 30.7 \\
\hline 1-day 10-year hydrologically based flow & 11.6 & 120 & 30.1 & 12.0 & 21.2 \\
\hline 3-day 2-year hydrologically based flow & 29.2 & 206 & 79.5 & 32.5 & 67.2 \\
\hline 3-day 5-year hydrologically based flow & 16.5 & 152 & 45.1 & 17.7 & 32.9 \\
\hline 3-day 10-year hydrologically based flow & 12.1 & 130 & 32.9 & 12.7 & 22.5 \\
\hline 7-day 2-year hydrologically based flow & 32.9 & 237 & 95.6 & 38.9 & 80.9 \\
\hline 7-day 5-year hydrologically based flow & 18.4 & 166 & 53.5 & 20.3 & 39.4 \\
\hline 7-day 10-year hydrologically based flow & 13.5 & 140 & 39.6 & 14.4 & 26.6 \\
\hline 14-day 2-year hydrologically based flow & 41.1 & 308 & 137 & 50.6 & 102 \\
\hline 14-day 5-year hydrologically based flow & 21.8 & 197 & 74.8 & 25.1 & 46.2 \\
\hline 14-day 10-year hydrologically based flow & 15.4 & 157 & 53.5 & 17.4 & 29.6 \\
\hline 30-day 2-year hydrologically based flow & 69.0 & 504 & 260 & 84.7 & 261 \\
\hline 30-day 5-year hydrologically based flow & 33.5 & 304 & 140 & 42.3 & 108 \\
\hline 30-day 10-year hydrologically based flow & 22.4 & 228 & 96.9 & 28.8 & 60.0 \\
\hline 1-day 3-year biologically based flow & 10.0 & 117 & 49.8 & 9.94 & 11.0 \\
\hline 4-day 3-year biologically based flow & 11.0 & 140 & 57.5 & 13.4 & 12.0 \\
\hline EPA harmonic-mean flow & 159 & 477 & 380 & 90.4 & 108 \\
\hline 5-percent-duration flow & 2,440 & 3,260 & 2,420 & 1,060 & 2,220 \\
\hline 10-percent-duration flow & 1,670 & 2,390 & 1,740 & 668 & 1,550 \\
\hline 15-percent-duration flow & 1,300 & 1,880 & 1,390 & 478 & 1,220 \\
\hline 20-percent-duration flow & 1,050 & 1,570 & 1,150 & 383 & 991 \\
\hline 25-percent-duration flow & 873 & 1,340 & 956 & 311 & 827 \\
\hline 30-percent-duration flow & 736 & 1,160 & 821 & 262 & 696 \\
\hline 35-percent-duration flow & 628 & 1,020 & 698 & 220 & 601 \\
\hline 40-percent-duration flow & 537 & 902 & 602 & 188 & 524 \\
\hline 45-percent-duration flow & 458 & 812 & 523 & 160 & 456 \\
\hline 50-percent-duration flow & 392 & 730 & 459 & 136 & 400 \\
\hline 55-percent-duration flow & 337 & 652 & 392 & 120 & 348 \\
\hline 60-percent-duration flow & 286 & 582 & 342 & 106 & 301 \\
\hline 65-percent-duration flow & 240 & 520 & 298 & 91.9 & 259 \\
\hline 70-percent-duration flow & 199 & 457 & 251 & 79.2 & 218 \\
\hline 75-percent-duration flow & 163 & 393 & 212 & 67.1 & 179 \\
\hline 80-percent-duration flow & 128 & 343 & 173 & 55.3 & 143 \\
\hline 85-percent-duration flow & 97.3 & 287 & 140 & 43.9 & 109 \\
\hline 90-percent-duration flow & 68.9 & 233 & 104 & 31.1 & 76.4 \\
\hline 95-percent-duration flow & 42.5 & 187 & 69.5 & 20.3 & 47.5 \\
\hline 99-percent-duration flow & 16.1 & 141 & 32.9 & 12.1 & 13.9 \\
\hline Variability index & 0.53 & 0.38 & 0.47 & 0.51 & 0.50 \\
\hline
\end{tabular}


Table 11. Selected annual and seasonal statistics for 77 stations in West Virginia representative of 1930-2002.—Continued

[Winter, January 1-March 31; spring, April 1-June 30; summer, July 1-September 30; fall, October 1-December 31; streamflow statistics are in cubic feet per second, except for variability index, which is dimensionless; the record period is in climatic years, April 1 through March 31 of the indicated ending year; EPA, U.S. Environmental Protection Agency]

\begin{tabular}{|c|c|c|c|c|c|}
\hline \multirow{2}{*}{ Streamflow statistic } & \multirow{2}{*}{ Annual } & \multicolumn{4}{|c|}{ Season } \\
\hline & & Winter & Spring & Summer & Fall \\
\hline \multicolumn{6}{|c|}{03195000 Elk River at Centralia, WV, 1940-1962 } \\
\hline 1-day 2-year hydrologically based flow & 15.3 & 209 & 85.6 & 17.6 & 23.5 \\
\hline 1-day 5-year hydrologically based flow & 5.71 & 141 & 59.1 & 7.24 & 8.50 \\
\hline 1-day 10-year hydrologically based flow & 3.33 & 113 & 49.5 & 4.50 & 4.78 \\
\hline 3-day 2-year hydrologically based flow & 16.7 & 220 & 95.0 & 19.4 & 28.9 \\
\hline 3-day 5-year hydrologically based flow & 6.20 & 147 & 65.0 & 7.99 & 10.0 \\
\hline 3-day 10-year hydrologically based flow & 3.59 & 117 & 54.1 & 4.94 & 5.35 \\
\hline 7-day 2-year hydrologically based flow & 19.3 & 247 & 117 & 24.2 & 35.2 \\
\hline 7-day 5-year hydrologically based flow & 6.90 & 157 & 79.4 & 9.31 & 11.8 \\
\hline 7-day 10-year hydrologically based flow & 3.95 & 123 & 65.2 & 5.52 & 6.16 \\
\hline 14-day 2-year hydrologically based flow & 24.1 & 322 & 164 & 33.1 & 47.4 \\
\hline 14-day 5-year hydrologically based flow & 8.46 & 189 & 111 & 12.2 & 14.6 \\
\hline 14-day 10-year hydrologically based flow & 4.86 & 141 & 90.2 & 6.96 & 7.43 \\
\hline 30-day 2-year hydrologically based flow & 37.3 & 535 & 296 & 61.3 & 100 \\
\hline 30-day 5-year hydrologically based flow & 13.5 & 348 & 199 & 22.2 & 30.6 \\
\hline 30-day 10 -year hydrologically based flow & 7.85 & 273 & 163 & 12.3 & 15.2 \\
\hline 1-day 3-year biologically based flow & 3.95 & 83.0 & 56.0 & 5.49 & 3.95 \\
\hline 4-day 3-year biologically based flow & 4.49 & 102 & 88.6 & 6.50 & 4.63 \\
\hline EPA harmonic-mean flow & 81.9 & 477 & 391 & 58.2 & 37.1 \\
\hline 5-percent-duration flow & 2,370 & 3,420 & 2,350 & 1,320 & 1,690 \\
\hline 10-percent-duration flow & 1,610 & 2,360 & 1,670 & 746 & 1,080 \\
\hline 15-percent-duration flow & 1,240 & 1,890 & 1,340 & 528 & 806 \\
\hline 20-percent-duration flow & 987 & 1,580 & 1,090 & 390 & 634 \\
\hline 25-percent-duration flow & 803 & 1,360 & 896 & 307 & 518 \\
\hline 30-percent-duration flow & 668 & 1,210 & 752 & 251 & 432 \\
\hline 35-percent-duration flow & 576 & 1,060 & 658 & 208 & 375 \\
\hline 40-percent-duration flow & 490 & 954 & 585 & 172 & 324 \\
\hline 45-percent-duration flow & 418 & 861 & 521 & 143 & 283 \\
\hline 50-percent-duration flow & 355 & 761 & 462 & 121 & 242 \\
\hline 55-percent-duration flow & 303 & 685 & 408 & 101 & 202 \\
\hline 60-percent-duration flow & 256 & 616 & 362 & 82.9 & 172 \\
\hline 65-percent-duration flow & 212 & 553 & 320 & 68.4 & 142 \\
\hline 70-percent-duration flow & 174 & 497 & 281 & 55.2 & 113 \\
\hline 75-percent-duration flow & 139 & 438 & 245 & 44.2 & 85.1 \\
\hline 80-percent-duration flow & 103 & 374 & 209 & 33.5 & 60.1 \\
\hline 85-percent-duration flow & 70.2 & 318 & 173 & 25.1 & 38.8 \\
\hline 90-percent-duration flow & 41.5 & 251 & 141 & 16.7 & 21.1 \\
\hline 95-percent-duration flow & 18.3 & 197 & 101 & 9.2 & 12.3 \\
\hline 99-percent-duration flow & 5.8 & 106 & 61.9 & 4.8 & 3.6 \\
\hline Variability index & 0.62 & 0.37 & 0.41 & 0.64 & 0.63 \\
\hline
\end{tabular}


Table 11. Selected annual and seasonal statistics for 77 stations in West Virginia representative of 1930-2002.—Continued

[Winter, January 1-March 31; spring, April 1-June 30; summer, July 1-September 30; fall, October 1-December 31; streamflow statistics are in cubic feet per second, except for variability index, which is dimensionless; the record period is in climatic years, April 1 through March 31 of the indicated ending year; EPA, U.S. Environmental Protection Agency]

\begin{tabular}{|c|c|c|c|c|c|}
\hline \multirow{2}{*}{ Streamflow statistic } & \multirow{2}{*}{ Annual } & \multicolumn{4}{|c|}{ Season } \\
\hline & & Winter & Spring & Summer & Fall \\
\hline \multicolumn{6}{|c|}{03195500 Elk River at Sutton, WV, 1940-1959 } \\
\hline 1-day 2-year hydrologically based flow & 27.8 & 335 & 135 & 28.4 & 40.5 \\
\hline 1-day 5-year hydrologically based flow & 7.94 & 218 & 92.2 & 11.9 & 10.8 \\
\hline 1-day 10 -year hydrologically based flow & 3.53 & 172 & 76.8 & 7.56 & 4.45 \\
\hline 3-day 2-year hydrologically based flow & 29.0 & 361 & 152 & 31.0 & 44.1 \\
\hline 3-day 5-year hydrologically based flow & 8.31 & 230 & 104 & 13.0 & 11.3 \\
\hline 3-day 10 -year hydrologically based flow & 3.72 & 178 & 86.6 & 8.25 & 4.61 \\
\hline 7-day 2-year hydrologically based flow & 32.6 & 420 & 190 & 38.0 & 51.4 \\
\hline 7-day 5-year hydrologically based flow & 9.03 & 255 & 129 & 15.3 & 12.7 \\
\hline 7-day 10 -year hydrologically based flow & 3.99 & 191 & 106 & 9.50 & 5.09 \\
\hline 14-day 2-year hydrologically based flow & 38.5 & 555 & 278 & 51.7 & 68.8 \\
\hline 14-day 5-year hydrologically based flow & 10.8 & 326 & 183 & 19.6 & 15.9 \\
\hline 14-day 10 -year hydrologically based flow & 5.03 & 242 & 145 & 11.6 & 6.47 \\
\hline 30-day 2-year hydrologically based flow & 57.4 & 960 & 474 & 94.4 & 138 \\
\hline 30-day 5-year hydrologically based flow & 17.6 & 620 & 313 & 33.3 & 36.2 \\
\hline 30-day 10-year hydrologically based flow & 9.03 & 477 & 255 & 18.5 & 15.8 \\
\hline 1-day 3-year biologically based flow & 7.77 & 130 & 82.7 & 9.50 & 7.77 \\
\hline 4-day 3-year biologically based flow & 8.27 & 160 & 137 & 10.9 & 8.27 \\
\hline EPA harmonic-mean flow & 90.6 & 781 & 626 & 91.3 & 32.9 \\
\hline 5-percent-duration flow & 3,730 & 5,570 & 3,600 & 2,040 & 2,780 \\
\hline 10-percent-duration flow & 2,610 & 3,840 & 2,640 & 1,300 & 1,790 \\
\hline 15-percent-duration flow & 2,040 & 3,080 & 2,170 & 893 & 1,290 \\
\hline 20-percent-duration flow & 1,680 & 2,610 & 1,800 & 644 & 980 \\
\hline 25-percent-duration flow & 1,380 & 2,250 & 1,520 & 491 & 800 \\
\hline 30-percent-duration flow & 1,160 & 2,010 & 1,310 & 402 & 666 \\
\hline 35-percent-duration flow & 965 & 1,820 & 1,140 & 329 & 567 \\
\hline 40-percent-duration flow & 822 & 1,650 & 982 & 272 & 483 \\
\hline 45-percent-duration flow & 689 & 1,490 & 853 & 225 & 412 \\
\hline 50-percent-duration flow & 577 & 1,350 & 752 & 184 & 348 \\
\hline 55-percent-duration flow & 483 & 1,220 & 663 & 153 & 290 \\
\hline 60-percent-duration flow & 406 & 1,100 & 583 & 125 & 242 \\
\hline 65-percent-duration flow & 332 & 988 & 515 & 102 & 199 \\
\hline 70-percent-duration flow & 263 & 880 & 456 & 81.1 & 155 \\
\hline 75-percent-duration flow & 207 & 775 & 402 & 64.1 & 112 \\
\hline 80-percent-duration flow & 152 & 658 & 339 & 49.9 & 73.7 \\
\hline 85-percent-duration flow & 99.1 & 539 & 273 & 36.2 & 48.4 \\
\hline 90-percent-duration flow & 57.0 & 412 & 223 & 27.4 & 29.2 \\
\hline 95-percent-duration flow & 27.9 & 303 & 165 & 15.0 & 14.2 \\
\hline 99-percent-duration flow & 9.1 & 165 & 92.2 & 8.7 & 2.6 \\
\hline Variability index & 0.64 & 0.37 & 0.41 & 0.65 & 0.68 \\
\hline
\end{tabular}


Table 11. Selected annual and seasonal statistics for 77 stations in West Virginia representative of 1930-2002.—Continued

[Winter, January 1-March 31; spring, April 1-June 30; summer, July 1-September 30; fall, October 1-December 31; streamflow statistics are in cubic feet per second, except for variability index, which is dimensionless; the record period is in climatic years, April 1 through March 31 of the indicated ending year; EPA, U.S. Environmental Protection Agency]

\begin{tabular}{|c|c|c|c|c|c|}
\hline \multirow{2}{*}{ Streamflow statistic } & \multirow{2}{*}{ Annual } & \multicolumn{4}{|c|}{ Season } \\
\hline & & Winter & Spring & Summer & Fall \\
\hline \multicolumn{6}{|c|}{03197000 Elk River at Queen Shoals, WV, 1932-1957 } \\
\hline 1-day 2-year hydrologically based flow & 48.8 & 653 & 256 & 48.9 & 64.5 \\
\hline 1-day 5-year hydrologically based flow & 13.3 & 393 & 144 & 20.6 & 16.7 \\
\hline 1-day 10-year hydrologically based flow & 5.45 & 288 & 102 & 13.2 & 6.52 \\
\hline 3-day 2-year hydrologically based flow & 49.7 & 718 & 278 & 52.7 & 65.6 \\
\hline 3-day 5-year hydrologically based flow & 14.1 & 434 & 154 & 22.1 & 17.8 \\
\hline 3-day 10-year hydrologically based flow & 6.11 & 317 & 107 & 14.0 & 7.36 \\
\hline 7-day 2-year hydrologically based flow & 53.2 & 842 & 314 & 63.2 & 72.3 \\
\hline 7-day 5-year hydrologically based flow & 16.1 & 486 & 174 & 25.6 & 20.6 \\
\hline 7-day 10-year hydrologically based flow & 7.65 & 346 & 121 & 16.0 & 9.36 \\
\hline 14-day 2-year hydrologically based flow & 63.9 & 1,130 & 428 & 87.4 & 101 \\
\hline 14-day 5-year hydrologically based flow & 19.5 & 638 & 241 & 33.8 & 28.8 \\
\hline 14-day 10 -year hydrologically based flow & 9.52 & 446 & 169 & 20.6 & 13.2 \\
\hline 30-day 2-year hydrologically based flow & 97.5 & 1,800 & 802 & 173 & 204 \\
\hline 30-day 5-year hydrologically based flow & 29.9 & 1,080 & 437 & 64.9 & 53.7 \\
\hline 30-day 10-year hydrologically based flow & 14.6 & 783 & 301 & 37.7 & 23.1 \\
\hline 1-day 3-year biologically based flow & 7.97 & 190 & 117 & 19.0 & 7.95 \\
\hline 4-day 3-year biologically based flow & 11.9 & 255 & 148 & 24.1 & 11.9 \\
\hline EPA harmonic-mean flow & 149 & 1,440 & 982 & 175 & 51.6 \\
\hline 5-percent-duration flow & 7,070 & 11,800 & 6,270 & 3,120 & 4,980 \\
\hline 10-percent-duration flow & 4,820 & 7,900 & 4,670 & 1,990 & 3,050 \\
\hline 15-percent-duration flow & 3,610 & 6,180 & 3,740 & 1,420 & 2,200 \\
\hline 20-percent-duration flow & 2,980 & 5,120 & 3,120 & 1,030 & 1,660 \\
\hline 25-percent-duration flow & 2,420 & 4,360 & 2,640 & 830 & 1,280 \\
\hline 30-percent-duration flow & 2,060 & 3,840 & 2,230 & 683 & 1,070 \\
\hline 35-percent-duration flow & 1,710 & 3,420 & 1,970 & 566 & 882 \\
\hline 40-percent-duration flow & 1,430 & 3,070 & 1,700 & 471 & 740 \\
\hline 45-percent-duration flow & 1,180 & 2,790 & 1,460 & 397 & 613 \\
\hline 50-percent-duration flow & 985 & 2,550 & 1,280 & 336 & 507 \\
\hline 55-percent-duration flow & 810 & 2,340 & 1,130 & 282 & 423 \\
\hline 60-percent-duration flow & 671 & 2,130 & 999 & 238 & 355 \\
\hline 65 -percent-duration flow & 543 & 1,910 & 873 & 197 & 286 \\
\hline 70-percent-duration flow & 429 & 1,680 & 759 & 160 & 220 \\
\hline 75-percent-duration flow & 334 & 1,470 & 656 & 129 & 172 \\
\hline 80-percent-duration flow & 247 & 1,260 & 551 & 101 & 113 \\
\hline 85-percent-duration flow & 170 & 1,040 & 448 & 73.9 & 73.8 \\
\hline 90-percent-duration flow & 103 & 782 & 343 & 49.6 & 47.5 \\
\hline 95-percent-duration flow & 48.8 & 571 & 237 & 28.4 & 22.7 \\
\hline 99-percent-duration flow & 15.7 & 233 & 118 & 15.2 & 2.8 \\
\hline Variability index & 0.65 & 0.38 & 0.43 & 0.61 & 0.70 \\
\hline
\end{tabular}


Table 11. Selected annual and seasonal statistics for 77 stations in West Virginia representative of 1930-2002.-Continued

[Winter, January 1-March 31; spring, April 1-June 30; summer, July 1-September 30; fall, October 1-December 31; streamflow statistics are in cubic feet per second, except for variability index, which is dimensionless; the record period is in climatic years, April 1 through March 31of the indicated ending year; EPA, U.S. Environmental Protection Agency]

\begin{tabular}{|c|c|c|c|c|c|}
\hline \multirow{2}{*}{ Streamflow statistic } & \multirow{2}{*}{ Annual } & \multicolumn{4}{|c|}{ Season } \\
\hline & & Winter & Spring & Summer & Fall \\
\hline \multicolumn{6}{|c|}{03198500 Big Coal River at Ashford, WV, 1932-2002 } \\
\hline 1-day 2-year hydrologically based flow & 19.0 & 159 & 73.9 & 21.3 & 25.3 \\
\hline 1-day 5-year hydrologically based flow & 8.03 & 80.0 & 38.9 & 8.88 & 11.7 \\
\hline 1-day 10-year hydrologically based flow & 4.76 & 52.1 & 25.9 & 5.23 & 7.53 \\
\hline 3-day 2-year hydrologically based flow & 19.9 & 179 & 81.3 & 22.4 & 26.3 \\
\hline 3-day 5-year hydrologically based flow & 8.56 & 88.3 & 42.7 & 9.50 & 12.3 \\
\hline 3-day 10-year hydrologically based flow & 5.14 & 56.6 & 28.3 & 5.66 & 8.06 \\
\hline 7-day 2-year hydrologically based flow & 21.7 & 222 & 97.2 & 25.4 & 29.5 \\
\hline 7-day 5-year hydrologically based flow & 9.61 & 107 & 50.3 & 11.0 & 14.1 \\
\hline 7-day 10-year hydrologically based flow & 5.91 & 66.3 & 33.4 & 6.61 & 9.39 \\
\hline 14-day 2-year hydrologically based flow & 24.7 & 289 & 124 & 31.1 & 35.5 \\
\hline 14-day 5-year hydrologically based flow & 11.6 & 140 & 64.1 & 14.0 & 16.7 \\
\hline 14-day 10-year hydrologically based flow & 7.51 & 87.1 & 43.1 & 8.86 & 11.0 \\
\hline 30-day 2-year hydrologically based flow & 31.7 & 508 & 190 & 46.8 & 53.8 \\
\hline 30-day 5-year hydrologically based flow & 15.3 & 261 & 96.7 & 21.8 & 24.7 \\
\hline 30-day 10-year hydrologically based flow & 10.3 & 165 & 65.5 & 14.2 & 16.5 \\
\hline 1-day 3-year biologically based flow & 3.40 & 34.9 & 44.9 & 4.18 & 4.25 \\
\hline 4-day 3-year biologically based flow & 4.24 & 48.0 & 54.7 & 6.11 & 6.70 \\
\hline EPA harmonic-mean flow & 74.4 & 272 & 286 & 47.1 & 39.7 \\
\hline 5-percent-duration flow & 1,910 & 3,100 & 2,040 & 613 & 1,230 \\
\hline 10-percent-duration flow & 1,260 & 2,080 & 1,340 & 357 & 769 \\
\hline 15-percent-duration flow & 952 & 1,620 & 1,040 & 254 & 559 \\
\hline 20-percent-duration flow & 757 & 1,350 & 843 & 197 & 420 \\
\hline 25-percent-duration flow & 612 & 1,160 & 705 & 159 & 322 \\
\hline 30-percent-duration flow & 514 & 1,010 & 602 & 133 & 250 \\
\hline 35-percent-duration flow & 424 & 902 & 524 & 114 & 195 \\
\hline 40-percent-duration flow & 345 & 801 & 458 & 97.4 & 155 \\
\hline 45-percent-duration flow & 285 & 727 & 402 & 84.6 & 125 \\
\hline 50-percent-duration flow & 231 & 653 & 350 & 73.2 & 103 \\
\hline 55-percent-duration flow & 184 & 595 & 309 & 64.9 & 85.6 \\
\hline 60-percent-duration flow & 147 & 537 & 270 & 56.7 & 71.8 \\
\hline 65-percent-duration flow & 117 & 479 & 235 & 49.5 & 60.0 \\
\hline 70-percent-duration flow & 92.7 & 422 & 203 & 42.7 & 51.4 \\
\hline 75-percent-duration flow & 73.0 & 364 & 173 & 36.9 & 43.2 \\
\hline 80-percent-duration flow & 57.2 & 309 & 140 & 31.2 & 35.5 \\
\hline 85-percent-duration flow & 43.5 & 255 & 112 & 25.6 & 28.8 \\
\hline 90-percent-duration flow & 31.8 & 194 & 86.2 & 19.5 & 22.1 \\
\hline 95-percent-duration flow & 20.3 & 123 & 61.0 & 12.1 & 15.5 \\
\hline 99-percent-duration flow & 7.8 & 37.1 & 30.5 & 4.7 & 6.5 \\
\hline Variability index & 0.62 & 0.40 & 0.46 & 0.50 & 0.59 \\
\hline
\end{tabular}


Table 11. Selected annual and seasonal statistics for 77 stations in West Virginia representative of 1930-2002.—Continued

[Winter, January 1-March 31; spring, April 1-June 30; summer, July 1-September 30; fall, October 1-December 31; streamflow statistics are in cubic feet per second, except for variability index, which is dimensionless; the record period is in climatic years, April 1 through March 31 of the indicated ending year; EPA, U.S. Environmental Protection Agency]

\begin{tabular}{|c|c|c|c|c|c|}
\hline \multirow{2}{*}{ Streamflow statistic } & \multirow{2}{*}{ Annual } & \multicolumn{4}{|c|}{ Season } \\
\hline & & Winter & Spring & Summer & Fall \\
\hline \multicolumn{6}{|c|}{03199000 Little Coal River at Danville, WV, 1934-1984 } \\
\hline 1-day 2-year hydrologically based flow & 9.88 & 102 & 49.5 & 11.1 & 14.4 \\
\hline 1-day 5-year hydrologically based flow & 3.36 & 47.8 & 21.9 & 3.82 & 5.11 \\
\hline 1-day 10-year hydrologically based flow & 1.77 & 29.7 & 12.5 & 2.04 & 2.82 \\
\hline 3-day 2-year hydrologically based flow & 10.6 & 118 & 54.0 & 12.0 & 15.3 \\
\hline 3-day 5-year hydrologically based flow & 3.85 & 54.4 & 23.8 & 4.37 & 5.49 \\
\hline 3-day 10-year hydrologically based flow & 2.13 & 33.0 & 13.5 & 2.44 & 3.05 \\
\hline 7-day 2-year hydrologically based flow & 11.9 & 142 & 63.8 & 13.7 & 17.3 \\
\hline 7-day 5-year hydrologically based flow & 4.44 & 65.2 & 28.4 & 5.14 & 6.25 \\
\hline 7-day 10-year hydrologically based flow & 2.53 & 39.2 & 16.3 & 2.93 & 3.48 \\
\hline 14-day 2-year hydrologically based flow & 14.3 & 183 & 80.1 & 17.6 & 20.7 \\
\hline 14-day 5-year hydrologically based flow & 5.68 & 85.3 & 34.6 & 7.00 & 7.75 \\
\hline 14-day 10 -year hydrologically based flow & 3.37 & 51.7 & 20.1 & 4.12 & 4.42 \\
\hline 30-day 2-year hydrologically based flow & 18.8 & 322 & 120 & 26.1 & 30.8 \\
\hline 30-day 5-year hydrologically based flow & 7.52 & 151 & 52.4 & 11.4 & 12.3 \\
\hline 30-day 10-year hydrologically based flow & 4.57 & 89.1 & 31.6 & 7.32 & 7.57 \\
\hline 1-day 3-year biologically based flow & 1.30 & 15.0 & 20.0 & 1.55 & 1.90 \\
\hline 4-day 3-year biologically based flow & 1.54 & 27.1 & 23.8 & 3.40 & 2.08 \\
\hline EPA harmonic-mean flow & 37.5 & 166 & 149 & 24.9 & 18.6 \\
\hline 5-percent-duration flow & 1,330 & 2,070 & 1,420 & 423 & 797 \\
\hline 10-percent-duration flow & 845 & 1,420 & 932 & 249 & 519 \\
\hline 15-percent-duration flow & 633 & 1,090 & 706 & 167 & 368 \\
\hline 20-percent-duration flow & 508 & 903 & 563 & 125 & 268 \\
\hline 25-percent-duration flow & 418 & 778 & 475 & 102 & 199 \\
\hline 30-percent-duration flow & 342 & 678 & 403 & 85.1 & 154 \\
\hline 35-percent-duration flow & 280 & 610 & 347 & 71.3 & 121 \\
\hline 40-percent-duration flow & 228 & 542 & 303 & 61.9 & 96.3 \\
\hline 45-percent-duration flow & 183 & 493 & 266 & 52.9 & 77.4 \\
\hline 50-percent-duration flow & 147 & 445 & 233 & 46.3 & 63.0 \\
\hline 55-percent-duration flow & 116 & 401 & 204 & 39.9 & 52.2 \\
\hline 60-percent-duration flow & 90.8 & 359 & 177 & 34.4 & 42.9 \\
\hline 65 -percent-duration flow & 71.0 & 317 & 153 & 29.2 & 35.6 \\
\hline 70-percent-duration flow & 55.3 & 275 & 129 & 25.4 & 29.4 \\
\hline 75-percent-duration flow & 43.9 & 236 & 106 & 21.6 & 24.2 \\
\hline 80-percent-duration flow & 33.5 & 196 & 84.4 & 18.0 & 19.8 \\
\hline 85-percent-duration flow & 25.0 & 159 & 66.2 & 14.2 & 15.4 \\
\hline 90-percent-duration flow & 17.7 & 120 & 49.4 & 10.1 & 11.2 \\
\hline 95-percent-duration flow & 10.2 & 69.4 & 32.8 & 5.9 & 6.7 \\
\hline 99-percent-duration flow & 3.0 & 20.0 & 16.3 & 2.2 & 2.2 \\
\hline Variability index & 0.66 & 0.42 & 0.49 & 0.54 & 0.64 \\
\hline
\end{tabular}


Table 11. Selected annual and seasonal statistics for 77 stations in West Virginia representative of 1930-2002.-Continued

[Winter, January 1-March 31; spring, April 1-June 30; summer, July 1-September 30; fall, October 1-December 31; streamflow statistics are in cubic feet per second, except for variability index, which is dimensionless; the record period is in climatic years, April 1 through March 31of the indicated ending year; EPA, U.S. Environmental Protection Agency]

\begin{tabular}{|c|c|c|c|c|c|}
\hline \multirow{2}{*}{ Streamflow statistic } & \multirow{2}{*}{ Annual } & \multicolumn{4}{|c|}{ Season } \\
\hline & & Winter & Spring & Summer & Fall \\
\hline \multicolumn{6}{|c|}{03200500 Coal River at Tornado, WV, 1963-1975 } \\
\hline 1-day 2-year hydrologically based flow & 127 & 662 & 366 & 167 & 202 \\
\hline 1-day 5-year hydrologically based flow & 41.3 & 465 & 240 & 57.5 & 80.8 \\
\hline 1-day 10-year hydrologically based flow & 19.7 & 368 & 187 & 26.9 & 43.8 \\
\hline 3-day 2-year hydrologically based flow & 137 & 735 & 396 & 178 & 221 \\
\hline 3-day 5-year hydrologically based flow & 45.7 & 511 & 260 & 63.8 & 88.1 \\
\hline 3-day 10-year hydrologically based flow & 22.2 & 399 & 202 & 30.6 & 47.2 \\
\hline 7-day 2-year hydrologically based flow & 155 & 837 & 456 & 205 & 243 \\
\hline 7-day 5-year hydrologically based flow & 54.1 & 612 & 302 & 75.8 & 99.2 \\
\hline 7-day 10-year hydrologically based flow & 26.7 & 500 & 233 & 37.0 & 54.1 \\
\hline 14-day 2-year hydrologically based flow & 176 & 1,000 & 530 & 235 & 265 \\
\hline 14-day 5-year hydrologically based flow & 69.0 & 750 & 345 & 104 & 111 \\
\hline 14-day 10-year hydrologically based flow & 37.7 & 613 & 260 & 59.1 & 60.8 \\
\hline 30-day 2-year hydrologically based flow & 239 & 1,330 & 689 & 287 & 383 \\
\hline 30-day 5-year hydrologically based flow & 109 & 942 & 452 & 144 & 191 \\
\hline 30-day 10-year hydrologically based flow & 65.0 & 738 & 343 & 93.0 & 111 \\
\hline 1-day 3-year biologically based flow & 9.97 & 259 & 250 & 39.8 & 12.0 \\
\hline 4-day 3-year biologically based flow & 17.8 & 310 & 273 & 67.9 & 21.2 \\
\hline EPA harmonic-mean flow & 419 & 1,230 & 1,080 & 302 & 223 \\
\hline 5-percent-duration flow & 4,240 & 6,550 & 4,260 & 1,580 & 3,100 \\
\hline 10-percent-duration flow & 2,910 & 4,450 & 3,060 & 1,060 & 2,220 \\
\hline 15-percent-duration flow & 2,320 & 3,530 & 2,460 & 848 & 1,700 \\
\hline 20-percent-duration flow & 1,940 & 3,050 & 2,080 & 730 & 1,340 \\
\hline 25-percent-duration flow & 1,640 & 2,680 & 1,800 & 644 & 1,150 \\
\hline 30-percent-duration flow & 1,440 & 2,390 & 1,580 & 576 & 1,010 \\
\hline 35-percent-duration flow & 1,250 & 2,180 & 1,420 & 523 & 896 \\
\hline 40-percent-duration flow & 1,100 & 1,990 & 1,260 & 475 & 779 \\
\hline 45-percent-duration flow & 960 & 1,830 & 1,130 & 433 & 662 \\
\hline 50-percent-duration flow & 847 & 1,670 & 1,030 & 396 & 585 \\
\hline 55-percent-duration flow & 738 & 1,530 & 934 & 360 & 518 \\
\hline 60-percent-duration flow & 636 & 1,430 & 846 & 333 & 469 \\
\hline 65-percent-duration flow & 555 & 1,330 & 764 & 306 & 422 \\
\hline 70-percent-duration flow & 484 & 1,230 & 690 & 272 & 384 \\
\hline 75-percent-duration flow & 419 & 1,130 & 627 & 232 & 345 \\
\hline 80-percent-duration flow & 357 & 1,030 & 578 & 205 & 306 \\
\hline 85-percent-duration flow & 303 & 929 & 503 & 164 & 259 \\
\hline 90-percent-duration flow & 239 & 811 & 420 & 122 & 186 \\
\hline 95-percent-duration flow & 142 & 639 & 316 & 92.1 & 106 \\
\hline 99-percent-duration flow & 51.8 & 366 & 175 & 31.9 & 23.7 \\
\hline Variability index & 0.44 & 0.30 & 0.34 & 0.36 & 0.42 \\
\hline
\end{tabular}


Table 11. Selected annual and seasonal statistics for 77 stations in West Virginia representative of 1930-2002.—Continued

[Winter, January 1-March 31; spring, April 1-June 30; summer, July 1-September 30; fall, October 1-December 31; streamflow statistics are in cubic feet per second, except for variability index, which is dimensionless; the record period is in climatic years, April 1 through March 31 of the indicated ending year; EPA, U.S. Environmental Protection Agency]

\begin{tabular}{|c|c|c|c|c|c|}
\hline \multirow{2}{*}{ Streamflow statistic } & \multirow{2}{*}{ Annual } & \multicolumn{4}{|c|}{ Season } \\
\hline & & Winter & Spring & Summer & Fall \\
\hline \multicolumn{6}{|c|}{03201000 Pocatalico River at Sissonville, WV, 1943-1978 } \\
\hline 1-day 2-year hydrologically based flow & 0.66 & 59.6 & 9.97 & 1.01 & 2.29 \\
\hline 1-day 5-year hydrologically based flow & 0.07 & 23.6 & 2.67 & 0.11 & 0.38 \\
\hline 1-day 10-year hydrologically based flow & 0.01 & 12.4 & 1.03 & 0.02 & 0.13 \\
\hline 3-day 2-year hydrologically based flow & 0.87 & 66.5 & 10.2 & 1.34 & 2.61 \\
\hline 3-day 5-year hydrologically based flow & 0.10 & 26.7 & 3.93 & 0.16 & 0.42 \\
\hline 3-day 10-year hydrologically based flow & 0.02 & 14.3 & 2.19 & 0.03 & 0.14 \\
\hline 7-day 2-year hydrologically based flow & 1.04 & 86.9 & 14.3 & 1.62 & 3.32 \\
\hline 7-day 5-year hydrologically based flow & 0.17 & 33.9 & 5.64 & 0.27 & 0.59 \\
\hline 7-day 10-year hydrologically based flow & 0.06 & 17.6 & 3.22 & 0.09 & 0.21 \\
\hline 14-day 2-year hydrologically based flow & 1.38 & 126 & 22.8 & 2.26 & 4.85 \\
\hline 14-day 5-year hydrologically based flow & 0.23 & 51.8 & 9.07 & 0.37 & 0.85 \\
\hline 14-day 10 -year hydrologically based flow & 0.08 & 28.6 & 5.22 & 0.12 & 0.30 \\
\hline 30-day 2-year hydrologically based flow & 2.97 & 251 & 46.0 & 6.25 & 14.4 \\
\hline 30-day 5-year hydrologically based flow & 0.60 & 118 & 20.3 & 1.20 & 2.53 \\
\hline 30-day 10-year hydrologically based flow & 0.25 & 70.2 & 12.6 & 0.46 & 0.88 \\
\hline 1-day 3-year biologically based flow & 0.00 & 6.00 & 5.14 & 0.00 & 0.00 \\
\hline 4-day 3-year biologically based flow & 0.00 & 6.68 & 6.84 & 0.00 & 0.00 \\
\hline EPA harmonic-mean flow & 1.90 & 74.4 & 62.5 & 1.09 & 0.86 \\
\hline 5-percent-duration flow & 1,440 & 2,360 & 1,270 & 390 & 1,070 \\
\hline 10-percent-duration flow & 796 & 1,530 & 751 & 168 & 531 \\
\hline 15-percent-duration flow & 518 & 1,090 & 492 & 97.1 & 335 \\
\hline 20-percent-duration flow & 367 & 826 & 354 & 64.7 & 230 \\
\hline 25-percent-duration flow & 285 & 657 & 266 & 47.8 & 165 \\
\hline 30-percent-duration flow & 219 & 547 & 204 & 36.5 & 124 \\
\hline 35-percent-duration flow & 166 & 462 & 168 & 27.8 & 96.3 \\
\hline 40-percent-duration flow & 131 & 391 & 136 & 22.1 & 73.6 \\
\hline 45-percent-duration flow & 101 & 343 & 114 & 17.7 & 55.9 \\
\hline 50-percent-duration flow & 80.0 & 299 & 94.3 & 14.6 & 43.1 \\
\hline 55-percent-duration flow & 61.3 & 265 & 81.3 & 11.9 & 32.7 \\
\hline 60-percent-duration flow & 45.1 & 232 & 68.3 & 9.6 & 25.1 \\
\hline 65 -percent-duration flow & 34.1 & 200 & 57.7 & 7.6 & 19.7 \\
\hline 70-percent-duration flow & 24.5 & 171 & 47.1 & 5.8 & 14.7 \\
\hline 75-percent-duration flow & 17.1 & 146 & 38.8 & 4.3 & 10.2 \\
\hline 80-percent-duration flow & 11.5 & 122 & 30.5 & 2.7 & 6.5 \\
\hline 85-percent-duration flow & 7.1 & 98.7 & 22.1 & 1.5 & 3.6 \\
\hline 90-percent-duration flow & 3.5 & 74.1 & 14.4 & 0.7 & 1.5 \\
\hline 95-percent-duration flow & 1.0 & 45.0 & 8.2 & 0.2 & 0.3 \\
\hline 99-percent-duration flow & 0.1 & 8.1 & 2.4 & 0.0 & 0.1 \\
\hline Variability index & 0.93 & 0.51 & 0.65 & 0.93 & 1.02 \\
\hline
\end{tabular}


Table 11. Selected annual and seasonal statistics for 77 stations in West Virginia representative of 1930-2002.-Continued

[Winter, January 1-March 31; spring, April 1-June 30; summer, July 1-September 30; fall, October 1-December 31; streamflow statistics are in cubic feet per second, except for variability index, which is dimensionless; the record period is in climatic years, April 1 through March 31of the indicated ending year; EPA, U.S. Environmental Protection Agency]

\begin{tabular}{|c|c|c|c|c|c|}
\hline \multirow{2}{*}{ Streamflow statistic } & \multirow{2}{*}{ Annual } & \multicolumn{4}{|c|}{ Season } \\
\hline & & Winter & Spring & Summer & Fall \\
\hline \multicolumn{6}{|c|}{03203600 Guyandotte River at Logan, WV, 1964-1975 } \\
\hline 1-day 2-year hydrologically based flow & 73.6 & 365 & 218 & 85.6 & 91.2 \\
\hline 1-day 5-year hydrologically based flow & 49.2 & 165 & 136 & 56.8 & 57.5 \\
\hline 1-day 10-year hydrologically based flow & 39.8 & 99.5 & 106 & 45.0 & 46.8 \\
\hline 3-day 2-year hydrologically based flow & 75.6 & 421 & 229 & 88.6 & 94.5 \\
\hline 3-day 5-year hydrologically based flow & 51.2 & 183 & 141 & 59.4 & 60.6 \\
\hline 3-day 10-year hydrologically based flow & 41.9 & 106 & 109 & 47.5 & 49.8 \\
\hline 7-day 2-year hydrologically based flow & 80.5 & 567 & 249 & 94.3 & 101 \\
\hline 7-day 5-year hydrologically based flow & 54.8 & 254 & 152 & 64.6 & 62.9 \\
\hline 7-day 10-year hydrologically based flow & 45.0 & 143 & 118 & 52.5 & 51.1 \\
\hline 14-day 2-year hydrologically based flow & 87.3 & 730 & 302 & 105 & 108 \\
\hline 14-day 5-year hydrologically based flow & 59.0 & 314 & 185 & 72.2 & 64.9 \\
\hline 14-day 10-year hydrologically based flow & 48.1 & 170 & 144 & 58.8 & 51.9 \\
\hline 30-day 2-year hydrologically based flow & 109 & 1,070 & 439 & 132 & 158 \\
\hline 30-day 5-year hydrologically based flow & 69.8 & 499 & 232 & 87.3 & 84.8 \\
\hline 30-day 10-year hydrologically based flow & 55.2 & 280 & 166 & 69.8 & 62.0 \\
\hline 1-day 3-year biologically based flow & 39.4 & 47.9 & 167 & 48.0 & 39.1 \\
\hline 4-day 3-year biologically based flow & 42.8 & 55.7 & 181 & 54.0 & 42.9 \\
\hline EPA harmonic-mean flow & 264 & 522 & 741 & 187 & 154 \\
\hline 5-percent-duration flow & 3,940 & 6,530 & 3,880 & 1,110 & 2,860 \\
\hline 10-percent-duration flow & 2,450 & 4,160 & 2,510 & 720 & 1,810 \\
\hline 15-percent-duration flow & 1,900 & 3,290 & 2,000 & 521 & 1,360 \\
\hline 20-percent-duration flow & 1,560 & 2,640 & 1,750 & 421 & 1,110 \\
\hline 25-percent-duration flow & 1,310 & 2,250 & 1,530 & 360 & 919 \\
\hline 30-percent-duration flow & 1,130 & 2,010 & 1,340 & 313 & 707 \\
\hline 35-percent-duration flow & 970 & 1,780 & 1,170 & 278 & 562 \\
\hline 40-percent-duration flow & 823 & 1,580 & 1,030 & 237 & 413 \\
\hline 45-percent-duration flow & 668 & 1,440 & 916 & 208 & 345 \\
\hline 50-percent-duration flow & 548 & 1,320 & 811 & 183 & 285 \\
\hline 55-percent-duration flow & 435 & 1,210 & 715 & 160 & 239 \\
\hline 60-percent-duration flow & 345 & 1,120 & 637 & 148 & 200 \\
\hline 65-percent-duration flow & 290 & 1,030 & 568 & 136 & 155 \\
\hline 70-percent-duration flow & 235 & 929 & 496 & 123 & 130 \\
\hline 75-percent-duration flow & 186 & 819 & 392 & 112 & 113 \\
\hline 80-percent-duration flow & 147 & 698 & 320 & 105 & 98.5 \\
\hline 85-percent-duration flow & 119 & 567 & 269 & 97.5 & 88.0 \\
\hline 90-percent-duration flow & 99.8 & 407 & 216 & 86.6 & 78.1 \\
\hline 95-percent-duration flow & 80.4 & 274 & 174 & 70.4 & 61.4 \\
\hline 99-percent-duration flow & 51.2 & 56.9 & 122 & 48.0 & 44.3 \\
\hline Variability index & 0.54 & 0.39 & 0.41 & 0.37 & 0.55 \\
\hline
\end{tabular}


Table 11. Selected annual and seasonal statistics for 77 stations in West Virginia representative of 1930-2002.—Continued

[Winter, January 1-March 31; spring, April 1-June 30; summer, July 1-September 30; fall, October 1-December 31; streamflow statistics are in cubic feet per second, except for variability index, which is dimensionless; the record period is in climatic years, April 1 through March 31 of the indicated ending year; EPA, U.S. Environmental Protection Agency]

\begin{tabular}{|c|c|c|c|c|c|}
\hline \multirow{2}{*}{ Streamflow statistic } & \multirow{2}{*}{ Annual } & \multicolumn{4}{|c|}{ Season } \\
\hline & & Winter & Spring & Summer & Fall \\
\hline \multicolumn{6}{|c|}{03204000 Guyandotte River at Branchland, WV, 1941-1979 } \\
\hline 1-day 2-year hydrologically based flow & 82.0 & 578 & 366 & 88.3 & 96.0 \\
\hline 1-day 5-year hydrologically based flow & 49.0 & 282 & 176 & 53.4 & 52.5 \\
\hline 1-day 10-year hydrologically based flow & 36.5 & 176 & 143 & 40.2 & 39.0 \\
\hline 3-day 2-year hydrologically based flow & 84.3 & 621 & 284 & 91.7 & 99.5 \\
\hline 3-day 5-year hydrologically based flow & 50.2 & 307 & 187 & 55.3 & 54.2 \\
\hline 3-day 10 -year hydrologically based flow & 37.4 & 193 & 150 & 41.7 & 40.2 \\
\hline 7-day 2-year hydrologically based flow & 90.1 & 733 & 320 & 100 & 106 \\
\hline 7-day 5-year hydrologically based flow & 53.7 & 357 & 209 & 60.2 & 57.5 \\
\hline 7-day 10-year hydrologically based flow & 40.1 & 221 & 169 & 45.2 & 42.8 \\
\hline 14-day 2-year hydrologically based flow & 100 & 948 & 398 & 117 & 121 \\
\hline 14-day 5-year hydrologically based flow & 58.7 & 460 & 254 & 69.7 & 65.2 \\
\hline 14-day 10-year hydrologically based flow & 43.6 & 283 & 204 & 52.0 & 48.8 \\
\hline 30-day 2-year hydrologically based flow & 125 & 1,490 & 583 & 164 & 173 \\
\hline 30-day 5-year hydrologically based flow & 70.9 & 758 & 350 & 94.4 & 87.6 \\
\hline 30-day 10-year hydrologically based flow & 52.2 & 488 & 270 & 69.5 & 63.3 \\
\hline 1-day 3-year biologically based flow & 30.9 & 83.7 & 200 & 41.0 & 30.9 \\
\hline 4-day 3-year biologically based flow & 35.4 & 111 & 221 & 48.0 & 35.7 \\
\hline EPA harmonic-mean flow & 306 & 890 & 959 & 214 & 161 \\
\hline 5-percent-duration flow & 5,970 & 9,820 & 6,050 & 1,620 & 3,990 \\
\hline 10-percent-duration flow & 3,930 & 6,780 & 4,110 & 1,000 & 2,600 \\
\hline 15-percent-duration flow & 2,980 & 5,260 & 3,130 & 762 & 1,840 \\
\hline 20-percent-duration flow & 2,370 & 4,450 & 2,550 & 602 & 1,410 \\
\hline 25-percent-duration flow & 1,930 & 3,820 & 2,150 & 498 & 1,070 \\
\hline 30-percent-duration flow & 1,600 & 3,350 & 1,850 & 426 & 803 \\
\hline 35-percent-duration flow & 1,340 & 2,960 & 1,600 & 367 & 624 \\
\hline 40-percent-duration flow & 1,100 & 2,650 & 1,390 & 315 & 495 \\
\hline 45-percent-duration flow & 899 & 2,380 & 1,220 & 279 & 401 \\
\hline 50-percent-duration flow & 729 & 2,120 & 1,080 & 245 & 324 \\
\hline 55-percent-duration flow & 583 & 1,920 & 950 & 213 & 266 \\
\hline 60-percent-duration flow & 468 & 1,720 & 841 & 192 & 221 \\
\hline 65-percent-duration flow & 378 & 1,540 & 740 & 172 & 184 \\
\hline 70-percent-duration flow & 303 & 1,370 & 642 & 156 & 157 \\
\hline 75-percent-duration flow & 239 & 1,200 & 543 & 140 & 135 \\
\hline 80-percent-duration flow & 187 & 1,020 & 456 & 125 & 115 \\
\hline 85-percent-duration flow & 148 & 813 & 383 & 108 & 97.0 \\
\hline 90-percent-duration flow & 113 & 584 & 315 & 85.4 & 81.1 \\
\hline 95-percent-duration flow & 76.8 & 360 & 241 & 60.8 & 60.2 \\
\hline 99-percent-duration flow & 40.8 & 119 & 168 & 38.2 & 36.3 \\
\hline Variability index & 0.77 & 0.40 & 0.43 & 0.42 & 0.58 \\
\hline
\end{tabular}


Table 11. Selected annual and seasonal statistics for 77 stations in West Virginia representative of 1930-2002.—Continued

[Winter, January 1-March 31; spring, April 1-June 30; summer, July 1-September 30; fall, October 1-December 31; streamflow statistics are in cubic feet per second, except for variability index, which is dimensionless; the record period is in climatic years, April 1 through March 31of the indicated ending year; EPA, U.S. Environmental Protection Agency]

\begin{tabular}{|c|c|c|c|c|c|}
\hline \multirow{2}{*}{ Streamflow statistic } & \multirow{2}{*}{ Annual } & \multicolumn{4}{|c|}{ Season } \\
\hline & & Winter & Spring & Summer & Fall \\
\hline \multicolumn{6}{|c|}{03204500 Mud River near Milton, WV, 1939-1980 } \\
\hline 1-day 2-year hydrologically based flow & 1.27 & 61.1 & 12.7 & 1.97 & 1.88 \\
\hline 1-day 5-year hydrologically based flow & 0.27 & 19.8 & 6.20 & 0.45 & 0.41 \\
\hline 1-day 10-year hydrologically based flow & 0.11 & 8.94 & 4.13 & 0.18 & 0.18 \\
\hline 3-day 2-year hydrologically based flow & 1.36 & 68.0 & 14.2 & 2.07 & 2.19 \\
\hline 3-day 5-year hydrologically based flow & 0.33 & 22.2 & 7.02 & 0.55 & 0.48 \\
\hline 3-day 10-year hydrologically based flow & 0.14 & 10.0 & 4.71 & 0.25 & 0.21 \\
\hline 7-day 2-year hydrologically based flow & 1.63 & 85.2 & 18.5 & 2.42 & 2.71 \\
\hline 7-day 5-year hydrologically based flow & 0.41 & 28.9 & 8.92 & 0.68 & 0.60 \\
\hline 7-day 10-year hydrologically based flow & 0.19 & 13.0 & 5.85 & 0.33 & 0.26 \\
\hline 14-day 2-year hydrologically based flow & 2.19 & 120 & 25.9 & 3.32 & 3.99 \\
\hline 14-day 5-year hydrologically based flow & 0.56 & 41.6 & 11.8 & 0.94 & 0.86 \\
\hline 14-day 10 -year hydrologically based flow & 0.25 & 19.5 & 7.55 & 0.46 & 0.36 \\
\hline 30-day 2-year hydrologically based flow & 3.57 & 209 & 50.0 & 6.36 & 8.28 \\
\hline 30-day 5-year hydrologically based flow & 0.95 & 88.5 & 21.8 & 1.81 & 1.54 \\
\hline 30-day 10-year hydrologically based flow & 0.46 & 49.8 & 13.9 & 0.94 & 0.61 \\
\hline 1-day 3-year biologically based flow & 0.07 & 2.96 & 7.28 & 0.48 & 0.07 \\
\hline 4-day 3-year biologically based flow & 0.09 & 3.24 & 10.2 & 0.62 & 0.09 \\
\hline EPA harmonic-mean flow & 4.29 & 50.5 & 75.9 & 3.75 & 1.53 \\
\hline 5-percent-duration flow & 1,300 & 2,220 & 1,410 & 341 & 812 \\
\hline 10-percent-duration flow & 727 & 1,380 & 729 & 169 & 402 \\
\hline 15-percent-duration flow & 487 & 1,010 & 501 & 100 & 248 \\
\hline 20-percent-duration flow & 352 & 772 & 361 & 66.9 & 187 \\
\hline 25-percent-duration flow & 272 & 609 & 283 & 48.0 & 140 \\
\hline 30-percent-duration flow & 214 & 516 & 230 & 36.0 & 104 \\
\hline 35-percent-duration flow & 169 & 441 & 189 & 27.7 & 77.1 \\
\hline 40-percent-duration flow & 134 & 384 & 160 & 21.8 & 57.6 \\
\hline 45-percent-duration flow & 103 & 335 & 135 & 17.9 & 42.8 \\
\hline 50-percent-duration flow & 78.8 & 294 & 114 & 14.4 & 30.7 \\
\hline 55-percent-duration flow & 59.0 & 257 & 96.3 & 11.6 & 21.9 \\
\hline 60-percent-duration flow & 42.9 & 228 & 80.5 & 9.1 & 15.7 \\
\hline 65-percent-duration flow & 30.3 & 199 & 66.7 & 7.4 & 11.0 \\
\hline 70-percent-duration flow & 21.2 & 173 & 54.8 & 5.9 & 7.5 \\
\hline 75-percent-duration flow & 14.7 & 147 & 43.9 & 4.5 & 5.2 \\
\hline 80-percent-duration flow & 9.5 & 121 & 34.2 & 3.3 & 3.5 \\
\hline 85-percent-duration flow & 5.8 & 92.4 & 25.7 & 2.4 & 2.1 \\
\hline 90-percent-duration flow & 3.3 & 61.3 & 18.1 & 1.6 & 1.1 \\
\hline 95-percent-duration flow & 1.4 & 34.2 & 11.1 & 0.9 & 0.4 \\
\hline 99-percent-duration flow & 0.3 & 4.7 & 4.9 & 0.2 & 0.1 \\
\hline Variability index & 0.91 & 0.52 & 0.62 & 0.77 & 1.01 \\
\hline
\end{tabular}


Table 11. Selected annual and seasonal statistics for 77 stations in West Virginia representative of 1930-2002.—Continued

[Winter, January 1-March 31; spring, April 1-June 30; summer, July 1-September 30; fall, October 1-December 31; streamflow statistics are in cubic feet per second, except for variability index, which is dimensionless; the record period is in climatic years, April 1 through March 31of the indicated ending year; EPA, U.S. Environmental Protection Agency]

\begin{tabular}{|c|c|c|c|c|c|}
\hline \multirow{2}{*}{ Streamflow statistic } & \multirow{2}{*}{ Annual } & \multicolumn{4}{|c|}{ Season } \\
\hline & & Winter & Spring & Summer & Fall \\
\hline \multicolumn{6}{|c|}{03213000 Tug Fork at Litwar, WV, 1934-1984 } \\
\hline 1-day 2-year hydrologically based flow & 47.9 & 183 & 153 & 56.6 & 52.4 \\
\hline 1-day 5-year hydrologically based flow & 33.1 & 85.9 & 100 & 38.2 & 34.9 \\
\hline 1-day 10-year hydrologically based flow & 27.7 & 54.8 & 78.2 & 31.2 & 29.2 \\
\hline 3-day 2-year hydrologically based flow & 49.9 & 207 & 161 & 58.8 & 54.5 \\
\hline 3-day 5-year hydrologically based flow & 34.7 & 96.6 & 106 & 39.9 & 36.5 \\
\hline 3-day 10 -year hydrologically based flow & 29.2 & 60.7 & 82.5 & 32.7 & 30.7 \\
\hline 7-day 2-year hydrologically based flow & 53.8 & 241 & 181 & 63.5 & 60.6 \\
\hline 7-day 5-year hydrologically based flow & 37.2 & 113 & 117 & 42.8 & 39.3 \\
\hline 7-day 10-year hydrologically based flow & 31.0 & 70.1 & 90.4 & 34.9 & 32.6 \\
\hline 14-day 2-year hydrologically based flow & 58.2 & 296 & 208 & 71.9 & 66.3 \\
\hline 14-day 5-year hydrologically based flow & 40.0 & 135 & 137 & 48.0 & 42.6 \\
\hline 14-day 10 -year hydrologically based flow & 33.3 & 83.2 & 108 & 38.9 & 35.3 \\
\hline 30-day 2-year hydrologically based flow & 69.1 & 463 & 270 & 93.0 & 84.1 \\
\hline 30-day 5-year hydrologically based flow & 46.4 & 216 & 172 & 63.0 & 51.2 \\
\hline 30-day 10 -year hydrologically based flow & 38.2 & 132 & 134 & 51.6 & 40.9 \\
\hline 1-day 3-year biologically based flow & 27.0 & 33.9 & 95.0 & 35.0 & 28.0 \\
\hline 4-day 3-year biologically based flow & 29.5 & 44.6 & 105 & 38.8 & 29.5 \\
\hline EPA harmonic-mean flow & 156 & 278 & 428 & 128 & 84.8 \\
\hline 5-percent-duration flow & 1,940 & 3,090 & 2,120 & 609 & 1,070 \\
\hline 10-percent-duration flow & 1,290 & 2,140 & 1,480 & 391 & 664 \\
\hline 15-percent-duration flow & 983 & 1,670 & 1,140 & 304 & 497 \\
\hline 20-percent-duration flow & 793 & 1,390 & 952 & 255 & 378 \\
\hline 25-percent-duration flow & 654 & 1,210 & 821 & 221 & 296 \\
\hline 30-percent-duration flow & 547 & 1,060 & 711 & 198 & 236 \\
\hline 35-percent-duration flow & 461 & 941 & 622 & 178 & 194 \\
\hline 40-percent-duration flow & 388 & 845 & 555 & 160 & 163 \\
\hline 45-percent-duration flow & 327 & 754 & 499 & 147 & 135 \\
\hline 50-percent-duration flow & 272 & 685 & 448 & 134 & 115 \\
\hline 55-percent-duration flow & 230 & 616 & 407 & 122 & 103 \\
\hline 60-percent-duration flow & 195 & 553 & 368 & 111 & 92.3 \\
\hline 65-percent-duration flow & 164 & 492 & 331 & 101 & 83.1 \\
\hline 70-percent-duration flow & 136 & 439 & 295 & 91.6 & 73.4 \\
\hline 75-percent-duration flow & 113 & 384 & 262 & 83.2 & 64.5 \\
\hline 80-percent-duration flow & 94.8 & 325 & 231 & 74.7 & 57.6 \\
\hline 85-percent-duration flow & 78.7 & 248 & 201 & 65.9 & 50.1 \\
\hline 90-percent-duration flow & 62.8 & 170 & 171 & 54.9 & 42.8 \\
\hline 95-percent-duration flow & 46.2 & 91.6 & 132 & 44.7 & 35.8 \\
\hline 99-percent-duration flow & 32.5 & 39.4 & 83.0 & 32.4 & 28.2 \\
\hline Variability index & 0.51 & 0.43 & 0.36 & 0.33 & 0.46 \\
\hline
\end{tabular}


Table 11. Selected annual and seasonal statistics for 77 stations in West Virginia representative of 1930-2002.—Continued

[Winter, January 1-March 31; spring, April 1-June 30; summer, July 1-September 30; fall, October 1-December 31; streamflow statistics are in cubic feet per second, except for variability index, which is dimensionless; the record period is in climatic years, April 1 through March 31 of the indicated ending year; EPA, U.S. Environmental Protection Agency]

\begin{tabular}{|c|c|c|c|c|c|}
\hline \multirow{2}{*}{ Streamflow statistic } & \multirow{2}{*}{ Annual } & \multicolumn{4}{|c|}{ Season } \\
\hline & & Winter & Spring & Summer & Fall \\
\hline \multicolumn{6}{|c|}{03213500 Panther Creek near Panther, WV, 1948-1978 } \\
\hline 1-day 2-year hydrologically based flow & 0.52 & 9.29 & 1.76 & 0.64 & 0.88 \\
\hline 1-day 5-year hydrologically based flow & 0.13 & 3.60 & 0.92 & 0.16 & 0.33 \\
\hline 1-day 10-year hydrologically based flow & 0.05 & 1.89 & 0.66 & 0.06 & 0.19 \\
\hline 3-day 2-year hydrologically based flow & 0.60 & 10.1 & 2.14 & 0.75 & 0.99 \\
\hline 3-day 5-year hydrologically based flow & 0.17 & 3.86 & 1.17 & 0.22 & 0.38 \\
\hline 3-day 10 -year hydrologically based flow & 0.07 & 2.01 & 0.85 & 0.09 & 0.22 \\
\hline 7-day 2-year hydrologically based flow & 0.71 & 12.4 & 2.89 & 0.90 & 1.24 \\
\hline 7-day 5-year hydrologically based flow & 0.26 & 4.64 & 1.63 & 0.32 & 0.50 \\
\hline 7-day 10-year hydrologically based flow & 0.15 & 2.37 & 1.21 & 0.17 & 0.31 \\
\hline 14-day 2-year hydrologically based flow & 0.97 & 17.2 & 4.09 & 1.30 & 1.66 \\
\hline 14-day 5-year hydrologically based flow & 0.39 & 6.62 & 2.28 & 0.51 & 0.63 \\
\hline 14-day 10 -year hydrologically based flow & 0.23 & 3.44 & 1.68 & 0.29 & 0.38 \\
\hline 30-day 2-year hydrologically based flow & 1.52 & 34.2 & 6.94 & 2.29 & 2.94 \\
\hline 30-day 5-year hydrologically based flow & 0.63 & 15.8 & 3.54 & 0.98 & 1.03 \\
\hline 30-day 10 -year hydrologically based flow & 0.38 & 8.51 & 2.52 & 0.58 & 0.58 \\
\hline 1-day 3-year biologically based flow & 0.00 & 0.59 & 1.07 & 0.00 & 0.09 \\
\hline 4-day 3-year biologically based flow & 0.00 & 0.66 & 1.51 & 0.16 & 0.24 \\
\hline EPA harmonic-mean flow & 2.58 & 10.2 & 12.0 & 1.55 & 1.38 \\
\hline 5-percent-duration flow & 145 & 268 & 144 & 33.7 & 96.5 \\
\hline 10-percent-duration flow & 84.7 & 158 & 88.2 & 19.9 & 54.1 \\
\hline 15-percent-duration flow & 61.7 & 115 & 64.5 & 13.8 & 37.0 \\
\hline 20-percent-duration flow & 47.5 & 91.3 & 52.1 & 10.3 & 28.0 \\
\hline 25-percent-duration flow & 36.8 & 74.8 & 42.9 & 7.9 & 21.4 \\
\hline 30-percent-duration flow & 30.3 & 64.4 & 34.5 & 6.6 & 16.3 \\
\hline 35-percent-duration flow & 24.2 & 56.4 & 28.8 & 5.4 & 13.0 \\
\hline 40-percent-duration flow & 19.9 & 49.4 & 24.1 & 4.6 & 10.4 \\
\hline 45-percent-duration flow & 16.1 & 44.0 & 20.3 & 3.8 & 8.1 \\
\hline 50-percent-duration flow & 12.7 & 39.3 & 17.3 & 3.2 & 6.4 \\
\hline 55-percent-duration flow & 9.7 & 35.2 & 14.7 & 2.7 & 5.0 \\
\hline 60-percent-duration flow & 7.3 & 31.6 & 12.3 & 2.4 & 3.9 \\
\hline 65-percent-duration flow & 5.6 & 28.0 & 9.9 & 2.0 & 3.1 \\
\hline 70-percent-duration flow & 4.2 & 24.9 & 8.3 & 1.7 & 2.5 \\
\hline 75-percent-duration flow & 3.2 & 21.8 & 6.8 & 1.4 & 2.0 \\
\hline 80-percent-duration flow & 2.4 & 18.9 & 5.3 & 1.1 & 1.5 \\
\hline 85-percent-duration flow & 1.8 & 15.9 & 4.0 & 0.9 & 1.1 \\
\hline 90-percent-duration flow & 1.2 & 12.1 & 3.0 & 0.6 & 0.7 \\
\hline 95-percent-duration flow & 0.6 & 5.7 & 2.0 & 0.4 & 0.5 \\
\hline 99-percent-duration flow & 0.2 & 0.8 & 0.9 & 0.1 & 0.1 \\
\hline Variability index & 0.73 & 0.47 & 0.57 & 0.57 & 0.71 \\
\hline
\end{tabular}


Table 11. Selected annual and seasonal statistics for 77 stations in West Virginia representative of 1930-2002._Continued

[Winter, January 1-March 31; spring, April 1-June 30; summer, July 1-September 30; fall, October 1-December 31; streamflow statistics are in cubic feet per second, except for variability index, which is dimensionless; the record period is in climatic years, April 1 through March 31of the indicated ending year; EPA, U.S. Environmental Protection Agency]

\begin{tabular}{|c|c|c|c|c|c|}
\hline \multirow{2}{*}{ Streamflow statistic } & \multirow{2}{*}{ Annual } & \multicolumn{4}{|c|}{ Season } \\
\hline & & Winter & Spring & Summer & Fall \\
\hline \multicolumn{6}{|c|}{03214000 Tug Fork near Kermit, WV, 1936-1985 } \\
\hline 1-day 2-year hydrologically based flow & 81.5 & 463 & 262 & 93.2 & 94.5 \\
\hline 1-day 5-year hydrologically based flow & 49.1 & 220 & 163 & 55.7 & 54.7 \\
\hline 1-day 10-year hydrologically based flow & 37.6 & 138 & 124 & 42.2 & 42.0 \\
\hline 3-day 2-year hydrologically based flow & 84.2 & 508 & 281 & 97.2 & 97.3 \\
\hline 3-day 5-year hydrologically based flow & 50.9 & 242 & 174 & 58.4 & 56.1 \\
\hline 3-day 10-year hydrologically based flow & 39.0 & 152 & 132 & 44.3 & 43.1 \\
\hline 7-day 2-year hydrologically based flow & 89.3 & 611 & 316 & 105 & 107 \\
\hline 7-day 5-year hydrologically based flow & 53.7 & 288 & 195 & 63.2 & 60.2 \\
\hline 7-day 10-year hydrologically based flow & 41.1 & 176 & 150 & 48.0 & 45.7 \\
\hline 14-day 2-year hydrologically based flow & 99.8 & 771 & 380 & 123 & 123 \\
\hline 14-day 5-year hydrologically based flow & 59.2 & 361 & 234 & 74.0 & 67.1 \\
\hline 14-day 10 -year hydrologically based flow & 45.0 & 219 & 181 & 56.3 & 50.1 \\
\hline 30-day 2-year hydrologically based flow & 125 & 1,300 & 533 & 171 & 167 \\
\hline 30-day 5-year hydrologically based flow & 72.0 & 633 & 316 & 103 & 86.5 \\
\hline 30-day 10 -year hydrologically based flow & 54.2 & 386 & 242 & 79.1 & 62.8 \\
\hline 1-day 3-year biologically based flow & 33.9 & 64.8 & 167 & 47.9 & 33.9 \\
\hline 4-day 3-year biologically based flow & 36.2 & 87.8 & 199 & 53.4 & 36.3 \\
\hline EPA harmonic-mean flow & 298 & 712 & 876 & 228 & 154 \\
\hline 5-percent-duration flow & 4,820 & 7,900 & 5,230 & 1,540 & 2,940 \\
\hline 10-percent-duration flow & 3,230 & 5,370 & 3,550 & 973 & 1,880 \\
\hline 15-percent-duration flow & 2,480 & 4,180 & 2,780 & 739 & 1,410 \\
\hline 20-percent-duration flow & 1,990 & 3,490 & 2,290 & 591 & 1,080 \\
\hline 25-percent-duration flow & 1,650 & 3,000 & 1,940 & 495 & 848 \\
\hline 30-percent-duration flow & 1,380 & 2,670 & 1,660 & 430 & 666 \\
\hline 35-percent-duration flow & 1,160 & 2,390 & 1,440 & 375 & 515 \\
\hline 40-percent-duration flow & 973 & 2,150 & 1,280 & 326 & 415 \\
\hline 45-percent-duration flow & 802 & 1,940 & 1,140 & 291 & 339 \\
\hline 50-percent-duration flow & 651 & 1,760 & 1,010 & 260 & 277 \\
\hline 55-percent-duration flow & 526 & 1,600 & 897 & 233 & 231 \\
\hline 60-percent-duration flow & 429 & 1,460 & 787 & 207 & 195 \\
\hline 65-percent-duration flow & 348 & 1,320 & 686 & 184 & 170 \\
\hline 70-percent-duration flow & 288 & 1,180 & 591 & 164 & 152 \\
\hline 75-percent-duration flow & 234 & 1,030 & 503 & 147 & 133 \\
\hline 80-percent-duration flow & 187 & 870 & 432 & 130 & 113 \\
\hline 85-percent-duration flow & 152 & 682 & 366 & 113 & 92.9 \\
\hline 90-percent-duration flow & 118 & 486 & 301 & 91.2 & 77.0 \\
\hline 95-percent-duration flow & 80.5 & 278 & 230 & 66.5 & 56.5 \\
\hline 99-percent-duration flow & 45.6 & 85.3 & 141 & 44.3 & 34.5 \\
\hline Variability index & 0.56 & 0.41 & 0.41 & 0.40 & 0.54 \\
\hline
\end{tabular}


Table 12. Selected annual and seasonal statistics for 40 stations in surrounding states near West Virginia representative of 1930-2002 (values do not supersede those determined for use by the particular state).

[Winter, January 1-March 31; spring, April 1-June 30; summer, July 1-September 30; fall, October 1-December 31; streamflow statistics are in cubic feet per second, except for variability index, which is dimensionless; the record period is in climatic years, April 1 through March 31of the indicated ending year; EPA, U.S. Environmental Protection Agency]

\begin{tabular}{|c|c|c|c|c|c|}
\hline \multirow{2}{*}{ Streamflow statistic } & \multirow{2}{*}{ Annual } & \multicolumn{4}{|c|}{$\begin{array}{l}\text { Season } \\
\end{array}$} \\
\hline & & Winter & Spring & Summer & Fall \\
\hline \multicolumn{6}{|c|}{01595000 North Branch Potomac River at Steyer, MD, 1958-1996 } \\
\hline 1-day 2-year hydrologically based flow & 9.68 & 65.2 & 29.1 & 10.6 & 16.0 \\
\hline 1-day 5-year hydrologically based flow & 6.08 & 44.9 & 17.2 & 6.33 & 9.06 \\
\hline 1-day 10-year hydrologically based flow & 4.74 & 36.4 & 12.7 & 4.80 & 6.92 \\
\hline 3-day 2-year hydrologically based flow & 10.8 & 68.9 & 32.4 & 11.8 & 17.7 \\
\hline 3-day 5-year hydrologically based flow & 6.76 & 47.6 & 19.4 & 6.97 & 10.2 \\
\hline 3-day 10 -year hydrologically based flow & 5.21 & 38.8 & 14.5 & 5.24 & 7.91 \\
\hline 7-day 2-year hydrologically based flow & 13.0 & 75.0 & 38.0 & 14.3 & 21.0 \\
\hline 7-day 5-year hydrologically based flow & 7.97 & 51.2 & 22.8 & 8.36 & 12.7 \\
\hline 7-day 10-year hydrologically based flow & 6.03 & 42.1 & 17.1 & 6.14 & 10.1 \\
\hline 14-day 2-year hydrologically based flow & 15.4 & 88.1 & 47.4 & 17.9 & 25.9 \\
\hline 14-day 5-year hydrologically based flow & 9.52 & 57.0 & 28.0 & 10.3 & 15.8 \\
\hline 14-day 10-year hydrologically based flow & 7.14 & 45.2 & 21.0 & 7.43 & 12.7 \\
\hline 30-day 2-year hydrologically based flow & 19.2 & 129 & 69.7 & 23.4 & 44.9 \\
\hline 30-day 5-year hydrologically based flow & 11.7 & 78.0 & 41.5 & 13.2 & 25.9 \\
\hline 30-day 10-year hydrologically based flow & 8.95 & 59.0 & 31.4 & 9.51 & 19.3 \\
\hline 1-day 3-year biologically based flow & 3.63 & 31.0 & 20.0 & 3.78 & 4.90 \\
\hline 4-day 3-year biologically based flow & 4.14 & 33.2 & 25.4 & 4.70 & 6.74 \\
\hline EPA harmonic-mean flow & 47.9 & 136 & 123 & 26.4 & 33.3 \\
\hline 5-percent-duration flow & 561 & 849 & 582 & 231 & 463 \\
\hline 10-percent-duration flow & 384 & 564 & 415 & 147 & 306 \\
\hline 15-percent-duration flow & 302 & 453 & 340 & 108 & 245 \\
\hline 20-percent-duration flow & 247 & 379 & 284 & 84.3 & 205 \\
\hline 25 -percent-duration flow & 210 & 326 & 245 & 68.9 & 175 \\
\hline 30-percent-duration flow & 182 & 283 & 215 & 56.9 & 153 \\
\hline 35-percent-duration flow & 158 & 253 & 190 & 48.9 & 135 \\
\hline 40-percent-duration flow & 138 & 229 & 168 & 42.9 & 119 \\
\hline 45-percent-duration flow & 120 & 207 & 149 & 38.2 & 104 \\
\hline 50-percent-duration flow & 104 & 191 & 132 & 34.3 & 90.3 \\
\hline 55-percent-duration flow & 88.2 & 176 & 117 & 30.7 & 77.3 \\
\hline 60-percent-duration flow & 73.9 & 159 & 103 & 27.5 & 65.5 \\
\hline 65-percent-duration flow & 61.6 & 144 & 89.9 & 24.5 & 54.9 \\
\hline 70-percent-duration flow & 51.1 & 131 & 77.1 & 21.8 & 45.6 \\
\hline 75-percent-duration flow & 41.3 & 117 & 67.2 & 19.3 & 38.0 \\
\hline 80-percent-duration flow & 33.6 & 103 & 57.8 & 16.7 & 30.8 \\
\hline 85 -percent-duration flow & 26.7 & 88.2 & 48.0 & 13.8 & 24.9 \\
\hline 90-percent-duration flow & 20.4 & 71.7 & 37.7 & 10.9 & 19.8 \\
\hline 95-percent-duration flow & 13.7 & 56.8 & 27.8 & 7.3 & 14.5 \\
\hline 99-percent-duration flow & 6.4 & 34.8 & 16.1 & 4.2 & 9.3 \\
\hline Variability index & 0.49 & 0.35 & 0.40 & 0.44 & 0.46 \\
\hline
\end{tabular}


Table 12. Selected annual and seasonal statistics for 40 stations in surrounding states near West Virginia representative of 1930-2002 (values do not supersede those determined for use by the particular state).-Continued

[Winter, January 1-March 31; spring, April 1-June 30; summer, July 1-September 30; fall, October 1-December 31; streamflow statistics are in cubic feet per second, except for variability index, which is dimensionless; the record period is in climatic years, April 1 through March 31of the indicated ending year; EPA, U.S. Environmental Protection Agency]

\begin{tabular}{|c|c|c|c|c|c|}
\hline \multirow{2}{*}{ Streamflow statistic } & \multirow{2}{*}{ Annual } & \multicolumn{4}{|c|}{ Season } \\
\hline & & Winter & Spring & Summer & Fall \\
\hline \multicolumn{6}{|c|}{01596000 North Branch Potomac River at Bloomington, MD, 1940-1950 } \\
\hline 1-day 2-year hydrologically based flow & 34.1 & 146 & 98.2 & 36.4 & 60.8 \\
\hline 1-day 5-year hydrologically based flow & 24.0 & 97.6 & 61.6 & 26.2 & 34.3 \\
\hline 1-day 10-year hydrologically based flow & 20.0 & 80.4 & 47.5 & 22.0 & 24.5 \\
\hline 3-day 2-year hydrologically based flow & 37.2 & 155 & 111 & 39.6 & 65.8 \\
\hline 3-day 5-year hydrologically based flow & 26.2 & 99.9 & 68.8 & 28.9 & 37.3 \\
\hline 3-day 10-year hydrologically based flow & 21.6 & 80.9 & 52.2 & 24.3 & 26.7 \\
\hline 7-day 2-year hydrologically based flow & 43.6 & 168 & 131 & 46.6 & 71.9 \\
\hline 7-day 5-year hydrologically based flow & 30.0 & 105 & 79.3 & 34.1 & 40.1 \\
\hline 7-day 10-year hydrologically based flow & 24.3 & 83.2 & 59.4 & 28.4 & 28.5 \\
\hline 14-day 2-year hydrologically based flow & 48.0 & 190 & 158 & 53.0 & 77.1 \\
\hline 14-day 5-year hydrologically based flow & 33.2 & 114 & 95.7 & 38.5 & 43.1 \\
\hline 14-day 10-year hydrologically based flow & 27.1 & 89.2 & 72.4 & 32.0 & 31.3 \\
\hline 30-day 2-year hydrologically based flow & 59.2 & 297 & 223 & 73.7 & 103 \\
\hline 30-day 5-year hydrologically based flow & 38.4 & 184 & 159 & 46.4 & 55.7 \\
\hline 30-day 10-year hydrologically based flow & 30.0 & 143 & 136 & 36.3 & 40.0 \\
\hline 1-day 3-year biologically based flow & 21.0 & 29.0 & 71.8 & 24.0 & 21.0 \\
\hline 4-day 3-year biologically based flow & 23.7 & 83.9 & 82.7 & 26.5 & 26.6 \\
\hline EPA harmonic-mean flow & 147 & 309 & 347 & 92.1 & 98.3 \\
\hline 5-percent-duration flow & 1,630 & 2,280 & 1,700 & 848 & 1,010 \\
\hline 10-percent-duration flow & 1,120 & 1,660 & 1,160 & 519 & 673 \\
\hline 15-percent-duration flow & 850 & 1,360 & 927 & 347 & 524 \\
\hline 20-percent-duration flow & 700 & 1,160 & 792 & 268 & 446 \\
\hline 25-percent-duration flow & 598 & 1,010 & 702 & 218 & 369 \\
\hline 30-percent-duration flow & 513 & 879 & 629 & 185 & 302 \\
\hline 35-percent-duration flow & 444 & 782 & 564 & 159 & 265 \\
\hline 40-percent-duration flow & 375 & 701 & 508 & 137 & 234 \\
\hline 45-percent-duration flow & 316 & 631 & 455 & 121 & 206 \\
\hline 50-percent-duration flow & 270 & 570 & 406 & 107 & 184 \\
\hline 55-percent-duration flow & 232 & 512 & 361 & 93.7 & 160 \\
\hline 60-percent-duration flow & 197 & 460 & 323 & 83.7 & 135 \\
\hline 65-percent-duration flow & 167 & 417 & 289 & 74.7 & 117 \\
\hline 70-percent-duration flow & 138 & 364 & 259 & 66.6 & 102 \\
\hline 75-percent-duration flow & 114 & 308 & 230 & 59.9 & 85.3 \\
\hline 80-percent-duration flow & 94.2 & 262 & 201 & 53.2 & 70.0 \\
\hline 85-percent-duration flow & 75.6 & 206 & 170 & 46.1 & 60.6 \\
\hline 90-percent-duration flow & 59.0 & 148 & 140 & 39.2 & 43.2 \\
\hline 95-percent-duration flow & 40.6 & 107 & 103 & 32.9 & 32.6 \\
\hline 99-percent-duration flow & 28.2 & 88.2 & 55.0 & 26.4 & 25.0 \\
\hline Variability index & 0.49 & 0.39 & 0.36 & 0.43 & 0.45 \\
\hline
\end{tabular}


Table 12. Selected annual and seasonal statistics for 40 stations in surrounding states near West Virginia representative of 1930-2002 (values do not supersede those determined for use by the particular state)._- Continued

[Winter, January 1-March 31; spring, April 1-June 30; summer, July 1-September 30; fall, October 1-December 31; streamflow statistics are in cubic feet per second, except for variability index, which is dimensionless; the record period is in climatic years, April 1 through March 31 of the indicated ending year; EPA, U.S. Environmental Protection Agency]

\begin{tabular}{|c|c|c|c|c|c|}
\hline \multirow{2}{*}{ Streamflow statistic } & \multirow{2}{*}{ Annual } & \multicolumn{4}{|c|}{ Season } \\
\hline & & Winter & Spring & Summer & Fall \\
\hline \multicolumn{6}{|c|}{01596500 Savage River near Barton, MD, 1950-1996 } \\
\hline 1-day 2-year hydrologically based flow & 2.03 & 21.5 & 8.32 & 2.12 & 3.90 \\
\hline 1-day 5-year hydrologically based flow & 1.14 & 12.6 & 5.05 & 1.15 & 2.12 \\
\hline 1-day 10-year hydrologically based flow & 0.84 & 9.00 & 3.92 & 0.83 & 1.56 \\
\hline 3-day 2-year hydrologically based flow & 2.13 & 22.4 & 9.07 & 2.24 & 4.13 \\
\hline 3-day 5-year hydrologically based flow & 1.20 & 13.1 & 5.43 & 1.22 & 2.25 \\
\hline 3-day 10 -year hydrologically based flow & 0.88 & 9.45 & 4.19 & 0.88 & 1.67 \\
\hline 7-day 2-year hydrologically based flow & 2.38 & 24.8 & 10.9 & 2.58 & 4.95 \\
\hline 7-day 5-year hydrologically based flow & 1.35 & 14.2 & 6.59 & 1.39 & 2.67 \\
\hline 7-day 10 -year hydrologically based flow & 1.00 & 10.2 & 5.14 & 1.00 & 1.98 \\
\hline 14-day 2-year hydrologically based flow & 2.77 & 29.4 & 14.3 & 3.06 & 5.90 \\
\hline 14-day 5-year hydrologically based flow & 1.54 & 16.0 & 8.58 & 1.61 & 3.04 \\
\hline 14-day 10 -year hydrologically based flow & 1.14 & 11.3 & 6.66 & 1.15 & 2.19 \\
\hline 30-day 2-year hydrologically based flow & 3.76 & 45.8 & 25.9 & 4.47 & 10.5 \\
\hline 30-day 5-year hydrologically based flow & 2.24 & 25.8 & 15.1 & 2.54 & 4.41 \\
\hline 30-day 10-year hydrologically based flow & 1.75 & 19.1 & 11.2 & 1.94 & 2.79 \\
\hline 1-day 3-year biologically based flow & 0.78 & 5.60 & 7.16 & 0.80 & 0.91 \\
\hline 4-day 3-year biologically based flow & 0.89 & 6.88 & 10.2 & 0.94 & 1.16 \\
\hline EPA harmonic-mean flow & 10.7 & 40.5 & 49.6 & 5.68 & 6.63 \\
\hline 5-percent-duration flow & 301 & 446 & 331 & 61.6 & 212 \\
\hline 10-percent-duration flow & 189 & 314 & 216 & 35.9 & 132 \\
\hline 15-percent-duration flow & 138 & 244 & 163 & 24.1 & 98.9 \\
\hline 20-percent-duration flow & 108 & 194 & 131 & 18.5 & 78.8 \\
\hline 25-percent-duration flow & 87.8 & 163 & 110 & 15.3 & 63.5 \\
\hline 30-percent-duration flow & 72.2 & 137 & 94.0 & 13.1 & 52.0 \\
\hline 35-percent-duration flow & 59.0 & 119 & 81.0 & 11.1 & 43.3 \\
\hline 40-percent-duration flow & 48.9 & 105 & 69.6 & 9.5 & 36.1 \\
\hline 45-percent-duration flow & 40.5 & 92.5 & 59.9 & 8.3 & 29.7 \\
\hline 50-percent-duration flow & 33.2 & 82.7 & 51.9 & 7.3 & 23.7 \\
\hline 55-percent-duration flow & 26.8 & 73.3 & 45.2 & 6.4 & 19.2 \\
\hline 60-percent-duration flow & 21.3 & 64.3 & 39.2 & 5.6 & 15.6 \\
\hline 65-percent-duration flow & 16.7 & 55.8 & 33.9 & 4.9 & 12.5 \\
\hline 70-percent-duration flow & 13.0 & 48.9 & 29.0 & 4.3 & 9.4 \\
\hline 75-percent-duration flow & 9.6 & 42.2 & 24.7 & 3.8 & 7.2 \\
\hline 80-percent-duration flow & 7.3 & 35.8 & 20.9 & 3.1 & 5.6 \\
\hline 85-percent-duration flow & 5.4 & 29.6 & 17.3 & 2.7 & 4.4 \\
\hline 90-percent-duration flow & 3.9 & 23.5 & 13.2 & 2.2 & 3.5 \\
\hline 95-percent-duration flow & 2.6 & 16.2 & 8.7 & 1.6 & 2.6 \\
\hline 99-percent-duration flow & 1.3 & 7.8 & 4.8 & 0.9 & 1.6 \\
\hline Variability index & 0.65 & 0.43 & 0.47 & 0.47 & 0.61 \\
\hline
\end{tabular}


Table 12. Selected annual and seasonal statistics for 40 stations in surrounding states near West Virginia representative of 1930-2002 (values do not supersede those determined for use by the particular state).-Continued

[Winter, January 1-March 31; spring, April 1-June 30; summer, July 1-September 30; fall, October 1-December 31; streamflow statistics are in cubic feet per second, except for variability index, which is dimensionless; the record period is in climatic years, April 1 through March 31 of the indicated ending year; EPA, U.S. Environmental Protection Agency]

\begin{tabular}{|c|c|c|c|c|c|}
\hline \multirow{2}{*}{ Streamflow statistic } & \multirow{2}{*}{ Annual } & \multicolumn{4}{|c|}{ Season } \\
\hline & & Winter & Spring & Summer & Fall \\
\hline \multicolumn{6}{|c|}{01597000 Crabtree Creek near Swanton, MD, 1950-1981 } \\
\hline 1-day 2-year hydrologically based flow & 1.55 & 7.72 & 5.09 & 1.64 & 1.99 \\
\hline 1-day 5-year hydrologically based flow & 1.13 & 4.55 & 3.43 & 1.19 & 1.29 \\
\hline 1-day 10-year hydrologically based flow & 0.97 & 3.39 & 2.76 & 1.01 & 1.08 \\
\hline 3-day 2-year hydrologically based flow & 1.60 & 8.08 & 5.47 & 1.70 & 2.07 \\
\hline 3-day 5-year hydrologically based flow & 1.17 & 4.81 & 3.67 & 1.22 & 1.36 \\
\hline 3-day 10 -year hydrologically based flow & 1.01 & 3.63 & 2.95 & 1.04 & 1.15 \\
\hline 7-day 2-year hydrologically based flow & 1.68 & 9.16 & 6.39 & 1.80 & 2.30 \\
\hline 7-day 5-year hydrologically based flow & 1.22 & 5.29 & 4.20 & 1.28 & 1.49 \\
\hline 7-day 10-year hydrologically based flow & 1.06 & 3.94 & 3.31 & 1.09 & 1.26 \\
\hline 14-day 2-year hydrologically based flow & 1.79 & 10.7 & 7.91 & 2.02 & 2.55 \\
\hline 14-day 5-year hydrologically based flow & 1.29 & 5.88 & 4.95 & 1.40 & 1.60 \\
\hline 14-day 10 -year hydrologically based flow & 1.13 & 4.29 & 3.82 & 1.18 & 1.32 \\
\hline 30-day 2-year hydrologically based flow & 2.01 & 17.7 & 11.8 & 2.46 & 3.74 \\
\hline 30-day 5-year hydrologically based flow & 1.44 & 10.1 & 7.11 & 1.68 & 1.95 \\
\hline 30-day 10-year hydrologically based flow & 1.27 & 7.50 & 5.45 & 1.41 & 1.45 \\
\hline 1-day 3-year biologically based flow & 0.85 & 2.40 & 4.04 & 0.96 & 0.98 \\
\hline 4-day 3-year biologically based flow & 0.96 & 2.84 & 4.95 & 1.09 & 1.14 \\
\hline EPA harmonic-mean flow & 5.89 & 15.6 & 22.0 & 3.58 & 3.45 \\
\hline 5-percent-duration flow & 113 & 169 & 122 & 23.9 & 71.5 \\
\hline 10-percent-duration flow & 72.3 & 120 & 82.8 & 13.7 & 44.2 \\
\hline 15-percent-duration flow & 54.0 & 96.2 & 64.9 & 9.6 & 33.0 \\
\hline 20-percent-duration flow & 42.1 & 78.3 & 53.9 & 7.8 & 25.1 \\
\hline 25-percent-duration flow & 34.4 & 64.5 & 44.9 & 6.5 & 20.0 \\
\hline 30-percent-duration flow & 28.3 & 54.7 & 38.7 & 5.6 & 16.2 \\
\hline 35-percent-duration flow & 23.1 & 47.9 & 33.9 & 4.9 & 13.7 \\
\hline 40-percent-duration flow & 19.0 & 42.2 & 29.6 & 4.3 & 11.6 \\
\hline 45-percent-duration flow & 15.8 & 37.3 & 26.2 & 3.9 & 9.4 \\
\hline 50-percent-duration flow & 13.1 & 33.4 & 23.2 & 3.5 & 7.8 \\
\hline 55-percent-duration flow & 10.7 & 29.6 & 20.4 & 3.2 & 6.1 \\
\hline 60-percent-duration flow & 8.4 & 26.1 & 18.1 & 3.0 & 4.8 \\
\hline 65-percent-duration flow & 6.7 & 22.7 & 16.0 & 2.7 & 3.9 \\
\hline 70-percent-duration flow & 5.2 & 19.3 & 14.0 & 2.5 & 3.2 \\
\hline 75-percent-duration flow & 4.1 & 16.3 & 12.2 & 2.2 & 2.8 \\
\hline 80-percent-duration flow & 3.3 & 13.6 & 10.4 & 2.0 & 2.4 \\
\hline 85-percent-duration flow & 2.7 & 11.2 & 8.5 & 1.8 & 2.1 \\
\hline 90-percent-duration flow & 2.2 & 8.4 & 6.9 & 1.6 & 1.8 \\
\hline 95-percent-duration flow & 1.7 & 6.0 & 4.9 & 1.4 & 1.5 \\
\hline 99-percent-duration flow & 1.2 & 3.2 & 3.1 & 1.0 & 1.2 \\
\hline Variability index & 0.59 & 0.44 & 0.42 & 0.37 & 0.55 \\
\hline
\end{tabular}


Table 12. Selected annual and seasonal statistics for 40 stations in surrounding states near West Virginia representative of 1930-2002 (values do not supersede those determined for use by the particular state).-Continued

[Winter, January 1-March 31; spring, April 1-June 30; summer, July 1-September 30; fall, October 1-December 31; streamflow statistics are in cubic feet per second, except for variability index, which is dimensionless; the record period is in climatic years, April 1 through March 31of the indicated ending year; EPA, U.S. Environmental Protection Agency]

\begin{tabular}{|c|c|c|c|c|c|}
\hline \multirow{2}{*}{ Streamflow statistic } & \multirow{2}{*}{ Annual } & \multicolumn{4}{|c|}{ Season } \\
\hline & & Winter & Spring & Summer & Fall \\
\hline \multicolumn{6}{|c|}{01598000 Savage River at Bloomington, MD, 1940-1950 } \\
\hline 1-day 2-year hydrologically based flow & 5.60 & 44.3 & 29.8 & 5.91 & 9.42 \\
\hline 1-day 5-year hydrologically based flow & 2.79 & 20.6 & 17.7 & 2.90 & 4.28 \\
\hline 1-day 10-year hydrologically based flow & 1.83 & 12.5 & 13.2 & 1.86 & 2.56 \\
\hline 3-day 2-year hydrologically based flow & 5.88 & 49.0 & 34.1 & 6.26 & 9.78 \\
\hline 3-day 5-year hydrologically based flow & 2.91 & 22.1 & 20.3 & 3.09 & 4.64 \\
\hline 3-day 10-year hydrologically based flow & 1.90 & 13.2 & 14.8 & 2.00 & 2.90 \\
\hline 7-day 2-year hydrologically based flow & 6.43 & 53.3 & 40.0 & 7.00 & 11.1 \\
\hline 7-day 5-year hydrologically based flow & 3.16 & 24.0 & 23.8 & 3.36 & 5.00 \\
\hline 7-day 10-year hydrologically based flow & 2.06 & 14.6 & 17.3 & 2.13 & 3.02 \\
\hline 14-day 2-year hydrologically based flow & 7.41 & 61.9 & 47.3 & 8.53 & 12.0 \\
\hline 14-day 5-year hydrologically based flow & 3.55 & 27.3 & 29.8 & 3.90 & 5.13 \\
\hline 14-day 10 -year hydrologically based flow & 2.25 & 16.5 & 23.0 & 2.37 & 3.18 \\
\hline 30-day 2-year hydrologically based flow & 9.70 & 102 & 70.4 & 12.6 & 17.8 \\
\hline 30-day 5-year hydrologically based flow & 4.36 & 54.9 & 47.5 & 5.50 & 8.16 \\
\hline 30-day 10-year hydrologically based flow & 2.65 & 37.8 & 39.1 & 3.25 & 5.49 \\
\hline 1-day 3-year biologically based flow & 1.18 & 1.38 & 20.0 & 1.14 & 1.15 \\
\hline 4-day 3-year biologically based flow & 1.18 & 7.13 & 23.4 & 2.27 & 1.39 \\
\hline EPA harmonic-mean flow & 22.8 & 57.9 & 112 & 13.9 & 13.1 \\
\hline 5-percent-duration flow & 619 & 894 & 747 & 224 & 373 \\
\hline 10-percent-duration flow & 392 & 625 & 463 & 125 & 242 \\
\hline 15-percent-duration flow & 302 & 495 & 357 & 87.1 & 180 \\
\hline 20-percent-duration flow & 246 & 400 & 299 & 63.2 & 139 \\
\hline 25-percent-duration flow & 198 & 339 & 264 & 49.0 & 104 \\
\hline 30-percent-duration flow & 165 & 297 & 230 & 40.3 & 82.6 \\
\hline 35-percent-duration flow & 137 & 265 & 197 & 33.7 & 68.1 \\
\hline 40-percent-duration flow & 112 & 236 & 168 & 28.7 & 57.9 \\
\hline 45-percent-duration flow & 91.2 & 209 & 149 & 24.7 & 47.6 \\
\hline 50-percent-duration flow & 76.4 & 185 & 133 & 21.5 & 39.2 \\
\hline 55-percent-duration flow & 63.6 & 166 & 117 & 19.1 & 32.1 \\
\hline 60-percent-duration flow & 51.8 & 148 & 102 & 16.8 & 25.8 \\
\hline 65-percent-duration flow & 41.1 & 128 & 90.0 & 14.9 & 20.5 \\
\hline 70-percent-duration flow & 32.0 & 109 & 79.9 & 13.1 & 17.1 \\
\hline 75-percent-duration flow & 23.7 & 92.6 & 70.7 & 11.3 & 14.4 \\
\hline 80-percent-duration flow & 18.1 & 77.8 & 61.4 & 9.1 & 11.8 \\
\hline 85-percent-duration flow & 14.0 & 64.4 & 52.8 & 6.8 & 9.0 \\
\hline 90-percent-duration flow & 9.8 & 44.8 & 44.3 & 4.8 & 6.8 \\
\hline 95-percent-duration flow & 5.8 & 22.7 & 32.4 & 3.1 & 5.2 \\
\hline 99-percent-duration flow & 1.8 & 8.6 & 17.0 & 1.4 & 1.6 \\
\hline Variability index & 0.63 & 0.46 & 0.41 & 0.54 & 0.59 \\
\hline
\end{tabular}


Table 12. Selected annual and seasonal statistics for 40 stations in surrounding states near West Virginia representative of 1930-2002 (values do not supersede those determined for use by the particular state).-Continued

[Winter, January 1-March 31; spring, April 1-June 30; summer, July 1-September 30; fall, October 1-December 31; streamflow statistics are in cubic feet per second, except for variability index, which is dimensionless; the record period is in climatic years, April 1 through March 31of the indicated ending year; EPA, U.S. Environmental Protection Agency]

\begin{tabular}{|c|c|c|c|c|c|}
\hline \multirow{2}{*}{ Streamflow statistic } & \multirow{2}{*}{ Annual } & \multicolumn{4}{|c|}{ Season } \\
\hline & & Winter & Spring & Summer & Fall \\
\hline \multicolumn{6}{|c|}{01600000 North Branch Potomac River at Pinto, MD, 1940-1950 } \\
\hline 1-day 2-year hydrologically based flow & 61.4 & 259 & 191 & 64.1 & 94.4 \\
\hline 1-day 5-year hydrologically based flow & 45.6 & 160 & 127 & 49.1 & 59.8 \\
\hline 1-day 10-year hydrologically based flow & 38.2 & 123 & 102 & 41.9 & 46.3 \\
\hline 3-day 2-year hydrologically based flow & 64.5 & 275 & 218 & 68.4 & 99.6 \\
\hline 3-day 5-year hydrologically based flow & 48.1 & 164 & 141 & 52.3 & 63.9 \\
\hline 3-day 10-year hydrologically based flow & 40.4 & 125 & 111 & 44.3 & 49.8 \\
\hline 7-day 2-year hydrologically based flow & 70.7 & 296 & 247 & 75.6 & 109 \\
\hline 7-day 5-year hydrologically based flow & 52.0 & 171 & 156 & 57.1 & 68.1 \\
\hline 7-day 10-year hydrologically based flow & 43.4 & 127 & 120 & 47.9 & 52.6 \\
\hline 14-day 2-year hydrologically based flow & 76.2 & 334 & 289 & 83.1 & 115 \\
\hline 14-day 5-year hydrologically based flow & 56.0 & 187 & 184 & 61.5 & 70.5 \\
\hline 14-day 10-year hydrologically based flow & 46.9 & 138 & 145 & 51.2 & 55.2 \\
\hline 30-day 2-year hydrologically based flow & 91.6 & 520 & 409 & 114 & 152 \\
\hline 30-day 5-year hydrologically based flow & 62.1 & 318 & 300 & 73.9 & 88.1 \\
\hline 30-day 10-year hydrologically based flow & 49.7 & 244 & 257 & 58.2 & 67.5 \\
\hline 1-day 3-year biologically based flow & 35.9 & 50.0 & 139 & 37.9 & 41.0 \\
\hline 4-day 3-year biologically based flow & 39.0 & 101 & 152 & 40.9 & 46.3 \\
\hline EPA harmonic-mean flow & 246 & 516 & 636 & 155 & 159 \\
\hline 5-percent-duration flow & 2,920 & 4,330 & 3,260 & 1,260 & 1,680 \\
\hline 10-percent-duration flow & 1,920 & 2,940 & 2,170 & 781 & 1,170 \\
\hline 15-percent-duration flow & 1,460 & 2,350 & 1,660 & 574 & 881 \\
\hline 20-percent-duration flow & 1,210 & 1,990 & 1,400 & 448 & 719 \\
\hline 25-percent-duration flow & 1,030 & 1,710 & 1,220 & 358 & 597 \\
\hline 30-percent-duration flow & 877 & 1,490 & 1,090 & 300 & 492 \\
\hline 35-percent-duration flow & 742 & 1,330 & 980 & 254 & 426 \\
\hline 40-percent-duration flow & 638 & 1,200 & 891 & 220 & 372 \\
\hline 45-percent-duration flow & 543 & 1,080 & 792 & 197 & 323 \\
\hline 50-percent-duration flow & 463 & 984 & 710 & 176 & 278 \\
\hline 55-percent-duration flow & 394 & 886 & 639 & 155 & 237 \\
\hline 60-percent-duration flow & 333 & 800 & 580 & 134 & 198 \\
\hline 65-percent-duration flow & 276 & 716 & 526 & 121 & 166 \\
\hline 70-percent-duration flow & 226 & 621 & 470 & 109 & 147 \\
\hline 75-percent-duration flow & 184 & 533 & 415 & 98.1 & 128 \\
\hline 80-percent-duration flow & 149 & 448 & 370 & 88.7 & 108 \\
\hline 85-percent-duration flow & 120 & 355 & 322 & 78.8 & 92.8 \\
\hline 90-percent-duration flow & 95.6 & 266 & 262 & 66.9 & 79.3 \\
\hline 95-percent-duration flow & 71.8 & 181 & 196 & 52.6 & 61.3 \\
\hline 99-percent-duration flow & 46.9 & 118 & 119 & 41.4 & 43.0 \\
\hline Variability index & 0.50 & 0.40 & 0.36 & 0.42 & 0.45 \\
\hline
\end{tabular}


Table 12. Selected annual and seasonal statistics for 40 stations in surrounding states near West Virginia representative of 1930-2002 (values do not supersede those determined for use by the particular state).-Continued

[Winter, January 1-March 31; spring, April 1-June 30; summer, July 1-September 30; fall, October 1-December 31; streamflow statistics are in cubic feet per second, except for variability index, which is dimensionless; the record period is in climatic years, April 1 through March 31of the indicated ending year; EPA, U.S. Environmental Protection Agency]

\begin{tabular}{|c|c|c|c|c|c|}
\hline \multirow{2}{*}{ Streamflow statistic } & \multirow{2}{*}{ Annual } & \multicolumn{4}{|c|}{ Season } \\
\hline & & Winter & Spring & Summer & Fall \\
\hline \multicolumn{6}{|c|}{01603000 North Branch Potomac River near Cumberland, MD, 1940-1950 } \\
\hline 1-day 2-year hydrologically based flow & 102 & 354 & 301 & 107 & 138 \\
\hline 1-day 5-year hydrologically based flow & 78.5 & 217 & 211 & 83.9 & 94.5 \\
\hline 1-day 10-year hydrologically based flow & 68.1 & 169 & 173 & 73.2 & 76.5 \\
\hline 3-day 2-year hydrologically based flow & 106 & 382 & 331 & 111 & 146 \\
\hline 3-day 5-year hydrologically based flow & 81.6 & 229 & 227 & 87.0 & 99.1 \\
\hline 3-day 10-year hydrologically based flow & 70.8 & 176 & 183 & 76.2 & 79.7 \\
\hline 7-day 2-year hydrologically based flow & 114 & 413 & 369 & 120 & 157 \\
\hline 7-day 5-year hydrologically based flow & 87.5 & 240 & 248 & 93.2 & 105 \\
\hline 7-day 10-year hydrologically based flow & 75.8 & 180 & 197 & 80.6 & 84.6 \\
\hline 14-day 2-year hydrologically based flow & 122 & 465 & 413 & 133 & 160 \\
\hline 14-day 5-year hydrologically based flow & 92.9 & 261 & 280 & 99.8 & 104 \\
\hline 14-day 10 -year hydrologically based flow & 80.0 & 194 & 227 & 84.8 & 86.7 \\
\hline 30-day 2-year hydrologically based flow & 139 & 717 & 562 & 171 & 211 \\
\hline 30-day 5-year hydrologically based flow & 100 & 433 & 451 & 119 & 133 \\
\hline 30-day 10-year hydrologically based flow & 84.0 & 332 & 413 & 97.5 & 108 \\
\hline 1-day 3-year biologically based flow & 62.9 & 79.9 & 207 & 74.9 & 64.9 \\
\hline 4-day 3-year biologically based flow & 69.5 & 151 & 260 & 76.4 & 73.6 \\
\hline EPA harmonic-mean flow & 370 & 728 & 942 & 244 & 236 \\
\hline 5-percent-duration flow & 4,110 & 5,850 & 4,710 & 1,700 & 2,330 \\
\hline 10-percent-duration flow & 2,710 & 4,230 & 3,120 & 1,030 & 1,680 \\
\hline 15-percent-duration flow & 2,120 & 3,440 & 2,400 & 741 & 1,250 \\
\hline 20-percent-duration flow & 1,760 & 2,870 & 2,070 & 586 & 1,010 \\
\hline 25-percent-duration flow & 1,490 & 2,490 & 1,830 & 487 & 804 \\
\hline 30-percent-duration flow & 1,270 & 2,190 & 1,640 & 420 & 657 \\
\hline 35-percent-duration flow & 1,070 & 1,930 & 1,470 & 362 & 560 \\
\hline 40-percent-duration flow & 883 & 1,730 & 1,320 & 312 & 487 \\
\hline 45-percent-duration flow & 743 & 1,570 & 1,180 & 281 & 414 \\
\hline 50-percent-duration flow & 636 & 1,410 & 1,030 & 255 & 362 \\
\hline 55-percent-duration flow & 541 & 1,280 & 912 & 230 & 314 \\
\hline 60-percent-duration flow & 457 & 1,160 & 811 & 202 & 268 \\
\hline 65-percent-duration flow & 382 & 1,030 & 735 & 186 & 226 \\
\hline 70-percent-duration flow & 318 & 856 & 667 & 171 & 204 \\
\hline 75-percent-duration flow & 262 & 735 & 594 & 157 & 182 \\
\hline 80-percent-duration flow & 216 & 627 & 528 & 141 & 160 \\
\hline 85-percent-duration flow & 181 & 490 & 461 & 124 & 139 \\
\hline 90-percent-duration flow & 147 & 354 & 397 & 109 & 120 \\
\hline 95-percent-duration flow & 113 & 259 & 310 & 88.6 & 102 \\
\hline 99-percent-duration flow & 78.5 & 173 & 195 & 73.3 & 71.0 \\
\hline Variability index & 0.49 & 0.40 & 0.35 & 0.39 & 0.43 \\
\hline
\end{tabular}


Table 12. Selected annual and seasonal statistics for 40 stations in surrounding states near West Virginia representative of 1930-2002 (values do not supersede those determined for use by the particular state).-Continued

[Winter, January 1-March 31; spring, April 1-June 30; summer, July 1-September 30; fall, October 1-December 31; streamflow statistics are in cubic feet per second, except for variability index, which is dimensionless; the record period is in climatic years, April 1 through March 31 of the indicated ending year; EPA, U.S. Environmental Protection Agency]

\begin{tabular}{|c|c|c|c|c|c|}
\hline \multirow{2}{*}{ Streamflow statistic } & \multirow{2}{*}{ Annual } & \multicolumn{4}{|c|}{ Season } \\
\hline & & Winter & Spring & Summer & Fall \\
\hline \multicolumn{6}{|c|}{01612500 Little Tonoloway Creek near Hancock, MD, 1949-1958 } \\
\hline 1-day 2-year hydrologically based flow & 0.00 & 3.38 & 0.61 & 0.00 & 0.24 \\
\hline 1-day 5-year hydrologically based flow & 0.00 & 1.11 & 0.33 & 0.00 & 0.00 \\
\hline 1-day 10-year hydrologically based flow & 0.00 & 0.54 & 0.24 & 0.00 & 0.00 \\
\hline 3-day 2-year hydrologically based flow & 0.05 & 3.76 & 0.70 & 0.05 & 0.26 \\
\hline 3-day 5-year hydrologically based flow & 0.00 & 1.31 & 0.36 & 0.00 & 0.00 \\
\hline 3-day 10-year hydrologically based flow & 0.00 & 0.67 & 0.26 & 0.00 & 0.00 \\
\hline 7-day 2-year hydrologically based flow & 0.09 & 4.47 & 1.07 & 0.09 & 0.48 \\
\hline 7-day 5-year hydrologically based flow & 0.00 & 1.68 & 0.56 & 0.00 & 0.16 \\
\hline 7-day 10-year hydrologically based flow & 0.00 & 0.96 & 0.38 & 0.00 & 0.09 \\
\hline 14-day 2-year hydrologically based flow & 0.08 & 6.27 & 1.62 & 0.09 & 0.65 \\
\hline 14-day 5-year hydrologically based flow & 0.00 & 2.34 & 1.09 & 0.00 & 0.21 \\
\hline 14-day 10-year hydrologically based flow & 0.00 & 1.30 & 0.89 & 0.00 & 0.11 \\
\hline 30-day 2-year hydrologically based flow & 0.14 & 11.5 & 3.62 & 0.16 & 1.28 \\
\hline 30-day 5-year hydrologically based flow & 0.00 & 4.98 & 2.03 & 0.00 & 0.33 \\
\hline 30-day 10-year hydrologically based flow & 0.00 & 2.97 & 1.55 & 0.00 & 0.15 \\
\hline 1-day 3-year biologically based flow & 0.00 & 0.14 & 0.56 & 0.00 & 0.00 \\
\hline 4-day 3-year biologically based flow & 0.00 & 0.27 & 0.76 & 0.00 & 0.00 \\
\hline EPA harmonic-mean flow & 0.81 & 5.03 & 5.76 & 0.35 & 0.52 \\
\hline 5-percent-duration flow & 71.3 & 116 & 72.5 & 14.5 & 54.0 \\
\hline 10-percent-duration flow & 42.0 & 73.6 & 41.9 & 6.2 & 26.5 \\
\hline 15-percent-duration flow & 28.9 & 54.2 & 27.9 & 3.6 & 19.2 \\
\hline 20-percent-duration flow & 22.3 & 44.9 & 21.6 & 2.2 & 14.2 \\
\hline 25-percent-duration flow & 17.6 & 38.0 & 17.4 & 1.6 & 10.8 \\
\hline 30-percent-duration flow & 14.0 & 32.1 & 14.7 & 1.1 & 7.7 \\
\hline 35-percent-duration flow & 11.6 & 28.6 & 12.7 & 0.9 & 6.2 \\
\hline 40-percent-duration flow & 9.1 & 25.3 & 11.0 & 0.6 & 4.6 \\
\hline 45-percent-duration flow & 6.8 & 22.5 & 9.5 & 0.4 & 3.2 \\
\hline 50-percent-duration flow & 5.1 & 19.7 & 8.3 & 0.3 & 2.5 \\
\hline 55-percent-duration flow & 3.9 & 17.8 & 6.9 & 0.3 & 2.0 \\
\hline 60-percent-duration flow & 2.7 & 15.9 & 5.7 & 0.2 & 1.6 \\
\hline 65-percent-duration flow & 1.9 & 14.0 & 4.9 & 0.2 & 1.4 \\
\hline 70-percent-duration flow & 1.3 & 11.9 & 4.2 & 0.1 & 1.2 \\
\hline 75-percent-duration flow & 1.0 & 9.8 & 3.5 & 0.1 & 1.0 \\
\hline 80-percent-duration flow & 0.6 & 8.2 & 2.8 & 0.1 & 0.7 \\
\hline 85-percent-duration flow & 0.3 & 5.8 & 2.0 & 0.1 & 0.5 \\
\hline 90-percent-duration flow & 0.1 & 4.2 & 1.4 & 0.1 & 0.2 \\
\hline 95-percent-duration flow & 0.1 & 1.8 & 0.9 & 0.0 & 0.1 \\
\hline 99-percent-duration flow & 0.0 & 0.6 & 0.3 & 0.0 & 0.1 \\
\hline Variability index & 0.90 & 0.51 & 0.56 & 0.56 & 0.80 \\
\hline
\end{tabular}


Table 12. Selected annual and seasonal statistics for 40 stations in surrounding states near West Virginia representative of 1930-2002 (values do not supersede those determined for use by the particular state)._- Continued

[Winter, January 1-March 31; spring, April 1-June 30; summer, July 1-September 30; fall, October 1-December 31; streamflow statistics are in cubic feet per second, except for variability index, which is dimensionless; the record period is in climatic years, April 1 through March 31of the indicated ending year; EPA, U.S. Environmental Protection Agency]

\begin{tabular}{|c|c|c|c|c|c|}
\hline \multirow{2}{*}{ Streamflow statistic } & \multirow{2}{*}{ Annual } & \multicolumn{4}{|c|}{ Season } \\
\hline & & Winter & Spring & Summer & Fall \\
\hline \multicolumn{6}{|c|}{01613900 Hogue Creek near Hayfield, VA, 1962-1986 } \\
\hline 1-day 2-year hydrologically based flow & 0.72 & 4.17 & 1.89 & 0.79 & 1.03 \\
\hline 1-day 5-year hydrologically based flow & 0.34 & 2.02 & 1.15 & 0.36 & 0.58 \\
\hline 1-day 10-year hydrologically based flow & 0.21 & 1.23 & 0.88 & 0.22 & 0.45 \\
\hline 3-day 2-year hydrologically based flow & 0.73 & 4.48 & 2.05 & 0.80 & 1.07 \\
\hline 3-day 5-year hydrologically based flow & 0.38 & 2.15 & 1.24 & 0.41 & 0.62 \\
\hline 3-day 10 -year hydrologically based flow & 0.27 & 1.30 & 0.94 & 0.28 & 0.49 \\
\hline 7-day 2-year hydrologically based flow & 0.78 & 4.78 & 2.39 & 0.86 & 1.19 \\
\hline 7-day 5-year hydrologically based flow & 0.48 & 2.34 & 1.38 & 0.51 & 0.73 \\
\hline 7-day 10 -year hydrologically based flow & 0.38 & 1.47 & 1.02 & 0.40 & 0.60 \\
\hline 14-day 2-year hydrologically based flow & 0.87 & 5.41 & 3.06 & 0.96 & 1.38 \\
\hline 14-day 5-year hydrologically based flow & 0.56 & 2.65 & 1.70 & 0.59 & 0.89 \\
\hline 14-day 10-year hydrologically based flow & 0.46 & 1.74 & 1.22 & 0.47 & 0.76 \\
\hline 30-day 2-year hydrologically based flow & 1.04 & 8.30 & 4.84 & 1.16 & 2.21 \\
\hline 30-day 5-year hydrologically based flow & 0.68 & 4.05 & 2.38 & 0.74 & 1.23 \\
\hline 30-day 10-year hydrologically based flow & 0.57 & 2.64 & 1.62 & 0.60 & 0.94 \\
\hline 1-day 3-year biologically based flow & 0.19 & 0.61 & 1.19 & 0.29 & 0.40 \\
\hline 4-day 3-year biologically based flow & 0.31 & 0.93 & 1.35 & 0.39 & 0.38 \\
\hline EPA harmonic-mean flow & 2.66 & 6.21 & 7.73 & 1.48 & 1.87 \\
\hline 5-percent-duration flow & 60.2 & 89.4 & 65.7 & 12.9 & 46.7 \\
\hline 10-percent-duration flow & 34.4 & 57.0 & 39.7 & 6.3 & 26.5 \\
\hline 15-percent-duration flow & 24.4 & 40.5 & 30.1 & 4.5 & 17.9 \\
\hline 20-percent-duration flow & 18.9 & 31.5 & 24.0 & 3.5 & 13.3 \\
\hline 25-percent-duration flow & 15.1 & 26.1 & 19.8 & 3.0 & 9.9 \\
\hline 30-percent-duration flow & 12.1 & 22.2 & 16.5 & 2.6 & 7.9 \\
\hline 35-percent-duration flow & 9.6 & 19.2 & 13.9 & 2.3 & 6.6 \\
\hline 40-percent-duration flow & 8.0 & 17.2 & 11.9 & 2.0 & 5.5 \\
\hline 45-percent-duration flow & 6.6 & 15.3 & 10.1 & 1.8 & 4.7 \\
\hline 50-percent-duration flow & 5.4 & 13.6 & 8.6 & 1.6 & 4.0 \\
\hline 55-percent-duration flow & 4.5 & 12.0 & 7.4 & 1.5 & 3.4 \\
\hline 60-percent-duration flow & 3.7 & 10.4 & 6.5 & 1.4 & 2.9 \\
\hline 65-percent-duration flow & 3.0 & 9.2 & 5.6 & 1.3 & 2.5 \\
\hline 70-percent-duration flow & 2.4 & 8.1 & 4.9 & 1.1 & 2.1 \\
\hline 75-percent-duration flow & 2.0 & 7.0 & 4.2 & 1.0 & 1.9 \\
\hline 80-percent-duration flow & 1.7 & 5.7 & 3.5 & 0.9 & 1.6 \\
\hline 85-percent-duration flow & 1.4 & 4.6 & 2.8 & 0.8 & 1.4 \\
\hline 90-percent-duration flow & 1.1 & 3.6 & 2.2 & 0.6 & 1.2 \\
\hline 95-percent-duration flow & 0.8 & 2.3 & 1.5 & 0.5 & 0.9 \\
\hline 99-percent-duration flow & 0.4 & 1.2 & 0.8 & 0.4 & 0.5 \\
\hline Variability index & 0.58 & 0.46 & 0.49 & 0.40 & 0.53 \\
\hline
\end{tabular}


Table 12. Selected annual and seasonal statistics for 40 stations in surrounding states near West Virginia representative of 1930-2002 (values do not supersede those determined for use by the particular state).-Continued

[Winter, January 1-March 31; spring, April 1-June 30; summer, July 1-September 30; fall, October 1-December 31; streamflow statistics are in cubic feet per second, except for variability index, which is dimensionless; the record period is in climatic years, April 1 through March 31 of the indicated ending year; EPA, U.S. Environmental Protection Agency]

\begin{tabular}{|c|c|c|c|c|c|}
\hline \multirow{2}{*}{ Streamflow statistic } & \multirow{2}{*}{ Annual } & \multicolumn{4}{|c|}{ Season } \\
\hline & & Winter & Spring & Summer & Fall \\
\hline \multicolumn{6}{|c|}{01615000 Opequon Creek near Berryville, VA, 1945-1996 } \\
\hline 1-day 2-year hydrologically based flow & 3.92 & 12.8 & 9.13 & 4.67 & 4.79 \\
\hline 1-day 5-year hydrologically based flow & 1.82 & 7.20 & 5.43 & 2.14 & 2.59 \\
\hline 1-day 10-year hydrologically based flow & 1.15 & 5.13 & 4.01 & 1.31 & 1.88 \\
\hline 3-day 2-year hydrologically based flow & 4.61 & 14.0 & 9.78 & 5.28 & 5.33 \\
\hline 3-day 5-year hydrologically based flow & 2.23 & 7.74 & 6.20 & 2.50 & 3.07 \\
\hline 3-day 10-year hydrologically based flow & 1.41 & 5.45 & 4.77 & 1.54 & 2.32 \\
\hline 7-day 2-year hydrologically based flow & 5.16 & 15.3 & 10.7 & 5.84 & 6.01 \\
\hline 7-day 5-year hydrologically based flow & 2.53 & 8.47 & 6.92 & 2.81 & 3.53 \\
\hline 7-day 10-year hydrologically based flow & 1.61 & 5.97 & 5.39 & 1.74 & 2.70 \\
\hline 14-day 2-year hydrologically based flow & 5.71 & 18.1 & 12.0 & 6.43 & 6.85 \\
\hline 14-day 5-year hydrologically based flow & 2.86 & 10.0 & 7.88 & 3.15 & 3.99 \\
\hline 14-day 10-year hydrologically based flow & 1.85 & 7.06 & 6.20 & 1.98 & 3.05 \\
\hline 30-day 2-year hydrologically based flow & 6.37 & 27.1 & 16.2 & 7.32 & 8.96 \\
\hline 30-day 5-year hydrologically based flow & 3.42 & 15.4 & 10.3 & 3.79 & 5.06 \\
\hline 30-day 10-year hydrologically based flow & 2.35 & 11.1 & 8.05 & 2.54 & 3.88 \\
\hline 1-day 3-year biologically based flow & 1.49 & 4.50 & 5.83 & 1.60 & 1.70 \\
\hline 4-day 3-year biologically based flow & 1.76 & 5.27 & 6.69 & 1.83 & 2.05 \\
\hline EPA harmonic-mean flow & 11.1 & 22.1 & 22.7 & 6.90 & 7.95 \\
\hline 5-percent-duration flow & 156 & 253 & 156 & 55.4 & 126 \\
\hline 10-percent-duration flow & 84.6 & 152 & 85.6 & 30.5 & 67.0 \\
\hline 15-percent-duration flow & 60.1 & 107 & 60.3 & 23.1 & 47.8 \\
\hline 20-percent-duration flow & 47.8 & 84.5 & 48.2 & 19.1 & 36.1 \\
\hline 25-percent-duration flow & 39.2 & 69.1 & 40.5 & 16.9 & 28.8 \\
\hline 30-percent-duration flow & 32.5 & 59.5 & 35.1 & 14.8 & 24.0 \\
\hline 35-percent-duration flow & 28.4 & 52.0 & 31.0 & 13.5 & 20.2 \\
\hline 40-percent-duration flow & 24.4 & 45.5 & 27.6 & 12.3 & 17.2 \\
\hline 45-percent-duration flow & 21.3 & 41.1 & 25.2 & 11.0 & 15.2 \\
\hline 50-percent-duration flow & 18.4 & 36.8 & 23.0 & 9.8 & 13.5 \\
\hline 55-percent-duration flow & 16.4 & 33.6 & 20.9 & 8.7 & 11.9 \\
\hline 60-percent-duration flow & 14.5 & 30.3 & 19.0 & 7.8 & 10.5 \\
\hline 65-percent-duration flow & 12.6 & 27.5 & 17.2 & 7.0 & 9.2 \\
\hline 70-percent-duration flow & 10.8 & 24.9 & 15.7 & 6.3 & 8.1 \\
\hline 75-percent-duration flow & 9.3 & 21.9 & 14.1 & 5.5 & 7.3 \\
\hline 80-percent-duration flow & 8.0 & 18.8 & 12.7 & 4.7 & 6.4 \\
\hline 85-percent-duration flow & 6.7 & 15.8 & 11.0 & 4.0 & 5.7 \\
\hline 90-percent-duration flow & 5.3 & 12.9 & 9.1 & 3.3 & 4.5 \\
\hline 95-percent-duration flow & 3.7 & 9.3 & 7.1 & 2.5 & 3.3 \\
\hline 99-percent-duration flow & 2.0 & 4.8 & 4.8 & 0.9 & 2.3 \\
\hline Variability index & 0.48 & 0.42 & 0.39 & 0.39 & 0.47 \\
\hline
\end{tabular}


Table 12. Selected annual and seasonal statistics for 40 stations in surrounding states near West Virginia representative of 1930-2002 (values do not supersede those determined for use by the particular state).-Continued

[Winter, January 1-March 31; spring, April 1-June 30; summer, July 1-September 30; fall, October 1-December 31; streamflow statistics are in cubic feet per second, except for variability index, which is dimensionless; the record period is in climatic years, April 1 through March 31of the indicated ending year; EPA, U.S. Environmental Protection Agency]

\begin{tabular}{|c|c|c|c|c|c|}
\hline \multirow{2}{*}{ Streamflow statistic } & \multirow{2}{*}{ Annual } & \multicolumn{4}{|c|}{ Season } \\
\hline & & Winter & Spring & Summer & Fall \\
\hline \multicolumn{6}{|c|}{01620500 North River near Stokesville, VA, 1948-1996 } \\
\hline 1-day 2-year hydrologically based flow & 0.64 & 7.53 & 3.07 & 0.70 & 1.29 \\
\hline 1-day 5-year hydrologically based flow & 0.31 & 3.74 & 1.73 & 0.32 & 0.53 \\
\hline 1-day 10-year hydrologically based flow & 0.20 & 2.36 & 1.25 & 0.21 & 0.33 \\
\hline 3-day 2-year hydrologically based flow & 0.66 & 7.96 & 3.29 & 0.72 & 1.36 \\
\hline 3-day 5-year hydrologically based flow & 0.32 & 4.03 & 1.88 & 0.34 & 0.54 \\
\hline 3-day 10-year hydrologically based flow & 0.21 & 2.58 & 1.37 & 0.22 & 0.34 \\
\hline 7-day 2-year hydrologically based flow & 0.71 & 9.28 & 3.83 & 0.78 & 1.53 \\
\hline 7-day 5-year hydrologically based flow & 0.34 & 4.75 & 2.20 & 0.37 & 0.58 \\
\hline 7-day 10-year hydrologically based flow & 0.23 & 3.02 & 1.62 & 0.25 & 0.35 \\
\hline 14-day 2-year hydrologically based flow & 0.77 & 10.9 & 4.91 & 0.89 & 1.83 \\
\hline 14-day 5-year hydrologically based flow & 0.38 & 5.40 & 2.85 & 0.43 & 0.68 \\
\hline 14-day 10 -year hydrologically based flow & 0.26 & 3.36 & 2.11 & 0.28 & 0.40 \\
\hline 30-day 2-year hydrologically based flow & 0.96 & 16.8 & 9.17 & 1.22 & 3.41 \\
\hline 30-day 5-year hydrologically based flow & 0.46 & 7.98 & 4.92 & 0.57 & 1.11 \\
\hline 30-day 10-year hydrologically based flow & 0.32 & 4.78 & 3.55 & 0.39 & 0.60 \\
\hline 1-day 3-year biologically based flow & 0.14 & 1.36 & 2.95 & 0.13 & 0.20 \\
\hline 4-day 3-year biologically based flow & 0.20 & 1.44 & 3.68 & 0.15 & 0.28 \\
\hline EPA harmonic-mean flow & 2.83 & 10.7 & 16.7 & 1.61 & 1.56 \\
\hline 5-percent-duration flow & 97.8 & 135 & 117 & 29.4 & 85.0 \\
\hline 10-percent-duration flow & 62.1 & 86.6 & 75.1 & 14.8 & 51.5 \\
\hline 15-percent-duration flow & 45.5 & 66.3 & 56.4 & 9.2 & 37.3 \\
\hline 20-percent-duration flow & 35.1 & 54.2 & 44.2 & 6.7 & 29.1 \\
\hline 25-percent-duration flow & 29.0 & 46.5 & 36.1 & 5.4 & 23.3 \\
\hline 30-percent-duration flow & 24.4 & 40.4 & 30.1 & 4.5 & 19.3 \\
\hline 35-percent-duration flow & 20.6 & 35.6 & 26.2 & 3.7 & 16.0 \\
\hline 40-percent-duration flow & 17.5 & 31.7 & 22.9 & 3.1 & 13.1 \\
\hline 45-percent-duration flow & 14.5 & 28.3 & 20.1 & 2.7 & 10.8 \\
\hline 50-percent-duration flow & 12.1 & 25.5 & 17.6 & 2.3 & 8.7 \\
\hline 55-percent-duration flow & 9.8 & 23.0 & 15.4 & 1.9 & 6.9 \\
\hline 60-percent-duration flow & 7.6 & 20.6 & 13.7 & 1.7 & 5.2 \\
\hline 65-percent-duration flow & 5.8 & 18.6 & 12.1 & 1.5 & 3.8 \\
\hline 70-percent-duration flow & 4.2 & 16.6 & 10.4 & 1.3 & 2.7 \\
\hline 75-percent-duration flow & 3.1 & 14.6 & 8.7 & 1.1 & 2.0 \\
\hline 80-percent-duration flow & 2.2 & 12.5 & 7.3 & 0.9 & 1.6 \\
\hline 85-percent-duration flow & 1.6 & 10.4 & 6.0 & 0.7 & 1.2 \\
\hline 90-percent-duration flow & 1.1 & 7.8 & 4.4 & 0.6 & 0.8 \\
\hline 95-percent-duration flow & 0.6 & 4.5 & 3.0 & 0.4 & 0.5 \\
\hline 99-percent-duration flow & 0.3 & 1.8 & 1.5 & 0.2 & 0.2 \\
\hline Variability index & 0.68 & 0.42 & 0.47 & 0.55 & 0.70 \\
\hline
\end{tabular}


Table 12. Selected annual and seasonal statistics for 40 stations in surrounding states near West Virginia representative of 1930-2002 (values do not supersede those determined for use by the particular state)._-Continued

[Winter, January 1-March 31; spring, April 1-June 30; summer, July 1-September 30; fall, October 1-December 31; streamflow statistics are in cubic feet per second, except for variability index, which is dimensionless; the record period is in climatic years, April 1 through March 31 of the indicated ending year; EPA, U.S. Environmental Protection Agency]

\begin{tabular}{|c|c|c|c|c|c|}
\hline \multirow{2}{*}{ Streamflow statistic } & \multirow{2}{*}{ Annual } & \multicolumn{4}{|c|}{ Season } \\
\hline & & Winter & Spring & Summer & Fall \\
\hline \multicolumn{6}{|c|}{01632000 North Fork Shenandoah River at Cootes Store, VA, 1930-2002 } \\
\hline 1-day 2-year hydrologically based flow & 2.60 & 36.1 & 17.1 & 2.90 & 5.42 \\
\hline 1-day 5-year hydrologically based flow & 1.02 & 15.7 & 9.24 & 1.09 & 2.13 \\
\hline 1-day 10-year hydrologically based flow & 0.61 & 9.23 & 6.40 & 0.64 & 1.31 \\
\hline 3-day 2-year hydrologically based flow & 2.78 & 38.5 & 18.9 & 3.13 & 5.80 \\
\hline 3-day 5 -year hydrologically based flow & 1.11 & 16.6 & 10.3 & 1.19 & 2.39 \\
\hline 3-day 10 -year hydrologically based flow & 0.67 & 9.77 & 7.17 & 0.70 & 1.52 \\
\hline 7-day 2-year hydrologically based flow & 3.05 & 43.6 & 22.5 & 3.51 & 6.90 \\
\hline 7-day 5-year hydrologically based flow & 1.23 & 18.1 & 12.5 & 1.33 & 2.83 \\
\hline 7-day 10-year hydrologically based flow & 0.76 & 10.6 & 8.93 & 0.78 & 1.81 \\
\hline 14-day 2-year hydrologically based flow & 3.48 & 51.3 & 29.8 & 4.28 & 8.09 \\
\hline 14-day 5-year hydrologically based flow & 1.40 & 20.6 & 16.3 & 1.55 & 3.17 \\
\hline 14-day 10-year hydrologically based flow & 0.86 & 12.0 & 11.6 & 0.89 & 2.00 \\
\hline 30-day 2-year hydrologically based flow & 4.46 & 86.5 & 58.5 & 6.53 & 14.4 \\
\hline 30-day 5-year hydrologically based flow & 1.73 & 34.1 & 29.1 & 2.06 & 4.75 \\
\hline 30-day 10-year hydrologically based flow & 1.05 & 19.5 & 19.6 & 1.12 & 2.70 \\
\hline 1-day 3-year biologically based flow & 0.49 & 4.99 & 12.0 & 0.48 & 0.80 \\
\hline 4-day 3-year biologically based flow & 0.56 & 5.81 & 15.4 & 0.51 & 1.08 \\
\hline EPA harmonic-mean flow & 11.0 & 44.8 & 87.3 & 5.24 & 7.32 \\
\hline 5-percent-duration flow & 753 & 1,070 & 893 & 295 & 543 \\
\hline 10-percent-duration flow & 438 & 663 & 523 & 142 & 288 \\
\hline 15-percent-duration flow & 307 & 475 & 384 & 84.5 & 196 \\
\hline 20-percent-duration flow & 230 & 377 & 298 & 56.9 & 144 \\
\hline 25-percent-duration flow & 181 & 317 & 237 & 41.7 & 110 \\
\hline 30-percent-duration flow & 147 & 267 & 193 & 32.1 & 87.0 \\
\hline 35-percent-duration flow & 117 & 232 & 165 & 25.6 & 68.2 \\
\hline 40-percent-duration flow & 96.4 & 201 & 140 & 20.6 & 53.2 \\
\hline 45-percent-duration flow & 77.9 & 177 & 119 & 17.2 & 41.7 \\
\hline 50-percent-duration flow & 62.2 & 154 & 104 & 14.2 & 32.7 \\
\hline 55-percent-duration flow & 49.4 & 134 & 90.2 & 11.8 & 24.8 \\
\hline 60-percent-duration flow & 38.2 & 116 & 78.2 & 9.6 & 18.4 \\
\hline 65-percent-duration flow & 29.0 & 100 & 66.8 & 7.5 & 13.9 \\
\hline 70-percent-duration flow & 21.6 & 84.6 & 57.2 & 5.8 & 10.8 \\
\hline 75-percent-duration flow & 15.9 & 69.0 & 48.2 & 4.5 & 8.5 \\
\hline 80-percent-duration flow & 10.9 & 54.6 & 39.8 & 3.3 & 6.7 \\
\hline 85-percent-duration flow & 7.5 & 41.7 & 32.2 & 2.4 & 5.2 \\
\hline 90-percent-duration flow & 4.7 & 28.5 & 24.7 & 1.6 & 3.9 \\
\hline 95-percent-duration flow & 2.5 & 15.3 & 16.5 & 1.0 & 2.9 \\
\hline 99-percent-duration flow & 0.8 & 6.7 & 7.3 & 0.5 & 1.4 \\
\hline Variability index & 0.76 & 0.53 & 0.52 & 0.74 & 0.72 \\
\hline
\end{tabular}


Table 12. Selected annual and seasonal statistics for 40 stations in surrounding states near West Virginia representative of 1930-2002 (values do not supersede those determined for use by the particular state).-Continued

[Winter, January 1-March 31; spring, April 1-June 30; summer, July 1-September 30; fall, October 1-December 31; streamflow statistics are in cubic feet per second, except for variability index, which is dimensionless; the record period is in climatic years, April 1 through March 31of the indicated ending year; EPA, U.S. Environmental Protection Agency]

\begin{tabular}{|c|c|c|c|c|c|}
\hline \multirow{2}{*}{ Streamflow statistic } & \multirow{2}{*}{ Annual } & \multicolumn{4}{|c|}{ Season } \\
\hline & & Winter & Spring & Summer & Fall \\
\hline \multicolumn{6}{|c|}{01632900 Smith Creek near New Market, VA, 1962-1989 } \\
\hline 1-day 2-year hydrologically based flow & 12.7 & 30.8 & 27.1 & 13.4 & 15.9 \\
\hline 1-day 5-year hydrologically based flow & 8.82 & 18.9 & 18.0 & 9.30 & 10.3 \\
\hline 1-day 10-year hydrologically based flow & 7.36 & 14.4 & 14.4 & 7.74 & 8.38 \\
\hline 3-day 2-year hydrologically based flow & 13.0 & 32.0 & 27.9 & 14.1 & 16.2 \\
\hline 3-day 5-year hydrologically based flow & 9.15 & 19.8 & 18.5 & 9.80 & 10.5 \\
\hline 3-day 10 -year hydrologically based flow & 7.68 & 15.1 & 14.8 & 8.11 & 8.63 \\
\hline 7-day 2-year hydrologically based flow & 13.4 & 35.6 & 29.2 & 14.6 & 16.7 \\
\hline 7-day 5-year hydrologically based flow & 9.43 & 21.7 & 19.4 & 10.1 & 10.6 \\
\hline 7-day 10-year hydrologically based flow & 7.93 & 16.4 & 15.6 & 8.33 & 8.81 \\
\hline 14-day 2-year hydrologically based flow & 14.0 & 39.8 & 31.8 & 15.6 & 17.6 \\
\hline 14-day 5 -year hydrologically based flow & 9.85 & 23.5 & 21.5 & 10.7 & 11.1 \\
\hline 14-day 10-year hydrologically based flow & 8.27 & 17.4 & 17.5 & 8.84 & 9.15 \\
\hline 30-day 2-year hydrologically based flow & 15.5 & 52.1 & 39.3 & 17.6 & 21.1 \\
\hline 30-day 5-year hydrologically based flow & 10.9 & 29.9 & 25.8 & 12.0 & 13.1 \\
\hline 30-day 10-year hydrologically based flow & 9.07 & 21.3 & 20.7 & 9.77 & 10.7 \\
\hline 1-day 3-year biologically based flow & 7.40 & 13.0 & 16.0 & 7.88 & 7.87 \\
\hline 4-day 3-year biologically based flow & 7.82 & 13.5 & 17.2 & 8.17 & 8.10 \\
\hline EPA harmonic-mean flow & 30.8 & 45.2 & 57.1 & 22.5 & 21.9 \\
\hline 5-percent-duration flow & 213 & 310 & 223 & 85.0 & 176 \\
\hline 10-percent-duration flow & 146 & 209 & 158 & 58.9 & 120 \\
\hline 15-percent-duration flow & 114 & 169 & 126 & 48.5 & 89.1 \\
\hline 20-percent-duration flow & 93.3 & 144 & 105 & 41.6 & 72.3 \\
\hline 25-percent-duration flow & 80.3 & 125 & 92.7 & 36.7 & 60.8 \\
\hline 30-percent-duration flow & 70.2 & 110 & 84.8 & 33.7 & 52.7 \\
\hline 35-percent-duration flow & 61.7 & 98.5 & 77.4 & 30.7 & 44.4 \\
\hline 40-percent-duration flow & 54.9 & 88.4 & 70.2 & 27.8 & 37.7 \\
\hline 45-percent-duration flow & 48.5 & 79.8 & 64.5 & 25.4 & 33.0 \\
\hline 50-percent-duration flow & 42.5 & 72.5 & 59.1 & 23.2 & 29.7 \\
\hline 55-percent-duration flow & 37.5 & 65.9 & 54.0 & 21.4 & 27.0 \\
\hline 60-percent-duration flow & 33.1 & 60.6 & 49.1 & 19.6 & 24.5 \\
\hline 65-percent-duration flow & 29.2 & 55.4 & 45.0 & 18.1 & 22.2 \\
\hline 70-percent-duration flow & 25.8 & 50.6 & 40.9 & 16.6 & 19.9 \\
\hline 75-percent-duration flow & 22.6 & 45.8 & 36.5 & 15.2 & 18.0 \\
\hline 80-percent-duration flow & 19.6 & 40.9 & 32.6 & 13.8 & 16.3 \\
\hline 85-percent-duration flow & 16.9 & 34.5 & 28.6 & 12.4 & 14.9 \\
\hline 90-percent-duration flow & 14.5 & 26.8 & 24.5 & 11.2 & 13.2 \\
\hline 95-percent-duration flow & 11.7 & 18.7 & 19.9 & 9.6 & 10.7 \\
\hline 99-percent-duration flow & 8.5 & 10.8 & 14.7 & 8.0 & 8.2 \\
\hline Variability index & 0.39 & 0.35 & 0.31 & 0.29 & 0.37 \\
\hline
\end{tabular}


Table 12. Selected annual and seasonal statistics for 40 stations in surrounding states near West Virginia representative of 1930-2002 (values do not supersede those determined for use by the particular state)._-Continued

[Winter, January 1-March 31; spring, April 1-June 30; summer, July 1-September 30; fall, October 1-December 31; streamflow statistics are in cubic feet per second, except for variability index, which is dimensionless; the record period is in climatic years, April 1 through March 31 of the indicated ending year; EPA, U.S. Environmental Protection Agency]

\begin{tabular}{|c|c|c|c|c|c|}
\hline \multirow{2}{*}{ Streamflow statistic } & \multirow{2}{*}{ Annual } & \multicolumn{4}{|c|}{ Season } \\
\hline & & Winter & Spring & Summer & Fall \\
\hline \multicolumn{6}{|c|}{01634500 Cedar Creek near Winchester, VA, 1939-1996 } \\
\hline 1-day 2-year hydrologically based flow & 7.09 & 30.7 & 17.1 & 7.30 & 9.24 \\
\hline 1-day 5-year hydrologically based flow & 4.87 & 18.1 & 11.0 & 4.97 & 6.67 \\
\hline 1-day 10-year hydrologically based flow & 3.99 & 13.3 & 8.57 & 4.04 & 5.93 \\
\hline 3-day 2-year hydrologically based flow & 7.38 & 33.0 & 18.3 & 7.58 & 9.61 \\
\hline 3-day 5 -year hydrologically based flow & 5.12 & 19.2 & 11.7 & 5.20 & 7.08 \\
\hline 3-day 10 -year hydrologically based flow & 4.22 & 13.9 & 9.13 & 4.25 & 6.36 \\
\hline 7-day 2-year hydrologically based flow & 7.89 & 36.8 & 21.3 & 8.11 & 10.9 \\
\hline 7-day 5-year hydrologically based flow & 5.46 & 20.7 & 13.7 & 5.58 & 7.83 \\
\hline 7-day 10-year hydrologically based flow & 4.49 & 14.8 & 10.7 & 4.59 & 6.92 \\
\hline 14-day 2-year hydrologically based flow & 8.55 & 41.9 & 26.3 & 8.97 & 12.8 \\
\hline 14-day 5-year hydrologically based flow & 5.97 & 23.6 & 16.6 & 6.18 & 8.72 \\
\hline 14-day 10-year hydrologically based flow & 4.96 & 17.0 & 13.0 & 5.09 & 7.46 \\
\hline 30-day 2-year hydrologically based flow & 9.94 & 58.2 & 41.1 & 10.8 & 17.6 \\
\hline 30-day 5-year hydrologically based flow & 6.98 & 33.0 & 24.2 & 7.35 & 10.9 \\
\hline 30-day 10-year hydrologically based flow & 5.85 & 23.9 & 18.2 & 6.05 & 8.94 \\
\hline 1-day 3-year biologically based flow & 3.58 & 12.0 & 13.9 & 3.55 & 5.38 \\
\hline 4-day 3-year biologically based flow & 4.02 & 13.3 & 16.1 & 4.04 & 5.72 \\
\hline EPA harmonic-mean flow & 24.2 & 51.7 & 66.4 & 14.1 & 16.8 \\
\hline 5-percent-duration flow & 342 & 504 & 422 & 94.5 & 232 \\
\hline 10-percent-duration flow & 211 & 312 & 274 & 51.8 & 140 \\
\hline 15-percent-duration flow & 153 & 240 & 201 & 37.9 & 99.3 \\
\hline 20-percent-duration flow & 119 & 196 & 158 & 30.8 & 76.1 \\
\hline 25-percent-duration flow & 98.7 & 165 & 130 & 25.7 & 61.6 \\
\hline 30-percent-duration flow & 82.5 & 140 & 111 & 22.4 & 51.6 \\
\hline 35-percent-duration flow & 69.0 & 124 & 97.3 & 19.9 & 43.4 \\
\hline 40-percent-duration flow & 59.5 & 111 & 85.5 & 18.0 & 36.0 \\
\hline 45-percent-duration flow & 50.4 & 101 & 76.1 & 16.0 & 29.9 \\
\hline 50-percent-duration flow & 41.8 & 90.9 & 67.7 & 14.8 & 25.7 \\
\hline 55-percent-duration flow & 34.8 & 82.0 & 60.5 & 13.5 & 22.4 \\
\hline 60-percent-duration flow & 29.0 & 73.6 & 54.2 & 12.5 & 19.8 \\
\hline 65-percent-duration flow & 24.2 & 65.6 & 48.3 & 11.6 & 17.7 \\
\hline 70-percent-duration flow & 20.2 & 58.6 & 42.8 & 10.6 & 16.0 \\
\hline 75-percent-duration flow & 17.2 & 51.9 & 37.4 & 9.4 & 14.6 \\
\hline 80-percent-duration flow & 14.7 & 43.6 & 32.4 & 8.3 & 13.3 \\
\hline 85-percent-duration flow & 12.6 & 35.0 & 27.1 & 7.5 & 12.0 \\
\hline 90-percent-duration flow & 10.2 & 28.3 & 21.9 & 6.7 & 10.7 \\
\hline 95-percent-duration flow & 7.8 & 21.8 & 16.5 & 5.6 & 8.9 \\
\hline 99-percent-duration flow & 5.2 & 12.0 & 10.3 & 4.0 & 6.4 \\
\hline Variability index & 0.51 & 0.40 & 0.42 & 0.36 & 0.44 \\
\hline
\end{tabular}


Table 12. Selected annual and seasonal statistics for 40 stations in surrounding states near West Virginia representative of 1930-2002 (values do not supersede those determined for use by the particular state).-Continued

[Winter, January 1-March 31; spring, April 1-June 30; summer, July 1-September 30; fall, October 1-December 31; streamflow statistics are in cubic feet per second, except for variability index, which is dimensionless; the record period is in climatic years, April 1 through March 31of the indicated ending year; EPA, U.S. Environmental Protection Agency]

\begin{tabular}{|c|c|c|c|c|c|}
\hline \multirow{2}{*}{ Streamflow statistic } & \multirow{2}{*}{ Annual } & \multicolumn{4}{|c|}{ Season } \\
\hline & & Winter & Spring & Summer & Fall \\
\hline \multicolumn{6}{|c|}{01637000 Little Catoctin Creek at Harmony, MD, 1949-1958 } \\
\hline 1-day 2-year hydrologically based flow & 0.96 & 4.03 & 2.55 & 0.96 & 1.21 \\
\hline 1-day 5-year hydrologically based flow & 0.62 & 2.04 & 1.81 & 0.62 & 0.73 \\
\hline 1-day 10-year hydrologically based flow & 0.48 & 1.36 & 1.50 & 0.48 & 0.54 \\
\hline 3-day 2-year hydrologically based flow & 1.00 & 4.36 & 2.67 & 1.02 & 1.24 \\
\hline 3-day 5-year hydrologically based flow & 0.66 & 2.24 & 1.85 & 0.67 & 0.76 \\
\hline 3-day 10-year hydrologically based flow & 0.52 & 1.50 & 1.51 & 0.53 & 0.58 \\
\hline 7-day 2-year hydrologically based flow & 1.08 & 4.80 & 3.08 & 1.11 & 1.41 \\
\hline 7-day 5-year hydrologically based flow & 0.70 & 2.46 & 2.06 & 0.73 & 0.86 \\
\hline 7-day 10-year hydrologically based flow & 0.55 & 1.66 & 1.64 & 0.56 & 0.64 \\
\hline 14-day 2-year hydrologically based flow & 1.20 & 5.65 & 3.69 & 1.27 & 1.50 \\
\hline 14-day 5-year hydrologically based flow & 0.78 & 2.76 & 2.41 & 0.84 & 0.92 \\
\hline 14-day 10-year hydrologically based flow & 0.60 & 1.80 & 1.90 & 0.66 & 0.71 \\
\hline 30-day 2-year hydrologically based flow & 1.39 & 7.80 & 5.38 & 1.61 & 2.01 \\
\hline 30-day 5-year hydrologically based flow & 0.94 & 4.18 & 3.32 & 1.08 & 1.31 \\
\hline 30-day 10-year hydrologically based flow & 0.75 & 2.84 & 2.57 & 0.85 & 1.05 \\
\hline 1-day 3-year biologically based flow & 0.32 & 1.26 & 2.12 & 0.40 & 0.48 \\
\hline 4-day 3-year biologically based flow & 0.50 & 1.43 & 2.39 & 0.54 & 0.62 \\
\hline EPA harmonic-mean flow & 3.11 & 6.80 & 8.92 & 1.90 & 2.00 \\
\hline 5-percent-duration flow & 30.9 & 38.8 & 31.2 & 14.7 & 23.6 \\
\hline 10-percent-duration flow & 23.3 & 31.3 & 23.9 & 7.9 & 15.6 \\
\hline 15-percent-duration flow & 19.3 & 26.9 & 21.1 & 5.2 & 12.3 \\
\hline 20-percent-duration flow & 16.2 & 24.2 & 18.7 & 3.9 & 9.2 \\
\hline 25-percent-duration flow & 13.7 & 21.9 & 16.6 & 3.2 & 7.5 \\
\hline 30-percent-duration flow & 12.0 & 19.9 & 15.2 & 2.9 & 5.4 \\
\hline 35-percent-duration flow & 10.2 & 17.5 & 13.8 & 2.7 & 4.6 \\
\hline 40-percent-duration flow & 8.4 & 15.6 & 12.6 & 2.5 & 3.9 \\
\hline 45-percent-duration flow & 7.2 & 14.2 & 11.5 & 2.3 & 3.4 \\
\hline 50-percent-duration flow & 5.5 & 13.1 & 10.1 & 2.1 & 3.0 \\
\hline 55-percent-duration flow & 4.4 & 12.3 & 9.1 & 2.0 & 2.6 \\
\hline 60-percent-duration flow & 3.5 & 11.2 & 8.4 & 1.8 & 2.4 \\
\hline 65-percent-duration flow & 2.9 & 9.8 & 7.3 & 1.7 & 2.1 \\
\hline 70-percent-duration flow & 2.5 & 8.7 & 6.1 & 1.5 & 2.0 \\
\hline 75-percent-duration flow & 2.2 & 7.9 & 5.2 & 1.4 & 1.8 \\
\hline 80-percent-duration flow & 1.9 & 6.4 & 4.3 & 1.2 & 1.6 \\
\hline 85-percent-duration flow & 1.6 & 5.2 & 3.6 & 1.1 & 1.3 \\
\hline 90-percent-duration flow & 1.3 & 3.2 & 2.9 & 0.9 & 1.1 \\
\hline 95-percent-duration flow & 1.0 & 2.2 & 2.5 & 0.7 & 1.0 \\
\hline 99-percent-duration flow & 0.6 & 1.5 & 1.6 & 0.6 & 0.6 \\
\hline Variability index & 0.49 & 0.36 & 0.35 & 0.37 & 0.44 \\
\hline
\end{tabular}


Table 12. Selected annual and seasonal statistics for 40 stations in surrounding states near West Virginia representative of 1930-2002 (values do not supersede those determined for use by the particular state).-Continued

[Winter, January 1-March 31; spring, April 1-June 30; summer, July 1-September 30; fall, October 1-December 31; streamflow statistics are in cubic feet per second, except for variability index, which is dimensionless; the record period is in climatic years, April 1 through March 31 of the indicated ending year; EPA, U.S. Environmental Protection Agency]

\begin{tabular}{|c|c|c|c|c|c|}
\hline \multirow{2}{*}{ Streamflow statistic } & \multirow{2}{*}{ Annual } & \multicolumn{4}{|c|}{ Season } \\
\hline & & Winter & Spring & Summer & Fall \\
\hline \multicolumn{6}{|c|}{01637500 Catoctin Creek near Middletown, MD, 1949-1996 } \\
\hline 1-day 2-year hydrologically based flow & 3.10 & 28.4 & 16.0 & 3.16 & 5.00 \\
\hline 1-day 5-year hydrologically based flow & 1.22 & 14.5 & 9.22 & 1.24 & 2.74 \\
\hline 1-day 10-year hydrologically based flow & 0.64 & 9.24 & 6.72 & 0.65 & 2.09 \\
\hline 3-day 2-year hydrologically based flow & 3.26 & 30.5 & 17.1 & 3.32 & 5.23 \\
\hline 3-day 5-year hydrologically based flow & 1.35 & 15.6 & 10.0 & 1.37 & 2.96 \\
\hline 3-day 10-year hydrologically based flow & 0.75 & 10.1 & 7.36 & 0.76 & 2.31 \\
\hline 7-day 2-year hydrologically based flow & 3.64 & 33.7 & 20.3 & 3.73 & 6.02 \\
\hline 7-day 5-year hydrologically based flow & 1.64 & 17.4 & 12.0 & 1.66 & 3.52 \\
\hline 7-day 10-year hydrologically based flow & 0.98 & 11.3 & 8.93 & 0.99 & 2.80 \\
\hline 14-day 2-year hydrologically based flow & 4.42 & 40.4 & 24.8 & 4.65 & 7.70 \\
\hline 14-day 5-year hydrologically based flow & 2.06 & 20.5 & 15.6 & 2.12 & 4.28 \\
\hline 14-day 10-year hydrologically based flow & 1.26 & 13.1 & 12.0 & 1.27 & 3.29 \\
\hline 30-day 2-year hydrologically based flow & 6.04 & 57.7 & 35.7 & 7.07 & 12.7 \\
\hline 30-day 5-year hydrologically based flow & 2.90 & 29.4 & 22.5 & 3.26 & 6.48 \\
\hline 30-day 10-year hydrologically based flow & 1.87 & 18.5 & 17.7 & 2.03 & 4.61 \\
\hline 1-day 3-year biologically based flow & 0.58 & 7.40 & 14.0 & 0.63 & 0.88 \\
\hline 4-day 3-year biologically based flow & 0.72 & 9.18 & 16.4 & 0.88 & 1.45 \\
\hline EPA harmonic-mean flow & 13.6 & 39.9 & 63.0 & 7.32 & 8.67 \\
\hline 5-percent-duration flow & 268 & 380 & 295 & 72.3 & 196 \\
\hline 10-percent-duration flow & 176 & 258 & 205 & 44.5 & 125 \\
\hline 15-percent-duration flow & 136 & 206 & 162 & 33.1 & 95.1 \\
\hline 20-percent-duration flow & 110 & 172 & 134 & 26.4 & 74.4 \\
\hline 25-percent-duration flow & 89.1 & 151 & 113 & 22.4 & 59.6 \\
\hline 30-percent-duration flow & 76.7 & 133 & 97.6 & 18.7 & 47.6 \\
\hline 35-percent-duration flow & 64.4 & 118 & 86.7 & 16.7 & 39.0 \\
\hline 40-percent-duration flow & 55.0 & 105 & 77.5 & 14.7 & 32.2 \\
\hline 45-percent-duration flow & 46.0 & 94.1 & 69.5 & 12.9 & 27.0 \\
\hline 50-percent-duration flow & 39.0 & 85.2 & 62.9 & 11.3 & 22.8 \\
\hline 55-percent-duration flow & 32.3 & 76.7 & 56.5 & 9.8 & 19.1 \\
\hline 60-percent-duration flow & 26.6 & 69.2 & 51.0 & 8.7 & 16.3 \\
\hline 65-percent-duration flow & 21.6 & 62.2 & 45.5 & 7.6 & 13.8 \\
\hline 70-percent-duration flow & 17.3 & 55.0 & 40.7 & 6.6 & 11.7 \\
\hline 75-percent-duration flow & 13.9 & 47.9 & 35.7 & 5.6 & 9.5 \\
\hline 80-percent-duration flow & 10.7 & 41.2 & 30.6 & 4.7 & 8.0 \\
\hline 85-percent-duration flow & 8.0 & 34.9 & 25.8 & 3.9 & 6.6 \\
\hline 90-percent-duration flow & 5.8 & 27.9 & 21.2 & 2.9 & 5.1 \\
\hline 95-percent-duration flow & 3.7 & 18.2 & 15.6 & 1.7 & 3.7 \\
\hline 99-percent-duration flow & 1.4 & 5.2 & 8.2 & 0.7 & 2.5 \\
\hline Variability index & 0.57 & 0.39 & 0.38 & 0.47 & 0.54 \\
\hline
\end{tabular}


Table 12. Selected annual and seasonal statistics for 40 stations in surrounding states near West Virginia representative of 1930-2002 (values do not supersede those determined for use by the particular state).-Continued

[Winter, January 1-March 31; spring, April 1-June 30; summer, July 1-September 30; fall, October 1-December 31; streamflow statistics are in cubic feet per second, except for variability index, which is dimensionless; the record period is in climatic years, April 1 through March 31of the indicated ending year; EPA, U.S. Environmental Protection Agency]

\begin{tabular}{|c|c|c|c|c|c|}
\hline \multirow{2}{*}{ Streamflow statistic } & \multirow{2}{*}{ Annual } & \multicolumn{4}{|c|}{ Season } \\
\hline & & Winter & Spring & Summer & Fall \\
\hline \multicolumn{6}{|c|}{01638480 Catoctin Creek at Taylorstown, VA, 1977-1989 } \\
\hline 1-day 2-year hydrologically based flow & 4.56 & 31.4 & 25.1 & 4.91 & 5.95 \\
\hline 1-day 5-year hydrologically based flow & 1.67 & 17.3 & 11.6 & 1.75 & 1.96 \\
\hline 1-day 10-year hydrologically based flow & 0.83 & 11.5 & 6.71 & 0.85 & 1.05 \\
\hline 3-day 2-year hydrologically based flow & 4.87 & 32.2 & 26.7 & 5.32 & 6.16 \\
\hline 3-day 5-year hydrologically based flow & 1.88 & 18.0 & 12.6 & 2.00 & 2.20 \\
\hline 3-day 10-year hydrologically based flow & 0.98 & 12.2 & 7.29 & 1.01 & 1.27 \\
\hline 7-day 2-year hydrologically based flow & 5.50 & 34.5 & 30.5 & 6.07 & 6.92 \\
\hline 7-day 5-year hydrologically based flow & 2.10 & 19.4 & 14.7 & 2.26 & 2.48 \\
\hline 7-day 10-year hydrologically based flow & 1.08 & 13.2 & 8.54 & 1.13 & 1.43 \\
\hline 14-day 2-year hydrologically based flow & 6.27 & 40.5 & 35.7 & 7.08 & 9.62 \\
\hline 14-day 5-year hydrologically based flow & 2.42 & 22.0 & 17.1 & 2.68 & 3.48 \\
\hline 14-day 10-year hydrologically based flow & 1.30 & 14.6 & 10.1 & 1.39 & 1.95 \\
\hline 30-day 2-year hydrologically based flow & 8.32 & 53.2 & 47.1 & 9.50 & 17.7 \\
\hline 30-day 5-year hydrologically based flow & 3.43 & 28.7 & 26.0 & 4.46 & 7.60 \\
\hline 30-day 10-year hydrologically based flow & 1.88 & 19.7 & 17.3 & 2.75 & 4.64 \\
\hline 1-day 3-year biologically based flow & 0.25 & 5.71 & 12.0 & 1.18 & 0.25 \\
\hline 4-day 3-year biologically based flow & 0.45 & 6.54 & 15.1 & 1.51 & 0.43 \\
\hline EPA harmonic-mean flow & 14.5 & 43.8 & 65.2 & 11.2 & 6.77 \\
\hline 5-percent-duration flow & 317 & 425 & 404 & 104 & 262 \\
\hline 10-percent-duration flow & 197 & 264 & 253 & 61.6 & 158 \\
\hline 15-percent-duration flow & 148 & 203 & 195 & 43.2 & 117 \\
\hline 20-percent-duration flow & 117 & 168 & 158 & 34.3 & 91.5 \\
\hline 25-percent-duration flow & 95.0 & 145 & 129 & 28.5 & 74.6 \\
\hline 30-percent-duration flow & 80.2 & 125 & 108 & 25.3 & 62.2 \\
\hline 35-percent-duration flow & 69.1 & 109 & 91.7 & 22.1 & 53.1 \\
\hline 40-percent-duration flow & 60.0 & 95.2 & 80.2 & 19.9 & 45.2 \\
\hline 45-percent-duration flow & 51.8 & 87.8 & 71.6 & 17.6 & 37.8 \\
\hline 50-percent-duration flow & 44.8 & 80.3 & 64.6 & 15.5 & 28.9 \\
\hline 55-percent-duration flow & 38.0 & 73.9 & 58.4 & 13.6 & 22.8 \\
\hline 60-percent-duration flow & 31.5 & 67.8 & 52.7 & 11.7 & 19.8 \\
\hline 65-percent-duration flow & 26.5 & 61.2 & 47.7 & 9.8 & 17.0 \\
\hline 70-percent-duration flow & 21.7 & 54.4 & 43.5 & 8.5 & 14.3 \\
\hline 75-percent-duration flow & 17.3 & 47.7 & 39.2 & 7.6 & 11.7 \\
\hline 80-percent-duration flow & 13.9 & 41.4 & 34.7 & 6.5 & 9.8 \\
\hline 85-percent-duration flow & 9.7 & 35.3 & 29.8 & 4.9 & 8.2 \\
\hline 90-percent-duration flow & 7.6 & 29.8 & 24.3 & 3.7 & 6.7 \\
\hline 95-percent-duration flow & 4.6 & 21.8 & 18.0 & 2.2 & 4.4 \\
\hline 99-percent-duration flow & 1.4 & 8.2 & 4.1 & 0.5 & 0.9 \\
\hline Variability index & 0.55 & 0.38 & 0.40 & 0.48 & 0.55 \\
\hline
\end{tabular}


Table 12. Selected annual and seasonal statistics for 40 stations in surrounding states near West Virginia representative of 1930-2002 (values do not supersede those determined for use by the particular state).-Continued

[Winter, January 1-March 31; spring, April 1-June 30; summer, July 1-September 30; fall, October 1-December 31; streamflow statistics are in cubic feet per second, except for variability index, which is dimensionless; the record period is in climatic years, April 1 through March 31 of the indicated ending year; EPA, U.S. Environmental Protection Agency]

\begin{tabular}{|c|c|c|c|c|c|}
\hline \multirow{2}{*}{ Streamflow statistic } & \multirow{2}{*}{ Annual } & \multicolumn{4}{|c|}{ Season } \\
\hline & & Winter & Spring & Summer & Fall \\
\hline \multicolumn{6}{|c|}{01643700 Goose Creek near Middleburg, VA, 1977-1989 } \\
\hline 1-day 2-year hydrologically based flow & 1.49 & 37.8 & 21.0 & 1.69 & 4.44 \\
\hline 1-day 5-year hydrologically based flow & 0.30 & 16.9 & 7.26 & 0.33 & 0.25 \\
\hline 1-day 10-year hydrologically based flow & 0.00 & 9.90 & 3.77 & 0.00 & 0.01 \\
\hline 3-day 2-year hydrologically based flow & 1.67 & 39.8 & 23.2 & 2.24 & 4.82 \\
\hline 3-day 5-year hydrologically based flow & 0.33 & 18.0 & 8.13 & 0.14 & 0.30 \\
\hline 3-day 10-year hydrologically based flow & 0.00 & 10.6 & 4.22 & 0.00 & 0.02 \\
\hline 7-day 2-year hydrologically based flow & 1.92 & 42.2 & 27.6 & 2.47 & 4.57 \\
\hline 7-day 5-year hydrologically based flow & 0.14 & 19.3 & 10.6 & 0.24 & 0.45 \\
\hline 7-day 10-year hydrologically based flow & 0.02 & 11.5 & 5.97 & 0.04 & 0.10 \\
\hline 14-day 2-year hydrologically based flow & 2.57 & 49.6 & 35.2 & 2.78 & 6.75 \\
\hline 14-day 5-year hydrologically based flow & 0.34 & 23.3 & 14.9 & 0.43 & 0.72 \\
\hline 14-day 10-year hydrologically based flow & 0.09 & 14.2 & 9.05 & 0.13 & 0.17 \\
\hline 30-day 2-year hydrologically based flow & 3.89 & 70.4 & 50.3 & 4.78 & 24.5 \\
\hline 30-day 5-year hydrologically based flow & 0.57 & 33.7 & 25.9 & 1.19 & 4.66 \\
\hline 30-day 10-year hydrologically based flow & 0.16 & 21.2 & 18.0 & 0.50 & 1.42 \\
\hline 1-day 3-year biologically based flow & 0.00 & 4.61 & 15.0 & 0.27 & 0.00 \\
\hline 4-day 3-year biologically based flow & 0.00 & 6.04 & 17.7 & 0.31 & 0.00 \\
\hline EPA harmonic-mean flow & 1.83 & 47.1 & 84.2 & 3.99 & 0.52 \\
\hline 5-percent-duration flow & 470 & 550 & 612 & 131 & 389 \\
\hline 10-percent-duration flow & 302 & 389 & 392 & 73.1 & 248 \\
\hline 15-percent-duration flow & 226 & 315 & 290 & 46.4 & 186 \\
\hline 20-percent-duration flow & 174 & 258 & 233 & 34.3 & 145 \\
\hline 25-percent-duration flow & 144 & 221 & 192 & 28.1 & 117 \\
\hline 30-percent-duration flow & 117 & 189 & 161 & 22.7 & 91.4 \\
\hline 35-percent-duration flow & 99.2 & 163 & 138 & 18.6 & 69.0 \\
\hline 40-percent-duration flow & 81.6 & 141 & 121 & 15.5 & 51.7 \\
\hline 45-percent-duration flow & 66.8 & 129 & 107 & 12.9 & 39.3 \\
\hline 50-percent-duration flow & 53.4 & 116 & 94.4 & 10.9 & 30.0 \\
\hline 55-percent-duration flow & 43.4 & 103 & 83.9 & 9.2 & 23.6 \\
\hline 60-percent-duration flow & 34.4 & 91.0 & 74.0 & 7.5 & 18.6 \\
\hline 65-percent-duration flow & 26.4 & 78.7 & 63.5 & 5.8 & 15.3 \\
\hline 70-percent-duration flow & 19.9 & 68.9 & 53.9 & 4.6 & 13.5 \\
\hline 75-percent-duration flow & 15.0 & 59.1 & 45.4 & 3.4 & 11.8 \\
\hline 80-percent-duration flow & 11.2 & 50.0 & 39.0 & 2.4 & 9.3 \\
\hline 85-percent-duration flow & 7.5 & 42.8 & 31.4 & 1.7 & 6.7 \\
\hline 90-percent-duration flow & 4.4 & 32.3 & 24.4 & 0.9 & 4.7 \\
\hline 95-percent-duration flow & 1.6 & 23.7 & 17.0 & 0.4 & 1.0 \\
\hline 99-percent-duration flow & 0.1 & 6.6 & 5.0 & 0.0 & 0.0 \\
\hline Variability index & 0.74 & 0.41 & 0.46 & 0.73 & 0.76 \\
\hline
\end{tabular}


Table 12. Selected annual and seasonal statistics for 40 stations in surrounding states near West Virginia representative of 1930-2002 (values do not supersede those determined for use by the particular state).-Continued

[Winter, January 1-March 31; spring, April 1-June 30; summer, July 1-September 30; fall, October 1-December 31; streamflow statistics are in cubic feet per second, except for variability index, which is dimensionless; the record period is in climatic years, April 1 through March 31of the indicated ending year; EPA, U.S. Environmental Protection Agency]

\begin{tabular}{|c|c|c|c|c|c|}
\hline \multirow{2}{*}{ Streamflow statistic } & \multirow{2}{*}{ Annual } & \multicolumn{4}{|c|}{ Season } \\
\hline & & Winter & Spring & Summer & Fall \\
\hline \multicolumn{6}{|c|}{01644000 Goose Creek near Leesburg, VA, 1931-2002 } \\
\hline 1-day 2-year hydrologically based flow & 8.42 & 110 & 61.2 & 8.73 & 20.3 \\
\hline 1-day 5-year hydrologically based flow & 2.70 & 50.1 & 30.3 & 2.76 & 6.13 \\
\hline 1-day 10-year hydrologically based flow & 1.42 & 30.1 & 20.2 & 1.44 & 3.11 \\
\hline 3-day 2-year hydrologically based flow & 9.02 & 122 & 66.7 & 9.47 & 21.5 \\
\hline 3-day 5-year hydrologically based flow & 2.88 & 57.0 & 33.9 & 2.96 & 6.53 \\
\hline 3-day 10 -year hydrologically based flow & 1.51 & 35.1 & 23.0 & 1.53 & 3.30 \\
\hline 7-day 2-year hydrologically based flow & 10.4 & 134 & 80.7 & 11.1 & 25.1 \\
\hline 7-day 5-year hydrologically based flow & 3.33 & 63.9 & 44.4 & 3.48 & 7.78 \\
\hline 7-day 10-year hydrologically based flow & 1.77 & 39.9 & 31.7 & 1.81 & 4.00 \\
\hline 14-day 2-year hydrologically based flow & 13.0 & 158 & 103 & 14.3 & 32.0 \\
\hline 14-day 5-year hydrologically based flow & 4.22 & 74.7 & 57.9 & 4.62 & 9.84 \\
\hline 14-day 10-year hydrologically based flow & 2.27 & 47.7 & 41.5 & 2.48 & 5.01 \\
\hline 30-day 2-year hydrologically based flow & 20.0 & 222 & 145 & 24.4 & 52.9 \\
\hline 30-day 5-year hydrologically based flow & 6.71 & 108 & 83.6 & 8.47 & 18.5 \\
\hline 30-day 10-year hydrologically based flow & 3.65 & 68.9 & 61.3 & 4.69 & 10.1 \\
\hline 1-day 3-year biologically based flow & 1.09 & 13.0 & 43.0 & 1.28 & 1.47 \\
\hline 4-day 3-year biologically based flow & 1.22 & 17.3 & 52.3 & 1.69 & 1.71 \\
\hline EPA harmonic-mean flow & 32.5 & 142 & 215 & 18.4 & 17.7 \\
\hline 5-percent-duration flow & 1,100 & 1,520 & 1,130 & 495 & 887 \\
\hline 10-percent-duration flow & 716 & 1,030 & 751 & 255 & 539 \\
\hline 15-percent-duration flow & 546 & 812 & 585 & 176 & 399 \\
\hline 20-percent-duration flow & 435 & 684 & 482 & 136 & 310 \\
\hline 25-percent-duration flow & 363 & 589 & 406 & 111 & 248 \\
\hline 30-percent-duration flow & 306 & 519 & 361 & 90.8 & 199 \\
\hline 35-percent-duration flow & 261 & 460 & 319 & 76.3 & 162 \\
\hline 40-percent-duration flow & 221 & 414 & 287 & 64.2 & 133 \\
\hline 45-percent-duration flow & 187 & 371 & 258 & 54.1 & 108 \\
\hline 50-percent-duration flow & 157 & 334 & 232 & 45.8 & 90.4 \\
\hline 55-percent-duration flow & 130 & 299 & 208 & 38.3 & 76.4 \\
\hline 60-percent-duration flow & 107 & 270 & 186 & 31.8 & 64.1 \\
\hline 65-percent-duration flow & 85.9 & 241 & 167 & 25.9 & 54.7 \\
\hline 70-percent-duration flow & 69.1 & 215 & 148 & 20.9 & 45.9 \\
\hline 75-percent-duration flow & 54.2 & 188 & 132 & 16.6 & 38.1 \\
\hline 80-percent-duration flow & 41.0 & 157 & 115 & 12.8 & 29.6 \\
\hline 85-percent-duration flow & 28.7 & 122 & 96.6 & 9.2 & 20.7 \\
\hline 90-percent-duration flow & 17.4 & 89.4 & 75.9 & 6.1 & 13.8 \\
\hline 95-percent-duration flow & 8.3 & 55.6 & 54.7 & 3.1 & 7.3 \\
\hline 99-percent-duration flow & 2.1 & 21.6 & 24.4 & 1.3 & 2.1 \\
\hline Variability index & 0.63 & 0.41 & 0.38 & 0.64 & 0.62 \\
\hline
\end{tabular}


Table 12. Selected annual and seasonal statistics for 40 stations in surrounding states near West Virginia representative of 1930-2002 (values do not supersede those determined for use by the particular state).-Continued

[Winter, January 1-March 31; spring, April 1-June 30; summer, July 1-September 30; fall, October 1-December 31; streamflow statistics are in cubic feet per second, except for variability index, which is dimensionless; the record period is in climatic years, April 1 through March 31 of the indicated ending year; EPA, U.S. Environmental Protection Agency]

\begin{tabular}{|c|c|c|c|c|c|}
\hline \multirow{2}{*}{ Streamflow statistic } & \multirow{2}{*}{ Annual } & \multicolumn{4}{|c|}{ Season } \\
\hline & & Winter & Spring & Summer & Fall \\
\hline \multicolumn{6}{|c|}{03072000 Dunkard Creek at Shannopin, PA, 1942-1980 } \\
\hline 1-day 2-year hydrologically based flow & 3.16 & 59.8 & 14.5 & 3.55 & 6.13 \\
\hline 1-day 5-year hydrologically based flow & 1.48 & 28.7 & 7.14 & 1.67 & 2.30 \\
\hline 1-day 10-year hydrologically based flow & 1.02 & 18.4 & 4.76 & 1.16 & 1.39 \\
\hline 3-day 2-year hydrologically based flow & 3.49 & 64.7 & 16.1 & 3.96 & 6.97 \\
\hline 3-day 5-year hydrologically based flow & 1.64 & 30.6 & 7.98 & 1.88 & 2.61 \\
\hline 3-day 10-year hydrologically based flow & 1.13 & 19.5 & 5.36 & 1.31 & 1.57 \\
\hline 7-day 2-year hydrologically based flow & 4.09 & 76.3 & 20.0 & 4.54 & 8.31 \\
\hline 7-day 5-year hydrologically based flow & 1.94 & 36.7 & 10.0 & 2.16 & 3.10 \\
\hline 7-day 10-year hydrologically based flow & 1.34 & 23.9 & 6.86 & 1.51 & 1.85 \\
\hline 14-day 2-year hydrologically based flow & 4.84 & 101 & 30.0 & 5.61 & 10.3 \\
\hline 14-day 5-year hydrologically based flow & 2.33 & 44.5 & 14.0 & 2.64 & 3.87 \\
\hline 14-day 10-year hydrologically based flow & 1.63 & 27.5 & 9.14 & 1.87 & 2.31 \\
\hline 30-day 2-year hydrologically based flow & 7.48 & 195 & 58.4 & 10.0 & 19.5 \\
\hline 30-day 5-year hydrologically based flow & 3.25 & 88.7 & 27.3 & 4.58 & 6.08 \\
\hline 30-day 10-year hydrologically based flow & 2.11 & 54.7 & 18.2 & 3.11 & 3.29 \\
\hline 1-day 3-year biologically based flow & 1.30 & 10.0 & 11.0 & 1.60 & 1.30 \\
\hline 4-day 3-year biologically based flow & 1.39 & 12.2 & 13.7 & 1.78 & 1.47 \\
\hline EPA harmonic-mean flow & 18.1 & 106 & 96.8 & 10.1 & 9.80 \\
\hline 5-percent-duration flow & 1,190 & 1,850 & 1,270 & 300 & 799 \\
\hline 10-percent-duration flow & 693 & 1,220 & 748 & 162 & 436 \\
\hline 15-percent-duration flow & 486 & 912 & 529 & 106 & 306 \\
\hline 20-percent-duration flow & 366 & 727 & 406 & 74.6 & 227 \\
\hline 25-percent-duration flow & 284 & 602 & 323 & 55.1 & 175 \\
\hline 30-percent-duration flow & 226 & 505 & 259 & 42.0 & 138 \\
\hline 35-percent-duration flow & 183 & 433 & 218 & 33.0 & 110 \\
\hline 40-percent-duration flow & 147 & 378 & 183 & 26.8 & 87.1 \\
\hline 45-percent-duration flow & 117 & 330 & 154 & 21.2 & 68.4 \\
\hline 50-percent-duration flow & 92.9 & 291 & 130 & 17.7 & 54.4 \\
\hline 55-percent-duration flow & 72.6 & 255 & 110 & 14.6 & 43.9 \\
\hline 60-percent-duration flow & 55.9 & 223 & 93.3 & 11.8 & 35.0 \\
\hline 65-percent-duration flow & 42.0 & 195 & 79.5 & 9.4 & 27.3 \\
\hline 70-percent-duration flow & 31.2 & 169 & 67.4 & 8.0 & 20.9 \\
\hline 75-percent-duration flow & 22.6 & 143 & 56.3 & 6.7 & 15.7 \\
\hline 80-percent-duration flow & 15.9 & 118 & 44.1 & 5.4 & 11.2 \\
\hline 85-percent-duration flow & 10.2 & 91.7 & 33.3 & 4.4 & 6.7 \\
\hline 90-percent-duration flow & 6.3 & 63.3 & 23.4 & 3.3 & 4.3 \\
\hline 95-percent-duration flow & 3.6 & 35.4 & 14.2 & 2.3 & 2.5 \\
\hline 99-percent-duration flow & 1.8 & 16.5 & 6.0 & 1.5 & 1.6 \\
\hline Variability index & 0.78 & 0.50 & 0.58 & 0.65 & 0.77 \\
\hline
\end{tabular}


Table 12. Selected annual and seasonal statistics for 40 stations in surrounding states near West Virginia representative of 1930-2002 (values do not supersede those determined for use by the particular state)._-Continued

[Winter, January 1-March 31; spring, April 1-June 30; summer, July 1-September 30; fall, October 1-December 31; streamflow statistics are in cubic feet per second, except for variability index, which is dimensionless; the record period is in climatic years, April 1 through March 31 of the indicated ending year; EPA, U.S. Environmental Protection Agency]

\begin{tabular}{|c|c|c|c|c|c|}
\hline \multirow{2}{*}{ Streamflow statistic } & \multirow{2}{*}{ Annual } & \multicolumn{4}{|c|}{ Season } \\
\hline & & Winter & Spring & Summer & Fall \\
\hline \multicolumn{6}{|c|}{03072590 Georges Creek at Smithfield, PA, 1965-1974 } \\
\hline 1-day 2-year hydrologically based flow & 0.29 & 5.24 & 1.14 & 0.29 & 0.53 \\
\hline 1-day 5-year hydrologically based flow & 0.00 & 2.81 & 0.54 & 0.00 & 0.25 \\
\hline 1-day 10-year hydrologically based flow & 0.00 & 1.95 & 0.36 & 0.00 & 0.17 \\
\hline 3-day 2-year hydrologically based flow & 0.27 & 5.50 & 1.34 & 0.28 & 0.59 \\
\hline 3-day 5-year hydrologically based flow & 0.11 & 3.08 & 0.62 & 0.12 & 0.32 \\
\hline 3-day 10 -year hydrologically based flow & 0.07 & 2.21 & 0.41 & 0.07 & 0.24 \\
\hline 7-day 2-year hydrologically based flow & 0.32 & 6.16 & 1.85 & 0.33 & 0.70 \\
\hline 7-day 5-year hydrologically based flow & 0.15 & 3.44 & 0.92 & 0.16 & 0.39 \\
\hline 7-day 10-year hydrologically based flow & 0.10 & 2.48 & 0.64 & 0.11 & 0.29 \\
\hline 14-day 2-year hydrologically based flow & 0.44 & 7.40 & 2.54 & 0.46 & 0.86 \\
\hline 14-day 5-year hydrologically based flow & 0.20 & 3.93 & 1.17 & 0.21 & 0.45 \\
\hline 14-day 10 -year hydrologically based flow & 0.14 & 2.77 & 0.79 & 0.14 & 0.32 \\
\hline 30-day 2-year hydrologically based flow & 0.65 & 10.9 & 4.82 & 0.67 & 1.23 \\
\hline 30-day 5-year hydrologically based flow & 0.33 & 6.00 & 2.57 & 0.34 & 0.54 \\
\hline 30-day 10-year hydrologically based flow & 0.24 & 4.30 & 1.84 & 0.24 & 0.36 \\
\hline 1-day 3-year biologically based flow & 0.10 & 1.73 & 1.28 & 0.10 & 0.10 \\
\hline 4-day 3-year biologically based flow & 0.08 & 2.08 & 1.99 & 0.09 & 0.14 \\
\hline EPA harmonic-mean flow & 1.46 & 10.7 & 10.8 & 0.68 & 0.93 \\
\hline 5-percent-duration flow & 65.8 & 90.9 & 81.5 & 15.0 & 49.0 \\
\hline 10-percent-duration flow & 42.4 & 59.4 & 53.9 & 7.9 & 29.1 \\
\hline 15-percent-duration flow & 32.1 & 45.8 & 43.0 & 5.4 & 21.0 \\
\hline 20-percent-duration flow & 25.4 & 38.8 & 34.3 & 4.3 & 16.1 \\
\hline 25-percent-duration flow & 20.4 & 33.8 & 27.9 & 3.3 & 12.4 \\
\hline 30-percent-duration flow & 16.8 & 30.0 & 23.8 & 2.7 & 9.8 \\
\hline 35-percent-duration flow & 14.0 & 26.8 & 20.3 & 2.1 & 7.9 \\
\hline 40-percent-duration flow & 11.4 & 24.0 & 17.5 & 1.8 & 6.6 \\
\hline 45-percent-duration flow & 9.0 & 21.4 & 15.3 & 1.5 & 5.6 \\
\hline 50-percent-duration flow & 7.3 & 18.9 & 13.3 & 1.2 & 4.7 \\
\hline 55-percent-duration flow & 5.8 & 17.1 & 11.6 & 1.0 & 4.0 \\
\hline 60-percent-duration flow & 4.7 & 15.1 & 9.6 & 0.8 & 3.2 \\
\hline 65-percent-duration flow & 3.7 & 13.4 & 8.1 & 0.7 & 2.4 \\
\hline 70-percent-duration flow & 2.6 & 12.1 & 6.9 & 0.5 & 1.8 \\
\hline 75-percent-duration flow & 1.8 & 10.7 & 5.8 & 0.5 & 1.2 \\
\hline 80-percent-duration flow & 1.2 & 9.1 & 4.7 & 0.4 & 0.9 \\
\hline 85-percent-duration flow & 0.8 & 7.1 & 3.4 & 0.3 & 0.6 \\
\hline 90-percent-duration flow & 0.5 & 5.3 & 2.3 & 0.2 & 0.5 \\
\hline 95-percent-duration flow & 0.3 & 3.9 & 1.3 & 0.1 & 0.4 \\
\hline 99-percent-duration flow & 0.1 & 2.3 & 0.5 & 0.1 & 0.2 \\
\hline Variability index & 0.73 & 0.40 & 0.53 & 0.63 & 0.67 \\
\hline
\end{tabular}


Table 12. Selected annual and seasonal statistics for 40 stations in surrounding states near West Virginia representative of 1930-2002 (values do not supersede those determined for use by the particular state).-Continued

[Winter, January 1-March 31; spring, April 1-June 30; summer, July 1-September 30; fall, October 1-December 31; streamflow statistics are in cubic feet per second, except for variability index, which is dimensionless; the record period is in climatic years, April 1 through March 31 of the indicated ending year; EPA, U.S. Environmental Protection Agency]

\begin{tabular}{|c|c|c|c|c|c|}
\hline \multirow{2}{*}{ Streamflow statistic } & \multirow{2}{*}{ Annual } & \multicolumn{4}{|c|}{ Season } \\
\hline & & Winter & Spring & Summer & Fall \\
\hline \multicolumn{6}{|c|}{03075500 Youghiogheny River near Oakland, MD, 1944-2002 } \\
\hline 1-day 2-year hydrologically based flow & 13.4 & 88.6 & 38.6 & 14.3 & 23.0 \\
\hline 1-day 5-year hydrologically based flow & 7.32 & 61.0 & 23.2 & 7.78 & 12.1 \\
\hline 1-day 10-year hydrologically based flow & 5.18 & 50.1 & 17.8 & 5.49 & 8.58 \\
\hline 3-day 2-year hydrologically based flow & 14.3 & 92.8 & 42.6 & 15.3 & 24.3 \\
\hline 3-day 5-year hydrologically based flow & 7.80 & 63.2 & 25.1 & 8.33 & 12.7 \\
\hline 3-day 10-year hydrologically based flow & 5.53 & 51.8 & 19.0 & 5.90 & 9.01 \\
\hline 7-day 2-year hydrologically based flow & 16.0 & 104 & 52.7 & 17.8 & 28.5 \\
\hline 7-day 5-year hydrologically based flow & 8.67 & 67.7 & 30.6 & 9.43 & 14.8 \\
\hline 7-day 10-year hydrologically based flow & 6.11 & 54.7 & 22.7 & 6.58 & 10.4 \\
\hline 14-day 2-year hydrologically based flow & 18.4 & 134 & 68.5 & 21.6 & 34.7 \\
\hline 14-day 5-year hydrologically based flow & 9.83 & 82.5 & 39.0 & 11.0 & 17.4 \\
\hline 14-day 10-year hydrologically based flow & 6.94 & 64.0 & 28.7 & 7.55 & 12.1 \\
\hline 30-day 2-year hydrologically based flow & 25.0 & 221 & 113 & 32.0 & 60.3 \\
\hline 30-day 5-year hydrologically based flow & 13.5 & 133 & 62.7 & 15.9 & 27.7 \\
\hline 30-day 10-year hydrologically based flow & 9.67 & 101 & 45.1 & 10.9 & 17.7 \\
\hline 1-day 3-year biologically based flow & 3.78 & 41.0 & 29.9 & 4.38 & 5.39 \\
\hline 4-day 3-year biologically based flow & 4.74 & 50.0 & 37.0 & 5.52 & 6.82 \\
\hline EPA harmonic-mean flow & 60.3 & 208 & 185 & 33.6 & 38.1 \\
\hline 5-percent-duration flow & 1,070 & 1,540 & 1,010 & 493 & 856 \\
\hline 10-percent-duration flow & 725 & 1,110 & 740 & 286 & 589 \\
\hline 15-percent-duration flow & 561 & 887 & 597 & 197 & 439 \\
\hline 20-percent-duration flow & 453 & 740 & 496 & 148 & 356 \\
\hline 25-percent-duration flow & 377 & 635 & 422 & 117 & 298 \\
\hline 30-percent-duration flow & 317 & 552 & 362 & 96.5 & 251 \\
\hline 35-percent-duration flow & 266 & 483 & 312 & 80.4 & 216 \\
\hline 40-percent-duration flow & 227 & 425 & 271 & 67.9 & 183 \\
\hline 45-percent-duration flow & 193 & 380 & 237 & 57.8 & 154 \\
\hline 50-percent-duration flow & 163 & 340 & 208 & 49.4 & 130 \\
\hline 55-percent-duration flow & 137 & 304 & 183 & 42.3 & 109 \\
\hline 60-percent-duration flow & 114 & 272 & 163 & 36.8 & 90.9 \\
\hline 65-percent-duration flow & 93.3 & 242 & 143 & 31.7 & 75.3 \\
\hline 70-percent-duration flow & 75.8 & 213 & 123 & 27.0 & 61.4 \\
\hline 75-percent-duration flow & 59.7 & 186 & 105 & 22.9 & 48.8 \\
\hline 80-percent-duration flow & 46.1 & 159 & 87.3 & 19.0 & 38.2 \\
\hline 85-percent-duration flow & 34.3 & 133 & 70.7 & 15.5 & 29.5 \\
\hline 90-percent-duration flow & 23.9 & 106 & 53.7 & 12.0 & 22.2 \\
\hline 95-percent-duration flow & 15.0 & 77.7 & 38.1 & 8.4 & 14.8 \\
\hline 99-percent-duration flow & 6.9 & 51.8 & 18.5 & 4.8 & 7.6 \\
\hline Variability index & 0.57 & 0.39 & 0.43 & 0.53 & 0.54 \\
\hline
\end{tabular}


Table 12. Selected annual and seasonal statistics for 40 stations in surrounding states near West Virginia representative of 1930-2002 (values do not supersede those determined for use by the particular state).-Continued

[Winter, January 1-March 31; spring, April 1-June 30; summer, July 1-September 30; fall, October 1-December 31; streamflow statistics are in cubic feet per second, except for variability index, which is dimensionless; the record period is in climatic years, April 1 through March 31of the indicated ending year; EPA, U.S. Environmental Protection Agency]

\begin{tabular}{|c|c|c|c|c|c|}
\hline \multirow{2}{*}{ Streamflow statistic } & \multirow{2}{*}{ Annual } & \multicolumn{4}{|c|}{ Season } \\
\hline & & Winter & Spring & Summer & Fall \\
\hline \multicolumn{6}{|c|}{03076600 Bear Creek at Friendsville, MD, 1984-2000 } \\
\hline 1-day 2-year hydrologically based flow & 4.74 & 34.6 & 14.5 & 5.28 & 7.51 \\
\hline 1-day 5-year hydrologically based flow & 2.94 & 21.9 & 9.42 & 3.13 & 3.90 \\
\hline 1-day 10-year hydrologically based flow & 2.35 & 16.4 & 7.50 & 2.40 & 2.84 \\
\hline 3-day 2-year hydrologically based flow & 4.94 & 36.2 & 15.6 & 5.54 & 7.84 \\
\hline 3-day 5-year hydrologically based flow & 3.14 & 22.5 & 9.88 & 3.32 & 4.12 \\
\hline 3-day 10-year hydrologically based flow & 2.54 & 16.7 & 7.73 & 2.56 & 3.03 \\
\hline 7-day 2-year hydrologically based flow & 5.27 & 39.0 & 18.8 & 6.03 & 8.61 \\
\hline 7-day 5-year hydrologically based flow & 3.41 & 23.8 & 11.7 & 3.66 & 4.57 \\
\hline 7-day 10-year hydrologically based flow & 2.80 & 17.9 & 9.03 & 2.85 & 3.40 \\
\hline 14-day 2-year hydrologically based flow & 6.00 & 48.1 & 21.9 & 6.91 & 9.77 \\
\hline 14-day 5-year hydrologically based flow & 3.92 & 30.7 & 14.0 & 4.23 & 5.16 \\
\hline 14-day 10-year hydrologically based flow & 3.27 & 24.4 & 11.0 & 3.34 & 3.82 \\
\hline 30-day 2-year hydrologically based flow & 7.23 & 79.2 & 31.8 & 9.09 & 14.9 \\
\hline 30-day 5-year hydrologically based flow & 4.51 & 48.1 & 19.4 & 5.20 & 7.24 \\
\hline 30-day 10-year hydrologically based flow & 3.71 & 36.6 & 14.7 & 3.99 & 5.14 \\
\hline 1-day 3-year biologically based flow & 2.38 & 15.0 & 15.0 & 2.40 & 2.45 \\
\hline 4-day 3-year biologically based flow & 2.54 & 15.7 & 18.4 & 2.87 & 2.70 \\
\hline EPA harmonic-mean flow & 21.1 & 65.4 & 71.2 & 12.9 & 12.1 \\
\hline 5-percent-duration flow & 342 & 460 & 340 & 155 & 297 \\
\hline 10-percent-duration flow & 246 & 335 & 250 & 76.9 & 189 \\
\hline 15-percent-duration flow & 179 & 281 & 188 & 56.0 & 132 \\
\hline 20-percent-duration flow & 138 & 242 & 148 & 42.0 & 106 \\
\hline 25-percent-duration flow & 112 & 206 & 121 & 33.4 & 90.5 \\
\hline 30-percent-duration flow & 94.7 & 174 & 104 & 27.4 & 77.6 \\
\hline 35-percent-duration flow & 80.4 & 152 & 89.8 & 23.2 & 67.3 \\
\hline 40-percent-duration flow & 68.8 & 134 & 78.8 & 20.2 & 59.3 \\
\hline 45-percent-duration flow & 60.0 & 120 & 69.3 & 17.5 & 51.5 \\
\hline 50-percent-duration flow & 52.0 & 107 & 62.7 & 15.0 & 44.8 \\
\hline 55-percent-duration flow & 44.6 & 94.8 & 56.3 & 13.0 & 38.8 \\
\hline 60-percent-duration flow & 37.8 & 84.7 & 50.2 & 11.2 & 32.2 \\
\hline 65-percent-duration flow & 31.4 & 76.1 & 44.3 & 9.7 & 26.9 \\
\hline 70-percent-duration flow & 25.2 & 67.6 & 39.1 & 8.6 & 20.6 \\
\hline 75-percent-duration flow & 19.4 & 59.6 & 34.4 & 7.6 & 13.0 \\
\hline 80-percent-duration flow & 14.0 & 53.3 & 29.6 & 6.5 & 9.7 \\
\hline 85-percent-duration flow & 10.3 & 46.2 & 24.0 & 5.4 & 7.6 \\
\hline 90-percent-duration flow & 7.5 & 39.6 & 19.1 & 4.5 & 6.5 \\
\hline 95-percent-duration flow & 5.2 & 32.1 & 13.9 & 3.5 & 4.9 \\
\hline 99-percent-duration flow & 3.1 & 18.1 & 8.4 & 2.6 & 3.1 \\
\hline Variability index & 0.56 & 0.36 & 0.42 & 0.50 & 0.56 \\
\hline
\end{tabular}


Table 12. Selected annual and seasonal statistics for 40 stations in surrounding states near West Virginia representative of 1930-2002 (values do not supersede those determined for use by the particular state).-Continued

[Winter, January 1-March 31; spring, April 1-June 30; summer, July 1-September 30; fall, October 1-December 31; streamflow statistics are in cubic feet per second, except for variability index, which is dimensionless; the record period is in climatic years, April 1 through March 31of the indicated ending year; EPA, U.S. Environmental Protection Agency]

\begin{tabular}{|c|c|c|c|c|c|}
\hline \multirow{2}{*}{ Streamflow statistic } & \multirow{2}{*}{ Annual } & \multicolumn{4}{|c|}{ Season } \\
\hline & & Winter & Spring & Summer & Fall \\
\hline \multicolumn{6}{|c|}{03085500 Chartiers Creek at Carnegie, PA, 1942-1980 } \\
\hline 1-day 2-year hydrologically based flow & 46.4 & 104 & 91.7 & 50.3 & 52.5 \\
\hline 1-day 5-year hydrologically based flow & 36.9 & 61.9 & 70.0 & 40.5 & 39.7 \\
\hline 1-day 10-year hydrologically based flow & 33.2 & 47.0 & 61.5 & 36.8 & 35.4 \\
\hline 3-day 2-year hydrologically based flow & 48.4 & 111 & 96.9 & 52.4 & 54.9 \\
\hline 3-day 5-year hydrologically based flow & 38.9 & 65.9 & 73.2 & 42.7 & 41.7 \\
\hline 3-day 10 -year hydrologically based flow & 35.4 & 50.5 & 63.8 & 39.2 & 37.3 \\
\hline 7-day 2-year hydrologically based flow & 51.7 & 121 & 107 & 55.7 & 58.8 \\
\hline 7-day 5-year hydrologically based flow & 42.3 & 71.8 & 80.2 & 45.6 & 45.1 \\
\hline 7-day 10-year hydrologically based flow & 38.8 & 55.0 & 69.8 & 42.1 & 40.5 \\
\hline 14-day 2-year hydrologically based flow & 55.1 & 142 & 124 & 61.7 & 64.8 \\
\hline 14-day 5-year hydrologically based flow & 44.2 & 80.3 & 91.1 & 48.3 & 48.5 \\
\hline 14-day 10-year hydrologically based flow & 40.3 & 59.5 & 78.5 & 43.7 & 42.7 \\
\hline 30-day 2-year hydrologically based flow & 61.7 & 204 & 154 & 70.4 & 77.7 \\
\hline 30-day 5-year hydrologically based flow & 48.2 & 113 & 112 & 53.3 & 55.0 \\
\hline 30-day 10-year hydrologically based flow & 43.3 & 81.0 & 95.6 & 47.6 & 46.7 \\
\hline 1-day 3-year biologically based flow & 34.0 & 38.9 & 73.9 & 39.0 & 33.9 \\
\hline 4-day 3-year biologically based flow & 37.0 & 41.7 & 80.4 & 42.7 & 38.0 \\
\hline EPA harmonic-mean flow & 123 & 179 & 243 & 93.4 & 82.5 \\
\hline 5-percent-duration flow & 885 & 1,350 & 975 & 385 & 502 \\
\hline 10-percent-duration flow & 610 & 939 & 666 & 247 & 353 \\
\hline 15-percent-duration flow & 476 & 756 & 534 & 193 & 261 \\
\hline 20-percent-duration flow & 393 & 646 & 453 & 163 & 213 \\
\hline 25-percent-duration flow & 331 & 571 & 399 & 140 & 179 \\
\hline 30-percent-duration flow & 282 & 508 & 354 & 124 & 154 \\
\hline 35-percent-duration flow & 241 & 453 & 316 & 112 & 133 \\
\hline 40-percent-duration flow & 210 & 403 & 285 & 103 & 117 \\
\hline 45-percent-duration flow & 182 & 364 & 259 & 94.5 & 105 \\
\hline 50-percent-duration flow & 158 & 331 & 235 & 88.7 & 95.7 \\
\hline 55-percent-duration flow & 137 & 300 & 214 & 83.1 & 87.9 \\
\hline 60-percent-duration flow & 119 & 270 & 197 & 77.8 & 80.5 \\
\hline 65-percent-duration flow & 104 & 243 & 180 & 72.8 & 74.8 \\
\hline 70-percent-duration flow & 93.0 & 214 & 163 & 68.0 & 69.2 \\
\hline 75-percent-duration flow & 82.9 & 182 & 148 & 63.8 & 64.1 \\
\hline 80-percent-duration flow & 74.0 & 150 & 133 & 59.6 & 59.2 \\
\hline 85-percent-duration flow & 65.9 & 120 & 119 & 55.6 & 54.3 \\
\hline 90-percent-duration flow & 57.9 & 89.6 & 104 & 51.6 & 49.3 \\
\hline 95-percent-duration flow & 49.5 & 68.9 & 86.5 & 46.3 & 43.9 \\
\hline 99-percent-duration flow & 39.8 & 45.6 & 65.0 & 38.9 & 37.0 \\
\hline Variability index & 0.40 & 0.38 & 0.32 & 0.28 & 0.33 \\
\hline
\end{tabular}


Table 12. Selected annual and seasonal statistics for 40 stations in surrounding states near West Virginia representative of 1930-2002 (values do not supersede those determined for use by the particular state).-Continued

[Winter, January 1-March 31; spring, April 1-June 30; summer, July 1-September 30; fall, October 1-December 31; streamflow statistics are in cubic feet per second, except for variability index, which is dimensionless; the record period is in climatic years, April 1 through March 31of the indicated ending year; EPA, U.S. Environmental Protection Agency]

\begin{tabular}{|c|c|c|c|c|c|}
\hline \multirow{2}{*}{ Streamflow statistic } & \multirow{2}{*}{ Annual } & \multicolumn{4}{|c|}{ Season } \\
\hline & & Winter & Spring & Summer & Fall \\
\hline \multicolumn{6}{|c|}{03108000 Raccoon Creek at Moffatts Mill, PA, 1943-1980 } \\
\hline 1-day 2-year hydrologically based flow & 12.1 & 59.3 & 38.4 & 12.4 & 18.2 \\
\hline 1-day 5-year hydrologically based flow & 8.07 & 32.1 & 26.0 & 8.25 & 11.5 \\
\hline 1-day 10-year hydrologically based flow & 6.56 & 22.4 & 21.3 & 6.70 & 9.38 \\
\hline 3-day 2-year hydrologically based flow & 12.2 & 64.6 & 41.4 & 12.7 & 19.6 \\
\hline 3-day 5-year hydrologically based flow & 8.24 & 33.9 & 28.2 & 8.51 & 12.2 \\
\hline 3-day 10 -year hydrologically based flow & 6.82 & 23.5 & 23.2 & 7.00 & 9.84 \\
\hline 7-day 2-year hydrologically based flow & 13.3 & 72.7 & 48.1 & 13.9 & 22.7 \\
\hline 7-day 5-year hydrologically based flow & 9.06 & 39.5 & 32.9 & 9.34 & 13.5 \\
\hline 7-day 10-year hydrologically based flow & 7.61 & 28.3 & 27.4 & 7.78 & 10.5 \\
\hline 14-day 2-year hydrologically based flow & 15.6 & 86.0 & 60.5 & 16.7 & 26.6 \\
\hline 14-day 5 -year hydrologically based flow & 10.4 & 45.8 & 40.7 & 10.8 & 15.2 \\
\hline 14-day 10-year hydrologically based flow & 8.51 & 32.6 & 33.5 & 8.88 & 11.5 \\
\hline 30-day 2-year hydrologically based flow & 19.1 & 132 & 86.4 & 22.0 & 35.9 \\
\hline 30-day 5-year hydrologically based flow & 12.6 & 71.9 & 56.8 & 14.1 & 19.9 \\
\hline 30-day 10-year hydrologically based flow & 10.3 & 51.9 & 46.5 & 11.4 & 14.6 \\
\hline 1-day 3-year biologically based flow & 6.06 & 13.0 & 30.9 & 6.29 & 7.58 \\
\hline 4-day 3-year biologically based flow & 7.10 & 14.6 & 39.9 & 7.87 & 8.29 \\
\hline EPA harmonic-mean flow & 49.5 & 107 & 150 & 30.2 & 31.7 \\
\hline 5-percent-duration flow & 660 & 1,030 & 723 & 201 & 404 \\
\hline 10-percent-duration flow & 451 & 720 & 514 & 127 & 275 \\
\hline 15-percent-duration flow & 345 & 571 & 409 & 96.3 & 201 \\
\hline 20-percent-duration flow & 276 & 480 & 339 & 76.1 & 156 \\
\hline 25-percent-duration flow & 228 & 413 & 286 & 63.9 & 127 \\
\hline 30-percent-duration flow & 190 & 362 & 248 & 55.5 & 107 \\
\hline 35-percent-duration flow & 159 & 319 & 221 & 48.7 & 88.9 \\
\hline 40-percent-duration flow & 133 & 285 & 197 & 43.2 & 75.5 \\
\hline 45-percent-duration flow & 111 & 255 & 175 & 38.6 & 65.0 \\
\hline 50-percent-duration flow & 93.3 & 227 & 155 & 34.3 & 56.2 \\
\hline 55-percent-duration flow & 77.6 & 204 & 138 & 31.0 & 49.0 \\
\hline 60-percent-duration flow & 65.0 & 180 & 122 & 27.7 & 43.8 \\
\hline 65-percent-duration flow & 54.2 & 158 & 108 & 24.7 & 38.6 \\
\hline 70-percent-duration flow & 46.0 & 136 & 96.5 & 22.0 & 33.4 \\
\hline 75-percent-duration flow & 38.8 & 113 & 84.5 & 19.9 & 28.9 \\
\hline 80-percent-duration flow & 31.8 & 93.2 & 73.1 & 17.8 & 24.8 \\
\hline 85-percent-duration flow & 25.5 & 73.9 & 62.4 & 15.6 & 20.4 \\
\hline 90-percent-duration flow & 19.7 & 57.4 & 50.9 & 13.4 & 16.7 \\
\hline 95-percent-duration flow & 14.6 & 41.2 & 40.3 & 10.8 & 13.2 \\
\hline 99-percent-duration flow & 8.7 & 24.3 & 25.6 & 7.3 & 8.2 \\
\hline Variability index & 0.52 & 0.42 & 0.38 & 0.38 & 0.46 \\
\hline
\end{tabular}


Table 12. Selected annual and seasonal statistics for 40 stations in surrounding states near West Virginia representative of 1930-2002 (values do not supersede those determined for use by the particular state).-Continued

[Winter, January 1-March 31; spring, April 1-June 30; summer, July 1-September 30; fall, October 1-December 31; streamflow statistics are in cubic feet per second, except for variability index, which is dimensionless; the record period is in climatic years, April 1 through March 31 of the indicated ending year; EPA, U.S. Environmental Protection Agency]

\begin{tabular}{|c|c|c|c|c|c|}
\hline \multirow{2}{*}{ Streamflow statistic } & \multirow{2}{*}{ Annual } & \multicolumn{4}{|c|}{ Season } \\
\hline & & Winter & Spring & Summer & Fall \\
\hline \multicolumn{6}{|c|}{03109500 Little Beaver Creek near East Liverpool, OH, 1930-2002 } \\
\hline 1-day 2-year hydrologically based flow & 35.5 & 175 & 107 & 36.0 & 52.4 \\
\hline 1-day 5-year hydrologically based flow & 23.2 & 100 & 65.2 & 23.4 & 33.9 \\
\hline 1-day 10-year hydrologically based flow & 18.7 & 71.2 & 49.2 & 18.9 & 27.9 \\
\hline 3-day 2-year hydrologically based flow & 36.6 & 182 & 115 & 37.2 & 54.8 \\
\hline 3-day 5-year hydrologically based flow & 24.0 & 104 & 69.5 & 24.2 & 35.3 \\
\hline 3-day 10 -year hydrologically based flow & 19.4 & 73.9 & 52.3 & 19.6 & 29.0 \\
\hline 7-day 2-year hydrologically based flow & 39.0 & 195 & 133 & 39.9 & 61.7 \\
\hline 7-day 5-year hydrologically based flow & 25.6 & 112 & 80.7 & 25.9 & 38.6 \\
\hline 7-day 10-year hydrologically based flow & 20.8 & 80.9 & 60.9 & 21.0 & 31.0 \\
\hline 14-day 2-year hydrologically based flow & 44.2 & 228 & 163 & 45.3 & 70.9 \\
\hline 14-day 5-year hydrologically based flow & 28.6 & 127 & 96.7 & 28.8 & 42.5 \\
\hline 14-day 10-year hydrologically based flow & 23.1 & 90.2 & 72.3 & 23.5 & 33.3 \\
\hline 30-day 2-year hydrologically based flow & 53.1 & 348 & 229 & 59.7 & 95.3 \\
\hline 30-day 5-year hydrologically based flow & 34.6 & 185 & 133 & 37.2 & 55.1 \\
\hline 30-day 10-year hydrologically based flow & 28.2 & 128 & 98.3 & 30.1 & 41.7 \\
\hline 1-day 3-year biologically based flow & 18.0 & 55.0 & 74.9 & 18.0 & 24.0 \\
\hline 4-day 3-year biologically based flow & 20.7 & 62.4 & 89.5 & 20.7 & 27.7 \\
\hline EPA harmonic-mean flow & 133 & 282 & 366 & 78.5 & 90.6 \\
\hline 5-percent-duration flow & 1,850 & 2,870 & 2,070 & 614 & 1,150 \\
\hline 10-percent-duration flow & 1,210 & 1,940 & 1,450 & 358 & 703 \\
\hline 15-percent-duration flow & 906 & 1,490 & 1,130 & 260 & 530 \\
\hline 20-percent-duration flow & 721 & 1,230 & 923 & 205 & 417 \\
\hline 25-percent-duration flow & 591 & 1,040 & 788 & 169 & 341 \\
\hline 30-percent-duration flow & 493 & 902 & 681 & 144 & 285 \\
\hline 35-percent-duration flow & 416 & 783 & 589 & 126 & 243 \\
\hline 40-percent-duration flow & 349 & 694 & 524 & 111 & 208 \\
\hline 45-percent-duration flow & 292 & 616 & 464 & 98.2 & 179 \\
\hline 50-percent-duration flow & 246 & 548 & 412 & 88.1 & 157 \\
\hline 55-percent-duration flow & 207 & 491 & 366 & 78.7 & 138 \\
\hline 60-percent-duration flow & 174 & 438 & 322 & 71.1 & 121 \\
\hline 65-percent-duration flow & 146 & 386 & 284 & 63.8 & 107 \\
\hline 70-percent-duration flow & 121 & 336 & 249 & 57.0 & 93.9 \\
\hline 75-percent-duration flow & 101 & 289 & 216 & 50.7 & 83.3 \\
\hline 80-percent-duration flow & 83.2 & 247 & 187 & 45.2 & 70.5 \\
\hline 85-percent-duration flow & 66.9 & 204 & 155 & 39.9 & 58.8 \\
\hline 90-percent-duration flow & 52.0 & 155 & 126 & 34.6 & 48.8 \\
\hline 95-percent-duration flow & 38.8 & 104 & 92.8 & 28.3 & 38.8 \\
\hline 99-percent-duration flow & 25.1 & 55.2 & 50.4 & 18.3 & 28.1 \\
\hline Variability index & 0.52 & 0.42 & 0.41 & 0.40 & 0.45 \\
\hline
\end{tabular}


Table 12. Selected annual and seasonal statistics for 40 stations in surrounding states near West Virginia representative of 1930-2002 (values do not supersede those determined for use by the particular state)._-Continued

[Winter, January 1-March 31; spring, April 1-June 30; summer, July 1-September 30; fall, October 1-December 31; streamflow statistics are in cubic feet per second, except for variability index, which is dimensionless; the record period is in climatic years, April 1 through March 31 of the indicated ending year; EPA, U.S. Environmental Protection Agency]

\begin{tabular}{|c|c|c|c|c|c|}
\hline \multirow{2}{*}{ Streamflow statistic } & \multirow{2}{*}{ Annual } & \multicolumn{4}{|c|}{ Season } \\
\hline & & Winter & Spring & Summer & Fall \\
\hline \multicolumn{6}{|c|}{03110000 Yellow Creek near Hammondsville, OH, 1942-1980 } \\
\hline 1-day 2-year hydrologically based flow & 4.56 & 53.0 & 25.1 & 4.59 & 10.1 \\
\hline 1-day 5-year hydrologically based flow & 2.30 & 26.5 & 15.9 & 2.28 & 4.56 \\
\hline 1-day 10-year hydrologically based flow & 1.58 & 17.0 & 12.5 & 1.58 & 2.93 \\
\hline 3-day 2-year hydrologically based flow & 4.85 & 55.7 & 27.3 & 4.85 & 11.2 \\
\hline 3-day 5-year hydrologically based flow & 2.43 & 27.2 & 17.2 & 2.40 & 5.17 \\
\hline 3-day 10 -year hydrologically based flow & 1.67 & 17.4 & 13.4 & 1.65 & 3.35 \\
\hline 7-day 2-year hydrologically based flow & 5.60 & 58.9 & 32.5 & 5.60 & 13.0 \\
\hline 7-day 5-year hydrologically based flow & 2.78 & 28.5 & 20.7 & 2.74 & 6.15 \\
\hline 7-day 10 -year hydrologically based flow & 1.89 & 18.2 & 16.3 & 1.88 & 4.10 \\
\hline 14-day 2-year hydrologically based flow & 6.62 & 69.5 & 41.7 & 6.72 & 16.1 \\
\hline 14-day 5-year hydrologically based flow & 3.41 & 32.5 & 25.9 & 3.36 & 7.86 \\
\hline 14-day 10 -year hydrologically based flow & 2.41 & 20.6 & 20.0 & 2.43 & 5.41 \\
\hline 30-day 2-year hydrologically based flow & 9.27 & 106 & 65.8 & 10.1 & 24.1 \\
\hline 30-day 5-year hydrologically based flow & 4.93 & 49.8 & 39.2 & 4.98 & 12.9 \\
\hline 30-day 10-year hydrologically based flow & 3.60 & 32.4 & 30.6 & 3.59 & 9.39 \\
\hline 1-day 3-year biologically based flow & 1.50 & 7.99 & 21.0 & 1.71 & 2.14 \\
\hline 4-day 3-year biologically based flow & 1.85 & 14.0 & 25.2 & 2.12 & 2.75 \\
\hline EPA harmonic-mean flow & 25.0 & 73.8 & 115 & 13.6 & 15.6 \\
\hline 5-percent-duration flow & 603 & 954 & 672 & 168 & 339 \\
\hline 10-percent-duration flow & 392 & 655 & 451 & 96.3 & 220 \\
\hline 15-percent-duration flow & 294 & 517 & 352 & 66.8 & 165 \\
\hline 20-percent-duration flow & 234 & 425 & 290 & 52.5 & 129 \\
\hline 25-percent-duration flow & 191 & 356 & 245 & 43.9 & 104 \\
\hline 30-percent-duration flow & 156 & 311 & 212 & 36.3 & 84.3 \\
\hline 35-percent-duration flow & 129 & 275 & 184 & 30.6 & 70.2 \\
\hline 40-percent-duration flow & 107 & 242 & 161 & 26.0 & 59.2 \\
\hline 45-percent-duration flow & 88.4 & 217 & 142 & 22.6 & 50.0 \\
\hline 50-percent-duration flow & 72.1 & 192 & 126 & 19.6 & 42.2 \\
\hline 55-percent-duration flow & 58.0 & 168 & 111 & 16.9 & 35.8 \\
\hline 60-percent-duration flow & 47.0 & 146 & 97.5 & 14.8 & 30.7 \\
\hline 65-percent-duration flow & 37.9 & 127 & 85.5 & 12.7 & 27.0 \\
\hline 70-percent-duration flow & 30.4 & 111 & 74.1 & 10.6 & 23.3 \\
\hline 75-percent-duration flow & 24.3 & 94.0 & 63.0 & 9.0 & 19.8 \\
\hline 80-percent-duration flow & 19.3 & 76.4 & 52.6 & 7.6 & 16.4 \\
\hline 85-percent-duration flow & 14.8 & 59.8 & 42.7 & 6.2 & 12.8 \\
\hline 90-percent-duration flow & 10.2 & 38.8 & 34.8 & 4.7 & 9.7 \\
\hline 95-percent-duration flow & 6.3 & 21.2 & 25.7 & 3.2 & 6.4 \\
\hline 99-percent-duration flow & 2.9 & 14.1 & 15.8 & 1.9 & 3.6 \\
\hline Variability index & 0.61 & 0.48 & 0.43 & 0.51 & 0.52 \\
\hline
\end{tabular}


Table 12. Selected annual and seasonal statistics for 40 stations in surrounding states near West Virginia representative of 1930-2002 (values do not supersede those determined for use by the particular state)._Continued

[Winter, January 1-March 31; spring, April 1-June 30; summer, July 1-September 30; fall, October 1-December 31; streamflow statistics are in cubic feet per second, except for variability index, which is dimensionless; the record period is in climatic years, April 1 through March 31 of the indicated ending year; EPA, U.S. Environmental Protection Agency]

\begin{tabular}{|c|c|c|c|c|c|}
\hline \multirow{2}{*}{ Streamflow statistic } & \multirow{2}{*}{ Annual } & \multicolumn{4}{|c|}{ Season } \\
\hline & & Winter & Spring & Summer & Fall \\
\hline \multicolumn{6}{|c|}{03111500 Short Creak near Dillonvale, OH, 1943-1980 } \\
\hline 1-day 2-year hydrologically based flow & 14.9 & 43.9 & 41.7 & 16.5 & 20.4 \\
\hline 1-day 5-year hydrologically based flow & 8.36 & 23.1 & 29.5 & 9.36 & 12.5 \\
\hline 1-day 10-year hydrologically based flow & 6.04 & 16.1 & 24.5 & 6.74 & 9.58 \\
\hline 3-day 2-year hydrologically based flow & 15.4 & 47.3 & 44.1 & 16.9 & 22.0 \\
\hline 3-day 5-year hydrologically based flow & 8.95 & 23.8 & 31.2 & 9.97 & 13.5 \\
\hline 3-day 10-year hydrologically based flow & 6.66 & 16.2 & 25.9 & 7.43 & 10.4 \\
\hline 7-day 2-year hydrologically based flow & 16.5 & 50.0 & 50.3 & 18.1 & 24.3 \\
\hline 7-day 5-year hydrologically based flow & 10.2 & 25.2 & 35.9 & 11.4 & 14.8 \\
\hline 7-day 10-year hydrologically based flow & 7.97 & 17.2 & 29.8 & 8.98 & 11.3 \\
\hline 14-day 2-year hydrologically based flow & 18.2 & 62.5 & 59.9 & 20.3 & 27.5 \\
\hline 14-day 5-year hydrologically based flow & 11.4 & 31.9 & 42.4 & 12.7 & 16.6 \\
\hline 14-day 10-year hydrologically based flow & 9.00 & 21.8 & 35.2 & 10.0 & 12.7 \\
\hline 30-day 2-year hydrologically based flow & 20.8 & 88.8 & 77.2 & 24.1 & 33.0 \\
\hline 30-day 5-year hydrologically based flow & 13.7 & 45.7 & 54.9 & 15.1 & 21.0 \\
\hline 30-day 10-year hydrologically based flow & 11.3 & 31.4 & 46.0 & 12.0 & 16.7 \\
\hline 1-day 3-year biologically based flow & 5.17 & 11.0 & 31.9 & 6.53 & 7.99 \\
\hline 4-day 3-year biologically based flow & 6.99 & 11.9 & 36.2 & 8.59 & 10.0 \\
\hline EPA harmonic-mean flow & 46.8 & 69.4 & 121 & 32.3 & 31.5 \\
\hline 5-percent-duration flow & 392 & 586 & 435 & 163 & 223 \\
\hline 10-percent-duration flow & 274 & 421 & 308 & 108 & 158 \\
\hline 15-percent-duration flow & 220 & 343 & 256 & 82.7 & 122 \\
\hline 20-percent-duration flow & 184 & 295 & 222 & 68.7 & 99.6 \\
\hline 25-percent-duration flow & 156 & 257 & 196 & 60.6 & 85.5 \\
\hline 30-percent-duration flow & 134 & 229 & 175 & 54.5 & 74.1 \\
\hline 35-percent-duration flow & 116 & 206 & 157 & 49.4 & 64.8 \\
\hline 40-percent-duration flow & 101 & 188 & 143 & 44.5 & 56.9 \\
\hline 45-percent-duration flow & 86.4 & 170 & 130 & 39.7 & 50.3 \\
\hline 50-percent-duration flow & 73.8 & 153 & 120 & 36.0 & 44.8 \\
\hline 55-percent-duration flow & 64.2 & 137 & 111 & 32.5 & 39.9 \\
\hline 60-percent-duration flow & 55.6 & 124 & 102 & 29.8 & 36.1 \\
\hline 65-percent-duration flow & 48.2 & 110 & 93.2 & 27.0 & 32.5 \\
\hline 70-percent-duration flow & 41.2 & 96.4 & 84.8 & 24.5 & 29.7 \\
\hline 75-percent-duration flow & 35.1 & 81.8 & 76.8 & 22.0 & 26.8 \\
\hline 80-percent-duration flow & 30.1 & 66.9 & 68.6 & 19.7 & 24.0 \\
\hline 85-percent-duration flow & 25.5 & 51.5 & 60.2 & 17.4 & 21.0 \\
\hline 90-percent-duration flow & 20.6 & 35.8 & 51.5 & 15.1 & 17.9 \\
\hline 95-percent-duration flow & 16.0 & 25.1 & 41.5 & 12.8 & 14.8 \\
\hline 99-percent-duration flow & 10.6 & 13.5 & 29.5 & 7.7 & 10.8 \\
\hline Variability index & 0.43 & 0.40 & 0.30 & 0.33 & 0.36 \\
\hline
\end{tabular}


Table 12. Selected annual and seasonal statistics for 40 stations in surrounding states near West Virginia representative of 1930-2002 (values do not supersede those determined for use by the particular state).-Continued

[Winter, January 1-March 31; spring, April 1-June 30; summer, July 1-September 30; fall, October 1-December 31; streamflow statistics are in cubic feet per second, except for variability index, which is dimensionless; the record period is in climatic years, April 1 through March 31of the indicated ending year; EPA, U.S. Environmental Protection Agency]

\begin{tabular}{|c|c|c|c|c|c|}
\hline \multirow{2}{*}{ Streamflow statistic } & \multirow{2}{*}{ Annual } & \multicolumn{4}{|c|}{ Season } \\
\hline & & Winter & Spring & Summer & Fall \\
\hline \multicolumn{6}{|c|}{03114000 Captina Creek at Armstrongs Mills, OH, 1960-1977 } \\
\hline 1-day 2-year hydrologically based flow & 0.74 & 46.5 & 10.8 & 0.75 & 4.04 \\
\hline 1-day 5-year hydrologically based flow & 0.00 & 24.6 & 4.56 & 0.00 & 1.05 \\
\hline 1-day 10-year hydrologically based flow & 0.00 & 17.2 & 2.78 & 0.00 & 0.43 \\
\hline 3-day 2-year hydrologically based flow & 0.87 & 47.7 & 12.0 & 0.88 & 5.00 \\
\hline 3-day 5-year hydrologically based flow & 0.00 & 25.3 & 5.35 & 0.00 & 1.42 \\
\hline 3-day 10 -year hydrologically based flow & 0.00 & 17.8 & 3.40 & 0.00 & 0.61 \\
\hline 7-day 2-year hydrologically based flow & 1.11 & 51.4 & 16.0 & 1.12 & 6.48 \\
\hline 7-day 5-year hydrologically based flow & 0.09 & 26.3 & 7.66 & 0.09 & 1.88 \\
\hline 7-day 10 -year hydrologically based flow & 0.00 & 18.3 & 5.14 & 0.00 & 0.81 \\
\hline 14-day 2-year hydrologically based flow & 1.67 & 64.0 & 21.1 & 1.57 & 11.1 \\
\hline 14-day 5-year hydrologically based flow & 0.25 & 30.1 & 10.7 & 0.25 & 2.18 \\
\hline 14-day 10 -year hydrologically based flow & 0.06 & 19.9 & 7.63 & 0.08 & 0.67 \\
\hline 30-day 2-year hydrologically based flow & 4.23 & 106 & 41.3 & 4.01 & 18.4 \\
\hline 30-day 5-year hydrologically based flow & 0.86 & 51.7 & 22.5 & 1.26 & 4.77 \\
\hline 30-day 10-year hydrologically based flow & 0.30 & 34.1 & 16.9 & 0.65 & 1.88 \\
\hline 1-day 3-year biologically based flow & 0.00 & 10.8 & 10.9 & 0.08 & 0.00 \\
\hline 4-day 3-year biologically based flow & 0.00 & 13.2 & 16.0 & 0.10 & 0.00 \\
\hline EPA harmonic-mean flow & 5.79 & 70.7 & 85.5 & 2.99 & 2.99 \\
\hline 5-percent-duration flow & 612 & 1,020 & 690 & 275 & 369 \\
\hline 10-percent-duration flow & 367 & 613 & 439 & 129 & 243 \\
\hline 15-percent-duration flow & 281 & 459 & 318 & 76.9 & 176 \\
\hline 20-percent-duration flow & 226 & 374 & 254 & 49.7 & 137 \\
\hline 25-percent-duration flow & 183 & 323 & 211 & 35.3 & 107 \\
\hline 30-percent-duration flow & 152 & 281 & 180 & 26.2 & 83.4 \\
\hline 35-percent-duration flow & 124 & 251 & 156 & 20.5 & 65.1 \\
\hline 40-percent-duration flow & 98.3 & 223 & 136 & 15.8 & 52.4 \\
\hline 45-percent-duration flow & 78.7 & 200 & 116 & 12.4 & 42.6 \\
\hline 50-percent-duration flow & 62.1 & 180 & 98.6 & 9.7 & 34.5 \\
\hline 55-percent-duration flow & 48.3 & 162 & 85.6 & 7.8 & 28.9 \\
\hline 60-percent-duration flow & 37.0 & 139 & 73.8 & 6.4 & 24.6 \\
\hline 65-percent-duration flow & 29.5 & 120 & 63.3 & 5.1 & 21.0 \\
\hline 70-percent-duration flow & 22.7 & 101 & 53.7 & 4.1 & 17.7 \\
\hline 75-percent-duration flow & 16.8 & 83.7 & 44.8 & 3.1 & 14.6 \\
\hline 80-percent-duration flow & 11.5 & 67.7 & 36.2 & 2.2 & 10.6 \\
\hline 85-percent-duration flow & 6.7 & 50.5 & 27.8 & 1.4 & 6.4 \\
\hline 90-percent-duration flow & 3.7 & 39.4 & 19.4 & 0.8 & 3.6 \\
\hline 95-percent-duration flow & 1.4 & 30.5 & 12.5 & 0.4 & 1.8 \\
\hline 99-percent-duration flow & 0.1 & 15.2 & 4.7 & 0.0 & 0.1 \\
\hline Variability index & 0.80 & 0.45 & 0.52 & 0.84 & 0.69 \\
\hline
\end{tabular}


Table 12. Selected annual and seasonal statistics for 40 stations in surrounding states near West Virginia representative of 1930-2002 (values do not supersede those determined for use by the particular state).-Continued

[Winter, January 1-March 31; spring, April 1-June 30; summer, July 1-September 30; fall, October 1-December 31; streamflow statistics are in cubic feet per second, except for variability index, which is dimensionless; the record period is in climatic years, April 1 through March 31 of the indicated ending year; EPA, U.S. Environmental Protection Agency]

\begin{tabular}{|c|c|c|c|c|c|}
\hline \multirow{2}{*}{ Streamflow statistic } & \multirow{2}{*}{ Annual } & \multicolumn{4}{|c|}{ Season } \\
\hline & & Winter & Spring & Summer & Fall \\
\hline \multicolumn{6}{|c|}{03115400 Little Muskingum River at Bloomfield, OH, 1960-1977 } \\
\hline 1-day 2-year hydrologically based flow & 0.95 & 52.1 & 9.93 & 1.02 & 2.76 \\
\hline 1-day 5-year hydrologically based flow & 0.30 & 22.2 & 4.21 & 0.32 & 0.79 \\
\hline 1-day 10-year hydrologically based flow & 0.13 & 13.3 & 2.58 & 0.14 & 0.42 \\
\hline 3-day 2-year hydrologically based flow & 1.03 & 55.1 & 11.2 & 1.13 & 3.20 \\
\hline 3-day 5-year hydrologically based flow & 0.27 & 22.9 & 4.70 & 0.30 & 0.90 \\
\hline 3-day 10 -year hydrologically based flow & 0.12 & 13.5 & 2.88 & 0.13 & 0.46 \\
\hline 7-day 2-year hydrologically based flow & 1.16 & 60.4 & 14.9 & 1.25 & 4.21 \\
\hline 7-day 5-year hydrologically based flow & 0.39 & 24.6 & 6.17 & 0.42 & 1.10 \\
\hline 7-day 10-year hydrologically based flow & 0.22 & 14.6 & 3.80 & 0.23 & 0.53 \\
\hline 14-day 2-year hydrologically based flow & 1.50 & 78.0 & 22.2 & 1.61 & 7.67 \\
\hline 14-day 5-year hydrologically based flow & 0.50 & 33.4 & 9.09 & 0.55 & 1.81 \\
\hline 14-day 10-year hydrologically based flow & 0.28 & 21.2 & 5.66 & 0.31 & 0.76 \\
\hline 30-day 2-year hydrologically based flow & 3.14 & 152 & 47.4 & 4.06 & 14.5 \\
\hline 30-day 5-year hydrologically based flow & 1.06 & 65.8 & 20.7 & 1.44 & 3.03 \\
\hline 30-day 10-year hydrologically based flow & 0.60 & 40.2 & 13.9 & 0.81 & 1.21 \\
\hline 1-day 3-year biologically based flow & 0.07 & 11.1 & 12.0 & 0.27 & 0.08 \\
\hline 4-day 3-year biologically based flow & 0.17 & 12.3 & 15.5 & 0.38 & 0.24 \\
\hline EPA harmonic-mean flow & 6.46 & 84.0 & 97.6 & 4.12 & 2.82 \\
\hline 5-percent-duration flow & 1,010 & 1,590 & 1,110 & 275 & 703 \\
\hline 10-percent-duration flow & 617 & 1,010 & 698 & 142 & 425 \\
\hline 15-percent-duration flow & 442 & 737 & 507 & 84.4 & 301 \\
\hline 20-percent-duration flow & 338 & 585 & 399 & 54.2 & 235 \\
\hline 25-percent-duration flow & 271 & 500 & 320 & 38.8 & 178 \\
\hline 30-percent-duration flow & 216 & 432 & 256 & 28.1 & 137 \\
\hline 35-percent-duration flow & 170 & 377 & 213 & 20.7 & 108 \\
\hline 40-percent-duration flow & 136 & 332 & 178 & 16.7 & 85.4 \\
\hline 45-percent-duration flow & 107 & 293 & 147 & 13.4 & 62.9 \\
\hline 50-percent-duration flow & 81.2 & 260 & 124 & 10.7 & 47.5 \\
\hline 55-percent-duration flow & 60.1 & 228 & 105 & 8.8 & 37.3 \\
\hline 60-percent-duration flow & 43.9 & 199 & 88.1 & 7.0 & 29.4 \\
\hline 65-percent-duration flow & 32.5 & 172 & 75.5 & 5.4 & 23.7 \\
\hline 70-percent-duration flow & 24.3 & 148 & 63.1 & 4.1 & 19.3 \\
\hline 75-percent-duration flow & 17.6 & 124 & 51.1 & 3.1 & 14.6 \\
\hline 80-percent-duration flow & 11.5 & 98.2 & 39.4 & 2.3 & 10.0 \\
\hline 85-percent-duration flow & 6.8 & 62.2 & 28.3 & 1.5 & 5.5 \\
\hline 90-percent-duration flow & 3.4 & 36.8 & 19.2 & 1.0 & 2.5 \\
\hline 95-percent-duration flow & 1.3 & 28.2 & 10.6 & 0.6 & 0.9 \\
\hline 99-percent-duration flow & 0.4 & 16.9 & 3.9 & 0.3 & 0.3 \\
\hline Variability index & 0.88 & 0.51 & 0.60 & 0.81 & 0.86 \\
\hline
\end{tabular}


Table 12. Selected annual and seasonal statistics for 40 stations in surrounding states near West Virginia representative of 1930-2002 (values do not supersede those determined for use by the particular state)._- Continued

[Winter, January 1-March 31; spring, April 1-June 30; summer, July 1-September 30; fall, October 1-December 31; streamflow statistics are in cubic feet per second, except for variability index, which is dimensionless; the record period is in climatic years, April 1 through March 31 of the indicated ending year; EPA, U.S. Environmental Protection Agency]

\begin{tabular}{|c|c|c|c|c|c|}
\hline \multirow{2}{*}{ Streamflow statistic } & \multirow{2}{*}{ Annual } & \multicolumn{4}{|c|}{ Season } \\
\hline & & Winter & Spring & Summer & Fall \\
\hline \multicolumn{6}{|c|}{03173000 Walker Creek at Bane, VA, 1939-1989 } \\
\hline 1-day 2-year hydrologically based flow & 40.6 & 114 & 85.1 & 42.5 & 47.7 \\
\hline 1-day 5-year hydrologically based flow & 32.3 & 66.9 & 63.5 & 33.8 & 35.4 \\
\hline 1-day 10-year hydrologically based flow & 29.0 & 48.7 & 54.4 & 30.7 & 31.2 \\
\hline 3-day 2-year hydrologically based flow & 41.7 & 122 & 88.8 & 43.3 & 49.8 \\
\hline 3-day 5-year hydrologically based flow & 33.7 & 73.5 & 65.9 & 34.4 & 37.1 \\
\hline 3-day 10 -year hydrologically based flow & 30.5 & 54.4 & 56.2 & 31.3 & 32.8 \\
\hline 7-day 2-year hydrologically based flow & 43.3 & 137 & 95.8 & 44.9 & 52.6 \\
\hline 7-day 5-year hydrologically based flow & 35.3 & 82.3 & 70.8 & 35.9 & 38.6 \\
\hline 7-day 10-year hydrologically based flow & 32.3 & 61.1 & 60.4 & 32.7 & 33.9 \\
\hline 14-day 2-year hydrologically based flow & 45.4 & 168 & 111 & 48.0 & 57.1 \\
\hline 14-day 5-year hydrologically based flow & 37.1 & 95.0 & 80.7 & 38.0 & 40.9 \\
\hline 14-day 10 -year hydrologically based flow & 33.9 & 68.3 & 68.2 & 34.6 & 35.4 \\
\hline 30-day 2-year hydrologically based flow & 50.5 & 247 & 151 & 53.8 & 70.0 \\
\hline 30-day 5-year hydrologically based flow & 41.1 & 131 & 106 & 43.3 & 46.2 \\
\hline 30-day 10-year hydrologically based flow & 37.5 & 89.6 & 88.2 & 40.5 & 38.8 \\
\hline 1-day 3-year biologically based flow & 29.0 & 38.0 & 75.8 & 31.9 & 30.0 \\
\hline 4-day 3-year biologically based flow & 31.0 & 43.5 & 82.2 & 34.2 & 33.2 \\
\hline EPA harmonic-mean flow & 113 & 198 & 252 & 79.7 & 72.9 \\
\hline 5-percent-duration flow & 1,120 & 1,710 & 1,210 & 345 & 744 \\
\hline 10-percent-duration flow & 725 & 1,180 & 804 & 217 & 469 \\
\hline 15-percent-duration flow & 548 & 914 & 620 & 160 & 349 \\
\hline 20-percent-duration flow & 435 & 754 & 499 & 132 & 271 \\
\hline 25-percent-duration flow & 361 & 649 & 424 & 114 & 217 \\
\hline 30-percent-duration flow & 303 & 569 & 370 & 100 & 174 \\
\hline 35-percent-duration flow & 258 & 507 & 326 & 92.0 & 146 \\
\hline 40-percent-duration flow & 221 & 451 & 291 & 84.5 & 123 \\
\hline 45-percent-duration flow & 189 & 402 & 264 & 78.5 & 107 \\
\hline 50-percent-duration flow & 161 & 364 & 241 & 72.7 & 94.0 \\
\hline 55-percent-duration flow & 137 & 327 & 220 & 68.3 & 83.1 \\
\hline 60-percent-duration flow & 117 & 292 & 200 & 63.9 & 74.3 \\
\hline 65-percent-duration flow & 99.8 & 260 & 182 & 60.0 & 67.0 \\
\hline 70-percent-duration flow & 86.2 & 231 & 164 & 56.3 & 61.0 \\
\hline 75-percent-duration flow & 74.4 & 205 & 148 & 52.6 & 55.4 \\
\hline 80-percent-duration flow & 64.7 & 176 & 131 & 49.3 & 50.7 \\
\hline 85-percent-duration flow & 56.5 & 149 & 116 & 45.9 & 46.6 \\
\hline 90-percent-duration flow & 48.9 & 116 & 101 & 42.3 & 43.4 \\
\hline 95-percent-duration flow & 42.7 & 76.1 & 82.0 & 38.2 & 39.5 \\
\hline 99-percent-duration flow & 34.5 & 44.8 & 57.5 & 30.8 & 34.2 \\
\hline Variability index & 0.45 & 0.39 & 0.35 & 0.28 & 0.41 \\
\hline
\end{tabular}


Table 12. Selected annual and seasonal statistics for 40 stations in surrounding states near West Virginia representative of 1930-2002 (values do not supersede those determined for use by the particular state).-Continued

[Winter, January 1-March 31; spring, April 1-June 30; summer, July 1-September 30; fall, October 1-December 31; streamflow statistics are in cubic feet per second, except for variability index, which is dimensionless; the record period is in climatic years, April 1 through March 31of the indicated ending year; EPA, U.S. Environmental Protection Agency]

\begin{tabular}{|c|c|c|c|c|c|}
\hline \multirow{2}{*}{ Streamflow statistic } & \multirow{2}{*}{ Annual } & \multicolumn{4}{|c|}{ Season } \\
\hline & & Winter & Spring & Summer & Fall \\
\hline \multicolumn{6}{|c|}{03175500 Wolf Creek near Narrows, VA, 1939-1989 } \\
\hline 1-day 2-year hydrologically based flow & 30.8 & 108 & 71.5 & 32.6 & 36.7 \\
\hline 1-day 5-year hydrologically based flow & 23.5 & 62.0 & 51.2 & 24.5 & 26.2 \\
\hline 1-day 10-year hydrologically based flow & 20.5 & 43.5 & 42.8 & 21.2 & 22.8 \\
\hline 3-day 2-year hydrologically based flow & 31.6 & 117 & 75.0 & 33.4 & 38.3 \\
\hline 3-day 5-year hydrologically based flow & 24.3 & 68.4 & 53.4 & 25.0 & 27.1 \\
\hline 3-day 10-year hydrologically based flow & 21.2 & 48.7 & 44.7 & 21.6 & 23.5 \\
\hline 7-day 2-year hydrologically based flow & 33.0 & 134 & 83.4 & 35.2 & 40.4 \\
\hline 7-day 5-year hydrologically based flow & 25.5 & 76.9 & 59.3 & 26.3 & 27.8 \\
\hline 7-day 10-year hydrologically based flow & 22.4 & 54.3 & 49.7 & 22.8 & 23.9 \\
\hline 14-day 2-year hydrologically based flow & 35.4 & 163 & 95.1 & 38.2 & 44.7 \\
\hline 14-day 5-year hydrologically based flow & 27.3 & 89.4 & 67.4 & 28.5 & 30.0 \\
\hline 14-day 10-year hydrologically based flow & 24.0 & 62.1 & 56.7 & 24.8 & 25.3 \\
\hline 30-day 2-year hydrologically based flow & 39.5 & 242 & 134 & 44.2 & 56.8 \\
\hline 30-day 5-year hydrologically based flow & 30.3 & 132 & 91.9 & 33.1 & 34.5 \\
\hline 30-day 10-year hydrologically based flow & 26.8 & 91.4 & 76.3 & 29.4 & 27.8 \\
\hline 1-day 3-year biologically based flow & 20.0 & 29.0 & 61.0 & 24.0 & 22.0 \\
\hline 4-day 3-year biologically based flow & 21.7 & 33.4 & 69.3 & 26.0 & 22.7 \\
\hline EPA harmonic-mean flow & 94.5 & 187 & 235 & 65.8 & 57.4 \\
\hline 5-percent-duration flow & 992 & 1,500 & 1,030 & 289 & 691 \\
\hline 10-percent-duration flow & 676 & 1,060 & 727 & 185 & 475 \\
\hline 15-percent-duration flow & 532 & 850 & 581 & 144 & 350 \\
\hline 20-percent-duration flow & 430 & 725 & 481 & 120 & 269 \\
\hline 25-percent-duration flow & 354 & 631 & 409 & 105 & 213 \\
\hline 30-percent-duration flow & 297 & 561 & 356 & 93.1 & 170 \\
\hline 35-percent-duration flow & 250 & 505 & 314 & 83.6 & 139 \\
\hline 40-percent-duration flow & 212 & 454 & 281 & 75.9 & 114 \\
\hline 45-percent-duration flow & 180 & 410 & 253 & 68.8 & 96.3 \\
\hline 50-percent-duration flow & 151 & 369 & 230 & 63.1 & 82.7 \\
\hline 55-percent-duration flow & 127 & 333 & 208 & 57.7 & 71.4 \\
\hline 60-percent-duration flow & 107 & 298 & 188 & 53.4 & 62.3 \\
\hline 65-percent-duration flow & 90.5 & 264 & 169 & 49.2 & 54.8 \\
\hline 70-percent-duration flow & 76.4 & 233 & 152 & 45.6 & 48.4 \\
\hline 75-percent-duration flow & 64.4 & 204 & 135 & 42.1 & 44.1 \\
\hline 80-percent-duration flow & 54.0 & 177 & 117 & 38.7 & 39.9 \\
\hline 85-percent-duration flow & 45.5 & 145 & 102 & 35.4 & 36.2 \\
\hline 90-percent-duration flow & 38.6 & 114 & 86.7 & 32.1 & 32.8 \\
\hline 95-percent-duration flow & 32.1 & 80.8 & 69.8 & 28.3 & 29.1 \\
\hline 99-percent-duration flow & 24.5 & 35.9 & 46.5 & 23.0 & 22.7 \\
\hline Variability index & 0.48 & 0.37 & 0.35 & 0.30 & 0.45 \\
\hline
\end{tabular}


Table 12. Selected annual and seasonal statistics for 40 stations in surrounding states near West Virginia representative of 1930-2002 (values do not supersede those determined for use by the particular state).-Continued

[Winter, January 1-March 31; spring, April 1-June 30; summer, July 1-September 30; fall, October 1-December 31; streamflow statistics are in cubic feet per second, except for variability index, which is dimensionless; the record period is in climatic years, April 1 through March 31of the indicated ending year; EPA, U.S. Environmental Protection Agency]

\begin{tabular}{|c|c|c|c|c|c|}
\hline \multirow{2}{*}{ Streamflow statistic } & \multirow{2}{*}{ Annual } & \multicolumn{4}{|c|}{ Season } \\
\hline & & Winter & Spring & Summer & Fall \\
\hline \multicolumn{6}{|c|}{03202000 Raccoon Creek at Adamsville, OH, 1940-1984 } \\
\hline 1-day 2-year hydrologically based flow & 13.4 & 160 & 67.9 & 15.4 & 19.9 \\
\hline 1-day 5-year hydrologically based flow & 5.26 & 64.8 & 40.5 & 6.57 & 6.61 \\
\hline 1-day 10-year hydrologically based flow & 3.07 & 35.2 & 30.6 & 4.09 & 3.54 \\
\hline 3-day 2-year hydrologically based flow & 14.2 & 170 & 72.4 & 16.5 & 20.6 \\
\hline 3-day 5-year hydrologically based flow & 5.61 & 69.5 & 43.1 & 7.08 & 6.92 \\
\hline 3-day 10-year hydrologically based flow & 3.30 & 38.4 & 32.6 & 4.42 & 3.76 \\
\hline 7-day 2-year hydrologically based flow & 15.9 & 193 & 86.1 & 18.5 & 23.1 \\
\hline 7-day 5-year hydrologically based flow & 6.39 & 79.7 & 49.6 & 8.04 & 7.89 \\
\hline 7-day 10-year hydrologically based flow & 3.79 & 45.5 & 37.1 & 5.07 & 4.31 \\
\hline 14-day 2-year hydrologically based flow & 18.5 & 247 & 111 & 22.6 & 27.9 \\
\hline 14-day 5-year hydrologically based flow & 7.41 & 99.8 & 61.8 & 9.84 & 9.39 \\
\hline 14-day 10 -year hydrologically based flow & 4.37 & 56.1 & 45.7 & 6.21 & 5.11 \\
\hline 30-day 2-year hydrologically based flow & 24.3 & 383 & 187 & 31.8 & 41.9 \\
\hline 30-day 5-year hydrologically based flow & 9.74 & 179 & 100 & 13.9 & 13.7 \\
\hline 30-day 10-year hydrologically based flow & 5.82 & 114 & 72.4 & 9.09 & 7.31 \\
\hline 1-day 3-year biologically based flow & 2.33 & 13.0 & 51.0 & 6.30 & 2.39 \\
\hline 4-day 3-year biologically based flow & 2.59 & 24.5 & 67.2 & 7.51 & 2.59 \\
\hline EPA harmonic-mean flow & 51.8 & 190 & 360 & 41.7 & 22.2 \\
\hline 5-percent-duration flow & 2,680 & 3,740 & 3,000 & 741 & 1,360 \\
\hline 10-percent-duration flow & 1,700 & 2,850 & 2,120 & 362 & 861 \\
\hline 15-percent-duration flow & 1,210 & 2,270 & 1,560 & 231 & 617 \\
\hline 20-percent-duration flow & 918 & 1,790 & 1,210 & 173 & 448 \\
\hline 25-percent-duration flow & 720 & 1,480 & 975 & 137 & 330 \\
\hline 30-percent-duration flow & 575 & 1,260 & 807 & 109 & 249 \\
\hline 35-percent-duration flow & 466 & 1,080 & 678 & 92.9 & 193 \\
\hline 40-percent-duration flow & 371 & 915 & 582 & 80.2 & 155 \\
\hline 45-percent-duration flow & 290 & 812 & 503 & 69.4 & 125 \\
\hline 50-percent-duration flow & 225 & 712 & 435 & 59.7 & 100 \\
\hline 55-percent-duration flow & 177 & 624 & 376 & 50.9 & 80.3 \\
\hline 60-percent-duration flow & 137 & 542 & 322 & 43.9 & 67.2 \\
\hline 65-percent-duration flow & 106 & 467 & 272 & 37.4 & 54.8 \\
\hline 70-percent-duration flow & 82.5 & 400 & 229 & 31.7 & 43.6 \\
\hline 75-percent-duration flow & 63.7 & 333 & 191 & 27.3 & 34.1 \\
\hline 80-percent-duration flow & 47.7 & 266 & 156 & 22.9 & 25.8 \\
\hline 85-percent-duration flow & 34.5 & 203 & 124 & 18.2 & 18.0 \\
\hline 90-percent-duration flow & 23.1 & 137 & 94.4 & 13.5 & 9.9 \\
\hline 95-percent-duration flow & 12.1 & 79.8 & 63.0 & 8.7 & 5.9 \\
\hline 99-percent-duration flow & 4.4 & 28.2 & 34.1 & 4.0 & 2.7 \\
\hline Variability index & 0.73 & 0.50 & 0.51 & 0.56 & 0.72 \\
\hline
\end{tabular}


Table 12. Selected annual and seasonal statistics for 40 stations in surrounding states near West Virginia representative of 1930-2002 (values do not supersede those determined for use by the particular state).-Continued

[Winter, January 1-March 31; spring, April 1-June 30; summer, July 1-September 30; fall, October 1-December 31; streamflow statistics are in cubic feet per second, except for variability index, which is dimensionless; the record period is in climatic years, April 1 through March 31of the indicated ending year; EPA, U.S. Environmental Protection Agency]

\begin{tabular}{|c|c|c|c|c|c|}
\hline \multirow{2}{*}{ Streamflow statistic } & \multirow{2}{*}{ Annual } & \multicolumn{4}{|c|}{ Season } \\
\hline & & Winter & Spring & Summer & Fall \\
\hline \multicolumn{6}{|c|}{03207500 Levisa Fork near Grundy, VA, 1956-1974 } \\
\hline 1-day 2-year hydrologically based flow & 7.40 & 82.5 & 25.2 & 7.99 & 12.3 \\
\hline 1-day 5-year hydrologically based flow & 3.18 & 38.2 & 14.1 & 3.38 & 5.74 \\
\hline 1-day 10-year hydrologically based flow & 1.92 & 23.4 & 10.4 & 2.00 & 3.98 \\
\hline 3-day 2-year hydrologically based flow & 7.87 & 89.1 & 28.1 & 8.52 & 13.3 \\
\hline 3-day 5-year hydrologically based flow & 3.36 & 41.1 & 15.6 & 3.58 & 6.35 \\
\hline 3-day 10-year hydrologically based flow & 2.03 & 25.0 & 11.6 & 2.12 & 4.47 \\
\hline 7-day 2-year hydrologically based flow & 8.99 & 112 & 34.1 & 9.80 & 15.4 \\
\hline 7-day 5-year hydrologically based flow & 3.75 & 48.7 & 18.9 & 4.02 & 7.07 \\
\hline 7-day 10-year hydrologically based flow & 2.21 & 28.4 & 13.8 & 2.31 & 4.89 \\
\hline 14-day 2-year hydrologically based flow & 10.9 & 153 & 46.0 & 13.0 & 18.8 \\
\hline 14-day 5-year hydrologically based flow & 4.42 & 63.9 & 26.4 & 5.01 & 8.31 \\
\hline 14-day 10-year hydrologically based flow & 2.61 & 35.9 & 19.7 & 2.82 & 5.65 \\
\hline 30-day 2-year hydrologically based flow & 15.8 & 278 & 78.6 & 24.0 & 31.8 \\
\hline 30-day 5-year hydrologically based flow & 6.81 & 138 & 40.5 & 9.84 & 12.9 \\
\hline 30-day 10-year hydrologically based flow & 4.22 & 83.8 & 28.6 & 5.57 & 7.99 \\
\hline 1-day 3-year biologically based flow & 1.18 & 14.0 & 17.9 & 1.19 & 3.66 \\
\hline 4-day 3-year biologically based flow & 1.20 & 15.2 & 23.0 & 1.81 & 3.93 \\
\hline EPA harmonic-mean flow & 33.8 & 132 & 134 & 20.6 & 18.3 \\
\hline 5-percent-duration flow & 1,110 & 1,780 & 1,230 & 315 & 776 \\
\hline 10-percent-duration flow & 674 & 1,120 & 762 & 189 & 439 \\
\hline 15-percent-duration flow & 496 & 862 & 567 & 132 & 322 \\
\hline 20-percent-duration flow & 396 & 705 & 440 & 100 & 245 \\
\hline 25-percent-duration flow & 323 & 599 & 364 & 78.1 & 194 \\
\hline 30-percent-duration flow & 264 & 523 & 302 & 62.8 & 155 \\
\hline 35-percent-duration flow & 219 & 469 & 254 & 52.8 & 123 \\
\hline 40-percent-duration flow & 182 & 420 & 217 & 44.6 & 96.5 \\
\hline 45-percent-duration flow & 147 & 379 & 185 & 37.7 & 74.8 \\
\hline 50-percent-duration flow & 116 & 341 & 163 & 32.4 & 60.0 \\
\hline 55-percent-duration flow & 92.7 & 306 & 140 & 27.7 & 47.9 \\
\hline 60-percent-duration flow & 72.5 & 274 & 118 & 24.1 & 38.7 \\
\hline 65-percent-duration flow & 56.3 & 247 & 102 & 21.0 & 31.4 \\
\hline 70-percent-duration flow & 43.7 & 222 & 87.1 & 18.5 & 25.5 \\
\hline 75-percent-duration flow & 33.4 & 197 & 72.6 & 16.2 & 21.3 \\
\hline 80-percent-duration flow & 24.8 & 172 & 59.3 & 14.0 & 18.0 \\
\hline 85-percent-duration flow & 19.3 & 140 & 47.7 & 11.6 & 14.9 \\
\hline 90-percent-duration flow & 14.9 & 99.5 & 36.1 & 8.7 & 11.8 \\
\hline 95-percent-duration flow & 9.8 & 59.4 & 22.2 & 5.9 & 7.3 \\
\hline 99-percent-duration flow & 3.8 & 16.1 & 13.3 & 1.2 & 3.8 \\
\hline Variability index & 0.65 & 0.42 & 0.52 & 0.52 & 0.63 \\
\hline
\end{tabular}


Table 12. Selected annual and seasonal statistics for 40 stations in surrounding states near West Virginia representative of 1930-2002 (values do not supersede those determined for use by the particular state).-Continued

[Winter, January 1-March 31; spring, April 1-June 30; summer, July 1-September 30; fall, October 1-December 31; streamflow statistics are in cubic feet per second, except for variability index, which is dimensionless; the record period is in climatic years, April 1 through March 31of the indicated ending year; EPA, U.S. Environmental Protection Agency]

\begin{tabular}{|c|c|c|c|c|c|}
\hline \multirow{2}{*}{ Streamflow statistic } & \multirow{2}{*}{ Annual } & \multicolumn{4}{|c|}{ Season } \\
\hline & & Winter & Spring & Summer & Fall \\
\hline \multicolumn{6}{|c|}{03208500 Russell Fork at Haysi, VA, 1930-2002 } \\
\hline 1-day 2-year hydrologically based flow & 8.10 & 82.6 & 40.9 & 9.64 & 12.5 \\
\hline 1-day 5-year hydrologically based flow & 2.70 & 36.6 & 16.7 & 3.62 & 4.32 \\
\hline 1-day 10-year hydrologically based flow & 1.36 & 21.5 & 8.52 & 2.03 & 2.26 \\
\hline 3-day 2-year hydrologically based flow & 8.65 & 92.2 & 44.8 & 10.4 & 13.4 \\
\hline 3-day 5-year hydrologically based flow & 2.96 & 40.5 & 19.0 & 3.91 & 4.78 \\
\hline 3-day 10-year hydrologically based flow & 1.53 & 23.6 & 10.0 & 2.18 & 2.56 \\
\hline 7-day 2-year hydrologically based flow & 9.74 & 114 & 53.3 & 12.1 & 15.8 \\
\hline 7-day 5-year hydrologically based flow & 3.44 & 49.0 & 22.8 & 4.59 & 5.49 \\
\hline 7-day 10-year hydrologically based flow & 1.83 & 27.9 & 12.6 & 2.56 & 2.91 \\
\hline 14-day 2-year hydrologically based flow & 12.1 & 151 & 63.5 & 16.3 & 20.3 \\
\hline 14-day 5-year hydrologically based flow & 4.48 & 65.3 & 31.1 & 6.20 & 6.80 \\
\hline 14-day 10-year hydrologically based flow & 2.43 & 37.7 & 20.3 & 3.42 & 3.53 \\
\hline 30-day 2-year hydrologically based flow & 17.6 & 268 & 104 & 28.5 & 30.8 \\
\hline 30-day 5-year hydrologically based flow & 6.96 & 123 & 52.7 & 11.5 & 10.5 \\
\hline 30-day 10-year hydrologically based flow & 3.92 & 73.1 & 36.8 & 6.46 & 5.69 \\
\hline 1-day 3-year biologically based flow & 0.79 & 9.50 & 17.0 & 1.45 & 1.23 \\
\hline 4-day 3-year biologically based flow & 1.10 & 12.8 & 20.0 & 2.11 & 1.71 \\
\hline EPA harmonic-mean flow & 29.9 & 119 & 149 & 20.8 & 14.3 \\
\hline 5-percent-duration flow & 1,210 & 2,190 & 1,280 & 452 & 714 \\
\hline 10-percent-duration flow & 746 & 1,310 & 787 & 240 & 425 \\
\hline 15-percent-duration flow & 553 & 990 & 619 & 164 & 294 \\
\hline 20-percent-duration flow & 431 & 793 & 502 & 123 & 218 \\
\hline 25-percent-duration flow & 343 & 666 & 415 & 96.1 & 169 \\
\hline 30-percent-duration flow & 285 & 568 & 355 & 80.9 & 133 \\
\hline 35-percent-duration flow & 232 & 497 & 305 & 68.1 & 104 \\
\hline 40-percent-duration flow & 195 & 436 & 263 & 57.8 & 82.3 \\
\hline 45-percent-duration flow & 159 & 391 & 231 & 49.6 & 66.8 \\
\hline 50-percent-duration flow & 129 & 348 & 199 & 42.6 & 54.8 \\
\hline 55-percent-duration flow & 103 & 313 & 177 & 37.4 & 45.7 \\
\hline 60-percent-duration flow & 80.0 & 279 & 155 & 32.8 & 38.1 \\
\hline 65-percent-duration flow & 63.9 & 248 & 134 & 28.4 & 31.5 \\
\hline 70-percent-duration flow & 50.4 & 218 & 114 & 24.5 & 27.2 \\
\hline 75-percent-duration flow & 39.2 & 190 & 95.4 & 20.5 & 23.1 \\
\hline 80-percent-duration flow & 31.0 & 161 & 78.0 & 16.6 & 18.2 \\
\hline 85-percent-duration flow & 23.5 & 128 & 61.4 & 13.0 & 13.0 \\
\hline 90-percent-duration flow & 16.1 & 90.9 & 45.8 & 8.8 & 8.5 \\
\hline 95-percent-duration flow & 8.3 & 49.5 & 29.9 & 4.4 & 4.4 \\
\hline 99-percent-duration flow & 2.1 & 15.6 & 12.4 & 1.6 & 1.4 \\
\hline Variability index & 0.66 & 0.47 & 0.48 & 0.57 & 0.66 \\
\hline
\end{tabular}


Table 12. Selected annual and seasonal statistics for 40 stations in surrounding states near West Virginia representative of 1930-2002 (values do not supersede those determined for use by the particular state).-Continued

[Winter, January 1-March 31; spring, April 1-June 30; summer, July 1-September 30; fall, October 1-December 31; streamflow statistics are in cubic feet per second, except for variability index, which is dimensionless; the record period is in climatic years, April 1 through March 31of the indicated ending year; EPA, U.S. Environmental Protection Agency]

\begin{tabular}{|c|c|c|c|c|c|}
\hline \multirow{2}{*}{ Streamflow statistic } & \multirow{2}{*}{ Annual } & \multicolumn{4}{|c|}{ Season } \\
\hline & & Winter & Spring & Summer & Fall \\
\hline \multicolumn{6}{|c|}{03208950 Cranes Nest River near Clintwood, VA, 1965-1974 } \\
\hline 1-day 2-year hydrologically based flow & 2.38 & 30.9 & 7.31 & 2.47 & 5.12 \\
\hline 1-day 5-year hydrologically based flow & 1.31 & 12.6 & 4.02 & 1.36 & 2.57 \\
\hline 1-day 10-year hydrologically based flow & 0.95 & 6.32 & 2.89 & 0.98 & 1.85 \\
\hline 3-day 2-year hydrologically based flow & 2.64 & 34.2 & 8.20 & 2.72 & 5.59 \\
\hline 3-day 5-year hydrologically based flow & 1.56 & 13.7 & 4.56 & 1.61 & 2.85 \\
\hline 3-day 10 -year hydrologically based flow & 1.19 & 6.70 & 3.29 & 1.23 & 2.07 \\
\hline 7-day 2-year hydrologically based flow & 3.19 & 40.7 & 10.8 & 3.69 & 6.35 \\
\hline 7-day 5-year hydrologically based flow & 1.87 & 16.2 & 5.77 & 2.14 & 3.23 \\
\hline 7-day 10 -year hydrologically based flow & 1.40 & 7.73 & 4.03 & 1.54 & 2.38 \\
\hline 14-day 2-year hydrologically based flow & 3.81 & 47.2 & 14.9 & 4.72 & 8.36 \\
\hline 14-day 5-year hydrologically based flow & 2.31 & 17.6 & 8.88 & 2.59 & 3.96 \\
\hline 14-day 10 -year hydrologically based flow & 1.81 & 8.25 & 6.71 & 1.93 & 2.82 \\
\hline 30-day 2-year hydrologically based flow & 5.45 & 74.8 & 23.9 & 6.87 & 13.6 \\
\hline 30-day 5-year hydrologically based flow & 3.72 & 31.2 & 12.4 & 4.02 & 6.72 \\
\hline 30-day 10-year hydrologically based flow & 3.15 & 15.3 & 9.01 & 3.28 & 4.73 \\
\hline 1-day 3-year biologically based flow & 0.84 & 1.90 & 4.28 & 1.25 & 0.90 \\
\hline 4-day 3-year biologically based flow & 1.08 & 2.13 & 5.44 & 1.86 & 1.69 \\
\hline EPA harmonic-mean flow & 14.0 & 31.4 & 39.6 & 9.22 & 8.31 \\
\hline 5-percent-duration flow & 282 & 431 & 308 & 126 & 228 \\
\hline 10-percent-duration flow & 174 & 278 & 182 & 75.1 & 135 \\
\hline 15-percent-duration flow & 131 & 210 & 132 & 49.3 & 97.1 \\
\hline 20-percent-duration flow & 104 & 173 & 108 & 35.7 & 77.0 \\
\hline 25-percent-duration flow & 88.5 & 148 & 92.6 & 28.5 & 61.2 \\
\hline 30-percent-duration flow & 75.2 & 128 & 80.6 & 23.4 & 50.8 \\
\hline 35-percent-duration flow & 63.7 & 115 & 70.7 & 19.0 & 43.3 \\
\hline 40-percent-duration flow & 55.0 & 103 & 62.9 & 15.8 & 37.0 \\
\hline 45-percent-duration flow & 46.7 & 94.0 & 56.0 & 13.4 & 30.5 \\
\hline 50-percent-duration flow & 38.8 & 86.7 & 49.7 & 11.5 & 24.7 \\
\hline 55-percent-duration flow & 31.8 & 79.5 & 44.2 & 9.8 & 20.2 \\
\hline 60-percent-duration flow & 25.7 & 72.9 & 38.8 & 8.4 & 16.6 \\
\hline 65-percent-duration flow & 20.4 & 66.3 & 33.4 & 7.3 & 13.3 \\
\hline 70-percent-duration flow & 15.9 & 59.8 & 27.6 & 6.4 & 10.0 \\
\hline 75-percent-duration flow & 12.2 & 53.5 & 23.1 & 5.6 & 7.8 \\
\hline 80-percent-duration flow & 8.8 & 46.2 & 18.8 & 4.8 & 6.4 \\
\hline 85-percent-duration flow & 6.5 & 37.8 & 14.8 & 4.1 & 5.3 \\
\hline 90-percent-duration flow & 5.0 & 28.4 & 12.1 & 3.4 & 4.6 \\
\hline 95-percent-duration flow & 3.7 & 17.7 & 7.2 & 2.5 & 3.8 \\
\hline 99-percent-duration flow & 2.0 & 2.2 & 3.8 & 1.3 & 2.1 \\
\hline Variability index & 0.59 & 0.39 & 0.47 & 0.52 & 0.57 \\
\hline
\end{tabular}


Table 12. Selected annual and seasonal statistics for 40 stations in surrounding states near West Virginia representative of 1930-2002 (values do not supersede those determined for use by the particular state).-Continued

[Winter, January 1-March 31; spring, April 1-June 30; summer, July 1-September 30; fall, October 1-December 31; streamflow statistics are in cubic feet per second, except for variability index, which is dimensionless; the record period is in climatic years, April 1 through March 31of the indicated ending year; EPA, U.S. Environmental Protection Agency]

\begin{tabular}{|c|c|c|c|c|c|}
\hline \multirow{2}{*}{ Streamflow statistic } & \multirow{2}{*}{ Annual } & \multicolumn{4}{|c|}{ Season } \\
\hline & & Winter & Spring & Summer & Fall \\
\hline \multicolumn{6}{|c|}{03210000 Johns Creek near Meta, KY, 1942-1980 } \\
\hline 1-day 2-year hydrologically based flow & 0.31 & 17.4 & 2.69 & 0.42 & 1.20 \\
\hline 1-day 5-year hydrologically based flow & 0.00 & 6.42 & 0.93 & 0.00 & 0.10 \\
\hline 1-day 10-year hydrologically based flow & 0.00 & 3.24 & 0.50 & 0.00 & 0.00 \\
\hline 3-day 2-year hydrologically based flow & 0.37 & 19.6 & 3.11 & 0.55 & 1.32 \\
\hline 3-day 5-year hydrologically based flow & 0.00 & 7.16 & 1.12 & 0.00 & 0.13 \\
\hline 3-day 10-year hydrologically based flow & 0.00 & 3.59 & 0.63 & 0.00 & 0.00 \\
\hline 7-day 2-year hydrologically based flow & 0.42 & 24.6 & 4.04 & 0.68 & 1.60 \\
\hline 7-day 5-year hydrologically based flow & 0.00 & 9.23 & 1.52 & 0.06 & 0.16 \\
\hline 7-day 10-year hydrologically based flow & 0.00 & 4.62 & 0.87 & 0.00 & 0.00 \\
\hline 14-day 2-year hydrologically based flow & 0.76 & 33.9 & 5.90 & 1.18 & 2.17 \\
\hline 14-day 5-year hydrologically based flow & 0.07 & 13.7 & 2.51 & 0.22 & 0.51 \\
\hline 14-day 10-year hydrologically based flow & 0.00 & 7.20 & 1.60 & 0.00 & 0.16 \\
\hline 30-day 2-year hydrologically based flow & 2.04 & 62.8 & 11.9 & 3.08 & 4.46 \\
\hline 30-day 5-year hydrologically based flow & 0.28 & 31.1 & 4.97 & 0.50 & 1.13 \\
\hline 30-day 10-year hydrologically based flow & 0.03 & 19.2 & 3.12 & 0.11 & 0.49 \\
\hline 1-day 3-year biologically based flow & 0.00 & 0.75 & 0.78 & 0.00 & 0.00 \\
\hline 4-day 3-year biologically based flow & 0.00 & 1.55 & 1.38 & 0.00 & 0.00 \\
\hline EPA harmonic-mean flow & 3.25 & 18.6 & 15.0 & 1.69 & 1.87 \\
\hline 5-percent-duration flow & 279 & 497 & 276 & 80.3 & 184 \\
\hline 10-percent-duration flow & 170 & 306 & 167 & 41.4 & 105 \\
\hline 15-percent-duration flow & 120 & 228 & 117 & 26.0 & 71.4 \\
\hline 20-percent-duration flow & 90.0 & 182 & 90.4 & 17.9 & 51.3 \\
\hline 25-percent-duration flow & 72.7 & 153 & 72.6 & 13.1 & 39.1 \\
\hline 30-percent-duration flow & 57.7 & 131 & 60.0 & 10.0 & 31.1 \\
\hline 35-percent-duration flow & 46.4 & 114 & 49.6 & 8.0 & 24.2 \\
\hline 40-percent-duration flow & 37.1 & 101 & 41.2 & 6.5 & 19.1 \\
\hline 45-percent-duration flow & 28.7 & 89.7 & 34.9 & 5.3 & 15.1 \\
\hline 50-percent-duration flow & 22.1 & 79.8 & 28.9 & 4.4 & 11.3 \\
\hline 55-percent-duration flow & 16.6 & 71.7 & 24.2 & 3.6 & 8.2 \\
\hline 60-percent-duration flow & 12.2 & 63.7 & 19.8 & 3.0 & 6.5 \\
\hline 65-percent-duration flow & 8.7 & 57.1 & 16.2 & 2.5 & 5.2 \\
\hline 70-percent-duration flow & 6.5 & 50.5 & 13.1 & 2.0 & 4.1 \\
\hline 75-percent-duration flow & 4.8 & 43.7 & 10.1 & 1.5 & 2.9 \\
\hline 80-percent-duration flow & 3.4 & 37.0 & 7.6 & 1.1 & 2.2 \\
\hline 85-percent-duration flow & 2.3 & 30.6 & 5.8 & 0.7 & 1.5 \\
\hline 90-percent-duration flow & 1.3 & 20.1 & 4.2 & 0.4 & 0.9 \\
\hline 95-percent-duration flow & 0.6 & 11.5 & 2.6 & 0.1 & 0.3 \\
\hline 99-percent-duration flow & 0.0 & 2.0 & 0.8 & 0.0 & 0.0 \\
\hline Variability index & 0.82 & 0.46 & 0.62 & 0.82 & 0.83 \\
\hline
\end{tabular}


Table 12. Selected annual and seasonal statistics for 40 stations in surrounding states near West Virginia representative of 1930-2002 (values do not supersede those determined for use by the particular state).-Continued

[Winter, January 1-March 31; spring, April 1-June 30; summer, July 1-September 30; fall, October 1-December 31; streamflow statistics are in cubic feet per second, except for variability index, which is dimensionless; the record period is in climatic years, April 1 through March 31 of the indicated ending year; EPA, U.S. Environmental Protection Agency]

\begin{tabular}{|c|c|c|c|c|c|}
\hline \multirow{2}{*}{ Streamflow statistic } & \multirow{2}{*}{ Annual } & \multicolumn{4}{|c|}{ Season } \\
\hline & & Winter & Spring & Summer & Fall \\
\hline \multicolumn{6}{|c|}{03211500 Johns Creek near Van Lear, KY, 1941-1981 } \\
\hline 1-day 2-year hydrologically based flow & 4.69 & 21.3 & 12.4 & 8.49 & 10.3 \\
\hline 1-day 5-year hydrologically based flow & 0.33 & 7.61 & 4.68 & 3.09 & 3.11 \\
\hline 1-day 10-year hydrologically based flow & 0.00 & 0.00 & 2.04 & 1.53 & 1.18 \\
\hline 3-day 2-year hydrologically based flow & 6.56 & 36.2 & 14.5 & 8.99 & 11.5 \\
\hline 3-day 5 -year hydrologically based flow & 1.66 & 14.8 & 7.06 & 3.39 & 3.98 \\
\hline 3-day 10-year hydrologically based flow & 0.35 & 7.09 & 4.42 & 1.72 & 1.88 \\
\hline 7-day 2-year hydrologically based flow & 8.54 & 63.5 & 17.6 & 9.89 & 12.9 \\
\hline 7-day 5-year hydrologically based flow & 3.29 & 28.7 & 8.51 & 3.92 & 4.35 \\
\hline 7-day 10 -year hydrologically based flow & 1.64 & 18.0 & 5.38 & 2.06 & 2.07 \\
\hline 14-day 2-year hydrologically based flow & 9.68 & 98.1 & 22.3 & 11.5 & 14.9 \\
\hline 14-day 5-year hydrologically based flow & 3.81 & 42.8 & 11.4 & 4.75 & 4.70 \\
\hline 14-day 10-year hydrologically based flow & 1.90 & 25.3 & 7.88 & 2.62 & 2.25 \\
\hline 30-day 2-year hydrologically based flow & 11.6 & 189 & 37.6 & 14.5 & 20.2 \\
\hline 30-day 5-year hydrologically based flow & 4.63 & 87.8 & 18.3 & 6.08 & 6.68 \\
\hline 30-day 10-year hydrologically based flow & 2.50 & 53.9 & 12.8 & 3.63 & 3.65 \\
\hline 1-day 3-year biologically based flow & 0.00 & 2.94 & 1.04 & 1.09 & 0.78 \\
\hline 4-day 3-year biologically based flow & 0.85 & 11.0 & 6.20 & 1.84 & 0.92 \\
\hline EPA harmonic-mean flow & 17.8 & 82.8 & 42.6 & 12.4 & 9.27 \\
\hline 5-percent-duration flow & 1,080 & 2,000 & 1,070 & 282 & 706 \\
\hline 10-percent-duration flow & 604 & 1,190 & 589 & 127 & 437 \\
\hline 15-percent-duration flow & 421 & 857 & 395 & 76.3 & 309 \\
\hline 20-percent-duration flow & 321 & 660 & 291 & 52.8 & 220 \\
\hline 25-percent-duration flow & 245 & 541 & 228 & 40.0 & 162 \\
\hline 30-percent-duration flow & 188 & 461 & 182 & 31.9 & 126 \\
\hline 35-percent-duration flow & 146 & 396 & 144 & 26.3 & 104 \\
\hline 40-percent-duration flow & 114 & 345 & 118 & 23.6 & 82.7 \\
\hline 45-percent-duration flow & 88.7 & 302 & 97.5 & 20.9 & 63.4 \\
\hline 50-percent-duration flow & 66.5 & 264 & 79.0 & 18.9 & 45.3 \\
\hline 55-percent-duration flow & 48.1 & 230 & 61.6 & 17.4 & 33.1 \\
\hline 60-percent-duration flow & 35.2 & 198 & 49.7 & 15.8 & 25.6 \\
\hline 65-percent-duration flow & 27.1 & 171 & 38.1 & 14.4 & 20.5 \\
\hline 70-percent-duration flow & 21.8 & 145 & 30.4 & 13.3 & 16.5 \\
\hline 75-percent-duration flow & 18.4 & 121 & 25.6 & 12.2 & 13.8 \\
\hline 80-percent-duration flow & 15.3 & 98.0 & 21.6 & 11.1 & 12.0 \\
\hline 85-percent-duration flow & 12.9 & 73.7 & 18.6 & 9.6 & 10.3 \\
\hline 90-percent-duration flow & 10.6 & 43.4 & 14.9 & 7.5 & 8.7 \\
\hline 95-percent-duration flow & 7.6 & 22.8 & 11.1 & 3.5 & 5.0 \\
\hline 99-percent-duration flow & 1.3 & 8.2 & 5.4 & 0.9 & 0.9 \\
\hline Variability index & 0.70 & 0.55 & 0.63 & 0.52 & 0.69 \\
\hline
\end{tabular}


Table 12. Selected annual and seasonal statistics for 40 stations in surrounding states near West Virginia representative of 1930-2002 (values do not supersede those determined for use by the particular state)._-Continued

[Winter, January 1-March 31; spring, April 1-June 30; summer, July 1-September 30; fall, October 1-December 31; streamflow statistics are in cubic feet per second, except for variability index, which is dimensionless; the record period is in climatic years, April 1 through March 31 of the indicated ending year; EPA, U.S. Environmental Protection Agency]

\begin{tabular}{|c|c|c|c|c|c|}
\hline \multirow{2}{*}{ Streamflow statistic } & \multirow{2}{*}{ Annual } & \multicolumn{4}{|c|}{ Season } \\
\hline & & Winter & Spring & Summer & Fall \\
\hline \multicolumn{6}{|c|}{03212000 Paint Creek at Staffordsville, KY, 1955-1975 } \\
\hline 1-day 2-year hydrologically based flow & 1.28 & 23.7 & 3.64 & 1.28 & 2.69 \\
\hline 1-day 5-year hydrologically based flow & 0.66 & 9.51 & 2.19 & 0.81 & 1.24 \\
\hline 1-day 10-year hydrologically based flow & 0.25 & 5.15 & 1.74 & 0.51 & 0.78 \\
\hline 3-day 2-year hydrologically based flow & 1.61 & 28.1 & 4.65 & 1.71 & 3.19 \\
\hline 3-day 5-year hydrologically based flow & 0.66 & 11.3 & 2.61 & 0.70 & 1.49 \\
\hline 3-day 10-year hydrologically based flow & 0.30 & 6.27 & 1.93 & 0.31 & 0.96 \\
\hline 7-day 2-year hydrologically based flow & 1.82 & 36.2 & 6.41 & 2.01 & 3.93 \\
\hline 7-day 5-year hydrologically based flow & 0.77 & 16.4 & 3.40 & 0.85 & 1.78 \\
\hline 7-day 10 -year hydrologically based flow & 0.38 & 9.83 & 2.44 & 0.41 & 1.11 \\
\hline 14-day 2-year hydrologically based flow & 2.42 & 51.2 & 10.6 & 3.16 & 5.46 \\
\hline 14-day 5-year hydrologically based flow & 0.95 & 22.1 & 5.47 & 1.18 & 2.23 \\
\hline 14-day 10 -year hydrologically based flow & 0.49 & 12.8 & 3.81 & 0.58 & 1.31 \\
\hline 30-day 2-year hydrologically based flow & 4.03 & 85.7 & 19.9 & 5.14 & 10.1 \\
\hline 30-day 5-year hydrologically based flow & 2.16 & 46.1 & 9.59 & 2.86 & 4.28 \\
\hline 30-day 10-year hydrologically based flow & 1.51 & 31.9 & 6.51 & 2.10 & 2.54 \\
\hline 1-day 3-year biologically based flow & 0.18 & 2.62 & 3.36 & 0.18 & 0.51 \\
\hline 4-day 3-year biologically based flow & 0.31 & 3.27 & 3.97 & 0.43 & 0.64 \\
\hline EPA harmonic-mean flow & 8.11 & 35.4 & 33.5 & 4.20 & 5.10 \\
\hline 5-percent-duration flow & 535 & 923 & 623 & 156 & 320 \\
\hline 10-percent-duration flow & 305 & 567 & 345 & 70.3 & 155 \\
\hline 15-percent-duration flow & 197 & 403 & 227 & 45.1 & 99.0 \\
\hline 20-percent-duration flow & 146 & 312 & 163 & 30.3 & 71.4 \\
\hline 25-percent-duration flow & 112 & 252 & 124 & 22.3 & 55.2 \\
\hline 30-percent-duration flow & 87.9 & 208 & 97.5 & 16.9 & 42.9 \\
\hline 35-percent-duration flow & 71.0 & 179 & 80.1 & 13.5 & 33.4 \\
\hline 40-percent-duration flow & 57.7 & 154 & 67.2 & 11.1 & 26.3 \\
\hline 45-percent-duration flow & 45.9 & 135 & 56.7 & 9.1 & 21.1 \\
\hline 50-percent-duration flow & 34.8 & 118 & 47.7 & 7.7 & 17.6 \\
\hline 55-percent-duration flow & 26.7 & 105 & 40.1 & 6.3 & 14.9 \\
\hline 60-percent-duration flow & 20.4 & 93.2 & 33.2 & 5.2 & 12.5 \\
\hline 65-percent-duration flow & 15.6 & 82.0 & 27.6 & 4.4 & 10.1 \\
\hline 70-percent-duration flow & 11.9 & 73.0 & 23.0 & 3.7 & 8.3 \\
\hline 75-percent-duration flow & 8.4 & 65.1 & 18.5 & 3.0 & 6.7 \\
\hline 80-percent-duration flow & 6.2 & 56.9 & 13.9 & 2.5 & 5.4 \\
\hline 85-percent-duration flow & 4.6 & 48.5 & 10.2 & 2.1 & 4.1 \\
\hline 90-percent-duration flow & 3.2 & 36.5 & 7.0 & 1.7 & 3.1 \\
\hline 95-percent-duration flow & 2.0 & 19.8 & 4.3 & 1.2 & 1.7 \\
\hline 99-percent-duration flow & 0.8 & 5.0 & 2.3 & 0.2 & 0.7 \\
\hline Variability index & 0.76 & 0.48 & 0.64 & 0.64 & 0.68 \\
\hline
\end{tabular}


Table 12. Selected annual and seasonal statistics for 40 stations in surrounding states near West Virginia representative of 1930-2002 (values do not supersede those determined for use by the particular state).—Continued

[Winter, January 1-March 31; spring, April 1-June 30; summer, July 1-September 30; fall, October 1-December 31; streamflow statistics are in cubic feet per second, except for variability index, which is dimensionless; the record period is in climatic years, April 1 through March 31of the indicated ending year; EPA, U.S. Environmental Protection Agency]

\begin{tabular}{|c|c|c|c|c|c|}
\hline \multirow{2}{*}{ Streamflow statistic } & \multirow{2}{*}{ Annual } & \multicolumn{4}{|c|}{ Season } \\
\hline & & Winter & Spring & Summer & Fall \\
\hline \multicolumn{6}{|c|}{03215500 Blaine Creek at Yatesville, KY, 1955-1975 } \\
\hline 1-day 2-year hydrologically based flow & 2.28 & 44.8 & 11.0 & 2.85 & 4.04 \\
\hline 1-day 5-year hydrologically based flow & 0.69 & 14.8 & 6.90 & 0.81 & 1.69 \\
\hline 1-day 10-year hydrologically based flow & 0.32 & 7.13 & 5.49 & 0.35 & 1.01 \\
\hline 3-day 2-year hydrologically based flow & 2.63 & 55.7 & 12.4 & 3.12 & 4.70 \\
\hline 3-day 5-year hydrologically based flow & 0.79 & 17.8 & 7.45 & 0.90 & 2.06 \\
\hline 3-day 10-year hydrologically based flow & 0.35 & 8.29 & 5.83 & 0.38 & 1.26 \\
\hline 7-day 2-year hydrologically based flow & 3.08 & 69.3 & 15.3 & 3.73 & 5.71 \\
\hline 7-day 5-year hydrologically based flow & 0.97 & 24.8 & 9.08 & 1.14 & 2.38 \\
\hline 7-day 10-year hydrologically based flow & 0.45 & 12.6 & 7.07 & 0.51 & 1.40 \\
\hline 14-day 2-year hydrologically based flow & 4.08 & 95.6 & 21.0 & 4.91 & 7.29 \\
\hline 14-day 5-year hydrologically based flow & 1.68 & 36.8 & 12.0 & 1.97 & 2.82 \\
\hline 14-day 10 -year hydrologically based flow & 0.96 & 19.4 & 9.12 & 1.12 & 1.59 \\
\hline 30-day 2-year hydrologically based flow & 6.18 & 168 & 45.7 & 7.57 & 11.8 \\
\hline 30-day 5-year hydrologically based flow & 2.92 & 86.1 & 23.4 & 3.57 & 4.21 \\
\hline 30-day 10 -year hydrologically based flow & 1.85 & 57.5 & 16.4 & 2.35 & 2.33 \\
\hline 1-day 3-year biologically based flow & 0.09 & 3.13 & 8.16 & 0.29 & 0.28 \\
\hline 4-day 3-year biologically based flow & 0.31 & 3.56 & 11.5 & 0.74 & 0.57 \\
\hline EPA harmonic-mean flow & 11.0 & 46.3 & 80.0 & 6.79 & 5.52 \\
\hline 5-percent-duration flow & 1,000 & 1,590 & 1,230 & 249 & 563 \\
\hline 10-percent-duration flow & 571 & 1,060 & 643 & 121 & 312 \\
\hline 15-percent-duration flow & 406 & 753 & 474 & 69.4 & 196 \\
\hline 20-percent-duration flow & 297 & 612 & 349 & 44.8 & 135 \\
\hline 25-percent-duration flow & 226 & 511 & 257 & 33.9 & 97.1 \\
\hline 30-percent-duration flow & 179 & 433 & 207 & 26.8 & 66.1 \\
\hline 35-percent-duration flow & 141 & 375 & 173 & 21.6 & 50.0 \\
\hline 40-percent-duration flow & 112 & 321 & 144 & 17.7 & 37.7 \\
\hline 45-percent-duration flow & 88.0 & 282 & 122 & 15.4 & 29.3 \\
\hline 50-percent-duration flow & 65.5 & 245 & 105 & 13.3 & 23.9 \\
\hline 55-percent-duration flow & 47.3 & 219 & 91.4 & 11.5 & 19.4 \\
\hline 60-percent-duration flow & 34.3 & 193 & 77.2 & 9.7 & 15.8 \\
\hline 65-percent-duration flow & 25.0 & 171 & 62.7 & 8.5 & 13.1 \\
\hline 70-percent-duration flow & 18.5 & 149 & 51.2 & 7.3 & 10.6 \\
\hline 75-percent-duration flow & 14.4 & 129 & 42.0 & 6.3 & 8.3 \\
\hline 80-percent-duration flow & 10.8 & 109 & 31.1 & 5.3 & 6.5 \\
\hline 85-percent-duration flow & 8.0 & 91.5 & 23.6 & 4.4 & 4.9 \\
\hline 90-percent-duration flow & 5.6 & 68.3 & 16.6 & 3.3 & 3.4 \\
\hline 95-percent-duration flow & 3.3 & 32.6 & 11.3 & 2.2 & 2.2 \\
\hline 99-percent-duration flow & 0.8 & 5.1 & 6.9 & 0.3 & 0.7 \\
\hline Variability index & 0.79 & 0.48 & 0.61 & 0.60 & 0.74 \\
\hline
\end{tabular}




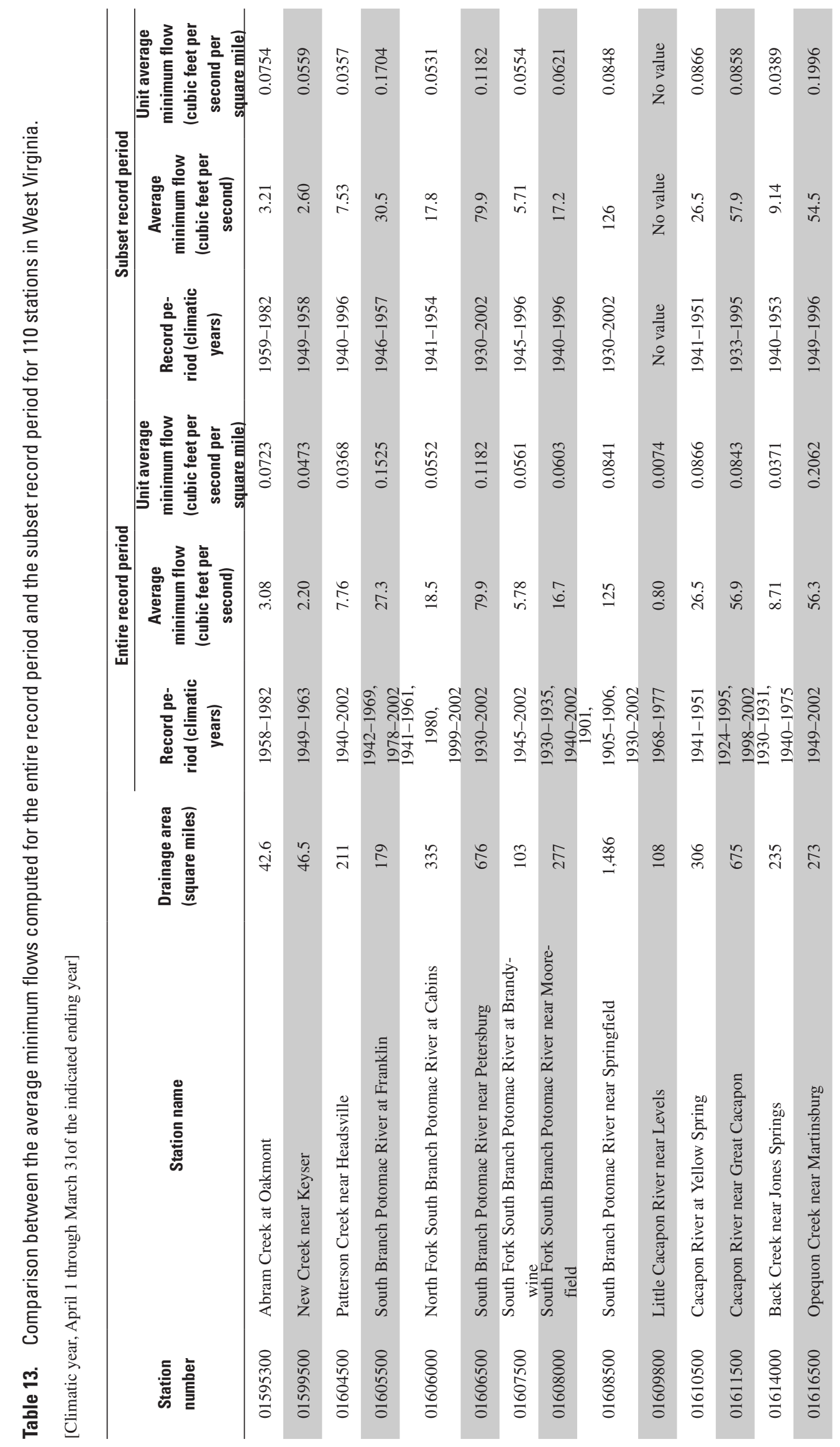




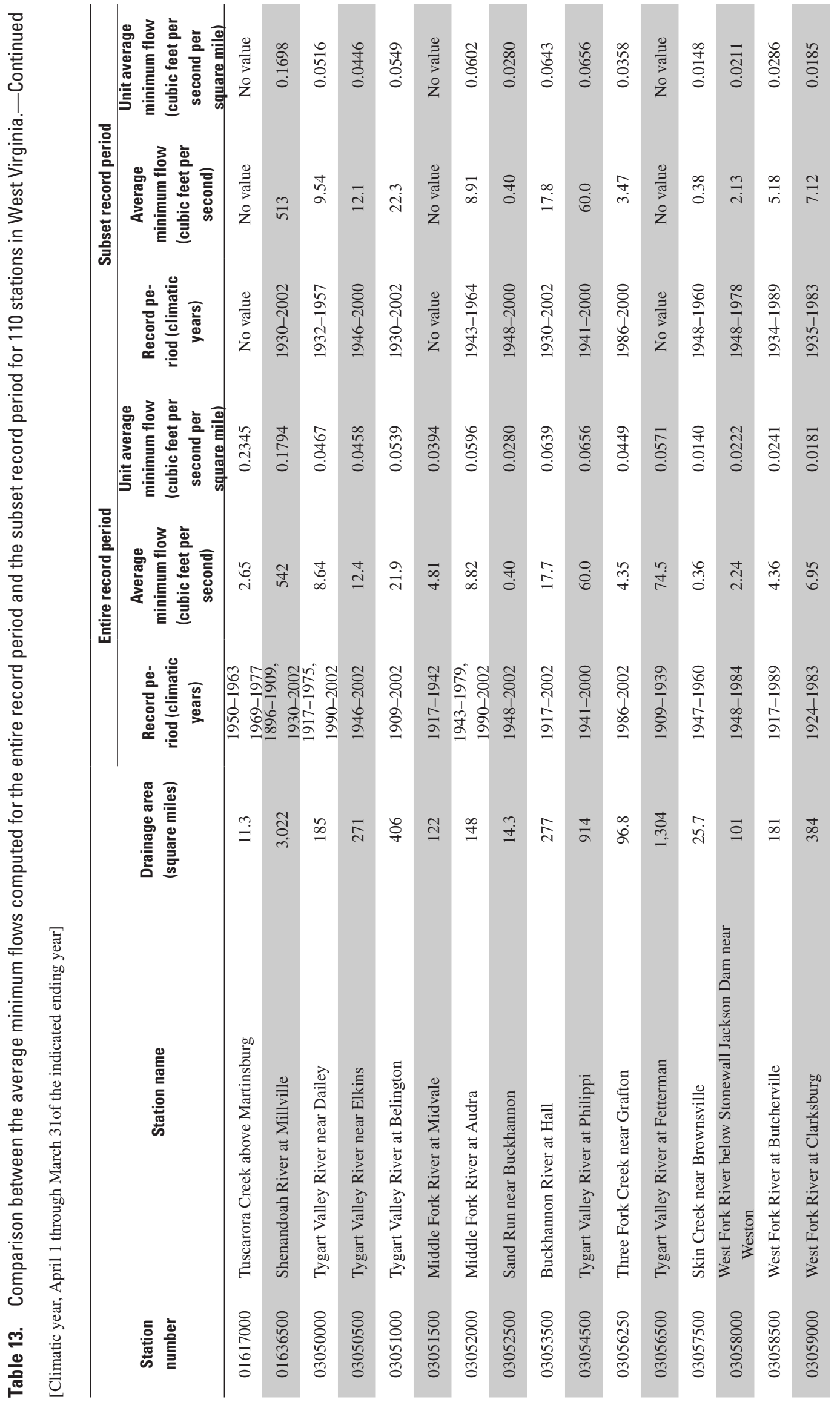




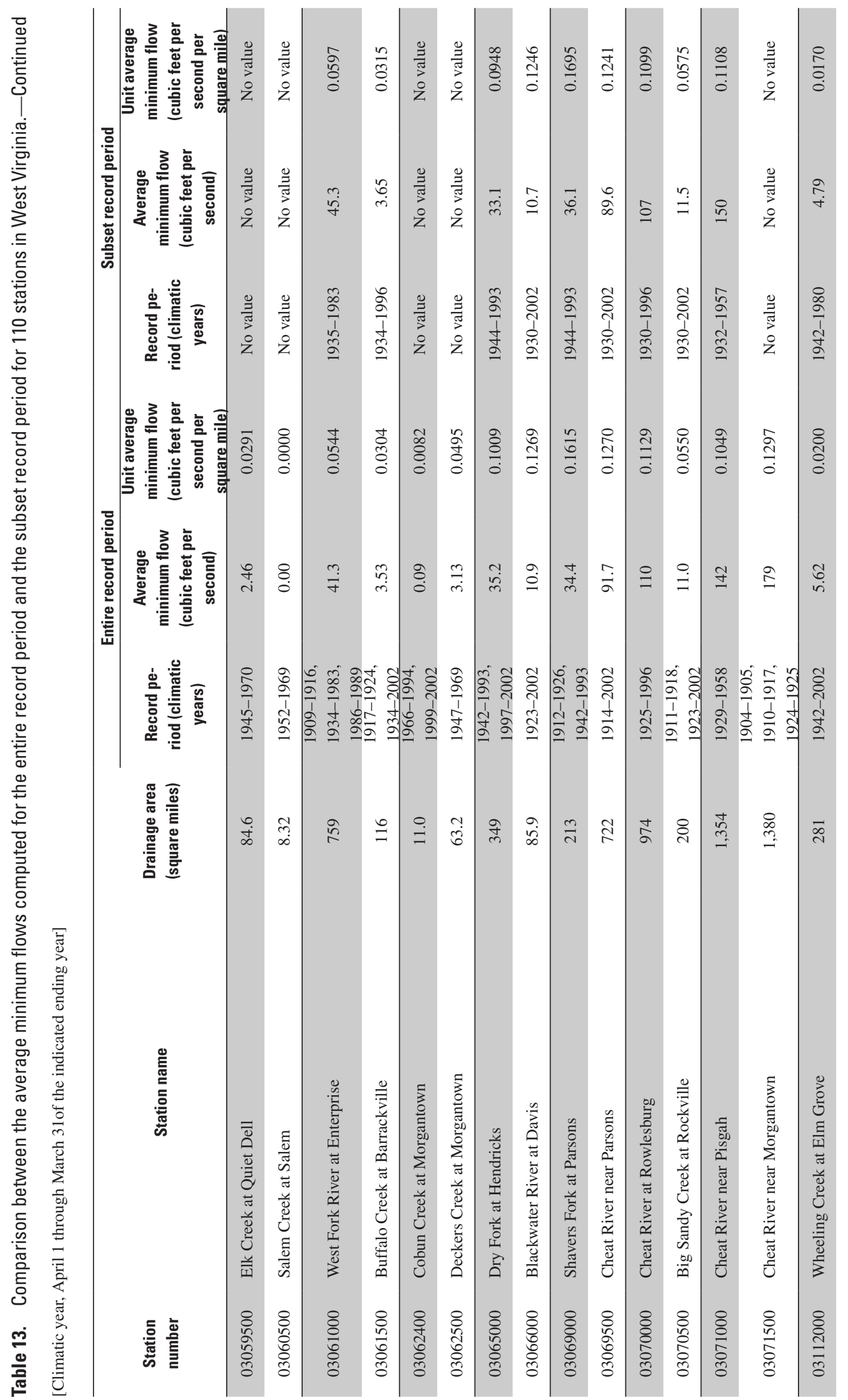




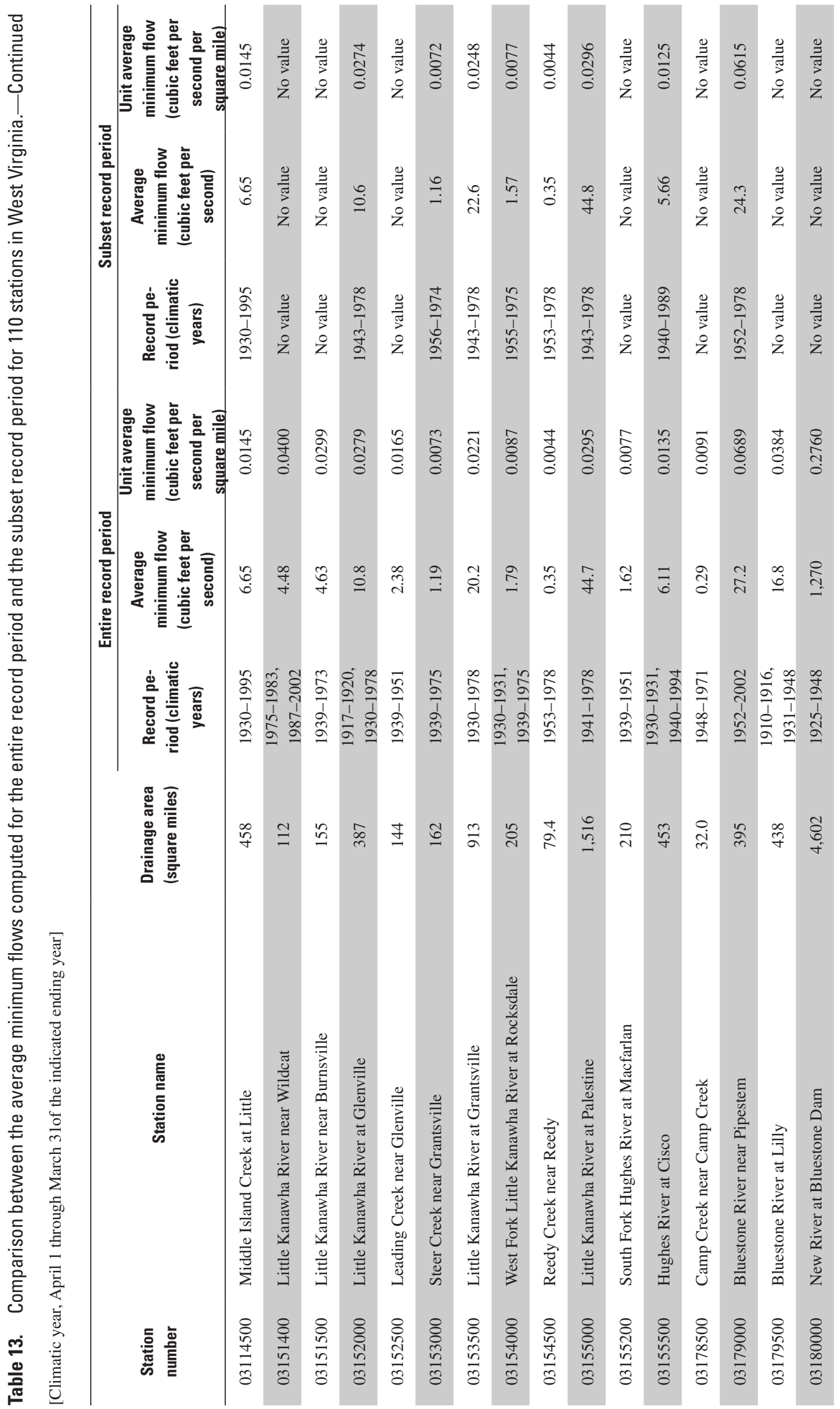




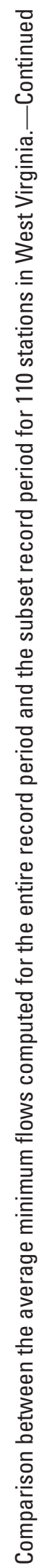

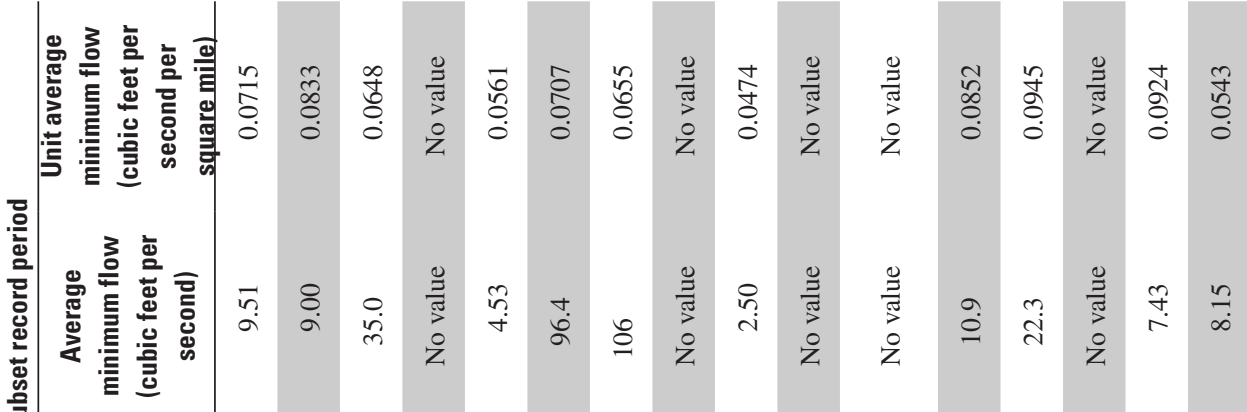

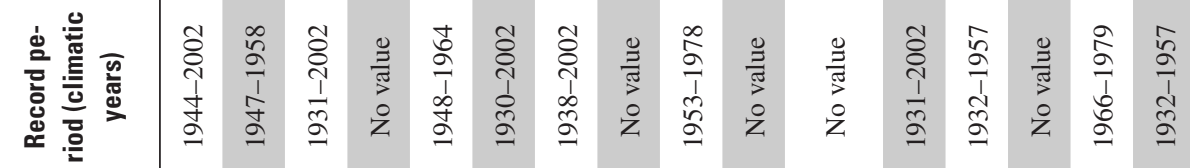

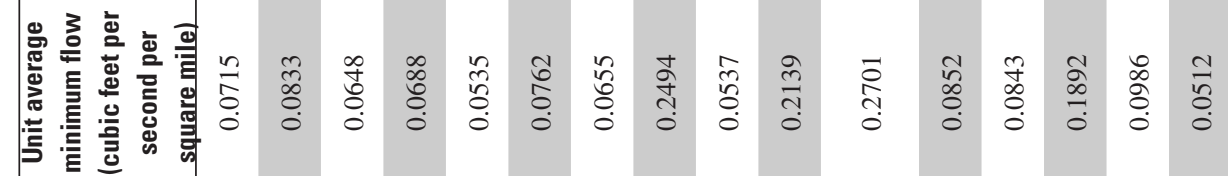
흔

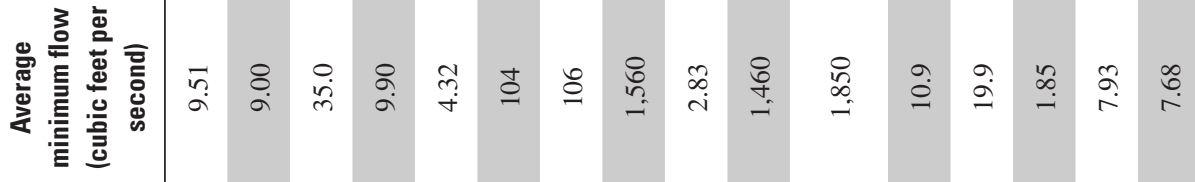

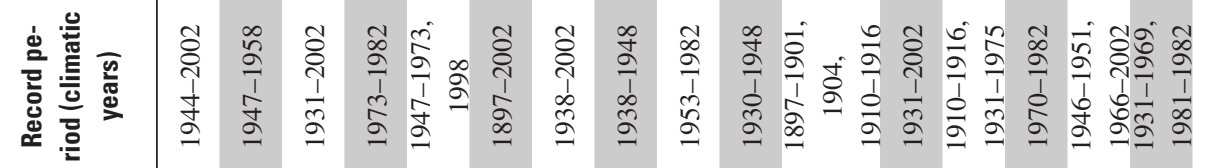

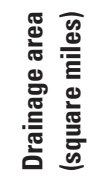

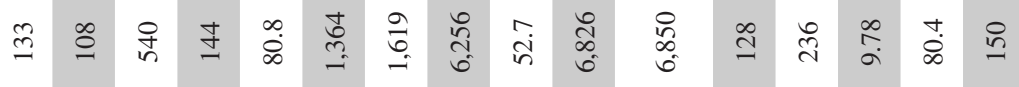

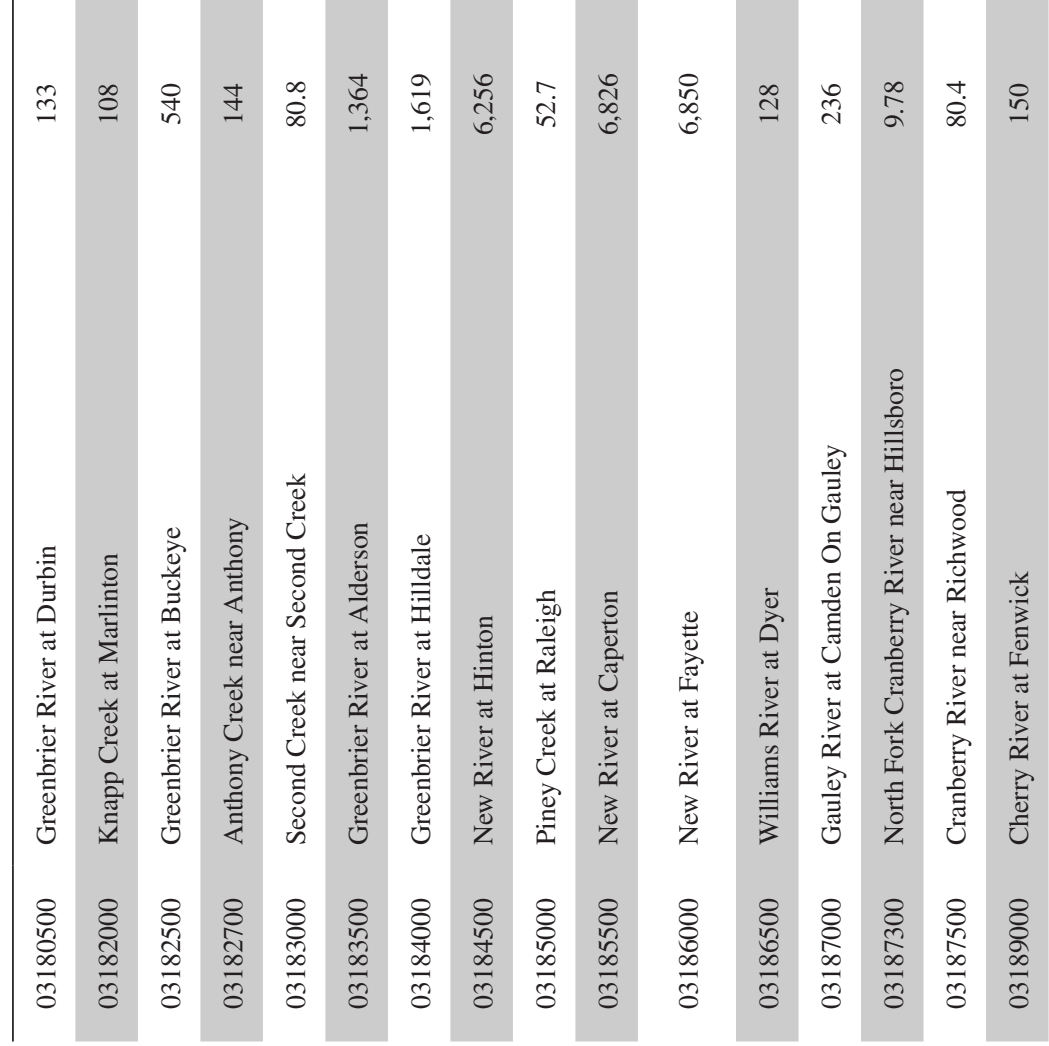




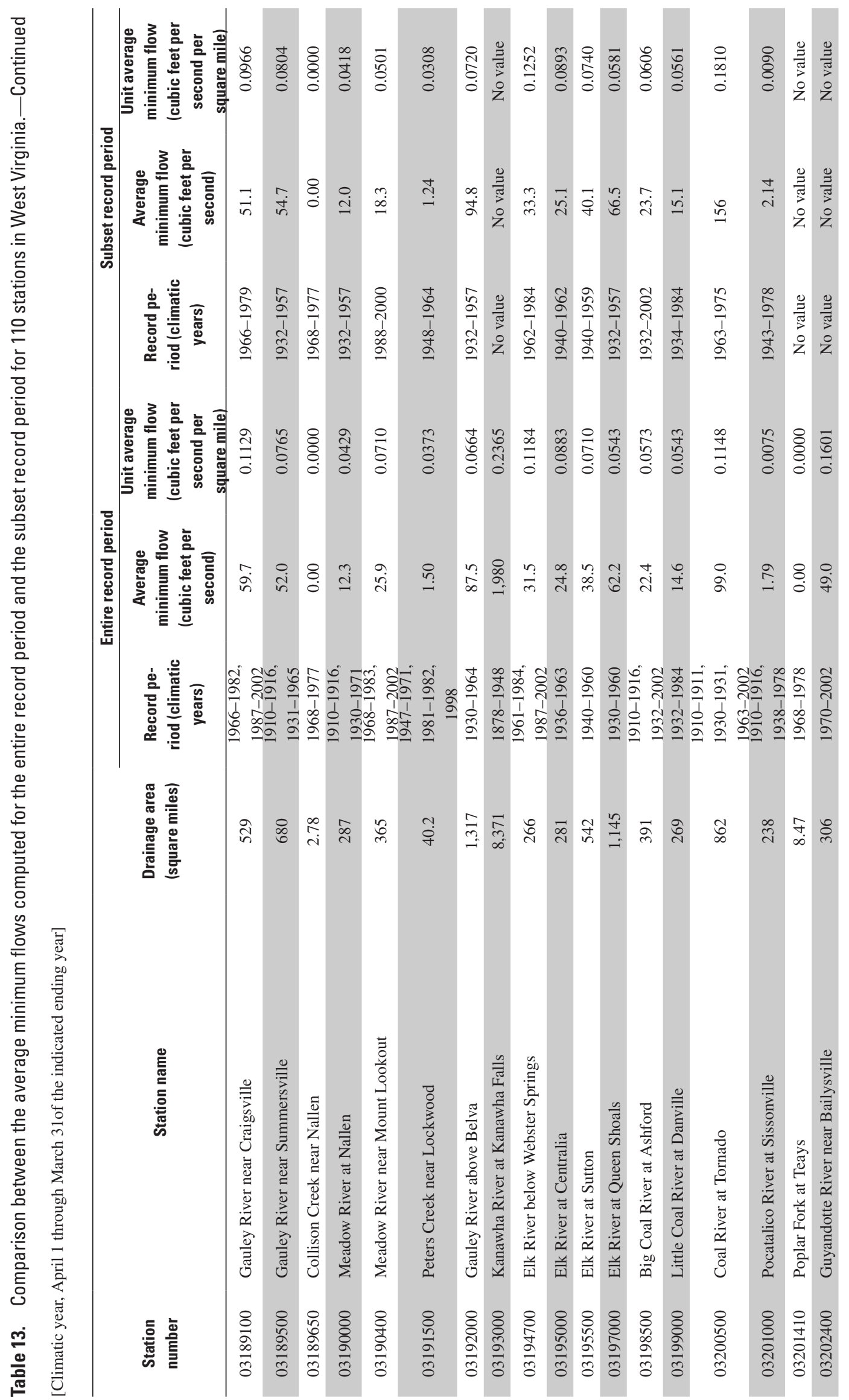




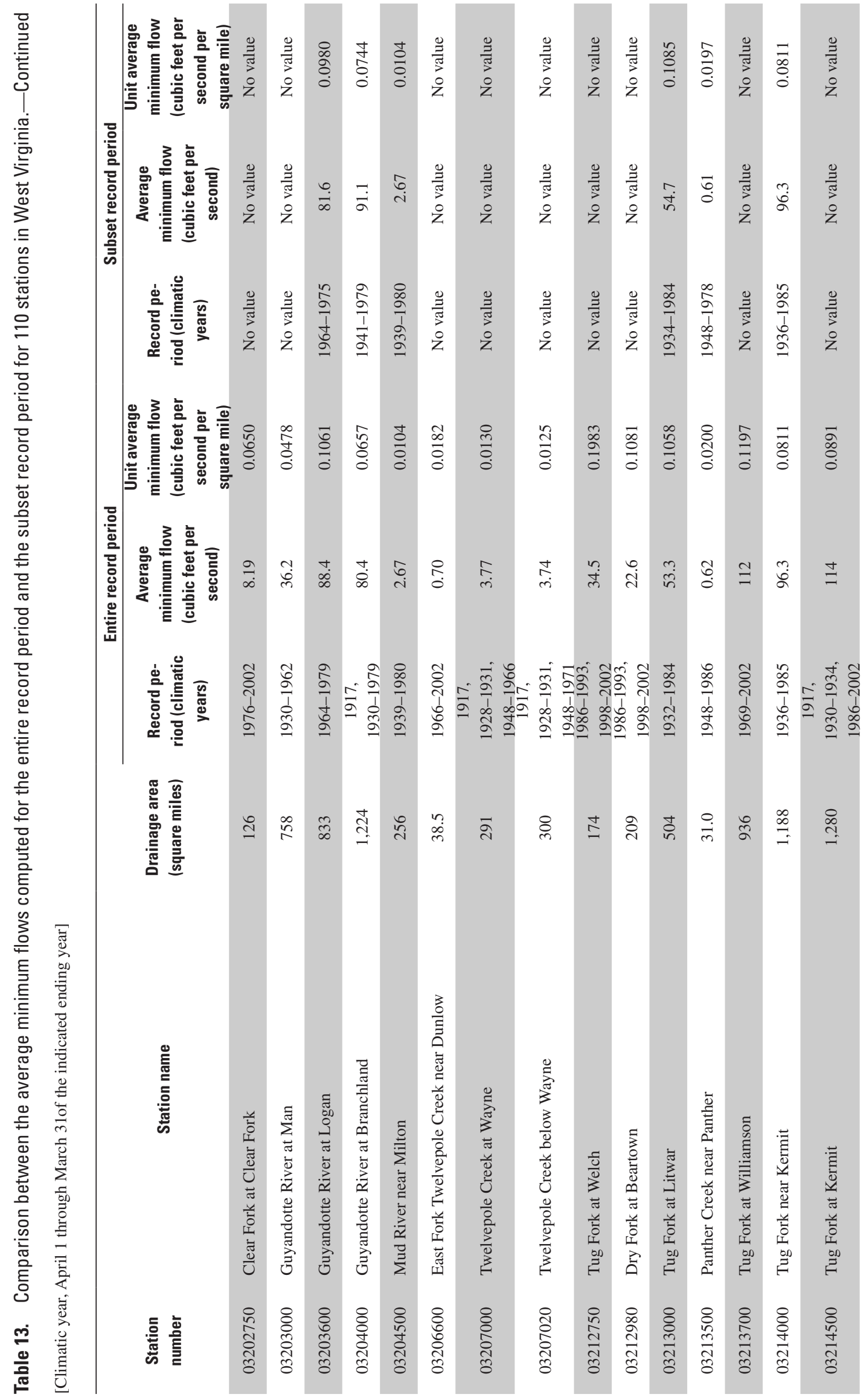


Printed on recycled paper 